\title{
Parametric Uncertainty Quantification in Coalescence Flutter
}

\author{
A thesis submitted to \\ the Faculty of Graduate and Postdoctorial Affairs \\ in Partial Fullfillment of the requirements for the degree \\ Master of Applied Science in the subject of Civil Engineering
}

by

Anton Matachniouk

Department of Civil and Environmental Engineering

Carleton University

The Ottawa-Carleton Institute of Civil and Environmental Engineering

Jan. 2013

C2013 Anton Matachniouk 
Library and Archives

Canada

Published Heritage

Branch

395 Wellington Street

Ottawa ON K1A ON4

Canada
Bibliothèque et

Archives Canada

Direction du

Patrimoine de l'édition

395 , rue Wellington

Ottawa ON K1A ON4

Canada
Your file Votre référence

ISBN: 978-0-494-94642-8

Our file Notre référence

ISBN: $978-0-494-94642-8$
NOTICE:

The author has granted a nonexclusive license allowing Library and Archives Canada to reproduce, publish, archive, preserve, conserve, communicate to the public by telecommunication or on the Internet, loan, distrbute and sell theses worldwide, for commercial or noncommercial purposes, in microform, paper, electronic and/or any other formats.

The author retains copyright ownership and moral rights in this thesis. Neither the thesis nor substantial extracts from it may be printed or otherwise reproduced without the author's permission.
AVIS:

L'auteur a accordé une licence non exclusive permettant à la Bibliothèque et Archives Canada de reproduire, publier, archiver, sauvegarder, conserver, transmettre au public par télécommunication ou par l'Internet, prêter, distribuer et vendre des thèses partout dans le monde, à des fins commerciales ou autres, sur support microforme, papier, électronique et/ou autres formats.

L'auteur conserve la propriété du droit d'auteur et des droits moraux qui protege cette thèse. $\mathrm{Ni}$ la thèse ni des extraits substantiels de celle-ci ne doivent être imprimés ou autrement reproduits sans son autorisation.
In compliance with the Canadian Privacy Act some supporting forms may have been removed from this thesis.

While these forms may be included in the document page count, their removal does not represent any loss of content from the thesis.
Conformément à la loi canadienne sur la protection de la vie privée, quelques formulaires secondaires ont été enlevés de cette thèse.

Bien que ces formulaires aient inclus dans la pagination, il n'y aura aucun contenu manquant. 


\begin{abstract}
Current safety certification procedures are, for the most part, structured within a conventional deterministic framework, which implicitly deals with the nominal values of parameters without systematically and rationally considering the associated uncertainties. The uncertainty is accounted via the use of safety factors. Such treatment of uncertainty raises some concerns, since the safety factors are empirically chosen. Furthermore, these safety factors cannot be directly related to the probability that the system response or the stability margin will exceed a predetermined critical level. On the other hand, a probabilistic approach provides a rational framework to deal with the uncertainty in the model and its parameters. The rational treatment of uncertainty and the assessment of associated risk and reliability levels permit a cost effective and optimal design and analysis of engineering systems at different stages in its life cycle.

In this thesis, a probabilistic methodology is applied to the analysis of the coalescence flutter, influenced by the parametric uncertainties associated with the structure. Coalescence flutter is a two mode aeroelastic dynamic instability, which can occur on flexible structures exposed to air flow, such as an aircraft, bridge or a wind turbine. In this thesis, a two degree-of-freedom elastically mounted rigid wing and a uniform cantilever wing models are considered for numerical investigations. For these systems, the effect of uncertainties is studied on the flutter speed and the pre-flutter modal parameters. Monte Carlo Simulation is performed to estimate the probability density functions of the output quantities of interest (i.e. modal parameters and flutter speed). Based on such probabilistic analysis, an optimal flutter speed may be selected for certification. In addition, appropriateness of the rigid wing model approximation of a cantilever wing is investigated. It is shown that this approximation is acceptable only for the cases, where the modes involved in the flutter mechanism can be adequately represented by a combination of the fundamental bending and torsional mode of a uniform cantilever without air flow. Furthermore, the effect of quasisteady and unsteady aerodynamic forcing is examined for both, the rigid wing model and a cantilever wing model, from a probabilistic prospective. The effect of statistical correlations among parameters is also examined on the flutter speed and the modal parameter estimates.
\end{abstract}




\section{Acknowledgments}

Before proceeding to the thesis I would first like to acknowledge some of the people who have made this learning process an exceptional experience. I would like to acknowledge both of my supervisors, Prof. Abhijit Sarkar and Prof. Dominique Poirel, for introducing me to the world of research and their motivation and encouragement. In addition, this experience would not have been the same without all of the colleagues and friends that I had the fortune to associate with during this time. I would like to acknowledge Mohammad Khalil and Dr. Waad Subber for assisting me in my research and spending their time to help me in my learning process. I would also like to acknowledge Amin Fereidooni, Adam Walker and Muhammad Rahman for their input in all of the discussions and brainstorming that took place. I have to especially acknowledge my family and my personal friends who have always supported me through all of my endeavors and helped me during the tougher times of this experience. To all, many thanks. 


\section{Contents}

1 Introduction 1

2 Literature Review $\quad 4$

2.1 Deterministic Aeroelastic Flutter . . . . . . . . . . . . . . 5

2.2 Uncertainty Quantification ................... 8

2.2.1 Monte Carlo Sampling (MCS) . . . . . . . . . . . . . . . 8

2.2.2 Perturbation Approach . . . . . . . . . . . . . 9

2.2.3 Spectral and Collocation Methods . . . . . . . . . . . . . 9

2.3 Probabilistic Flutter Speed Estimation . . . . . . . . . . . . . . . . . 10

2.4 Probabilistic Characterization of Modal Parameters . . . . . . . . . . . 13

3 A Two Degree-of-freedom Airfoil: Flutter Speed 14

3.1 Introduction . . . . . . . . . . . . . . . . . . 14

3.2 Deterministic Formulation . . . . . . . . . . . . . . 15

3.2.1 Quasisteady Aerodynamics . . . . . . . . . . . . . 17

3.2.2 Types of Coalescence Flutter . . . . . . . . . . . . . 27

3.2.3 Unsteady Aerodynamics ................... 29

3.3 Probabilistic Formulation . . . . . . . . . . . . . . . . . 34

3.4 Flutter Speed Estimation . . . . . . . . . . . . . . . . 35

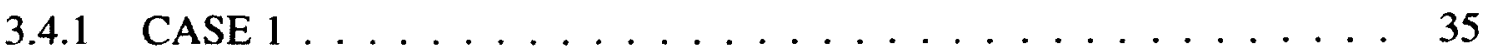

3.4 .2 CASE $2 \ldots \ldots \ldots \ldots \ldots$

3.4 .3 CASE $3 \ldots \ldots \ldots \ldots$ 
3.5 Closing Remarks . . . . . . . . . . . . . . . . . 65

4 A Two Degree-of-freedom Airfoil: Characteristic Roots 67

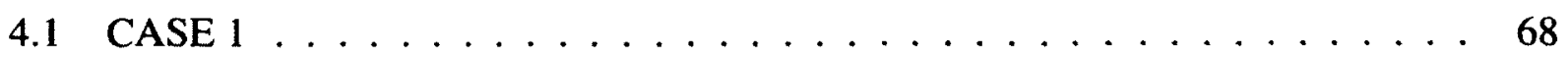

4.1 .1 Quasisteady Results $\ldots \ldots \ldots \ldots$. . . . . . . . 68

4.1 .2 Unsteady Results . . . . . . . . . . . . . . . . . . 78

4.1.3 Comparison of Quasisteady and Unsteady Results _. . . . . . . 87

4.2 CASE $2 \ldots \ldots \ldots \ldots \ldots \ldots \ldots$

4.2 .1 Quasisteady Results . . . . . . . . . . . . . 88

4.2 .2 Unsteady Results . . . . . . . . . . . . . . . . . . . 96

4.2.3 Comparison of Quasisteady and Unsteady Results _ . . . . . . . . 103

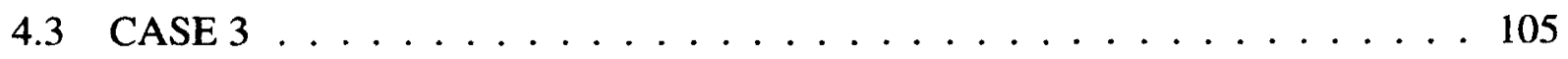

4.3.1 Quasisteady Results . . . . . . . . . . . . . . . 105

4.4 Closing Remarks $\ldots \ldots \ldots \ldots$

5 A Cantilever Wing: Flutter Speed $\quad 114$

5.1 Deterministic Formulation . . . . . . . . . . . . . . . . 115

5.1 .1 Discrete Model Representation . . . . . . . . . . . . . 116

5.1 .2 Quasisteady Aerodynamics . . . . . . . . . . . . . 117

5.1 .3 Unsteady Aerodynamics . . . . . . . . . . . . . . . . 121

5.2 Flutter Speed Estimation $\ldots \ldots \ldots \ldots$

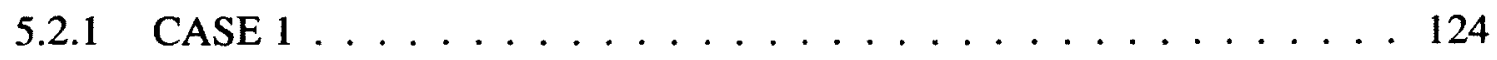

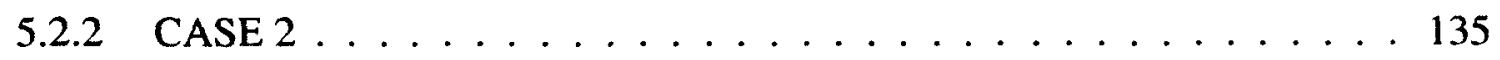

6 A Cantilever Wing: Characteristic Roots 147

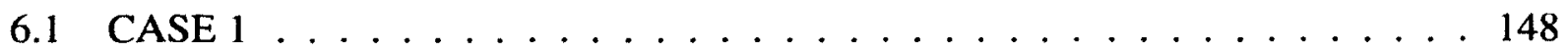

6.1 .1 Quasisteady Results . . . . . . . . . . . . . . . 148

6.1 .2 Unsteady Results . . . . . . . . . . . . . . . . . . 163

6.1.3 Comparison of Quasisteady and Unsteady Results . . . . . . . . 177

6.2 Comparison of Cantilever and Rigid Wing Model Results . . . . . . . . . 177 
6.3 CASE $2 \ldots \ldots \ldots \ldots \ldots$

6.3.1 Quasisteady Results .................... 178

6.3 .2 Unsteady Results . . . . . . . . . . . . . . . . 193

6.3.3 Comparison of Quasisteady and Unsteady Aerodynamics . . . . . . . . 201

6.4 Comparison of Cantilever and Rigid Wing Models for Quasisteady Aerodynamics 208

6.5 Comparison of Cantilever and Rigid Wing Models for Unsteady Aerodynamics 208

7 Conclusion $\quad 209$

$\begin{array}{ll}\text { Appendices } & 211\end{array}$

A Equations of Motion for Rigid Wing Model

B State Space Transformation $\quad 220$

$\begin{array}{ll}\text { C System Parameters } & 222\end{array}$

\begin{tabular}{l|l} 
D Analytical Expression for Flutter Speed & 224
\end{tabular}

$\begin{array}{lr}\text { E Continuous Aeroelastic System } & 226\end{array}$

$\begin{array}{llr}\text { F Basis Functions } & 232\end{array}$

$\begin{array}{lr}\text { References } & 234\end{array}$ 


\section{List of Tables}

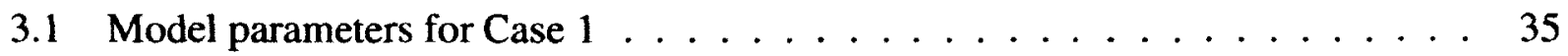

3.2 Results illustrating the uncertainty in the flutter speed due to independent uncertainty in the structural parameters $\ldots \ldots \ldots \ldots \ldots$

3.3 Results illustrating the uncertainty in the flutter speed due to correlated uncertainty in the structural parameters . . . . . . . . . . . . . 39

3.4 Results illustrating the uncertainty in the flutter speed due to independent uncertainty in the structural parameters $\ldots \ldots \ldots \ldots \ldots$

3.5 Results illustrating the uncertainty in the flutter speed due to correlated uncertainty in the structural parameters . . . . . . . . . . . . . . . . 44

3.6 Model parameters for Case $2 \ldots \ldots \ldots$. . . . . . . . . . . 46

3.7 Results illustrating the uncertainty in the flutter speed due to independent uncertainty in the structural parameters $\ldots \ldots \ldots \ldots \ldots \ldots$

3.8 Results illustrating the uncertainty in the flutter speed due to correlated uncertainty in the structural parameters $\ldots \ldots \ldots \ldots \ldots$

3.9 Results illustrating the uncertainty in the flutter speed due to independent uncertainty in the structural parameters $\ldots \ldots \ldots \ldots \ldots \ldots$

3.10 Results illustrating the uncertainty in the flutter speed due to correlated uncertainty in the structural parameters . . . . . . . . . . . 53

3.11 Model parameters for Case $3 \ldots \ldots \ldots 6$

3.12 Results illustrating the uncertainty in the flutter speed due to independent uncertainty in the structural parameters $\ldots \ldots \ldots \ldots \ldots$ 
3.13 Results illustrating the uncertainty in the flutter speed due to correlated uncertainty in the structural parameters . . . . . . . . . . . . . 59

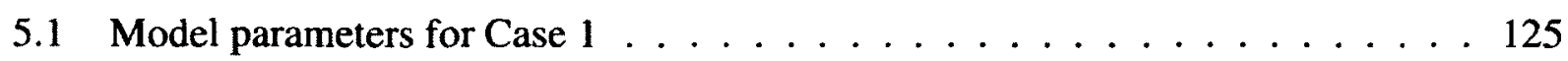

5.2 Results illustrating the uncertainty in the flutter speed due to independent uncertainty in the structural parameters . . . . . . . . . . . . . 128

5.3 Results illustrating the uncertainty in the flutter speed due to correlated uncertainty in the structural parameters . . . . . . . . . . . . . . 128

5.4 Results illustrating the uncertainty in the flutter speed due to independent uncertainty in the structural parameters . . . . . . . . . . . . 133

5.5 Results illustrating the uncertainty in the flutter speed due to correlated uncertainty in the structural parameters . . . . . . . . . . . . . . 133

5.6 Model parameters for Case $2 \ldots \ldots \ldots$. . . . . . . . . . . . 136

5.7 Results illustrating the uncertainty in the flutter speed due to independent uncertainty in the structural parameters . . . . . . . . . . . . 139

5.8 Results illustrating the uncertainty in the flutter speed due to correlated uncertainty in the structural parameters . . . . . . . . . . . . . . 139

5.9 Results illustrating the uncertainty in the flutter speed due to independent uncertainty in the structural parameters . . . . . . . . . . . . . 144

5.10 Results illustrating the uncertainty in the flutter speed due to correlated uncertainty in the structural parameters . . . . . . . . . . . . . . . 144

C.1 Model parameters for Case $1 \ldots \ldots 22$

C.2 Model parameters for Case 2 . . . . . . . . . . . . . 223

C.3 Model parameters for Case $3 \ldots \ldots$. . . . . . . . . . . . 223 


\section{List of Figures}

3.1 Example of an airfoil under study $\ldots \ldots \ldots \ldots$

3.2 Theodorsen's function, $\mathrm{C}(\mathrm{k})$, at top $\mathrm{F}(\mathrm{k})$ and bottom $\mathrm{G}(\mathrm{k})[1] \ldots \ldots$

3.3 Symmetric airfoil illustrating important geometric parameters . . . . . . . . . . . . . 19

3.4 Oscillatory motion producing positive work . . . . . . . . . . . . . . . 22

3.5 Evolution of the oscillatory modes' modal parameters with respect to increasing airspeed. Top plot describes modal decay rates; bottom plot describes damped modal frequencies . . . . . .

3.6 Figures representing stable, marginally stable and unstable responses, respectively from top to

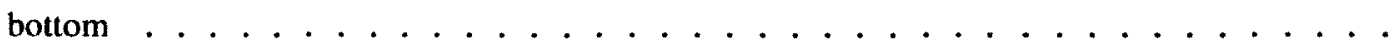

3.7 Examples of mild flutter, moderate flutter and explosive flutter respectively from top to bottom. Solid lines represent the original modal parameters, dashed lines represent modal parameters obtained using manipulated damping ratios $\ldots \ldots \ldots$

3.8 Wagner's Function represents a lag in reaching the steady aerodynamics . . . . . . . . 30

3.9 Evolution of the oscillatory modes' modal parameters with respect to increasing airspeed. Top plot describes modal decay rates; bottom plot describes damped modal frequencies . . . . . . 33

3.10 Evolution of modal parameters with respect to airspeed $\ldots \ldots \ldots$

3.11 real part, imaginary part, and magnitude of eigenvectors of two modes, illustrating relative strength of each component . . . . . . . . . . . . . . . . 37

3.12 The functional relationships among the flutter speed and structural parameters . . . . . . 37

3.13 Flutter speed pdfs (case 1 airfoil, quasisteady, $10 \%$ COV) . . . . . . . . . . . . . 40

3.14 Flutter speed pdfs (case 1 airfoil, quasisteady, $10 \%$ COV) . . . . . . . . . . . . . 41

3.15 Evolution of modal parameters with respect to airspeed . . . . . . . . . . . . . 42 
3.16 real part, imaginary part, and magnitude of eigenvectors of two modes, illustrating relative strength of each component . . . . . . . . . . . . . . . . . 43

3.17 Flutter speed pdfs (case 1 airfoil, unsteady, 10\% COV) . . . . . . . . . . . . . 44

3.18 Flutter speed pdfs (case 1 airfoil, unsteady, 10\% COV) . . . . . . . . . . . 45

3.19 Evolution of modal parameters with respect to airspeed . . . . . . . . . . . . . . 47

3.20 real part, imaginary part, and magnitude of eigenvectors of two modes, illustrating relative strength of each component . . . . . . . . . . . . . . . . . 47

3.21 The functional relationships among the flutter speed and structural parameters . . . . . . . 48

3.22 Flutter speed pdfs (case 2 airfoil, quasisteady, $10 \%$ COV) . . . . . . . . . . . . . 50

3.23 Flutter speed pdfs (case 2 airfoil, quasisteady, $10 \%$ COV) . . . . . . . . . . . 51

3.24 Evolution of modal parameters with respect to airspeed . . . . . . . . . . . . . 52

3.25 real part, imaginary part, and magnitude of eigenvectors of two modes, illustrating relative

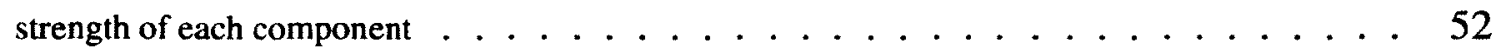

3.26 Flutter speed pdfs (case 2 airfoil, unsteady, $10 \%$ COV) . . . . . . . . . . . . 54

3.27 Flutter speed pdfs (case 2 airfoil, unsteady, $10 \%$ COV) . . . . . . . . . . . . . 55

3.28 Evolution of modal parameters with respect to airspeed . . . . . . . . . . . . . 57

3.29 real part, imaginary part, and magnitude of eigenvectors of two modes, illustrating relative

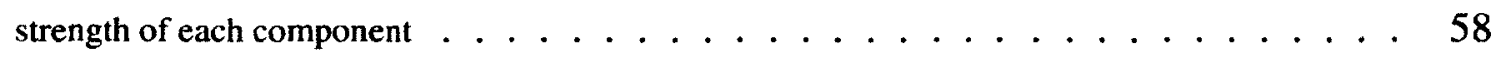

3.30 The functional relationships among the flutter speed and structural parameters $\ldots \ldots$. . . 58

3.31 Flutter speed pdfs (case 3 airfoil, quasisteady, $2.5 \% \mathrm{COV}$ ) . . . . . . . . . . . 60

3.32 Flutter speed pdfs (case 3 airfoil, quasisteady, $2.5 \%$ COV) . . . . . . . . . . . 61

3.33 Flutter speed pdfs (case 3 airfoil, quasisteady, $17.5 \% \mathrm{COV}) \ldots \ldots 2$

3.34 Flutter speed pdfs (case 3 airfoil, quasisteady, $17.5 \%$ COV) $\ldots \ldots$. . . . . . . . 63

4.1 Modal parameter pdfs resulting from randomness in mass with $10 \% \mathrm{COV}$, obtained at 3 test airspeeds: $0 \mathrm{~m} / \mathrm{s}, 69 \mathrm{~m} / \mathrm{s}, 96.9 \mathrm{~m} / \mathrm{s} \ldots \ldots \ldots \ldots$. . . . . . . . . . 69

4.2 Modal parameter pdfs resulting from randomness in mass moment of inertia with $10 \% \mathrm{COV}$, obtained at 3 test airspeeds: $0 \mathrm{~m} / \mathrm{s}, 69 \mathrm{~m} / \mathrm{s}, 96.9 \mathrm{~m} / \mathrm{s} \ldots \ldots \ldots$ 
4.3 Modal parameter pdfs resulting from randomness in heave stiffness with $10 \% \mathrm{COV}$, obtained at 3 test airspeeds: $0 \mathrm{~m} / \mathrm{s}, 69 \mathrm{~m} / \mathrm{s}, 96.9 \mathrm{~m} / \mathrm{s} \ldots \ldots \ldots \ldots \ldots$

4.4 Modal parameter pdfs resulting from randomness in pitch stiffness with $10 \% \mathrm{COV}$, obtained at 3 test airspeeds: $0 \mathrm{~m} / \mathrm{s}, 69 \mathrm{~m} / \mathrm{s}, 96.9 \mathrm{~m} / \mathrm{s} \ldots \ldots \ldots \ldots$

4.5 Modal parameter pdfs resulting from randomness in $1^{\text {st }}$ structural damping ratio with $10 \%$ COV, obtained at 3 test airspeeds: $0 \mathrm{~m} / \mathrm{s}, 69 \mathrm{~m} / \mathrm{s}, 96.9 \mathrm{~m} / \mathrm{s} \ldots \ldots \ldots$

4.6 Modal parameter pdfs resulting from randomness in $2^{\text {nd }}$ structural damping ratio with $10 \%$ COV, obtained at 3 test airspeeds: $0 \mathrm{~m} / \mathrm{s}, 69 \mathrm{~m} / \mathrm{s}, 96.9 \mathrm{~m} / \mathrm{s} \ldots \ldots \ldots$

4.7 Modal parameter pdfs resulting from randomness in the uncorrelated inertia parameters with $10 \% \mathrm{COV}$, obtained at 3 test airspeeds: $0 \mathrm{~m} / \mathrm{s}, 69 \mathrm{~m} / \mathrm{s}, 96.9 \mathrm{~m} / \mathrm{s} \ldots \ldots \ldots$

4.8 Modal parameter pdfs resulting from randomness in the uncorrelated stiffness parameters with $10 \%$ COV, obtained at 3 test airspeeds: $0 \mathrm{~m} / \mathrm{s}, 69 \mathrm{~m} / \mathrm{s}, 96.9 \mathrm{~m} / \mathrm{s} \ldots \ldots \ldots$

4.9 Modal parameter pdfs resulting from randomness in the uncorrelated damping parameters with $10 \%$ COV, obtained at 3 test airspeeds: $0 \mathrm{~m} / \mathrm{s}, 69 \mathrm{~m} / \mathrm{s}, 96.9 \mathrm{~m} / \mathrm{s} \ldots \ldots \ldots$

4.10 Modal parameter pdfs resulting from randomness in the correlated inertia parameters with $10 \%$ COV, obtained at 3 test airspeeds: $0 \mathrm{~m} / \mathrm{s}, 69 \mathrm{~m} / \mathrm{s}, 96.9 \mathrm{~m} / \mathrm{s} \ldots \ldots \ldots$

4.11 Modal parameter pdfs resulting from randomness in the correlated stiffness parameters with $10 \% \mathrm{COV}$, obtained at 3 test airspeeds: $0 \mathrm{~m} / \mathrm{s}, 69 \mathrm{~m} / \mathrm{s}, 96.9 \mathrm{~m} / \mathrm{s} \ldots \ldots \ldots$

4.12 Modal parameter pdfs resulting from randomness correlated damping parameters with $10 \%$ COV, obtained at 3 test airspeeds: $0 \mathrm{~m} / \mathrm{s}, 69 \mathrm{~m} / \mathrm{s}, 96.9 \mathrm{~m} / \mathrm{s} \ldots \ldots \ldots$

4.13 Modal parameter pdfs resulting from randomness in mass with $10 \% \mathrm{COV}$, obtained at 3 test airspeeds: $\approx 0 \mathrm{~m} / \mathrm{s}, 100 \mathrm{~m} / \mathrm{s}, 141 \mathrm{~m} / \mathrm{s} \ldots \ldots \ldots \ldots$

4.14 Modal parameter pdfs resulting from randomness in mass moment of inertia with $10 \% \mathrm{COV}$, obtained at 3 test airspeeds: $\approx 0 \mathrm{~m} / \mathrm{s}, 100 \mathrm{~m} / \mathrm{s}, 141 \mathrm{~m} / \mathrm{s} \ldots \ldots \ldots$

4.15 Modal parameter pdfs resulting from randomness in heave stiffness with $10 \% \mathrm{COV}$, obtained at 3 test airspeeds: $\approx 0 \mathrm{~m} / \mathrm{s}, 100 \mathrm{~m} / \mathrm{s}, 141 \mathrm{~m} / \mathrm{s} \ldots \ldots \ldots \ldots$

4.16 Modal parameter pdfs resulting from randomness in pitch stiffness with $10 \% \mathrm{COV}$, obtained at 3 test airspeeds: $\approx 0 \mathrm{~m} / \mathrm{s}, 100 \mathrm{~m} / \mathrm{s}, 141 \mathrm{~m} / \mathrm{s} \ldots \ldots \ldots \ldots$ 
4.17 Modal parameter pdfs resulting from randomness in the $1^{\text {st }}$ modal damping ratio with $10 \%$ COV, obtained at 3 test airspeeds: $\approx 0 \mathrm{~m} / \mathrm{s}, 100 \mathrm{~m} / \mathrm{s}, 141 \mathrm{~m} / \mathrm{s} \ldots \ldots \ldots$

4.18 Modal parameter pdfs resulting from randomness in the $2^{\text {nd }}$ modal damping ratio with $10 \%$ COV, obtained at 3 test airspeeds: $\approx 0 \mathrm{~m} / \mathrm{s}, 100 \mathrm{~m} / \mathrm{s}, 141 \mathrm{~m} / \mathrm{s} \ldots \ldots \ldots$

4.19 Modal parameter pdfs resulting from randomness in the uncorrelated inertia parameters with $10 \% \mathrm{COV}$, obtained at 3 test airspeeds: $\approx 0 \mathrm{~m} / \mathrm{s}, 100 \mathrm{~m} / \mathrm{s}, 141 \mathrm{~m} / \mathrm{s} \ldots \ldots$

4.20 Modal parameter pdfs resulting from randomness in the uncorrelated stiffness parameters with $10 \% \mathrm{COV}$, obtained at 3 test airspeeds: $\approx 0 \mathrm{~m} / \mathrm{s}, 100 \mathrm{~m} / \mathrm{s}, 141 \mathrm{~m} / \mathrm{s} \ldots \ldots$

4.21 Modal parameter pdfs resulting from randomness in the uncorrelated damping parameters with $10 \%$ COV, obtained at 3 test airspeeds: $\approx 0 \mathrm{~m} / \mathrm{s}, 100 \mathrm{~m} / \mathrm{s}, 141 \mathrm{~m} / \mathrm{s} \ldots \ldots$

4.22 Modal parameter pdfs resulting from randomness in the correlated inertia parameters with $10 \%$ COV, obtained at 3 test airspeeds: $\approx 0 \mathrm{~m} / \mathrm{s}, 100 \mathrm{~m} / \mathrm{s}, 141 \mathrm{~m} / \mathrm{s} \ldots \ldots \ldots$

4.23 Modal parameter pdfs resulting from randomness in the correlated stiffness parameters with $10 \% \mathrm{COV}$, obtained at 3 test airspeeds: $\approx 0 \mathrm{~m} / \mathrm{s}, 100 \mathrm{~m} / \mathrm{s}, 141 \mathrm{~m} / \mathrm{s} \ldots \ldots$

4.24 Modal parameter pdfs resulting from randomness correlated damping parameters with $10 \%$ COV, obtained at 3 test airspeeds: $\approx 0 \mathrm{~m} / \mathrm{s}, 100 \mathrm{~m} / \mathrm{s}, 141 \mathrm{~m} / \mathrm{s} \ldots \ldots \ldots$

4.25 Modal parameter pdfs resulting from randomness in mass with $10 \% \mathrm{COV}$, obtained at 3 test airspeeds: $0 \mathrm{~m} / \mathrm{s}, 68 \mathrm{~m} / \mathrm{s}, 95 \mathrm{~m} / \mathrm{s} \ldots \ldots \ldots \ldots \ldots$

4.26 Modal parameter pdfs resulting from randomness in mass moment of inertia with $10 \% \mathrm{COV}$, obtained at 3 test airspeeds: $0 \mathrm{~m} / \mathrm{s}, 68 \mathrm{~m} / \mathrm{s}, 95 \mathrm{~m} / \mathrm{s} \ldots \ldots \ldots \ldots$

4.27 Modal parameter pdfs resulting from randomness in heave stiffness with $10 \% \mathrm{COV}$, obtained at 3 test airspeeds: $0 \mathrm{~m} / \mathrm{s}, 68 \mathrm{~m} / \mathrm{s}, 95 \mathrm{~m} / \mathrm{s} \ldots \ldots \ldots \ldots$

4.28 Modal parameter pdfs resulting from randomness in pitch stiffness with $10 \% \mathrm{COV}$, obtained at 3 test airspeeds: $0 \mathrm{~m} / \mathrm{s}, 68 \mathrm{~m} / \mathrm{s}, 95 \mathrm{~m} / \mathrm{s} \ldots \ldots \ldots \ldots$

4.29 Modal parameter pdfs resulting from randomness in the $1^{\text {st }}$ modal damping ratio with $10 \%$ COV, obtained at 3 test airspeeds: $0 \mathrm{~m} / \mathrm{s}, 68 \mathrm{~m} / \mathrm{s}, 95 \mathrm{~m} / \mathrm{s} \ldots \ldots \ldots$

4.30 Modal parameter pdfs resulting from randomness in the $2^{\text {nd }}$ modal damping ratio with $10 \%$ COV, obtained at 3 test airspeeds: $0 \mathrm{~m} / \mathrm{s}, 68 \mathrm{~m} / \mathrm{s}, 95 \mathrm{~m} / \mathrm{s} \ldots \ldots \ldots \ldots$ 
4.31 Modal parameter pdfs resulting from randomness in the uncorrelated inertia parameters with $10 \%$ COV, obtained at 3 test airspeeds: $0 \mathrm{~m} / \mathrm{s}, 68 \mathrm{~m} / \mathrm{s}, 95 \mathrm{~m} / \mathrm{s} \ldots \ldots$. . . . . . . . .

4.32 Modal parameter pdfs resulting from randomness in the uncorrelated stiffness parameters with $10 \%$ COV, obtained at 3 test airspeeds: $0 \frac{\mathrm{m}}{\mathrm{s}}, 68 \frac{\mathrm{m}}{\mathrm{s}}, 95 \frac{\mathrm{m}}{\mathrm{s}} \ldots \ldots \ldots$

4.33 Modal parameter pdfs resulting from randomness in the uncorrelated damping parameters with $10 \%$ COV, obtained at 3 test airspeeds: $0 \mathrm{~m} / \mathrm{s}, 68 \mathrm{~m} / \mathrm{s}, 95 \mathrm{~m} / \mathrm{s} \ldots \ldots$. . . . . . . .

4.34 Modal parameter pdfs resulting from randomness in the correlated inertia parameters with $10 \%$ COV, obtained at 3 test airspeeds: $0 \mathrm{~m} / \mathrm{s}, 68 \mathrm{~m} / \mathrm{s}, 95 \mathrm{~m} / \mathrm{s} \ldots \ldots \ldots$

4.35 Modal parameter pdfs resulting from randomness in the correlated stiffness parameters with $10 \%$ COV, obtained at 3 test airspeeds: $0 \mathrm{~m} / \mathrm{s}, 68 \mathrm{~m} / \mathrm{s}, 95 \mathrm{~m} / \mathrm{s} \ldots \ldots$. . . . . . . .

4.36 Modal parameter pdfs resulting from randomness correlated damping parameters with $10 \%$ COV, obtained at 3 test airspeeds: $0 \mathrm{~m} / \mathrm{s}, 68 \mathrm{~m} / \mathrm{s}, 95 \mathrm{~m} / \mathrm{s} \ldots \ldots \ldots \ldots$

4.37 Modal parameter pdfs resulting from randomness in mass with $10 \% \mathrm{COV}$, obtained at 3 test airspeeds $: \approx 0 \mathrm{~m} / \mathrm{s}, 98 \mathrm{~m} / \mathrm{s}, 137 \mathrm{~m} / \mathrm{s} \ldots \ldots \ldots \ldots$

4.38 Modal parameter pdfs resulting from randomness in mass moment of inertia with $10 \% \mathrm{COV}$, obtained at 3 test airspeeds: $\approx 0 \mathrm{~m} / \mathrm{s}, 98 \mathrm{~m} / \mathrm{s}, 137 \mathrm{~m} / \mathrm{s} \ldots \ldots \ldots$

4.39 Modal parameter pdfs resulting from randomness in heave stiffness with $10 \% \mathrm{COV}$, obtained at 3 test airspeeds: $\approx 0 \mathrm{~m} / \mathrm{s}, 98 \mathrm{~m} / \mathrm{s}, 137 \mathrm{~m} / \mathrm{s} \ldots \ldots \ldots \ldots$

4.40 Modal parameter pdfs resulting from randomness in pitch stiffness with $10 \% \mathrm{COV}$, obtained at 3 test airspeeds: $\approx 0 \mathrm{~m} / \mathrm{s}, 98 \mathrm{~m} / \mathrm{s}, 137 \mathrm{~m} / \mathrm{s} \ldots \ldots \ldots \ldots$

4.41 Modal parameter pdfs resulting from randomness in the $1^{\text {st }}$ modal damping ratio with $10 \%$ COV, obtained at 3 test airspeeds: $\approx 0 \mathrm{~m} / \mathrm{s}, 98 \mathrm{~m} / \mathrm{s}, 137 \mathrm{~m} / \mathrm{s} \ldots \ldots \ldots$

4.42 Modal parameter pdfs resulting from randomness in the $2^{\text {nd }}$ modal damping ratio with $10 \%$ COV, obtained at 3 test airspeeds: $\approx 0 \mathrm{~m} / \mathrm{s}, 98 \mathrm{~m} / \mathrm{s}, 137 \mathrm{~m} / \mathrm{s}$

4.43 Modal parameter pdfs resulting from randomness in the uncorrelated inertia parameters with $10 \%$ COV, obtained at 3 test airspeeds: $\approx 0 \mathrm{~m} / \mathrm{s}, 98 \mathrm{~m} / \mathrm{s}, 137 \mathrm{~m} / \mathrm{s} \ldots \ldots \ldots$

4.44 Modal parameter pdfs resulting from randomness in the uncorrelated stiffness parameters with $10 \% \mathrm{COV}$, obtained at 3 test airspeeds: $\approx 0 \mathrm{~m} / \mathrm{s}, 98 \mathrm{~m} / \mathrm{s}, 137 \mathrm{~m} / \mathrm{s} \ldots \ldots \ldots$ 
4.45 Modal parameter pdfs resulting from randomness in the uncorrelated damping parameters with $10 \%$ COV, obtained at 3 test airspeeds: $\approx 0 \mathrm{~m} / \mathrm{s}, 98 \mathrm{~m} / \mathrm{s}, 137 \mathrm{~m} / \mathrm{s} \ldots \ldots 2$

4.46 Modal parameter pdfs resulting from randomness in the correlated inertia parameters with $10 \%$ COV, obtained at 3 test airspeeds: $\approx 0 \mathrm{~m} / \mathrm{s}, 98 \mathrm{~m} / \mathrm{s}, 137 \mathrm{~m} / \mathrm{s} \ldots \ldots \ldots$

4.47 Modal parameter pdfs resulting from randomness in the correlated stiffness parameters with $10 \%$ COV, obtained at 3 test airspeeds: $\approx 0 \mathrm{~m} / \mathrm{s}, 98 \mathrm{~m} / \mathrm{s}, 137 \mathrm{~m} / \mathrm{s} \ldots \ldots$

4.48 Modal parameter pdfs resulting from randomness correlated damping parameters with $10 \%$ COV, obtained at 3 test airspeeds: $\approx 0 \mathrm{~m} / \mathrm{s}, 98 \mathrm{~m} / \mathrm{s}, 137 \mathrm{~m} / \mathrm{s} \ldots \ldots \ldots$

4.49 Modal parameter pdfs resulting from randomness in mass with $17.5 \% \mathrm{COV}$, obtained at 3 test airspeeds: $0 \mathrm{~m} / \mathrm{s}, 33 \mathrm{~m} / \mathrm{s}, 47 \mathrm{~m} / \mathrm{s} \ldots \ldots \ldots \ldots \ldots$

4.50 Modal parameter pdfs resulting from randomness in mass moment of inertia with $17.5 \% \mathrm{COV}$, obtained at 3 test airspeeds: $0 \mathrm{~m} / \mathrm{s}, 33 \mathrm{~m} / \mathrm{s}, 47 \mathrm{~m} / \mathrm{s} \ldots \ldots \ldots$. . . . . . . 106

4.51 Modal parameter pdfs resulting from randomness in heave stiffness with $17.5 \% \mathrm{COV}$, obtained at 3 test airspeeds: $0 \mathrm{~m} / \mathrm{s}, 33 \mathrm{~m} / \mathrm{s}, 47 \mathrm{~m} / \mathrm{s} \ldots \ldots \ldots \ldots \ldots$

4.52 Modal parameter pdfs resulting from randomness in pitch stiffness with $17.5 \% \mathrm{COV}$, obtained at 3 test airspeeds: $0 \mathrm{~m} / \mathrm{s}, 33 \mathrm{~m} / \mathrm{s}, 47 \mathrm{~m} / \mathrm{s} \ldots \ldots \ldots \ldots$

4.53 Modal parameter pdfs resulting from randomness in the $1^{\text {st }}$ modal damping ratio with $17.5 \%$ COV, obtained at 3 test airspeeds: $0 \mathrm{~m} / \mathrm{s}, 33 \mathrm{~m} / \mathrm{s}, 47 \mathrm{~m} / \mathrm{s} \ldots \ldots \ldots$

4.54 Modal parameter pdfs resulting from randomness in the $2^{\text {nd }}$ modal damping ratio with $17.5 \%$ COV, obtained at 3 test airspeeds: $0 \mathrm{~m} / \mathrm{s}, 33 \mathrm{~m} / \mathrm{s}, 47 \mathrm{~m} / \mathrm{s} \ldots \ldots \ldots$

4.55 Modal parameter pdfs resulting from randomness in the uncorrelated inertia parameters with $17.5 \% \mathrm{COV}$, obtained at 3 test airspeeds: $0 \mathrm{~m} / \mathrm{s}, 33 \mathrm{~m} / \mathrm{s}, 47 \mathrm{~m} / \mathrm{s} \ldots \ldots \ldots$

4.56 Modal parameter pdfs resulting from randomness in the uncorrelated stiffness parameters with $17.5 \% \mathrm{COV}$, obtained at 3 test airspeeds: $0 \mathrm{~m} / \mathrm{s}, 33 \mathrm{~m} / \mathrm{s}, 47 \mathrm{~m} / \mathrm{s} \ldots \ldots$

4.57 Modal parameter pdfs resulting from randomness in the uncorrelated damping parameters with $17.5 \% \mathrm{COV}$, obtained at 3 test airspeeds: $0 \mathrm{~m} / \mathrm{s}, 33 \mathrm{~m} / \mathrm{s}, 47 \mathrm{~m} / \mathrm{s} \ldots \ldots \ldots$

4.58 Modal parameter pdfs resulting from randomness in the correlated inertia parameters with $17.5 \%$ COV, obtained at 3 test airspeeds: $0 \mathrm{~m} / \mathrm{s}, 33 \mathrm{~m} / \mathrm{s}, 47 \mathrm{~m} / \mathrm{s} \ldots \ldots \ldots$ 
4.59 Modal parameter pdfs resulting from randomness in the correlated stiffness parameters with $17.5 \%$ COV, obtained at 3 test airspeeds: $0 \mathrm{~m} / \mathrm{s}, 33 \mathrm{~m} / \mathrm{s}, 47 \mathrm{~m} / \mathrm{s} \ldots \ldots \ldots$

4.60 Modal parameter pdfs resulting from randomness correlated damping parameters with $17.5 \%$ COV, obtained at 3 test airspeeds: $0 \mathrm{~m} / \mathrm{s}, 33 \mathrm{~m} / \mathrm{s}, 47 \mathrm{~m} / \mathrm{s} \ldots \ldots \ldots$

5.1 Lift reduction factor as function of normalized span $\ldots \ldots \ldots \ldots$

5.2 Evolution of the modal parameters with respect to increasing airspeed. Top plot describes modal decay rates; bottom plot describes damped modal frequencies . . . . . . . . . . . . 120

5.3 Evolution of the modal parameters with respect to increasing airspeed. Top plot describes modal decay rates; bottom plot describes damped modal frequencies . . . . . . . . . . . .

5.4 Evolution of the oscillatory modes' modal parameters with respect to increasing airspeed. Top plot describes modal decay rates; bottom plot describes damped modal frequencies . . . . . .

5.5 Evolution of the oscillatory modes' modal parameters with respect to increasing airspeed. Top plot describes modal decay rates; bottom plot describes damped modal frequencies . . . . . . 124

5.6 Evolution of the modal parameters with respect to airspeed . . . . . . . . . . 125

5.7 Magnified view of evolution of the modal parameters with respect to increasing airspeed . . 126

5.8 real part, imaginary part, and magnitude of eigenvectors of the vibration modes, illustrating relative strength of each component . . . . . . . . . . . . . . . . . 127

5.9 Flutter speed pdfs (case 1 wing, quasisteady, 10\% COV) . . . . . . . . . . . . 129

5.10 Flutter speed pdfs (case 1 wing, quasisteady, 10\% COV) . . . . . . . . . . . 130

5.11 Evolution of modal parameters with respect to airspeed $\ldots \ldots \ldots 131$

5.12 Magnified view of evolution of the modal parameters with respect to increasing airspeed . . 131

5.13 real part, imaginary part, and magnitude of eigenvectors of the vibration modes, illustrating relative strength of each component . . . . . . . . . . . . . . . 132

5.14 Flutter speed pdfs (case 1 wing, unsteady, 10\% COV) . . . . . . . . . . . . . 134

5.15 Flutter speed pdfs (case 1 wing, unsteady, 10\% COV) . . . . . . . . . . . . . 135

5.16 Evolution of modal parameters with respect to airspeed $\ldots \ldots \ldots$. . . . . . . $\ldots$

5.17 Magnified view of evolution of the modal parameters with respect to increasing airspeed . . 137 
5.18 real part, imaginary part, and magnitude of eigenvectors of the vibration modes, illustrating relative strength of each component . . . . . . . . . . . . . 138

5.19 Flutter speed pdfs (case 2 wing, quasisteady, 10\% COV) . . . . . . . . . . 140

5.20 Flutter speed pdfs (case 2 wing, quasisteady, 10\% COV) . . . . . . . . . . . 141

5.21 Evolution of modal parameters with respect to airspeed . . . . . . . . . . . . 141

5.22 Magnified view of evolution of the modal parameters with respect to increasing airspeed $\ldots 142$

5.23 real part, imaginary part, and magnitude of eigenvectors of the vibration modes, illustrating relative strength of each component . . . . . . . . . . . . . 143

5.24 Flutter speed pdfs (case 2 wing, unsteady, $10 \%$ COV) . . . . . . . . . . . . 145

5.25 Flutter speed pdfs (case 2 wing, unsteady, $10 \%$ COV) . . . . . . . . . . . . 146

6.1 Modal parameter pdfs resulting from randomness in mass with $10 \% \mathrm{COV}$, obtained at 3 test airspeeds: $0 \mathrm{~m} / \mathrm{s}, 109 \mathrm{~m} / \mathrm{s}, 155.13 \mathrm{~m} / \mathrm{s} \ldots \ldots \ldots \ldots$

6.2 Magnified view of coalescing mode's modal parameter pdfs for uncertainty in mass $\ldots$. . . 149

6.3 Modal parameter pdfs resulting from randomness in mass moment of inertia with $10 \% \mathrm{COV}$, obtained at 3 test airspeeds: $0 \mathrm{~m} / \mathrm{s}, 109 \mathrm{~m} / \mathrm{s}, 155.13 \mathrm{~m} / \mathrm{s} \ldots \ldots \ldots$

6.4 Magnified view of coalescing mode's modal parameter pdfs for uncertainty in mass moment of

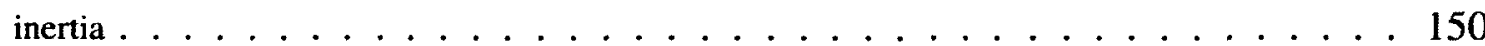

6.5 Modal parameter pdfs resulting from randomness in bending stiffness with $10 \% \mathrm{COV}$, obtained at 3 test airspeeds: $0 m / s, 109 m / s, 155.13 m / s \ldots \ldots \ldots 152$

6.6 Magnified view of coalescing mode's modal parameter pdfs for uncertainty in bending stiffness

6.7 Modal parameter pdfs resulting from randomness in torsional stiffness with $10 \% \mathrm{COV}$, obtained at 3 test airspeeds: $0 \mathrm{~m} / \mathrm{s}, 109 \mathrm{~m} / \mathrm{s}, 155.13 \mathrm{~m} / \mathrm{s} \ldots \ldots \ldots \ldots$

6.8 Magnified view of coalescing mode's modal parameter pdfs for uncertainty in torsional stiffness

6.9 Modal parameter pdfs resulting from randomness in first structural damping ratio with $10 \%$ COV, obtained at 3 test airspeeds: $0 \mathrm{~m} / \mathrm{s}, 109 \mathrm{~m} / \mathrm{s}, 155.13 \mathrm{~m} / \mathrm{s} \ldots \ldots \ldots$

6.10 Magnified view of coalescing mode's modal parameter pdfs for uncertainty in first structural damping ratio 
6.11 Modal parameter pdfs resulting from randomness in second structural damping ratio with $10 \%$ COV, obtained at 3 test airspeeds: $0 \mathrm{~m} / \mathrm{s}, 109 \mathrm{~m} / \mathrm{s}, 155.13 \mathrm{~m} / \mathrm{s} \ldots \ldots \ldots$

6.12 Magnified view of coalescing mode's modal parameter pdfs for uncertainty in second structural

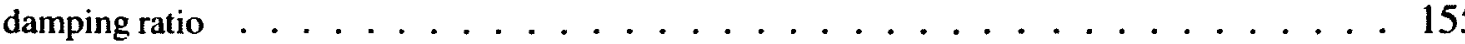

6.13 Modal parameter pdfs resulting from randomness in uncorrelated inertia terms with $10 \% \mathrm{COV}$, obtained at 3 test airspeeds: $0 \mathrm{~m} / \mathrm{s}, 109 \mathrm{~m} / \mathrm{s}, 155.13 \mathrm{~m} / \mathrm{s} \ldots \ldots \ldots$

6.14 Magnified view of coalescing mode's modal parameter pdfs for uncertainty in uncorrelated inertia terms $\ldots \ldots \ldots \ldots$

6.15 Modal parameter pdfs resulting from randomness in uncorrelated stiffness terms with $10 \%$ COV, obtained at 3 test airspeeds: $0 \mathrm{~m} / \mathrm{s}, 109 \mathrm{~m} / \mathrm{s}, 155.13 \mathrm{~m} / \mathrm{s} \ldots \ldots \ldots$

6.16 Magnified view of coalescing mode's modal parameter pdfs for uncertainty in uncorrelated stiffness terms . . . . . . . . . . . . . . . . . 158

6.17 Modal parameter pdfs resulting from randomness in uncorrelated damping terms with $10 \%$ COV, obtained at 3 test airspeeds: $0 \mathrm{~m} / \mathrm{s}, 109 \mathrm{~m} / \mathrm{s}, 155.13 \mathrm{~m} / \mathrm{s} \ldots \ldots \ldots \ldots$

6.18 Magnified view of coalescing mode's modal parameter pdfs for uncertainty in uncorrelated damping terms $\ldots \ldots \ldots \ldots \ldots \ldots \ldots$

6.19 Modal parameter pdfs resulting from randomness in correlated inertia terms with $10 \% \mathrm{COV}$, obtained at 3 test airspeeds: $0 \frac{m}{s}, 109 \frac{m}{s}, 155.13 \frac{m}{s} \ldots \ldots \ldots 160$

6.20 Magnified view of coalescing mode's modal parameter pdfs for uncertainty in correlated inertia

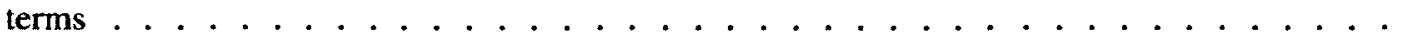

6.21 Modal parameter pdfs resulting from randomness in correlated stiffness terms with $10 \%$ COV, obtained at 3 test airspeeds: $0 \frac{m}{s}, 109 \frac{m}{s}, 155.13 \frac{m}{s} \ldots \ldots \ldots \ldots$

6.22 Magnified view of coalescing mode's modal parameter pdfs for uncertainty in correlated stiffness terms $\ldots \ldots \ldots \ldots$

6.23 Modal parameter pdfs resulting from randomness in correlated damping terms with $10 \% \mathrm{COV}$, obtained at 3 test airspeeds: $0 \frac{m}{s}, 109 \frac{m}{s}, 155.13 \frac{m}{s} \ldots \ldots \ldots 162$

6.24 Magnified view of coalescing mode's modal parameter pdfs for uncertainty in correlated damping term 
6.25 Modal parameter pdfs resulting from randomness in mass with $10 \% \mathrm{COV}$, obtained at 3 test airspeeds: $\approx 0 \mathrm{~m} / \mathrm{s}, 135.0 \mathrm{~m} / \mathrm{s}, 190.2 \mathrm{~m} / \mathrm{s} \ldots \ldots \ldots 3$

6.26 Magnified view of coalescing mode's modal parameter pdfs for uncertainty in mass . . . . 164

6.27 Modal parameter pdfs resulting from randomness in mass moment of inertia with $10 \% \mathrm{COV}$, obtained at 3 test airspeeds: $\approx 0 \mathrm{~m} / \mathrm{s}, 135.0 \mathrm{~m} / \mathrm{s}, 190.2 \mathrm{~m} / \mathrm{s} \ldots \ldots \ldots 4$

6.28 Magnified view of coalescing mode's modal parameter pdfs for uncertainty in mass moment of

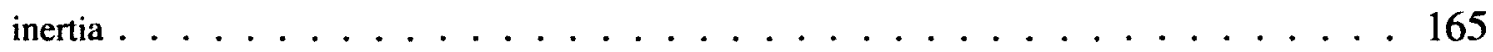

6.29 Modal parameter pdfs resulting from randomness in bending stiffness with $10 \% \mathrm{COV}$, obtained at 3 test airspeeds: $\approx 0 \mathrm{~m} / \mathrm{s}, 135.0 \mathrm{~m} / \mathrm{s}, 190.2 \mathrm{~m} / \mathrm{s} \ldots \ldots \ldots \ldots$

6.30 Magnified view of coalescing mode's modal parameter pdfs for uncertainty in bending stiffness 166

6.31 Modal parameter pdfs resulting from randomness in torsional stiffness with $10 \% \mathrm{COV}$, obtained at 3 test airspeeds: $\approx 0 \mathrm{~m} / \mathrm{s}, 135.0 \mathrm{~m} / \mathrm{s}, 190.2 \mathrm{~m} / \mathrm{s} \ldots \ldots \ldots 7$

6.32 Magnified view of coalescing mode's modal parameter pdfs for uncertainty in torsional stiffness 167

6.33 Modal parameter pdfs resulting from randomness in first structural damping ratio with $10 \%$ COV, obtained at 3 test airspeeds: $\approx 0 \mathrm{~m} / \mathrm{s}, 135.0 \mathrm{~m} / \mathrm{s}, 190.2 \mathrm{~m} / \mathrm{s} \ldots \ldots 168$

6.34 Magnified view of coalescing mode's modal parameter pdfs for uncertainty in first structural damping ratio $\ldots \ldots \ldots \ldots \ldots \ldots$

6.35 Modal parameter pdfs resulting from randomness in second structural damping ratio with $10 \%$ COV, obtained at 3 test airspeeds: $\approx 0 \mathrm{~m} / \mathrm{s}, 135.0 \mathrm{~m} / \mathrm{s}, 190.2 \mathrm{~m} / \mathrm{s} \ldots \ldots \ldots 9$

6.36 Magnified view of coalescing mode's modal parameter pdfs for uncertainty in second structural

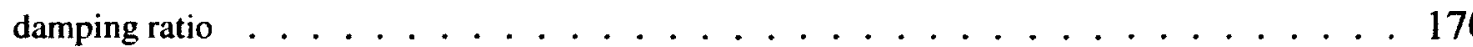

6.37 Modal parameter pdfs resulting from randomness in uncorrelated inertia terms with $10 \% \mathrm{COV}$, obtained at 3 test airspeeds: $\approx 0 \mathrm{~m} / \mathrm{s}, 135.0 \mathrm{~m} / \mathrm{s}, 190.2 \mathrm{~m} / \mathrm{s} \ldots \ldots \ldots$

6.38 Magnified view of coalescing mode's modal parameter pdfs for uncertainty in uncorrelated

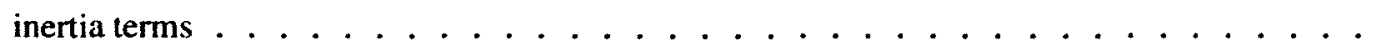

6.39 Modal parameter pdfs resulting from randomness in uncorrelated stiffness terms with $10 \%$ COV, obtained at 3 test airspeeds: $\approx 0 \mathrm{~m} / \mathrm{s}, 135.0 \mathrm{~m} / \mathrm{s}, 190.2 \mathrm{~m} / \mathrm{s} \ldots \ldots \ldots$ 
6.40 Magnified view of coalescing mode's modal parameter pdfs for uncertainty in uncorrelated stiffness terms $\ldots \ldots \ldots \ldots \ldots \ldots$

6.41 Modal parameter pdfs resulting from randomness in uncorrelated damping terms with $10 \%$ COV, obtained at 3 test airspeeds: $\approx 0 \mathrm{~m} / \mathrm{s}, 135.0 \mathrm{~m} / \mathrm{s}, 190.2 \mathrm{~m} / \mathrm{s} \ldots \ldots$

6.42 Magnified view of coalescing mode's modal parameter pdfs for uncertainty in uncorrelated

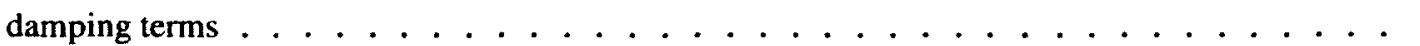

6.43 Modal parameter pdfs resulting from randomness in correlated inertia terms with $10 \% \mathrm{COV}$, obtained at 3 test airspeeds: $\approx 0 \mathrm{~m} / \mathrm{s}, 135.0 \mathrm{~m} / \mathrm{s}, 190.2 \mathrm{~m} / \mathrm{s} \ldots \ldots \ldots$

6.44 Magnified view of coalescing mode's modal parameter pdfs for uncertainty in correlated inertia

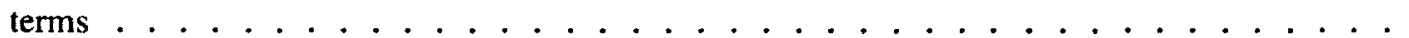

6.45 Modal parameter pdfs resulting from randomness in correlated stiffness terms with $10 \% \mathrm{COV}$, obtained at 3 test airspeeds: $\approx 0 \mathrm{~m} / \mathrm{s}, 135.0 \mathrm{~m} / \mathrm{s}, 190.2 \mathrm{~m} / \mathrm{s} \ldots \ldots \ldots$

6.46 Magnified view of coalescing mode's modal parameter pdfs for uncertainty in correlated stiffness terms $\ldots \ldots \ldots \ldots \ldots$

6.47 Modal parameter pdfs resulting from randomness in correlated damping terms with $10 \% \mathrm{COV}$, obtained at 3 test airspeeds: $\approx 0 \mathrm{~m} / \mathrm{s}, 135.0 \mathrm{~m} / \mathrm{s}, 190.2 \mathrm{~m} / \mathrm{s} \ldots \ldots \ldots$

6.48 Magnified view of coalescing mode's modal parameter pdfs for uncertainty in correlated damping terms $\ldots \ldots \ldots \ldots \ldots \ldots$

6.49 Modal parameter pdfs resulting from randomness in mass with $10 \% \mathrm{COV}$, obtained at 3 test airspeeds: $0 \mathrm{~m} / \mathrm{s}, 112 \mathrm{~m} / \mathrm{s}, 160.21 \mathrm{~m} / \mathrm{s} \ldots \ldots \ldots \ldots \ldots \ldots$

6.50 Magnified view of coalescing mode's modal parameter pdfs for uncertainty in mass . . . . .

6.51 Modal parameter pdfs resulting from randomness in mass moment of inertia with $10 \% \mathrm{COV}$, obtained at 3 test airspeeds: $0 \mathrm{~m} / \mathrm{s}, 112 \mathrm{~m} / \mathrm{s}, 160.21 \mathrm{~m} / \mathrm{s} \ldots \ldots \ldots$

6.52 Magnified view of coalescing mode's modal parameter pdfs for uncertainty in mass moment of inertia ..........................

6.53 Modal parameter pdfs resulting from randomness in bending stiffness with $10 \% \mathrm{COV}$, obtained at 3 test airspeeds: $0 \mathrm{~m} / \mathrm{s}, 112 \mathrm{~m} / \mathrm{s}, 160.21 \mathrm{~m} / \mathrm{s} \ldots \ldots \ldots \ldots$

6.54 Magnified view of coalescing mode's modal parameter pdfs for uncertainty in bending stiffness 
6.55 Modal parameter pdfs resulting from randomness in torsional stiffness with $10 \% \mathrm{COV}$, obtained at 3 test airspeeds: $0 \mathrm{~m} / \mathrm{s}, 112 \mathrm{~m} / \mathrm{s}, 160.21 \mathrm{~m} / \mathrm{s} \ldots \ldots \ldots \ldots$

6.56 Magnified view of coalescing mode's modal parameter pdfs for uncertainty in torsional stiffness 183

6.57 Modal parameter pdfs resulting from randomness in first structural damping ratio with $10 \%$ COV, obtained at 3 test airspeeds: $0 \mathrm{~m} / \mathrm{s}, 112 \mathrm{~m} / \mathrm{s}, 160.21 \mathrm{~m} / \mathrm{s} \ldots \ldots$. . . . . . . . . .

6.58 Magnified view of coalescing mode's modal parameter pdfs for uncertainty in first structural damping ratio $\ldots \ldots \ldots \ldots \ldots \ldots \ldots$

6.59 Modal parameter pdfs resulting from randomness in second structural damping ratio with $10 \%$ COV, obtained at 3 test airspeeds: $0 \mathrm{~m} / \mathrm{s}, 112 \mathrm{~m} / \mathrm{s}, 160.21 \mathrm{~m} / \mathrm{s} \ldots \ldots \ldots \ldots$

6.60 Magnified view of coalescing mode's modal parameter pdfs for uncertainty in second structural damping ratio $\ldots \ldots \ldots \ldots \ldots \ldots \ldots$

6.61 Modal parameter pdfs resulting from randomness in uncorrelated inertia terms with $10 \% \mathrm{COV}$, obtained at 3 test airspeeds: $0 \mathrm{~m} / \mathrm{s}, 112 \mathrm{~m} / \mathrm{s}, 160.21 \mathrm{~m} / \mathrm{s} \ldots \ldots \ldots$

6.62 Magnified view of coalescing mode's modal parameter pdfs for uncertainty in uncorrelated

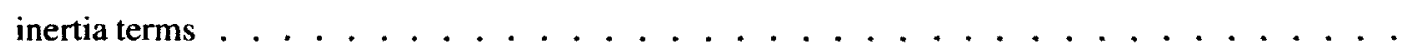

6.63 Modal parameter pdfs resulting from randomness in uncorrelated stiffness terms with $10 \%$ COV, obtained at 3 test airspeeds: $0 \mathrm{~m} / \mathrm{s}, 112 \mathrm{~m} / \mathrm{s}, 160.21 \mathrm{~m} / \mathrm{s} \ldots \ldots \ldots \ldots$

6.64 Magnified view of coalescing mode's modal parameter pdfs for uncertainty in uncorrelated stiffness terms $\ldots \ldots \ldots \ldots \ldots \ldots$

6.65 Modal parameter pdfs resulting from randomness in uncorrelated damping terms with $10 \%$ COV, obtained at 3 test airspeeds: $0 \mathrm{~m} / \mathrm{s}, 112 \mathrm{~m} / \mathrm{s}, 160.21 \mathrm{~m} / \mathrm{s} \ldots \ldots \ldots \ldots$

6.66 Magnified view of coalescing mode's modal parameter pdfs for uncertainty in uncorrelated

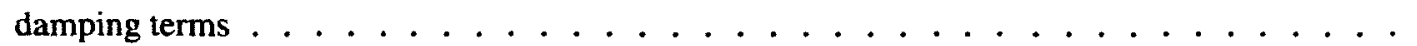

6.67 Modal parameter pdfs resulting from randomness in correlated inertia terms with $10 \% \mathrm{COV}$, obtained at 3 test airspeeds: $0 \mathrm{~m} / \mathrm{s}, 112 \mathrm{~m} / \mathrm{s}, 160.21 \mathrm{~m} / \mathrm{s} \ldots \ldots \ldots \ldots$

6.68 Magnified view of coalescing mode's modal parameter pdfs for uncertainty in correlated inertia 
6.69 Modal parameter pdfs resulting from randomness in correlated stiffness terms with $10 \% \mathrm{COV}$, obtained at 3 test airspeeds: $0 \mathrm{~m} / \mathrm{s}, 112 \mathrm{~m} / \mathrm{s}, 160.21 \mathrm{~m} / \mathrm{s} \ldots \ldots \ldots$

6.70 Magnified view of coalescing mode's modal parameter pdfs for uncertainty in correlated stiffness terms $\ldots \ldots \ldots \ldots \ldots \ldots \ldots$

6.71 Modal parameter pdfs resulting from randomness in correlated damping terms with $10 \% \mathrm{COV}$, obtained at 3 test airspeeds: $0 \mathrm{~m} / \mathrm{s}, 112 \mathrm{~m} / \mathrm{s}, 160.21 \mathrm{~m} / \mathrm{s} \ldots \ldots \ldots \ldots$

6.72 Magnified view of coalescing mode's modal parameter pdfs for uncertainty in correlated damping terms $\ldots \ldots \ldots \ldots \ldots \ldots$

6.73 Modal parameter pdfs resulting from randomness in mass with $10 \% \mathrm{COV}$, obtained at 3 test airspeeds: $\approx 0 m / s, 131.0 \mathrm{~m} / \mathrm{s}, 187.91 \mathrm{~m} / \mathrm{s} \ldots \ldots \ldots \ldots$

6.74 Magnified view of coalescing mode's modal parameter pdfs for uncertainty in mass $\ldots$. . . 194

6.75 Modal parameter pdfs resulting from randomness in mass moment of inertia with $10 \% \mathrm{COV}$, obtained at 3 test airspeeds: $\approx 0 \mathrm{~m} / \mathrm{s}, 131.0 \mathrm{~m} / \mathrm{s}, 187.91 \mathrm{~m} / \mathrm{s} \ldots \ldots \ldots$

6.76 Magnified view of coalescing mode's modal parameter pdfs for uncertainty in mass moment of

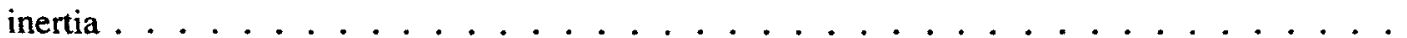

6.77 Modal parameter pdfs resulting from randomness in bending stiffness with $10 \% \mathrm{COV}$, obtained at 3 test airspeeds: $\approx 0 \mathrm{~m} / \mathrm{s}, 131.0 \mathrm{~m} / \mathrm{s}, 187.91 \mathrm{~m} / \mathrm{s} \ldots \ldots \ldots$

6.78 Magnified view of coalescing mode's modal parameter pdfs for uncertainty in bending stiffness

6.79 Modal parameter pdfs resulting from randomness in torsional stiffness with $10 \% \mathrm{COV}$, obtained at 3 test airspeeds: $\approx 0 \mathrm{~m} / \mathrm{s}, 131.0 \mathrm{~m} / \mathrm{s}, 187.91 \mathrm{~m} / \mathrm{s} \ldots \ldots \ldots \ldots$

6.80 Magnified view of coalescing mode's modal parameter pdfs for uncertainty in torsional stiffness

6.81 Modal parameter pdfs resulting from randomness in first structural damping ratio with $10 \%$ $\mathrm{COV}$, obtained at 3 test airspeeds: $\approx 0 \mathrm{~m} / \mathrm{s}, 131.0 \mathrm{~m} / \mathrm{s}, 187.91 \mathrm{~m} / \mathrm{s} \ldots \ldots \ldots$

6.82 Magnified view of coalescing mode's modal parameter pdfs for uncertainty in first structural damping ratio $\ldots \ldots \ldots \ldots \ldots$

6.83 Modal parameter pdfs resulting from randomness in second structural damping ratio with $10 \%$ COV, obtained at 3 test airspeeds: $\approx 0 \mathrm{~m} / \mathrm{s}, 131.0 \mathrm{~m} / \mathrm{s}, 187.91 \mathrm{~m} / \mathrm{s}$ 
6.84 Magnified view of coalescing mode's modal parameter pdfs for uncertainty in second structural

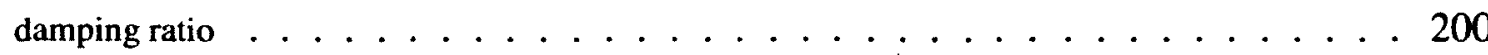

6.85 Modal parameter pdfs resulting from randomness in uncorrelated inertia terms with $10 \% \mathrm{COV}$, obtained at 3 test airspeeds: $\approx 0 \mathrm{~m} / \mathrm{s}, 131.0 \mathrm{~m} / \mathrm{s}, 187.91 \mathrm{~m} / \mathrm{s} \ldots \ldots 202$

6.86 Magnified view of coalescing mode's modal parameter pdfs for uncertainty in uncorrelated

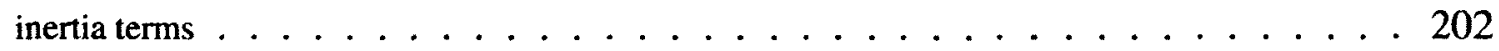

6.87 Modal parameter pdfs resulting from randomness in uncorrelated stiffness terms with $10 \%$ COV, obtained at 3 test airspeeds: $\approx 0 \mathrm{~m} / \mathrm{s}, 131.0 \mathrm{~m} / \mathrm{s}, 187.91 \mathrm{~m} / \mathrm{s} \ldots \ldots 203$

6.88 Magnified view of coalescing mode's modal parameter pdfs for uncertainty in uncorrelated stiffness terms . . . . . . . . . . . . . . . . . . 203

6.89 Modal parameter pdfs resulting from randomness in uncorrelated damping terms with $10 \%$ COV, obtained at 3 test airspeeds: $\approx 0 \mathrm{~m} / \mathrm{s}, 131.0 \mathrm{~m} / \mathrm{s}, 187.91 \mathrm{~m} / \mathrm{s} \ldots \ldots 204$

6.90 Magnified view of coalescing mode's modal parameter pdfs for uncertainty in uncorrelated

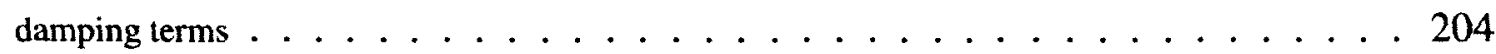

6.91 Modal parameter pdfs resulting from randomness in correlated inertia terms with $10 \% \mathrm{COV}$, obtained at 3 test airspeeds: $\approx 0 \mathrm{~m} / \mathrm{s}, 131.0 \mathrm{~m} / \mathrm{s}, 187.91 \mathrm{~m} / \mathrm{s} \ldots \ldots$. . . . . . 205

6.92 Magnified view of coalescing mode's modal parameter pdfs for uncertainty in correlated inertia

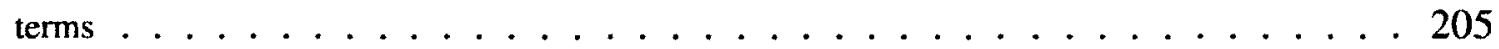

6.93 Modal parameter pdfs resulting from randomness in correlated stiffness terms with $10 \% \mathrm{COV}$, obtained at 3 test airspeeds: $\approx 0 \mathrm{~m} / \mathrm{s}, 131.0 \mathrm{~m} / \mathrm{s}, 187.91 \mathrm{~m} / \mathrm{s} \ldots \ldots 206$

6.94 Magnified view of coalescing mode's modal parameter pdfs for uncertainty in correlated stiff-

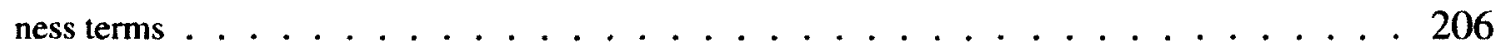

6.95 Modal parameter pdfs resulting from randomness in correlated damping terms with $10 \% \mathrm{COV}$, obtained at 3 test airspeeds: $\approx 0 \mathrm{~m} / \mathrm{s}, 131.0 \mathrm{~m} / \mathrm{s}, 187.91 \mathrm{~m} / \mathrm{s} \ldots \ldots$. . . . . . . 207

6.96 Magnified view of coalescing mode's modal parameter pdfs for uncertainty in correlated damp-

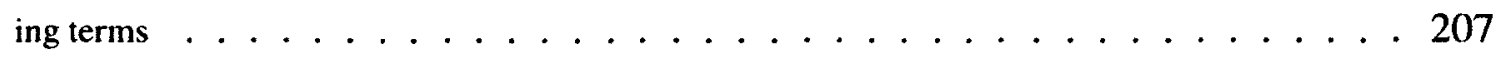

A.1 Airfoil with definition of geometric parameters . . . . . . . . . . . . . 212

A.2 Airfoil with definition of the translational and rotational springs . . . . . . . . . 212 
A.3 Definition of the forcing acting on airfoil, lift and moment, as well as the degrees of freedom in heave and pitch about elastic axis . . . . . . . . . . . . . . 213

A.4 Redefinition of lift and moment about aerodynamic center into equivalent forces about elastic axis 213

A.5 Illustration of airfoil and component of velocity that represents downwash $\ldots \ldots \ldots$

D.1 Theodorsen's function, $\mathrm{C}(\mathrm{k})$, at top $\mathrm{F}(\mathrm{k})$ and bottom $\mathrm{G}(\mathrm{k})[1] \ldots \ldots 28$

D.1 Top figure depicts first 3 flexural mode shapes; bottom figure depicts first 3 torsional

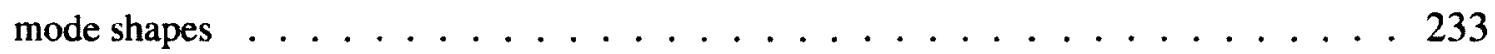




\section{Chapter 1}

\section{Introduction}

In a typical design practice, system parameters are selected to optimize the system performance and simultaneously satisfy all of the failure criteria. Effectively, an appropriate failure criterion should strive to avoid the possibility of failure throughout the life-cycle of the engineering system. With this in mind, all sources of uncertainty inherent to the model should be incorporated into such failure criteria. In many cases, the effect of uncertainty can be significant in modeling and simulation of engineering systems. Therefore, the effect of uncertainty must be considered for the robust design of these systems. For aircraft, for instance, the sources of uncertainty include (but not limited to) the production process of components, the flight conditions, aging process, etc.. In order to account for uncertainty in the aircraft certification process, the airworthiness criteria includes a safety factor that is empirically chosen from experience and flight tests. As this factor does not include a measure of risk associated to the selected value, it implicitly assumes that $100 \%$ of the aircraft contained in a certification batch would fall within the acceptable safety brackets. Although the safety factor approach has traditionally been used for the certification of an aircraft fleet, it has certain drawbacks. First, as already noted, it does not carry within itself any explicit information regarding the risk of failure. For instance, the critical speed for flutter instability that is obtained using a safety factor may be considered to be too conservative for a cost effective and optimal aircraft design. Second, neither this method or other methods could realistically guarantee absolute safety against failure and therefore, points toward the need to assess the risk associated with the chosen value of a design parameter for the 
failure analysis.

The focus of this work is the application of a probabilistic method for flutter analysis. This study focuses exclusively on the dynamic instability due to the coalescence flutter phenomenon. Such dynamic instability originates from the coupling mechanism between the structure and the fluid, whereby the airflow begins to feed energy to the structure, leading to the self-sustained growing oscillatory motions[1], which sometimes lead to a "benign" limit cycle oscillations or to catastrophic failure. Coalescence flutter requires at least two degrees-of-freedom with appropriate phase difference between the elements of aeroelastic modes. Using a probabilistic approach, the uncertainty in the flutter speed is estimated due to the random variability of the system parameters. In contrast to the safety factor approach, the probabilistic method provides a probability density function (pdf) of the flutter speed, from which the risk of failure can be estimated. The pdf of flutter speed could then be used to prescribe the limiting flight speeds for a prescribed probability of failure. The flight speed below this limiting speed would be deemed safe and at the same time, the design will not be overly conservative. The probabilistic method offers many advantages over the currently employed certification methods. The probabilistic approach provides a rational basis for estimating the flutter speed of an entire fleet of aircraft based on the statistical information regarding the system parameters. The current investigation focuses only on the uncertainty of the structural parameters, but the methodology can be easily extended to account for the random variability of the aerodynamic parameters. Furthermore, since coalescence flutter is a problem that can occur on any flexible structure exposed to airflow, the analysis covers a range of engineering applications, such as aircraft, bridges and wind turbines.

The main objectives of the thesis are summarized as follows:

1. For a two degree-of-freedom (dof) elastically mounted rigid airfoil, a detailed probabilistic analysis is carried out using Monte Carlo Simulation (MCS) for both, the quasisteady and unsteady aerodynamic loadings. The robustness of the flutter speed with respect to random variabilities of the structural parameters are investigated in detail. Furthermore, the probabilistic characteristics of the aeroelastic modal parameters (e.g. modal frequen- 
cies and decay rates) at pre-flutter airspeeds are studied in detail.

2. Next, the probabilistic flutter analysis is extended to the more realistic case of a continuous wing modeled as a uniform cantilever for the quasisteady and unsteady aerodynamic loads. Using the cantilever beam modes (without flow) as the basis functions, the coupled bending and torsional aeroelastic oscillations of the continuous wing are investigated from the probabilistic point of view. The effect of parametric uncertainties of the structural models is investigated in the flutter speed and the pre-flutter aeroelastic modal frequencies and decay rates.

3. From the probabilistic prospective, this analysis identifies the similarities and differences between the rigid and cantilever wing models and examines the effects of parameter correlation.

The layout of this thesis is summarized as follows:

Chapter 1 provides an introduction of the thesis. Chapter 2 involves a brief literature review relevant to the probabilistic analysis of aeroelastic flutter. Chapter 3 introduces the basic mechanism of the coalescence flutter using a two dof elastically mounted rigid model of a wing for both, the quasisteady and unsteady aerodynamics in a deterministic setting. It is then followed by the probabilistic analysis to consider the uncertainty effects in the inertia, stiffness and damping coefficients of the aeroelastic system. In particular, the non-linear transformation effects of the structural parameter uncertainties are investigated in detail on the flutter speed and the pre-flutter aeroelastic modal parameters. Then, Chapter 4 details the coupled bending and torsional aeroelastic vibration of a uniform cantilever subject to quasisteady and unsteady aerodynamics. Consequently, using the Galerkin Method, a coupled set of ordinary differential equations (odes) are derived from the original partial differential equations describing the bending-torsional motion of the cantilever. A detailed numerical investigation is conducted to study the influence of structural parameter uncertainty on the flutter speed. The significant effect of uncertainty is also highlighted in the aeroelastic modal frequencies and decay rates at the subcritical (i.e. pre-flutter) airspeed. 


\section{Chapter 2}

\section{Literature Review}

When structural dynamics and dynamic instabilities became an issue in the modern designs of aircraft, the subject of aeroelasticity has been an active area of research. Most of the early works have been dedicated towards the mathematical development and physical understanding of the aeroelastic phenomena in the deterministic setting in order to assess and improve the aircraft safety $[2,3,4]$. These deterministic methods however fail to take into account the random variability of the aircraft properties and the atmospheric conditions due to manufacturing process and the operational and environmental conditions. An assessment of all properties of the individual aircraft flying at specific flight conditions is neither possible nor practical due to excessive time consumption and expense associated with the aircraft testing [5]. As a result, a batch of aircraft must be certified based on testing of only a limited number of aircraft at a limited number of flight conditions. To take into account the inherent uncertainties into the design and certification of these aircraft, two different methods are currently applied. The earlier method, which is still widely applied in practice, uses an empirically chosen safety factor to incorporate this uncertainty [5], maintaining a deterministic framework. An empirical choice of a safety factor, however, does not always guarantee safety [5] and the hard limit set on the failure criteria may be too conservative for specific types of missions or aircraft. In contrast to the safety factor approach, the second method poses the problem in a probabilistic setting. This method permits the user to select a maximum flight speed based on a prescribed level of acceptable risk of failure for a specific scenario without being overly conservative. Recently 
the research initiative has been directed towards probabilistic uncertainty analysis in various engineering disciplines $[6,7,8,9,10,11]$. A brief literature review is provided in this chapter relevant to the fields of aeroelasticity, uncertainty quantification (UQ) and their applications to the problem of coalescence flutter. Initially, a brief introduction to the deterministic problem is provided, which is then followed by relevant literature in the uncertainty quantification.

Historically, the aircraft have gone through a multitude of changes in their overall look and design in order to accommodate and advance their functionality and performance. Generally, all aircraft underwent a significant reduction in the structural weight of the lifting surfaces (relative to the size of the aircraft) and a complete change in their geometry, rendering a much more complex in design. These enhancements were primarily accomplished by improving the original design concepts and developing techniques to have the ability to use better and lighter materials with higher strengths (e.g. composite materials) [12]. Such enhancements have contributed in achieving an increase in operational speeds, much greater maneuverability and other functional advancements in the fighter aircraft. In civilian aircraft, the increases in material strength permitted a design of lifting surfaces of high aspect ratio, making it possible to have larger aircraft flying at relatively fast speeds. As a result, wing flexibility increased, which led to amplification of aeroelastic effects. As the operational speeds have increased, the aircraft became prone to instabilities, which became a major issue in the design process. More care had to be given to these instabilities within the airworthiness criteria to achieve safety over the entire flight envelope. [3]

\subsection{Deterministic Aeroelastic Flutter}

Most recent research initiatives are devoted towards the development of techniques to analyze the stability of aeroelastic systems in a deterministic setting. Starting with the early works in the 1920 s to 1940 s, stability of a simplified rigid wing model, i.e. airfoil, has been extensively studied by Wagner [4] and Theodorsen [13, 14] and later, Goland proposed an analytical solution of a simple uniform cantilever wing model, known as the Goland Wing [15]. For simplicity, only linear structural and aerodynamic systems were considered, i.e. small deflections and sub- 
sonic flow were assumed. Since then, other researchers have conducted similar investigations with various modifications to the general Goland wing model. Some examples of such works include wings that are swept and tapered with camber effects [16], performing maneuvers and holding payload [17], in transonic region [18].

For complex geometrical configurations and variation of the material properties the numerical methods such as finite element method (FEM) are commonly used. The aerodynamic models for inviscid flow around the wing rely upon the potential flow theory $[13,19,20]$. For viscous flow, the Navier Stokes equations are solved numerically, using a Computational Fluid Dynamics (CFD) method [21], to obtain the aerodynamic loading on the structural model. A few papers report the solutions to the dynamic response and stability problems using FEM $[22,23,24]$, where the forcing can be accurately described by the potential flow theory. The first two references $[22,23]$ examine the response of a cable stayed bridge to gust loading and the third reference [24] examines stability of a model aircraft and its wings. In situations where the potential flow theory does not apply due to nonlinear effects (e.g. viscosity, large deformations or material plasticity, etc.), seen in references $[25,26,27]$, CFD methods are used to solve the full Navier Stokes equations to obtain the forcing expressions.

Although this review mostly focuses on the applications in aircraft design, similar aeroelastic effects occur in many other fields, including for instance cable-stayed suspension bridges and wind turbines $[28,29]$.

The aeroelastic coupling between the fluid and structure leads to a non-self adjoint operator for flutter analysis. In its discrete representation, the non-self adjoint operator gives rise to non-symmetric mass, stiffness and damping matrices. The classical (normal) modes do not exist for such systems. Stated differently, the mode shapes of such systems are complex and non-orthogonal. Yang [30] and Eldred [31] provide a few examples of non-self adjoint systems in structural dynamics. Similar to the coalescence flutter problem in aeroelasticity, where the fluid flow changes the mass, stiffness and damping properties of the aeroelastic system, the dynamical systems involving gyroscopic forces also possess non-symmetric damping and stiffness matrices. 
Hoen [32] and Ewins [33] provide a physical interpretation of the complex modes. They illustrate how the complex nature of these modes relates to a phase difference between the elements of each mode shape vector. For aeroelastic systems, the phase lag effect may eventually lead to the dynamic instability. An elementary case is illustrated by Zimmerman [34], who studies an airfoil in a steady flight. Although this method provides a solution, it has inherent limitations for the analysis of non-self adjoint operators. Yang [30] demonstrates how the eigenvalue analysis of non-self adjoint operators leads to non-orthogonal eigenvectors. Yang notes that the modal analysis with non-orthogonal eigenvectors does not guarantee the convergence of solution as the eigenvectors may not constitute a complete basis. Yang adopts an eigenfunction expansion of the Green's function for the non-self adjoint system. However, several papers report the usefulness of the modal analysis using these complex modes [32] [22].

In the aeroelastic computation, inherent modeling uncertainties present in the structural and aerodynamic systems and inaccuracies in the measurements demands uncertainty quantification. As a result, the probabilistic methods have recently received considerable amount of attention $[35,36,37,12,38,39]$. Some of these methods include simple Monte Carlo Simulation (MCS), perturbation method and spectral methods $[11,40]$. A recent paper by Pettit [35] stresses on the need for uncertainty quantification in aeroelasticity and outlines some of the benefits of the probabilistic analysis. Pettit reviews some of the currently available and developing uncertainty quantification techniques and discusses the major challenges associated with the implementation of these techniques in aeroelastic design of the aircraft. He points out the lack of coordination among teams from different engineering fields, lack of a unified method for uncertainty definitions and its incorporation into the analysis and a preference, or persistence, of researchers and professionals to stay within a traditional framework of the deterministic analysis techniques. Nonetheless, all of these challenges will be resolved as the probabilistic methods achieve further advancements $[35,41]$. 


\subsection{Uncertainty Quantification}

Next, a short overview of the UQ methods and some of their applications in the field of aeroelasticity is provided. Due to large computational costs associated with the probabilistic analysis, some authors prefer to conduct a sensitivity analysis in order to identify the most significant parameters influencing the output $[36,42,43]$. This method is based on a Taylor Series expansion of the response around the baseline parameter value. There are two weaknesses associated with the sensitivity analysis. Firstly, the sensitivity of the solution is computed only at the baseline parameter value using (first and second order) Taylor Series expansion and encounters difficulty for large deviations of the parameters. Secondly, the parameter correlation effects cannot be taken into account by the sensitivity analysis.

\subsubsection{Monte Carlo Sampling (MCS)}

Schueller et al. [11] reviews the most commonly used methods for UQ to propagate the uncertainty in complex structures. They introduce the perturbation method, various forms of spectral methods and MCS. The simplest method to propagate the uncertainty is to apply a direct MCS technique. In MCS, a large set of deterministic problems is solved using the samples of input random vectors that are generated from their joint pdf. From the ensemble of output, the statistics of the response (including the joint pdf) can be estimated. Although MCS is versatile it is also computationally inefficient, therefore it is typically used as a base of comparison for other methods $[5,42,44,6,45,46]$. Lindsley et al. applied MCS to propagate stiffness and boundary condition (BC) uncertainty in a plate to analyze the probabilistic effects in Limit Cycle Oscillation (LCO) [47, 48]. Murugan et al. [49] applied MCS to investigate the effects of uncertainty in the helicopter composite blade stiffness on the response. Siva et al. [50] extended this study to consider the effect of uncertainty in the blade and rotor geometric and aerodynamic properties on the thrust and power coefficients.

An earlier study by Millwater et al. [51] has explored the advantages of the probabilistic assessment in structural failure detection. Using the historical measurement data and MCS to describe uncertainty in the structural parameters, the authors have illustrated the importance of 
the uncertainty quantification and sensitivity analysis for improvement of the design concept with a reduced probability of failure. Similar effects have been explored by Sexstone [52], where he describes the ELAPS method (developed and used by NASA) to provide a good early design concept. In these designs, the structural weight plays a major role in establishing the design parameters. Therefore a probabilistic analysis using MCS is conducted to probabilistically estimate the total structural weight due to random variability of the individual components.

\subsubsection{Perturbation Approach}

As the MCS is computationally inefficient, other efficient methods for uncertainty propagation were developed. The Perturbation technique that uses a Taylor Series expansion around a mean (baseline) value parameter can efficiently capture the mean and covariances of the solution. Moss [7,53] examines a simple undamped discrete structural system to show the effectiveness of perturbation technique for an inverse modal analysis problem using a first and second order Taylor series expansion with uniform distribution of input. The results of this method show good agreement when compared with the MCS technique, but only for low uncertainty in the input. The perturbation method proves to be much more efficient than the MCS for low levels of uncertainty $[54,53,55]$. Oh and Librescu [56] present a method based on the Perturbation technique in order to obtain a measure of randomness within the modal parameters based on uncertainty contained within the structural properties of a composite cantilever wing. These authors focus strictly on the analysis of a system with no flow effects (with self-adjoint operators). Ganesan [57] has treated a system with non-self adjoint operators, where he uses a Perturbation approach for uncertainty quantification where the material properties and loading conditions are considered as random. Ganesan considers only to undamped systems with low level of uncertainty.

\subsubsection{Spectral and Collocation Methods}

The spectral and collocation methods (e.g. $[58,59])$ prove to be much more efficient than the MCS and do not suffer from an assumption of low uncertainty of the Perturbation technique. 
These methods are based on a spectral decomposition of the random input process by a set of deterministic shape functions multiplying a random amplitude and the associated solution obtained by expanding the solution by the polynomial chaos expansion (e.g. $[58,59,60]$. The Collocation methods (pseudo-spectral methods) include a variety of different collocation techniques for random variables based on global [61] or multi-element collocation [62, 63]. Witteveen, Loeven and Bijl $[64,65,66,61]$ compare the efficiency of several such methods. In [64] a new collocation method is proposed, where a piecewise cubic interpolation is used, instead of a typical global collocation. In this work, the effectiveness of the new approach is examined against the Galerkin (intrusive) Polynomial Chaos Expansion (PCE) and the typical Stochastic Collocation. Similarly, other two investigations $[66,65]$ propose a Probabilistic Collocation (PC) method, effectiveness of which is compared against Stochastic Collocation and the intrusive and non-intrusive PCE. The probabilistic representation of a bifurcation point is considered in [66] when the external torque is considered a random quantity. The probabilistic representation of the lift and drag forces and dynamic pressures acting on the airfoil are reported in [65] where the free stream airspeed is considered a random quantity. Accuracy of all methods is validated using the MCS approach. Then, Witteveen et al. [61] have proposed an extension to the general non-intrusive PCE method in order to avoid the problem of growing variance in the unsteady analysis of Limit Cycle Oscillations. This method, referred to as PCLCO, bounds the variance for long-term results, where it obtains results comparable with the MCS. For short-term response, ordinary Probabilistic Collocation (PC) provides very good results.

Although both spectral and collocation methods are very powerful, they share a single weakness. For multi-modal non-Gaussian pdfs, the order of chaos basis and collocation points increases significantly, thereby increasing the problem size [67].

\subsection{Probabilistic Flutter Speed Estimation}

The probabilistic estimation of the coalescence flutter speed has been previously examined by numerous authors. For example, Kurdi [42] examines the effects of uncertainty in the geomet- 
rical dimensions of isolated wing components, i.e. thickness and area, on the flutter onset at different flight conditions. Initially, Kurdi performs a sensitivity analysis on the baseline wing in order to eliminate the parameters that prove to be insignificant in controlling the natural frequencies. Consequentially, Kurdi optimizes the baseline wing parameters to achieve maximum values of the natural frequencies. Using MCS for uncertainty quantification, Kurdi compares the flutter speed statistics of the baseline and optimized wings with or without a tip store mass. In the absence of a tip store mass, the small uncertainty in geometric parameters does not lead to large result uncertainty in the flutter speed for the entire range of subsonic and transonic flight regime. As the tip store mass is attached to the wing, the uncertainty in the flutter speed remains very similar to the case with no tip store mass within the subsonic region. For the transonic region, the flutter speed experiences large uncertainty and its pdf becomes multi-modal. Pitt [5] studies the effects of uncertainty in the material properties (mass, stiffness and center of gravity), the natural frequencies (bending and torsion) and the air density on variations of flutter speed in an airfoil and a beam-rod wing FEM model. Only effects of independent parameter uncertainty on the flutter speed were examined. Pitt uses the MCS technique for uncertainty propagation of the random input parameters described by a Gaussian distribution. He identifies the practical benefits of the probabilistic approach over the currently used safety margin approach, which may be too conservative. For an airfoil, the probabilistic results illustrate that the most critical parameters (i.e. ones producing the significant sensitivity in the flutter speed) prove to be the torsion frequency, mass and air density. In this case $+1-15 \%$ variation in all parameters resulted in a failure of the safety margin requirement set forth by the current military standard. Next, a 3-D wing was examined using a beam-rod analogy modeled using FEM. In this analysis, element mass, stiffness and modeshapes (by use of Modal Assurance Criteria) were varied. When all of these parameters were varied together, mass $+/-5 \%$, stiffness $+/-0.1 \%$, modeshapes $+/-5 \%$, the resulting flutter speed retained a Gaussian distribution and varied by $+/-4 \%$, which is acceptable level of uncertainty in the current military standards. Bansal et al. [44] also investigated the effects of independent uncertainty in the material and geometrical properties of a tapered wing on the sensitivity and uncertainty in the flutter speed. Using MCS 
Bansal et al. found that the chord-wise location of mass center and torsional rigidity had the most influence on the uncertainty of the flutter speed. The effects of structural uncertainty were also studied by Borello et al. [36], whereby different reliability methods are compared with respect to the results of MCS. Using a rigid wing model, this work investigated the influence of different amounts of structural parameter uncertainty on the flutter speed to assess the accuracy of the reliability methods. Moreover, the validity of a rigid wing model (airfoil) was established based on the comparison of the deterministic flutter speed of the uniform Goland wing model [15] and an equivalent rigid wing model. Furthermore, the importance of the sensitivity analysis was also illustrated in this investigation. For an isotropic uniform wing, elastic modulus was shown to have negligible sensitivity on the flutter speed, and hence was treated as a deterministic variable. Uncertainty in the flutter speed was also investigated by Chung et al. [68], who uses the $\mu$-method to study the effects of structural and aerodynamic parameter uncertainty on the flutter of a uniform wing. In this approach a weight value, which relates to the level of uncertainty in the system, is assigned to each parameter of the modal matrices and the real and imaginary parts of the Theodorsen function. The $\mu$-method, however, determines only the worse case scenario of the flutter phenomenon, and hence it does not actually quantify the risk of failure. The probabilistic analysis of the flutter phenomenon was also conducted by Witteveen et al. [61] due to the random variability of the free flow airspeed. Although only a low amount of input uncertainty was selected, the results have shown a very substantial probability of flutter failure. Castravete and Ibrahim [69] investigated the effects of uncertainty in stiffness on the flutter speed using a perturbation approach with application of the KarhunenLoeve expansion. For a large input variance, the limitation of the perturbation approach was identified in this investigation.

The effect of uncertainties are also investigated based on the widely used Flutter Margin approach, which was originally established by Zimmerman and Weissenburger [70]. This method evaluates the stability based on the Flutter Margin (FM) measure, which is evaluated for a specific airspeed using the modal parameter values. Then a quadratic relationship is used to extrapolate a flutter speed based on a few measurements of modal parameters $[70,71]$. Several 
researchers adopted this method to study the probabilistic effects $[72,73,74]$.

\subsection{Probabilistic Characterization of Modal Parameters}

Substantial amount of research has been devoted to examine the influence of the parameter uncertainties on the modal parameters of the structural systems. The early work of Shinozuka et al. [46] investigated the modal parameter uncertainty using the perturbation approach. Benaroya [53] provides the probabilistic representation of the modal properties based on the perturbation approach. Similarly, Adhikari [75] has used an asymptotic approach to examine the probabilistic characteristics of eigenvalues. Oh and Librescu [56] have used the perturbation approach to perform risk analysis based on the eigenvalue analysis of a composite wing. In another work by Adhikari [76], the eigenvalue density plots were used to obtain a representation of the modal parameter pdfs of a structural system. The eigenvalue density plots eliminate the need for eigenvalue sorting for closely spaced modes. A PCE approach was used to study the effects of the uncertainty on the modal parameters by Ghosh and Ghanem [37, 58], Ghosh et al. [40], Meidani and Ghanem [77]. They validated their results based on MCS results. Mingolet and Soize [78] have investigated a case with non-parametric uncertainty in the wing flexible boundary conditions. They used the random matrix representation of the structural system matrices to model such uncertainty. The results have shown a multi-modal pdfs of the flutter speed and the aeroelastic modal frequency in the flutter mode. Khodaparast et al. [79] conducted the probabilistic analysis (using interval arithmetic and fuzzy set theory) to examine the effect of parameter uncertainty on the pre-flutter modal parameters and the flutter speed of an aeroelastic system. Reddy et al.[80] have investigated the probabilistic properties of aeroelastic modal parameters for different values of reduced frequencies for two aeroelastic configurations (one stable configuration and the other unstable configuration). The sensitivity of the aeroelastic system modal parameters is also investigated by Plaut and Adhikari [81, 82] based on derivatives of complex modes. With this brief overview of literature, this section is complete. The following section begins the probabilistic investigation of the flutter problem. 


\section{Chapter 3}

\section{A Two Degree-of-freedom Airfoil: Flutter Speed}

\subsection{Introduction}

A realistic representation of the dynamic behaviour of an arbitrary wing is generally established with a 3-D structural and aerodynamic model, which takes into account the 3-D flow effects. A direct analysis of this 3-D model of the wing, however, poses substantial theoretical, analytical and computational challenges. Complexities arise in the development of the analytical (when they are available) and numerical solutions of the problem. Furthermore, a relatively large computational time is required to obtain an accurate numerical solution to the problem.

In this chapter, the analysis of flutter in a 3-D cantilever wing is simplified using a 2-D rigid wing model, also known as a typical section. This two degree-of-freedom model captures the essential aeroelastic effects without introducing the theoretical and computational complexities in its formulation and solution [1]. Consequently, the 2-D analysis is used to introduce the basic physics of the coalescence flutter phenomenon. Furthermore, the rigid wing model analysis also simplifies the experimental verification of its numerical predictions.

In this chapter, a detailed derivation of the 2-D airfoil dynamics is formulated for both, the quasisteady and unsteady aerodynamic loads. Then, the probabilistic solution of the flutter problem is obtained in time domain. The probabilistic analysis is then interpreted based on the 
physics of coalescence flutter for the deterministic system. Effectively, the probabilistic results are compared to those obtained from the deterministic case. The probabilistic results are then contrasted for the cases of quasisteady and unsteady aerodynamics. Lastly, a detailed numerical investigation is carried out for the random eigenvalue problem relating to the natural frequencies and decay rates of the aeroelastic system at the subcritical (pre-flutter) airspeeds.

\subsection{Deterministic Formulation}

The airfoil under investigation and the associated elastic springs are shown in fig. (3.1) below. To simplify the derivations, the airfoil considered in this analysis maintains a

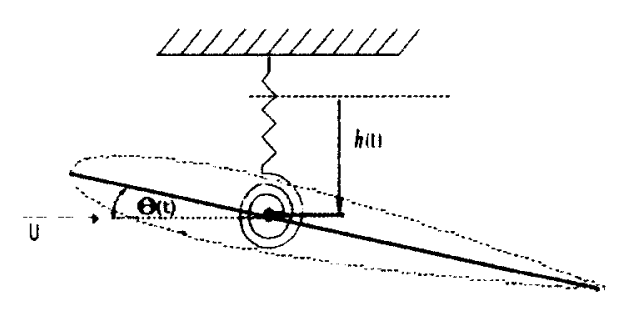

Figure 3.1: Example of an airfoil under study

symmetric geometry about the chord, hence it is free of camber effects. Regardless of this assumption, camber of the section does not effect the stability of a linear aeroelastic system [1]. The dynamics of a rigid wing model are derived based on the following assumptions:

- the structural vibration is limited to small amplitude motion

- the validity of the model is restricted to lower Mach numbers, relating to subsonic flow regime

- the Reynolds number at flutter speed is assumed to be very high, leading to negligibly small viscous effects

These assumptions justify the linear analysis [1]. Validity and limitations of the linear modeling of structural and aerodynamic subsystems will be discussed in more detail later in this chapter.

The coupled linear ordinary differential equations (ODEs) representing the motions of the airfoil in heave and pitch are: 


$$
\begin{aligned}
& m \ddot{h}+m b x_{\theta} \ddot{\theta}+B_{1} \dot{h}+B_{2} \dot{\theta}+k_{h} h=-L \\
& I_{e a} \ddot{\theta}+m b x_{\theta} \ddot{h}+B_{3} \dot{h}+B_{4} \dot{\theta}+k_{\theta} \theta=M_{e a}
\end{aligned}
$$

where $m$ and $I_{e a}$ terms represent the structural mass and the mass moment of inertia, respectively (note that $I_{e a}=m b^{2}\left(r_{g}^{2}+x_{\theta}^{2}\right)$, where $r_{g}$ is a non-dimensional radius of gyration); $k_{h}$ and $k_{\theta}$ terms represent the spring stiffnesses in heave and pitch, respectively; $B_{1}, B_{3}, B_{2}$ and $B_{4}$ are the elements of the structural damping matrix. The structural damping is assumed to be a proportional damping $\left(\right.$ i.e. $\left.B_{s t r}=d_{1} M_{s t r}+d_{2} K_{s t r}=\left[\begin{array}{cc}B_{1} & B_{2} \\ B_{3} & B_{4}\end{array}\right]\right)$, which is set by the prescribed modal damping ratios; $b$ and $x_{\theta}$ are geometric parameters representing a half-chord length and the mass offset aft of the elastic axis normalized by the half-chord length, respectively; $h$ and $\theta$ are the two degrees of freedom representing the heave and pitch motions of the elastic axis of the airfoil, respectively; $L$ and $M_{e a}$ represent the aerodynamic lift and moment about the elastic axis respectively. Since the model is represented in 2-D, all of the model parameters and loading terms are stated as per meter span values. A detailed derivation of the equations of motion is presented in Appendix A.

In the matrix form, eqs. (3.1)-(3.2) can be represented as follows:

$$
\left[M_{s t r}\right]\left\{\begin{array}{c}
\ddot{h} \\
\ddot{\theta}
\end{array}\right\}+\left[B_{s t r}\right]\left\{\begin{array}{l}
\dot{h} \\
\dot{\theta}
\end{array}\right\}+\left[K_{s t r}\right]\left\{\begin{array}{l}
h \\
\theta
\end{array}\right\}=\left\{\begin{array}{c}
-L \\
M_{e a}
\end{array}\right\}
$$

where $\left[M_{s t r}\right],\left[B_{s t r}\right]$ and $\left[K_{s t r}\right]$ are the structural mass, damping and stiffness matrices, respectively. These structural matrices are symmetric and positive-definite. For the aerodynamic loading, both quasisteady and unsteady aerodynamics are considered in this investigation. The next section formulates the two types of aerodynamics and points out when it is appropriate to simplify the more general unsteady aerodynamics to the quasisteady counterpart. The derivation of aerodynamics is established based on the Thin Airfoil theory. 


\subsubsection{Quasisteady Aerodynamics}

This section provides a brief overview of the formulation of quasisteady aerodynamics. The detailed derivation of equations of aerodynamic load for a typical section are provided in Appendix A. These derivations are taken from texts by Hodges and Pierce [83] and Fung [1].

The general quasisteady aerodynamic forcing considers the effects of $\theta, \dot{h}$ and $\dot{\theta}$ motions, which alter the angle of attack. In addition, the added mass effects will be included together with the quasisteady aerodynamics. The added mass effects are function of acceleration terms $\ddot{h}$ and $\ddot{\theta}$. Typically, the added mass does not appear with the quasisteady aerodynamics model, however, its inclusion does not invalidate the quasisteady model assumption. The reason for its inclusion here is simple: when contrasting the effects of quasisteady and unsteady aerodynamics, we would like to focus strictly on the effect of addition of the circulatory force component, which will be introduced shortly.

Effectively, the quasisteady lift and moment are functions of the airspeed, heave and pitch motions as denoted by:

$$
\begin{aligned}
& L=L(U, \theta, \dot{h}, \dot{\theta}, \ddot{h}, \ddot{\theta}) \\
& M_{e a}=M_{e a}(U, \theta, \dot{h}, \dot{\theta}, \ddot{h}, \ddot{\theta})
\end{aligned}
$$

The aerodynamic load vector in eq. (3.3) can be recast as:

$$
\left\{\begin{array}{c}
-L \\
M_{e a}
\end{array}\right\}=\left[M_{\text {aero }}\right]\left\{\begin{array}{c}
\ddot{h} \\
\ddot{\theta}
\end{array}\right\}+\left[B_{\text {aero }}(U)\right]\left\{\begin{array}{c}
\dot{h} \\
\dot{\theta}
\end{array}\right\}+\left[K_{\text {aero }}(U)\right]\left\{\begin{array}{c}
h \\
\theta
\end{array}\right\}
$$

where $\left[M_{\text {aero }}\right],\left[B_{\text {aero }}\right]$ and $\left[K_{\text {aero }}\right]$ are the aerodynamic mass, damping and stiffness matrices respectively. Only the damping and stiffness matrices are influenced by the airflow. The aerodynamic damping and stiffness matrices are non-symmetric.

In the general case of unsteady aerodynamics, the aerodynamic damping and stiffness can be represented as a function of a Theodorsen's function, $C(k)$, which in itself is a function of the reduced frequency $k=\frac{\omega b}{U_{\infty}}$, where $\omega, b$ and $U_{\infty}$ are the frequency of oscillation, the halfchord length (see fig. (3.1)) and free flow airspeed. Typical graphs of the real and imaginary 
parts of the Theodorsen's function: $C(k)=F(k)+i G(k)$ are plotted below in fig. (3.2). This function represents the effects of trailing vortices, which are time dependent [1]. Such effects are reflected by a lag between the forcing and the response. Naturally, unsteady aerodynamics would have to be used for analysis of a general case. However, the unsteady (circulatory force) effects are negligible in some cases, meaning that a steady condition is reached instantly, leading to the case of quasisteady aerodynamics, where the
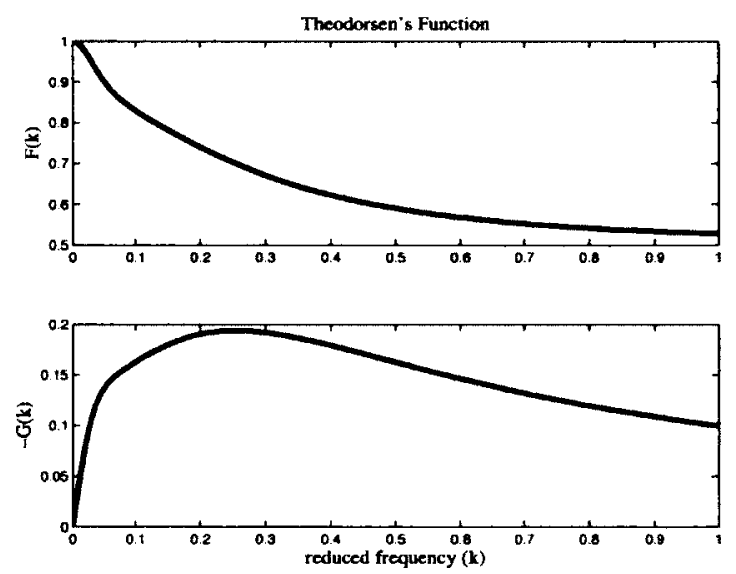

Figure 3.2: Theodorsen's function, $C(k)$, at top $F(k)$ and bottom $G(k)[1]$

Theodorsen's function takes a value of 1.0. Such condition occurs only when the value of reduced frequency is 0 , where the real component is 1.0 and the imaginary component is 0 , as evident from fig. (3.2). Zero imaginary component implies that there is no lag between the response and the forcing. This condition occurs only when either of two conditions are met: the frequency of vibration is 0 or the free stream air flow speed is $\infty$. Physically, the first condition can be interpreted as steady motion aerodynamics, where the angle of attack remains constant. The second condition implies that the wing is moving at such a high speed, that the unsteady circulatory effects move far downstream before the wing could actually register these effects. In reality, however, these conditions are impractical. If the aircraft is moving at a very high speed and/or the response vibrations are of very low frequency range, the quasisteady aerodynamics could approximate the unsteady aerodynamics with reasonable accuracy. In this case, the quasisteady treatment of aerodynamics removes the dependence of aerodynamic loading on the Theodorsen's function (removing the explicit time dependence), since it has a unit value. 
Next, eq. (3.3) can be recast as:

$$
\left[M_{a e}\right]\left\{\begin{array}{c}
\ddot{h} \\
\ddot{\theta}
\end{array}\right\}+\left[B_{a e}\right]\left\{\begin{array}{c}
\dot{h} \\
\dot{\theta}
\end{array}\right\}+\left[K_{a e}\right]\left\{\begin{array}{l}
h \\
\theta
\end{array}\right\}=\left\{\begin{array}{l}
0 \\
0
\end{array}\right\}
$$

where the mass, damping and stiffness matrices are shown next, with their full development provided in Appendix A.

$$
\begin{aligned}
& {\left[M_{a e}\right]=\left[\begin{array}{cc}
m+\frac{1}{2} \rho b^{2} C_{L \alpha} & m b x_{\theta}-\frac{1}{2} \rho b^{3} C_{L \alpha} a_{h} \\
m b x_{\theta}-\frac{1}{2} \rho b^{3} C_{L \alpha} a_{h} & I_{e a}+\frac{1}{2} \rho b^{4} C_{L \alpha}\left(\frac{1}{8}+a_{h}^{2}\right)
\end{array}\right]} \\
& {\left[B_{a e}\right]=\left[\begin{array}{cc}
B_{1}+\rho U b C_{L \alpha} & B_{2}+\rho U b^{2} C_{L \alpha}\left(1-a_{h}\right) \\
B_{3}-\rho U b^{2} C_{L \alpha}\left(\frac{1}{2}+a_{h}\right) & B_{4}-\rho U b^{3} C_{L \alpha} a_{h}\left(\frac{1}{2}-a_{h}\right)
\end{array}\right]} \\
& {\left[K_{a e}\right]=\left[\begin{array}{cc}
k_{h} & \rho U^{2} b C_{L \alpha} \\
0 & k_{\theta}-\rho U^{2} b^{2} C_{L \alpha}\left(a_{h}+\frac{1}{2}\right)
\end{array}\right]}
\end{aligned}
$$

where $\rho$ is the air density, $C_{L \alpha}$ is the lift curve slope and $a_{h}$ is a geometrical dimension normalized by the half-chord length, representing elastic axis offset aft of mid-chord. The important geometric parameters are illustrated in fig. (3.3) below.

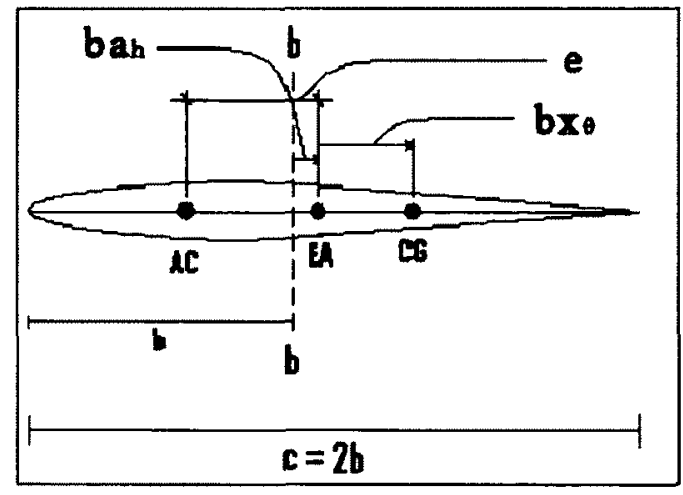

Figure 3.3: Symmetric airfoil illustrating important geometric parameters

\section{Mach Correction Factor}

In the subsonic flow, the compressibility effects are generally considered negligible for very low airspeeds. As the airspeed increases and the Mach number passes a value of 0.3 , the compressibility effects become more significant and therefore they have to be accounted for. Fortunately, a correction factor exists to include these effects. A Prandtl-Glauert Mach Correction Factor 
[84] is used in the lift curve slope to consider these effects for the subsonic regime. This factor is applied as follows:

$$
C_{L \alpha}=\frac{\bar{C}_{L \alpha}}{\sqrt{1-M_{\infty}^{2}}}
$$

where $\bar{C}_{L \alpha}$ is the lift curve slope at $M_{\infty}=0$ and $M_{\infty}$ is the free flow Mach number being a function of the free flow airspeed and a speed of sound $a$, stated as $M_{\infty}=\frac{U_{\infty}}{a}$. With this correction, the compressibility effects have been included in the aeroelastic analysis.

\section{Physical Explanation of Coalescence Flutter}

The fluid-structure interaction problem in aeroelasticity involves a non-self adjoint (partial) differential operator describing the dynamics of the structure. The finite dimensional discretization of such differential operator leads to a non-symmetric stiffness and damping matrices. This fact leads to a change in the system stability characteristics, which may lead to the loss of stability of the structure due to static (divergence) and dynamic (flutter) instabilities $[85,86]$. In this thesis, we investigate the dynamic instability phenomenon leading to the coalescence flutter instability.

From another prospective, the classical theory of structural dynamics generally involves a symmetric, undamped or proportionally damped structural system with the absence of fluid. Such dynamical system produces classical modes that are real. In this case, when the structure vibrates with one of its (damped) natural frequencies, all the dof of the system vibrate in the same phase. Such oscillations produce standing waves. On the contrary, the classical modes do not exist for the aeroelastic system due to non-symmetric nature of its system matrices. These modes will be defined as aeroelastic modes which are generally complex. The vibration of a system with the frequencies that are associated with these complex modes will lead to traveling wave motions, whereby the dofs will contain phase differences $[34,32]$. This very phase lag effect introduces the coalescence flutter instability.

For better visualization of a link between the phase difference and the decay variations associated with the coalescence flutter phenomenon, work produced by the airflow will be examined next. As already mentioned, changes in the coupling properties that are incurred due to increase in airspeed lead to changes in the phase differences between individual modal components of 
the modes that are involved in the flutter mechanism. Exactly at the flutter point, the coupling interactions of the aeroelastic system cause the phases in the modal components of the flutter mode to align in such a way, where the total work induced onto the structural subsystem by aerodynamic loading would be zero, resulting in self-excited oscillation of the airfoil. Once this point of instability is passed, in absence of any external forcing terms, the total work term becomes positive, and in effect the airfoil, which was modeled as a linear system, would experience diverging oscillations. For clarity purposes, an illustrative example taken from Fung's theory [1] is provided next. With the same definition for the airfoil parameters, shown in fig. (3.1) and same predefined degrees of freedom, assuming steady aerodynamics and simple harmonic motion (response at flutter point) for both heave and pitch, work due to lift can be obtained through the following expression:

$$
\partial W_{L}=-L \partial h
$$

obtaining total work produced within a cycle of oscillation by integrating it over one cycle of motion bounded between $h_{1}$ and $h_{2}$ :

$$
W_{L}=-\int_{h_{1}}^{h_{2}} L \partial h
$$

$W_{L}$ is the work generated by aerodynamic lift through the displacement in heave. According to steady aerodynamics, lift $L \propto \theta$. Restating lift and changing the integral to temporal depiction of oscillation cycle, work is depicted as:

$$
W_{L} \propto-\int_{h_{1}}^{h_{2}} \theta \partial h=-\int_{t_{1}}^{t_{2}} \theta \frac{\partial h}{\partial t} d t=-\int_{t_{1}}^{t_{2}} \theta \dot{h} d t
$$

Together with the above equation eq. (3.13), a hypothetical example is shown below for clarity, illustrating a case where the steady aerodynamic loading would lead to positive work. 


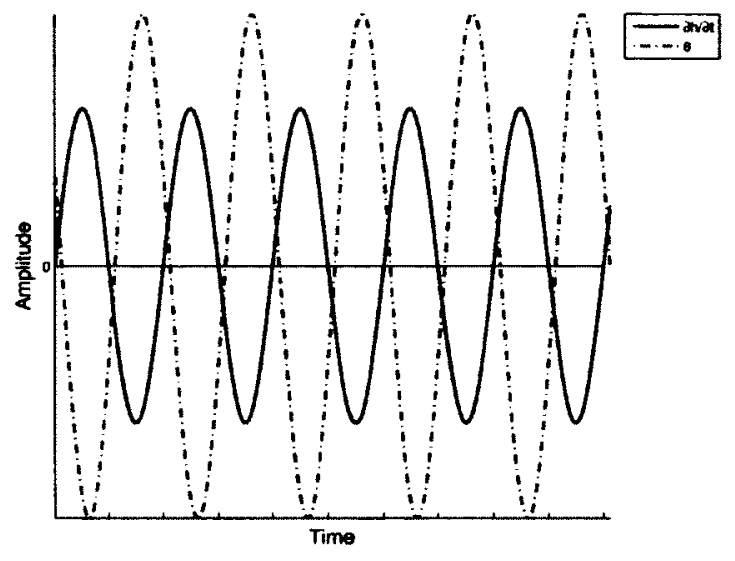

Figure 3.4: Oscillatory motion producing positive work

The dashed line shows the pitch motion and solid line the heave velocity. Since $\theta$ and $\dot{h}$ have an opposite sign for majority of the oscillation, the integration of eq. (3.13) produces a negative result, translating to positive work due to lift. At this instance, the airflow is feeding energy to the structural subsystem, leading to a self-sustained growing dynamic oscillation, a phenomenon that was described as coalescence flutter. The above example, and more generally eq. (3.13), were used to illustrate the cause of coalescence flutter phenomenon. From this simple example, it should now be clear that the coalescence flutter arises as a direct consequence of the phase difference between the degrees of freedom of the flutter mode.

In his work, Zimmerman [34] uses the energy principle to look further into the physical mechanism of the flutter phenomenon. In the pre-flutter region, he explains, the mechanical energy from all of the modes is absorbed by the air. Then, as the system enters the post flutter region, he states that the air acts as a constant source of energy that feeds the structural subsystem through the vibration of the flutter mode, and at the same time the stable modes keep transferring their mechanical energy to the air. Once again it will be noted that the air provides an energy feeding mechanism through the flutter mode because the phases of individual modal component within the flutter mode align in a particular way.

It should be mentioned that the coalescence flutter phenomenon takes its name from a feature that is very distinct of this type of instability, where the modes that are involved in the flutter mechanism experience frequency coalescence. This observation has been made in both theory 
and practice [34]. As the aeroelastic system approaches the flutter point, the inter-coupling and interaction between the modes promotes a modal energy exchange mechanism by which the modal frequencies coalesce. A simple example is shown by Zimmerman [34], where he examines the transformation of characteristic roots as well as the energy exchange mechanism of an oscillating airfoil that is affected by steady aerodynamics. For this simple case, Zimmerman shows that the flutter point occurs when the two frequencies meet, where the decay of one of the modes is on the brink of becoming positive, signifying the onset of instability. This work examines the transformations of the characteristic roots in detail, illustrating that the approach to instability is associated to the coalescence of the modal frequencies of the interacting modes. Even for a more general type of aerodynamics, he explains that the frequency coalescence is essential for the instability to set in. In a sense, the frequency coalescence can be attributed to the growing coupling effects between the interacting modes as the airflow strengthens, which translates to an energy exchange between these modes.

Effectively, for a general aeroelastic model of an airfoil, where the structural damping and the additional effects of non-steady aerodynamics are present, similar conclusions apply. With increasing air speed, the frequency coalescence escalates, and once the flutter speed is reached, the instability sets in. This instability results in a growing oscillatory motion of the wing, which may eventually lead to fatigue failure or a destructive failure of the entire wing. Since there exists a possibility of a rapid and catastrophic failure in an aircraft due to coalescence flutter, an accurate estimation of the flutter speed is necessary to achieve maximized performance with increased safety. All of these aforementioned deductions will become more evident once the probabilistic study is accomplished. However, before proceeding to the probabilistic analysis, the next section presents a commonly used approach to carry out a deterministic analysis of the coalescence flutter phenomenon by means of analyzing the system characteristic roots (eigenvalues). 


\section{Eigenvalue Analysis for the Modal Properties of the System}

This section aims to interpret the eigenvalues and eigenvectors relevant to the properties of the typical aeroelastic systems. Consider the steady state harmonic motion of the aeroelastic system defined by:

$$
\left\{\begin{array}{l}
h \\
\theta
\end{array}\right\}=\{X\} \mathbf{e}^{\lambda t}
$$

Substituting eq. (3.14) into eq. (3.6) of the aeroelastic system, we obtain:

$$
\left[\lambda^{2} M_{a e}+\lambda B_{a e}+K_{a e}\right]\{\mathbf{X}\} \mathbf{e}^{\lambda t}=\left\{\begin{array}{l}
0 \\
0
\end{array}\right\}
$$

which leads to the following Quadratic Eigenvalue Problem [87]:

$$
\left[\lambda^{2} M_{a e}+\lambda B_{a e}+K_{a e}\right]\{\mathbf{X}\}=\left\{\begin{array}{l}
0 \\
0
\end{array}\right\}
$$

Solution of this equation yields a set of eigenvalues, $\lambda$ 's, known as the characteristic roots, and an eigenvector $\{\mathbf{X}\}$ that is associated with each of the characteristic roots.

If the state space representation of the system is used (see Appendix B), the Quadratic Eigenvalue Problem can be transformed into a Standard Eigenvalue Problem defined as:

$$
[A]\{X\}=\lambda\{X\}
$$

where $[A]=\left[\begin{array}{cc}{[0]} & {[I]} \\ -\left[M_{a e}\right]^{-1}\left[K_{a e}\right] & -\left[M_{a e}\right]^{-1}\left[B_{a e}\right]\end{array}\right]$ and $\{X\}^{T}=\{h \theta \dot{h} \dot{\theta}\}$. The eigenvalues of a representative system generally appear in complex conjugate pairs, given by:

$$
\lambda_{i}=-\beta_{i} \pm j\left(\omega_{d}\right)_{i}
$$

where $\beta_{i}$ and $\left(\omega_{d}\right)_{i}$ are the modal decay (damping) rate and the modal frequency of the $i^{\text {th }}$ mode. 
For the flutter problem, the negative modal decay dictates that the loss of stability of the system has occurred, leading to an exponential growth of oscillation. Although the decay rate reveals the loss of stability, the modal frequency has to be studied carefully, since the coalescence of the two modal frequencies is the primary mechanism of the coalescence flutter, as investigated in the thesis. Generally, it is assumed that the decay is only a manifestation of the frequency coalescence phenomenon, which actually controls the mechanism of flutter. This does not necessarily mean that the decay or damping rate of the mode is not important to detect the onset of flutter, it just means that it is of secondary significance. The importance of the decay rate, or damping, on the flutter mechanism will be investigated in detail in the subsequent section that describes various types of flutter.

In order to illustrate the physics in a representative case of coalescence flutter problem, a particular set of system parameters was selected to model this representative airfoil. These parameters are supplied in Appendix C. Their selection criteria will be reviewed further with the discussion of unsteady aerodynamics. For the aeroelastic system that is described by the representative parameters, the resulting modal properties of both vibrating modes are plotted below, in fig. (3.5), as a function of airspeed.
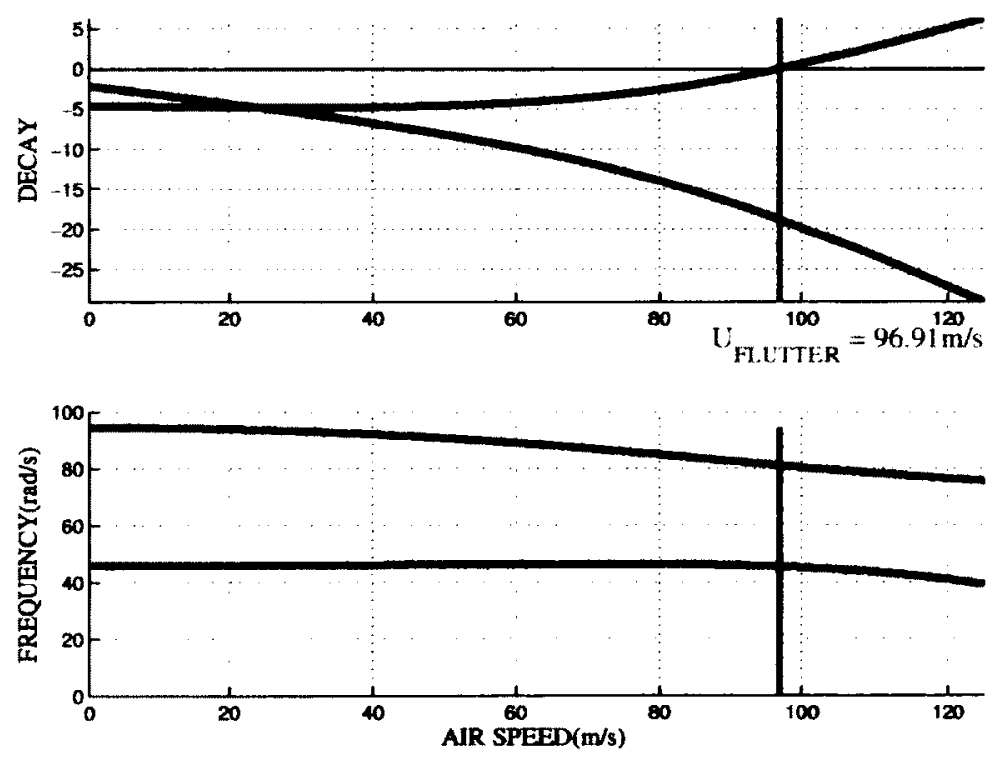

Figure 3.5: Evolution of the oscillatory modes' modal parameters with respect to increasing airspeed. Top plot describes modal decay rates; bottom plot describes damped modal frequencies 
The top plot in the figure depicts the modal decays. The bottom plot shows the modal frequencies. This figure plots the evolution of modal properties as a function of airspeed.

For this representative case, fig. (3.5) illustrates that an increase in airspeed causes the decay of one mode to increase and the second mode to decrease. As the decays rates spread apart, the two frequencies coalesce. This phenomenon is largely attributed to the increased coupling between the two modes. The relative spread of the decay rates is not a necessary condition, as shown in a later example for instability. On the other hand, the frequency coalescence is an essential condition for flutter. As was mentioned earlier, the frequency coalescence is directly associated with the increase in coupling attributed to the energy exchange mechanism between the coalescing modes. Therefore, it is worth mentioning that the onset of flutter is controlled by the frequency coalescence phenomenon. Thus, the parameters that have the greatest effect on the modal frequencies control the flutter. We again reiterate that the decay is only a manifestation of the frequency coalescence, and hence serves mainly as an indicator of the flutter occurrence.

As one of the modal decay rates approaches a value of zero, just before going to the negative value, the system is said to have reached its stability boundary. The airspeed where this instability occurs is identified as the flutter speed, which is shown by a vertical red line in fig. (3.5). Fig. (3.6) below contains three time-history plots at various airspeeds, illustrating examples of a stable response in the pre-flutter region, a neutrally stable response at a flutter point and an unstable response in the post-flutter region, given in the same order from top plot to the bottom plot. The stable response shows a positive value of decay in both modes leading to decaying oscillation. The response at neutral stability contains one mode that has a large positive value of decay, which damps out very quickly and another mode that will have a zero decay rate. Hence it does not damp out, leading to persistent oscillations. Lastly, the bottom plot shows an unstable response at a point slightly higher than the flutter speed, where similar to the previous case, a very largely positive decay value of one mode indicates its rapid decay, yet a slightly negative value of the decay rate of the second mode leads to a diverging oscillation. These response plots only show the heave oscillations, but it should be mentioned that the response of the pitch 

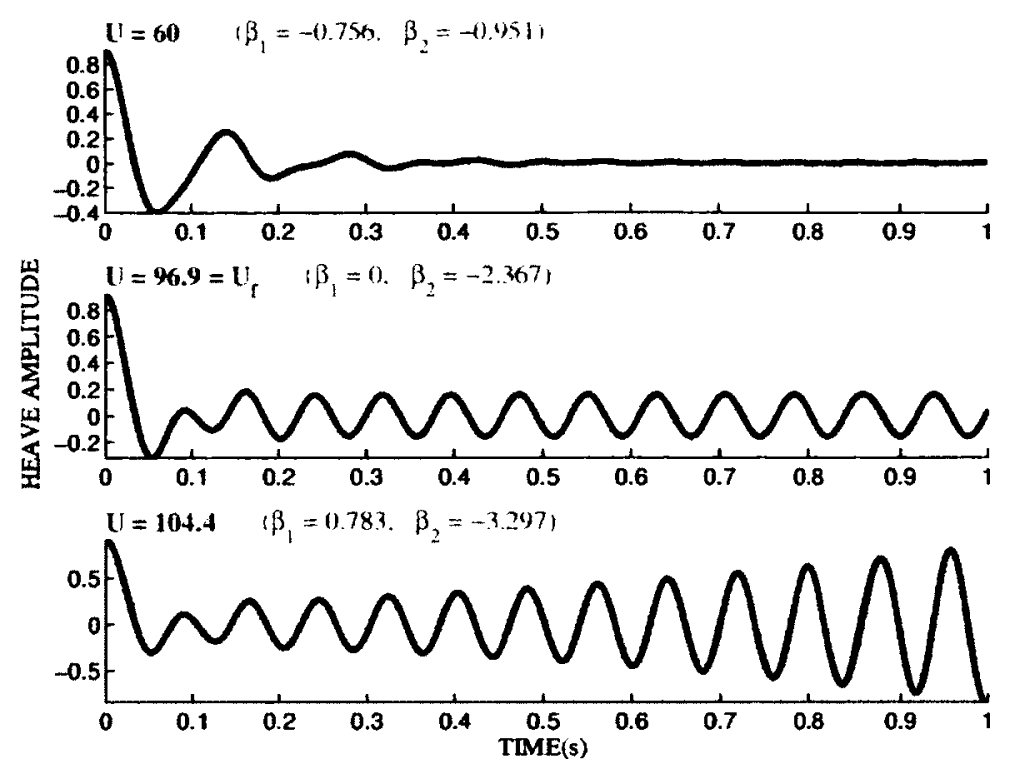

Figure 3.6: Figures representing stable, marginally stable and unstable responses, respectively from top to bottom

oscillations is of similar behaviour, but contains a phase difference relative to the heave oscillations.

For the unstable response analysis, small amplitude vibration assumption is invalid as the diverging oscillations will have very large amplitude as the time increases. Thus, the plot of unstable response is unrealistic due to the modeling errors. If proper post-flutter analysis needs to be pursued, the aeroelastic system must then take into account the non-linear effects into both structural and aerodynamic models. As we are only interested in the pre-flutter results, the linear system assumption is considered to be sufficient.

\subsubsection{Types of Coalescence Flutter}

Next, different classifications of flutter will be reviewed. Each classification is based on the explosiveness of flutter, which in effect defines the sensitivity of flutter speed to the damping variations. Generally, there exist three types of flutter: mild, moderate and explosive. In fig. (3.7) the top, middle and bottom plots illustrate the mild, moderate and explosive types of flutter, respectively. The definition of each type is based on a relative interval of air speed, represented by variable $\mathrm{x}$ in the figure, between the most stable point and the point of instability onset in the 
flutter mode [70]. The physical interpretation of each flutter type is explained in details in the work by Zimmerman [34], where he explains that the explosiveness of flutter depends on the relative magnitude of the quadrature lift component (relating to $\dot{h}, \dot{\theta}, \ddot{h}$ and $\ddot{\theta}$ ) with respect to the in-phase lift component (relating only to $\theta$ ). The in-phase lift comes mainly from the steady aerodynamics. The quadrature component comes from the additional quasisteady and unsteady components. When the quadrature component is low, an explosive flutter is experienced. On the contrary, if a larger quadrature component is experienced, the flutter type becomes milder. As it was already stated, the flutter mechanism is controlled by the frequency coalescence, therefore a more explosive nature of decay rates is representative of a much stronger frequency coalescence effects in vicinity of the flutter region. In effect, the quadrature component reduces the rate of frequency coalescence, which reduces the rate of the flutter mode decay growth to instability. Therefore, for milder cases of flutter, the coupling effects do not have to be as strong for the

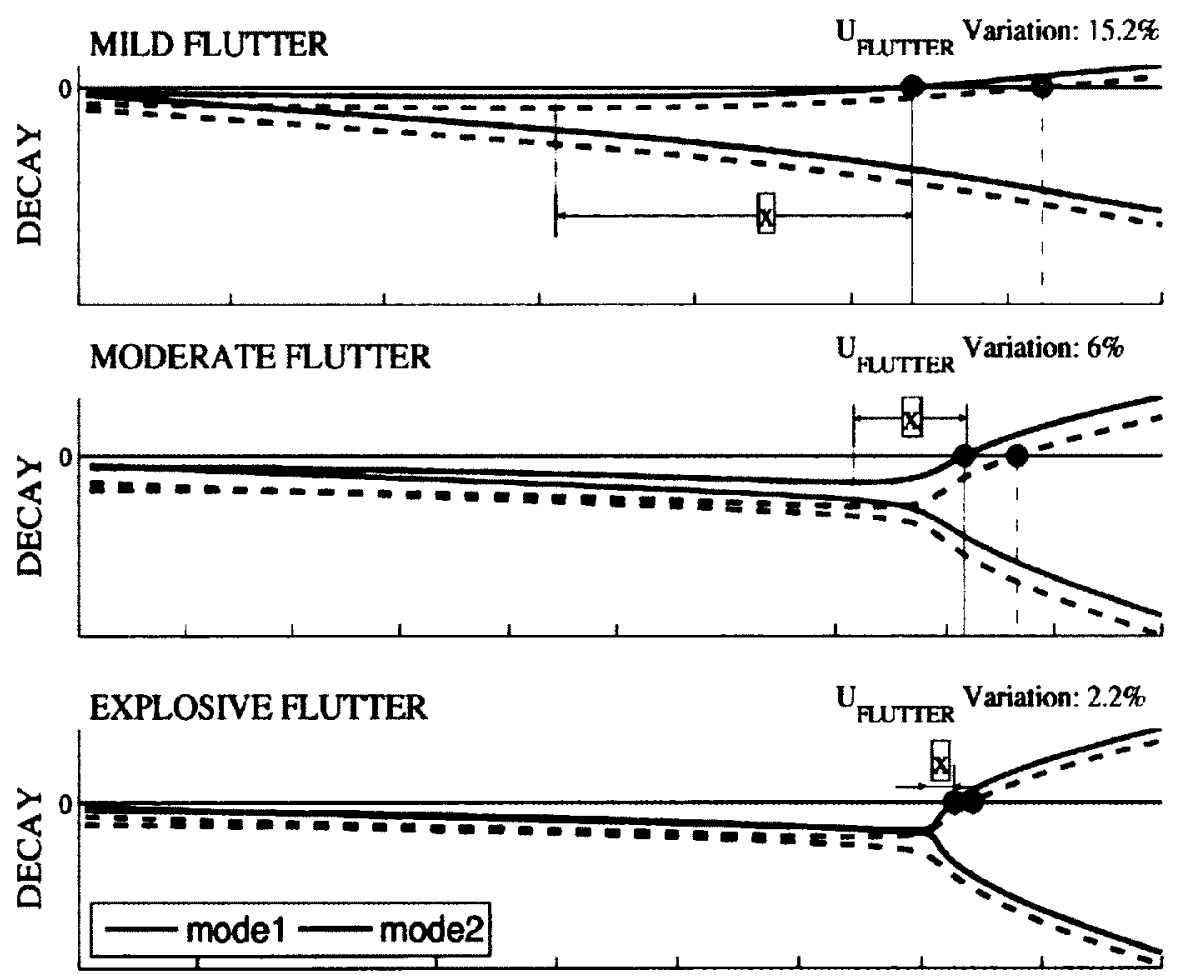

AIR SPEED $(\mathrm{m} / \mathrm{s})$

Figure 3.7: Examples of mild flutter, moderate flutter and explosive flutter respectively from top to bottom. Solid lines represent the original modal parameters, dashed lines represent modal parameters obtained using manipulated damping ratios 
onset of instability.

Due to a completely distinct nature of the parameters chosen to represent each flutter type, the width $\mathrm{x}$ is only relative in fig. (3.7), therefore it displays only qualitative information exhibiting the three various types of flutter. It has been observed that the systems experiencing a mild flutter are sensitive to the variations in the structural damping. In order to establish a quantitative comparison between each type of flutter, the percentage variation of flutter speed is given in each plot when the structural damping ratios experience an equal change for each of the three scenarios. In fig. (3.7) it is evident that the flutter speed is most sensitive to the structural damping variations in the case of mild flutter. By changing both modal damping ratios from $1 \%$ to $3 \%$, in the mild flutter scenario, the flutter speed varied by $15.2 \%$, however in the explosive flutter scenario, the flutter speed has changed only by $2.2 \%$.

\subsubsection{Unsteady Aerodynamics}

This section introduces the effect of unsteady aerodynamics. In general, the unsteady aerodynamics include two additional components to the typical quasisteady aerodynamics due to the added inertia terms and the circulatory effects due to the vortex shedding. However, in this study the added inertia terms were already included in the quasisteady aerodynamics, as mentioned previously. Therefore, only additional effect introduced by the circulatory forcing needs to be accounted for by the unsteady aerodynamics. The circulatory forcing accounts for the lag effects originating from the starting vortices traveling downstream [1]. Previously, while formulating the quasisteady aerodynamics, a brief introduction to the unsteady effects was provided. A solution of flutter speed is obtained in the frequency domain, using a Theodorsen's function to account for the effect of these circulatory forces. Although this method proves to be very fast and powerful, it assumes that the aerodynamic loads are influenced only by the flutter frequency and the effects of the other modes are neglected. The time domain approach based on the Wagner's method relaxes this assumption. The Wagner's method is detailed in Appendix A. In this approach, the unsteady effects are incorporated into the model by introducing an additional degree of freedom in $z$. This new parameter considers the contribution of the lag effects 
from the so-called starting vortices [1]. As the starting vortex travels downstream of the airfoil, the influence of the resulting vortex shedding force on the wing diminishes with increasing distance between this individual vortex and the airfoil. Within the effective influence region of ten chord-lengths, for the incompressible flow, Wagner's Function closely approximates the effect of lag due to the vortex shedding effects [1]. A two term approximation of this function, developed by Jones [88], as given below:

$$
\Phi(\tau)=1-A_{1} \mathrm{e}^{-0.0455 \tau}-A_{2} \mathrm{e}^{-0.3 \tau}
$$

where $A_{1}=0.165, A_{2}=0.335$ and $\tau=\frac{U t}{b}$ is a dimensionless time parameter, $U$ is the flow speed and $b$ is half-chord length. In the dimensionless form, the Wagner's Function is given by:

$$
\Phi(t)=1-A_{1} \mathbf{e}^{-b_{1} t}-A_{2} \mathbf{e}^{-b_{2} t}
$$

where $b_{1}=0.0455 \frac{U}{b}$ and $b_{2}=0.3 \frac{U}{b}$. For the non-dimensional form, the Wagner's Function is plotted in fig. (3.8).

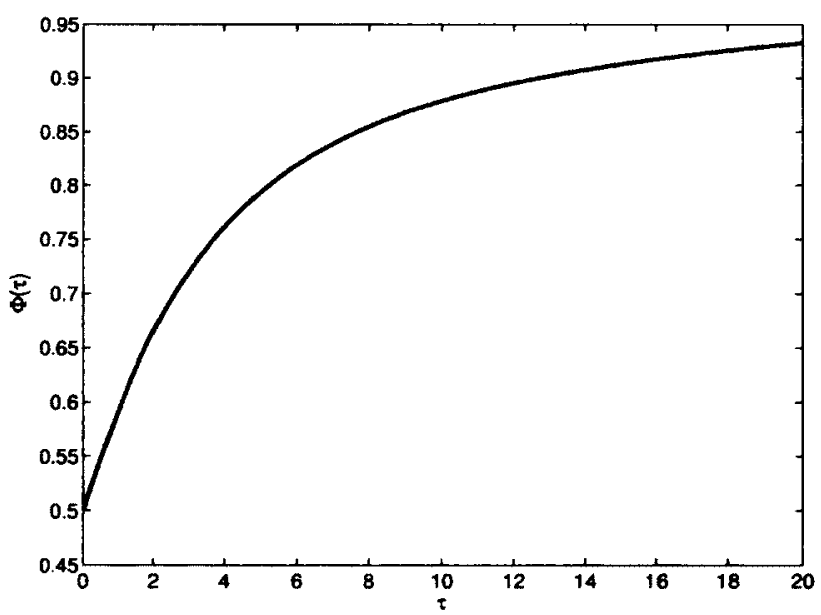

Figure 3.8: Wagner's Function represents a lag in reaching the steady aerodynamics

In the Wagner's method, the vortex is thought to have negligible effect on the airfoil past ten chord lengths downstream of it. The two term approximate of the Wagner's function still retains 
some of the effect of vortex shedding beyond ten chords. However, if there is a need for a better approximation, more terms could be included in the Wagner's function. The number of terms used in the approximation reflects the balance between accuracy and computational efficiency. In general, a two term approximate provides acceptable results [84].

When the unsteady aerodynamics are considered, an additional equation needs to be included to describe the dynamics of the system as follows:

$$
\ddot{z}(t)+\left(b_{1}+b_{2}\right) \dot{z}(t)+\left(b_{1} b_{2}\right) \dot{z}(t)=w_{\frac{3}{4} c}
$$

where the term $w_{\frac{3}{4}}$ (described in Appendix A) represents the effective downwash at the threequarter chord point. Consequently, the dynamics of the coupled aeroelastic system is given by:

$$
\left[\begin{array}{cc}
M_{s t r} & 0 \\
0 & 1
\end{array}\right]\left\{\begin{array}{c}
\ddot{h} \\
\ddot{\theta} \\
\ddot{z}
\end{array}\right\}+\left[\begin{array}{cc}
B_{s t r} & 0 \\
0 & b_{1}+b_{2}
\end{array}\right]\left\{\begin{array}{c}
\dot{h} \\
\dot{\theta} \\
\dot{z}
\end{array}\right\}+\left[\begin{array}{cc}
K_{s t r} & 0 \\
0 & b_{1} b_{2}
\end{array}\right]\left\{\begin{array}{c}
h \\
\theta \\
z
\end{array}\right\}=\left\{\begin{array}{c}
-L \\
M_{e a} \\
w_{\frac{3}{4} c}
\end{array}\right\}
$$

where $\left[M_{s t r}\right],\left[B_{s t r}\right],\left[K_{s t r}\right]$ are the structural matrices as defined in previous section; $L$ and $M_{e a}$ represent the aerodynamic lift and moment about elastic axis respectively (see Appendix A). $h$ and $\theta$ represent the heave and pitch degrees of freedom of the airfoil; $z$ is a new degree of freedom, which accounts for the lag effects. In unsteady analysis, the forcing terms become explicit functions of time, acceleration, velocity, displacement and airspeed [89]:

$$
\begin{aligned}
& L=L(U, \theta, \dot{h}, \dot{\theta}, \ddot{h}, \ddot{\theta}, t) \\
& M_{e a}=M_{e a}(U, \theta, \dot{h}, \dot{\theta}, \ddot{h}, \ddot{\theta}, t) \\
& w_{\frac{3}{4} c}=w_{\frac{3}{4} c}(\theta, \dot{h}, \dot{\theta})
\end{aligned}
$$

Restating all of the aerodynamic loading terms in a matrix form leads to the following relation: 


$$
\left\{\begin{array}{c}
-L \\
M_{e a} \\
w_{\frac{3}{4} c}
\end{array}\right\}=\left[M_{\text {aero }}\right]\left\{\begin{array}{c}
\ddot{h} \\
\ddot{\theta} \\
\ddot{z}
\end{array}\right\}+\left[B_{\text {aero }}(U)\right]\left\{\begin{array}{c}
\dot{h} \\
\dot{\theta} \\
\dot{z}
\end{array}\right\}+\left[K_{\text {aero }}(U)\right]\left\{\begin{array}{c}
h \\
\theta \\
z
\end{array}\right\}
$$

where $\left[M_{\text {aero }}\right],\left[B_{\text {aero }}\right]$ and $\left[K_{\text {aero }}\right]$ are the aerodynamic mass, damping and stiffness matrices, respectively. A detailed derivation of these equations of motion for unsteady aerodynamics with all of the system matrices can be found in Appendix A. Consequently, the equation of motion can be written as:

$$
\left[M_{a e}\right]\left\{\begin{array}{c}
\ddot{h} \\
\ddot{\theta} \\
\ddot{z}
\end{array}\right\}+\left[B_{a e}(U)\right]\left\{\begin{array}{c}
\dot{h} \\
\dot{\theta} \\
\dot{z}
\end{array}\right\}+\left[K_{a e}(U)\right]\left\{\begin{array}{l}
h \\
\theta \\
z
\end{array}\right\}=\left\{\begin{array}{l}
0 \\
0 \\
0
\end{array}\right\}
$$

Using Appendix A, the expressions of the system matrices are given by:

$$
\begin{aligned}
& {\left[M_{a+e}\right]=\left[\begin{array}{ccc}
m+\frac{1}{2} \rho_{\infty} C_{L \alpha} b^{2} & m b x_{\theta}-\frac{1}{2} \rho_{\infty} C_{L \alpha} b^{3} a_{h} & 0 \\
m b x_{\theta}-\frac{1}{2} \rho_{\infty} C_{L \alpha} b^{3} a_{h} & I_{e a}+\frac{1}{2} \rho_{\infty} C_{L \alpha} b^{4}\left(\frac{1}{8}+a_{h}{ }^{2}\right) & 0 \\
0 & 0 & 1
\end{array}\right]} \\
& {\left[B_{a+1}\right]=\left[\begin{array}{ccc}
B_{1}+\frac{1}{2} \rho_{\infty} U_{\infty} C_{L \alpha} b & B_{2}+\frac{1}{2} \rho_{\infty} U_{\infty} C_{L \alpha} b^{2}\left(\frac{3}{2}-a_{h}\right) & \rho_{\infty} U_{\infty} C_{L \alpha} b\left(A_{1} b_{1}+A_{2} b_{2}\right) \\
B_{3}-\frac{1}{2} \rho_{\infty} U_{\infty} C_{L \alpha} b^{2}\left(\frac{1}{2}+a_{h}\right) & B_{4}+\frac{1}{2} \rho_{\infty} U_{\infty} C_{L \alpha} b\left(b\left(\frac{1}{2}-a_{h}\right)\right)^{2} & -\rho_{\infty} U_{\infty} C_{L \alpha} b^{2}\left(\frac{1}{2}+a_{h}\right)\left(A_{1} b_{1}+A_{2} b_{2}\right) \\
0 & 0 & b_{1}+b_{2}
\end{array}\right]} \\
& {\left[K_{a E}\right]=\left[\begin{array}{ccc}
k_{h} & \frac{1}{2} \rho_{\infty} U_{\infty}^{2} C_{L \alpha} b & \rho_{\infty} U_{\infty} C_{L \alpha} b\left(A_{1}+A_{2}\right) b_{1} b_{2} \\
0 & k_{\theta}-\frac{1}{2} \rho_{\infty} U_{\infty}^{2} C_{L \alpha} b^{2}\left(\frac{1}{2}+a_{h}\right) & -\rho_{\infty} U_{\infty} C_{L \alpha} b^{2}\left(\frac{1}{2}+a_{h}\right)\left(A_{1}+A_{2}\right) b_{1} b_{2} \\
0 & 0 & b_{1} b_{2}
\end{array}\right]}
\end{aligned}
$$

\section{Eigenvalue Analysis for Modal Properties of the System}

In contrast to the quasisteady aerodynamics, the eigenvalue analysis now yields two additional characteristic roots in addition to the original two complex conjugate pairs of eigenvalues, that represent the two oscillatory aeroelastic modes. These new roots are distinct and purely real. Each complex conjugate pair of roots represents the modal decay and the frequency for the aeroelastic mode. The two purely real roots represent two overdamped modes that arise from the addition of an extra degree of freedom $z$, which introduces the unsteady effects into the aerodynamic forcing.

Fig. (3.9) shows the change in modal parameters for the two oscillating modes as a function 

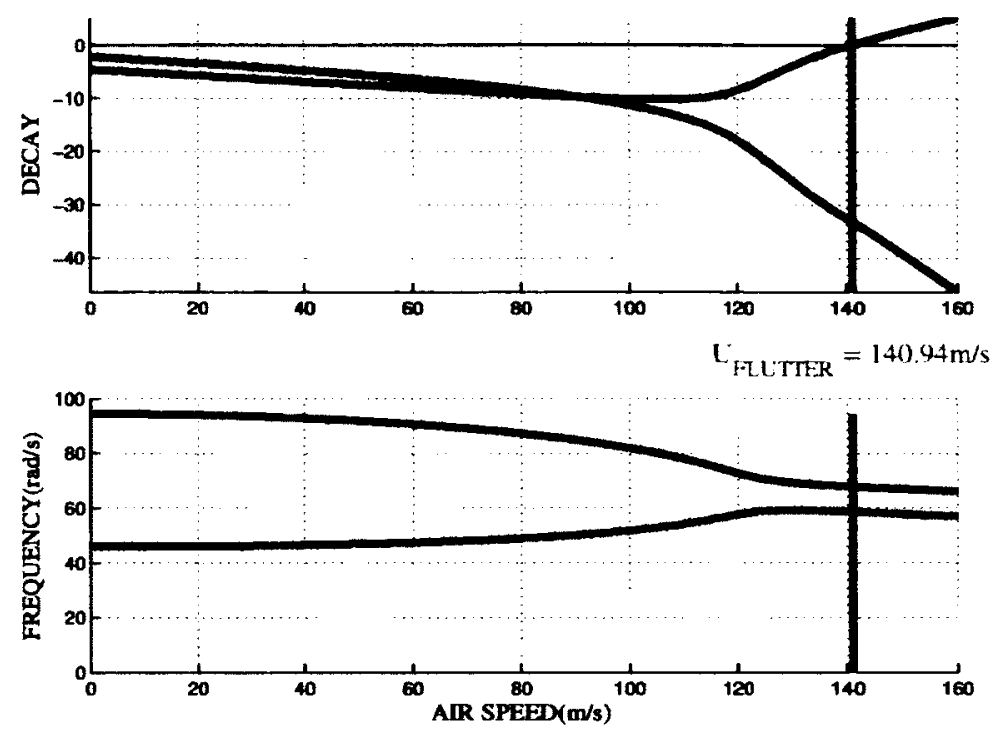

Figure 3.9: Evolution of the oscillatory modes' modal parameters with respect to increasing airspeed. Top plot describes modal decay rates; bottom plot describes damped modal frequencies

of airspeed. The modal properties of the overdamped modes are excluded from the figure as these modes do not exhibit information indicative of the occurrence of flutter. The parameters that were used to generate the modal parameter results for this representative airfoil, are shown in Appendix C. They were chosen to resemble the basic dynamics of a general Uniform Goland Wing, a case that has been widely examined in literature. Reference [36] provides the basis for the parameter selection that draws equivalence between the cantilever wing model and its equivalent rigid wing model. The equivalence between these two models is based on matching of the mass ratios and natural frequencies of the two coalescing modes. The results of the equivalent rigid model have shown good match with the cantilever model for the case with unsteady aerodynamics.

The following section examines the effect of structural uncertainties on the flutter speed estimate, and in process, identifies the most critical structural parameters to effect the flutter speed. Then, further analysis develops a physical interpretation of the leading causes of the flutter speed sensitivity. 


\subsection{Probabilistic Formulation}

The previous section treated the baseline deterministic problem, where exact values of all of the parameters are known from the start. From analysis of this problem, we are able to discern a basic understanding of how the structure works, how different forces affect it and how certain assumptions implicate the analysis. In reality, however, the parameters are never initially known, since there is always a degree of randomness present within them. Through proper sampling methods, the randomness within these parameters can be captured and described using a probability density function (pdf). Further, implementing statistical techniques together with the physical model of the system, which was developed in deterministic setting, establishes a direct link to study a probabilistic problem. Statistical techniques, if applied properly, aid in treatment of uncertainty contained within the input parameters of the problem. In our case, solving a problem in a probabilistic sense accomplishes two objectives: one, it provides a straight forward way to assign an amount of risk associated to a specific choice of the controlled parameter; two, it uncovers some hidden physics of the problem, which are not obvious when dealing with a deterministically set problem, for example a minimum flutter speed. These two factors, and many others, make the probabilistic assessment of many problems very tempting, but nonetheless, the probabilistic techniques have to be well understood for their successful implementation.

In the subsequent sections we conduct a detailed probabilistic analysis for (a) the flutter speed and (b) the characteristic roots for the following distinct cases:

Case-1: The parameters for this system relate to the canonical model widely used in the literature $[68,36,15,17]$. This system has been used to compare the aeroelastic behaviour of an elastically mounted rigid wing and a continuous wing that is modeled by a uniform cantilever. For this case, a single cantilever bending and a single torsional beam basis function reasonably approximate the coalescing aeroelastic modes for the flutter analysis.

Case-2: The parameters of this system are similar to the parameters of Case 1, representative of a canonical model of a cantilever wing, with exception of increased value of static imbalance and the elastic axis offset. As a result of these parameter changes, the off-diagonal terms in the 
aeroelastic system matrices become more dominant, leading to greater coupling. Effectively, for this case multiple bending and torsional beam basis functions are required to accurately model the two coalescing aeroelastic modes for the flutter analysis.

Case-3: This aeroelastic system demonstrates how uncertainty in the system parameters can dramatically influence the probabilistic behaviour of the flutter speed and characteristic roots due to the strong non-linear mapping between the input and output, as delineated later.

The two different analytical functions, chosen to represent input parameter distributions, are namely Gaussian and uniform probability density functions.

\subsection{Flutter Speed Estimation}

\subsubsection{CASE 1}

The baseline system parameters are taken from a study by Borello et al. [36], who derived this 2-D simplified airfoil model to approximate the dynamics of a 3-D model of a uniform cantilever wing, originally studied by Goland [15]. In [36], the equivalence between the 2-D rigid airfoil and the uniform wing is established by matching the first two natural frequencies of the uncoupled system without air flow. One of these modes becomes unstable when the flow speed is increased, leading to the occurrence of coalescence flutter. The parameters describing

\begin{tabular}{||l|c|l||}
\hline PARAMETER & Symbol & Value \\
\hline \hline mass & $m$ & $35.7187 \mathrm{~kg} / \mathrm{m}$ \\
normalized radius of gyration & $r_{g}$ & 0.5 \\
heave stiffness & $k_{h}$ & $8.75 \times 10^{4} \mathrm{~N} / \mathrm{m}^{2}$ \\
pitch stiffness & $k_{\theta}$ & $6.57 \times 10^{4} \mathrm{~N} / \mathrm{rad}$ \\
chord length & $c$ & $1.8288 \mathrm{~m}$ \\
span & $L$ & $6.096 \mathrm{~m}$ \\
normalized static imbalance & $x_{\theta}$ & 0.2 \\
damping ratio 1 & $\xi_{1}$ & 0.05 \\
damping ratio 2 & $\xi_{2}$ & 0.05 \\
free stream fluid density & $\rho_{\infty}$ & $1.225 \mathrm{~kg} / \mathrm{m}^{3}$ \\
normalized distance btw EA and mid-chord & $a_{h}$ & -0.34 \\
lift coefficient gradient & $\bar{C}_{l \alpha}$ & $2 \pi$ \\
\hline \hline Num. of Samples & - & 50000 \\
\hline
\end{tabular}

Table 3.1: Model parameters for Case 1 
this airfoil model are stated in tbl. (3.1), and additionally they are provided in Appendix C along with the parameters of an equivalent 3-D model.

\section{Quasisteady Analysis: Baseline (Deterministic) Case}

Fig. (3.10) shows the modal decays and the modal frequencies for the specified range of air speeds. As evident from fig. (3.10), the second mode (higher frequency) becomes unstable through a mild flutter having a flutter speed of $96.91 \mathrm{~m} / \mathrm{s}$.
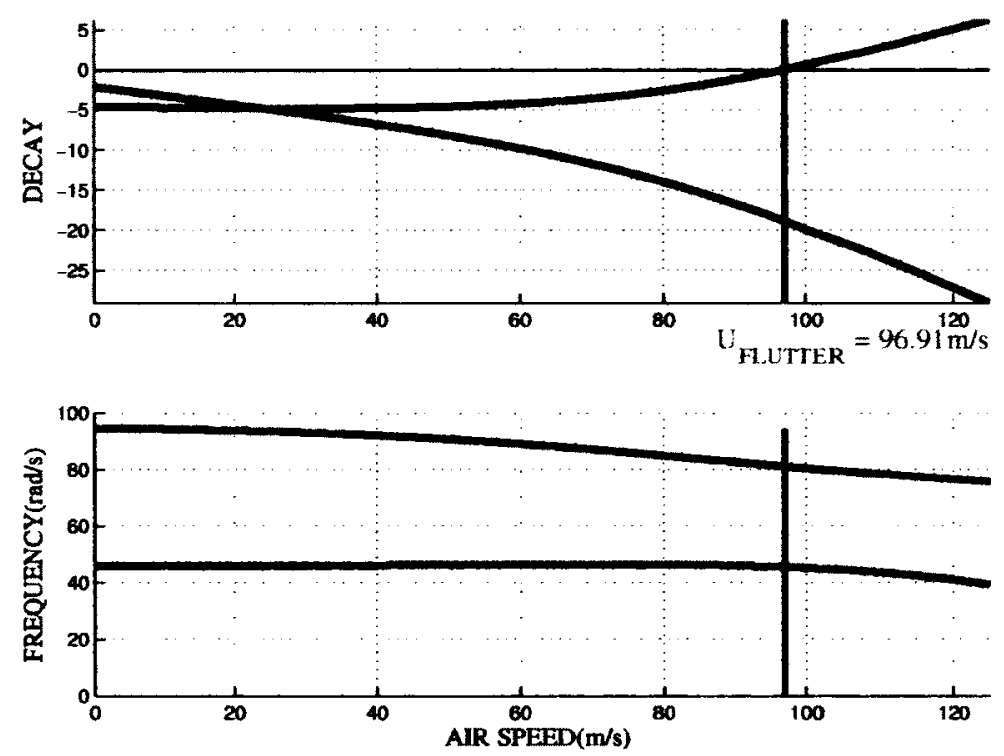

Figure 3.10: Evolution of modal parameters with respect to airspeed

Fig. (3.11) demonstrates the relative magnitude of the elements of the eigenvectors of each mode at no flow condition and at the proximity of the flutter speed. The imaginary part of the mode relates to the relative phase between the individual components. For Case 1, it can be seen that the relative strength of each eigenvector changes with an increase in airspeed. Initially, the first mode exhibits strong heave dominance, and the second mode, pitch dominance. At flutter point, both of the modes lose the relative strength of dominant components. This change in the modal components may be indicative of the energy exchange between the two interacting modes due to aeroelastic coupling.

Lastly, using the nominal system parameters for the quasisteady case, the functions relating the flutter speed to the mass, stiffness and damping parameters are plotted in fig. (3.12). These 

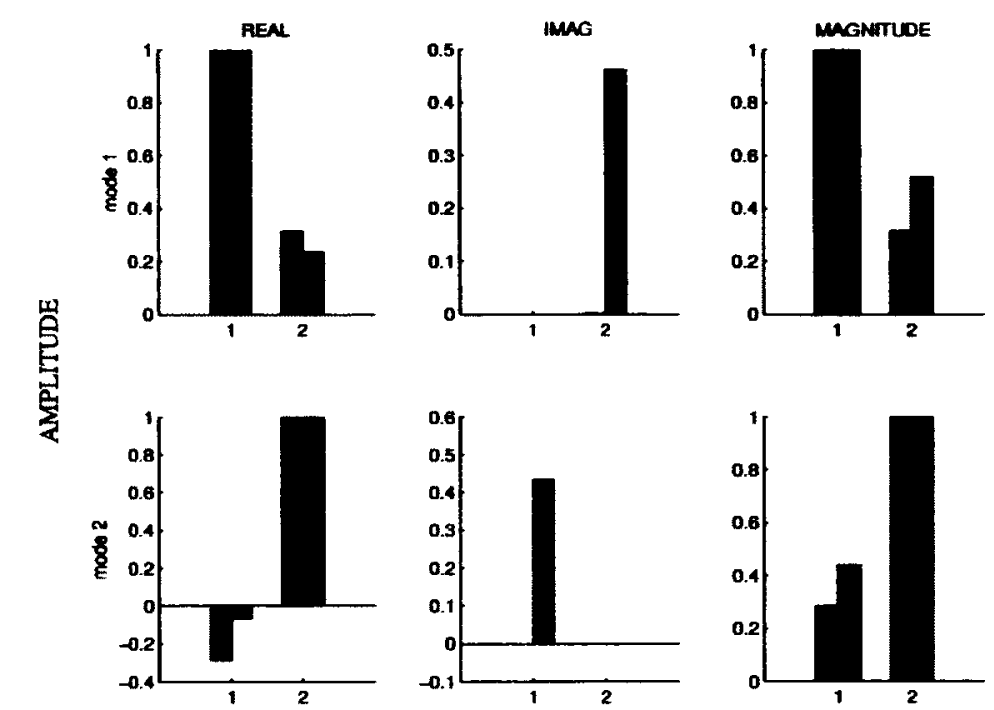

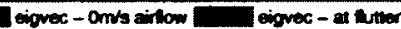
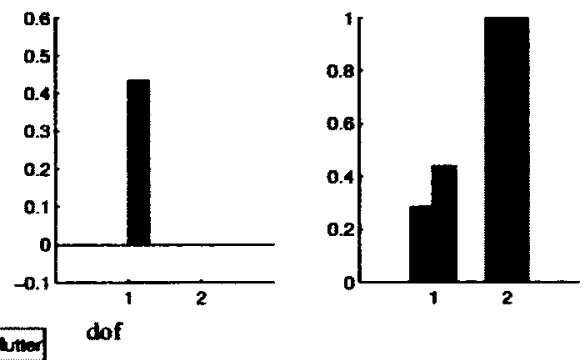

Figure 3.11: real part, imaginary part, and magnitude of eigenvectors of two modes, illustrating relative strength of each component
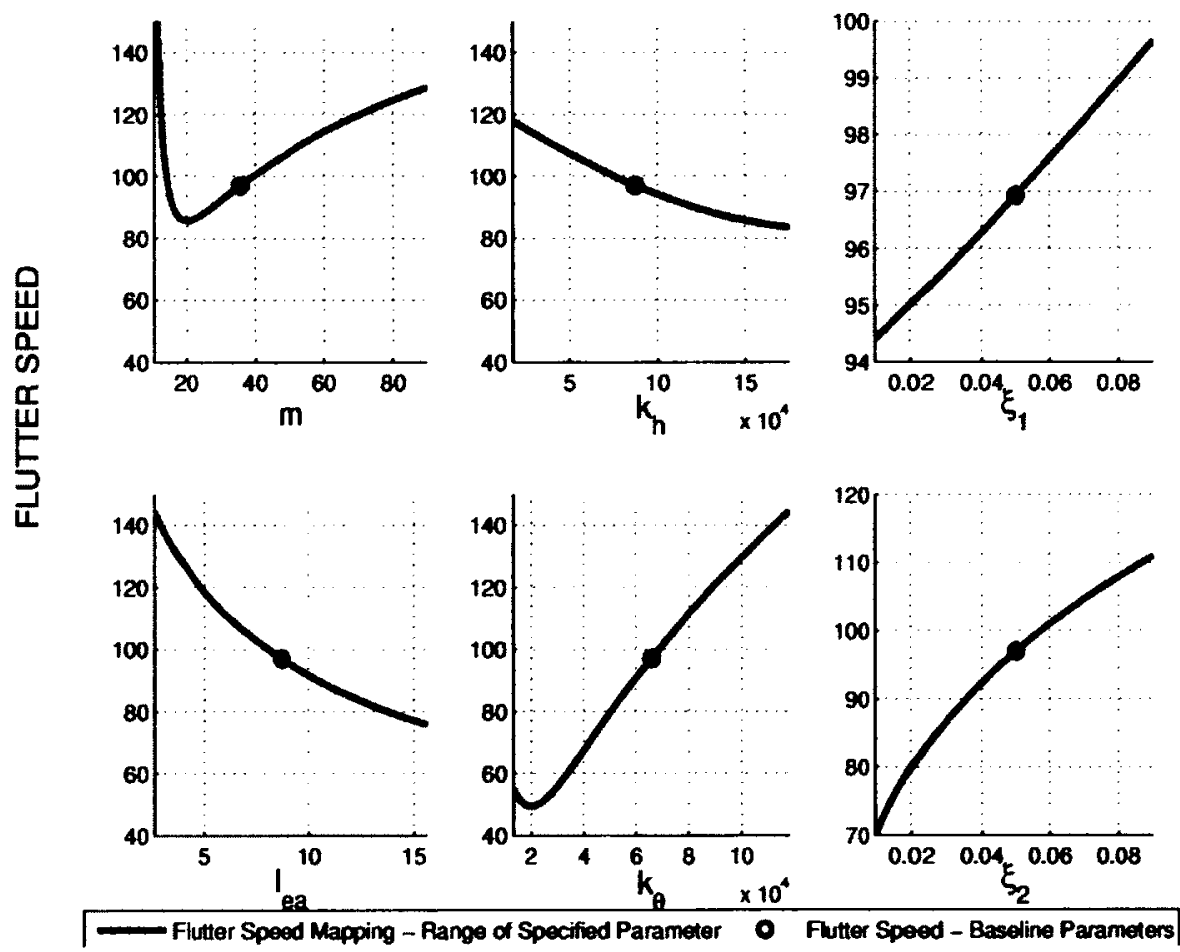

Figure 3.12: The functional relationships among the flutter speed and structural parameters

results are obtained in an analytical form via Routh-Hurwitz Stability Criteria, derivation of which is described in the Appendix D. When generating the flutter speed mapping plots, a base- 
line case Prandtl-Glauert factor (accounting for the compressibility effects in subsonic region) is applied to the entire range of the uncertain system parameters. Although this approximation introduces some error, it simplifies the analysis. Nonetheless, the error due to this approximation is very small in the vicinity of the baseline parameter. These flutter speed mapping plots provide an insight into the transformation of the input uncertainty to that of the flutter speed.

Assume a random vector $x$ with pdf $p(x)$, which transforms into a random vector $\mathrm{y}$ through the relationship: $y=f(x)$. The pdf of $y$ can be written as $p(y)=\sum_{i=1}^{n}|J|_{x_{i}=f^{-1}(y)}^{-1} p(x)$ where $J$ is the Jacobian matrix (i.e. for this case $\frac{\partial y}{\partial x}$ ) and $|*|$ denotes its determinant [90]. If $x$ represents an uncertain structural parameter and $y$ is the flutter speed, the $|J|$ parameter relates to the slope of the curve at $x_{i}=f^{-1}(y)$. If the mapping is linear, the output pdf $y$ will resemble the pdf of $x$.

\section{Quasisteady Analysis: Probabilistic Case}

Tbls. (3.2) and (3.3), provide a summary of the results of probabilistic flutter speed estimation for Case 1 with quasisteady aerodynamics.

All of the resulting flutter speed pdfs obtained for cases with low uncertainty in the structural parameters indicate an almost linear mapping between the flutter speed and the structural parameter. For such a case, the information provided in tbls. (3.2) and (3.3) is sufficient to

\begin{tabular}{|c|c|c|c|}
\hline \multicolumn{2}{|c|}{ Input COV } & $2.5 \%$ & $10 \%$ \\
\hline Random Parameter & Distribution & \multicolumn{2}{c|}{ Flutter Speed COV \% } \\
\hline \hline \multirow{2}{*}{ mass } & Gaussian & 0.753 & 2.99 \\
\cline { 2 - 4 } & uniform & 0.753 & 3.00 \\
\hline \multirow{2}{*}{ radius of gyration } & Gaussian & 0.958 & 3.87 \\
\cline { 2 - 4 } & uniform & 0.958 & 3.85 \\
\hline \multirow{2}{*}{ heave stiffness } & Gaussian & 0.526 & 2.10 \\
\cline { 2 - 4 } & uniform & 0.526 & 2.10 \\
\hline \multirow{2}{*}{ pitch stiffness } & Gaussian & 1.73 & 6.95 \\
\cline { 2 - 4 } & uniform & 1.73 & 6.95 \\
\hline \multirow{2}{*}{ damping ratio 1 } & Gaussian & 0.0817 & 0.327 \\
\cline { 2 - 4 } & uniform & 0.0817 & 0.327 \\
\hline \multirow{2}{*}{ damping ratio 2 } & Gaussian & 0.536 & 2.16 \\
\cline { 2 - 4 } & uniform & 0.536 & 2.15 \\
\hline
\end{tabular}

Table 3.2: Results illustrating the uncertainty in the flutter speed due to independent uncertainty in the structural parameters 


\begin{tabular}{|c|c|c|c|c|c|}
\hline \multicolumn{2}{|c|}{ Input COV } & $2.5 \%$ & $10 \%$ & $2.5 \%$ & $10 \%$ \\
\hline Random Parameter & Distribution & \multicolumn{3}{c|}{ Flutter Speed COV (\%) } \\
\hline \hline \multicolumn{1}{|c|}{} & \multicolumn{3}{|c|}{ Uncorrelated Parameters } & Fully Correlated Parameters \\
\hline \hline $\begin{array}{c}\text { mass \& } \\
\text { radius of gyration }\end{array}$ & $\begin{array}{c}\text { Gaussian } \\
\text { Gaussian }\end{array}$ & 1.22 & 4.88 & 0.206 & 0.884 \\
\hline $\begin{array}{c}\text { mass \& } \\
\text { radius of gyration }\end{array}$ & $\begin{array}{c}\text { uniform } \\
\text { uniform }\end{array}$ & 1.22 & 4.87 & 0.205 & 0.852 \\
\hline $\begin{array}{c}\text { heave stiffness \& } \\
\text { pitch stiffness }\end{array}$ & $\begin{array}{c}\text { Gaussian } \\
\text { Gaussian }\end{array}$ & 1.81 & 7.23 & 1.21 & 4.85 \\
\hline $\begin{array}{c}\text { heave stiffness \& } \\
\text { pitch stiffness }\end{array}$ & $\begin{array}{c}\text { uniform } \\
\text { uniform }\end{array}$ & 1.81 & 7.22 & 1.21 & 4.84 \\
\hline $\begin{array}{c}\text { damping ratio 1 \& } \\
\text { damping ratio 2 }\end{array}$ & $\begin{array}{c}\text { Gaussian } \\
\text { Gaussian }\end{array}$ & 0.543 & 2.19 & 0.618 & 2.49 \\
\hline $\begin{array}{c}\text { damping ratio 1 \& } \\
\text { damping ratio 2 }\end{array}$ & $\begin{array}{c}\text { uniform } \\
\text { uniform }\end{array}$ & 0.543 & 2.18 & 0.618 & 2.48 \\
\hline \hline
\end{tabular}

Table 3.3: Results illustrating the uncertainty in the flutter speed due to correlated uncertainty in the structural parameters

establish the conclusions from these cases. Thus, the full results will be examined only for cases experiencing large uncertainty $(10 \% \mathrm{COV})$ in the input.

\section{(A) Truncated Gaussian Distribution with $\mathrm{COV}=10 \%$}

Fig. (3.13) and tbls. (3.2) and (3.3) show the resulting flutter speed pdfs in blue. For the truncated Gaussian probability densities of the structural parameters with $10 \% \mathrm{COV}$, the COV of the flutter speed pdfs are shown in the boxes of each respective parameter variation plot. The mean of each flutter speed distribution and the baseline flutter speed, are depicted by a vertical green line and a vertical red line respectively. The equivalent Gaussian pdfs of flutter speed are also plotted using a green line. The equivalent Gaussian pdf, which is based on the mean and the variance obtained from the actual flutter speed pdf, provides a qualitative identification of the non-linear transformation features (i.e. skew effects). This effect will be examined in more detail in a later study.

In fig. (3.13) note the following observations:

- Although the uncertainties in the inertia (with exception of fully correlated inertia parameters) and stiffness contribute significantly to the uncertainty in the flutter speed, the uncertainty in the structural damping of the flutter mode also influences the randomness 

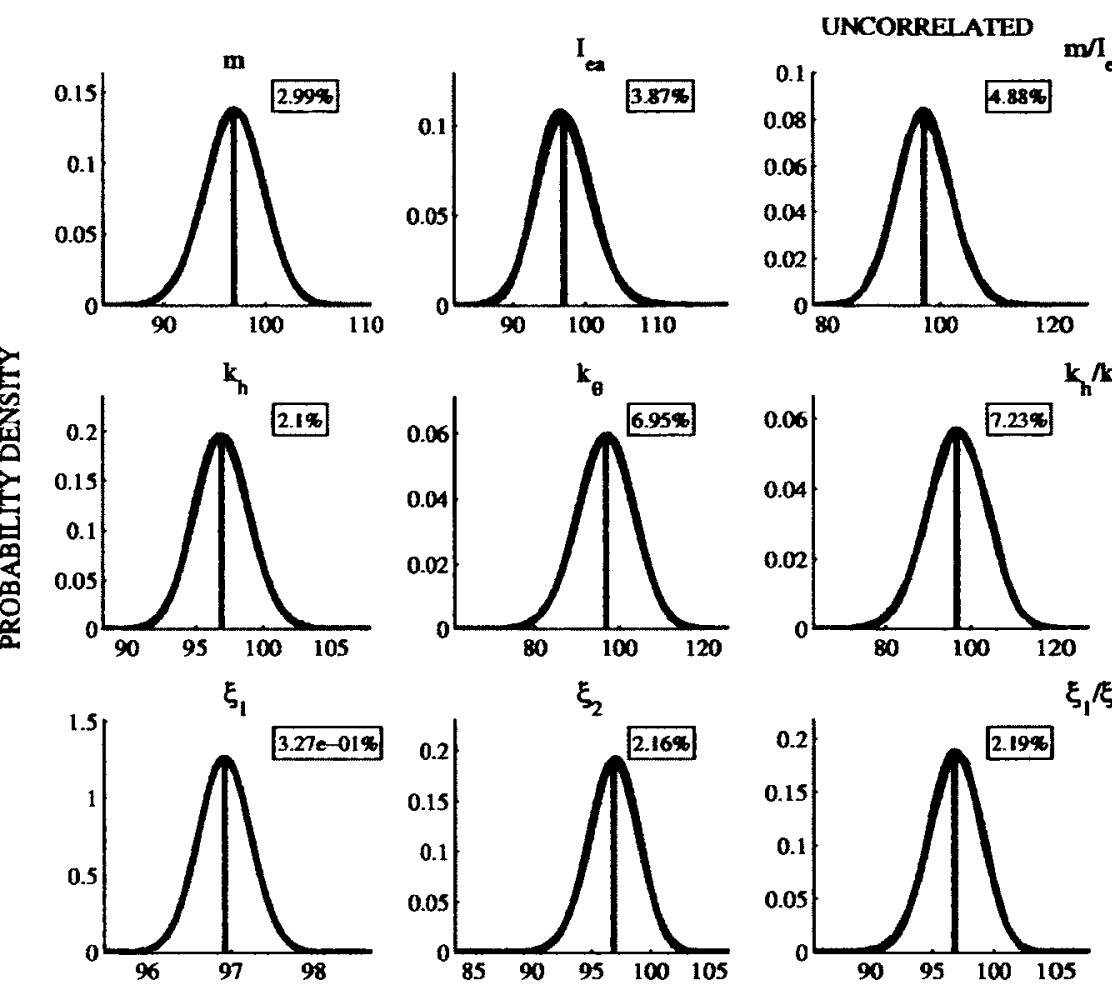

CORRFLATED
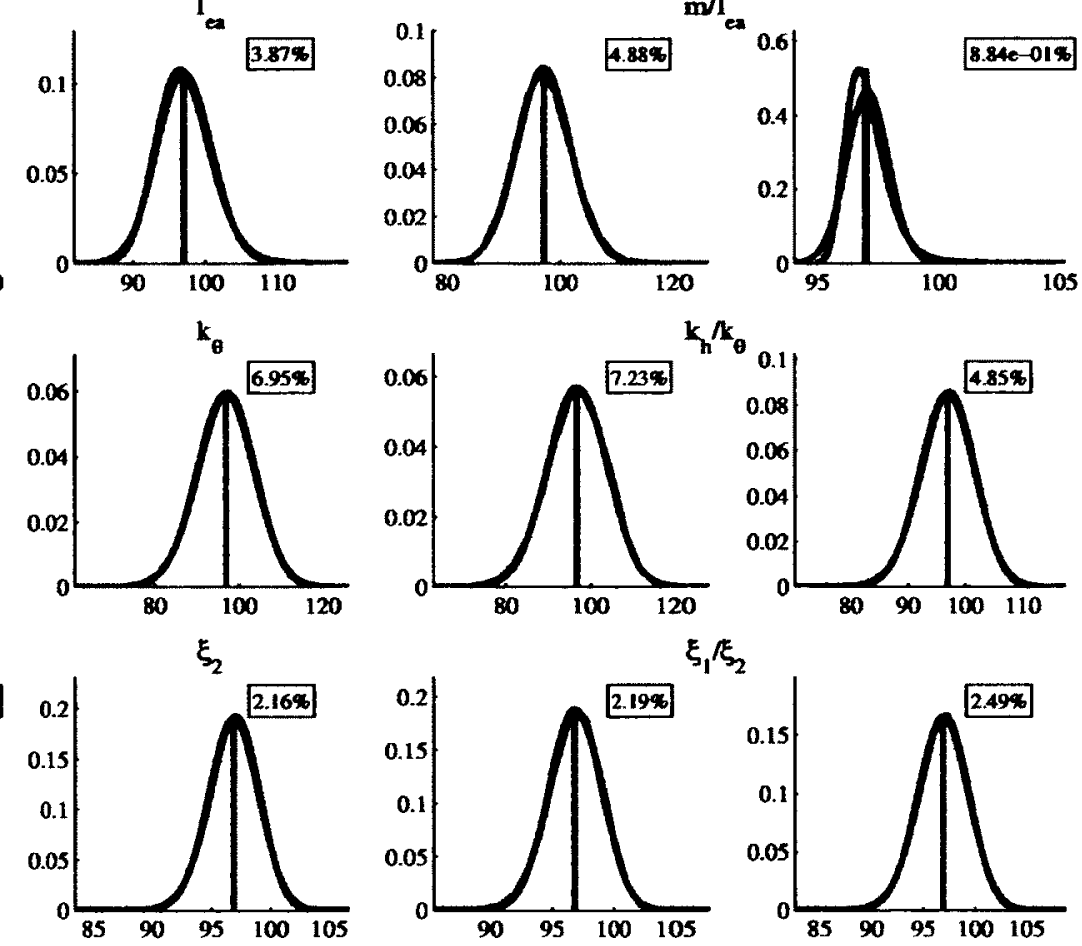

FLUTTER SPEED

Figure 3.13: Flutter speed pdfs (case 1 airfoil, quasisteady, 10\% COV)

in the flutter speed due to the mild nature of the coalescence flutter for this system.

- When the uncertainty is present in multiple uncorrelated random parameters, the flutter speed uncertainty increases.

- Although the correlation among the mass and stiffness decreases uncertainty in the flutter speed, the comrelated damping parameters lead to an increase of the uncertainty in the flutter speed.

- Some of the flutter speed pdfs have a slightly non-Gaussian distribution, indicating a mild non-linear mapping between the relevant structural parameter pdf and the flutter speed pdf. For such cases, the mean flutter speed no longer matches the baseline flutter speed.

(B) Uniform Distribution with $\mathrm{COV}=10 \%$

For fig. (3.14), the observations are very similar to the case of Truncated Gaussian input pdfs. We highlight the following points: 

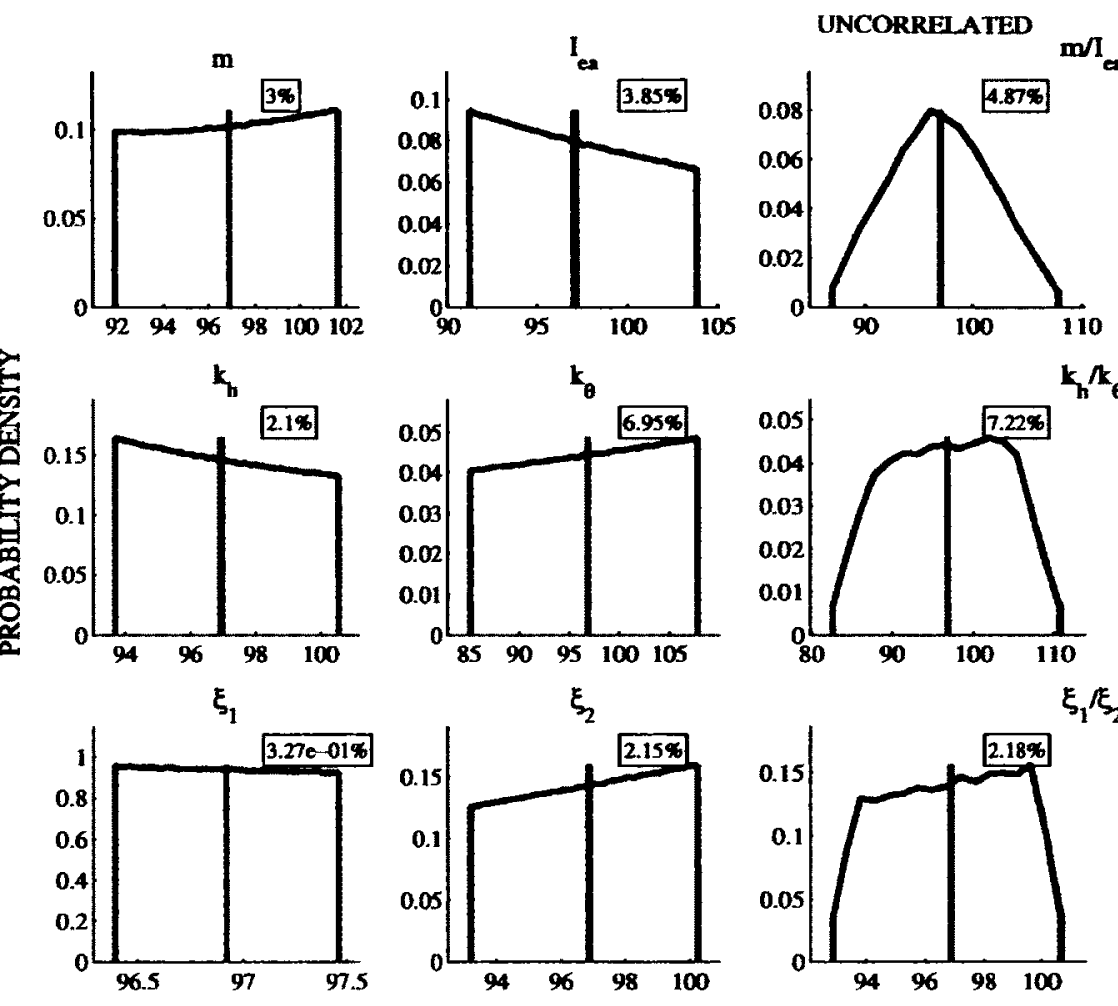

CORREIATED
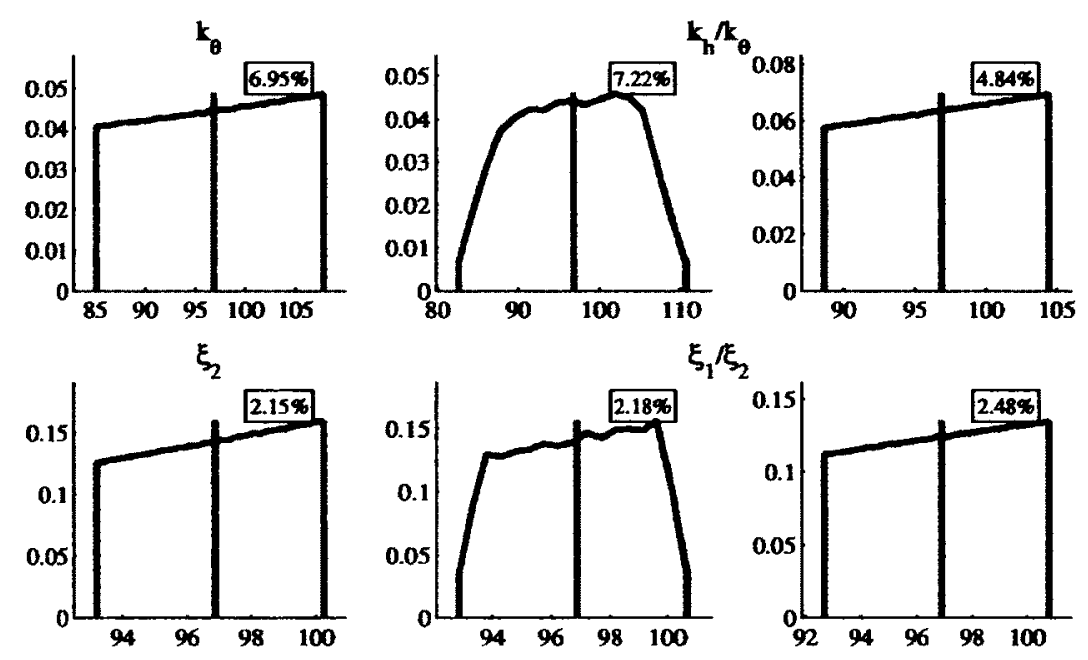

FLUTTER SPEED

Figure 3.14: Flutter speed pdfs (case 1 airfoil, quasisteady, $10 \% \mathrm{COV}$ )

- Although the uncertainties in the inertia and stiffness contribute significantly to the uncertainty in the flutter speed, the uncertainty in the structural damping of the flutter mode also influences the randomness in the flutter speed due to the mild nature of the coalescence flutter for this system.

- When the uncertainty is present in multiple uncorrelated random parameters, the flutter speed uncertainty increases.

- Although the correlation among the mass and stiffness decreases uncertainty in the flutter speed, the correlated damping parameters lead to an increase in the uncertainty in the flutter speed.

- Some of the flutter speed pdfs no longer have a uniform distribution, indicating a mild non-linear mapping between the relevant structural parameter pdf and the flutter speed pdf. For such cases, the mean flutter speed no longer matches the baseline flutter speed. 


\section{Unsteady Analysis: Baseline (Deterministic) Case}

Fig. (3.15) shows the modal properties as a function of airspeed of the baseline system for the case of unsteady aerodynamics as a function of increasing air speed. Similarly to the quasisteady case, the second mode becomes unstable. In contrast to the quasisteady case, the flutter type is no longer mild and the flutter speed increases to $140.94 \mathrm{~m} / \mathrm{s}$.
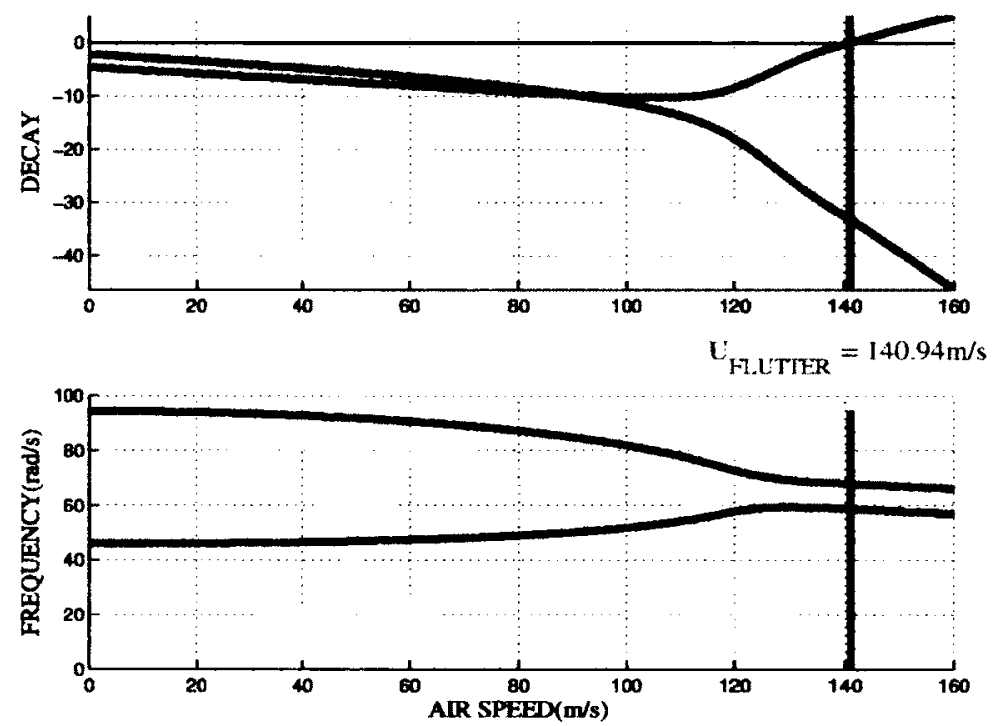

Figure 3.15: Evolution of modal parameters with respect to airspeed

Fig. (3.16) shows the relative magnitude of the elements of the eigenvectors of each mode for the no flow condition and at the flutter speed. The relative strength of individual components in each eigenvector changes with increase in airspeed. Initially, the first and second modes exhibit heave and pitch dominance, respectively, similarly to the quasisteady case. At flutter point, however, the dominance of the first and second aeroelastic modes switches to become heave and pitch dominant, respectively.

\section{Unsteady Analysis: Probabilistic Case}

Tbls. (3.4) and (3.5) provide a summary of the probabilistic flutter speed identification results.

Then, the flutter speed pdfs are examined in more detail. Similarly to the study of quasisteady results, only the results for large ( $10 \% \mathrm{COV})$ input uncertainty will be examined, since the low parameter uncertainty produces almost linear flutter speed transformation features. 

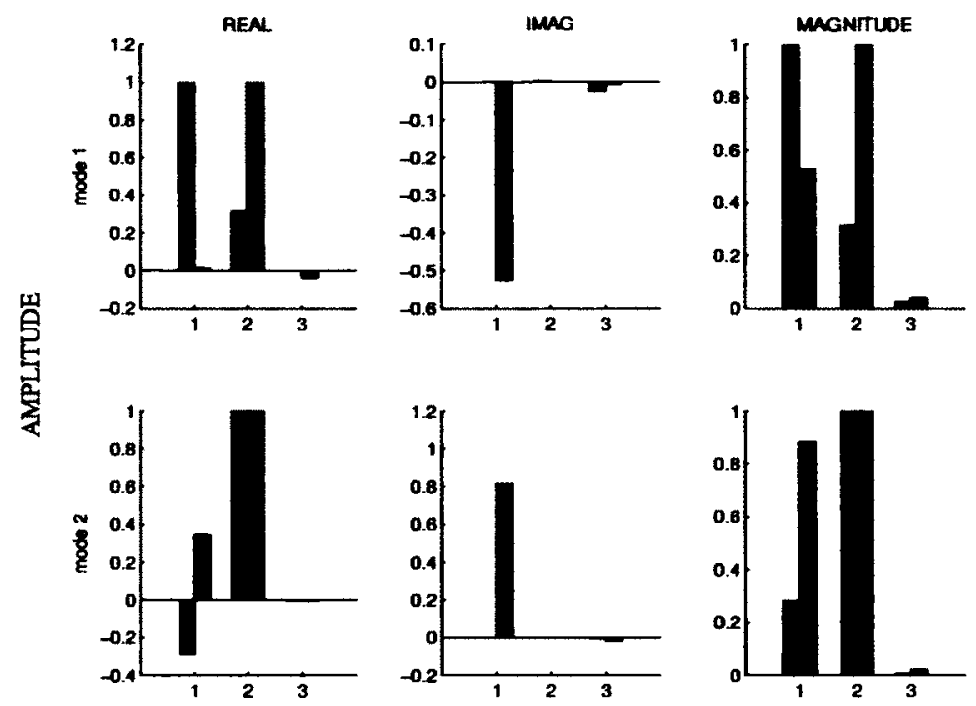

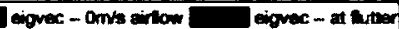
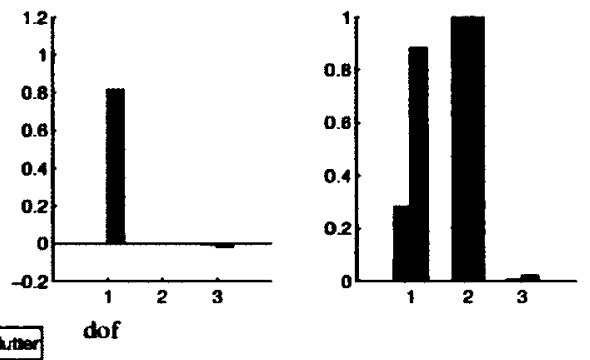

Figure 3.16: real part, imaginary part, and magnitude of eigenvectors of two modes, illustrating relative strength of each component

\begin{tabular}{|c|c|c|c|}
\hline \multicolumn{2}{|c|}{ Input COV } & $2.5 \%$ & $10 \%$ \\
\hline Random Parameter & Distribution & \multicolumn{2}{c|}{ Flutter Speed COV \% } \\
\hline \hline \multirow{2}{*}{ mass } & Gaussian & 0.380 & 1.52 \\
\cline { 2 - 4 } & uniform & 0.380 & 1.52 \\
\hline radius of gyration & Gaussian & 0.811 & 3.25 \\
\cline { 2 - 4 } & uniform & 0.811 & 3.25 \\
\hline \multirow{2}{*}{ heave stiffness } & Gaussian & 0.334 & 1.34 \\
\cline { 2 - 4 } & uniform & 0.334 & 1.33 \\
\hline \multirow{2}{*}{ pitch stiffness } & Gaussian & 1.51 & 6.05 \\
\cline { 2 - 4 } & uniform & 1.51 & 6.05 \\
\hline \multirow{2}{*}{ damping ratio 1 } & Gaussian & 0.0914 & 0.365 \\
\cline { 2 - 4 } & uniform & 0.0914 & 0.365 \\
\hline damping ratio 2 & Gaussian & 0.119 & 0.475 \\
\cline { 2 - 4 } & uniform & 0.119 & 0.475 \\
\hline
\end{tabular}

Table 3.4: Results illustrating the uncertainty in the flutter speed due to independent uncertainty in the structural parameters

\section{(A) Truncated Gaussian Distribution with $\mathrm{COV}=10 \%$}

From fig. (3.17) the following observations are made:

- Randomness in inertia (with exception of fully correlated inertia parameters) and stiffness parameters introduce large uncertainty in the flutter speed. As expected for the moderate/explosive flutter, the effect of damping is not significant. 


\begin{tabular}{|c|c|c|c|c|c|}
\hline \multicolumn{2}{|c|}{ Input COV } & $2.5 \%$ & $10 \%$ & $2.5 \%$ & $10 \%$ \\
\hline Random Parameter & Distribution & \multicolumn{3}{c|}{ Flutter Speed COV (\%) } \\
\hline \hline \multicolumn{1}{|c|}{$\begin{array}{c}\text { mass \& } \\
\text { radius of gyration }\end{array}$} & $\begin{array}{c}\text { Gaussian } \\
\text { Gaussian }\end{array}$ & 0.896 & 3.60 & 0.433 & 1.80 \\
\hline $\begin{array}{c}\text { mass \& } \\
\text { radius of gyration }\end{array}$ & $\begin{array}{c}\text { uniform } \\
\text { uniform }\end{array}$ & 0.896 & 3.60 & 0.432 & 1.77 \\
\hline $\begin{array}{c}\text { heave stiffness \& } \\
\text { pitch stiffness }\end{array}$ & $\begin{array}{c}\text { Gaussian } \\
\text { Gaussian }\end{array}$ & 1.54 & 6.17 & 1.17 & 4.73 \\
\hline $\begin{array}{c}\text { heave stiffness \& } \\
\text { pitch stiffness }\end{array}$ & $\begin{array}{c}\text { uniform } \\
\text { uniform }\end{array}$ & 1.54 & 6.17 & 1.17 & 4.71 \\
\hline $\begin{array}{c}\text { damping ratio 1 \& } \\
\text { damping ratio 2 }\end{array}$ & $\begin{array}{c}\text { Gaussian } \\
\text { Gaussian }\end{array}$ & 0.150 & 0.599 & 0.210 & 0.841 \\
\hline $\begin{array}{c}\text { damping ratio 1 \& } \\
\text { damping ratio 2 }\end{array}$ & $\begin{array}{c}\text { uniform } \\
\text { uniform }\end{array}$ & 0.150 & 0.599 & 0.210 & 0.840 \\
\hline \hline
\end{tabular}

Table 3.5: Results illustrating the uncertainty in the flutter speed due to correlated uncertainty in the structural parameters
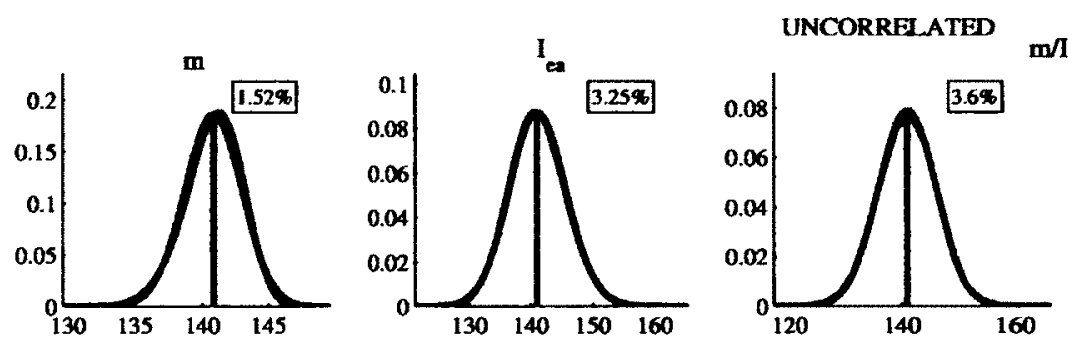

CORRELATED
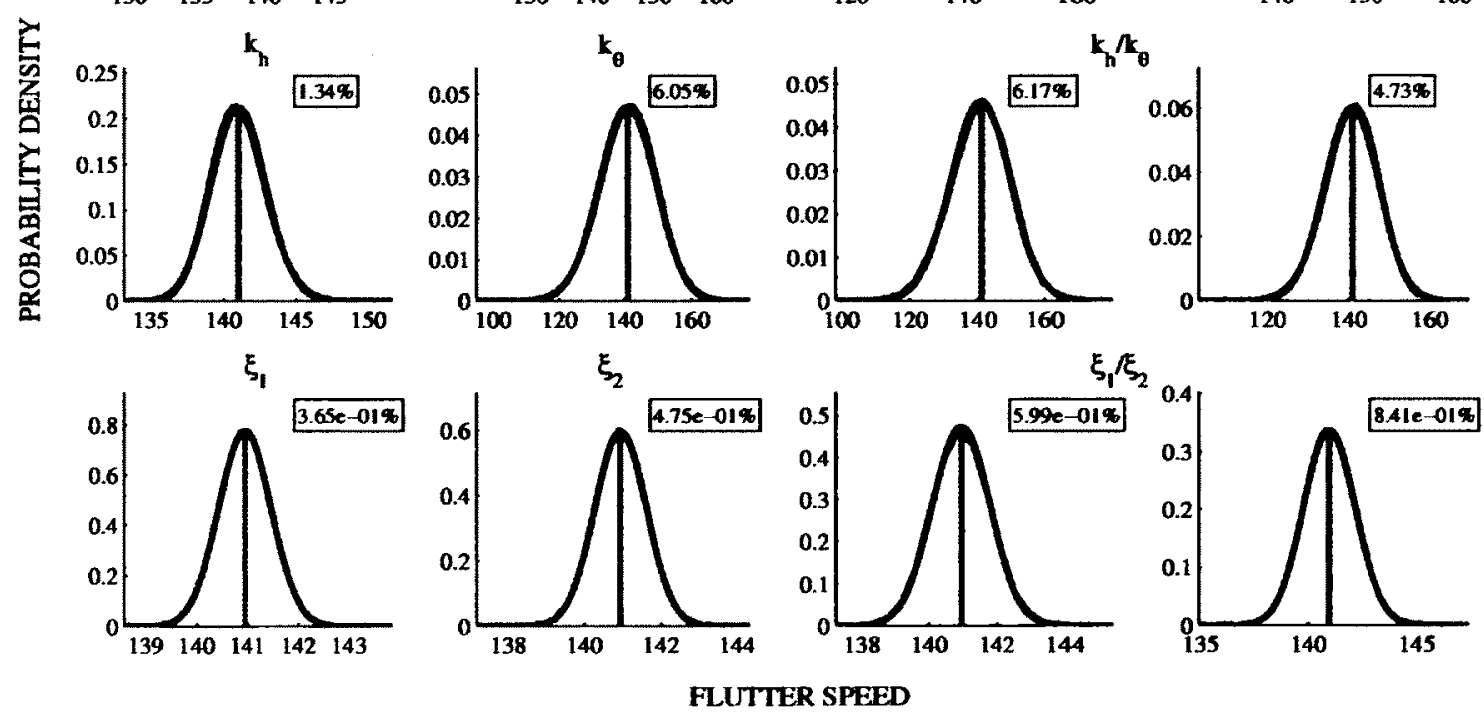

Figure 3.17: Flutter speed pdfs (case 1 airfoil, unsteady, 10\% COV)

The remainder of the observations are identical to the analysis with quasisteady aerodynamic 
forcing.

\section{(B) Uniform Distribution with $\mathrm{COV}=10 \%$}

The observations from fig. (3.18) indicate similar conclusions as for quasisteady case, except that the effect of uncertainty in damping on the flutter speed is significantly reduced, as expected with a more explosive flutter type.

In this study, the effect of an uncertainty increase and an input distribution change did not effect the robustness of the results due to approximately a linear mapping between the flutter speed and the structural parameters. Furthermore, when comparing the probabilistic results of the analysis with the quasisteady and unsteady aerodynamics, in general (but not always), the effects of uncertainty are significantly smaller for the unsteady case. The exceptions to this observation are the cases of uncertainty in the structural damping of the non-flutter mode and the correlated inertia parameters.
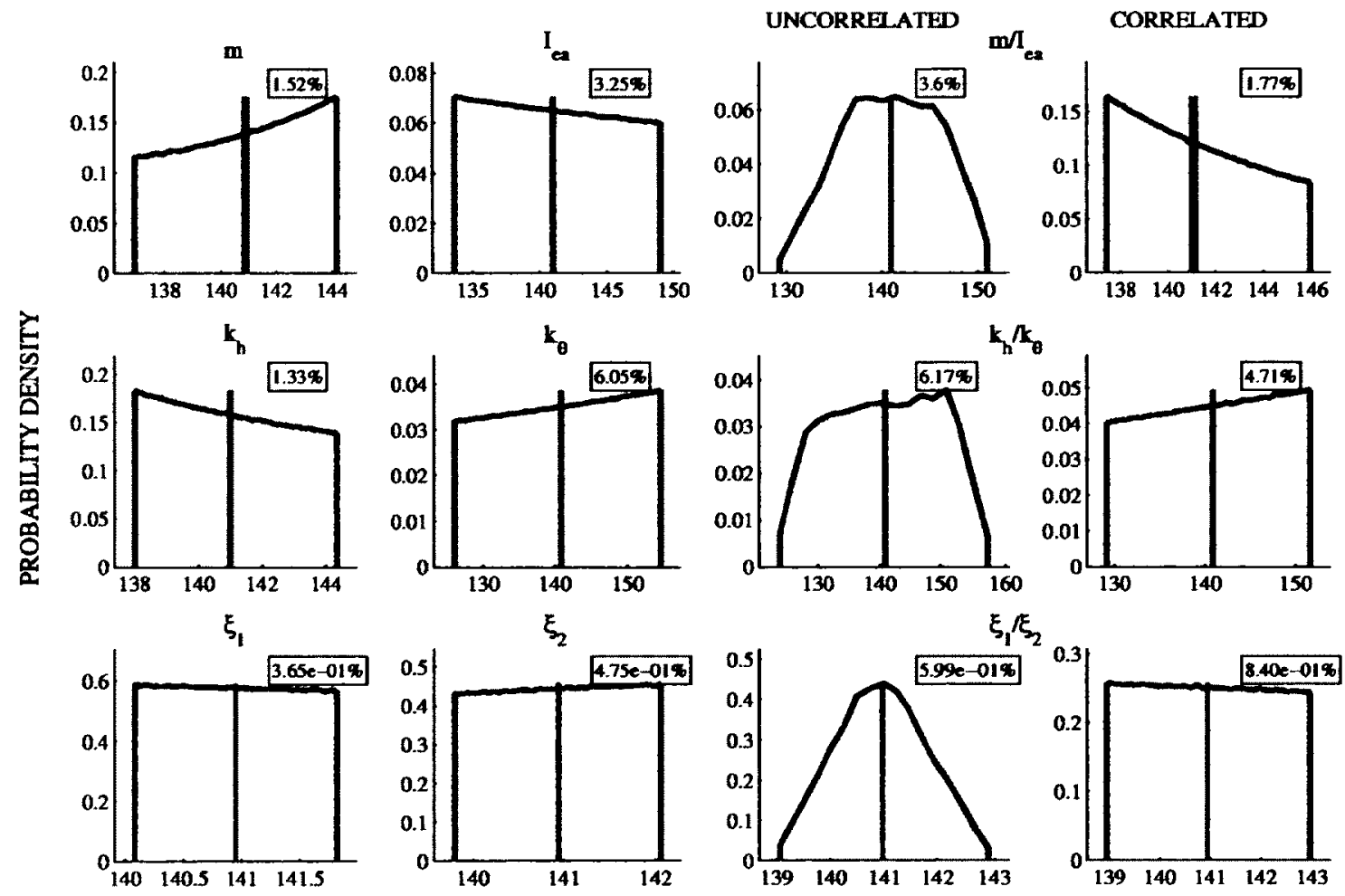

FLUTTER SPEED

Figure 3.18: Flutter speed pdfs (case 1 airfoil, unsteady, 10\% COV) 


\subsubsection{CASE 2}

The parameters of the baseline system are shown in tbl. (3.6). As mentioned previously, this case will be used later to compare the dynamics of a rigid 2-D model of a wing and the dynamics of a continuous cantilever model.

\section{Quasisteady Analysis: Baseline Case}

Fig. (3.19) illustrates the modal decays and the modal frequencies for the specified range of air speeds. As evident from this figure, the second mode (higher frequency) becomes

\begin{tabular}{||l|c|l||}
\hline PARAMETER & Symbol & Value \\
\hline \hline mass & $m$ & $35.7187 \mathrm{~kg} / \mathrm{m}$ \\
normalized radius of gyration & $r_{g}$ & 1.05 \\
heave stiffness & $k_{h}$ & $8.74608 \times 10^{4} \mathrm{~N} / \mathrm{m}^{2}$ \\
pitch stiffness & $k_{\theta}$ & $2.62688 \times 10^{4} \mathrm{~N} / \mathrm{rad}$ \\
chord length & $c$ & $1.8288 \mathrm{~m}$ \\
span & $L$ & $6.096 \mathrm{~m}$ \\
normalized static imbalance & $x_{\theta}$ & 0.95 \\
damping ratio 1 & $\xi_{1}$ & 0.04 \\
damping ratio 2 & $\xi_{2}$ & 0.04 \\
free stream fluid density & $\rho_{\infty}$ & $1.225 \mathrm{~kg} / \mathrm{m}^{3}$ \\
normalized distance btw EA and mid-chord & $a_{h}$ & -1.0 \\
lift coefficient gradient & $\bar{C}_{l \alpha}$ & $2 \pi$ \\
\hline \hline Num. of Samples & - & 50000 \\
\hline
\end{tabular}

Table 3.6: Model parameters for Case 2

unstable through a mild flutter having a flutter speed of $95.19 \mathrm{~m} / \mathrm{s}$.

Fig. (3.20) demonstrates the relative magnitude of the elements of the eigenvectors of each mode at no flow condition and at the proximity of the flutter speed. In Case 2, it can be seen that the relative strength of each eigenvector changes with an increase in airspeed. Initially, the first mode exhibits strong pitch dominance, and the second mode, heave dominance. At flutter point, both of the modes lose the relative strength of dominant components. 

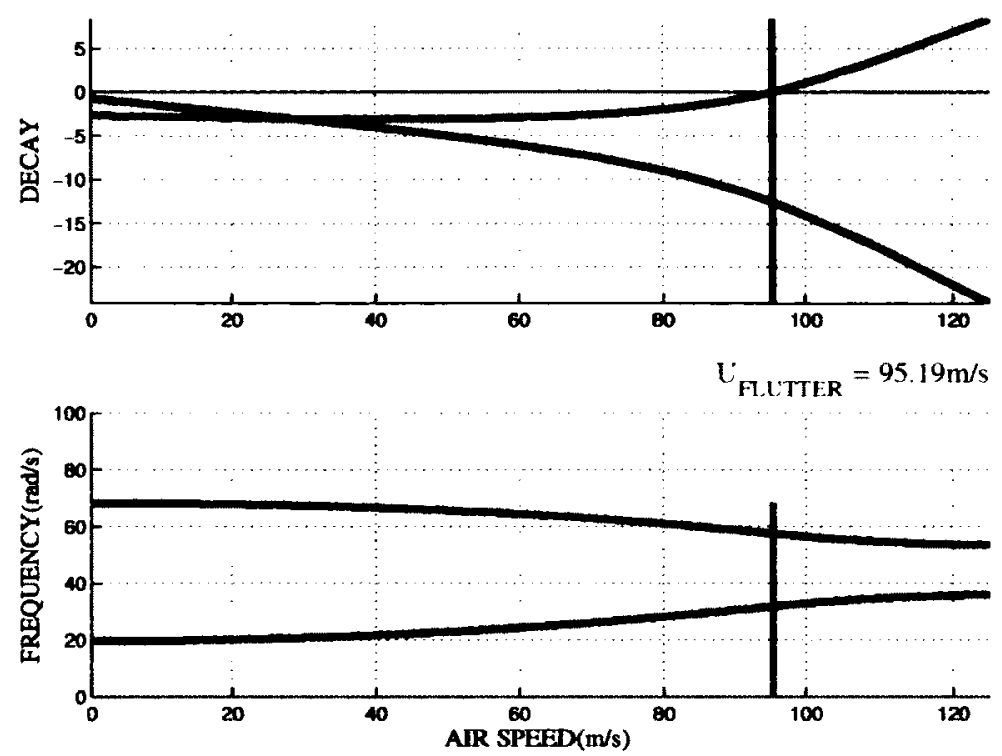

Figure 3.19: Evolution of modal parameters with respect to airspeed
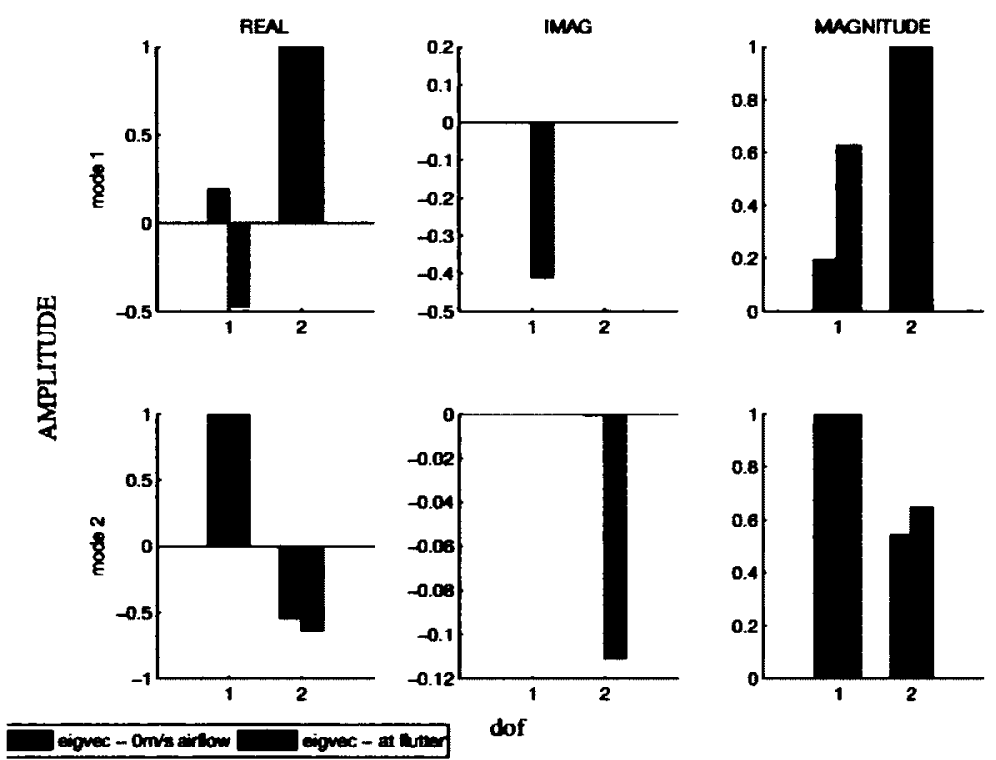

Figure 3.20: real part, imaginary part, and magnitude of eigenvectors of two modes, illustrating relative strength of each component

Similar to the previous case study, the mappings of flutter speed and the structural parameters are shown in fig. (3.21). 


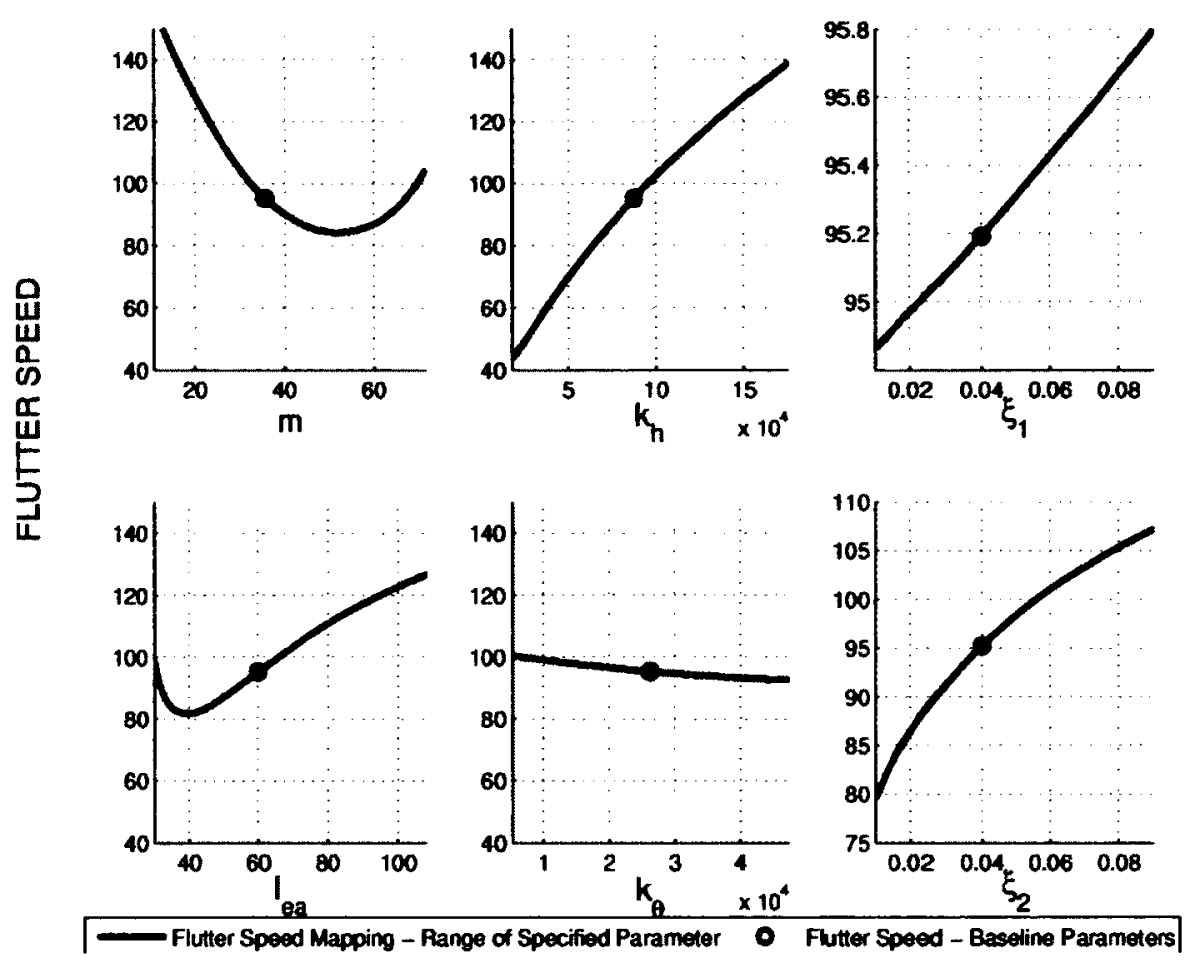

Figure 3.21: The functional relationships among the flutter speed and structural parameters

\section{Quasisteady Analysis: Probabilistic Case}

The summary of the probabilistic flutter speed results is reported in tbls. (3.7) and (3.8).

Similarly to the analysis of Case 1 results, the resulting flutter speed pdfs with effects of low uncertainty will not be illustrated in this section, since they show almost linear features. Therefore, all of the significant features in these cases can be obtained from the information provided in tbls. (3.7) and (3.8).

Figs. (3.22) and (3.23) illustrate the probabilistic flutter speed estimate resulting from a truncated Gaussian and a uniform distributions of the random structural parameters, respectively, with a $10 \% \mathrm{COV}$. The results of Case 2 show similar qualitative features as the results of Case 1 study. From these figures the following observations are obtained:

- Although the uncertainties in the inertia (with exception of fully correlated inertia parameters) and stiffness contribute significantly to the uncertainty in the flutter speed, the uncertainty in the structural damping of the flutter mode also influences the randomness 


\begin{tabular}{|c|c|c|c|}
\hline \multicolumn{2}{|c|}{ Input COV } & $2.5 \%$ & $10 \%$ \\
\hline Random Parameter & Distribution & \multicolumn{2}{c|}{ Flutter Speed COV \% } \\
\hline \hline \multirow{2}{*}{ mass } & Gaussian & 1.25 & 5.03 \\
\cline { 2 - 4 } & uniform & 1.25 & 5.00 \\
\hline radius of gyration & Gaussian & 1.28 & 4.95 \\
\cline { 2 - 4 } & uniform & 1.28 & 5.02 \\
\hline heave stiffness & Gaussian & 1.32 & 5.31 \\
\cline { 2 - 4 } & uniform & 1.32 & 5.30 \\
\hline \multirow{2}{*}{ pitch stiffness } & Gaussian & 0.123 & 0.493 \\
\cline { 2 - 4 } & uniform & 0.123 & 0.492 \\
\hline \multirow{2}{*}{ damping ratio 1 } & Gaussian & 0.0115 & 0.0460 \\
\cline { 2 - 4 } & uniform & 0.0115 & 0.0460 \\
\hline \multirow{2}{*}{ damping ratio 2 } & Gaussian & 0.348 & 1.40 \\
\cline { 2 - 4 } & uniform & 0.348 & 1.40 \\
\hline
\end{tabular}

Table 3.7: Results illustrating the uncertainty in the flutter speed due to independent uncertainty in the structural parameters

\begin{tabular}{|c|c|c|c|c|c|}
\hline \multicolumn{2}{|c|}{ Input COV } & $2.5 \%$ & $10 \%$ & $2.5 \%$ & $10 \%$ \\
\hline Random Parameter & Distribution & \multicolumn{3}{c|}{ Flutter Speed COV (\%) } \\
\hline \hline \multicolumn{2}{|c|}{} & \multicolumn{2}{|c|}{ Uncorrelated Parameters } & Fully Correlated Parameters \\
\hline \hline $\begin{array}{c}\text { mass \& } \\
\text { radius of gyration }\end{array}$ & $\begin{array}{c}\text { Gaussian } \\
\text { Gaussian }\end{array}$ & 1.78 & 6.87 & 0.0268 & 0.104 \\
\hline $\begin{array}{c}\text { mass \& } \\
\text { radius of gyration }\end{array}$ & $\begin{array}{c}\text { uniform } \\
\text { uniform }\end{array}$ & 1.78 & 6.88 & 0.0268 & 0.105 \\
\hline $\begin{array}{c}\text { heave stiffness \& } \\
\text { pitch stiffness }\end{array}$ & $\begin{array}{c}\text { Gaussian } \\
\text { Gaussian }\end{array}$ & 1.33 & 5.33 & 1.20 & 4.82 \\
\hline $\begin{array}{c}\text { heave stiffness \& } \\
\text { pitch stiffness }\end{array}$ & $\begin{array}{c}\text { uniform } \\
\text { uniform }\end{array}$ & 1.33 & 5.32 & 1.20 & 4.81 \\
\hline $\begin{array}{c}\text { damping ratio 1 \& } \\
\text { damping ratio 2 }\end{array}$ & $\begin{array}{c}\text { Gaussian } \\
\text { Gaussian }\end{array}$ & 0.348 & 1.40 & 0.360 & 1.45 \\
\hline $\begin{array}{c}\text { damping ratio 1 \& } \\
\text { damping ratio 2 }\end{array}$ & $\begin{array}{c}\text { uniform } \\
\text { uniform }\end{array}$ & 0.348 & 1.40 & 0.359 & 1.44 \\
\hline \hline
\end{tabular}

Table 3.8: Results illustrating the uncertainty in the flutter speed due to correlated uncertainty in the structural parameters

in the flutter speed due to the mild nature of the coalescence flutter for this system.

- Although the correlation among the mass and stiffness decreases uncertainty in the flutter speed, the correlated damping parameters lead to an increase of the uncertainty in the flutter speed. 
- Some of the flutter speed pdfs show mildly non-linear mapping trend between the flutter speed and the random structural parameter. For such cases, the mean flutter speed does not match the baseline flutter speed.

- Variation of a pitch stiffness parameter proves to be less influential on the variation of flutter speed in comparison to the effect of variation of damping in the flutter mode. In fig. (3.21), the gradient of flutter speed with respect to the pitch stiffness variation is very low, confirming that the flutter speed should not be very sensitive to this parameter.
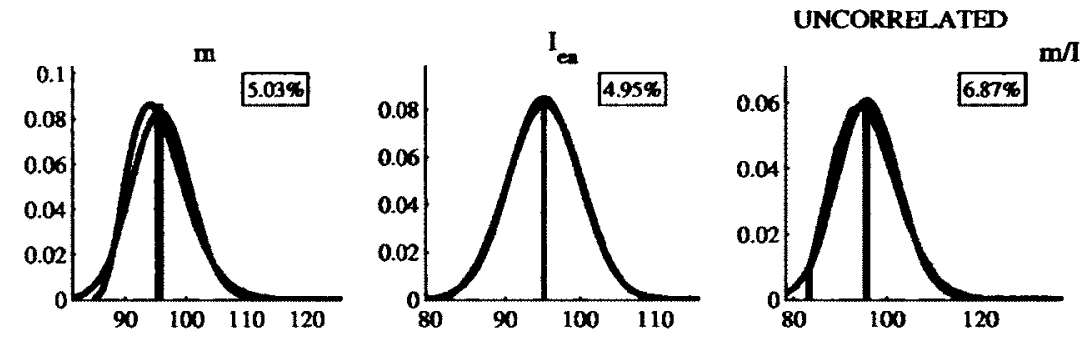

CORRELATED
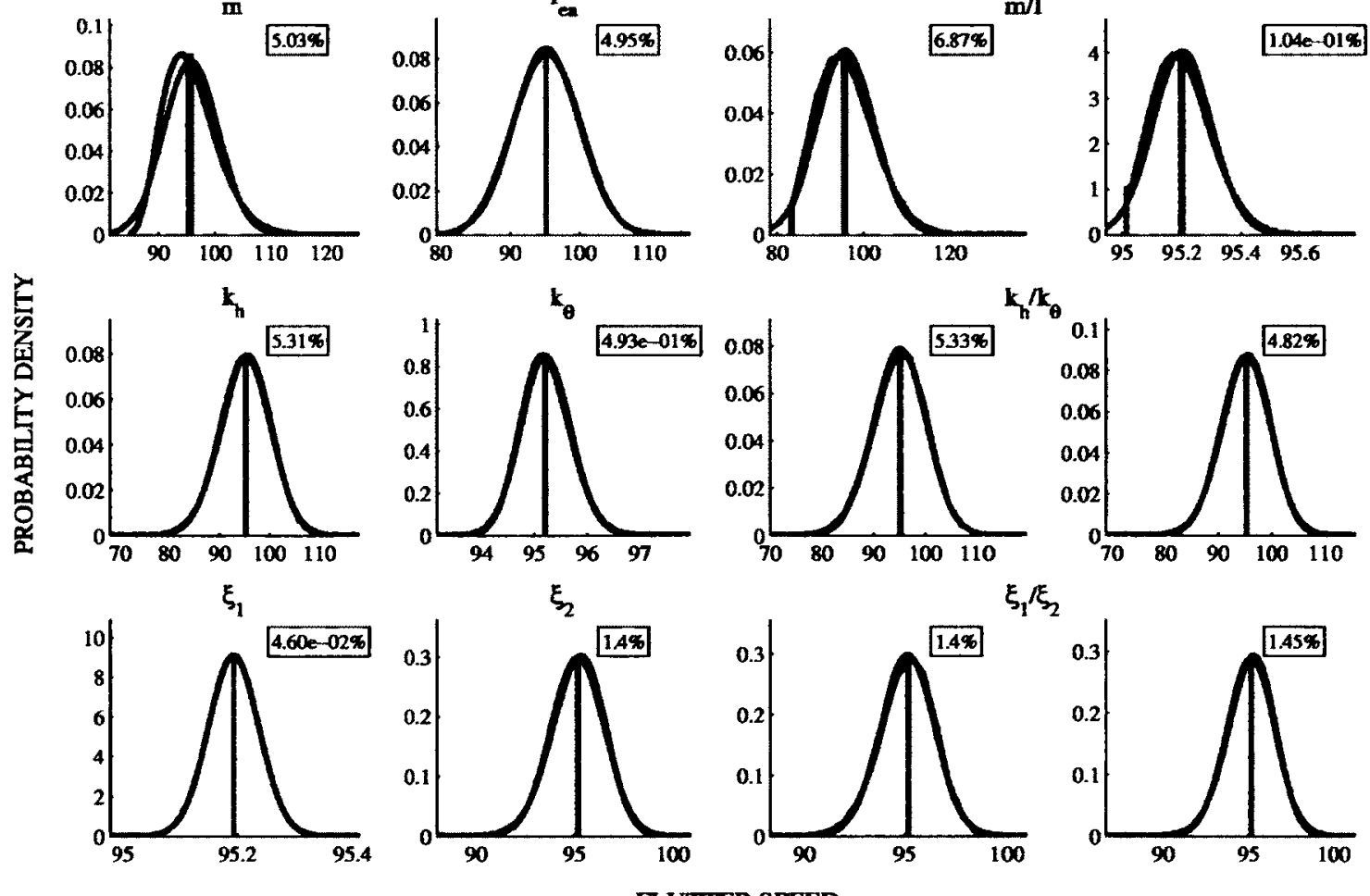

Figure 3.22: Flutter speed pdfs (case 2 airfoil, quasisteady, 10\% COV) 

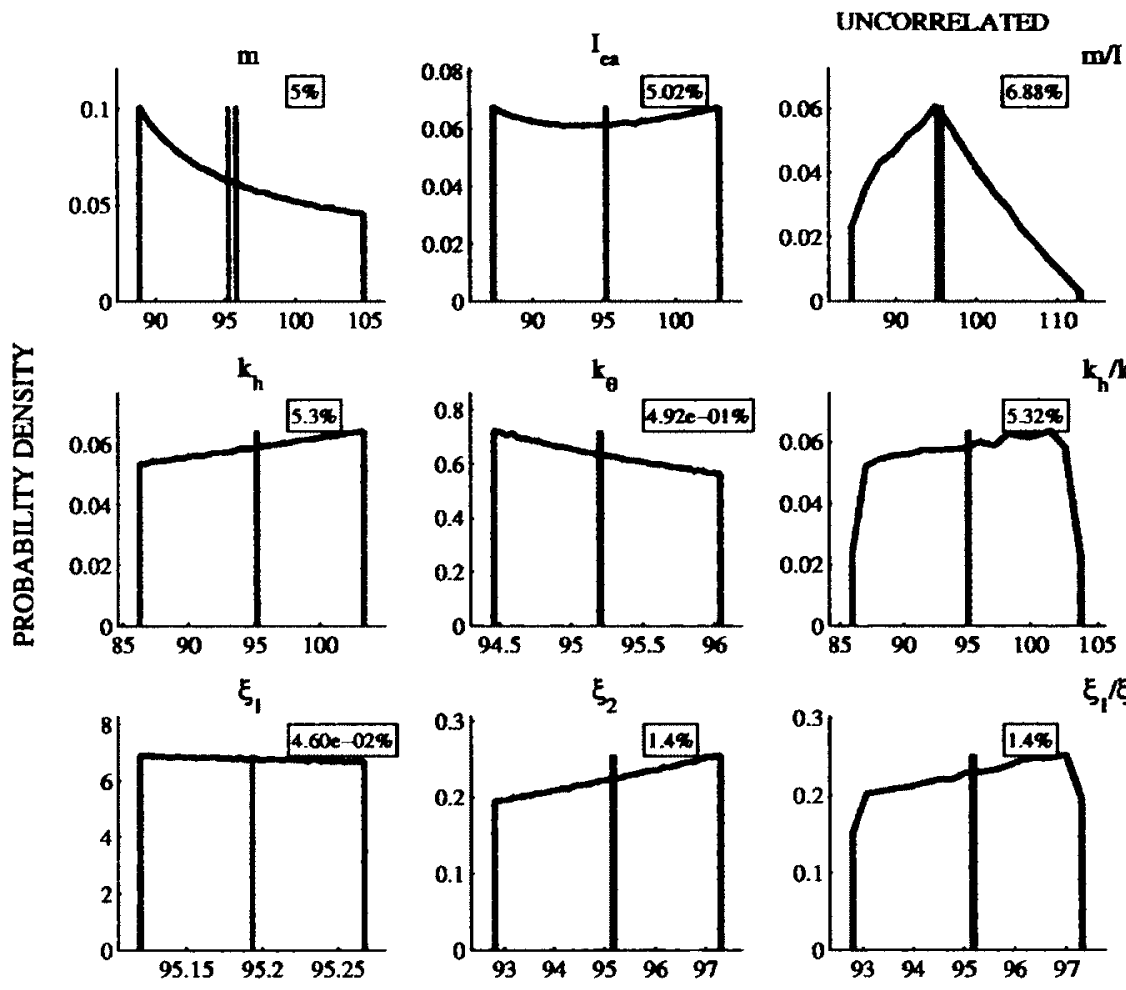

CORRELATED
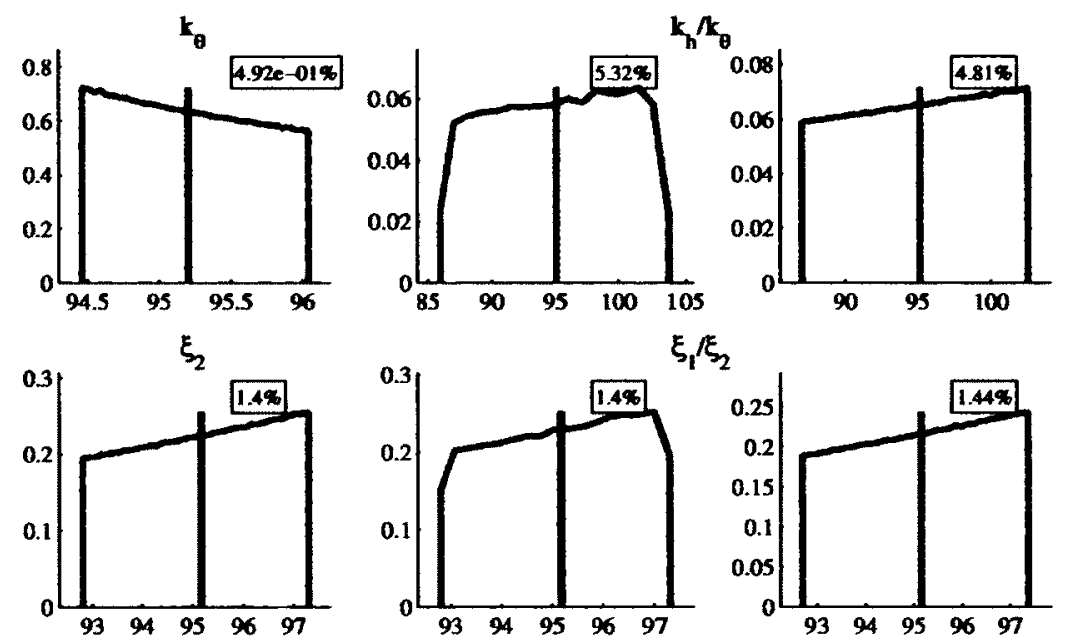

FLUTTER SPEED

Figure 3.23: Flutter speed pdfs (case 2 airfoil, quasisteady, $10 \%$ COV)

\section{Unsteady Analysis: Baseline Case}

Fig. (3.24) shows the modal properties as a function of airspeed of the baseline system for the case of unsteady aerodynamics as a function of increasing air speed. In this case, the second mode becomes unstable, the flutter type changes to moderate-explosive the flutter speed increases to $130.37 \mathrm{~m} / \mathrm{s}$.

Fig. (3.25) shows the relative magnitude of the elements of the eigenvectors of each mode for the no flow condition and at the flutter speed. The relative strength of individual components in each eigenvector changes with increase in airspeed. Initially, the first mode and second modes exhibit pitch and heave dominance, respectively. Despite the diminishing strength of the dominant component of each mode, the modal dominance of the aeroelastic modes does not switch, as it did in the previous case study with unsteady aerodynamics. 

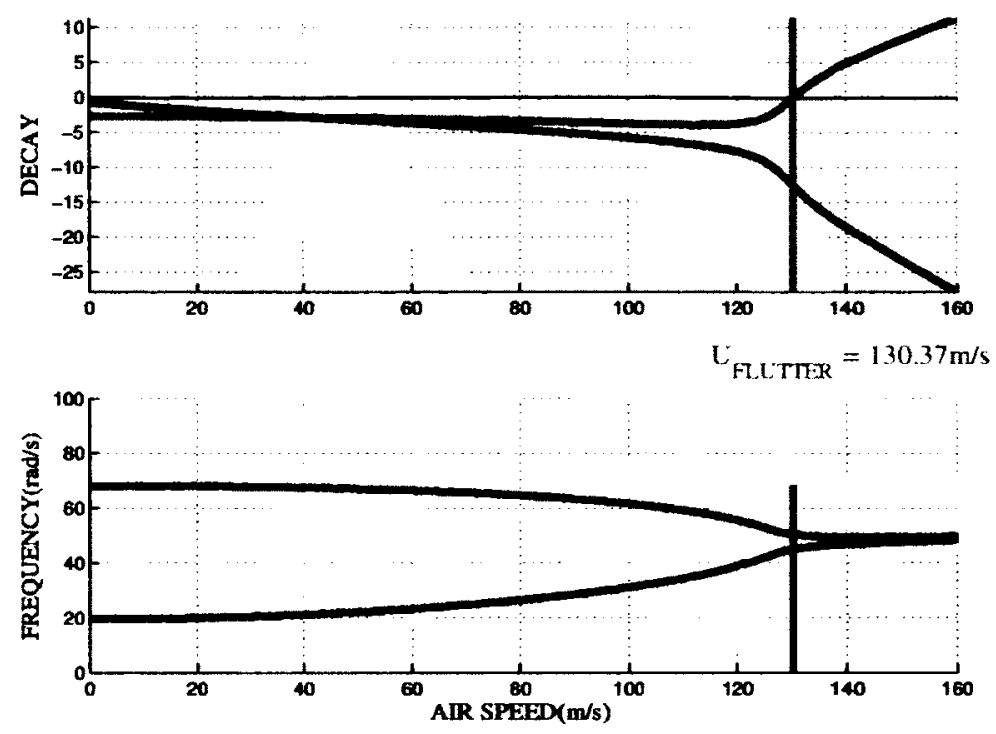

Figure 3.24: Evolution of modal parameters with respect to airspeed
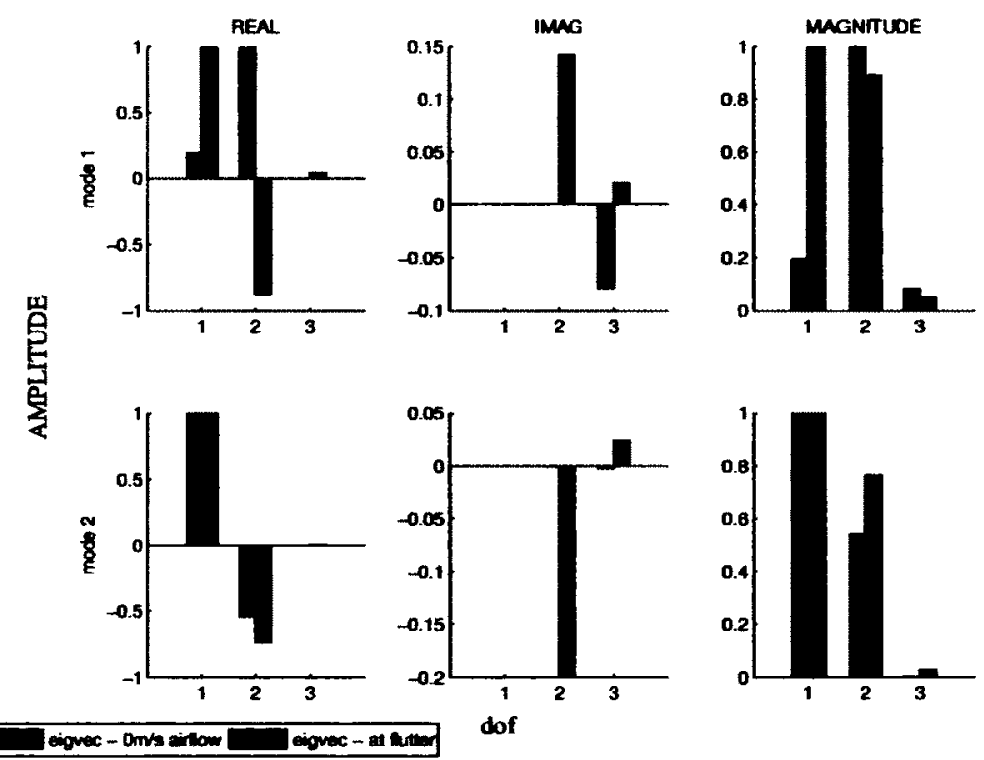

Figure 3.25: real part, imaginary part, and magnitude of eigenvectors of two modes, illustrating relative strength of each component

\section{Unsteady Analysis: Probabilistic Case}

Tbls. (3.9) and (3.10), provide a summary of the results of probabilistic flutter speed estimation for Case 1 with quasisteady aerodynamics.

Similarly to all of the prior flutter speed results, the effects of low uncertainty will not be 
illustrated because they show almost linear features, since all of the significant features for these cases could be directly gathered from the information provided in tbls. (3.7) and (3.8).

Figs. (3.26) and (3.27) illustrate the probabilistic flutter speed estimate resulting from the truncated Gaussian and a uniform distributions of the random structural parameters, respectively, with a $10 \%$ COV. Once again, the probabilistic results of the Case 2 study are

\begin{tabular}{|c|c|c|c|}
\hline \multicolumn{2}{|c|}{ Input COV } & $2.5 \%$ & $10 \%$ \\
\hline Random Parameter & Distribution & \multicolumn{2}{c|}{ Flutter Speed COV \% } \\
\hline \hline \multirow{2}{*}{ mass } & Gaussian & 0.455 & 1.89 \\
\cline { 2 - 4 } & uniform & 0.454 & 1.84 \\
\hline radius of gyration & Gaussian & 0.280 & 0.981 \\
\cline { 2 - 4 } & uniform & 0.281 & 1.02 \\
\hline heave stiffness & Gaussian & 1.20 & 4.82 \\
\cline { 2 - 4 } & uniform & 1.20 & 4.81 \\
\hline \multirow{2}{*}{ pitch stiffness } & Gaussian & 0.0325 & 0.130 \\
\cline { 2 - 4 } & uniform & 0.0325 & 0.130 \\
\hline damping ratio 1 & Gaussian & 0.0127 & 0.0508 \\
\cline { 2 - 4 } & uniform & 0.0127 & 0.0508 \\
\hline damping ratio 2 & Gaussian & 0.0698 & 0.282 \\
\cline { 2 - 4 } & uniform & 0.0698 & 0.281 \\
\hline
\end{tabular}

Table 3.9: Results illustrating the uncertainty in the flutter speed due to independent uncertainty in the structural parameters

\begin{tabular}{|c|c|c|c|c|c|}
\hline \multicolumn{2}{|c|}{ Input COV } & $2.5 \%$ & \multicolumn{1}{c|}{$10 \%$} & $2.5 \%$ & $10 \%$ \\
\hline Random Parameter & Distribution & \multicolumn{4}{c|}{ Flutter Speed COV (\%) } \\
\hline \hline \multicolumn{2}{|c|}{} & \multicolumn{2}{|c|}{ Uncorrelated Parameters } & Fully Correlated Parameters \\
\hline \hline $\begin{array}{c}\text { mass \& } \\
\text { radius of gyration }\end{array}$ & $\begin{array}{c}\text { Gaussian } \\
\text { Gaussian }\end{array}$ & 0.531 & 2.06 & 0.171 & 0.678 \\
\hline $\begin{array}{c}\text { mass \& } \\
\text { radius of gyration }\end{array}$ & $\begin{array}{c}\text { uniform } \\
\text { uniform }\end{array}$ & 0.532 & 1.99 & 0.171 & 0.680 \\
\hline $\begin{array}{c}\text { heave stiffness \& } \\
\text { pitch stiffness }\end{array}$ & $\begin{array}{c}\text { Gaussian } \\
\text { Gaussian }\end{array}$ & 1.20 & 4.82 & 1.16 & 4.69 \\
\hline $\begin{array}{c}\text { heave stiffness \& } \\
\text { pitch stiffness }\end{array}$ & $\begin{array}{c}\text { uniform } \\
\text { uniform }\end{array}$ & 1.20 & 4.81 & 1.16 & 4.68 \\
\hline $\begin{array}{c}\text { damping ratio 1 \& } \\
\text { damping ratio 2 }\end{array}$ & $\begin{array}{c}\text { Gaussian } \\
\text { Gaussian }\end{array}$ & 0.0709 & 0.287 & 0.0826 & 0.332 \\
\hline $\begin{array}{c}\text { damping ratio 1 \& } \\
\text { damping ratio 2 }\end{array}$ & $\begin{array}{c}\text { uniform } \\
\text { uniform }\end{array}$ & 0.0709 & 0.285 & 0.0825 & 0.331 \\
\hline \hline
\end{tabular}

Table 3.10: Results illustrating the uncertainty in the flutter speed due to correlated uncertainty in the structural parameters 
very similar to the results of Case 1 study. Therefore, the observations from these figures indicate similar conclusions as for the quasisteady aerodynamics case:

- Uncertainty in inertia and stiffness parameters (excluding the cases with uncertainty in the fully correlated inertia parameters and the individual pitch stiffness) produce larger effects on the flutter speed estimate. As expected from a more explosive flutter type, the effect of damping is not very significant.

- Uncertainty in the pitch stiffness parameter proves to be non-influential on the variation of the flutter speed. Fig. (3.21) indicates that the gradient of the flutter speed with respect to the pitch stiffness variation is very low, confirming that the flutter speed should not be very sensitive to this parameter.

- Although the correlation among the inertia and stiffness decreases uncertainty in the flutter speed, the correlated damping parameters lead to an increase in the uncertainty of
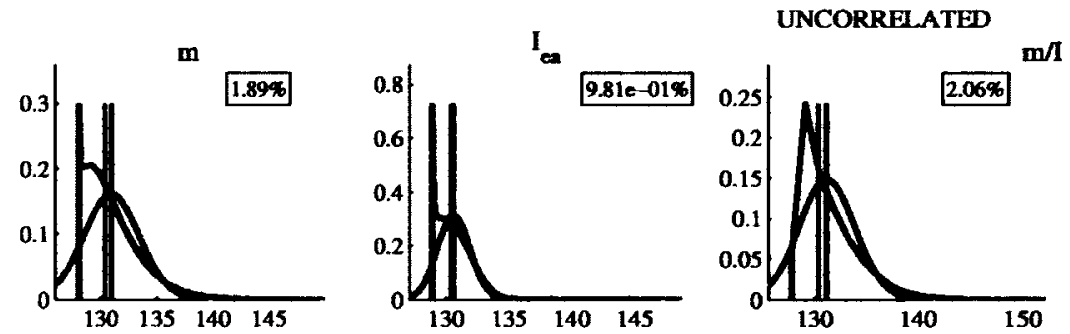

CORRELATED
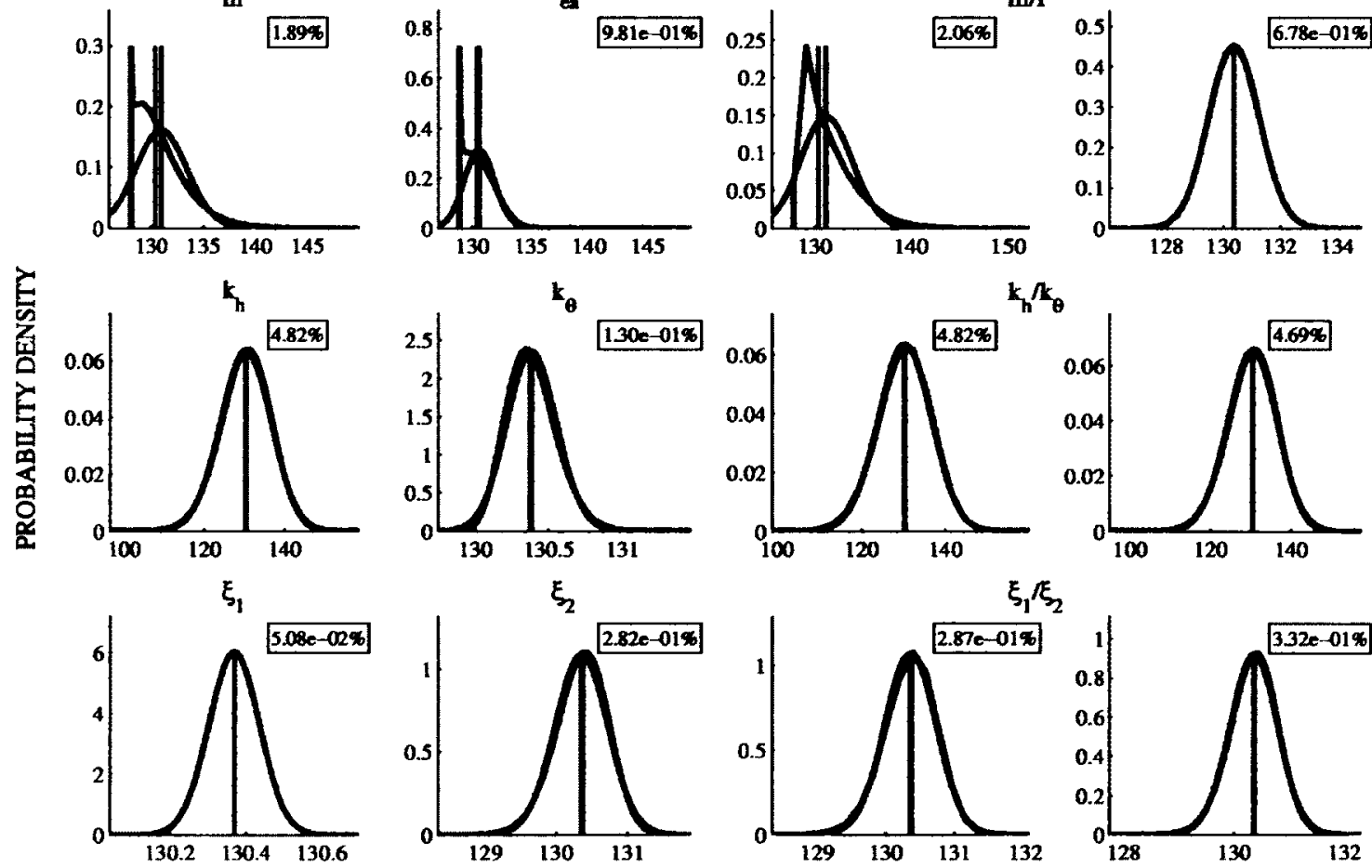

FLUTTER SPEED

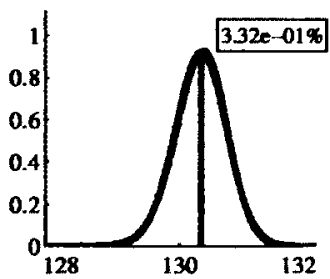

Figure 3.26: Flutter speed pdfs (case 2 airfoil, unsteady, 10\% COV) 

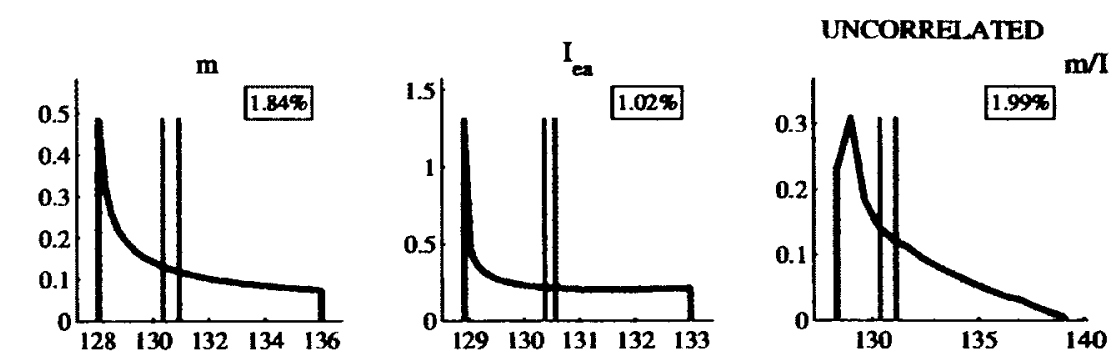

CORRELATED
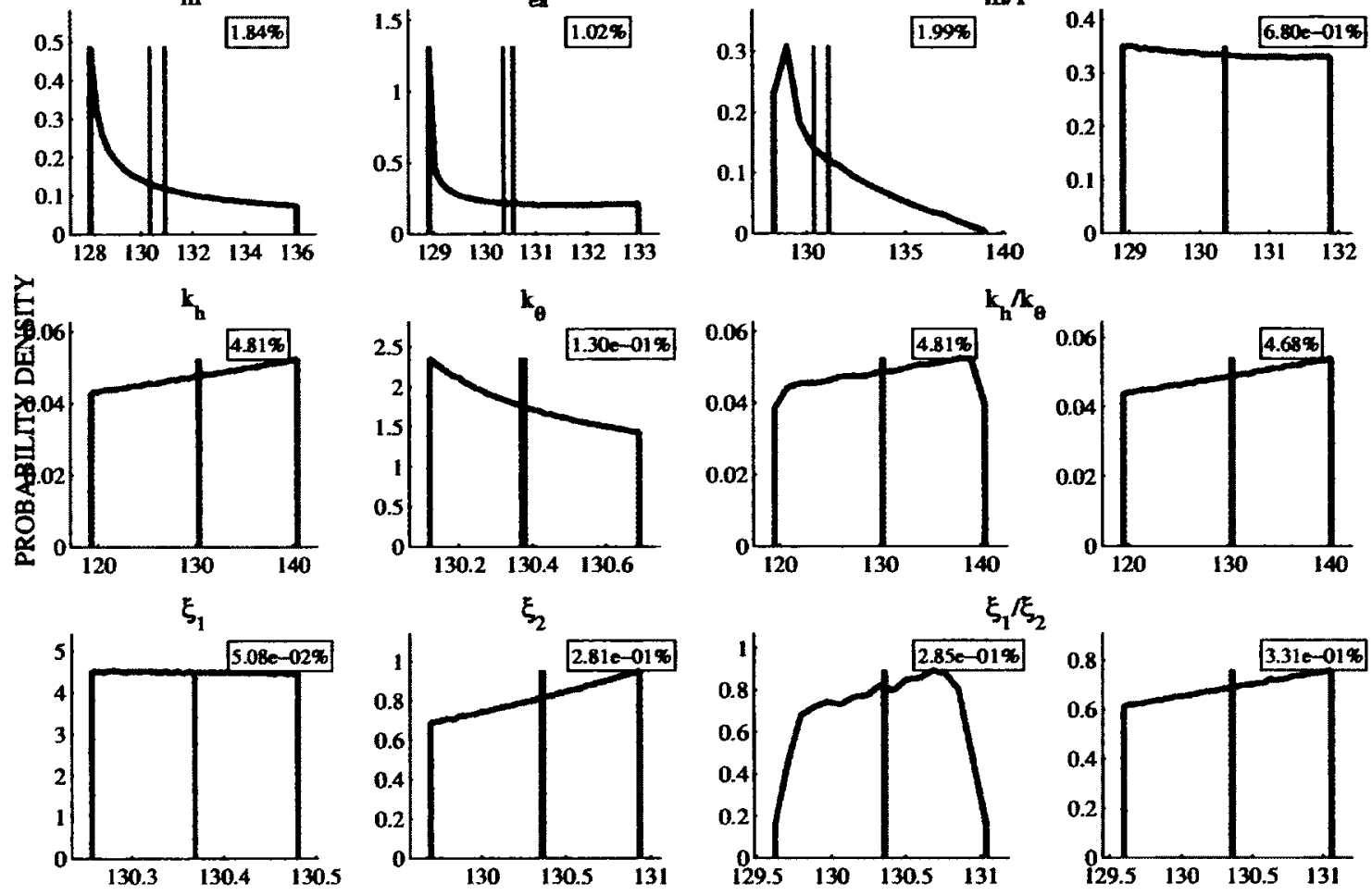

FLUTTER SPEED

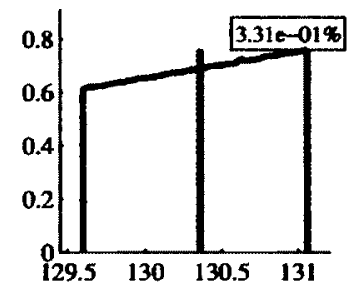

Figure 3.27: Flutter speed pdfs (case 2 airfoil, unsteady, 10\% COV)

the flutter speed.

Moreover, several other features should be examined:

- For the result cases where the mapping trends between the flutter speed and the random structural parameters are almost linear, the robustness of the results is not effected with a change in the uncertainty amount or the distribution.

- In contrast, for a larger uncertainty in the inertia parameters, most of the flutter speed pdfs experience strong positive skew effects and a minimum cutoff flutter speed. These effects are related to the non-linear features of the mapping plots, provided in fig. (3.21). Such non-linear features have profound effects on the solution robustness with respect to the increase in uncertainty and the distribution change. These effects are case specific and will be thoroughly covered in the analysis of the next case study. For cases that experience skew effects, the mean flutter speed does not match the baseline flutter speed. 
- While comparing the probabilistic results of the analysis with the quasisteady and unsteady aerodynamics, in general (but not always), the effects of uncertainty are significantly smaller for the unsteady case. The exceptions to this observation are the cases of uncertainty in the structural damping of the stable mode and the correlated inertia parameters.

\subsubsection{CASE 3}

This case is intended to demonstrate the effects of strong non-linear relationship among the flutter speed and the structural parameters as manifested in the probability density functions of the flutter speed mappings. The parameters selected for this case highlight the non-linear among the uncertain system parameters and the output quantities of interest (e.g. flutter speed and modal parameters). The parameters of the baseline system are stated in tbl. (3.11).

\begin{tabular}{||l|c|l||}
\hline PARAMETER & Symbol & Value \\
\hline \hline mass & $m$ & $75.0 \mathrm{~kg} / \mathrm{m}$ \\
normalized radius of gyration & $r_{g}$ & 0.47 \\
heave stiffness & $k_{h}$ & $7.50 \times 10^{4} \mathrm{~N} / \mathrm{m}^{2}$ \\
pitch stiffness & $k_{\theta}$ & $2.12 \times 10^{4} \mathrm{~N} / \mathrm{rad}$ \\
chord length & $c$ & $1.8288 \mathrm{~m}$ \\
span & $L$ & $6.096 \mathrm{~m}$ \\
normalized static imbalance & $x_{\theta}$ & 0.2 \\
damping ratio 1 & $\xi_{1}$ & 0.05 \\
damping ratio 2 & $\xi_{2}$ & 0.05 \\
free stream fluid density & $\rho_{\infty}$ & $1.225 \mathrm{~kg} / \mathrm{m}^{3}$ \\
normalized distance btw EA and mid-chord & $a_{h}$ & -0.34 \\
lift coefficient gradient & $\bar{C}_{l \alpha}$ & $2 \pi$ \\
\hline \hline Num. of Samples & - & 50000 \\
\hline
\end{tabular}

Table 3.11: Model parameters for Case 3

The analysis of Case 3 is limited to the quasisteady aerodynamic loading.

\section{Quasisteady Analysis: Baseline Case}

Fig. (3.28) shows the modal properties as a function of airspeed of the baseline system for the case of quasisteady aerodynamics. The instability occurred in the second mode at $47.14 \mathrm{~m} / \mathrm{s}$, 
through a mild type of flutter.
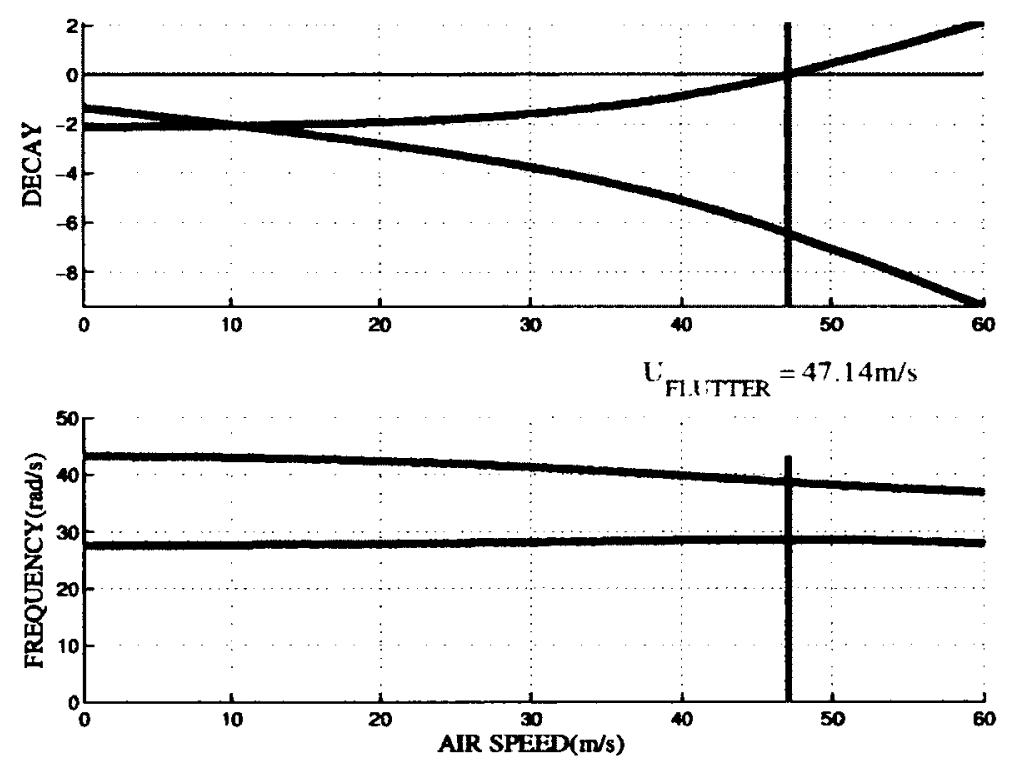

Figure 3.28: Evolution of modal parameters with respect to airspeed

Fig. (3.29) shows the relative magnitude of the elements of the eigenvectors of each mode at no flow condition and at the proximity of the flutter speed. The relative strength of each eigenvector changes with an increase in airspeed. Initially, the first mode exhibits strong heave dominance, and the second mode pitch dominance. At flutter point, both of the modes lose the relative strength of dominant components, but the first mode remains slightly heave dominant and the second, slightly pitch dominant.

The mappings of the flutter speed with respect to each random structural parameters are shown in fig. (3.30). In this case the baseline parameters were selected in a highly non-linear region of the mapping plots. For minor amounts of uncertainty, a linear form of mapping may hold, however, as the uncertainty increases to a moderate amount, the linearized depiction of this mapping would fail to capture the actual curve of the mapping. Therefore, in Case 3 study the non-linear mapping features of the flutter speed mapping results should produce greater non-linear mapping transformation effects in the flutter speed pdfs. 

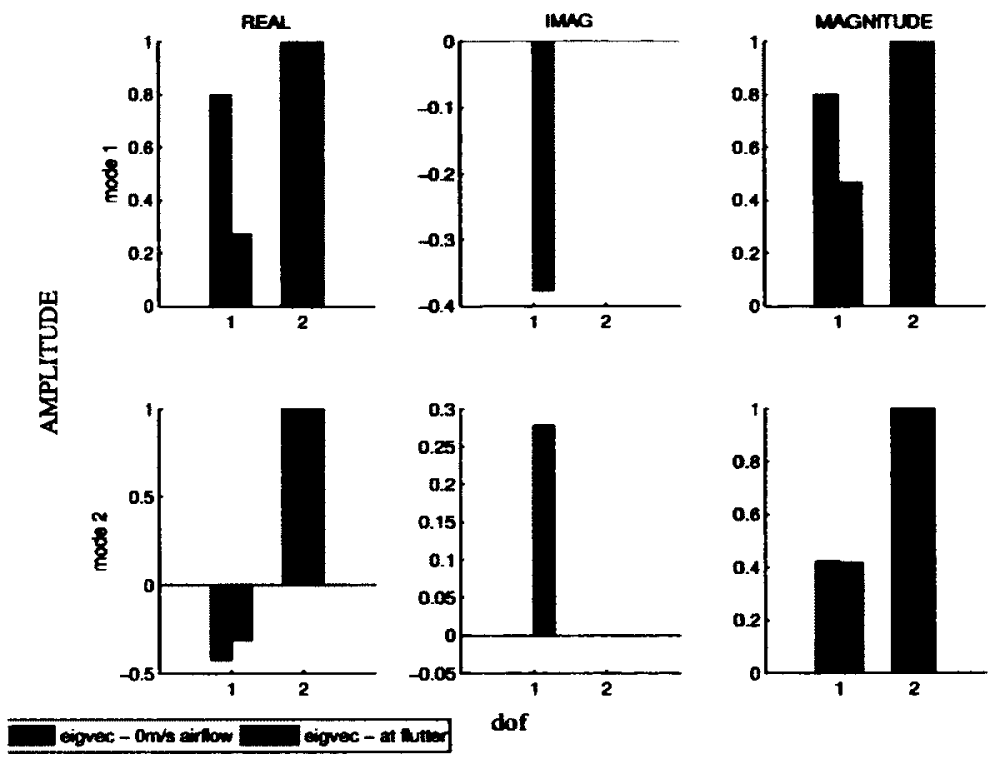

Figure 3.29: real part, imaginary part, and magnitude of eigenvectors of two modes, illustrating relative strength of each component

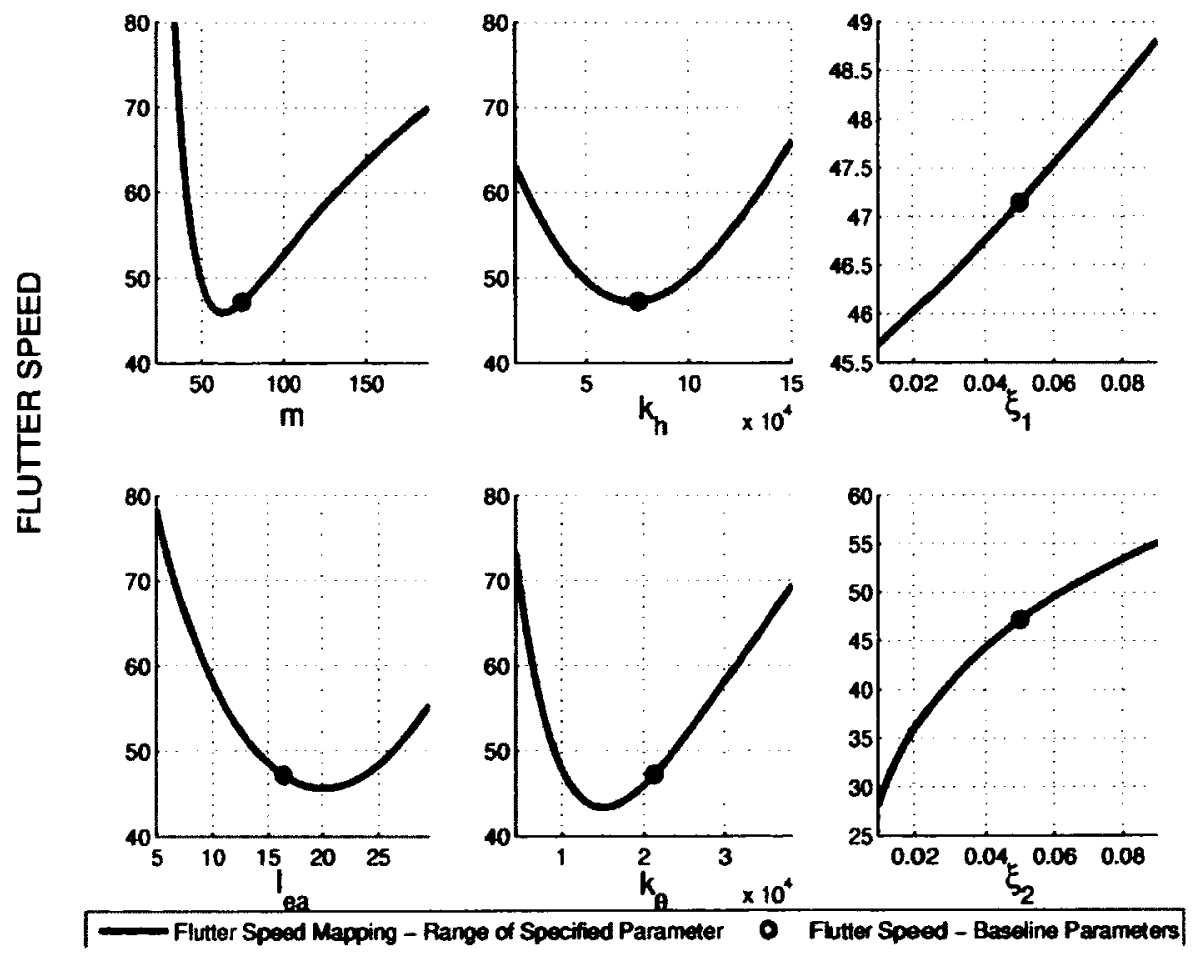

Figure 3.30: The functional relationships among the flutter speed and structural parameters 


\section{Quasisteady Analysis: Probabilistic Case}

The summary of the probabilistic flutter speed results for Case 3 with quasisteady aerodynamics is reported in tbls. (3.12) and (3.13) below.

\begin{tabular}{|c|c|c|c|}
\hline \multicolumn{2}{|c|}{ Input COV } & $2.5 \%$ & $17.5 \%$ \\
\hline Random Parameter & Distribution & \multicolumn{2}{c|}{ Flutter Speed COV \% } \\
\hline \hline \multirow{2}{*}{ mass } & Gaussian & 0.689 & 6.40 \\
\cline { 2 - 4 } & uniform & 0.691 & 3.94 \\
\hline radius of gyration & Gaussian & 0.738 & 5.99 \\
\cline { 2 - 4 } & uniform & 0.736 & 5.45 \\
\hline \multirow{2}{*}{ heave stiffness } & Gaussian & 0.0949 & 2.20 \\
\cline { 2 - 4 } & uniform & 0.0883 & 1.46 \\
\hline \multirow{2}{*}{ pitch stiffness } & Gaussian & 1.15 & 7.34 \\
\cline { 2 - 4 } & uniform & 1.15 & 7.49 \\
\hline \multirow{2}{*}{ damping ratio 1 } & Gaussian & 0.103 & 0.720 \\
\cline { 2 - 4 } & uniform & 0.103 & 0.720 \\
\hline damping ratio 2 & Gaussian & 0.671 & 4.89 \\
\cline { 2 - 4 } & uniform & 0.671 & 4.81 \\
\hline
\end{tabular}

Table 3.12: Results illustrating the uncertainty in the flutter speed due to independent uncertainty in the structural parameters

\begin{tabular}{|c|c|c|c|c|c|}
\hline \multicolumn{2}{|c|}{ Input COV } & $2.5 \%$ & $17.5 \%$ & $2.5 \%$ & $17.5 \%$ \\
\hline Random Parameter & Distribution & \multicolumn{3}{c|}{ Flutter Speed COV (\%) } \\
\hline \hline \multicolumn{2}{|c|}{} & \multicolumn{2}{|c|}{ Uncorrelated Parameters } & Fully Correlated Parameters \\
\hline \hline $\begin{array}{c}\text { mass \& } \\
\text { radius of gyration }\end{array}$ & $\begin{array}{c}\text { Gaussian } \\
\text { Gaussian }\end{array}$ & 1.01 & 8.81 & 0.0422 & 0.484 \\
\hline $\begin{array}{c}\text { mass \& } \\
\text { radius of gyration }\end{array}$ & $\begin{array}{c}\text { uniform } \\
\text { uniform }\end{array}$ & 1.01 & 6.78 & 0.0420 & 0.368 \\
\hline $\begin{array}{c}\text { heave stiffness \& } \\
\text { pitch stiffness }\end{array}$ & $\begin{array}{c}\text { Gaussian } \\
\text { Gaussian }\end{array}$ & 1.16 & 8.11 & 1.24 & 8.83 \\
\hline $\begin{array}{c}\text { heave stiffness \& } \\
\text { pitch stiffness }\end{array}$ & $\begin{array}{c}\text { uniform } \\
\text { uniform }\end{array}$ & 1.16 & 8.06 & 1.24 & 8.77 \\
\hline $\begin{array}{c}\text { damping ratio 1 \& } \\
\text { damping ratio 2 }\end{array}$ & $\begin{array}{c}\text { Gaussian } \\
\text { Gaussian }\end{array}$ & 0.680 & 4.95 & 0.774 & 5.60 \\
\hline $\begin{array}{c}\text { damping ratio 1 \& } \\
\text { damping ratio 2 }\end{array}$ & $\begin{array}{c}\text { uniform } \\
\text { uniform }\end{array}$ & 0.678 & 4.86 & 0.774 & 5.52 \\
\hline \hline
\end{tabular}

Table 3.13: Results illustrating the uncertainty in the flutter speed due to correlated uncertainty in the structural parameters

Figs. (3.31) and (3.32) show the probabilistic flutter speed results for low structural parameter uncertainty with $\mathrm{COV}=2.5 \%$. Each figure, respectively, represents the effect of a truncated 
Gaussian and a uniform distributions of the structural parameters.

From these figures the following observations are revealed:

- Although the uncertainties in the inertia (with exception of fully correlated inertia parameters) and stiffness contribute significantly to the uncertainty in the flutter speed, the uncertainty in the structural damping of the flutter mode also influences the randomness in the flutter speed due to the mild nature of the coalescence flutter for this system.

- The correlation among the inertia terms decreases uncertainty in the flutter speed, yet the correlation between the stiffness parameters and the damping parameters leads to an increase of the uncertainty in the flutter speed.

- Uncertainty in all structural parameters, with exception of the heave stiffness, show approximately a Gaussian or a uniform flutter speed distributions, for their respective case of input distribution. This means that their flutter speed mapping with respect to the random parameter is almost linear.
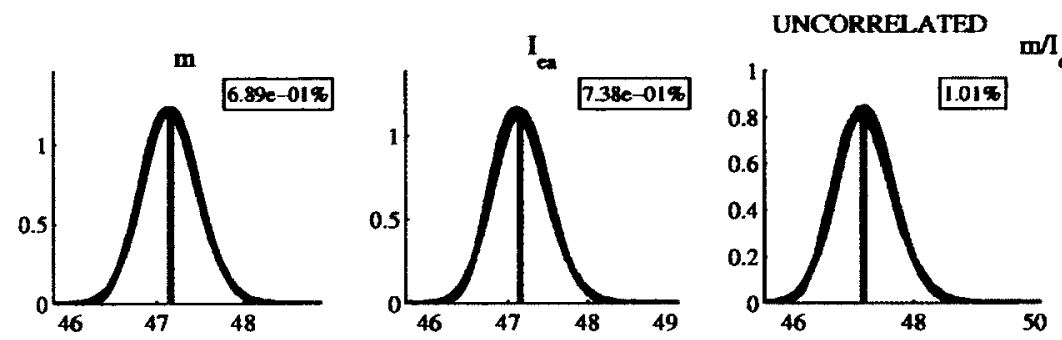

CORRELATED
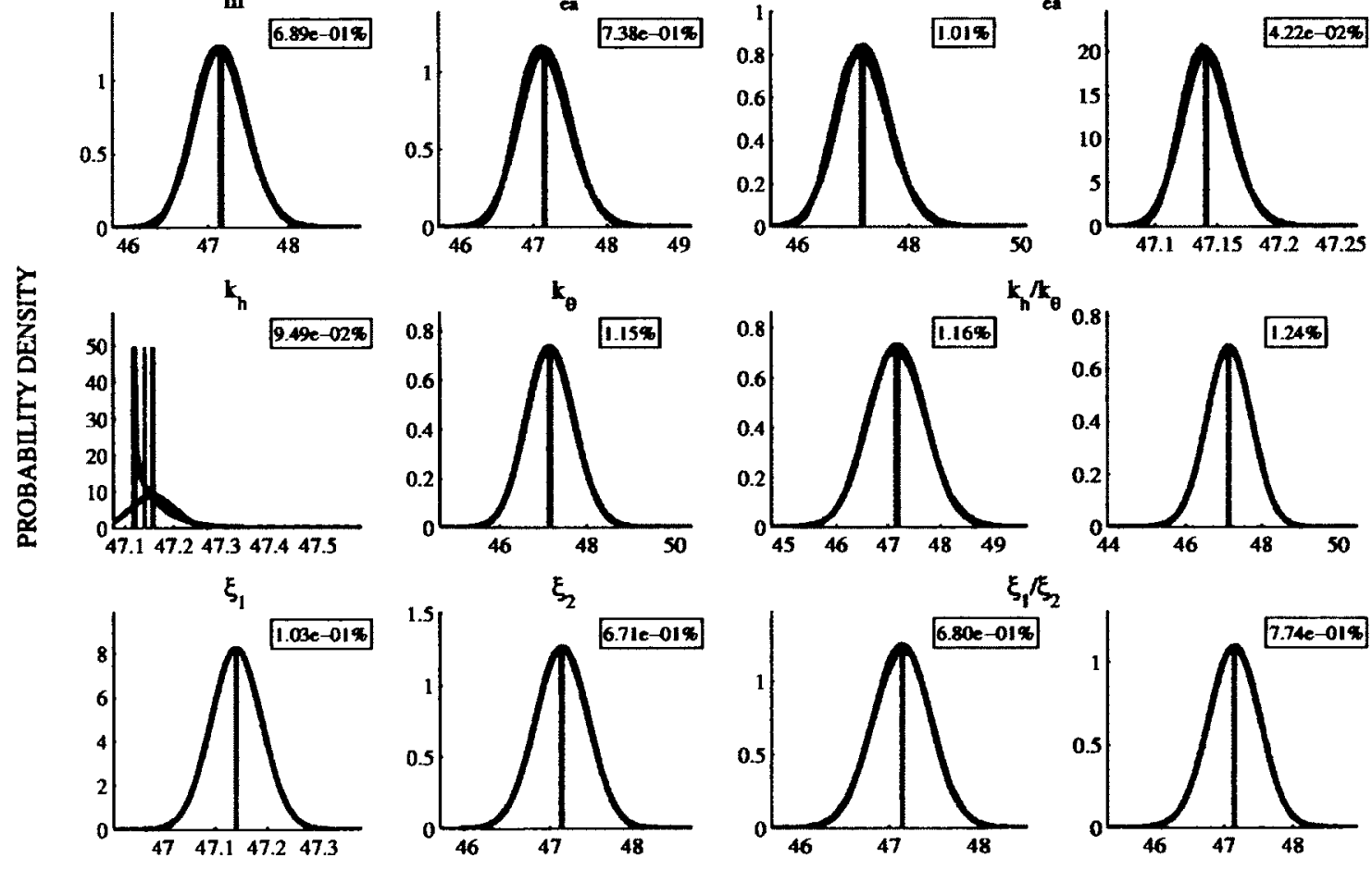

FLUTTER SPEED

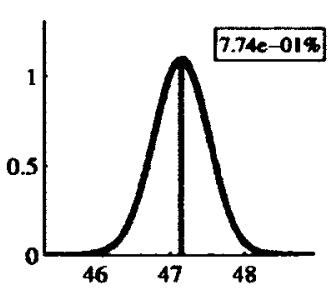

Figure 3.31: Flutter speed pdfs (case 3 airfoil, quasisteady, $2.5 \% \mathrm{COV}$ ) 

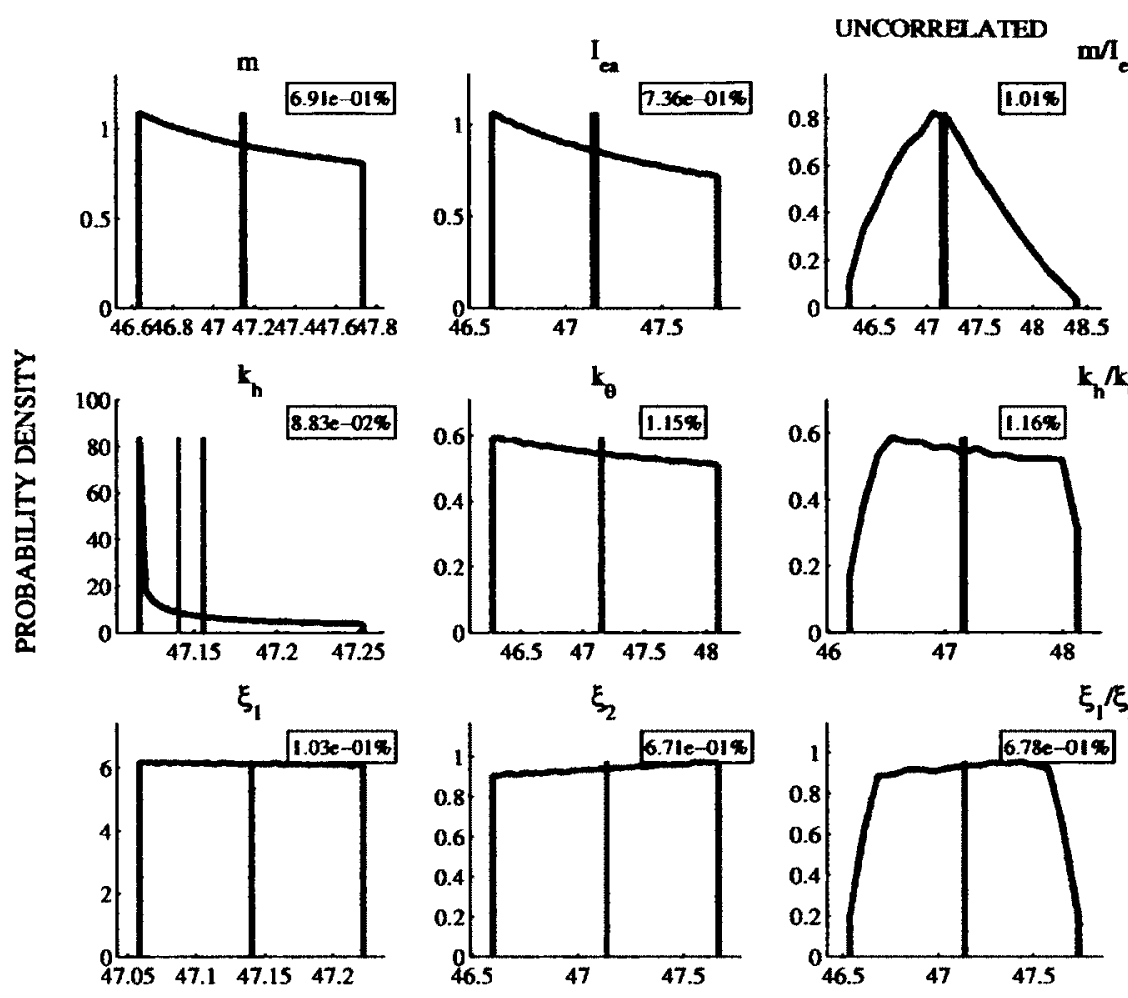

CORRELATED
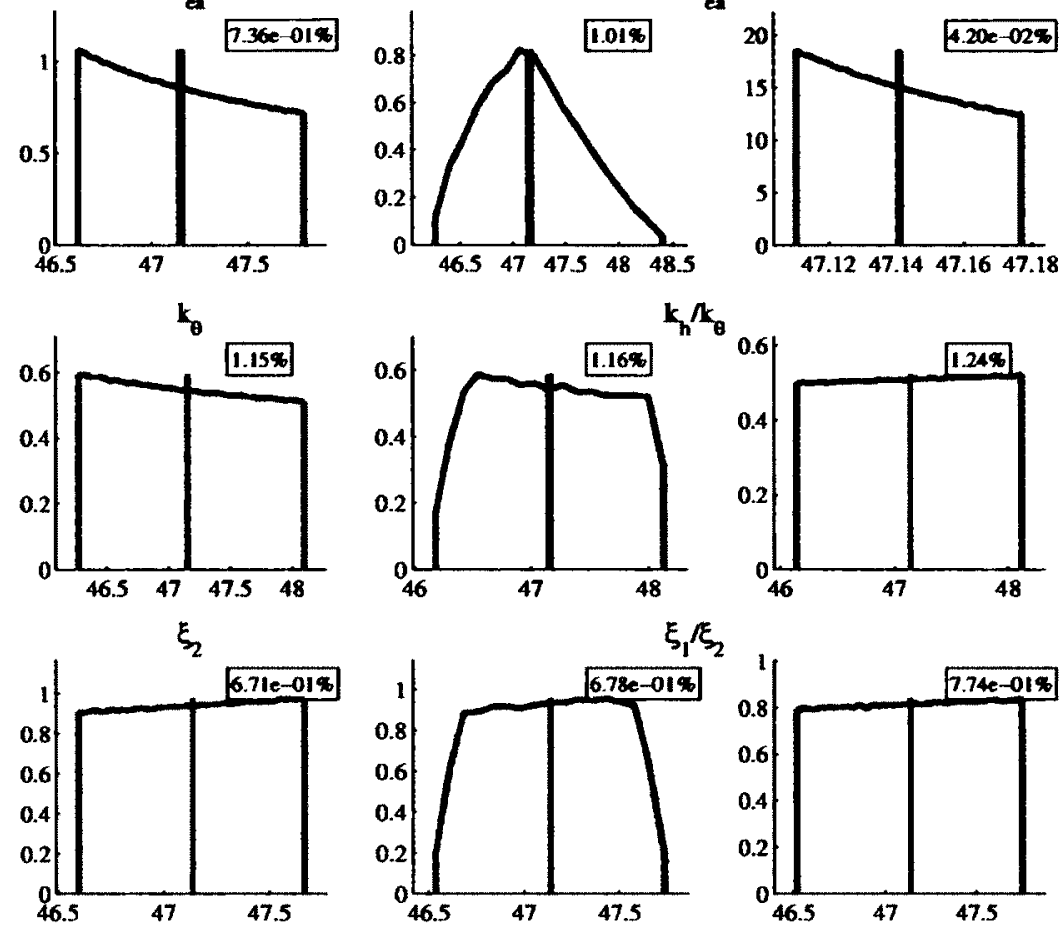

FLUTTER SPEED

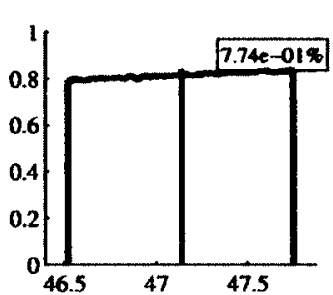

Figure 3.32: Flutter speed pdfs (case 3 airfoil, quasisteady, $2.5 \% \mathrm{COV}$ )

- The uncertainty of the flutter speed pdfs for all scenarios is reflected by the gradient within the parameter range in the mapping plots. A large gradient of the flutter speed with respect to the parameter, for example the pitch stiffness, results in a high uncertainty of flutter speed. On the contrary, a flat, or very low, gradient, for example with heave stiffness, has only minor effect on flutter speed.

- Uncertainty in the heave stiffness parameter produces very strong skewness. A sharp cutoff in the flutter speed pdf implies a strong non-linear relationship between the flutter speed and the heave stiffness. It occurs at a minimum flutter speed. These non-linear effects will be examined in greater detail in the probabilistic analysis of the modal parameters.

- For the cases when the non-linear effects are significant, the mean and mode of theflutter speed pdf does not generally coincide with the baseline flutter speed. 
Next, the effect of large structural parameter uncertainty $(\mathrm{COV}=17.5 \%)$ is examined. The figs. (3.33) and (3.34) show the effect of the truncated Gaussian and the uniform distributions. The main conclusions are noted below:

- Although the uncertainties in the inertia (except for fully correlated inertia parameters) and stiffness contribute significantly to the uncertainty in the flutter speed, the uncertainty in the structural damping of the flutter mode also influences the randomness in the flutter speed due to the mild nature of the coalescence flutter for this system.

- The correlation among the inertia terms decreases uncertainty in the flutter speed, yet the correlation between the stiffness parameters and the damping parameters leads to an increase of the uncertainty in the flutter speed.

- The uncertainty of the flutter speed pdfs for all uncertainty scenarios is reflected by the gradient of the flutter speed mapping in the range of structural parameter uncertainty.
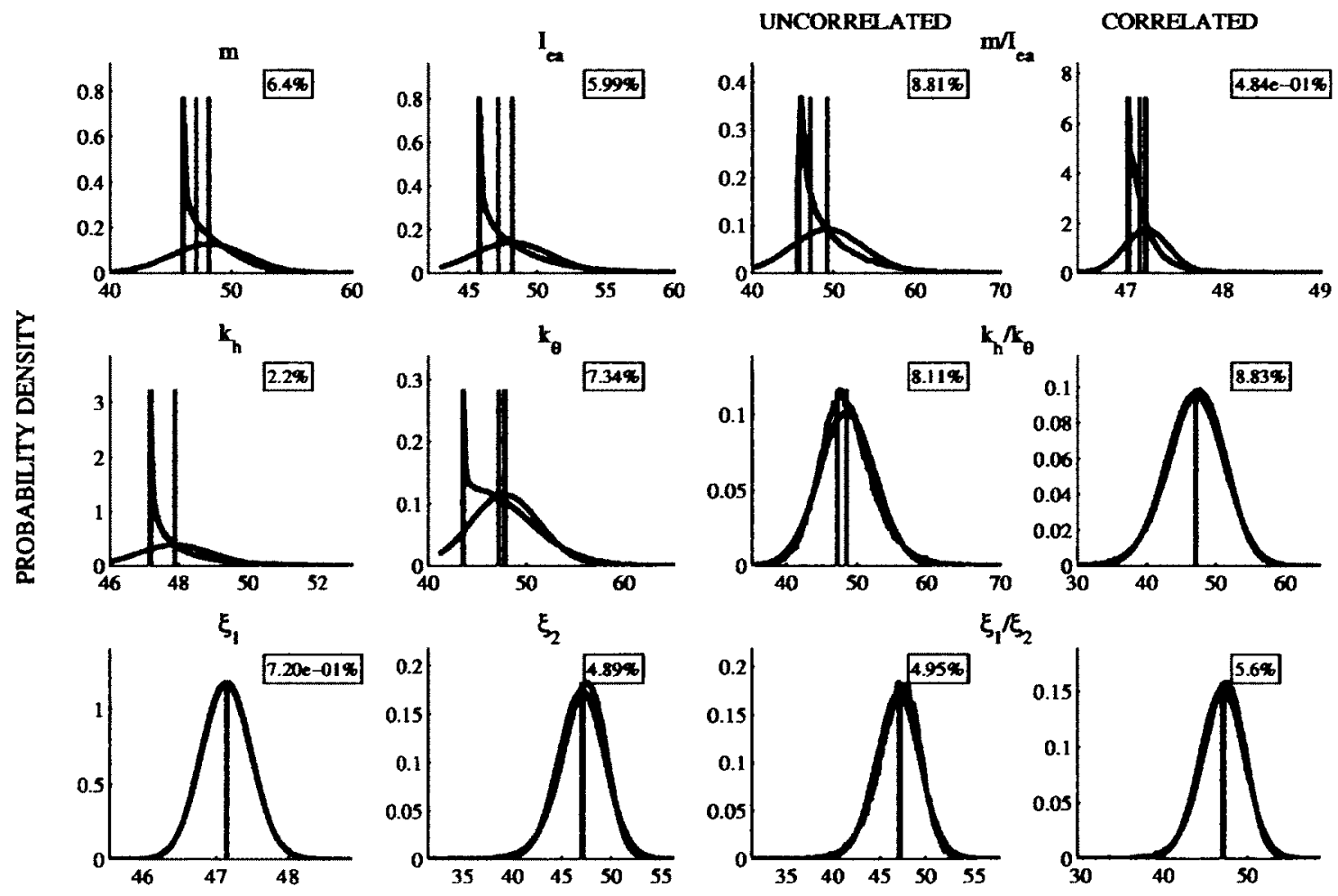

$1 \xi_{2}$

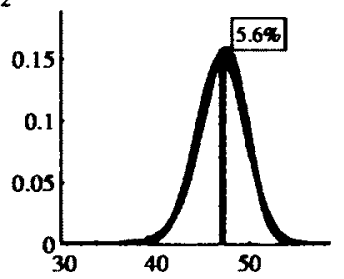

FLUTTER SPEED

Figure 3.33: Flutter speed pdfs (case 3 airfoil, quasisteady, 17.5\% COV) 


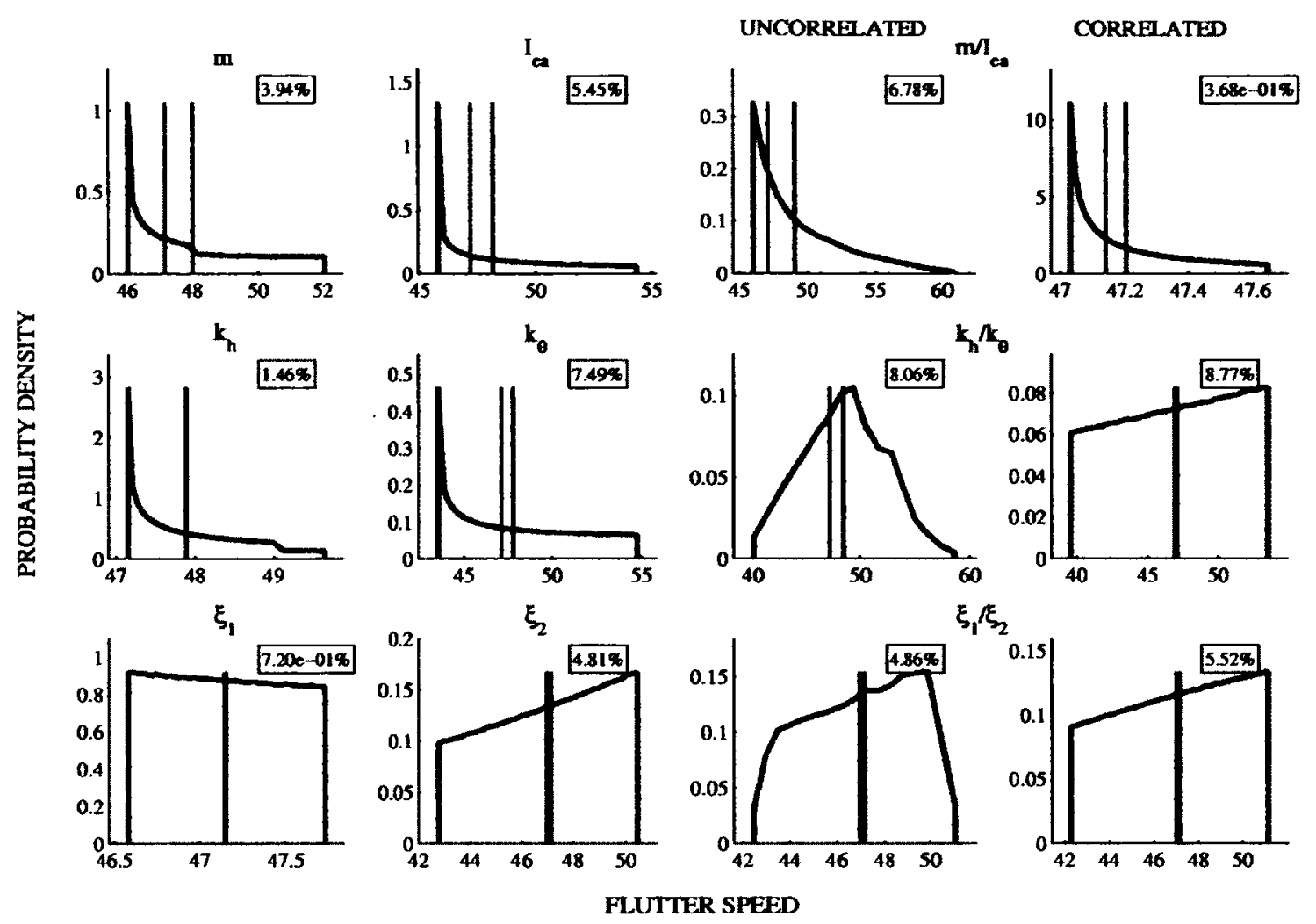

Figure 3.34: Flutter speed pdfs (case 3 airfoil, quasisteady, 17.5\% COV)

- The flutter speed pdfs that relate to strong non-linear dependence between the flutter speed and the random structural parameters result in mean and mode flutter speeds that do not match the baseline flutter speed.

However, one difference is obvious:

- Most of the cases with uncertainty in inertia and stiffness parameters produce flutter speed distributions that undergo strong non-linear transformations. These flutter speed distributions experience large positive skewness and substantial cutoffs at a minimum flutter speed.

A closer look at the fig. (3.30) of the flutter speed mapping confirms the strong non-linear features.

A more detailed investigation of effects of the non-linear mapping on the flutter speed pdfs is provided next. Some general conclusions are made based on figs. (3.30)-(3.34): 
- The skew effects and localized minima/maxima in the flutter speed pdfs relate to changes in the gradient of the flutter speed and the rate of change in this gradient around the baseline value of the structural parameter. The positive skew effects indicate a reduction of slope towards the decreasing values of the random parameter and an approaching minimum flutter speed. The effects of negative skew are opposite, indicating a reduction of the slope for increasing values of the random parameter and an approaching maximum flutter speed.

- A zero slope in the flutter speed mapping plot corresponds to either a minimum or a maximum flutter speed.

- An increasing magnitude of the slope (or gradient), which represents the Jacobian (J) described in section 3.4.1, around the baseline value of the random structural parameter is reflected by a larger sensitivity of the flutter speed with respect to the uncertain structural parameter. A decreasing gradient implies the opposite effect.

An example of these effects is clearly evident in fig. (3.30) for the pitch stiffness parameter. For the pitch stiffness range of approximately $1.5 \times 10^{4}-2.12 \times 10^{4}$ the flutter speed experiences a reduction in gradient, which would imply a positive skew effect in the flutter speed pdf. At the pitch stiffness value of approximately $1.5 \times 10^{4}$, a minimum flutter speed is observed. For the pitch stiffness values below the $1.5 \times 10^{4}$ and above the baseline parameter value, the flutter speed shows an increasing gradient, which would imply that the skew effects would increase towards the regions of larger flutter speed. All of these effects are confirmed within the resulting flutter speed pdf with uncertainty in the pitch stiffness in fig. (3.33).

For an increased amount of structural parameter uncertainty, fig. (3.30) shows that the range of parameter uncertainty increases to include more of the non-linear features. As uncertainty is increased, some of the general observations are noted below:

- If the mapping between the flutter speed and the uncertain parameter remains linear, the increase of flutter speed variance is proportional to the increase in structural parameter uncertainty. 
- If the gradient of the flutter speed mapping experiences mostly a reduction around the baseline value of the parameter within the increased range of parameter uncertainty, the variance of the flutter speed pdf would result in a proportionally reduced variance with respect to the increased parameter uncertainty.

- Conversely, if the gradient of the flutter speed mapping experiences mostly a growing trend around the baseline value of the parameter within the increased range of parameter uncertainty, the variance of the flutter speed pdf would result in a proportionally increased variance with respect to the increased parameter uncertainty.

- Since most cases experience all of these effects, the resulting effects of the non-linear features on the flutter speed variance depend on which gradient varying trends tend to be strongest.

- Lastly, since the support of the uniform distribution of the random parameter is smaller than that of the Gaussian distribution, the non-linear features between the system parameter and the flutter speed are less significant. Therefore, the effect of non-linear features is less influential on the variance of the flutter speed pdf for a uniform distribution of the random parameter.

\subsection{Closing Remarks}

The purpose of this chapter was to introduce the basic principles of the coalescence flutter phenomenon for an elastically mounted rigid wing in a deterministic setting and then extend the analysis to a probabilistic setting. The probabilistic results confirm the deterministic interpretation of the coalescence flutter mechanism. The results indicate that typically the inertia and stiffness parameter uncertainties dominantly influence the frequency coalescence mechanism. The structural damping uncertainty produces only minimal effects. The effect of uncertainty on the flutter speed depends on the explosiveness of the flutter. In addition, these particular case studies have indicated that the effect of additional circulatory force due to unsteady aerodynamics has been stabilizing, whereby the flutter speed increases as the flutter type becomes more 
explosive. For correlations in the frequency controlling parameters, a reduction of uncertainty was noted in the flutter speed pdfs, and in contrast, the correlation between the damping parameters typically increases the uncertainty. The next chapter continues this investigation from the prospective of characteristic roots describing modal frequencies and damping. 


\section{Chapter 4}

\section{A Two Degree-of-freedom Airfoil: Characteristic Roots}

In this chapter, the effect of structural parameter uncertainty on the aeroelastic modal parameters is investigated in detail. Note that:

- The modal decay rates $\left(\beta_{i}(U), i=1,2\right)$ and the modal frequencies $\left(\omega_{d, i}(U), i=1,2\right)$ are complicated non-linear functions of the structural inertia, stiffness and damping parameters. For analysis with the unsteady aerodynamic forcing, there are additional two non-oscillatory modes that arise due to circulatory forcing but they will not be considered in this probabilistic analysis.

- The physical mechanism of coalescence flutter dictates a strong relationship among the decay rate of the modes and spacing between the coalescing frequencies.

- It is therefore natural to expect that the decay rates and frequencies will exhibit strong statistical correlation due to uncertainty in the system parameters.

- Furthermore, the modal frequencies and decay rates may exhibit strong non-stationary trend with change in airspeed as the random scatter in these parameters display substantial growth or decay. 
A truncated Gaussian distribution will be used to represent the structural parameters, since it is likely to exhibit a fairly accurate representation of the realistic parameter distribution. In addition, a larger amount of uncertainty in random parameters will be considered (i.e. $10 \% \mathrm{COV}$ ). Lastly, the modal parameter pdfs will be obtained only for three test airspeeds at $0 \%, 70 \%$ and $100 \%$ of the baseline flutter speed.

In this section, most of the effort is devoted to understanding the complex statistical interplay among the modal frequencies and decays. For a conceptual difficulty of sorting complex aeroelastic modes, we avoid tracking the individual modes. Instead, the density plots are used to study the statistics of the modal decay rates and modal frequencies (e.g. see Adhikari et al. [76]). The density plots are especially convenient and useful when the modal parameters exhibit significant statistical overlap (i.e. their pdfs contain significant overlapping region). With this being said, the analysis begins with the presentation of Case 1 results.

\subsection{CASE 1}

\subsubsection{Quasisteady Results}

For uncertainty in the mass, the modal decays are shown in the left plot and the modal frequencies in the right plot in fig. (4.1). In black, the figures outline the baseline case modal parameters, and in red, the associated probabilistic modal parameter pdfs. Several notable features are observed. The uncertainty in the mass parameter produces greater influence on the frequency of the heave-dominant, first mode; The pdf of the first modal frequency also shows a nearly stationary trend with an increasing airspeed, meaning that the modal frequency pdfs do not change significantly with increasing airspeed. The pdf of the second (i.e. flutter) modal frequency, shows some non-stationary trends, whereby the frequency pdf is approximately Gaussian for the no flow case, but its pdf becomes strongly skewed as the airspeed increases. As the nonstationary trends in the frequency pdfs of the coalescing modes are only mild, the modal decay pdf of the flutter mode experiences significant non-stationary trend with growing variance as a function of airspeed. 

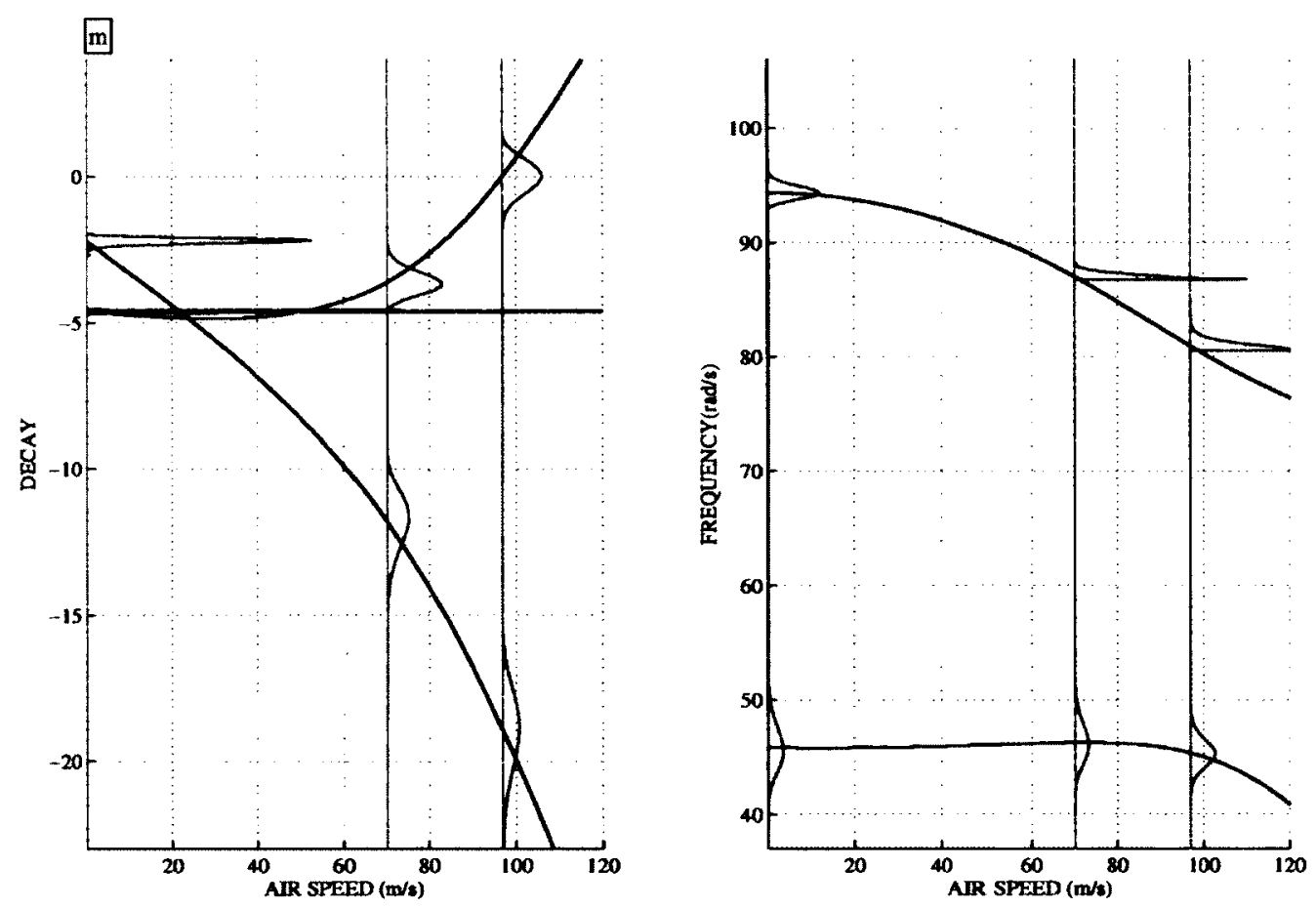

Figure 4.1: Modal parameter pdfs resulting from randomness in mass with $10 \% \mathrm{COV}$, obtained at 3 test airspeeds: $0 \mathrm{~m} / \mathrm{s}, 69 \mathrm{~m} / \mathrm{s}, 96.9 \mathrm{~m} / \mathrm{s}$

Next, the effects of uncertainty in the mass moment of inertia parameter are examined in fig. (4.2). The observations are very similar to the effects induced by the uncertainty of mass. The uncertainty in the mass moment of inertia produces a greater influence on the uncertainty of the frequency pdf of the second, pitch-dominant, flutter mode and an almost stationary trend with increasing airspeed. The frequency pdf of the first mode, shows some non-stationary trends, where the frequency pdf becomes skewed as the airspeed increases. Both frequency pdfs experience most significant non-stationarity in the flutter region. As the non-stationary trends in the frequency pdfs of the coalescing modes are only mild, the modal decay pdf of the flutter mode experiences significant non-stationary trend with growing variance as a function of airspeed. A slight skew in the decay pdfs is noted near flutter speed, but nonetheless, the flutter mode decay remains almost Gaussian. 

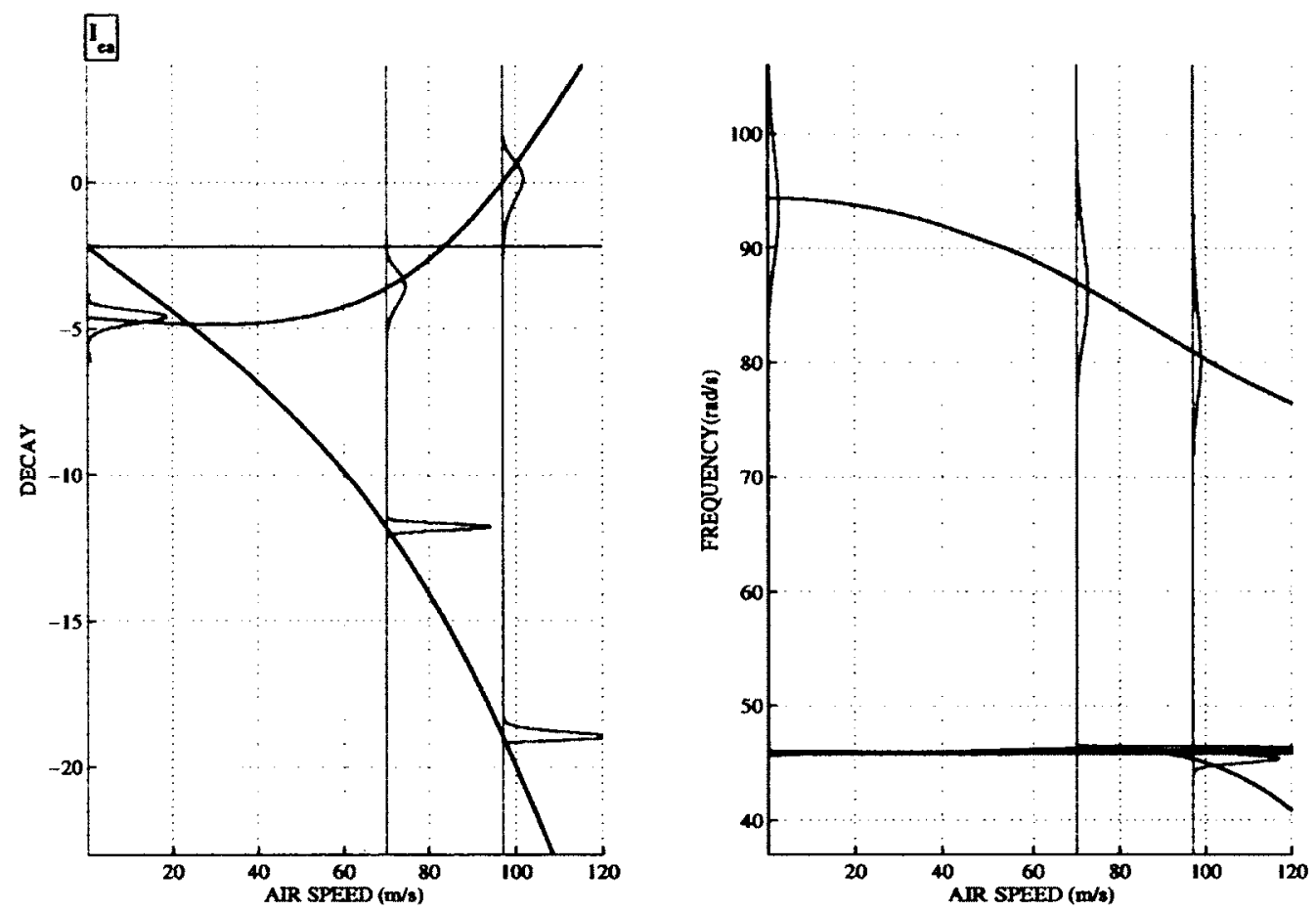

Figure 4.2: Modal parameter pdfs resulting from randomness in mass moment of inertia with $10 \% \mathrm{COV}$, obtained at 3 test airspeeds: $0 \mathrm{~m} / \mathrm{s}, 69 \mathrm{~m} / \mathrm{s}, 96.9 \mathrm{~m} / \mathrm{s}$

Then, the effects of uncertainty in the stiffness parameters are shown next. The observations are very similar to those obtained for uncertainty in the inertia parameters. Fig. (4.3) illustrates the modal parameter pdfs produced by randomness in the heave stiffness parameter. The uncertainty in heave stiffness parameter has a greater influence on the uncertainty of frequency of the first, heave-dominant mode. Both of the modal frequency pdfs shows an almost stationary trend with an increasing airspeed. The frequency pdfs are approximately Gaussian. The modal decay pdf of the flutter mode shows a significant non-stationary trend with increasing airspeed, but it retain an approximately Gaussian distribution.

Then, fig. (4.4) illustrates the modal parameter pdfs produced by uncertainty in the pitch stiffness parameter. The uncertainty in this parameter produces a greater influence on the uncertainty of the frequency pdf of the second, pitch-dominant, flutter mode and an almost stationary trend with increasing airspeed. The frequency pdf of the first mode, shows a non-stationary transformation trend, where the frequency pdf is heavily skewed. Although only minor, but 

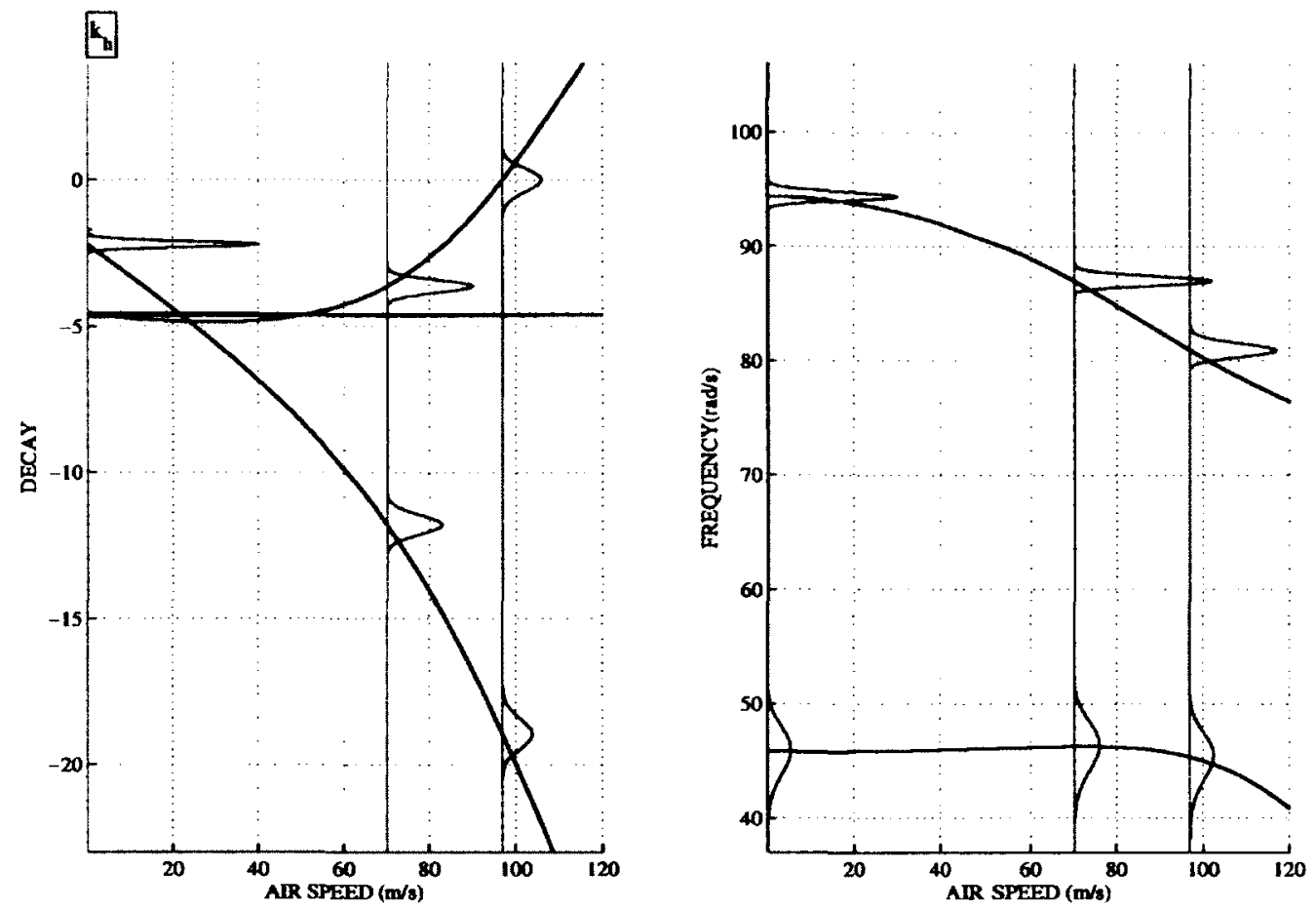

Figure 4.3: Modal parameter pdfs resulting from randomness in heave stiffness with $10 \%$ COV, obtained at 3 test airspeeds: $0 \mathrm{~m} / \mathrm{s}, 69 \mathrm{~m} / \mathrm{s}, 96.9 \mathrm{~m} / \mathrm{s}$
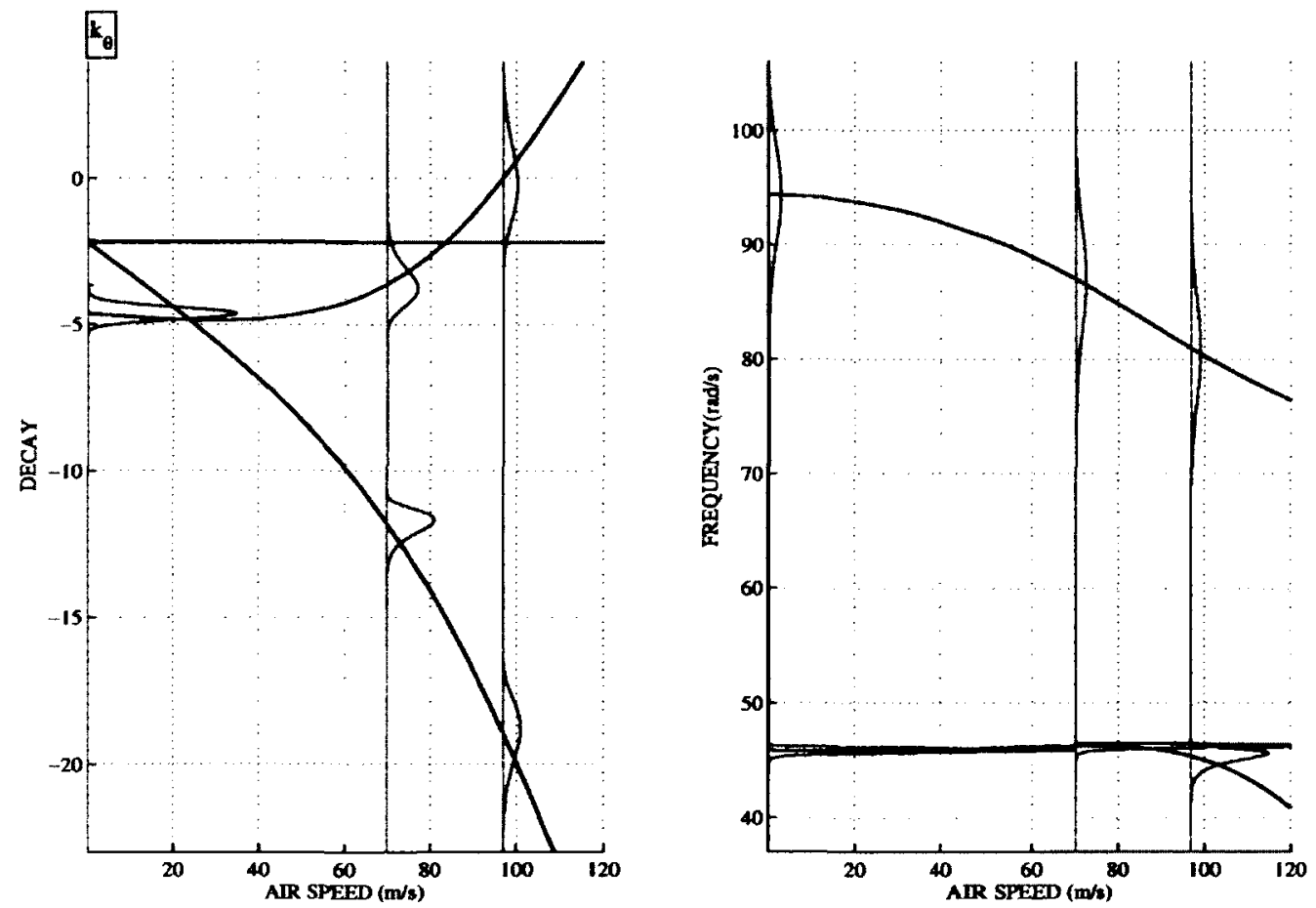

Figure 4.4: Modal parameter pdfs resulting from randomness in pitch stiffness with $10 \%$ COV, obtained at 3 test airspeeds: $0 \mathrm{~m} / \mathrm{s}, 69 \mathrm{~m} / \mathrm{s}, 96.9 \mathrm{~m} / \mathrm{s}$ 
most significant non-stationary effects occur in the flutter region. The modal decay pdf of the flutter mode shows a significant non-stationary trend with increasing airspeed and shows slight signs of skewness.

Next, the effect of uncertainty in the damping parameters on the modal properties is examined. It was already described that the variations of the structural damping ratios do not produce considerable effects on the modal frequencies of system and thus does not affect the flutter mechanism. As such, the uncertainty in the structural damping reflects in the flutter only through change in the energy dissipation.

First, the effect of uncertainty in the first mode structural damping ratio are examined in fig. (4.5). The resulting observations confirm the anticipated trend. There are no notable uncertainty effects on the modal frequencies. Most significant uncertainty is experienced in the decay of the first (non-flutter) mode. The variance of the second mode is almost negligible, but increases near flutter point. Both modal decay distributions remain almost Gaussian. A very low and almost constant variance in the decay of the flutter mode leads to very low uncertainty in the
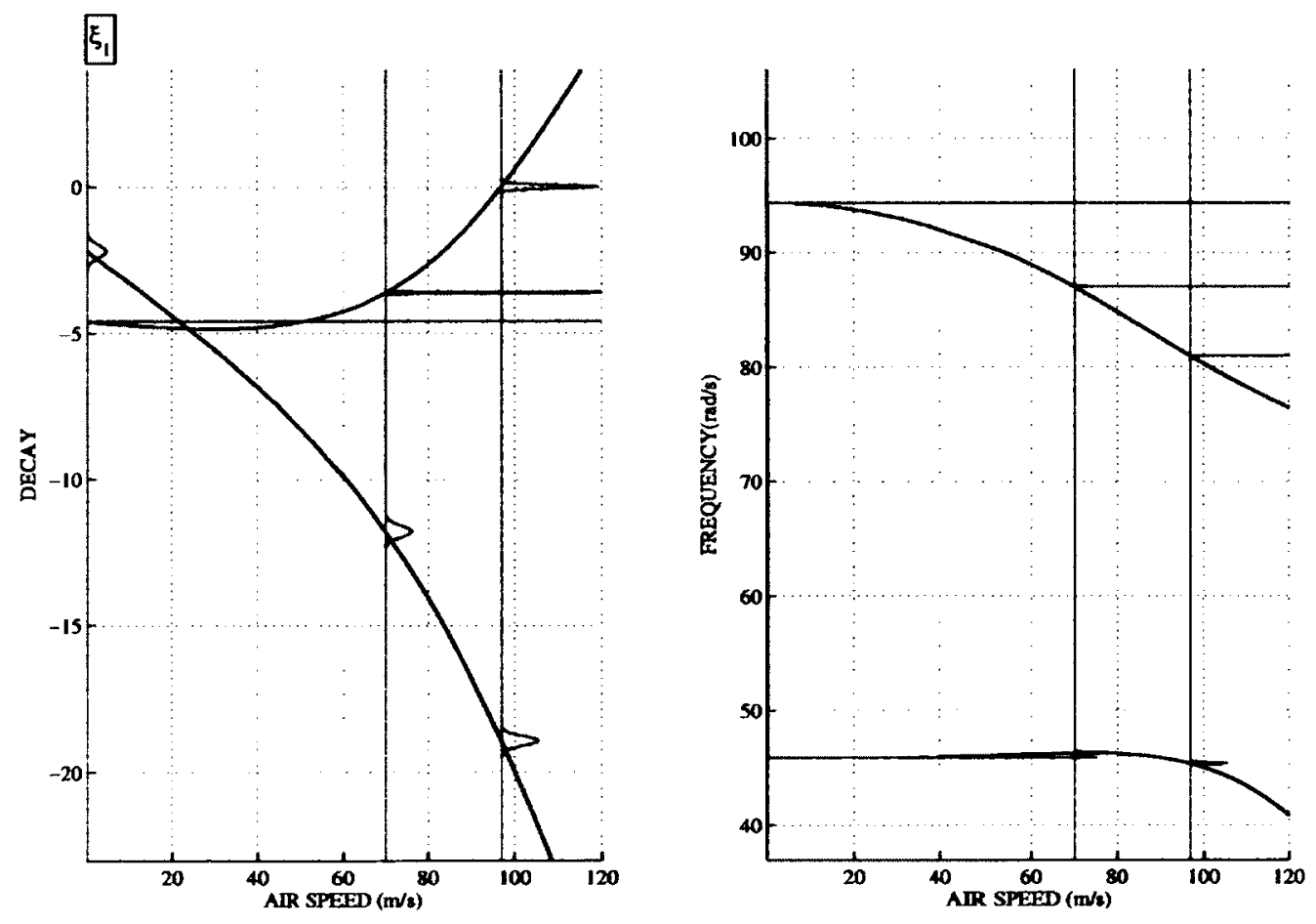

Figure 4.5: Modal parameter pdfs resulting from randomness in $1^{s t}$ structural damping ratio with $10 \% \mathrm{COV}$, obtained at 3 test airspeeds: $0 \mathrm{~m} / \mathrm{s}, 69 \mathrm{~m} / \mathrm{s}, 96.9 \mathrm{~m} / \mathrm{s}$ 
flutter speed pdf, as discussed previously.

Then, fig. (4.6) illustrates the effect of uncertainty in the second mode structural damping ratio. Once again, there are no notable uncertainty effects on the modal frequencies. Most significant variance is experienced in the decay of the second (flutter) mode. The variance of
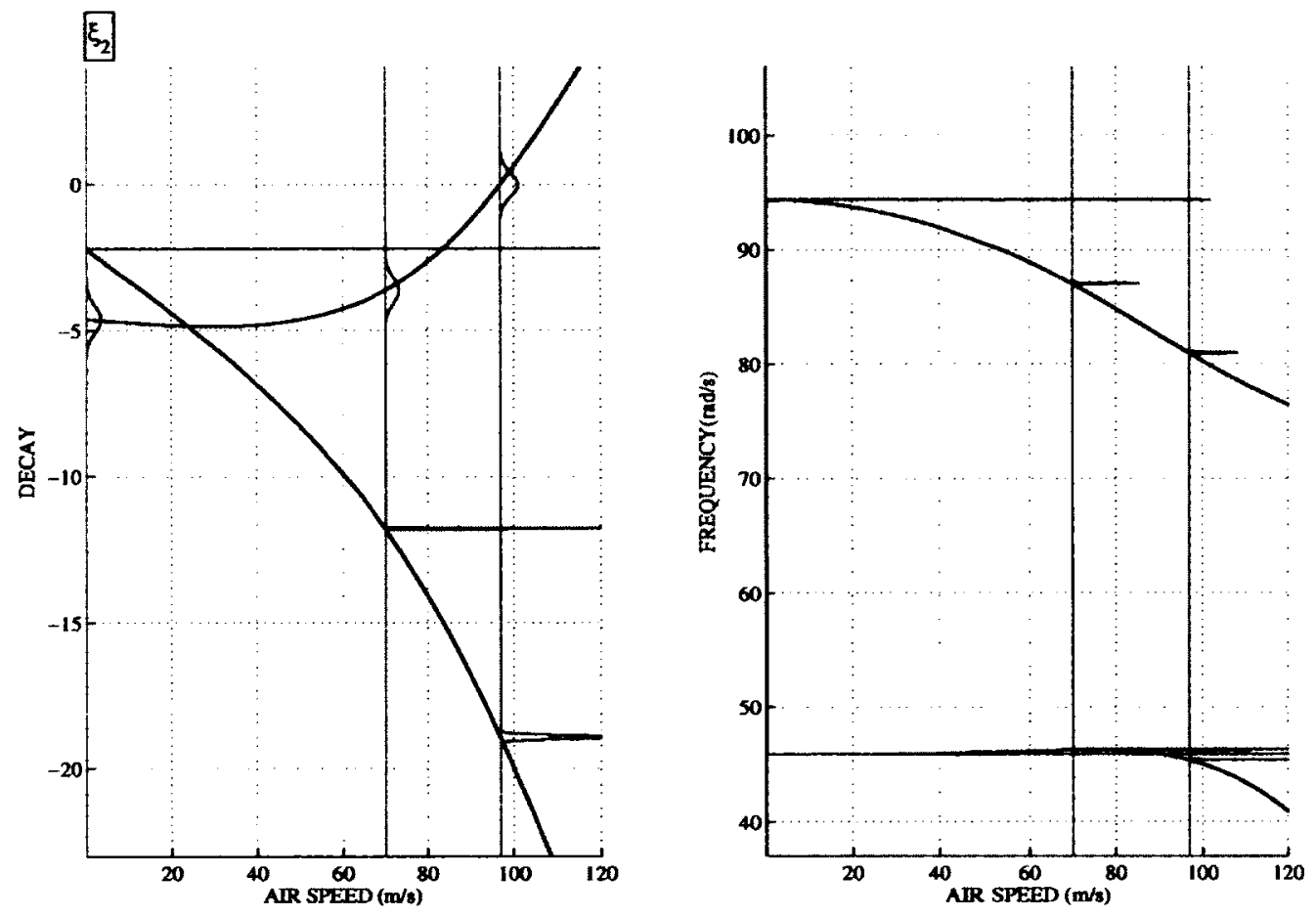

Figure 4.6: Modal parameter pdfs resulting from randomness in $2^{\text {nd }}$ structural damping ratio with $10 \% \mathrm{COV}$, obtained at 3 test airspeeds: $0 \mathrm{~m} / \mathrm{s}, 69 \mathrm{~m} / \mathrm{s}, 96.9 \mathrm{~m} / \mathrm{s}$

the first mode is almost negligible, but increases slightly near the flutter point. Both modal decay distributions remain almost Gaussian. A moderate and almost constant variance in the decay of the flutter mode leads to a moderate uncertainty in the flutter speed pdf for a case with mild flutter type.

\section{Multiple Parameter Uncertainty}

The analysis now turns to the study of effects of multiple parameter variations where the random parameters are fully uncorrelated. Figs. (4.7)-(4.9) show the resulting pdfs of modal parameters and its transformations as a function of airspeed for random inertia, stiffness and damping parameters. All of the results indicate that the combined effects of uncertainty for uncorrelated 

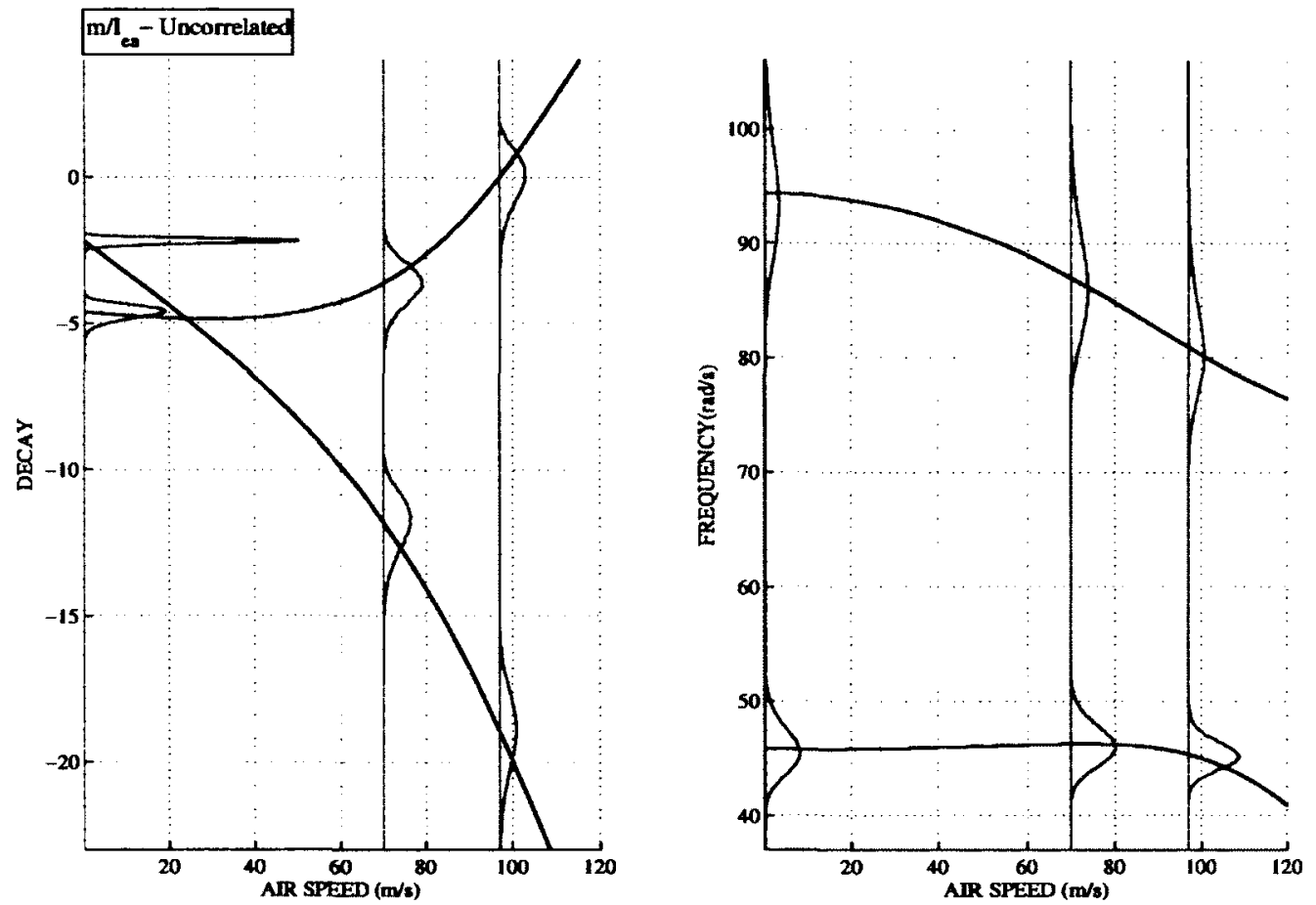

Figure 4.7: Modal parameter pdfs resulting from randomness in the uncorrelated inertia parameters with $10 \% \mathrm{COV}$, obtained at 3 test airspeeds: $0 \mathrm{~m} / \mathrm{s}, 69 \mathrm{~m} / \mathrm{s}, 96.9 \mathrm{~m} / \mathrm{s}$
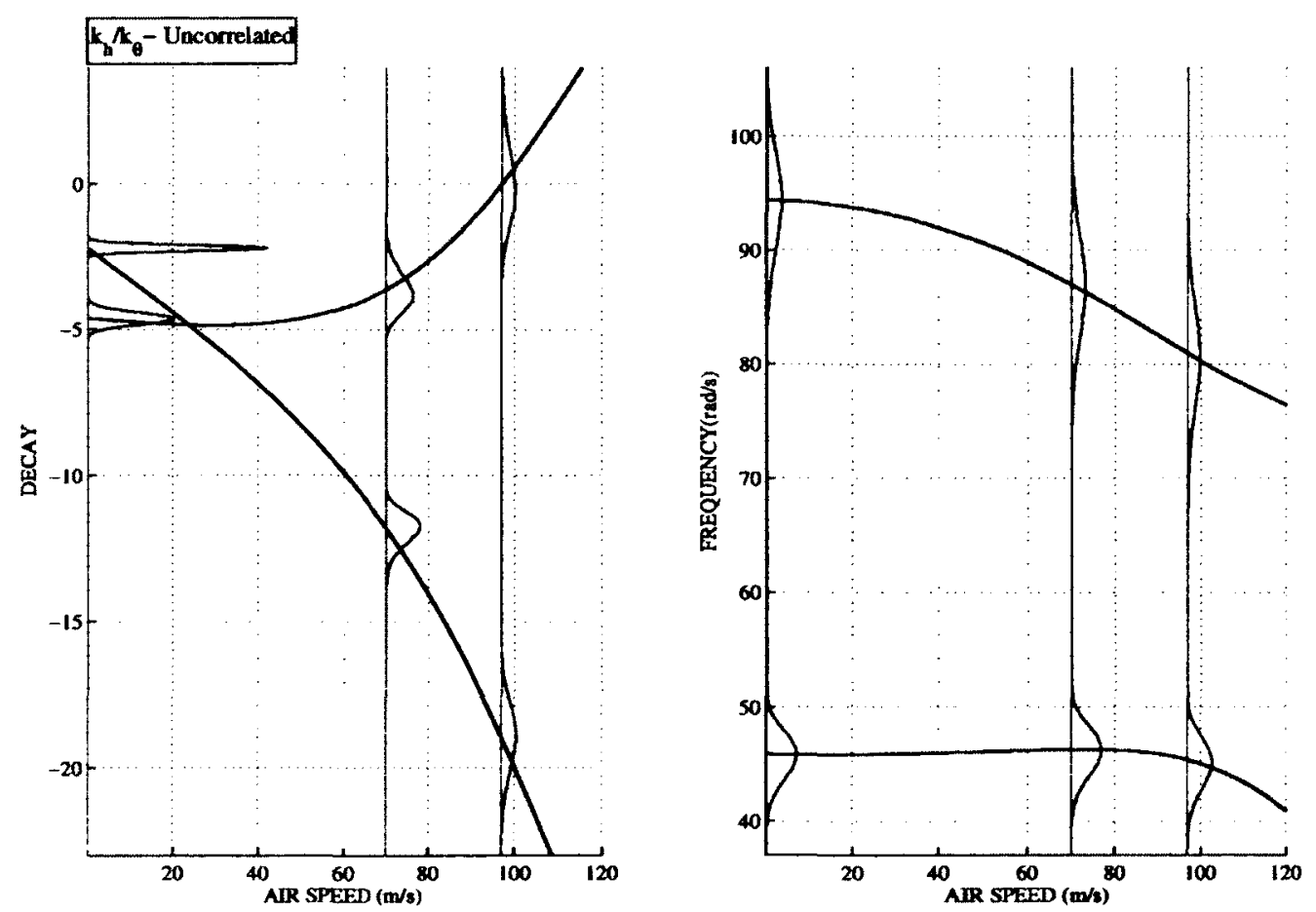

Figure 4.8: Modal parameter pdfs resulting from randomness in the uncorrelated stiffness parameters with $10 \% \mathrm{COV}$, obtained at 3 test airspeeds: $0 \mathrm{~m} / \mathrm{s}, 69 \mathrm{~m} / \mathrm{s}, 96.9 \mathrm{~m} / \mathrm{s}$ 

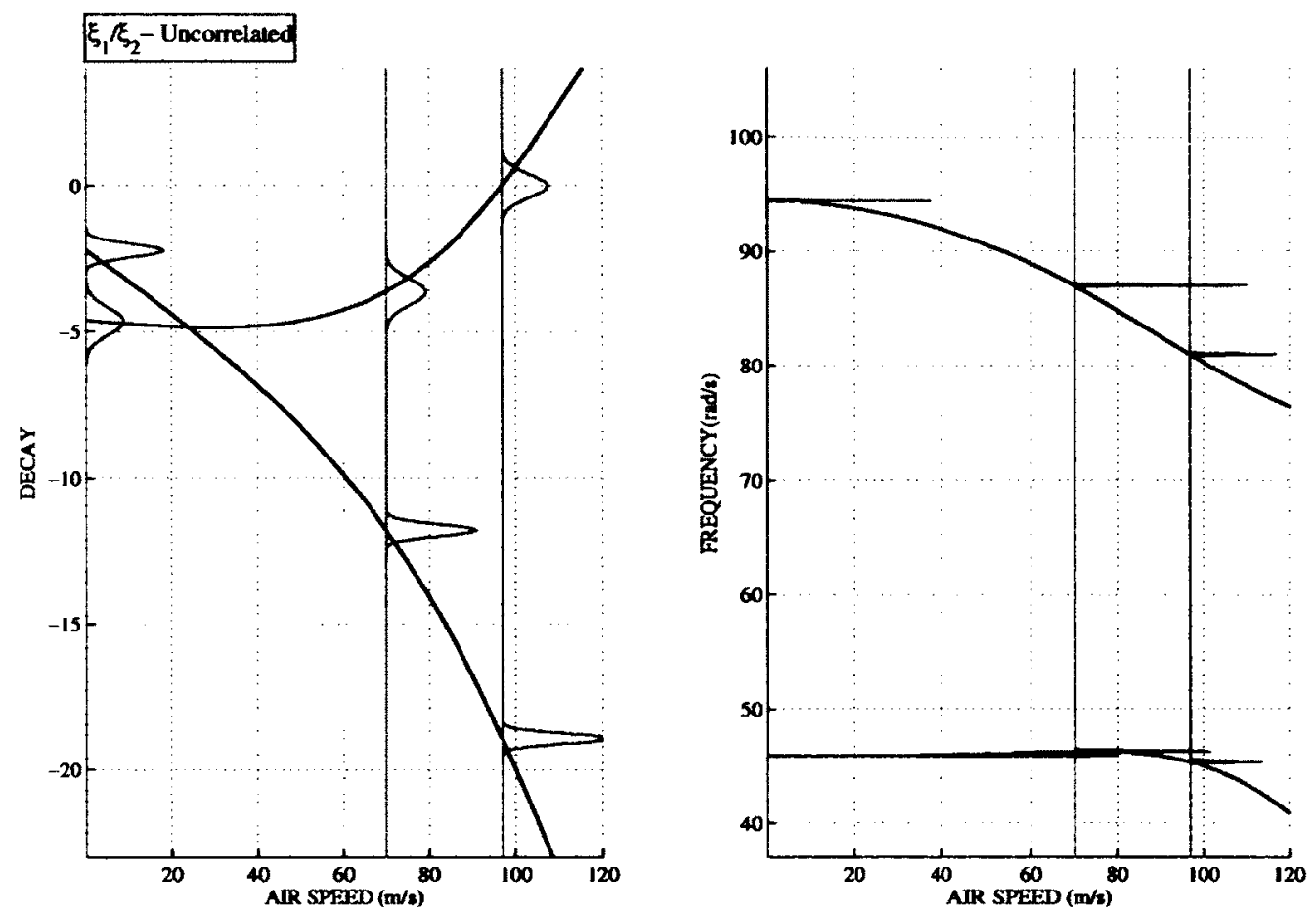

Figure 4.9: Modal parameter pdfs resulting from randomness in the uncorrelated damping parameters with $10 \%$ COV, obtained at 3 test airspeeds: $0 \mathrm{~m} / \mathrm{s}, 69 \mathrm{~m} / \mathrm{s}, 96.9 \mathrm{~m} / \mathrm{s}$

parameters produce a larger variance in all of the modal parameter pdfs, when compared to the cases of individual parameter variations. In effect, the resulting larger variance of modal decay of the flutter mode produces a larger variance in the flutter speed, as evident in fig. (3.13). In certain cases that involve a linear transformation of the modal parameters, a convolution principle [90] could be applied to establish the probabilistic results of multiple independent parameter variations based on the results of individual structural parameter variations.

In most realistic scenarios, there usually exists some correlation between the random parameters. Next, the effects of having a full correlation between the random parameters are examined. Although a full correlation may be unrealistic, since the actual parameter correlation information is not available, the case of full correlation may be used in order to get a fundamental understanding of the correlation effects.

Figs. (4.10)-(4.12) illustrate the probabilistic modal parameter transformation results for fully correlated inertia, stiffness and damping parameters, respectively. From figs. (4.10) and (3.13), the following observations are noted. The uncertainty of the frequency parameters does 
not significantly depend on the correlation between the random inertia parameters. The correlation between the random inertia parameters leads to certain correlation effects between the modal frequencies and the modal decays: in this case, the correlation between the modal frequencies reduces uncertainty in the frequency spacing between the coalescing modes, leading to a reduced growth of variance in the modal decays (as function of airspeed). Due to a reduced variance growth of decay in the flutter mode, the flutter speed pdf shows a lower variance for uncertainty in the correlated inertia parameters.

Then, figs. (4.11) and (3.13) indicate that a similar argument holds for the uncertainty effects of the correlated stiffness parameters. The uncertainty of the frequency parameters does not significantly depend on the correlation between the random stiffness parameters. The correlation between random stiffness parameters leads to correlation effects between the modal frequencies and the modal decays: the correlation between modal frequencies reduces uncertainty in the frequency spacing between the coalescing modes, leading to a reduced growth of variance in the modal decays (as function of airspeed). As a result of a reduced variance growth in the
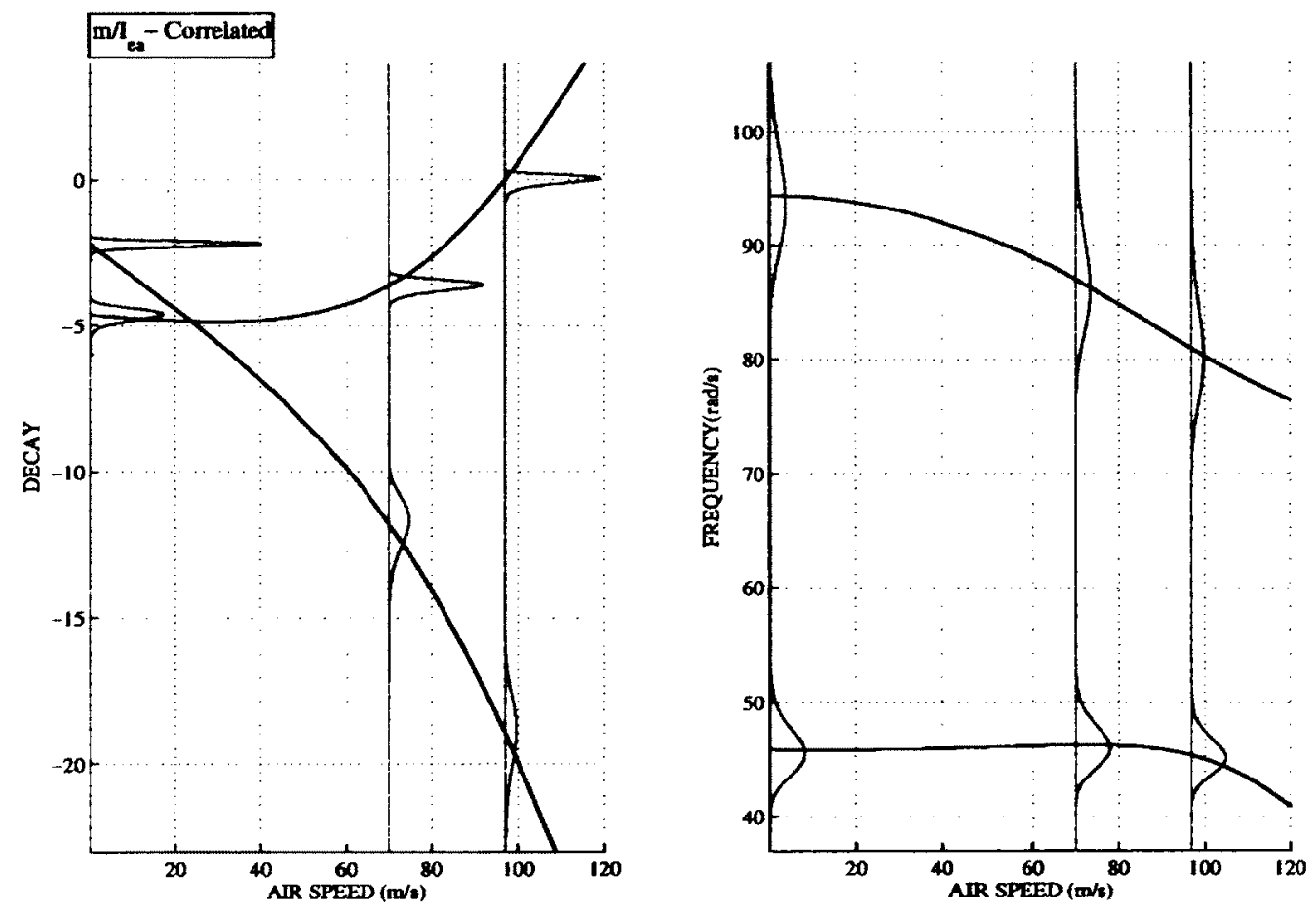

Figure 4.10: Modal parameter pdfs resulting from randomness in the correlated inertia parameters with $10 \% \mathrm{COV}$, obtained at 3 test airspeeds: $0 \mathrm{~m} / \mathrm{s}, 69 \mathrm{~m} / \mathrm{s}, 96.9 \mathrm{~m} / \mathrm{s}$ 

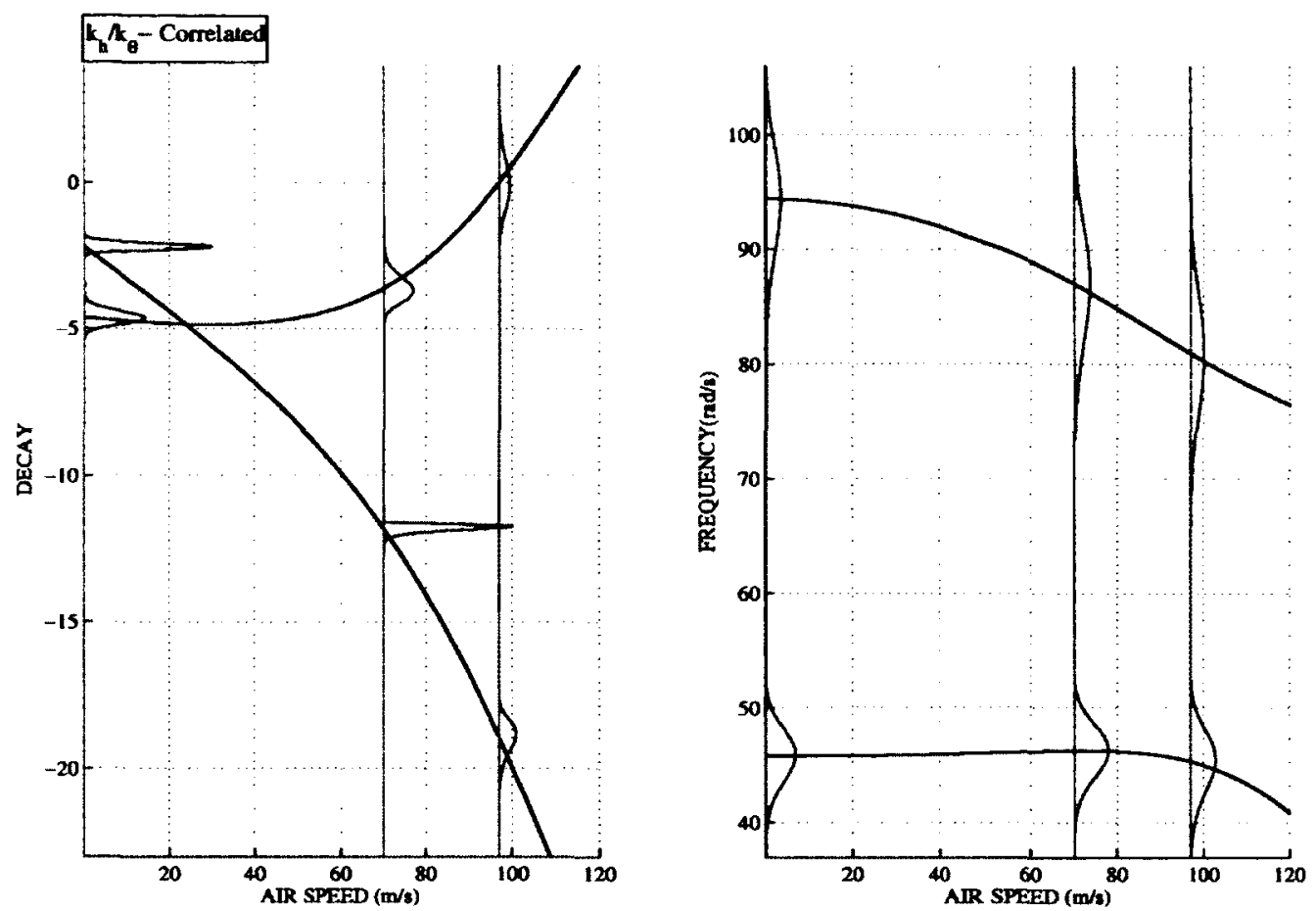

Figure 4.11: Modal parameter pdfs resulting from randomness in the correlated stiffness parameters with $10 \% \mathrm{COV}$, obtained at 3 test airspeeds: $0 \mathrm{~m} / \mathrm{s}, 69 \mathrm{~m} / \mathrm{s}, 96.9 \mathrm{~m} / \mathrm{s}$
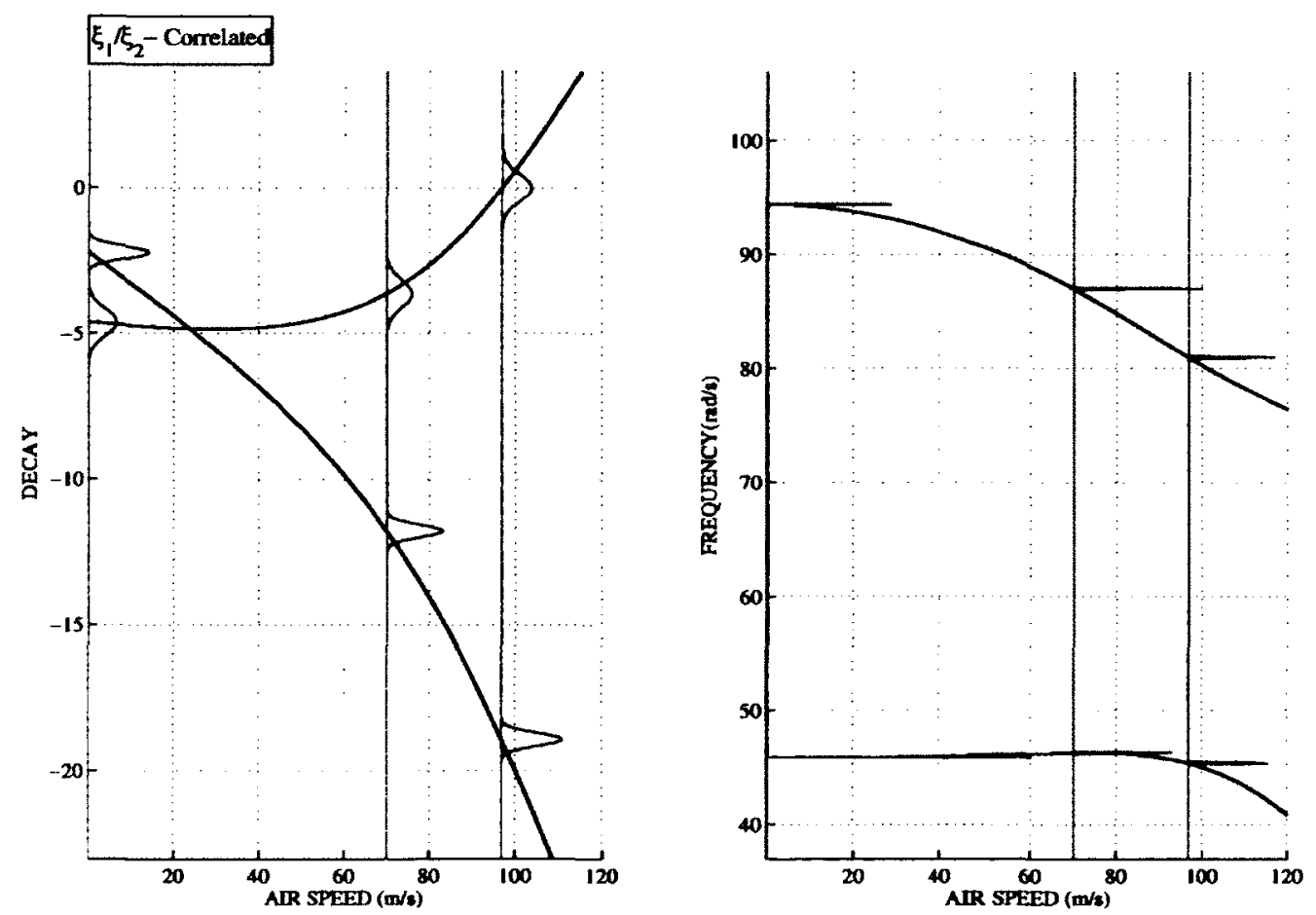

Figure 4.12: Modal parameter pdfs resulting from randomness correlated damping parameters with $10 \% \mathrm{COV}$, obtained at 3 test airspeeds: $0 \mathrm{~m} / \mathrm{s}, 69 \mathrm{~m} / \mathrm{s}, 96.9 \mathrm{~m} / \mathrm{s}$ 
decay of the flutter mode, the flutter speed pdf yields a smaller variance for a case of correlated stiffness parameters.

Lastly, from figs. (4.12) and (3.13) the observations from the effect of uncertainty in the damping parameters are obtained. The uncertainty effects in the modal frequencies are almost invariant to the uncertainty contained in the structural damping parameters, therefore the effects of parameter correlation are negligible in frequency pdfs. The correlation between random structural damping parameters leads to correlation effects between the modal decays, leading to a consistent increase in the variance of the modal decays in the coalescing modes for all airspeeds. An increased variance of the decay of the flutter mode results in an increased variance of the flutter speed pdf for correlated damping parameters.

\subsubsection{Unsteady Results}

Analysis of the effects of structural uncertainty on the probabilistic transformations of the modal parameters of a system with unsteady aerodynamics is provided next. The resulting effects of randomness in the independent parameters are presented first. A close attention has to be paid to the effect of a more explosive flutter type, which is experienced in this case due to unsteady aerodynamics.

The uncertainty in the modal parameters due to randomness in the mass parameter are shown in fig. (4.13). Only modal parameter pdfs of the oscillating modes are illustrated. For this case, the modal decay pdfs experience overlapping effects. In the modal frequency pdfs, the uncertainty of the mass parameter produces a greater influence on the uncertainty of the heavedominant mode. Both modal frequency pdfs indicate a non-stationary trend, especially significant in the flutter region. It should be noted that such a dramatic increase of uncertainty in the non-flutter mode and a dramatic decrease in uncertainty of the flutter mode may be linked to a change in the dominance of the aeroelastic eigenvectors (see fig. 3.16). However, where the frequency pdfs are approximately stationary, a growing variance of the decay in the coalescing modes is observed. As a result of a fairly large and changing variance in the modal decay as a function of airspeed, flutter speed pdf with a moderate variance is produced. 

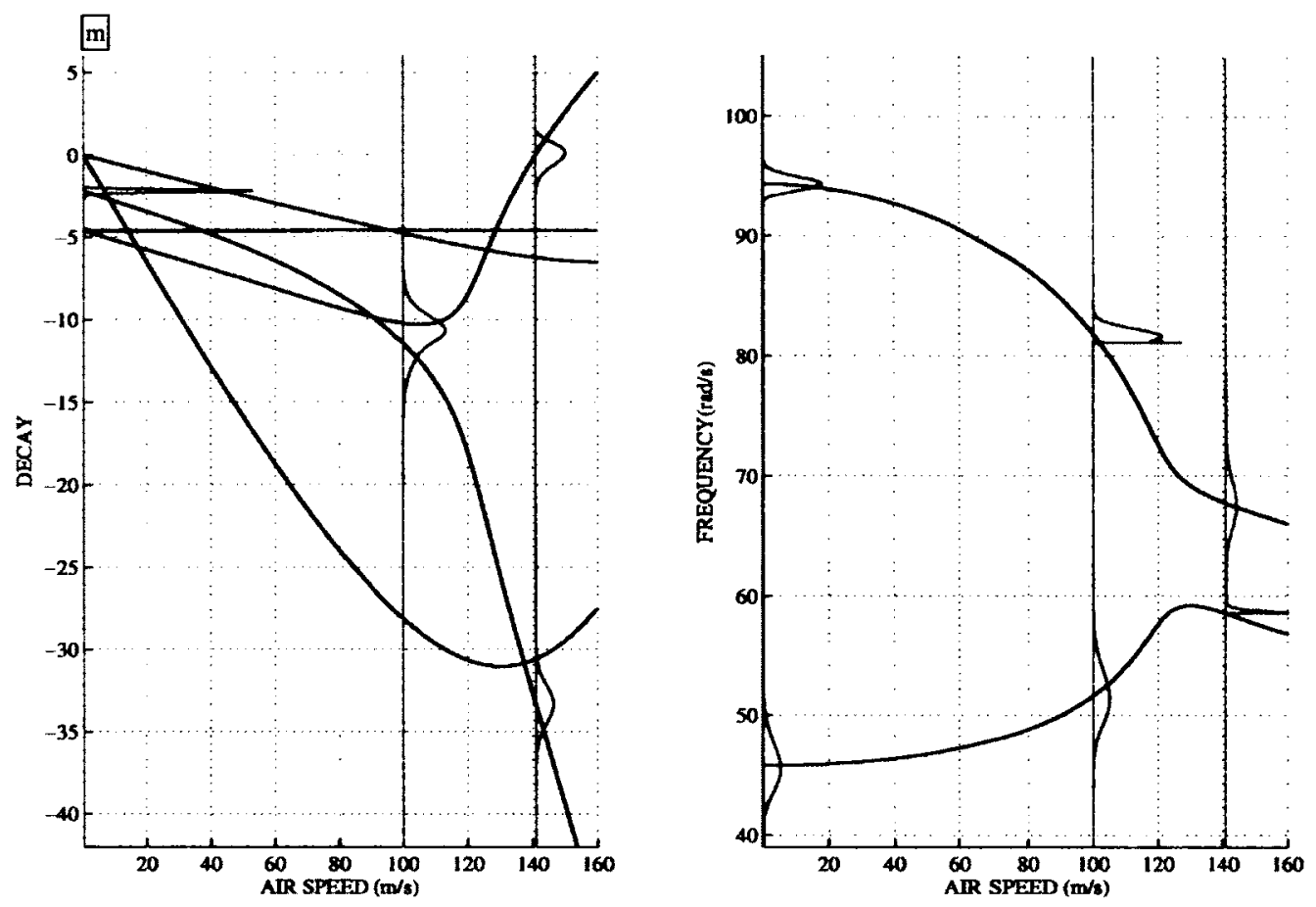

Figure 4.13: Modal parameter pdfs resulting from randomness in mass with $10 \% \mathrm{COV}$, obtained at 3 test airspeeds: $\approx 0 \mathrm{~m} / \mathrm{s}, 100 \mathrm{~m} / \mathrm{s}, 141 \mathrm{~m} / \mathrm{s}$

Next, the effects of uncertainty in the mass moment of inertia parameter are examined in fig. (4.14). The observations are very similar to the effects induced by the uncertainty of mass, but these effects have a greater influence in the pitch dominant mode. The modal decay pdfs experience overlapping effects. In the modal frequency pdfs, the uncertainty of the mass moment of inertia parameter produces a greater influence on the uncertainty ofthe pitch-dominant mode. Both modal frequency pdfs indicate a non-stationary trend, especially significant in the flutter region. A dramatic decrease of uncertainty in the non-flutter mode and a dramatic increase in the uncertainty of the flutter mode should be noted in the flutter region, which may be linked to a change in the dominance of the aeroelastic eigenvectors. As a result of a large and changing variance in the modal decay, a large variance is produced in the flutter speed pdf.

The effects of parameter uncertainty in the structural stiffness are shown next. Figs. (4.15) and (4.16) illustrate the modal parameter pdf transformations produced by uncertainty in the heave stiffness and pitch stiffness parameters, respectively. Similarly to the observations from effects of uncertainty in the inertia parameter, but most notably, the uncertainty in heave stiffness 

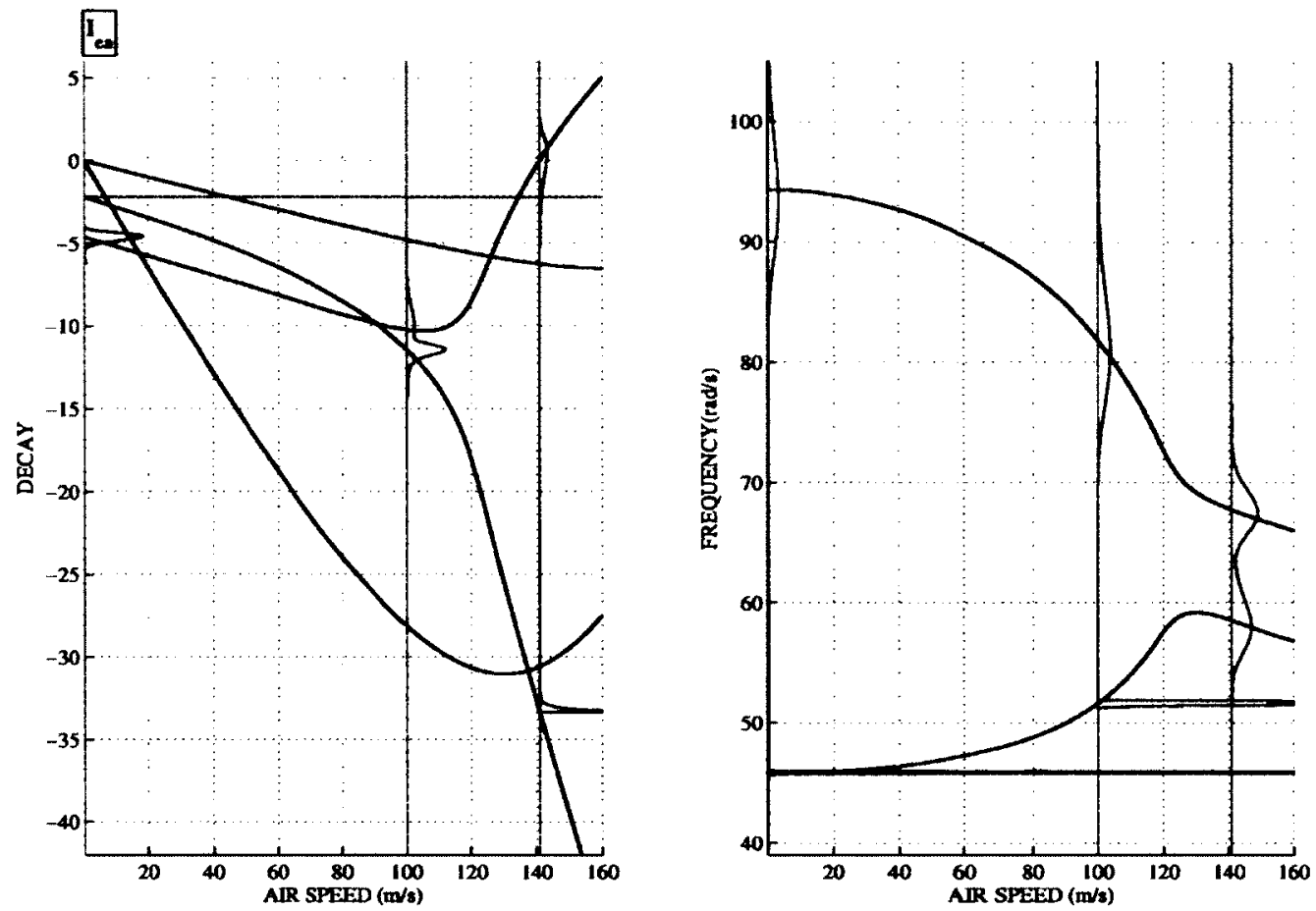

Figure 4.14: Modal parameter pdfs resulting from randomness in mass moment of inertia with $10 \%$ COV, obtained at 3 test airspeeds: $\approx 0 \mathrm{~m} / \mathrm{s}, 100 \mathrm{~m} / \mathrm{s}, 141 \mathrm{~m} / \mathrm{s}$
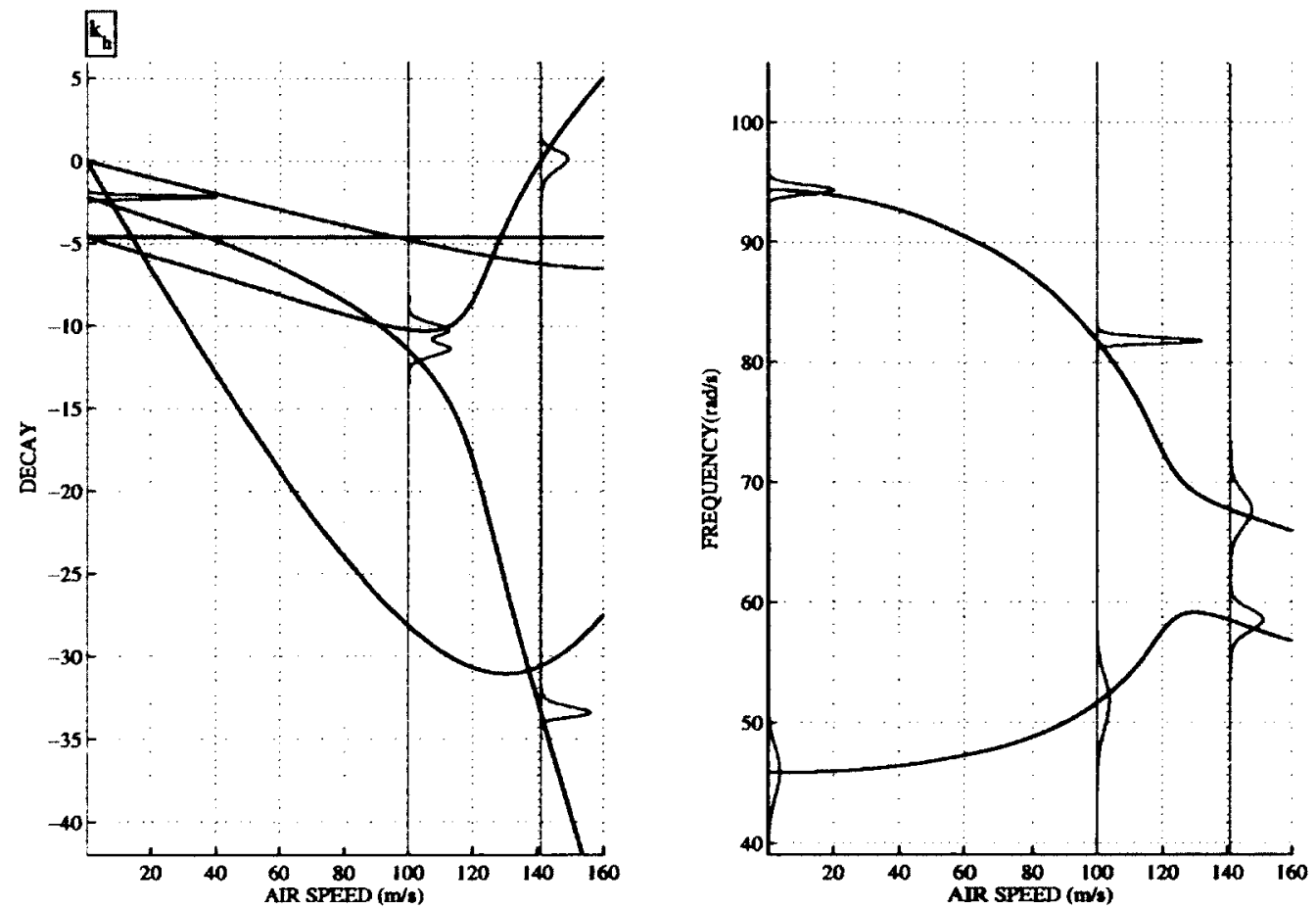

Figure 4.15: Modal parameter pdfs resulting from randomness in heave stiffness with $10 \%$ COV, obtained at 3 test airspeeds: $\approx 0 \mathrm{~m} / \mathrm{s}, 100 \mathrm{~m} / \mathrm{s}, 141 \mathrm{~m} / \mathrm{s}$ 

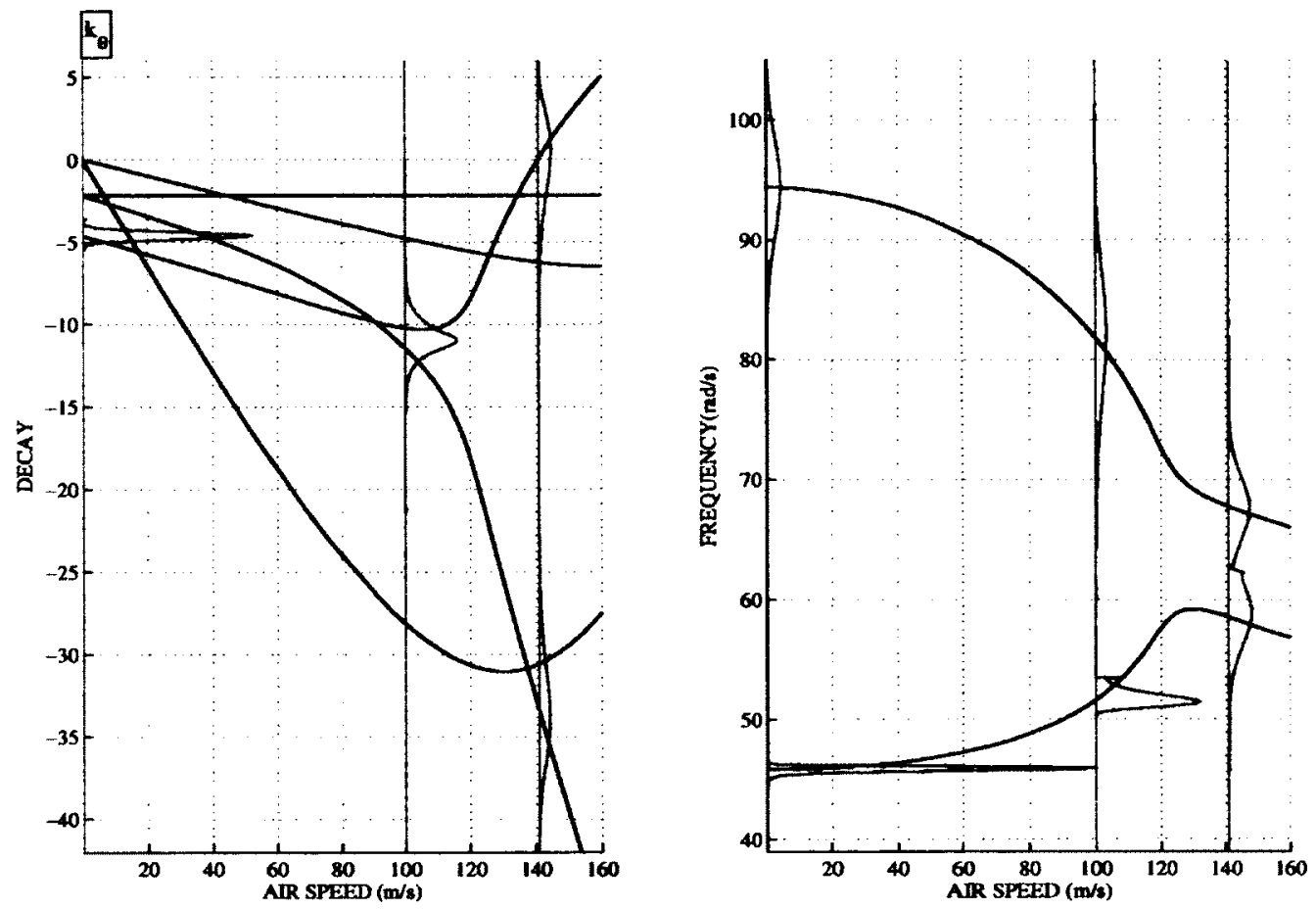

Figure 4.16: Modal parameter pdfs resulting from randomness in pitch stiffness with $10 \%$ COV, obtained at 3 test airspeeds: $\approx 0 \mathrm{~m} / \mathrm{s}, 100 \mathrm{~m} / \mathrm{s}, 141 \mathrm{~m} / \mathrm{s}$

parameter has a greater influence on the uncertainty of the frequency pdf of the heave-dominant mode. In contrast, the uncertainty in the pitch stiffness parameter has a greater influence on the uncertainty of the frequency pdf of the pitch-dominant mode. In both cases, the modal frequency pdfs show a non-stationary trend with an increasing airspeed, especially noticeable in the flutter region, where dramatic changes in variances of frequencies are experienced in both modes. For case of uncertainty in the heave stiffness, a non-stationary trend in the modal decays leads to a moderate variance in the flutter mode decay pdf at flutter point. With uncertainty in the pitch stiffness, similar effects lead to a large variance in the decay pdf at flutter point.

In the analysis of the probabilistic flutter speed results for this case, it was noted that the uncertainty of the structural damping parameters become much less significant for the case of unsteady aerodynamics, since the flutter type became more explosive. The probabilistic results of the modal parameter transformations remain very similar to the results for quasisteady aerodynamic forcing. The one distinguishable feature observed in the modal parameter pdfs is a greater change in the variances of the decay pdfs in the flutter region, where the greatest 
frequency coalescence occurs. It is assumed that the changes in dominance of the eigenvectors contribute to this effect.

Figs. (4.17) and (4.18) illustrate the effects of uncertainty in the first and the second mode structural damping ratios, respectively. There are no notable uncertainty effects on the modal frequencies. The uncertainty in the first structural damping ratio, produces greater variance in the heave-dominant mode. Similarly, for the uncertainty in the second structural damping ratio, a pitch-dominant mode experiences the greater variance. As the dominance of the eigenvector switches near the flutter point, so does the relative magnitude of variance in the modal decays. With an explosive flutter type, a fairly low variance in the decay of the flutter mode, which does not vary significantly as a function of airspeed, leads to very low uncertainty in the flutter speed pdf.
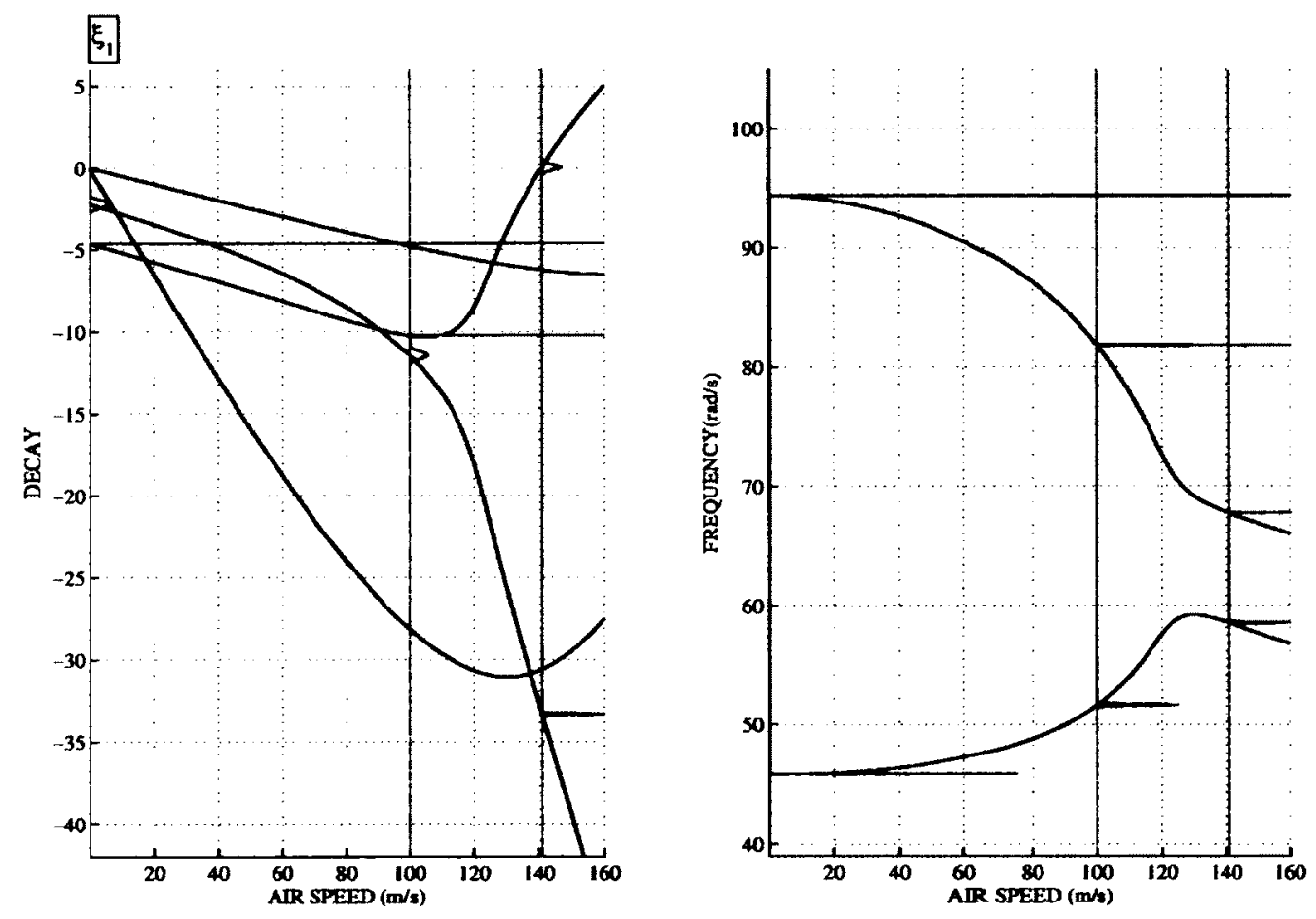

Figure 4.17: Modal parameter pdfs resulting from randomness in the $1^{\text {st }}$ modal damping ratio with $10 \%$ COV, obtained at 3 test airspeeds: $\approx 0 \mathrm{~m} / \mathrm{s}, 100 \mathrm{~m} / \mathrm{s}, 141 \mathrm{~m} / \mathrm{s}$ 

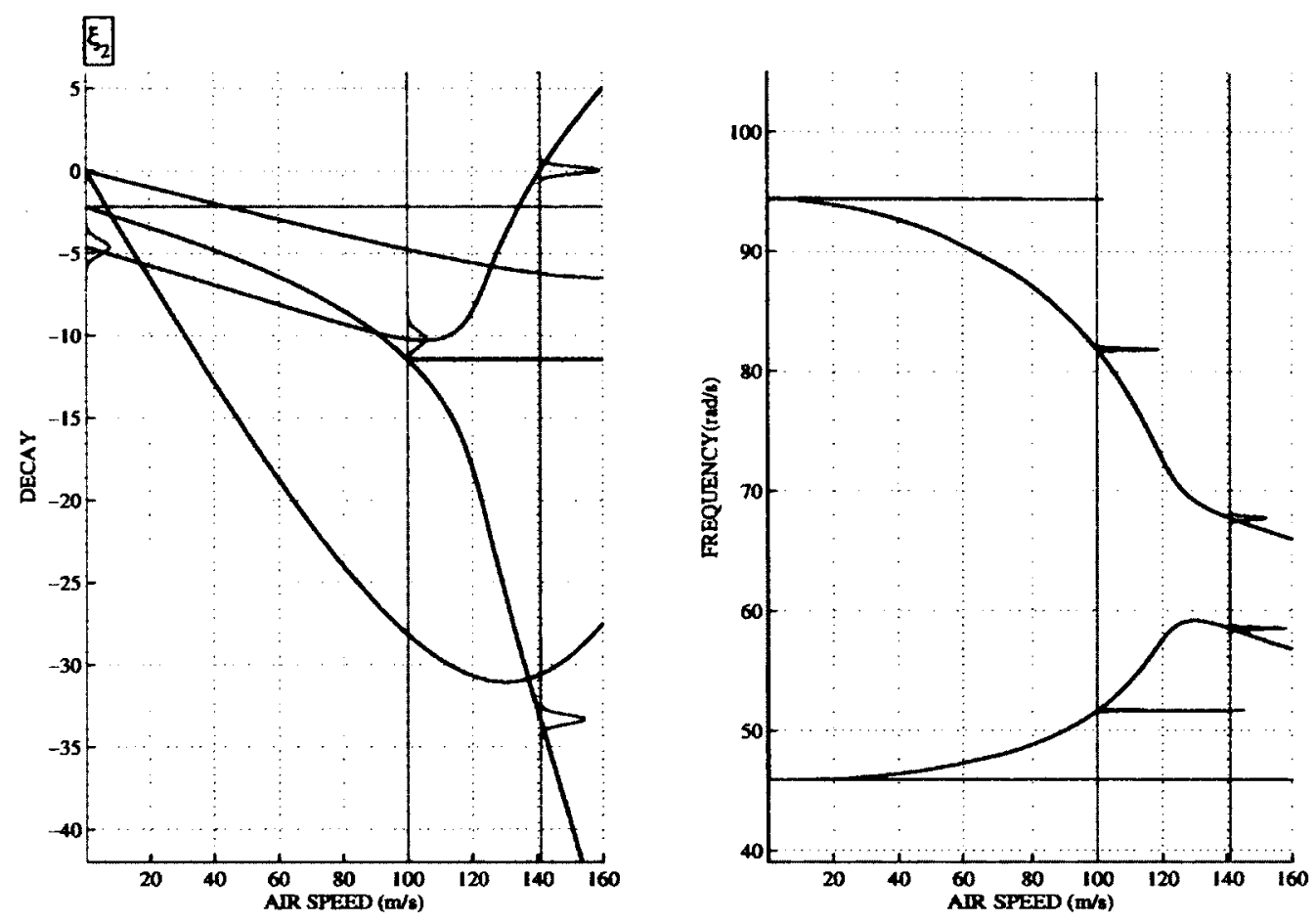

Figure 4.18: Modal parameter pdfs resulting from randomness in the $2^{\text {nd }}$ modal damping ratio with $10 \% \mathrm{COV}$, obtained at 3 test airspeeds: $\approx 0 \mathrm{~m} / \mathrm{s}, 100 \mathrm{~m} / \mathrm{s}, 141 \mathrm{~m} / \mathrm{s}$

\section{Multiple Parameter Uncertainty}

The effect of multiple parameter variations will be examined next. First, the results for fully uncorrelated random parameters are introduced. Figs. (4.19)-(4.21) show the probabilistic results of the modal parameters and their transformations as a function of airspeed for random inertia, stiffness and damping parameters, respectively. All of the observations are identical to the observations from the quasisteady aerodynamic forcing: the combined effects of uncertainty in the uncorrelated parameters produce a larger variance in all of the modal parameter pdfs, when compared to the cases of individual parameter variations. In effect, due to a larger variance of the modal decay of the flutter mode, the flutter speed pdfs experience a larger variance as well (evident from fig. (3.17)).

Next, the effects of full parameter correlation are examined when the uncertainty in the same random parameters is considered. Figs. (4.22)-(4.24) illustrate the probabilistic results for the modal parameters for fully correlated inertia, stiffness and damping parameters, respectively. 

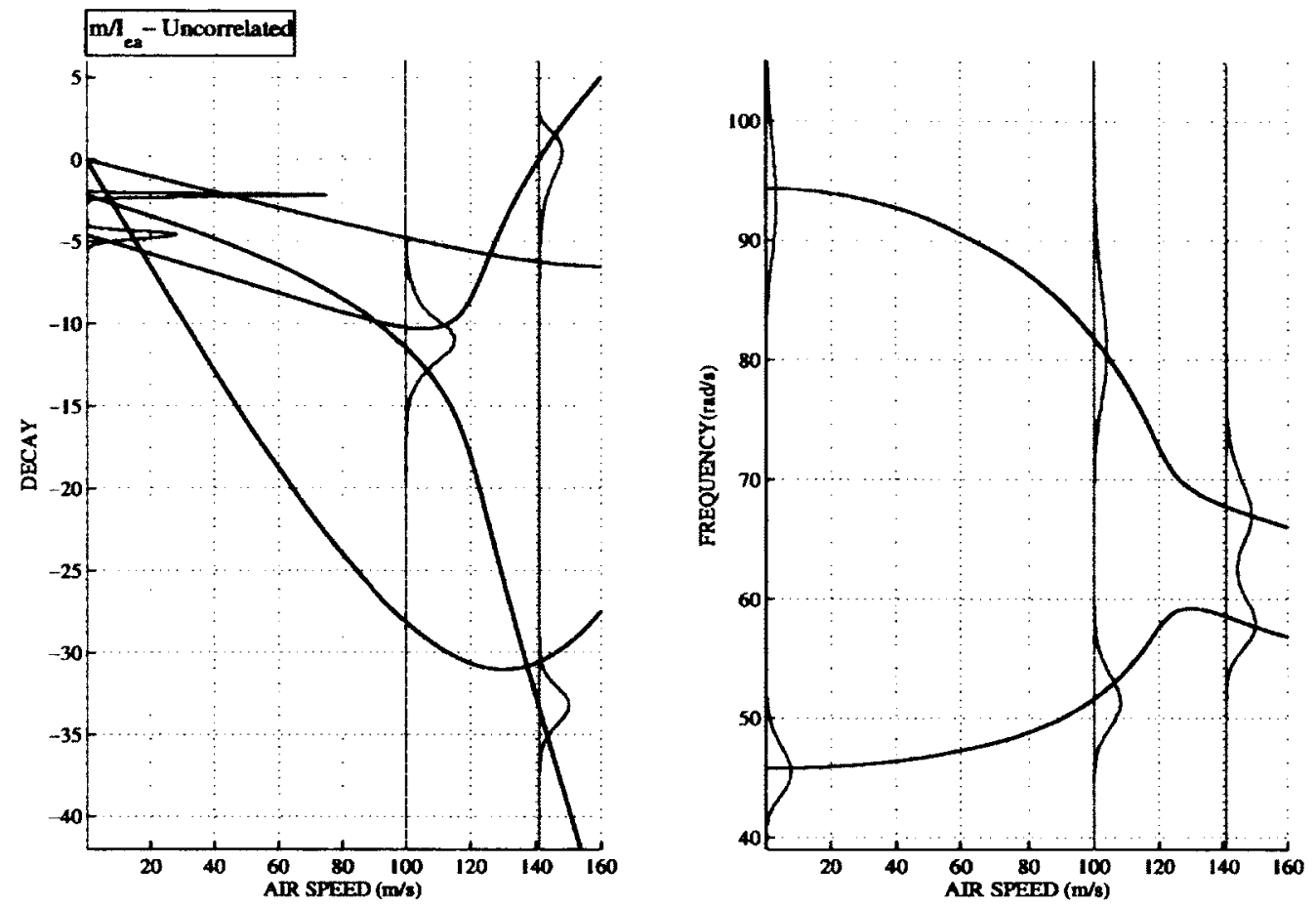

Figure 4.19: Modal parameter pdfs resulting from randomness in the uncorrelated inertia parameters with $10 \% \mathrm{COV}$, obtained at 3 test airspeeds: $\approx 0 \mathrm{~m} / \mathrm{s}, 100 \mathrm{~m} / \mathrm{s}, 141 \mathrm{~m} / \mathrm{s}$
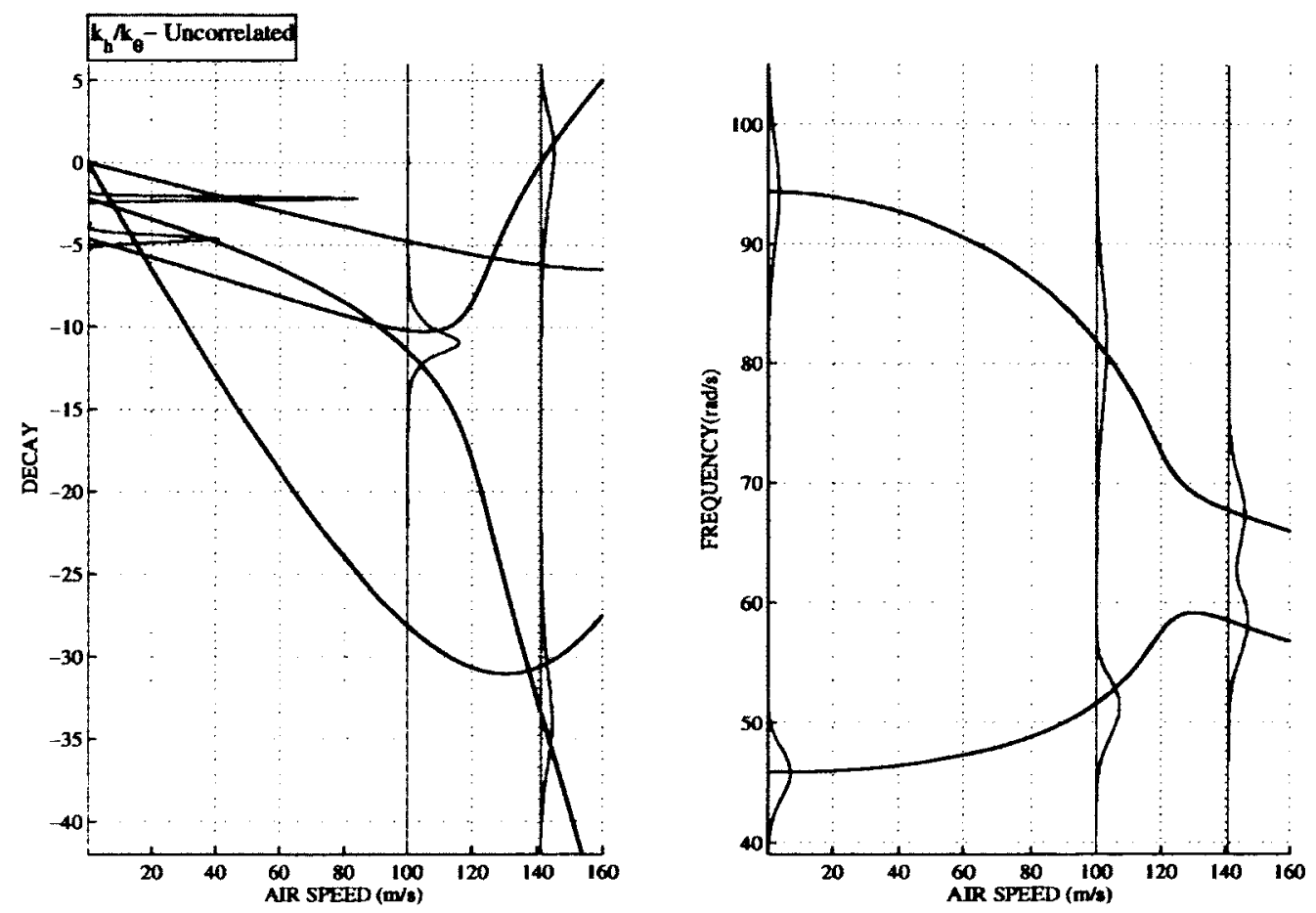

Figure 4.20: Modal parameter pdfs resulting from randomness in the uncorrelated stiffness parameters with $10 \% \mathrm{COV}$, obtained at 3 test airspeeds: $\approx 0 \mathrm{~m} / \mathrm{s}, 100 \mathrm{~m} / \mathrm{s}, 141 \mathrm{~m} / \mathrm{s}$ 

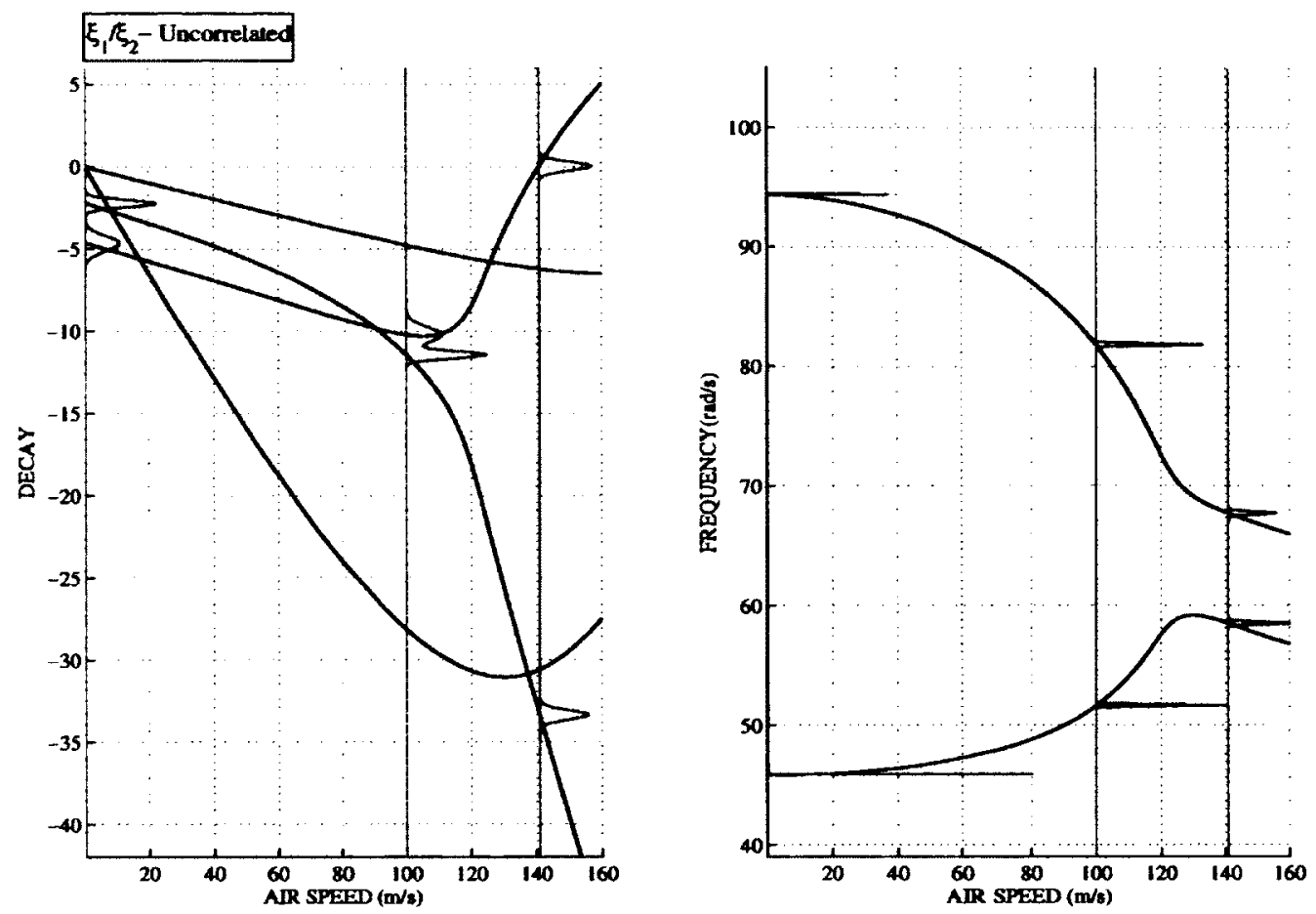

Figure 4.21: Modal parameter pdfs resulting from randomness in the uncorrelated damping parameters with $10 \% \mathrm{COV}$, obtained at 3 test airspeeds: $\approx 0 \mathrm{~m} / \mathrm{s}, 100 \mathrm{~m} / \mathrm{s}, 141 \mathrm{~m} / \mathrm{s}$

From figs. (4.22) and (4.23), along with the fig. (3.17), the following are the noted observations for the effects of inertia and stiffness parameter uncertainties. The uncertainty in the frequency parameters does not depend significantly on the correlation between the random inertia or stiffness parameters. For uncertainty in the inertia or stiffness, the correlation between these parameters leads to correlation effects between the modal frequencies, and in effect, the modal decays. Similarly with the quasisteady analysis, in this case the correlation between the modal frequencies reduces the uncertainty growth of the modal decays prior to the frequency coalescence region, which leads to a smaller variance in the flutter speed pdf.

Then, from fig. (4.12) the effects of correlation in the structural damping parameters are examined. Following is the list of made observations. The effects of uncertainty of the correlated damping parameters are negligible in the frequency pdfs. However, the correlation between random structural damping parameters leads to correlation effects between the modal decays, which leads to a consistent increase in the variance of the modal decays in the coalescing modes for all airspeeds. This increase of flutter mode decay variance results in an increased variance 

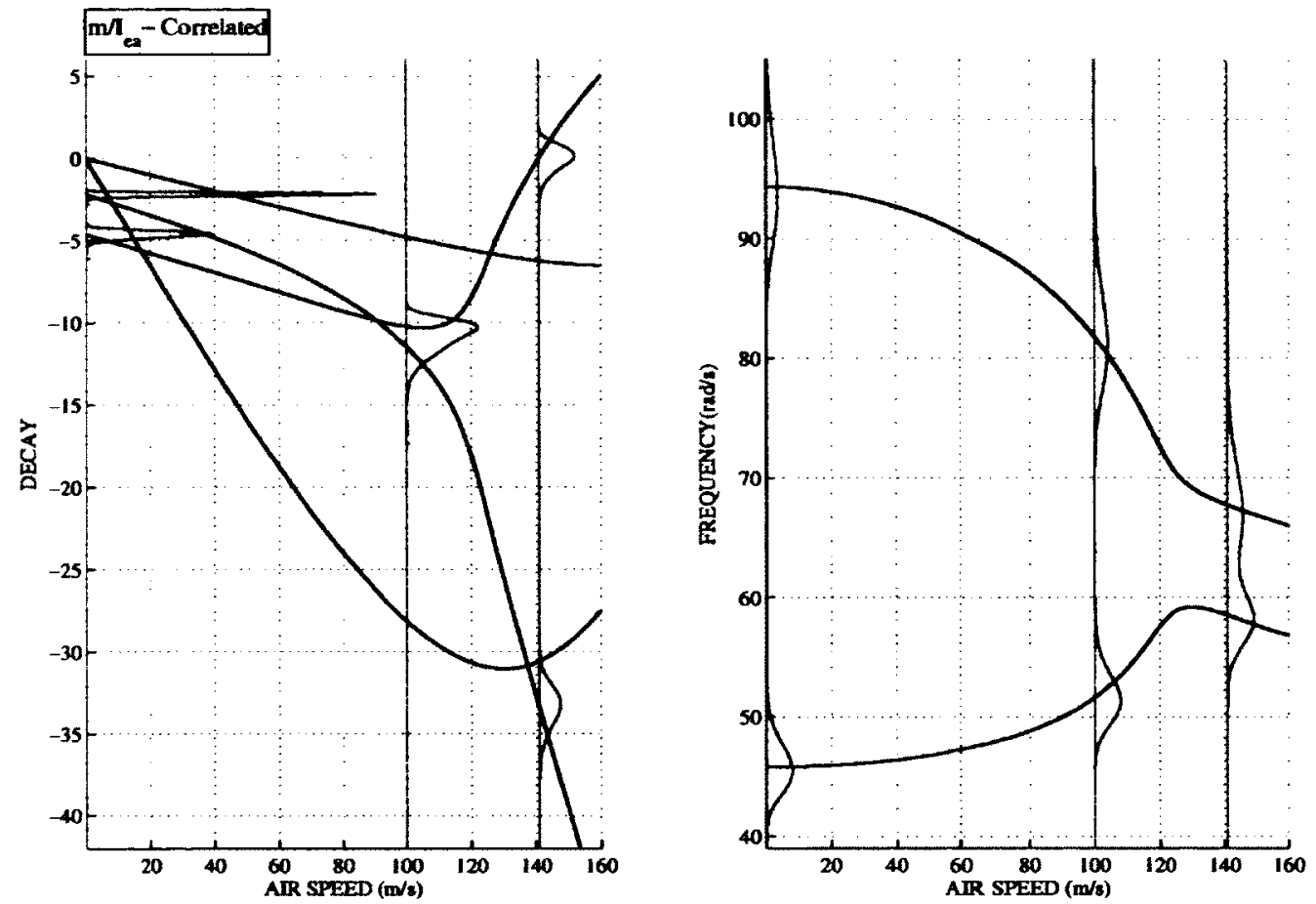

Figure 4.22: Modal parameter pdfs resulting from randomness in the correlated inertia parameters with $10 \% \mathrm{COV}$, obtained at 3 test airspeeds: $\approx 0 \mathrm{~m} / \mathrm{s}, 100 \mathrm{~m} / \mathrm{s}, 141 \mathrm{~m} / \mathrm{s}$
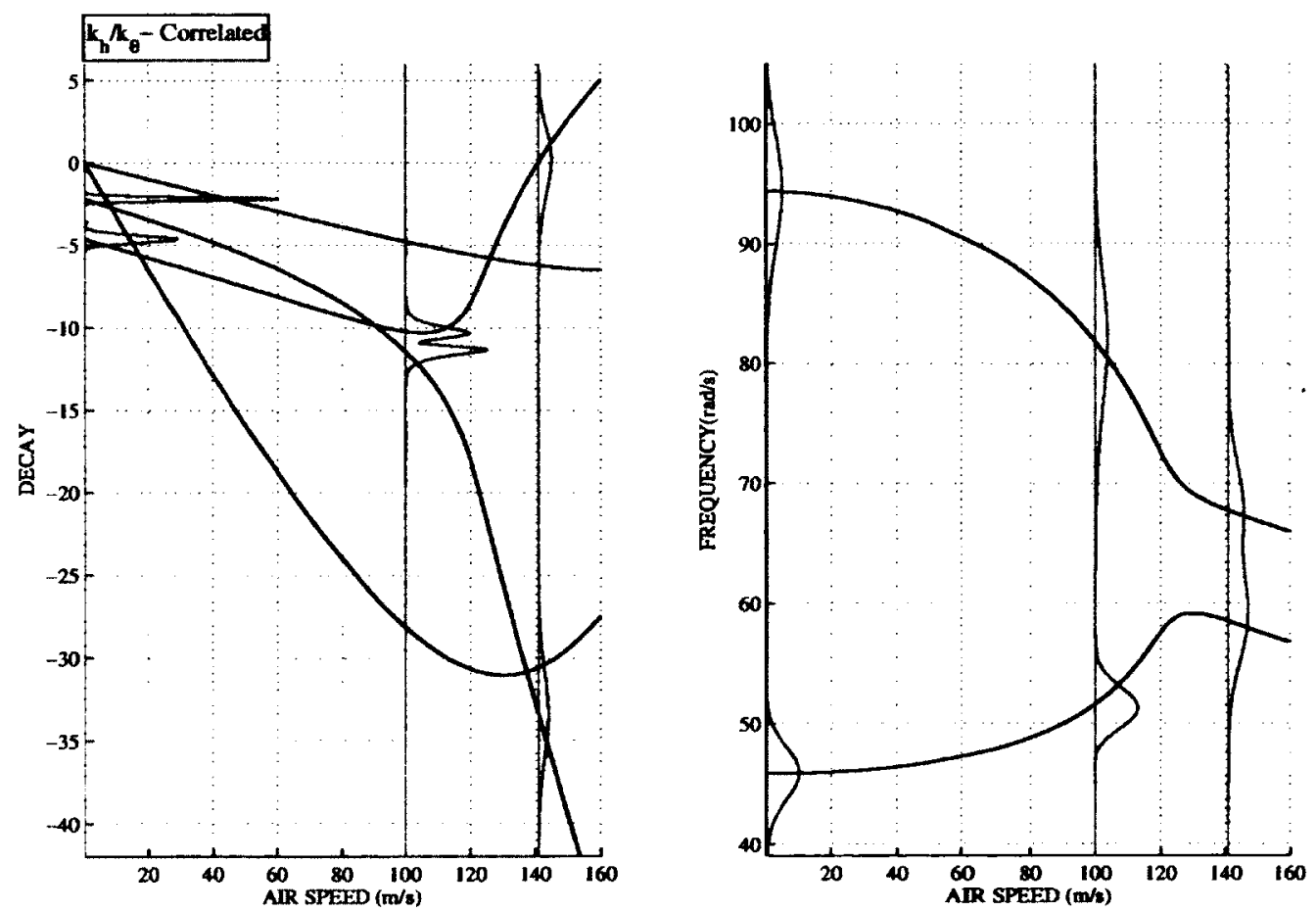

Figure 4.23: Modal parameter pdfs resulting from randomness in the correlated stiffness parameters with $10 \% \mathrm{COV}$, obtained at 3 test airspeeds: $\approx 0 \mathrm{~m} / \mathrm{s}, 100 \mathrm{~m} / \mathrm{s}, 141 \mathrm{~m} / \mathrm{s}$ 

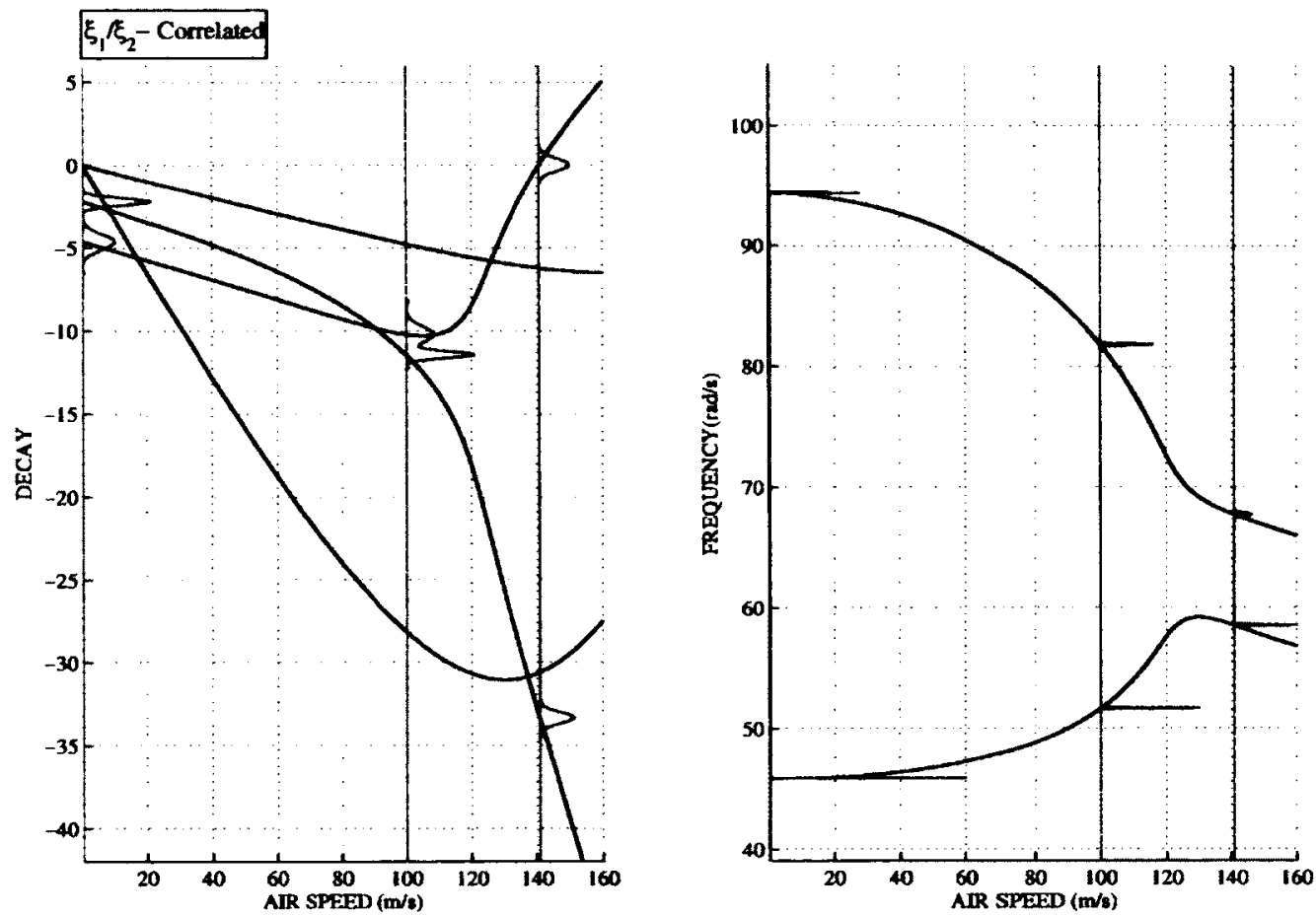

Figure 4.24: Modal parameter pdfs resulting from randomness correlated damping parameters with $10 \% \mathrm{COV}$, obtained at 3 test airspeeds: $\approx 0 \mathrm{~m} / \mathrm{s}, 100 \mathrm{~m} / \mathrm{s}, 141 \mathrm{~m} / \mathrm{s}$

of the flutter speed pdf.

\subsubsection{Comparison of Quasisteady and Unsteady Results}

A comparison of the probabilistic results of unsteady aerodynamics with the results of quasisteady aerodynamics shows certain differences. The modal frequencies of the coalescing modes experience statistical overlaps with unsteady aerodynamics due to a significant frequency coalescence (related to a more explosive flutter type). In this case, due to a more explosive flutter, a greater non-stationary trend of the aeroelastic frequencies are observed between the coalescing modes in vicinity of flutter. 


\subsection{CASE 2}

\subsubsection{Quasisteady Results}

Case 2 rigid wing model presents an alternate system, which is represented by a different set of structural parameters. This section focuses on interpreting the probabilistic results based on the probabilistic trends of the modal parameter transformations. The main observations from these results are very similar to the observations of Case 1, implying that the physical mechanism of flutter remains almost the same. Therefore, only a brief summary of the results will be provided. Figs. (4.25) and (4.26) illustrate the probabilistic results of modal parameters for uncertain mass and mass moment of inertia parameters, respectively. The following observations are noted. Uncertainty in the mass parameter leads to a fairly small variance and nearly stationary trend in both of the modal frequency pdfs, which cause a significant growth of variance in the modal decay pdfs. The frequency pdf of the second mode experiences positive skewness and a minimum cutoff and slight negative skewness in the decay pdf. In contrast, the uncertainty in the mass moment of inertia has a greater influence on the second, heave-dominant, mode and results in large variance and a near stationarity in the modal frequency pdfs, which leads to a growing variance of the decay of the flutter mode. For both uncertainty cases, the growth of variance in the flutter mode decay, with a large variance at the flutter point results in a large variance of the flutter speed pdf.

Next, figs. (4.27) and (4.28) illustrate the effects of uncertainty of the stiffness parameters. The uncertainty in heave stiffness parameter has a greater influence on the uncertainty of the frequency pdf of the second, heave-dominant mode. Both of the modal frequency pdfs show an almost stationary trend with an increasing airspeed with approximately Gaussian distributions, which results in a growth of variance in the modal decays of the coalescing modes as function of increasing airspeed. This leads to a large variance of the flutter speed pdf. The decay of the flutter mode develops a light positive skewness. The uncertainty in the pitch stiffness parameter has a greater influence on the uncertainty of the frequency pdf of the first, pitch-dominant mode. The modal frequency pdfs show an almost stationary trend with increasing airspeed with 

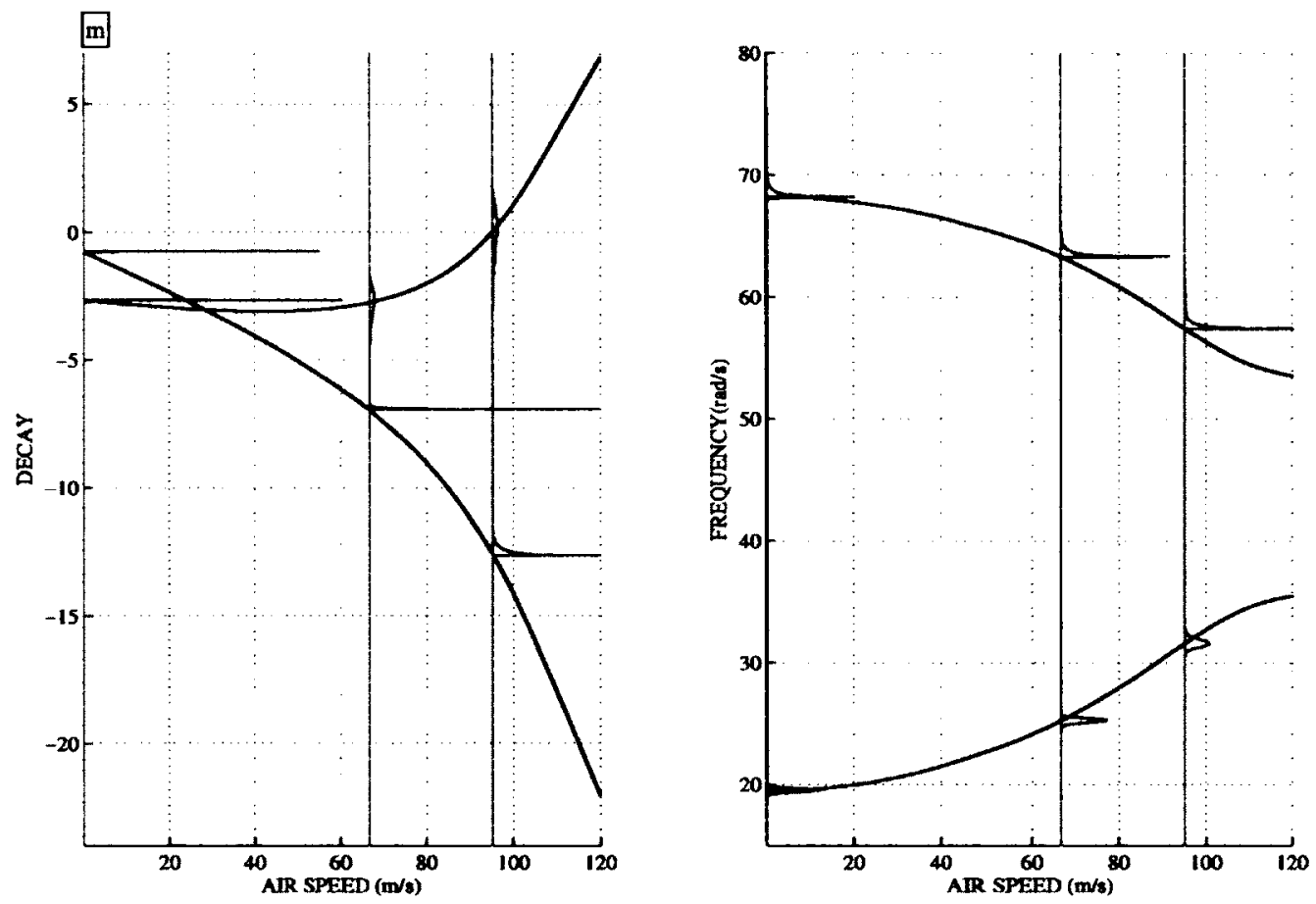

Figure 4.25: Modal parameter pdfs resulting from randomness in mass with $10 \% \mathrm{COV}$, obtained at 3 test airspeeds: $0 \mathrm{~m} / \mathrm{s}, 68 \mathrm{~m} / \mathrm{s}, 95 \mathrm{~m} / \mathrm{s}$
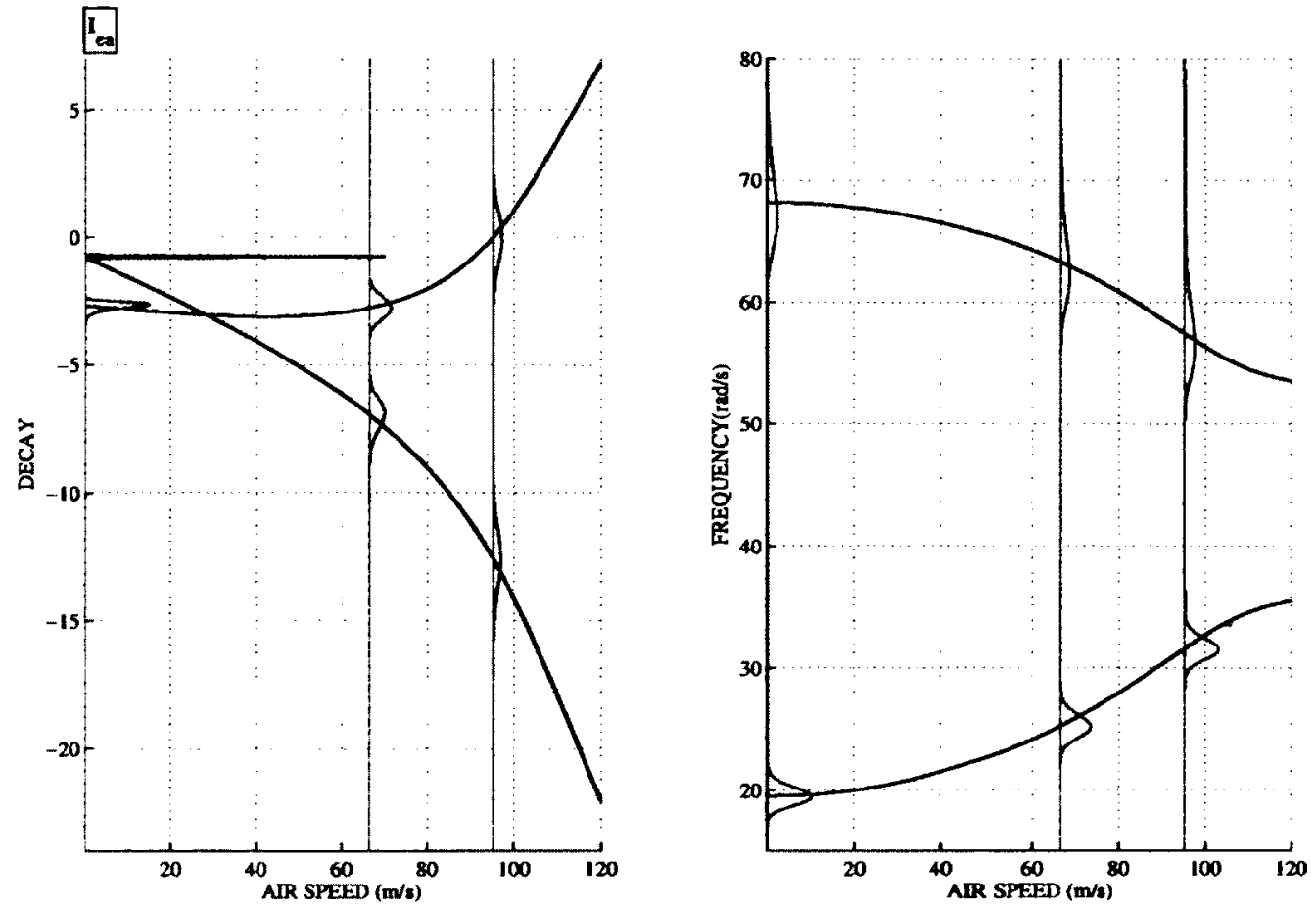

Figure 4.26: Modal parameter pdfs resulting from randomness in mass moment of inertia with $10 \%$ COV, obtained at 3 test airspeeds: $0 \mathrm{~m} / \mathrm{s}, 68 \mathrm{~m} / \mathrm{s}, 95 \mathrm{~m} / \mathrm{s}$ 
approximately Gaussian distributions. Only minor growth of variance in the modal decays of the coalescing modes is noticeable. The flutter speed pdf shows very minor variance.

The effects of uncertainty in the structural damping ratios are reported in figs. (4.29) and (4.30). The resulting observations are as anticipated. There are no notable effects of uncertainty on the modal frequencies. For case of uncertainty in the first structural modal damping ratio, the most significant effect appears in the decay of the first (non-flutter) mode. The variance of the second mode decay is almost negligible, but increases slightly near the flutter point. Both modal decay distributions remain almost Gaussian. Since the variance of the flutter mode decay is almost negligible, the variance of the flutter speed is also negligible. For the case of uncertainty in the second structural modal damping ratio, the most significant effect appears in the decay of the second (flutter) mode. The variance of the second mode is remains moderate, but decreases slightly near the flutter point. Both modal decay distributions remain almost Gaussian. Due to a mild flutter type and only a moderate and almost constant variance of the flutter mode decay, the variance of the flutter speed is moderately large.
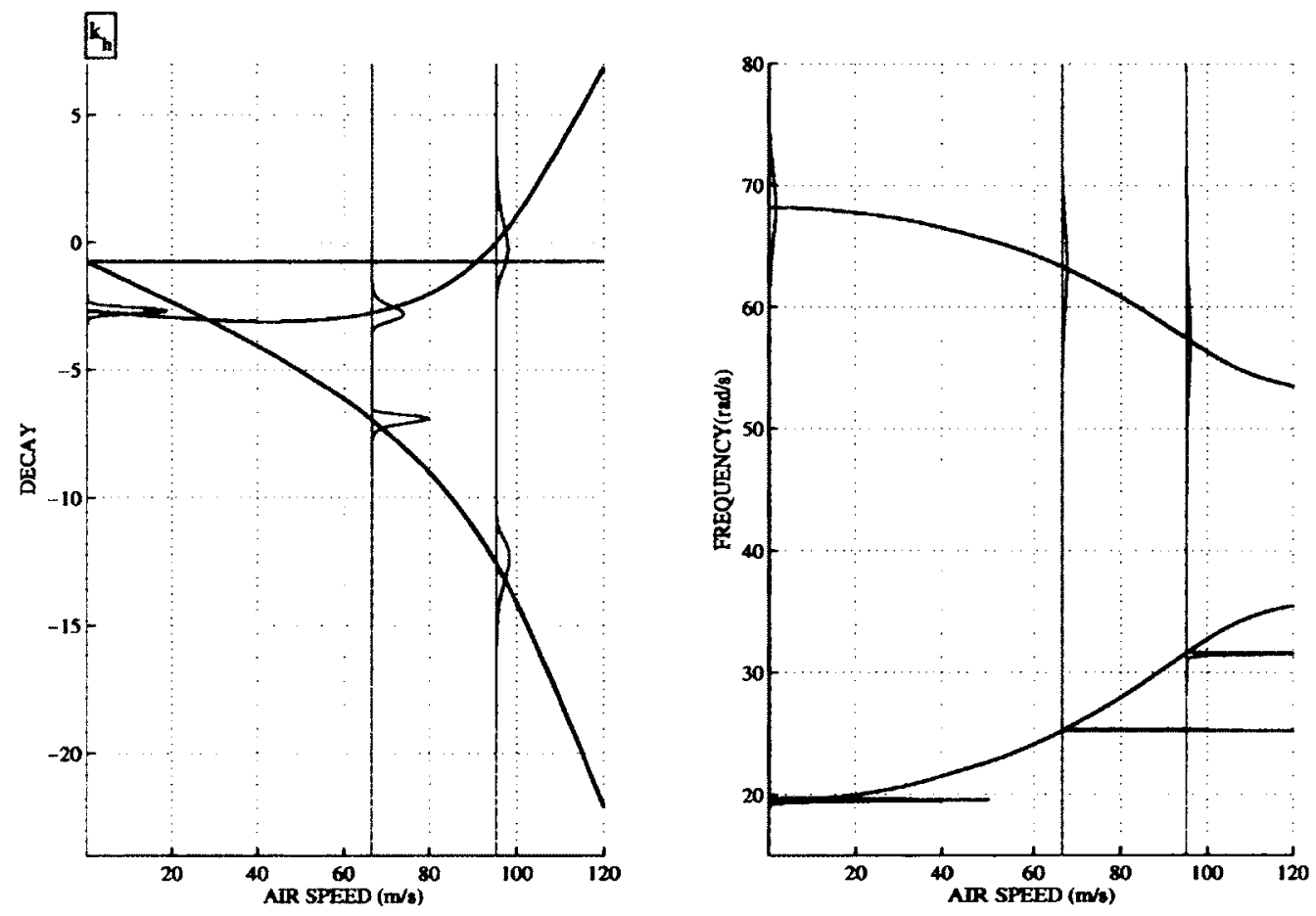

Figure 4.27: Modal parameter pdfs resulting from randomness in heave stiffness with $10 \%$ COV, obtained at 3 test airspeeds: $0 \mathrm{~m} / \mathrm{s}, 68 \mathrm{~m} / \mathrm{s}, 95 \mathrm{~m} / \mathrm{s}$ 

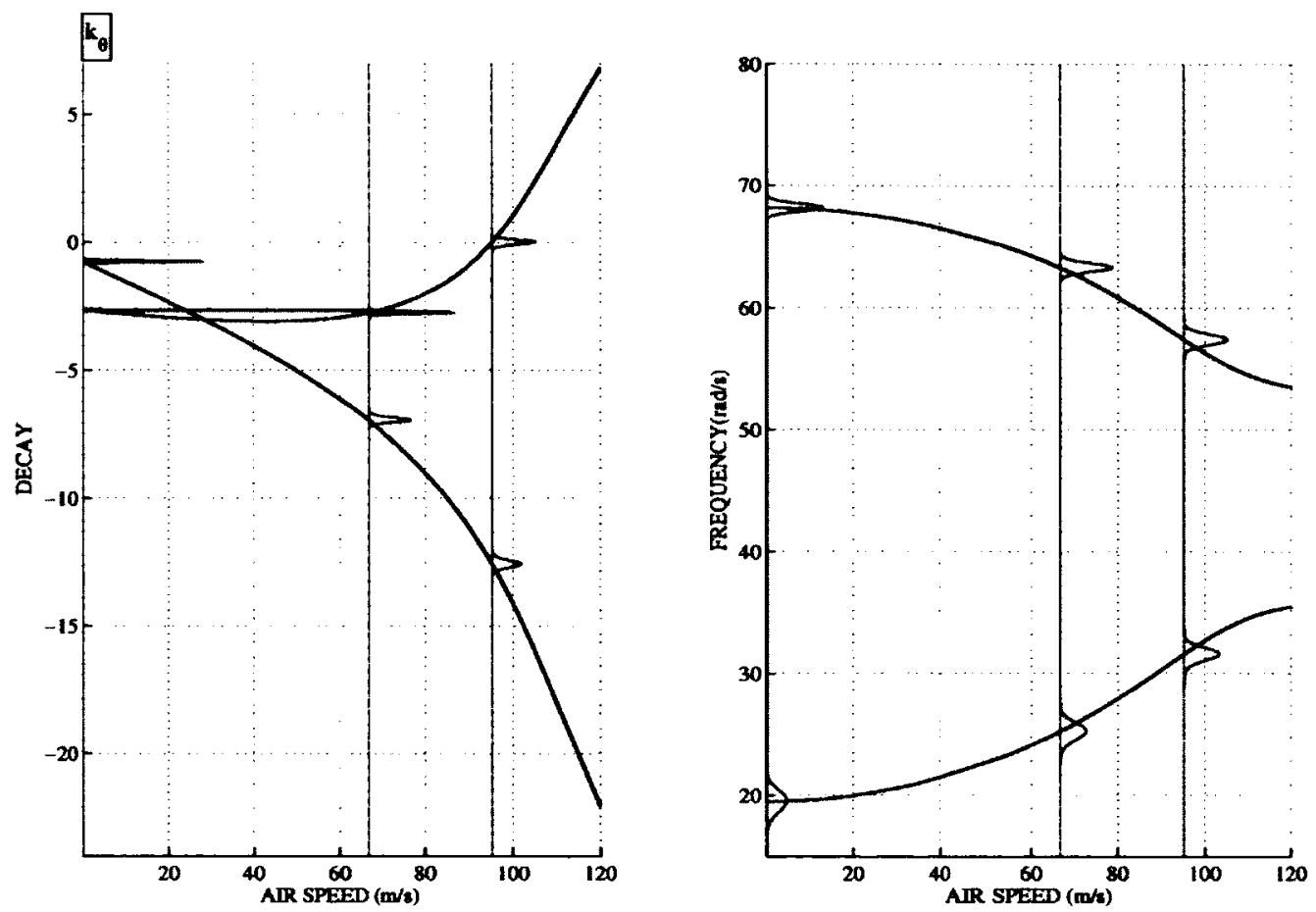

Figure 4.28: Modal parameter pdfs resulting from randomness in pitch stiffness with $10 \%$ COV, obtained at 3 test airspeeds: $0 \mathrm{~m} / \mathrm{s}, 68 \mathrm{~m} / \mathrm{s}, 95 \mathrm{~m} / \mathrm{s}$
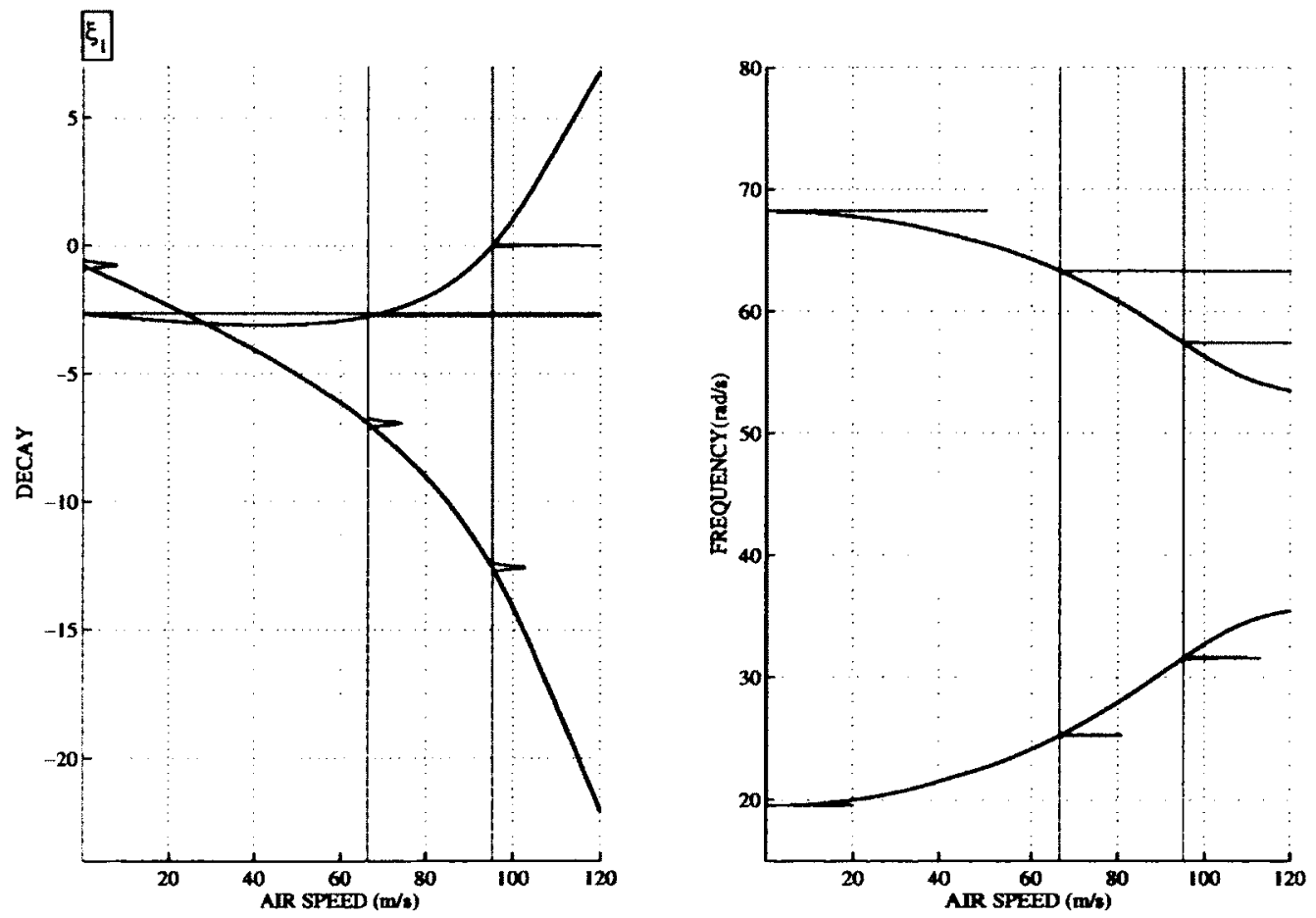

Figure 4.29: Modal parameter pdfs resulting from randomness in the $1^{\text {st }}$ modal damping ratio with $10 \% \mathrm{COV}$, obtained at 3 test airspeeds: $0 \mathrm{~m} / \mathrm{s}, 68 \mathrm{~m} / \mathrm{s}, 95 \mathrm{~m} / \mathrm{s}$ 

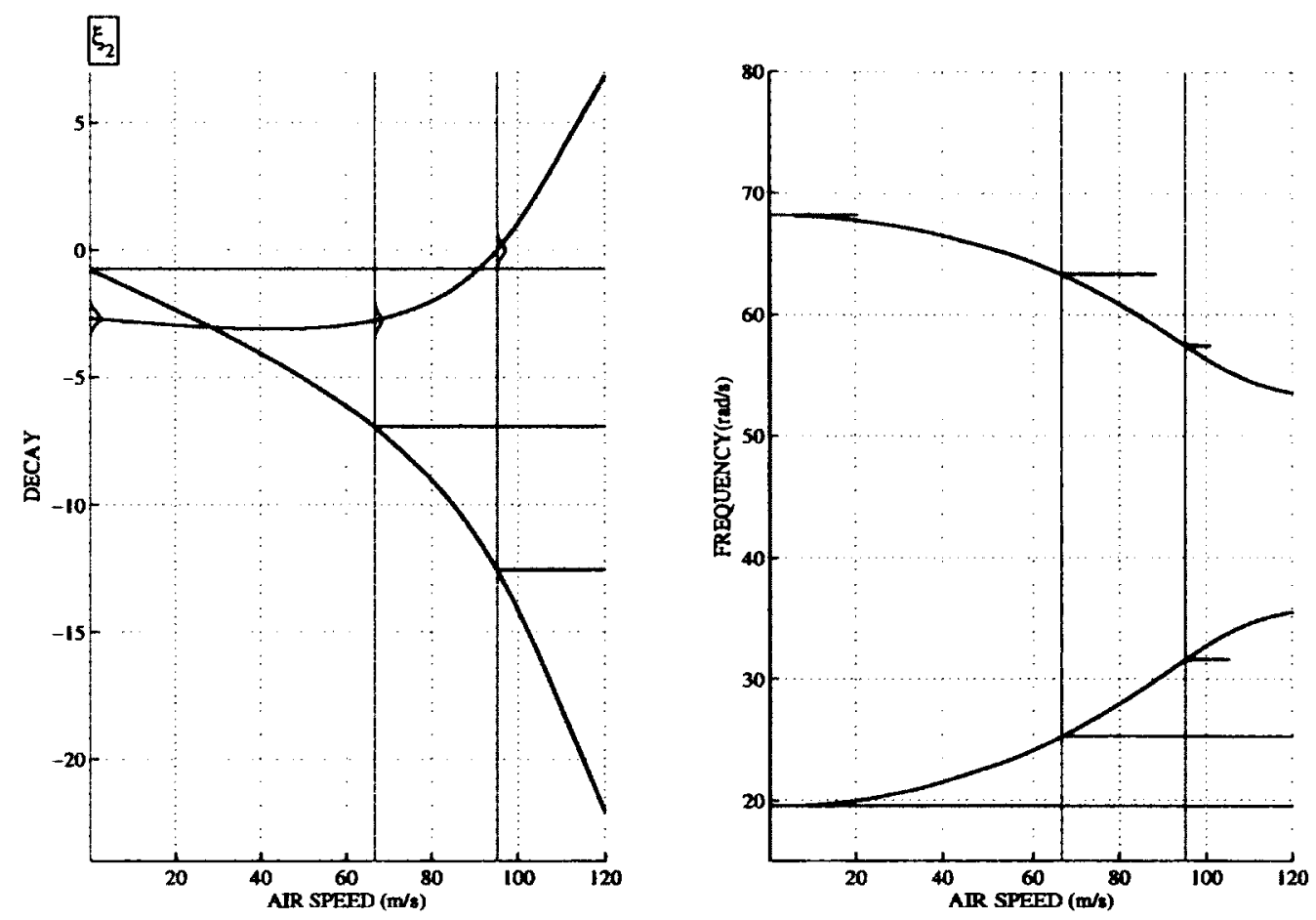

Figure 4.30: Modal parameter pdfs resulting from randomness in the $2^{\text {nd }}$ modal damping ratio with $10 \% \mathrm{COV}$, obtained at 3 test airspeeds: $0 \mathrm{~m} / \mathrm{s}, 68 \mathrm{~m} / \mathrm{s}, 95 \mathrm{~m} / \mathrm{s}$

\section{Multiple Parameter Uncertainty}

The effects of multiple parameter variations are shown next. Figs. (4.31)-(4.33) show the resulting pdfs of modal parameters and their transformations as a function of airspeed for fully uncorrelated inertia, stiffness and damping parameters, respectively. Similar to the previous case study, these results indicate that uncertainty in multiple uncorrelated parameters produces a larger variance in all of the modal parameter pdfs in comparison to the individual random parameter effects. Therefore, a larger variance in the flutter speed pdfs is obtained.

The effect of full correlation between the random structural parameters is also examined. Similarly, figs.(4.34)-(4.36) illustrate the probabilistic results for modal parameters of fully correlated inertia, stiffness and damping parameters, respectively. From these figures, the following observations are noted. The uncertainty in the frequency parameters does not depend significantly on the correlation between the random structural parameters. The correlation between the random inertia and stiffness parameters leads to certain correlation effects between the modal 
93
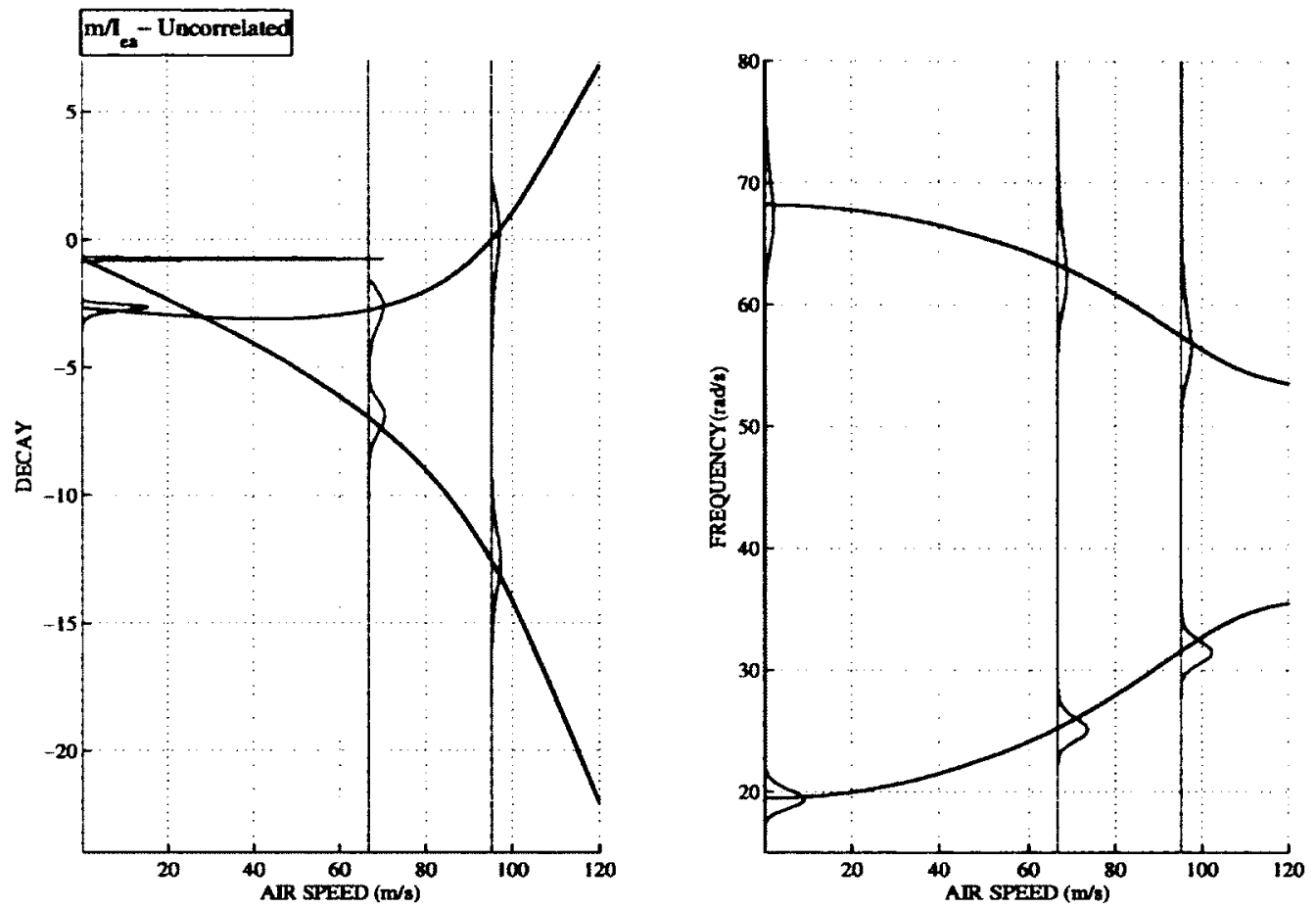

Figure 4.31: Modal parameter pdf resulting from randomness in the uncorrelated inertia parameters with $10 \%$ COV, obtained at 3 test airspeeds: $0 \mathrm{~m} / \mathrm{s}, 68 \mathrm{~m} / \mathrm{s}, 95 \mathrm{~m} / \mathrm{s}$
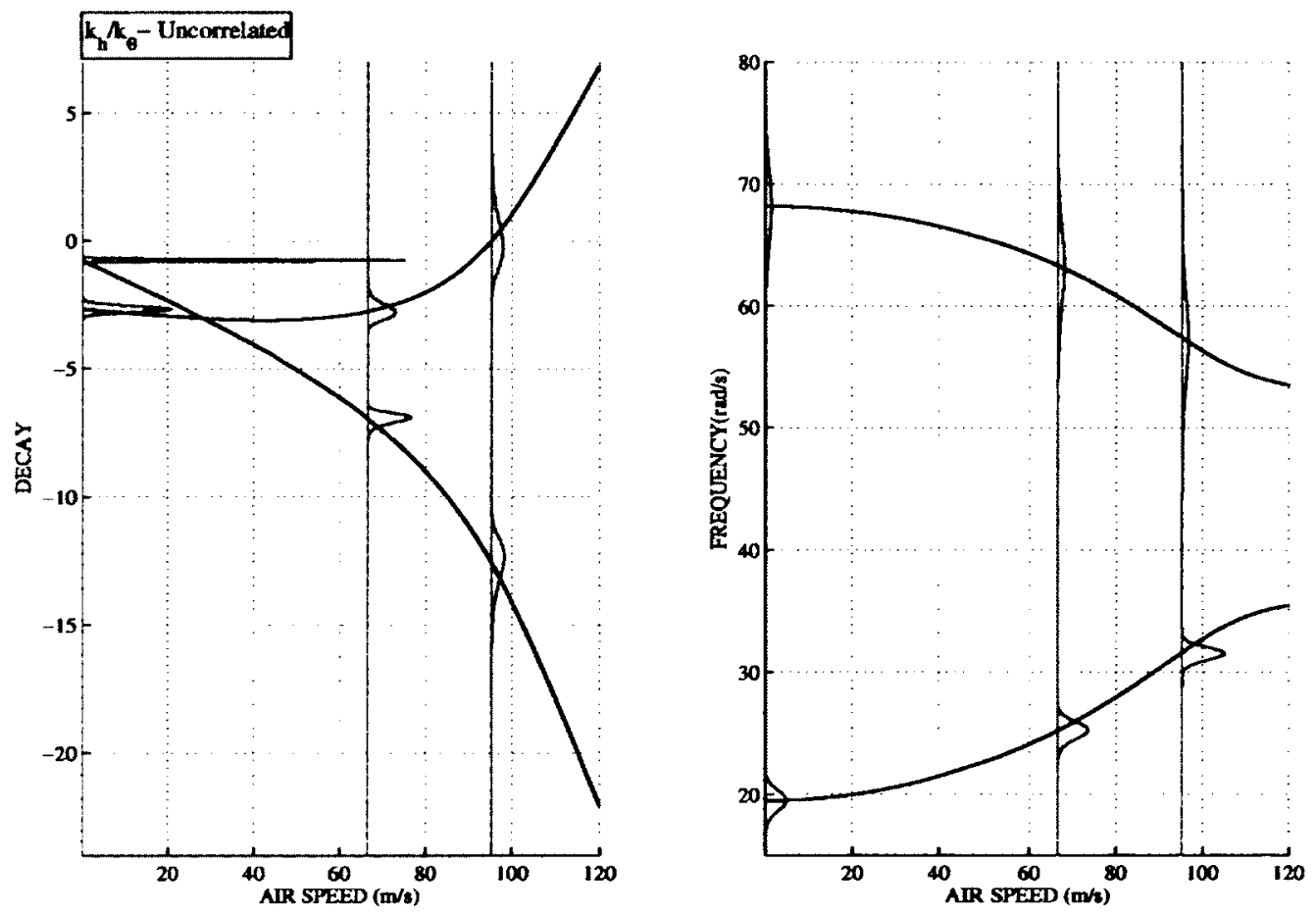

Figure 4.32: Modal parameter pdfs resulting from randomness in the uncorrelated stiffness parameters with $10 \%$ COV, obtained at 3 test airspeeds: $0 \frac{\mathrm{m}}{\mathrm{s}}, 68 \frac{\mathrm{m}}{\mathrm{s}}, 95 \frac{\mathrm{m}}{\mathrm{s}}$ 

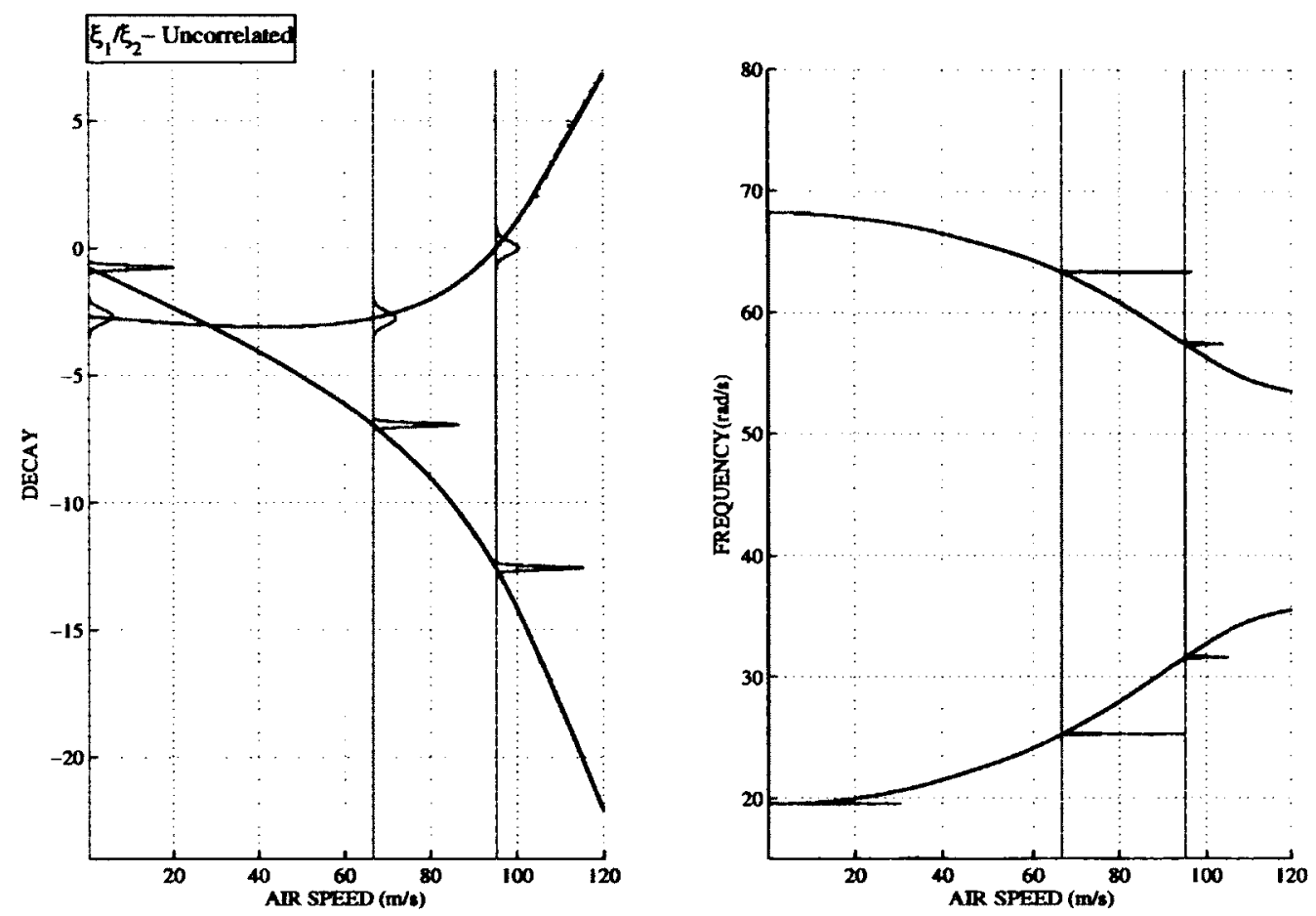

Figure 4.33: Modal parameter pdfs resulting from randomness in the uncorrelated damping parameters with $10 \%$ COV, obtained at 3 test airspeeds: $0 \mathrm{~m} / \mathrm{s}, 68 \mathrm{~m} / \mathrm{s}, 95 \mathrm{~m} / \mathrm{s}$
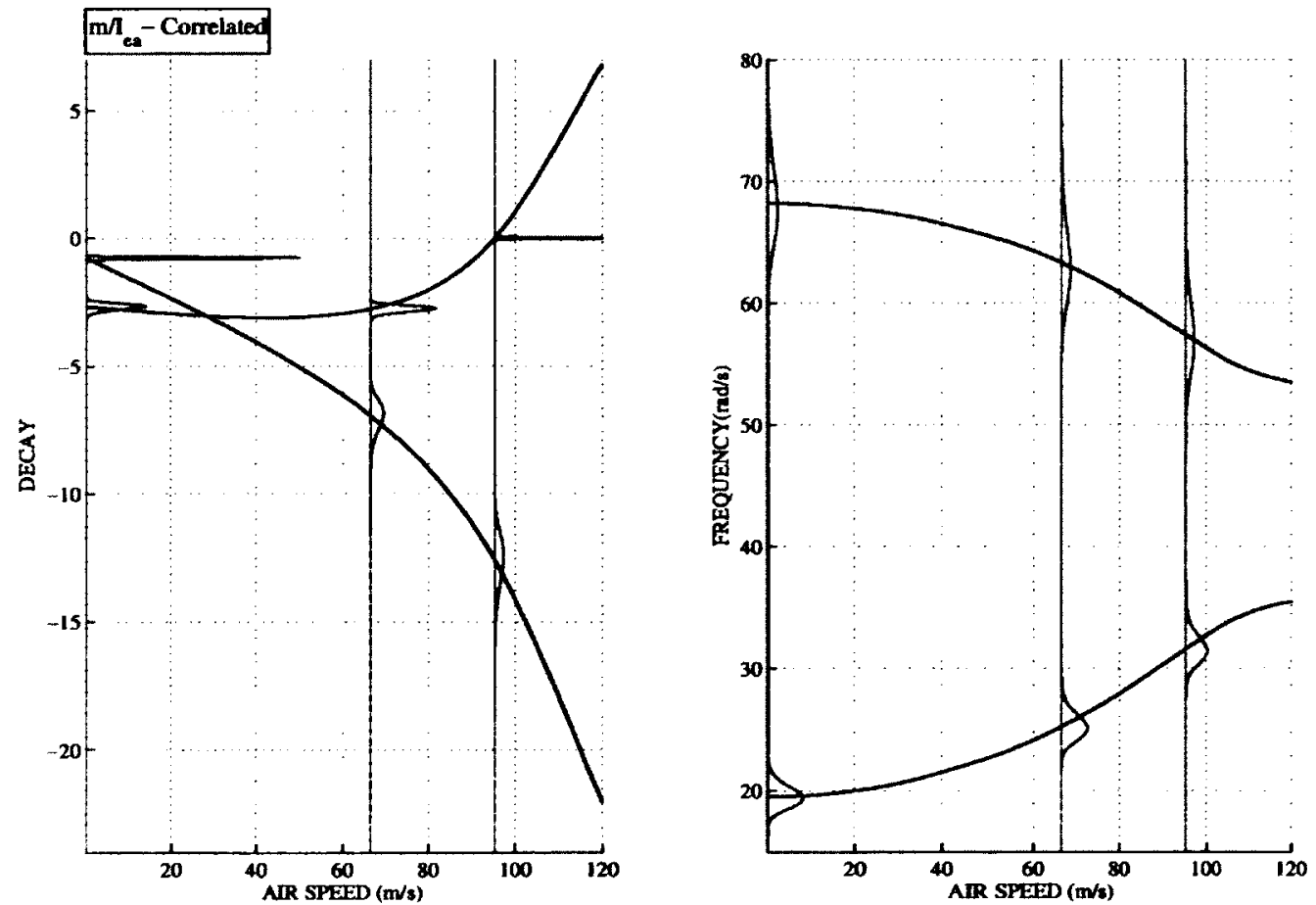

Figure 4.34: Modal parameter pdfs resulting from randomness in the correlated inertia parameters with $10 \% \operatorname{COV}$, obtained at 3 test airspeeds: $0 \mathrm{~m} / \mathrm{s}, 68 \mathrm{~m} / \mathrm{s}, 95 \mathrm{~m} / \mathrm{s}$ 

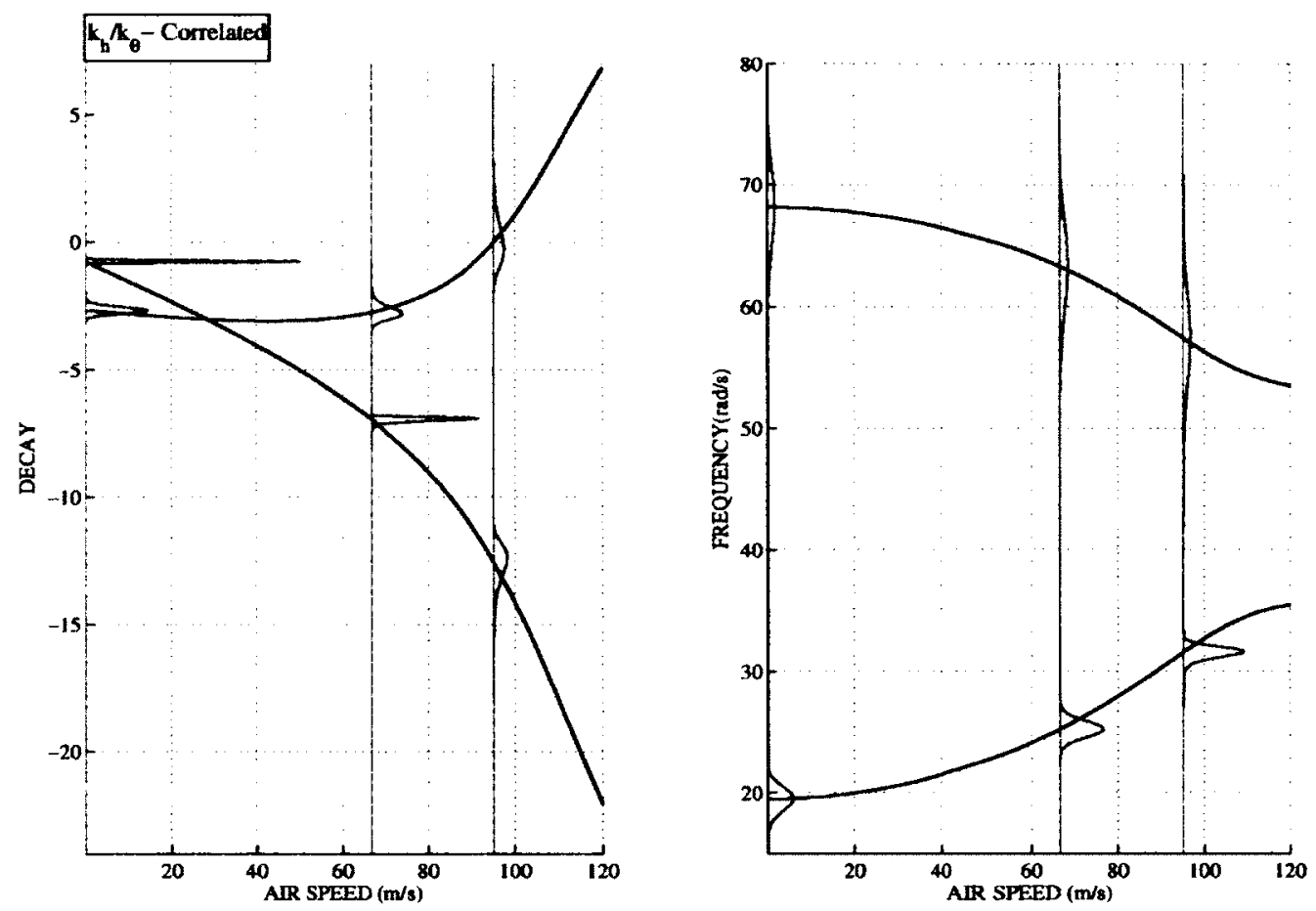

Figure 4.35: Modal parameter pdfs resulting from randomness in the correlated stiffness parameters with $10 \% \mathrm{COV}$, obtained at 3 test airspeeds: $0 \mathrm{~m} / \mathrm{s}, 68 \mathrm{~m} / \mathrm{s}, 95 \mathrm{~m} / \mathrm{s}$
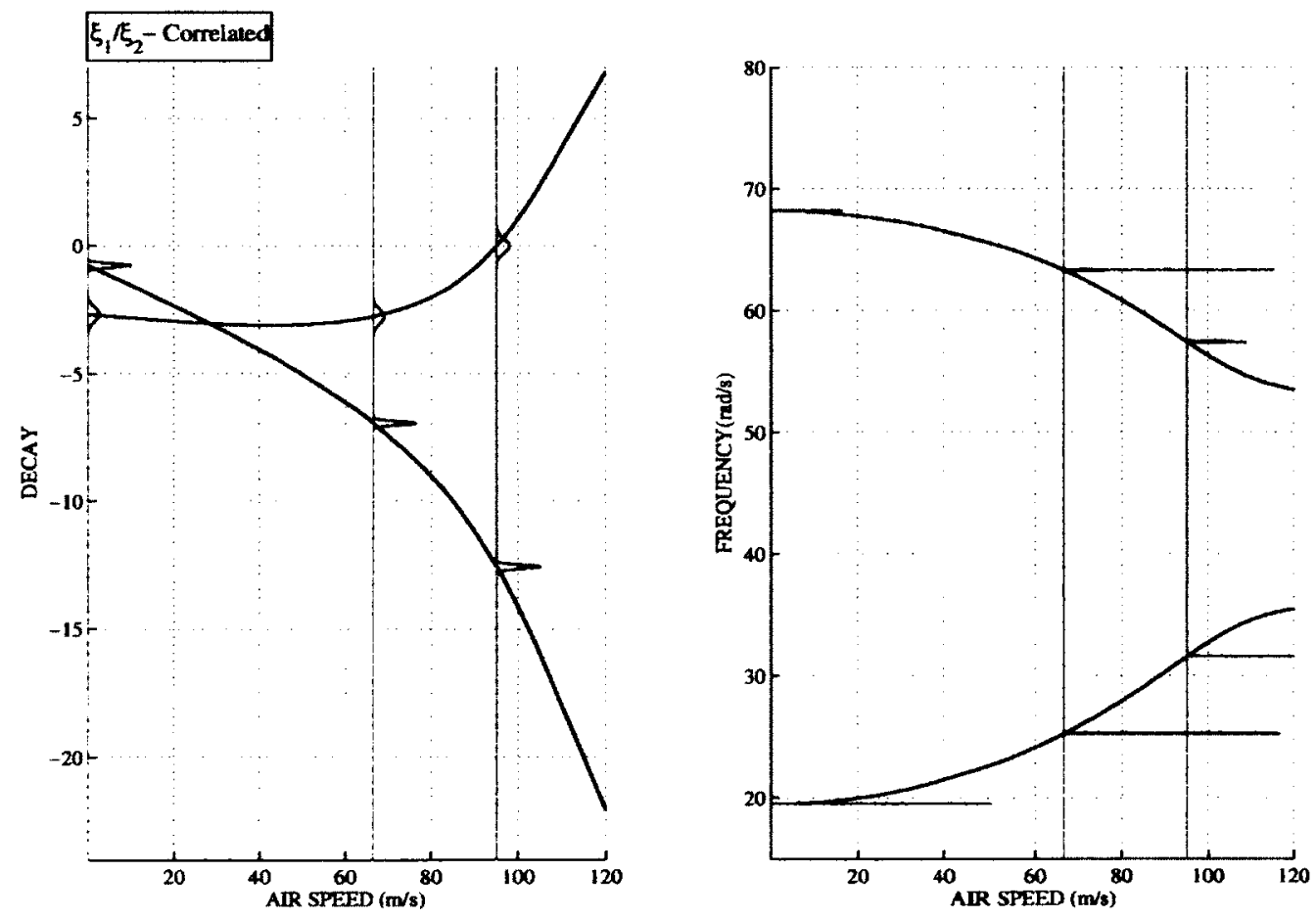

Figure 4.36: Modal parameter pdfs resulting from randomness correlated damping parameters with $10 \% \mathrm{COV}$, obtained at 3 test airspeeds: $0 \mathrm{~m} / \mathrm{s}, 68 \mathrm{~m} / \mathrm{s}, 95 \mathrm{~m} / \mathrm{s}$ 
frequencies and the modal decays, which leads to a reduced growth of variance in the modal decays with increasing airspeed. As a result, the flutter speed pdf yields a smaller variance for a fully correlated set of either inertia or stiffness parameters. The effects of correlation between the damping parameters are outlined next. Since the effects uncertainty in the modal frequencies are almost negligible for random damping parameters, the correlation between structural damping parameters only leads to correlation effects between the modal decays. In effect, a very slight increase of variance in the modal decays of coalescing modes is experienced, which leads to a slightly larger variance in the flutter speed pdf.

\subsubsection{Unsteady Results}

The probabilistic analysis of the effects of structural uncertainty on the transformations of the modal parameters of a system with unsteady aerodynamics is provided next. Once again, these results are very similar with the results for quasisteady aerodynamics, however, there is some influence that arises from a more explosive flutter type due to unsteady aerodynamics. The resulting observations for independent parameter variation are presented first.

Figs. (4.37) and (4.38) show the resulting transformations for random inertia parameters. The modal frequency and decay pdfs experience very large non-linear transformation effects and significant non-stationarity. The non-stationarity of the frequency pdfs is especially evident in the frequency coalescence region. For the case of uncertainty in the mass moment of inertia, as the modal frequencies coalesce, their pdfs produce overlapping effects. Uncertainty of inertia parameters produces more variance in the modal frequency pdfs of the heave dominant mode. Although the variance of the modal frequencies is much larger in the case with uncertainty in the mass moment of inertia parameter, it is expected that the substantial non-linear transformation trends as well as specific frequency correlation trends for the random mass parameter produce greater growth of variance in the decay of the flutter mode. The large negative skewness and a maximum cutoff value in the flutter mode decay near the flutter speed are assumed to be the cause of the minimum flutter speed cutoff and a positive skew in the flutter speed pdf. The variance of flutter speed is very small for both cases of uncertainty due to very large non-linear 

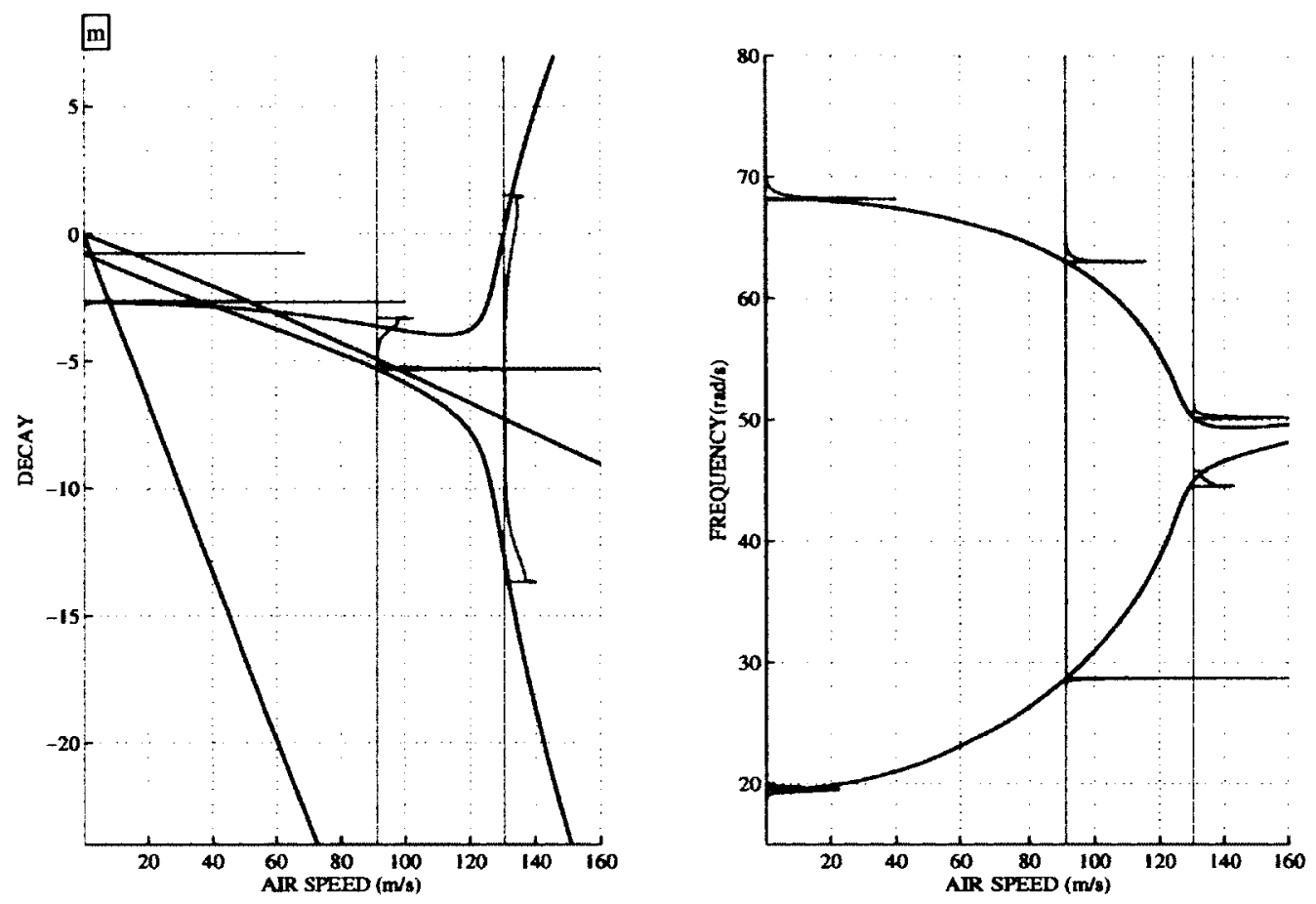

Figure 4.37: Modal parameter pdfs resulting from randomness in mass with $10 \% \mathrm{COV}$, obtained at 3 test airspeeds: $\approx 0 \mathrm{~m} / \mathrm{s}, 98 \mathrm{~m} / \mathrm{s}, 137 \mathrm{~m} / \mathrm{s}$
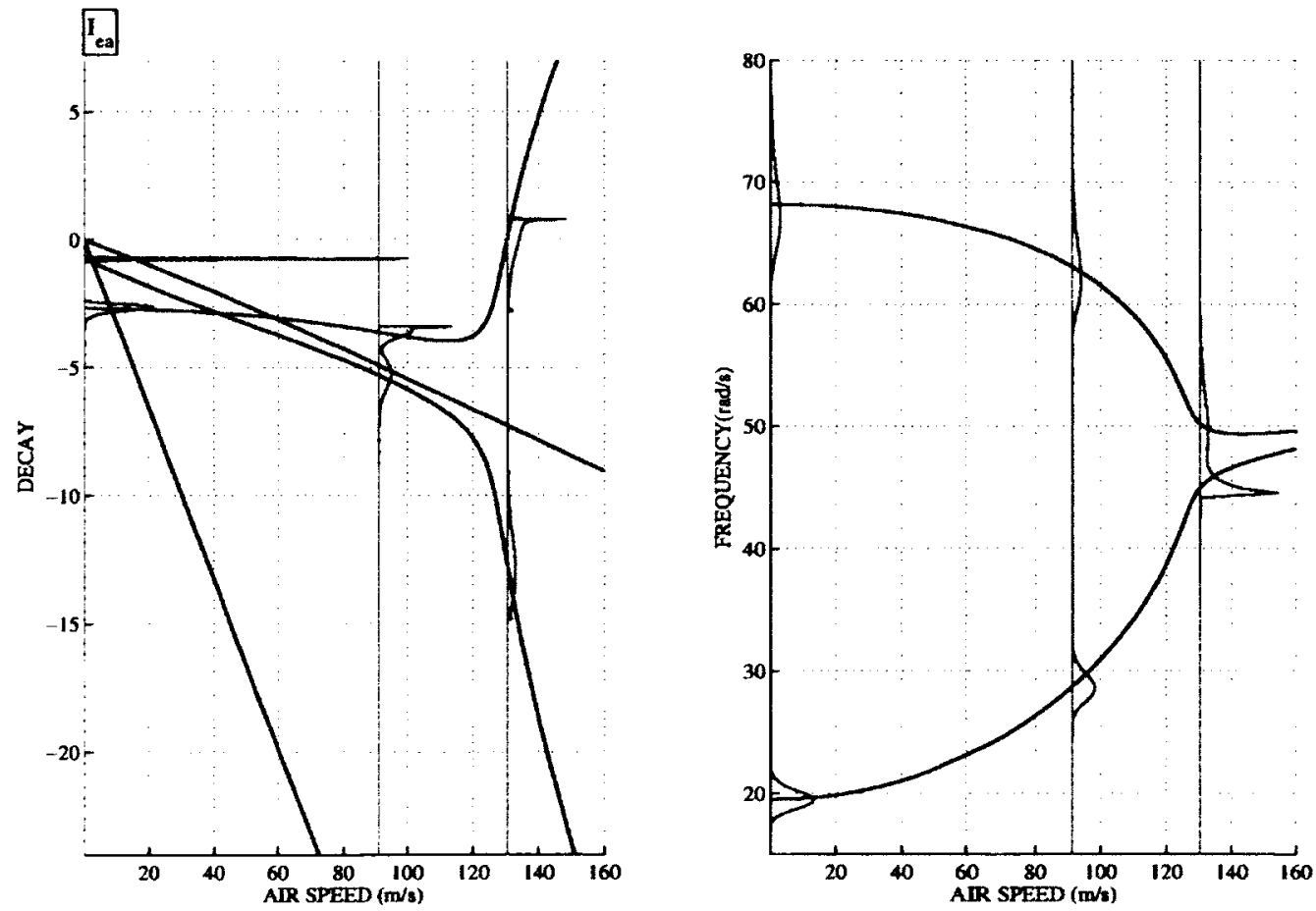

Figure 4.38: Modal parameter pdfs resulting from randomness in mass moment of inertia with $10 \%$ COV, obtained at 3 test airspeeds: $\approx 0 \mathrm{~m} / \mathrm{s}, 98 \mathrm{~m} / \mathrm{s}, 137 \mathrm{~m} / \mathrm{s}$ 
mapping features.

The effects of parameter uncertainty in the structural stiffness are illustrated next. Figs. (4.39) and (4.40) illustrate the modal parameter pdf transformations produced by uncertainty in the heave stiffness and pitch stiffness parameters, respectively. Prior to the flutter region, the uncertainty in heave stiffness parameter has a greater influence on the uncertainty in the frequency pdf of the heave-dominant (second) mode. Within the same region, the uncertainty in the pitch stiffness parameter has a greater influence on the uncertainty of the frequency pdf of the pitch-dominant (first) mode. For both cases, the modal frequency pdfs show a strong nonstationary trend in the flutter region, where the most significant frequency coalescence effects take place and dramatic changes in variances of frequencies are experienced in both modes. For case of uncertainty in the heave stiffness parameter, substantial non-linear transformation effects are noted in the first mode frequency pdf, in addition to a strong positive skewness in the decay of the flutter mode. A very significant growth of flutter mode decay variance in the flutter region produces a large variance in the flutter speed pdf. For the case of uncertainty in the pitch
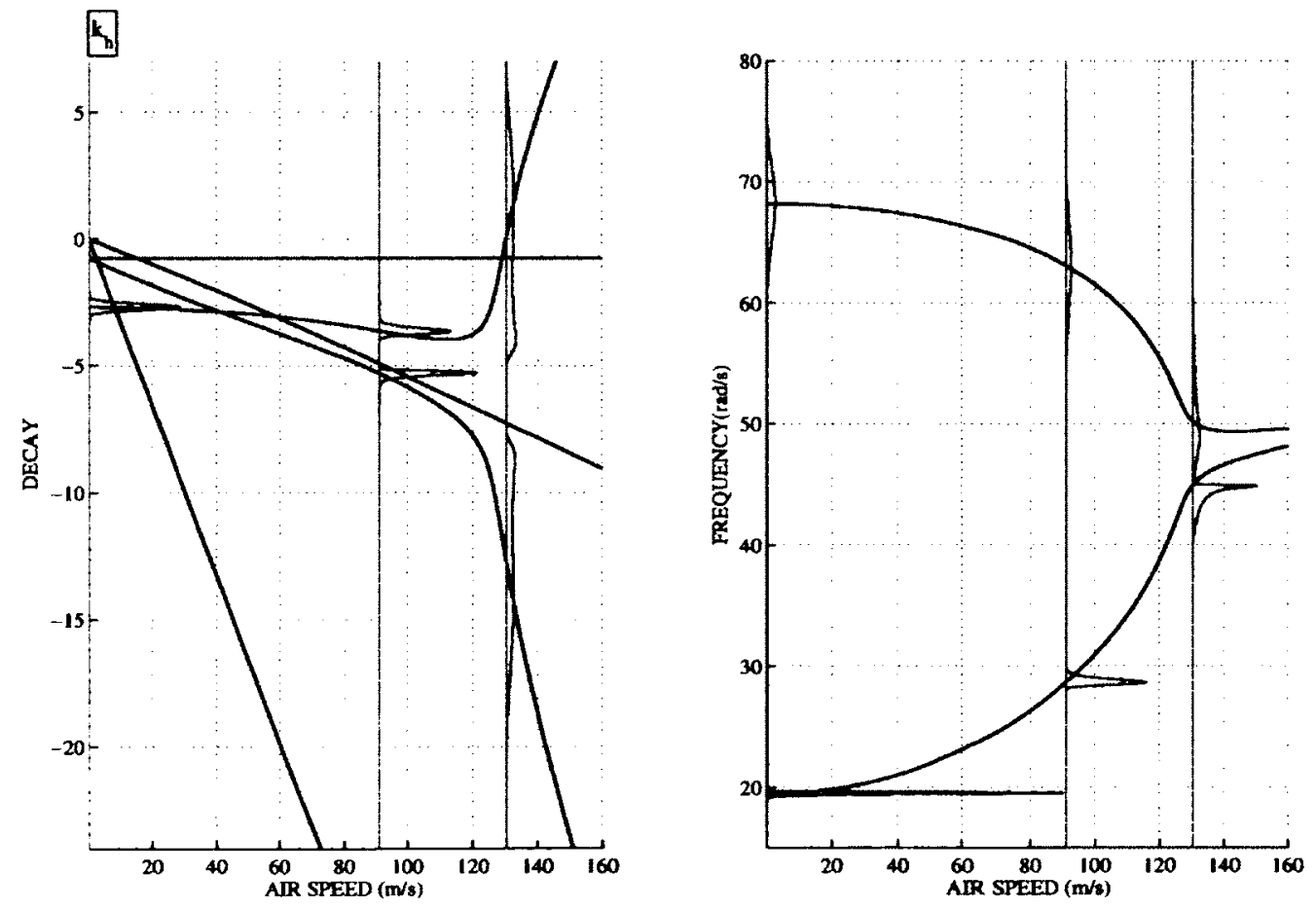

Figure 4.39: Modal parameter pdfs resulting from randomness in heave stiffness with $10 \%$ COV, obtained at 3 test airspeeds: $\approx 0 \mathrm{~m} / \mathrm{s}, 98 \mathrm{~m} / \mathrm{s}, 137 \mathrm{~m} / \mathrm{s}$ 

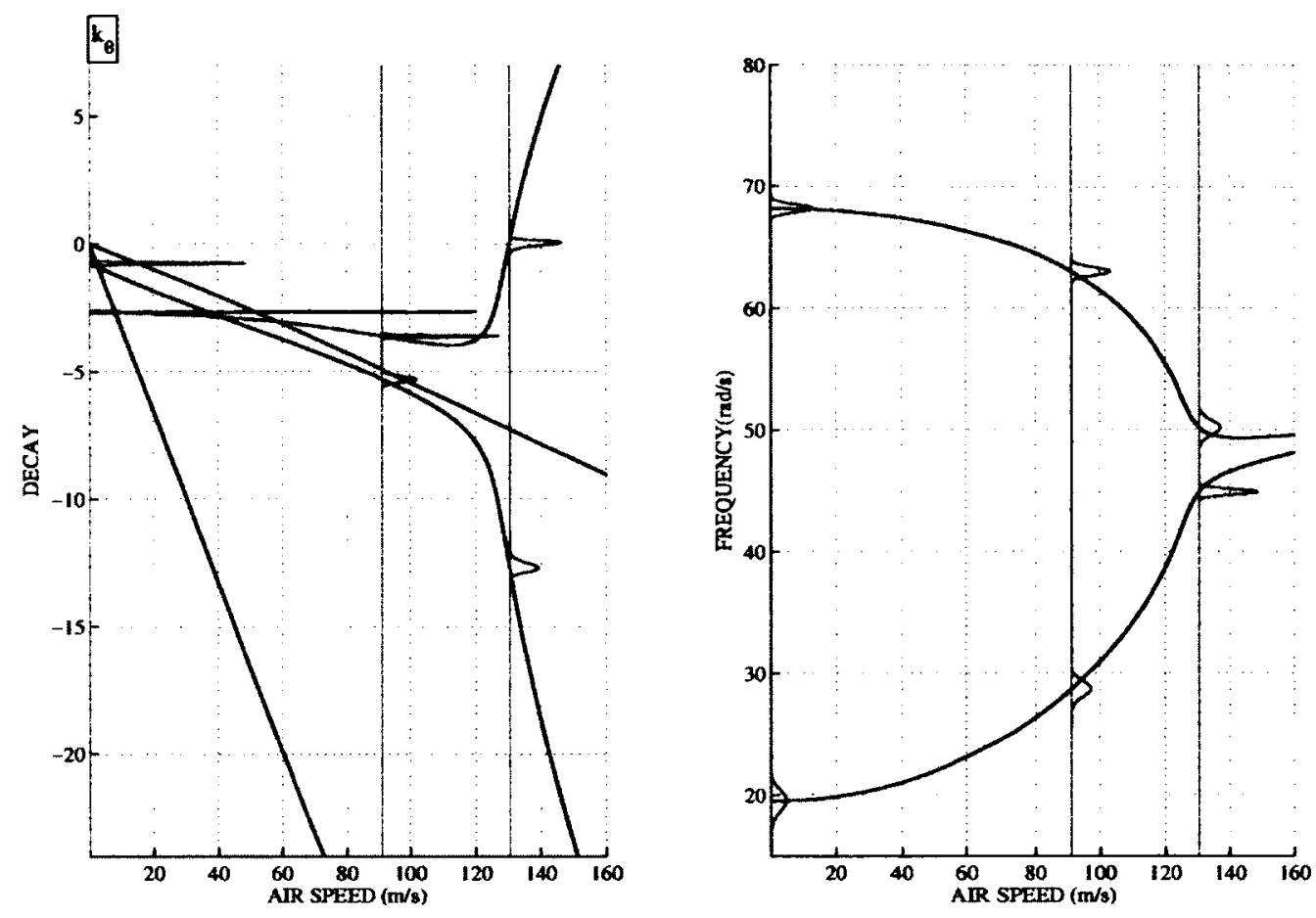

Figure 4.40: Modal parameter pdfs resulting from randomness in pitch stiffness with $10 \%$ COV, obtained at 3 test airspeeds: $\approx 0 \mathrm{~m} / \mathrm{s}, 98 \mathrm{~m} / \mathrm{s}, 137 \mathrm{~m} / \mathrm{s}$

stiffness, due to a minor variance in the flutter mode decay pdf, the flutter speed pdf produces only low variance.

Next, figs. (4.41) and (4.42) illustrate the effect of uncertainty in the structural damping ratio parameters. There are no significant uncertainty effects on the modal frequencies. For the uncertainty in the structural damping ratio of the first mode, the first aeroelastic mode experiences the greater variance. The second mode experiences the greater variance for uncertainty in the second structural damping ratio. As the dominant modal features of the eigenvectors change near the flutter region, so does the relative magnitude of variance in the modal decays. Lastly, due to the explosive nature of flutter type, and a fairly low and almost constant variance in the modal decay of the flutter mode the flutter speed pdf results in a low variance. 

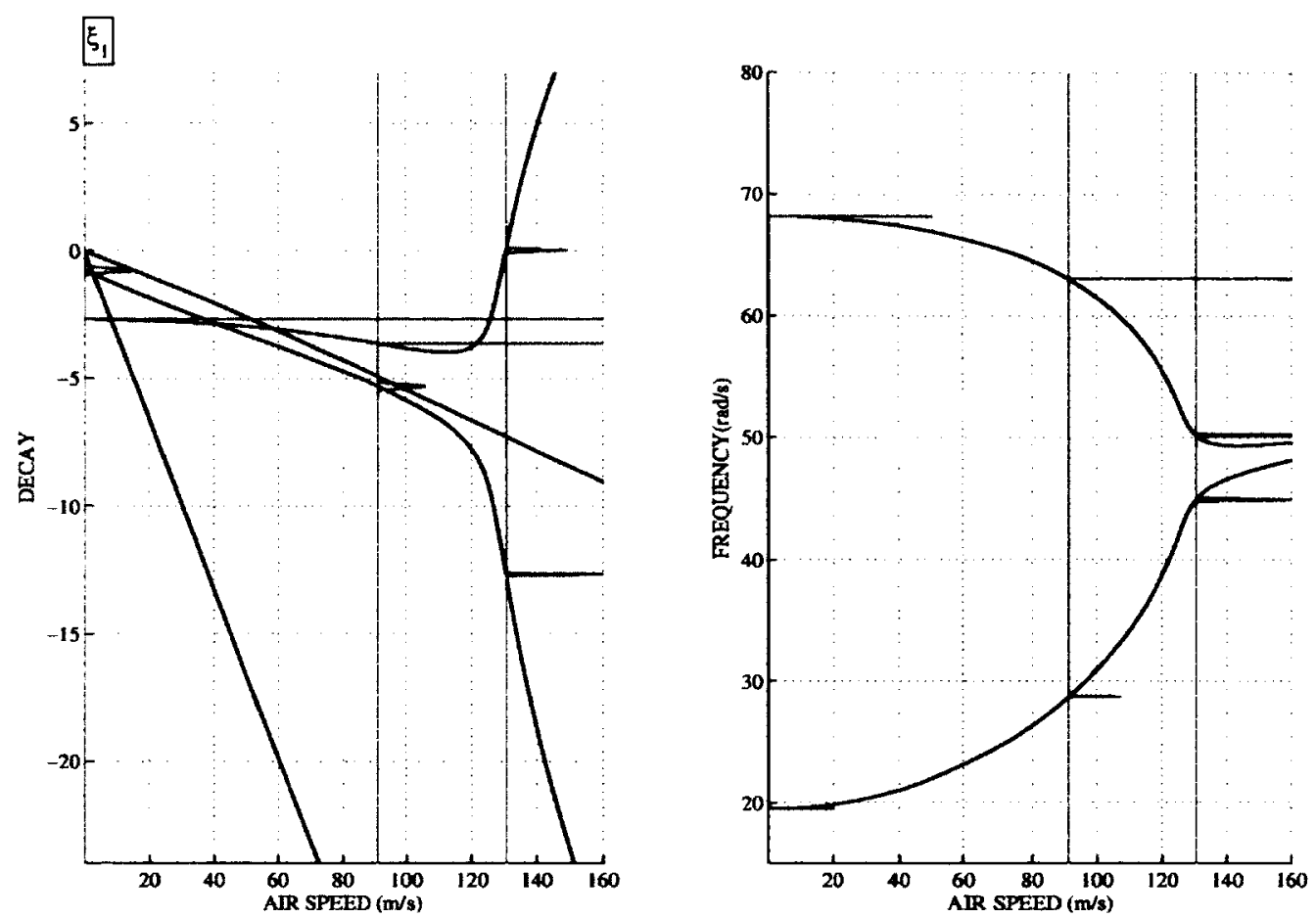

Figure 4.41: Modal parameter pdfs resulting from randomness in the $1^{\text {st }}$ modal damping ratio with $10 \%$ COV, obtained at 3 test airspeeds: $\approx 0 \mathrm{~m} / \mathrm{s}, 98 \mathrm{~m} / \mathrm{s}, 137 \mathrm{~m} / \mathrm{s}$
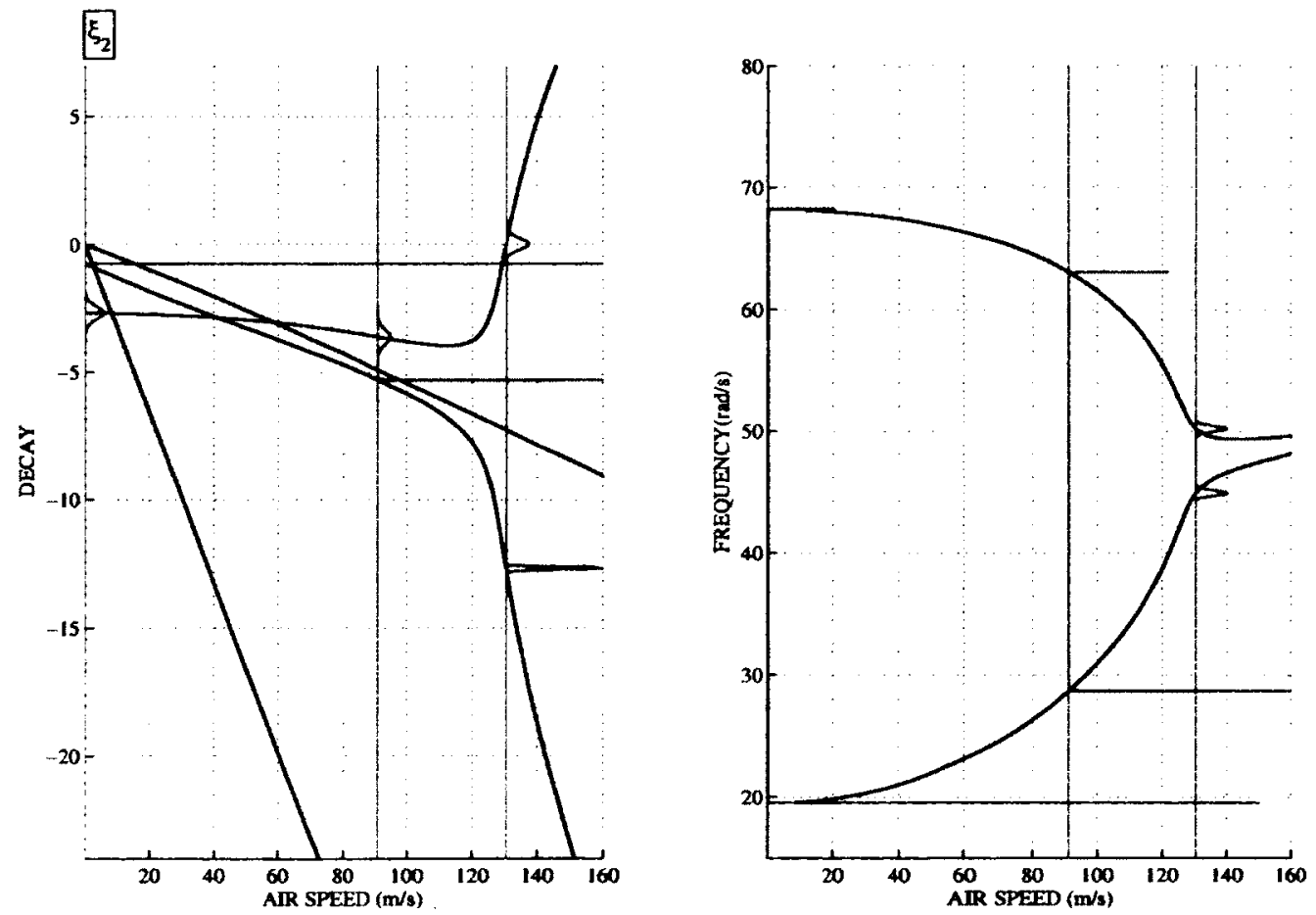

Figure 4.42: Modal parameter pdfs resulting from randomness in the $2^{\text {nd }}$ modal damping ratio with $10 \%$ COV, obtained at 3 test airspeeds: $\approx 0 \mathrm{~m} / \mathrm{s}, 98 \mathrm{~m} / \mathrm{s}, 137 \mathrm{~m} / \mathrm{s}$ 


\section{Multiple Parameter Uncertainty}

The effect of combined uncertainty in the pair of parameters will be examined next. First, the results for fully uncorrelated random parameters are shown. Figs. (4.43)-(4.45) show the pdfs of the modal parameters as a function of airspeed for random inertia, stiffness and damping parameters, respectively. When uncertain parameters are uncorrelated, the variance in the modal parameters increases in relation to the cases of individual uncertain parameters.

Now, the effects of full parameter correlation are examined. Figs. (4.46)-(4.48) illustrate the probabilistic modal parameter transformation results for fully correlated inertia, stiffness and damping parameters, respectively. From figs. (4.46), (4.47) and (3.26), the following observations for inertia and stiffness parameter uncertainties are noted. The uncertainty in the aeroelastic frequencies does not depend significantly on the correlation between the random inertia or stiffness parameters. While considering the uncertainty in the inertia or stiffness properties, the assumed correlation effects between the modal parameters produce a reduced growth of variance in the modal decays, showing a smaller variance in the flutter speed pdfs.
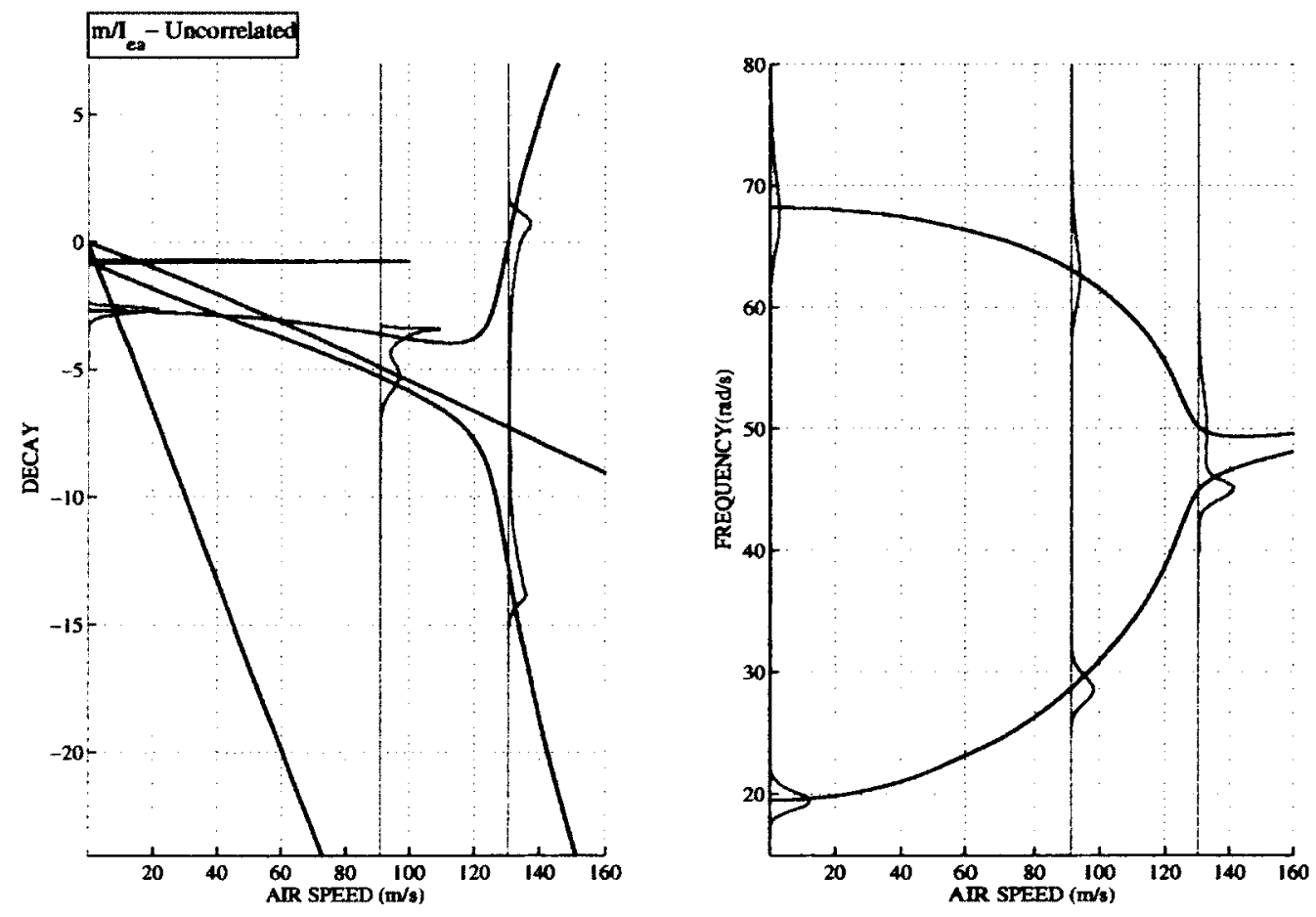

Figure 4.43: Modal parameter pdfs resulting from randomness in the uncorrelated inertia parameters with $10 \%$ COV, obtained at 3 test airspeeds: $\approx 0 \mathrm{~m} / \mathrm{s}, 98 \mathrm{~m} / \mathrm{s}, 137 \mathrm{~m} / \mathrm{s}$ 

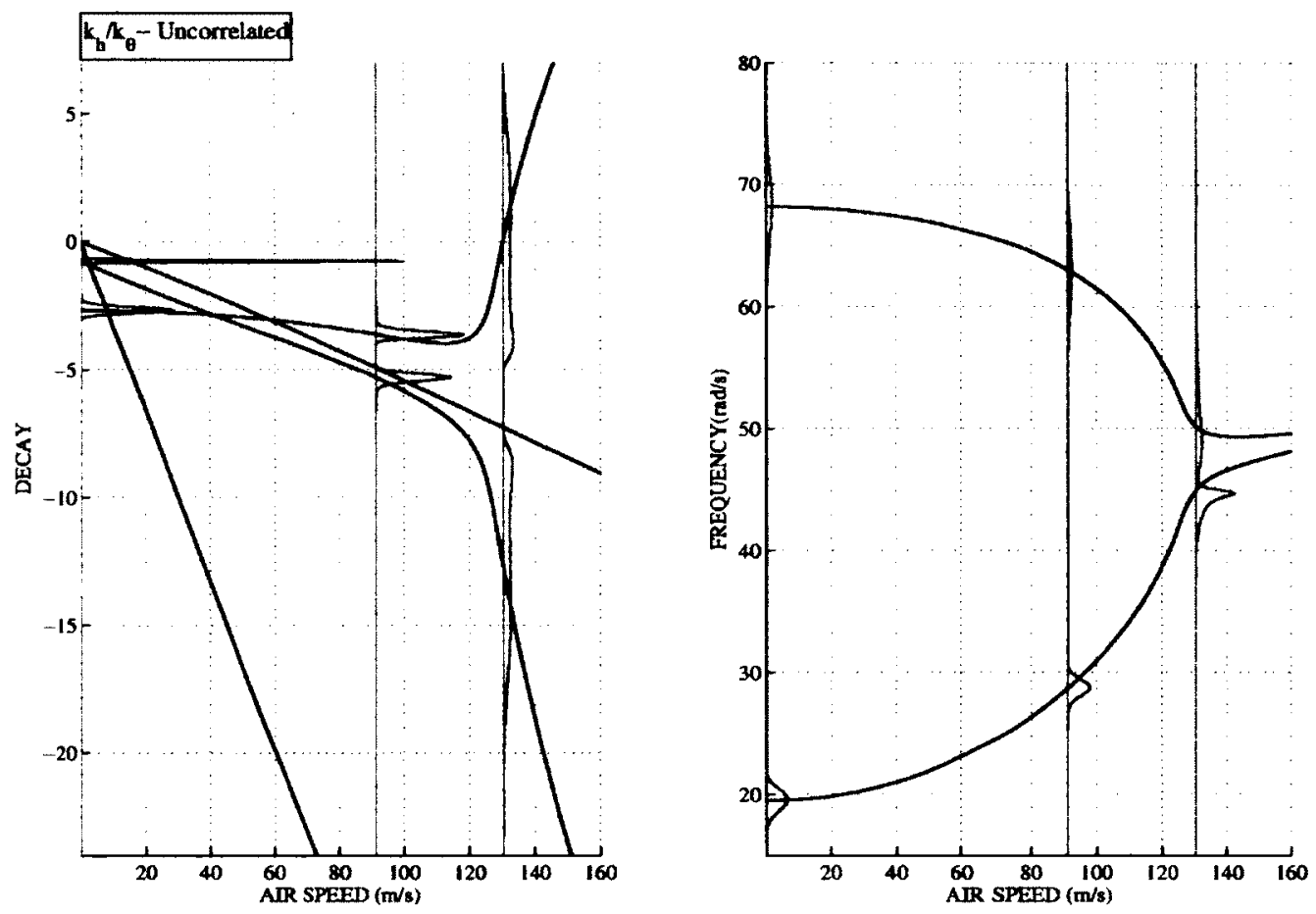

Figure 4.44: Modal parameter pdfs resulting from randomness in the uncorrelated stiffness parameters with $10 \% \mathrm{COV}$, obtained at 3 test airspeeds: $\approx 0 \mathrm{~m} / \mathrm{s}, 98 \mathrm{~m} / \mathrm{s}, 137 \mathrm{~m} / \mathrm{s}$
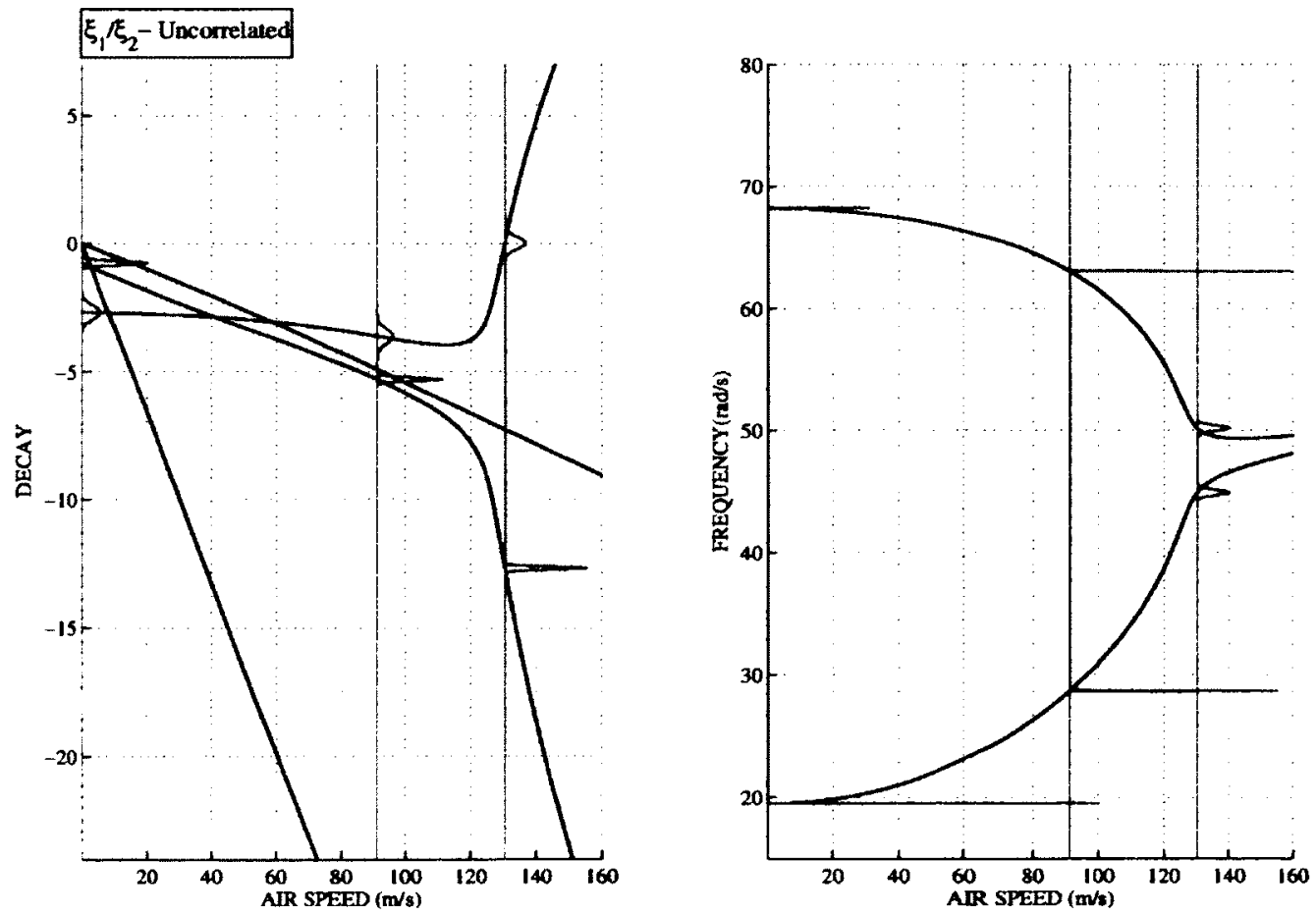

Figure 4.45: Modal parameter pdfs resulting from randomness in the uncorrelated damping parameters with $10 \% \mathrm{COV}$, obtained at 3 test airspeeds: $\approx 0 \mathrm{~m} / \mathrm{s}, 98 \mathrm{~m} / \mathrm{s}, 137 \mathrm{~m} / \mathrm{s}$ 
Additionally, it should be noted that correlation between the inertia parameters eliminates the strong non-linear transformation effects that were observed for the independent parameter variations.

From fig. (4.48) and fig. (3.26), the effects of correlation in the structural damping parameters are examined. The following observations are made. The uncertainty effects in the modal frequencies are almost negligible, thus the effects of parameter correlation are negligible in the frequency pdfs. However, there are certain correlation effects in the modal decays due to correlation between the structural damping parameters, which result in an increased variance of the modal decay of the flutter mode at all airspeeds. Such increase of variance the flutter mode decay produces an increased variance of the flutter speed pdf.

\subsubsection{Comparison of Quasisteady and Unsteady Results}

Similar to Case 1, a comparison of the effects of unsteady and quasisteady aerodynamic forcing indicates that the modal frequencies of the coalescing modes experience statistical overlaps for
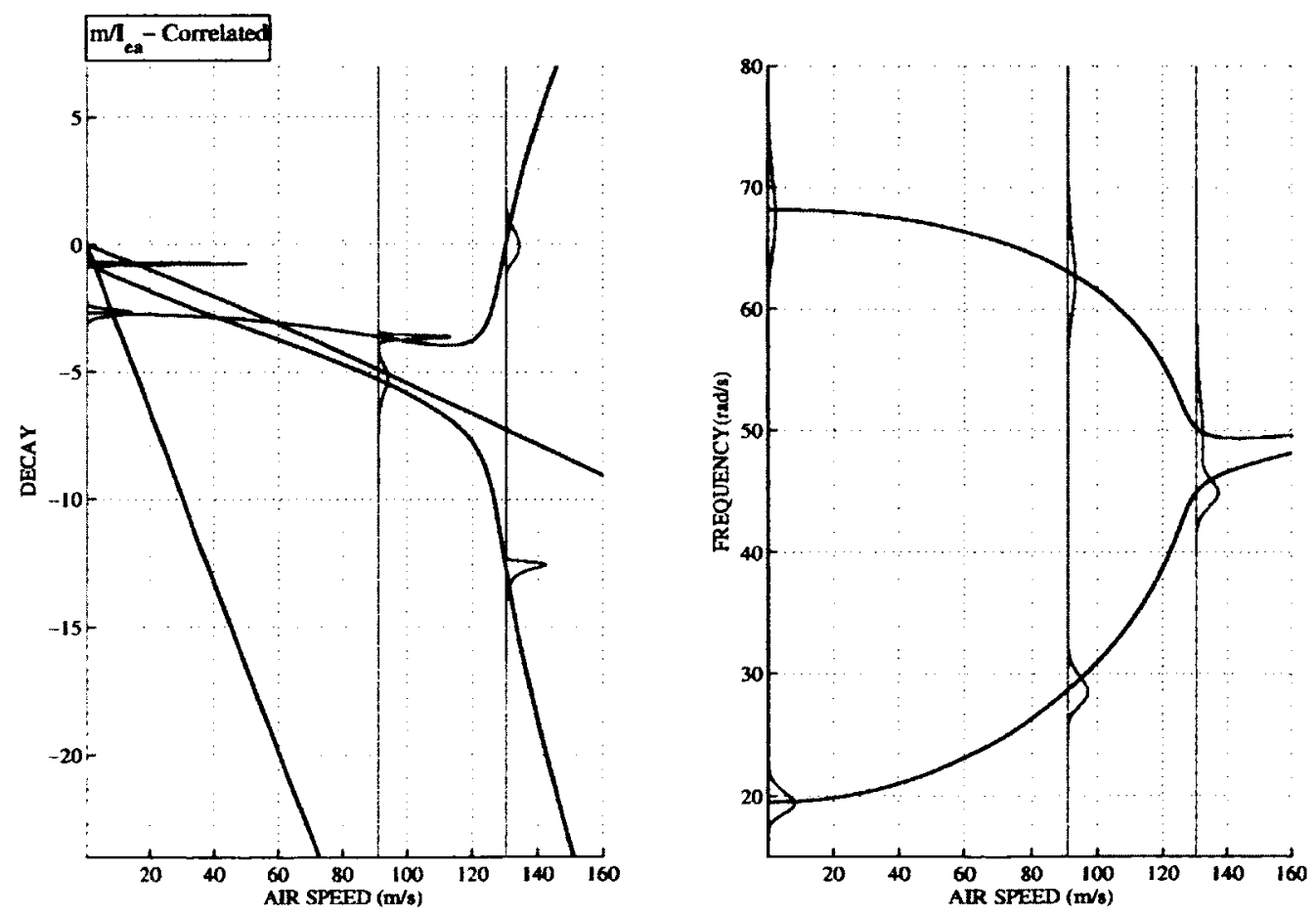

Figure 4.46: Modal parameter pdfs resulting from randomness in the correlated inertia parameters with $10 \% \mathrm{COV}$, obtained at 3 test airspeeds: $\approx 0 \mathrm{~m} / \mathrm{s}, 98 \mathrm{~m} / \mathrm{s}, 137 \mathrm{~m} / \mathrm{s}$ 

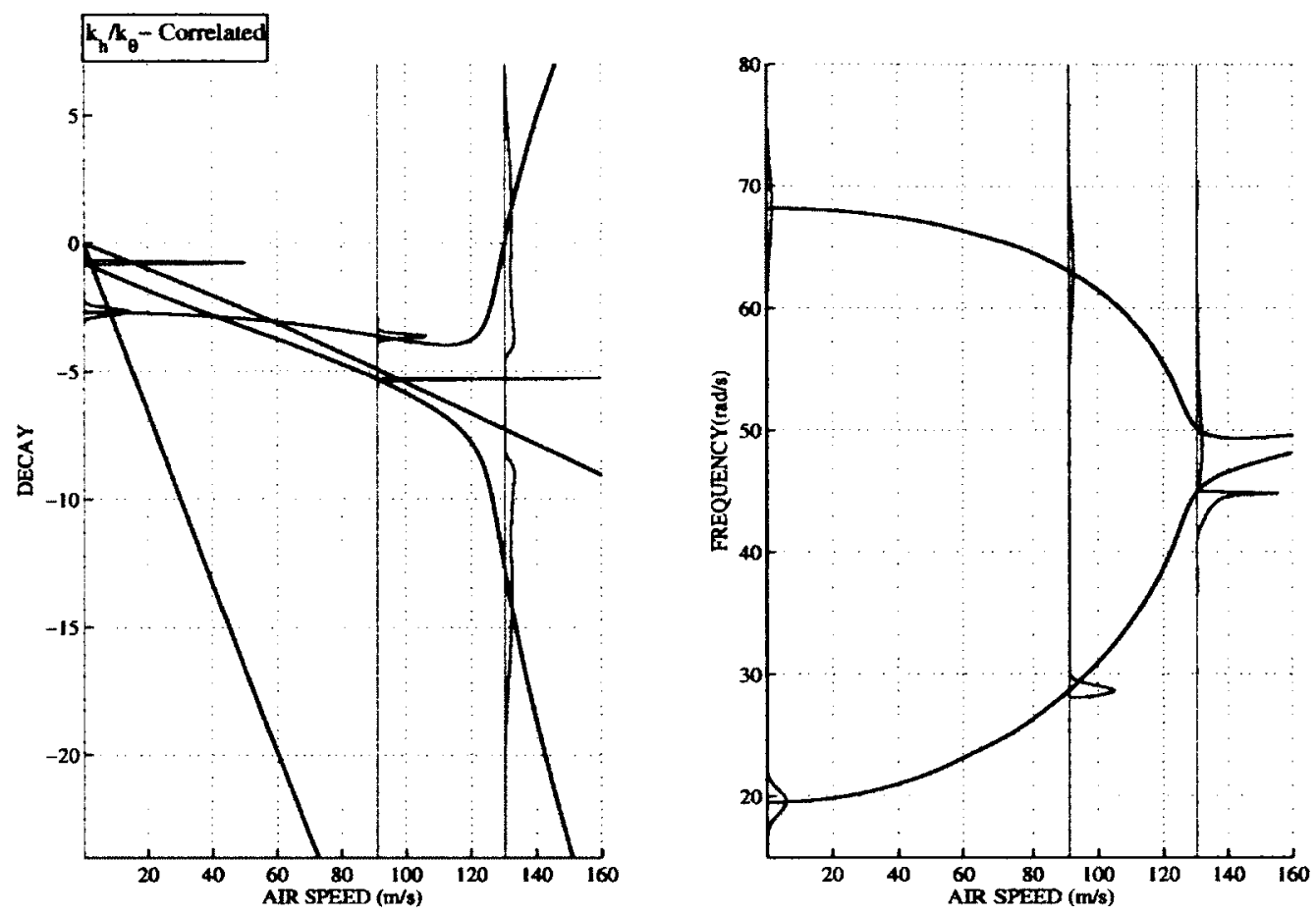

Figure 4.47: Modal parameter pdfs resulting from randomness in the correlated stiffness parameters with $10 \% \mathrm{COV}$, obtained at 3 test airspeeds: $\approx 0 \mathrm{~m} / \mathrm{s}, 98 \mathrm{~m} / \mathrm{s}, 137 \mathrm{~m} / \mathrm{s}$
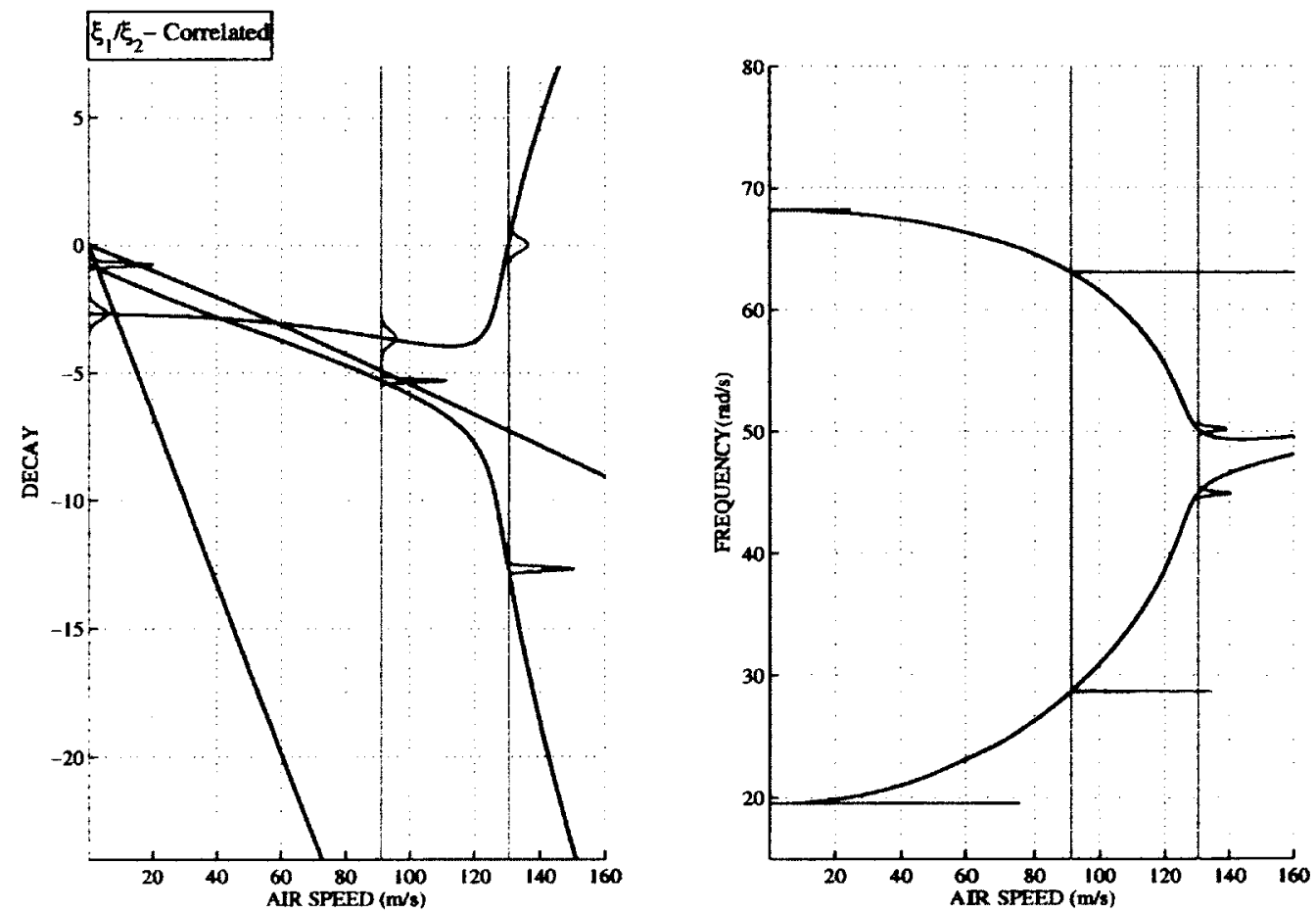

Figure 4.48: Modal parameter pdfs resulting from randomness correlated damping parameters with $10 \% \mathrm{COV}$, obtained at 3 test airspeeds: $\approx 0 \mathrm{~m} / \mathrm{s}, 98 \mathrm{~m} / \mathrm{s}, 137 \mathrm{~m} / \mathrm{s}$ 
unsteady aerodynamics. Additionally, a non-stationary trend of the aeroelastic frequencies of the coalescing modes near the flutter point. Lastly, significant non-linear transformation trends in the modal parameters produced by uncertainty in the inertia parameters leads to low variance in the flutter speed pdfs.

\subsection{CASE 3}

\subsubsection{Quasisteady Results}

This case is devoted primarily to highlight the strong non-linear dependence among the flutter speed and the structural parameters and how this effect manifests in the probabilistic characteristics of the modal parameters and the flutter speed. For brevity, this case study will only focus on the analysis with quasisteady aerodynamics.

Figs.(4.49)-(4.54) illustrate the effects of random inertia, stiffness and damping parameters, respectively, on the pdfs of the modal parameters as function of airspeed. The probabilistic flutter speed results from fig. (3.33) are examined in parallel. Significant non-linear effects are evident in the modal decays for cases of random inertia and stiffness parameters. For uncertainty in the mass and mass moment of inertia, depicted by figs. (4.49) and (4.50), the following observations are noted. The modal frequency pdfs show almost stationary trend, showing only some signs of non-stationarity in the most significant frequency coalescence region, where substantial statistical overlaps are observed. The modal decay pdfs show very strong non-stationary trends, with a growth of variance near the flutter region. For uncertainty in the mass parameter, the second (flutter) mode frequency distribution is non-Gaussian, experiencing a minimum frequency cutoff and a significant positive skewness. The first mode frequency distribution is approximately Gaussian. The modal decay pdf of the flutter mode has a heavy negative skewness with a cutoff at maximum decay value. For a random mass moment of inertia parameter, both modal frequency distributions are approximately Gaussian. The modal decay pdf of the flutter mode has a heavy negative skewness with a cutoff at maximum decay value. An assumption is made that such non-linear mapping features along with a growth of variance of the 
106
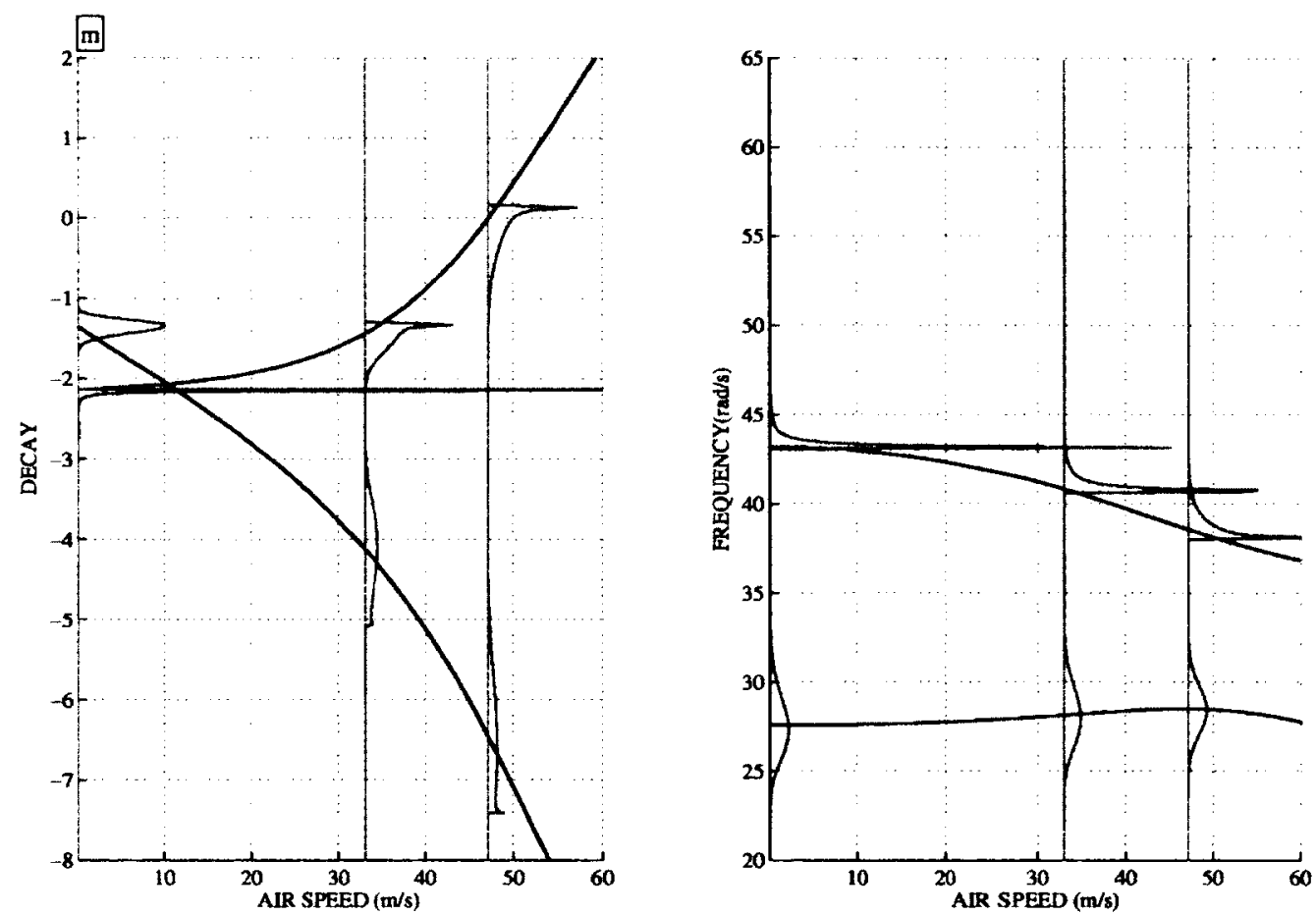

Figure 4.49: Modal parameter pdf resulting from randomness in mass with $17.5 \% \mathrm{COV}$, obtained at 3 test airspeeds: $0 \mathrm{~m} / \mathrm{s}, 33 \mathrm{~m} / \mathrm{s}, 47 \mathrm{~m} / \mathrm{s}$
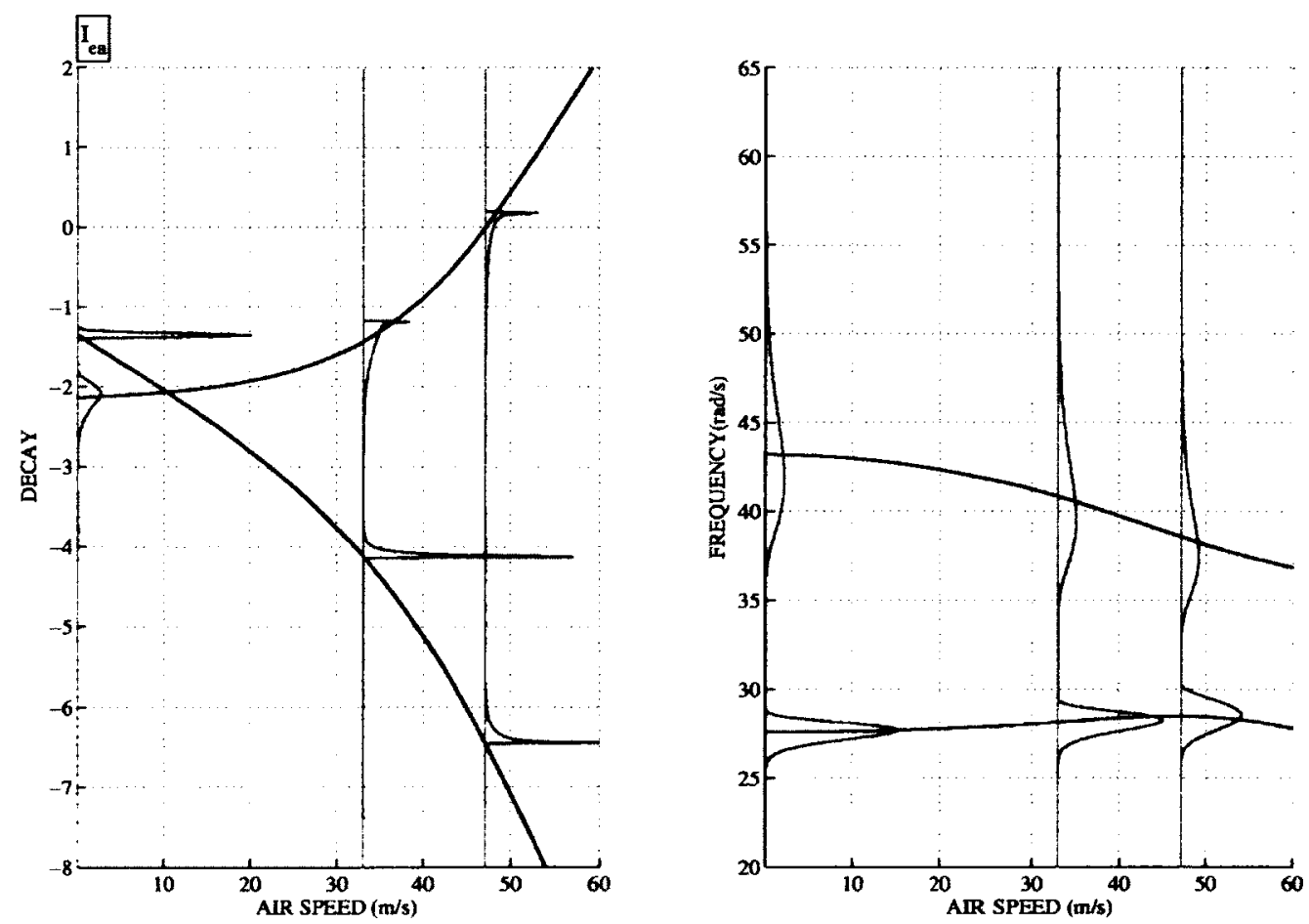

Figure 4.50: Modal parameter pdfs resulting from randomness in mass moment of inertia with $17.5 \% \mathrm{COV}$, obtained at 3 test airspeeds: $0 \mathrm{~m} / \mathrm{s}, 33 \mathrm{~m} / \mathrm{s}, 47 \mathrm{~m} / \mathrm{s}$ 
flutter mode decay in the flutter region correspond to a flutter speed pdf with a minimum cutoff, large positive skewness and a significant variance.

For the random heave and pitch stiffness parameters, the results are provided in figs. (4.51)(4.52). The modal frequency pdfs show an almost stationary trend, showing only some signs of non-stationarity in the region of frequency coalescence. The modal frequency distributions contain only slight skewness. For a random heave stiffness parameter, the modal decay pdfs show non-stationary trends with significant non-linear effects. The modal decay pdf of the flutter mode has a heavy negative skewness with a cutoff at maximum decay value. Such non-linear features correspond to a flutter speed pdf with a minimum cutoff and large positive skewness. The flutter mode decay pdf does not experience significant growth of variance in the flutter region, producing a low variance in the flutter speed pdf. For a random pitch stiffness parameter, the modal decay pdfs show very strong non-stationary trends, with a growth of variance near the flutter region. At flutter point, the modal decay pdf of the flutter mode has a heavy negative skewness with a cutoff at a maximum decay value. Such non-linear features and the growth of
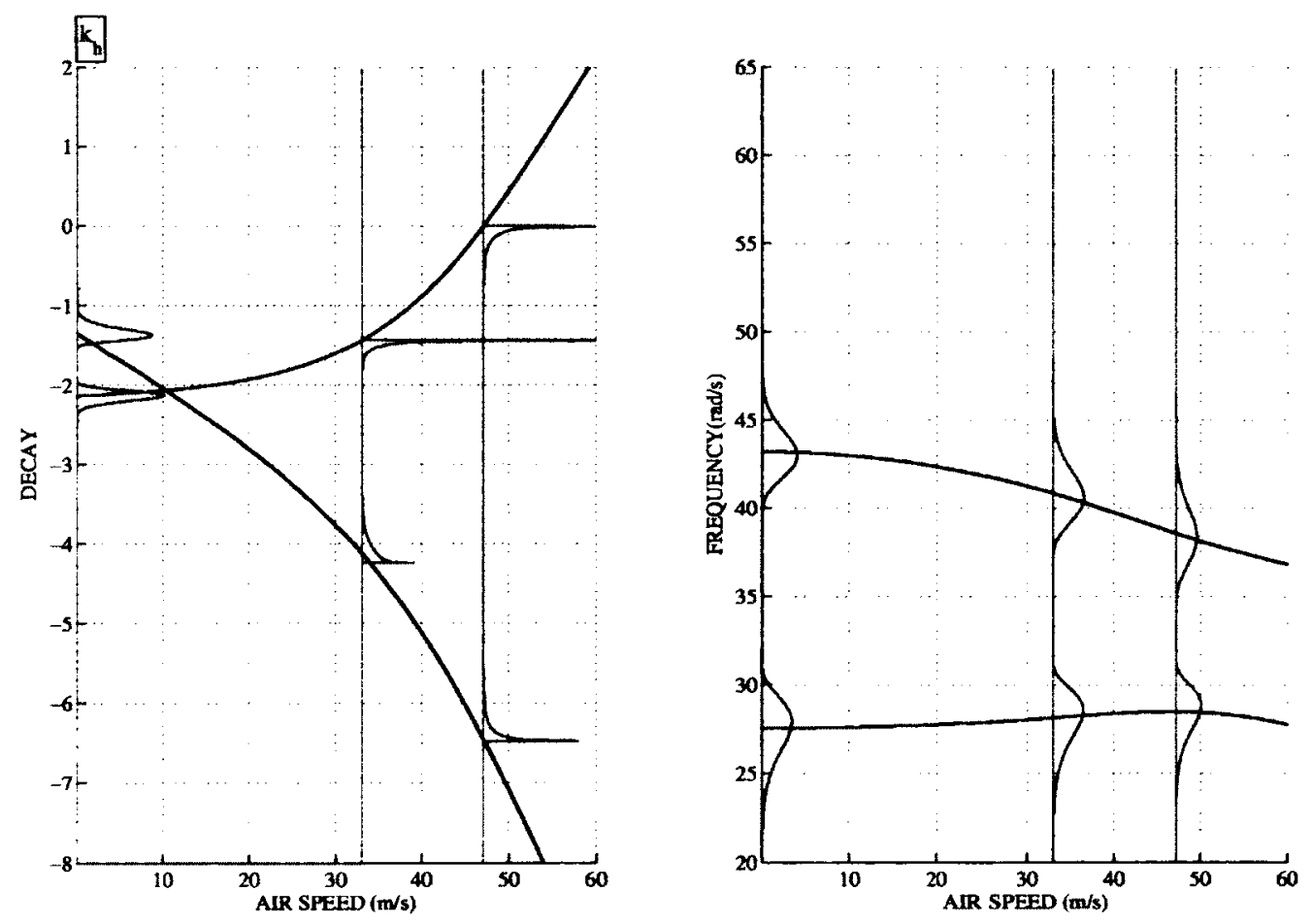

Figure 4.51: Modal parameter pdfs resulting from randomness in heave stiffness with $17.5 \%$ COV, obtained at 3 test airspeeds: $0 \mathrm{~m} / \mathrm{s}, 33 \mathrm{~m} / \mathrm{s}, 47 \mathrm{~m} / \mathrm{s}$ 

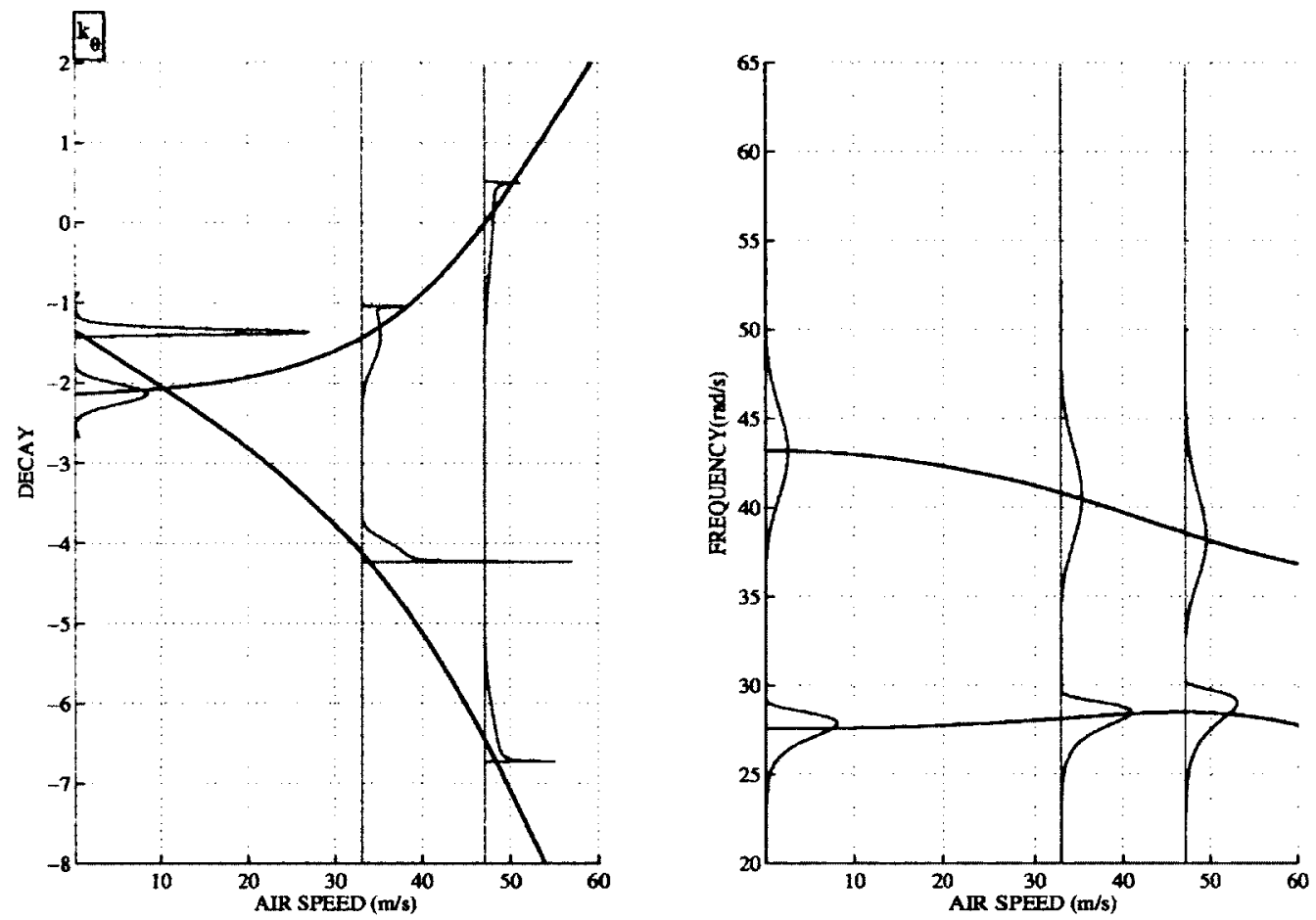

Figure 4.52: Modal parameter pdfs resulting from randomness in pitch stiffness with $17.5 \%$ COV, obtained at 3 test airspeeds: $0 \mathrm{~m} / \mathrm{s}, 33 \mathrm{~m} / \mathrm{s}, 47 \mathrm{~m} / \mathrm{s}$

variance in the flutter region of the flutter mode decay correspond to a flutter speed pdf with a minimum cutoff, large positive skewness and a significant variance, as evident in fig. (3.33).

The results of uncertainty in the damping parameters are examined in figs. (4.53) and (4.54). The observations are similar to Case 1 or Case 2 studies with quasisteady aerodynamics. There are no significant uncertainty effects on the modal frequencies. For uncertainty in the first structural damping ratio, a first mode experiences the greater variance. Similarly, for uncertainty in the second structural damping ratio, a second mode experiences the greater variance. As the dominant modal features of the eigenvectors changes near the flutter region, so does the relative magnitude of variance in the modal decays. Although the variance in decay of the flutter mode is almost constant, due to a mild flutter type the variance of the flutter speed pdf is fairly large. 

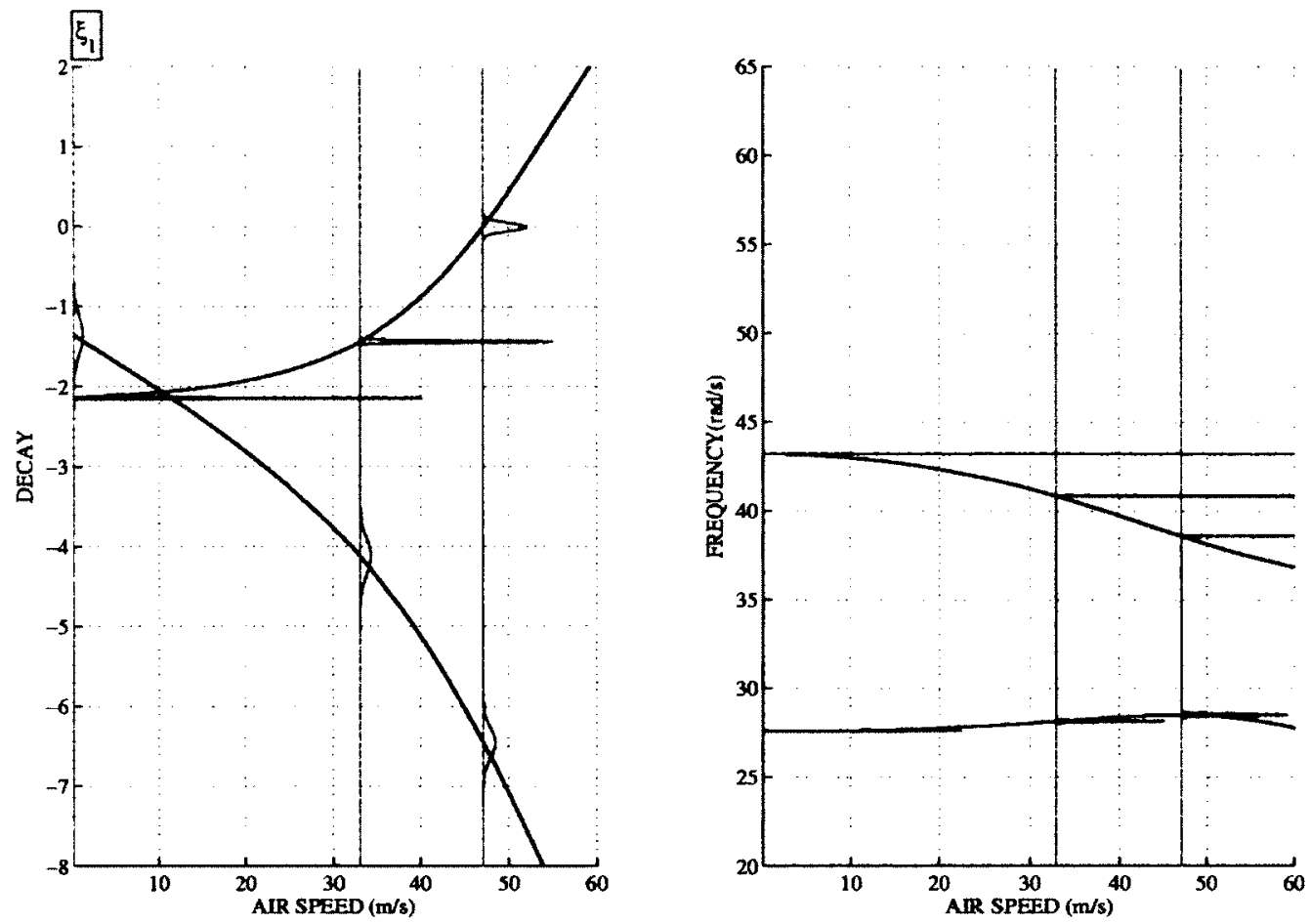

Figure 4.53: Modal parameter pdfs resulting from randomness in the $1^{\text {st }}$ modal damping ratio with $17.5 \% \mathrm{COV}$, obtained at 3 test airspeeds: $0 \mathrm{~m} / \mathrm{s}, 33 \mathrm{~m} / \mathrm{s}, 47 \mathrm{~m} / \mathrm{s}$
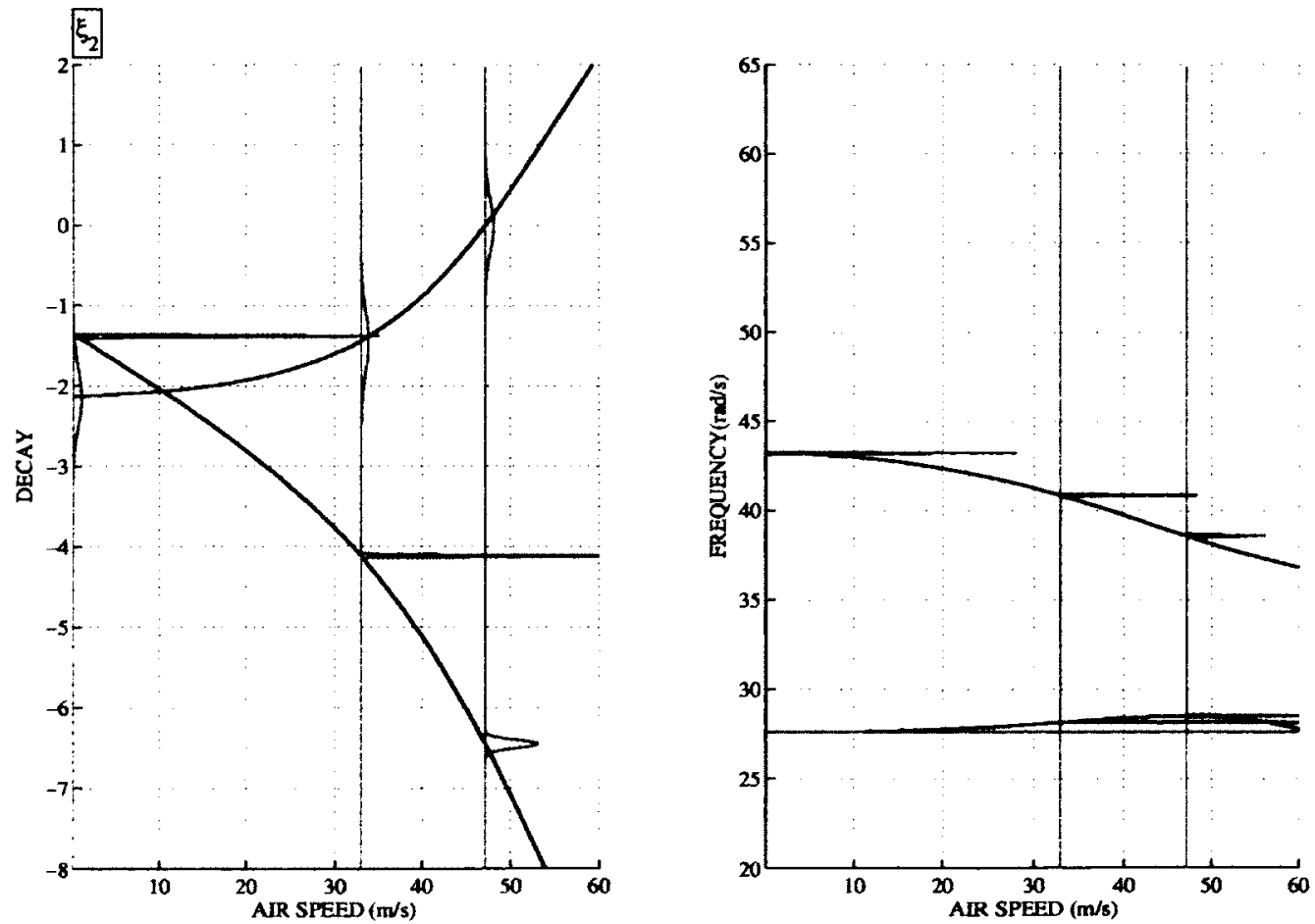

Figure 4.54: Modal parameter pdfs resulting from randomness in the $2^{\text {nd }}$ modal damping ratio with $17.5 \% \mathrm{COV}$, obtained at 3 test airspeeds: $0 \mathrm{~m} / \mathrm{s}, 33 \mathrm{~m} / \mathrm{s}, 47 \mathrm{~m} / \mathrm{s}$ 


\section{Multiple Parameter Uncertainty}

The uncertainty for parameter pairs will be illustrated next with the probabilistic results. Figs. (4.55)-(4.57) illustrate the effect of uncertainty in the uncorrelated structural inertia, stiffness and damping parameters. Figs. (4.58)-(4.60) show the effect of correlation between the same random structural parameters. For this case, the effects of uncertainty in multiple random parameters becomes more complex due to very strong non-linear effects. The uncertainty in the uncorrelated random inertia, stiffness and damping parameters produces a larger uncertainty in the modal parameters, which leads to a variance increase in the flutter speed pdf. For stiffness uncertainty, the non-linear mapping effects in the flutter mode decay pdf vanish.

The resulting observations from the effect of parameter correlation are summarized below. For inertia parameter uncertainty, the variance of the flutter mode decay pdfs decreases, perhaps leading to a low variance in the flutter speed pdf. For uncertainty in the stiffness parameters, the variance of the modal frequency and the flutter mode decay pdfs increases, perhaps resulting in a larger variance of the flutter speed pdf. The non-linear mapping effects in the flutter mode
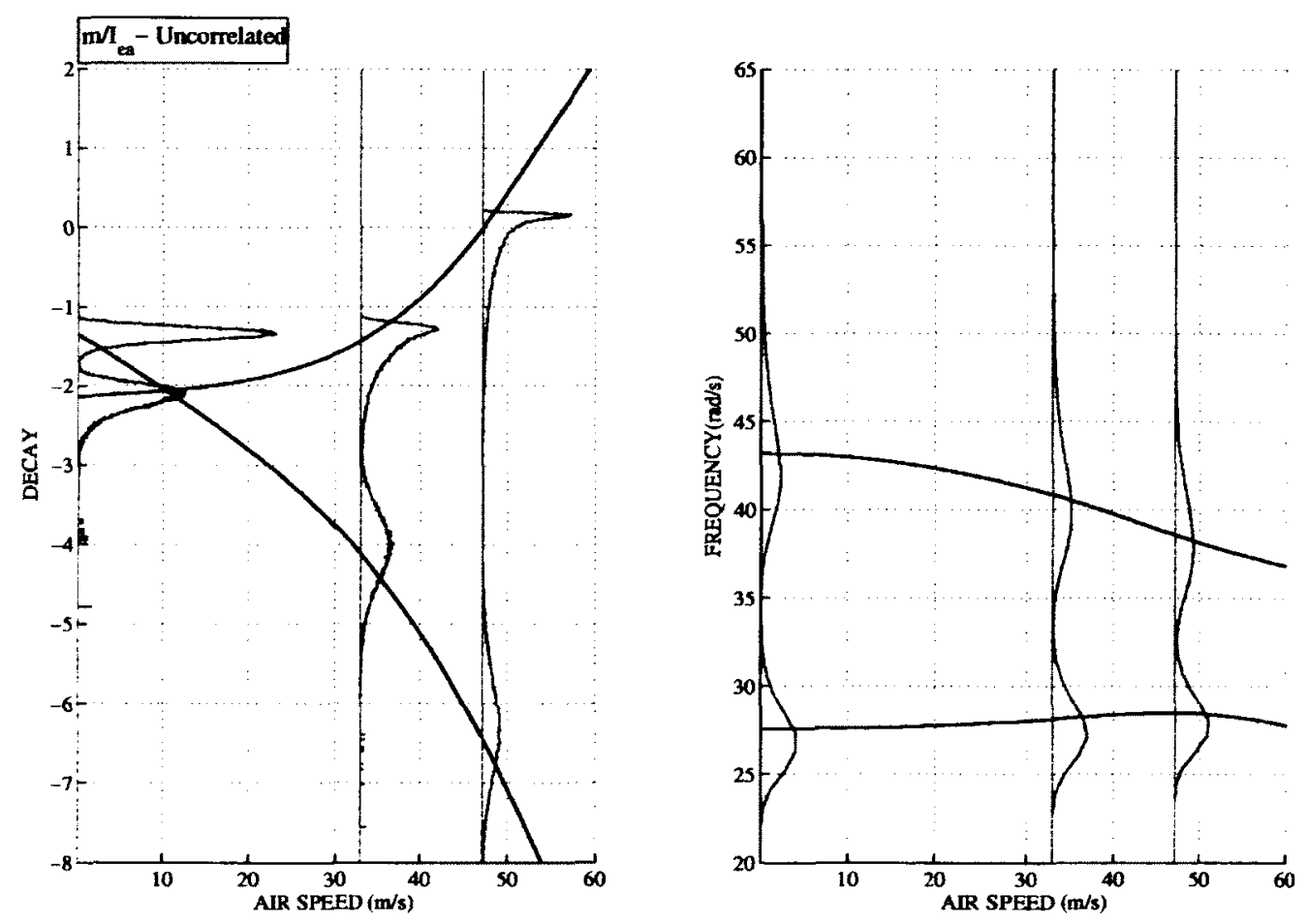

Figure 4.55: Modal parameter pdfs resulting from randomness in the uncorrelated inertia parameters with $17.5 \% \mathrm{COV}$, obtained at 3 test airspeeds: $0 \mathrm{~m} / \mathrm{s}, 33 \mathrm{~m} / \mathrm{s}, 47 \mathrm{~m} / \mathrm{s}$ 

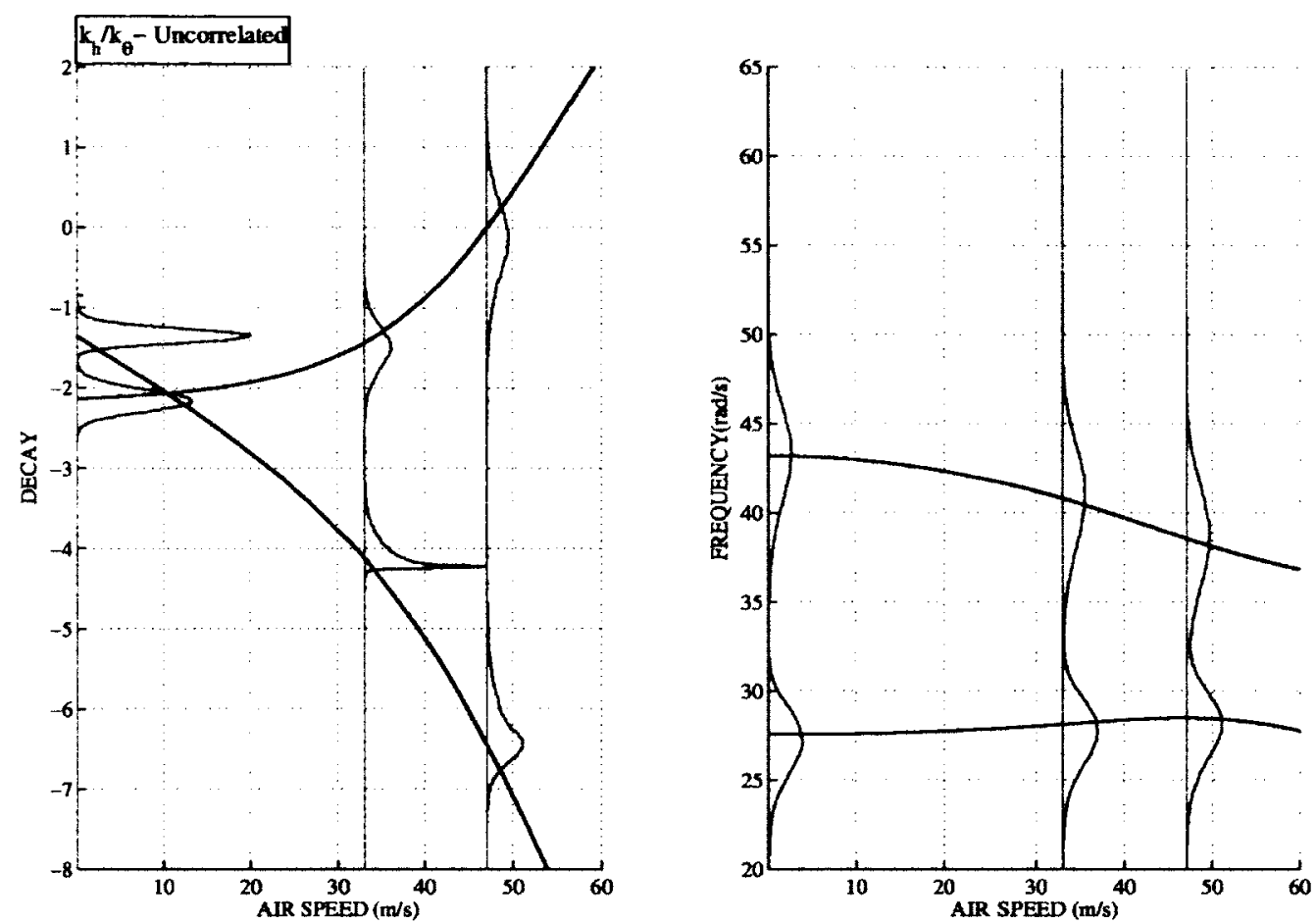

Figure 4.56: Modal parameter pdfs resulting from randomness in the uncorrelated stiffness parameters with $17.5 \% \mathrm{COV}$, obtained at 3 test airspeeds: $0 \mathrm{~m} / \mathrm{s}, 33 \mathrm{~m} / \mathrm{s}, 47 \mathrm{~m} / \mathrm{s}$
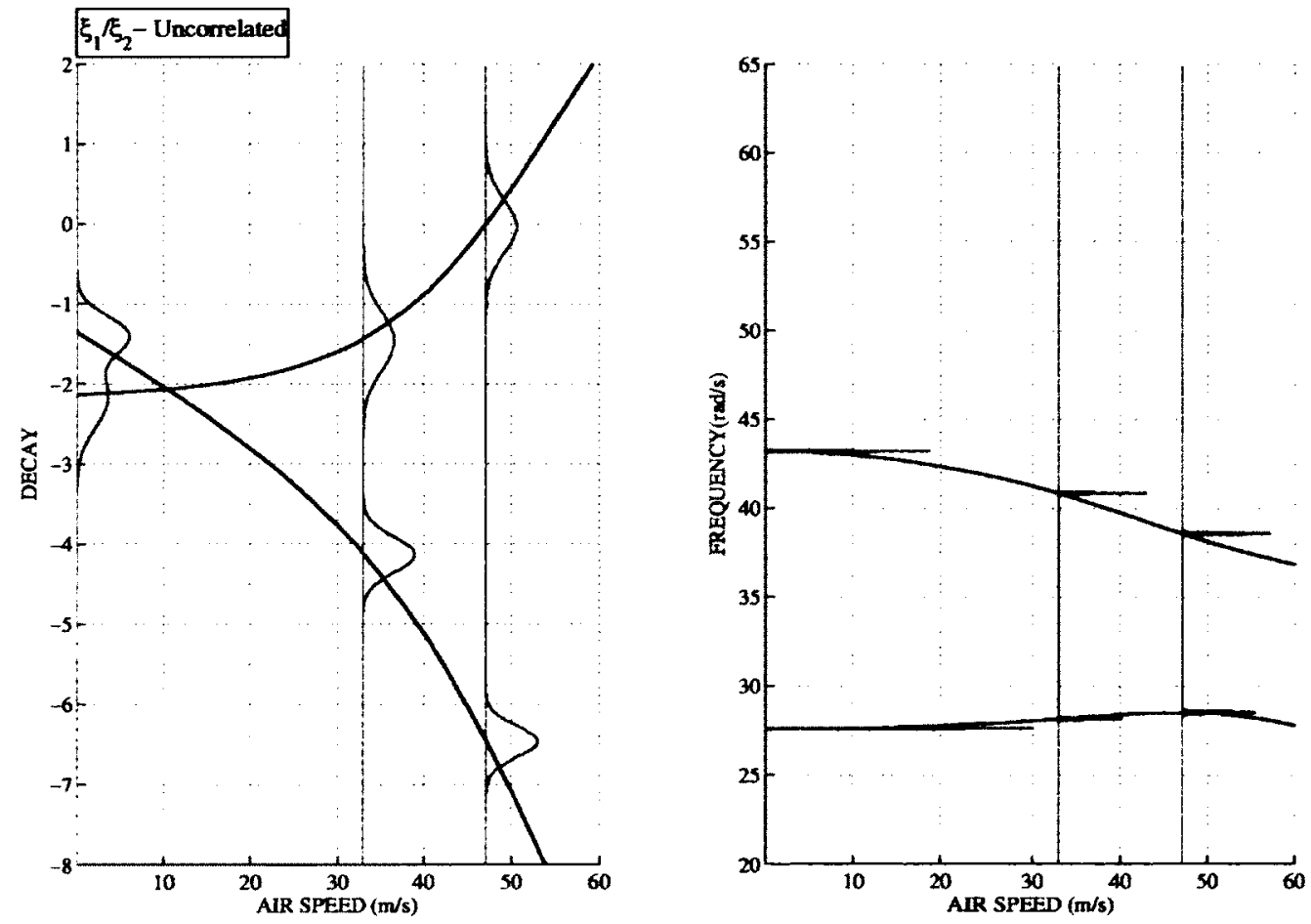

Figure 4.57: Modal parameter pdfs resulting from randomness in the uncorrelated damping parameters with $17.5 \% \mathrm{COV}$, obtained at 3 test airspeeds: $0 \mathrm{~m} / \mathrm{s}, 33 \mathrm{~m} / \mathrm{s}, 47 \mathrm{~m} / \mathrm{s}$ 

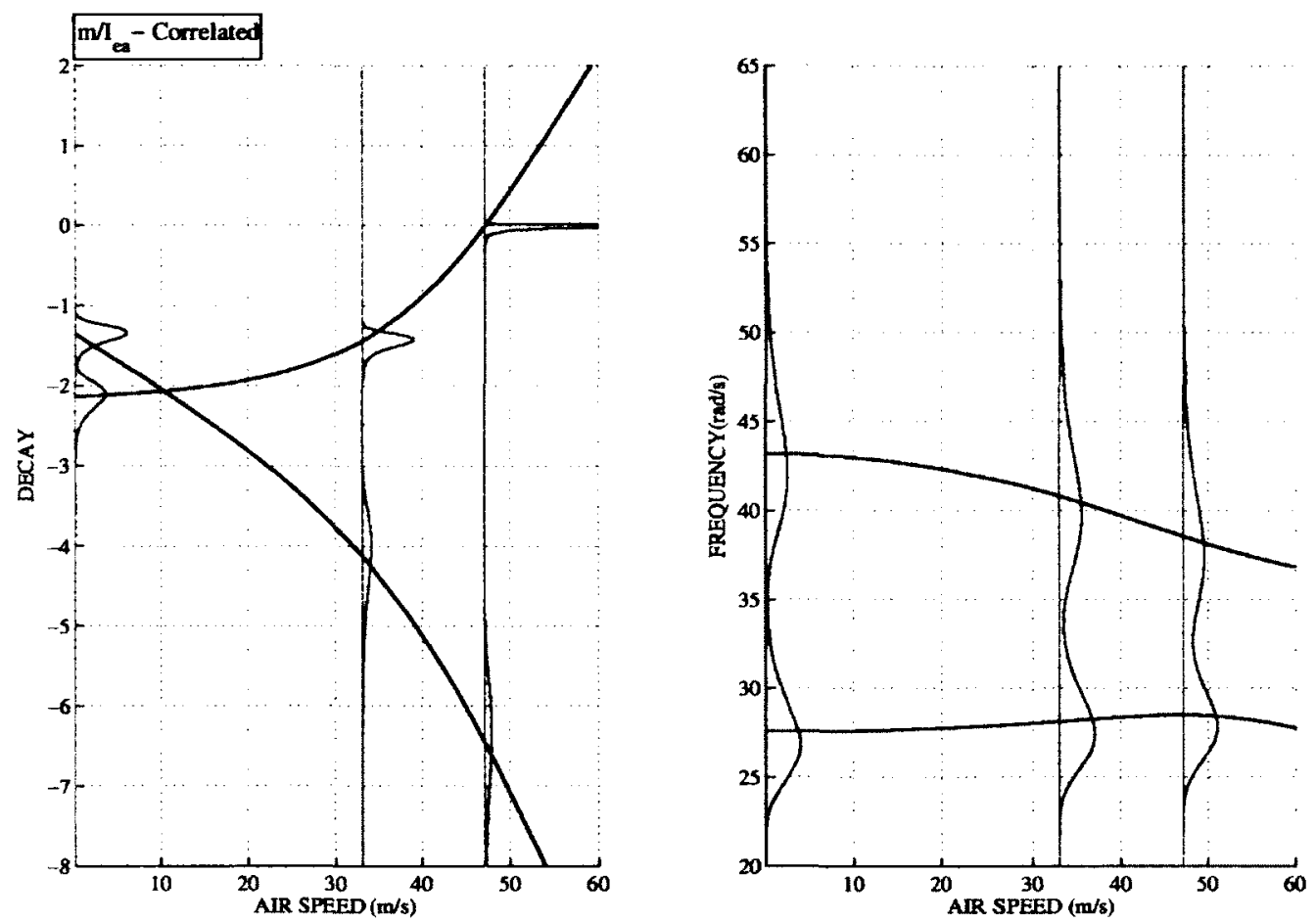

Figure 4.58: Modal parameter pdfs resulting from randomness in the correlated inertia parameters with $17.5 \% \mathrm{COV}$, obtained at 3 test airspeeds: $0 \mathrm{~m} / \mathrm{s}, 33 \mathrm{~m} / \mathrm{s}, 47 \mathrm{~m} / \mathrm{s}$
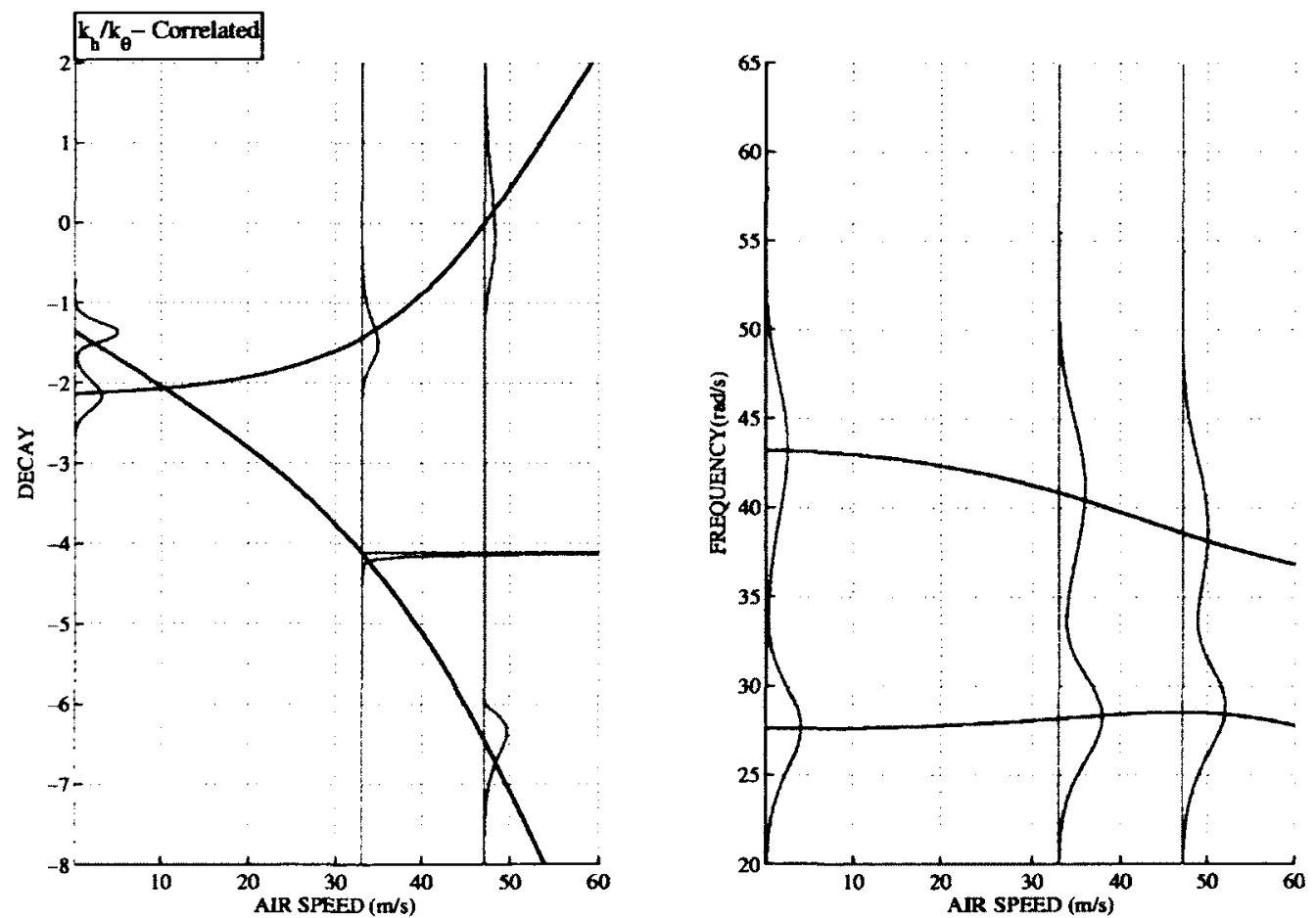

Figure 4.59: Modal parameter pdfs resulting from randomness in the correlated stiffness parameters with $17.5 \%$ COV, obtained at 3 test airspeeds: $0 \mathrm{~m} / \mathrm{s}, 33 \mathrm{~m} / \mathrm{s}, 47 \mathrm{~m} / \mathrm{s}$ 

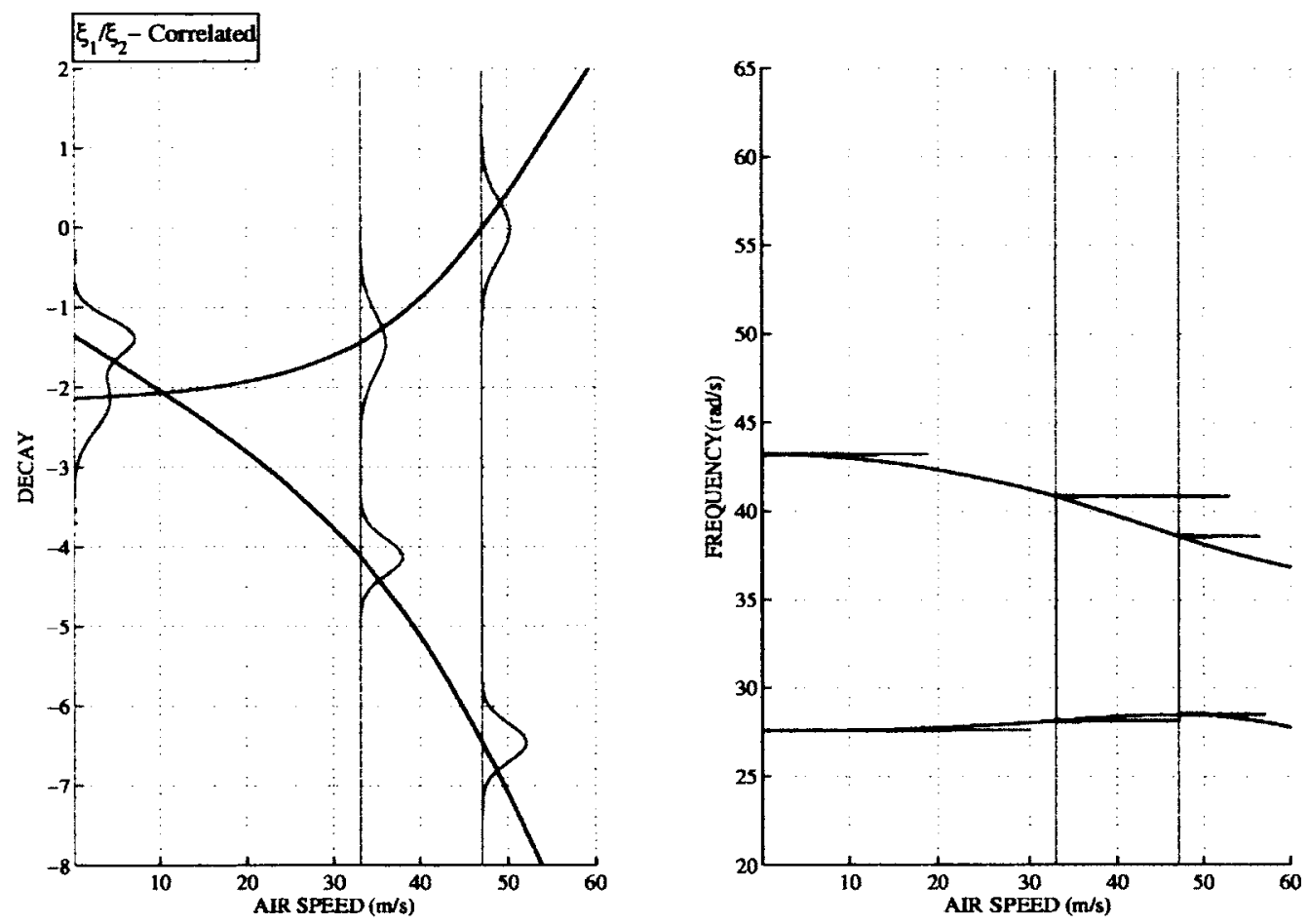

Figure 4.60: Modal parameter pdfs resulting from randomness correlated damping parameters with $17.5 \% \mathrm{COV}$, obtained at 3 test airspeeds: $0 \mathrm{~m} / \mathrm{s}, 33 \mathrm{~m} / \mathrm{s}, 47 \mathrm{~m} / \mathrm{s}$

decay pdf vanish. For damping parameter uncertainty, the variance of the modal decay pdfs increases, leading to a larger uncertainty in the flutter speed pdf.

\subsection{Closing Remarks}

The results indicate that typically the inertia and stiffness parameter uncertainties dominantly influence the characteristic roots, describing modal frequencies and decays. Furthermore, uncertainty in the modal parameters typically decreases for unsteady aerodynamics. The effect of parameter correlations has proven to be significant, altering the correlations among the modal parameters. 


\section{Chapter 5}

\section{A Cantilever Wing: Flutter Speed}

A two dimensional rigid wing model of a wing provides considerable insight into the coalescence flutter induced aeroelastic instability. Nevertheless, a realistic aeroelastic model must also consider the three dimensional effects from (a) the structural dynamics and (b) the aerodynamics point of view due to the following reasons:

(a) A rigid wing model cannot adequately capture the spacially varying heave and pitch motion (as described for the 2-D airfoil model) due to bending and torsional motions exhibited by a realistic aircraft wing.

(b) From aerodynamics prospective, a complex three dimensional flow around the wing produces highly complex aerodynamic loading along the span of the wing, which cannot be represented with the rigid airfoil model. There are two main factors that contribute to the 3-D flow effects: one, the highly varying nature of the wing motions arising from the flexural and torsional effects, and two, the span-wise reduction of lift due to the effect of tip vortices. An example of a lift reduction factor that reflects the span-wise reduction of lift due to the effect of tip vortices is illustrated in fig. (5.1), where $\eta$ represents a normalized wing span. The lift coefficient varies along the span, reducing to a value of zero at the free-end.

For a rigid wing model, only two dofs, namely the pitch and heave motions contribute to the dynamics of the two coalescing modes inducing flutter. On the contrary, the cantilever system having an infinite number of dofs, must be approximated by a finite dimensional representation. 


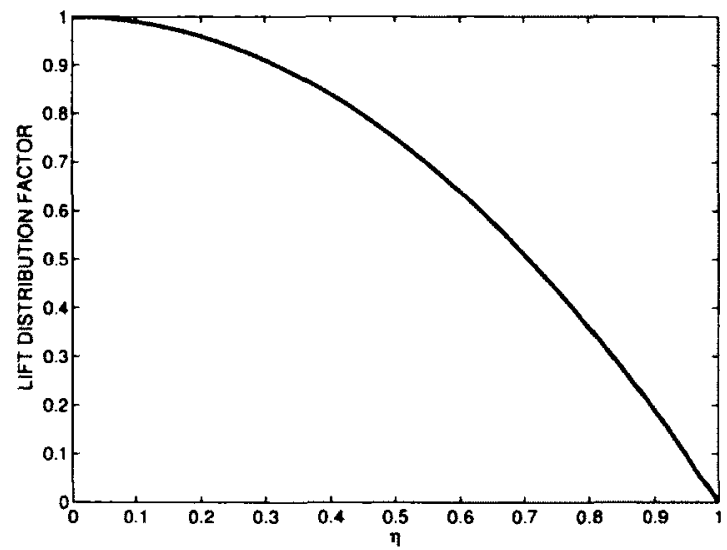

Figure 5.1: Lift reduction factor as function of normalized span

In this case, a larger number of generalized coordinates, or dofs, can contribute to two coalescing aeroelastic modes that induce flutter. For closely spaced aeroelastic modes, the modal frequencies may exhibit substantial statistical overlap, which produces significant difficulty in tracking the aeroelastic modes.

\subsection{Deterministic Formulation}

In this investigation, the baseline (deterministic) continuous wing is idealized by a uniform cantilever model. The flutter speed and characteristic roots for the baseline system are investigated for both, the quasisteady and unsteady aerodynamics. It is then followed by the probabilistic analysis of the flutter speed and characteristic roots. From the probabilistic prospective, the similarities and differences between the cantilever and equivalent rigid airfoil models are pointed out.

The coupled bending and torsional motions of the uniform cantilever wing are given by the following system of partial differential equations (pdes):

$$
\begin{gathered}
m \frac{\partial^{2} h(x, t)}{\partial t^{2}}+m b x_{\theta} \frac{\partial^{2} \theta(x, t)}{\partial t^{2}}+B_{1} \frac{\partial h(x, t)}{\partial t}+B_{2} \frac{\partial \theta(x, t)}{\partial t}+B_{3} \frac{\partial^{5} h(x, t)}{\partial x^{4} \partial t}+E I \frac{\partial^{4} h(x, t)}{\partial x^{4}}=-L(x, t) \\
I_{e a} \frac{\partial^{2} \theta(x, t)}{\partial t^{2}}+m b x_{\theta} \frac{\partial^{2} h(x, t)}{\partial t^{2}}+B_{4} \frac{\partial h(x, t)}{\partial t}+B_{5} \frac{\partial \theta(x, t)}{\partial t}+B_{6} \frac{\partial^{3} \theta(x, t)}{\partial x^{2} \partial t}-G J \frac{\partial^{2} \theta(x, t)}{\partial x^{2}}=M_{e a}(x, t)
\end{gathered}
$$


where $x$ is a span-wise Cartesian coordinate that specifies the distance from the root of the wing. The $m$ and $I_{e a}$ represent distributed mass and mass moment of inertia about the axis of rotation, respectively; $B_{1}-B_{6}$ represent the proportional structural damping coefficients due to bending and torsional motions; $E$ and $G$ are the modulus of elasticity and the shear modulus, respectively; $I$ and $J$ are the second moment of area and the polar moment of inertia, respectively; $b$ is the half-chord length and $x_{\theta}$ is a geometric measure of offset between the center of gravity and the axis of rotation; $h$ and $\theta$ are the vertical (bending) and rotational (torsional) displacements; $L$ and $M_{e a}$ are the distributed lift and moment forces, respectively, acting on the wing; and $t$ is a time coordinate.

\subsubsection{Discrete Model Representation}

In order to obtain a solution to the above pdes, Eqs. (5.1) and (5.2), the continuous model of a cantilever wing is discretized using the resultant modes of an uncoupled and undamped cantilever system in vacuum (with no influence of surrounding air) as the projection bases. The discretization was achieved using the Galerkin Method [91]. Then, the resulting vertical and rotational motions of the elastic axis can be represented as:

$$
\begin{aligned}
& h(x, t)=\sum_{i=1}^{\infty} \Psi_{i}(x) \xi_{i}(t) \approx \sum_{i=1}^{n} \Psi_{i}(x) \xi_{i}(t) \\
& \theta(x, t)=\sum_{i=1}^{\infty} \Omega_{i}(x) \gamma_{i}(t) \approx \sum_{i=1}^{m} \Omega_{i}(x) \gamma_{i}(t)
\end{aligned}
$$

where $\Psi_{i}(x)$ and $\Omega_{i}(x)$ are the bending and torsional projection basis and $\xi_{i}(t)$ and $\gamma_{i}(t)$ are their corresponding generalized coordinates, respectively. Only a sufficient number of basis functions was used to satisfy the flutter speed convergence criteria for accurate computation of flutter speed. The $n$ and $m$ parameters specify the truncated number of basis functions used to represent the bending and torsional motions, respectively. Due to the geometrical simplicity of a uniform cantilever model, only a few basis functions were needed to obtain an accurate representation of the two modes that participate in the flutter mechanism. In particular, only 3 bending and 3 torsional functions were needed to represent the coupled bending and torsional 
motion. A detailed description of the basis functions and the associated finite dimensional model is provided in Appendices $\mathrm{E}$ and $\mathrm{F}$.

From the discretization of pdes, the following set of ordinary differential equations (odes) is formulated:

$$
\left[M_{s t r}\right]\left\{\begin{array}{c}
\ddot{\xi} \\
\ddot{\gamma}
\end{array}\right\}+\left[B_{s t r}\right]\left\{\begin{array}{c}
\dot{\xi} \\
\dot{\gamma}
\end{array}\right\}+\left[K_{s t r}\right]\left\{\begin{array}{c}
\xi \\
\gamma
\end{array}\right\}=\left\{\begin{array}{c}
L^{b} \\
M_{e a}^{t}
\end{array}\right\}
$$

where $\left[M_{s t r}\right],\left[B_{s t r}\right]$ and $\left[K_{s t r}\right]$ are the structural inertia, damping and stiffness matrices respectively; $\left\{L^{b}\right\}$ and $\left\{M_{e a}^{t}\right\}$ are vectors that represent the bending and torsional aerodynamic forcing onto the wing, obtained from the distributed lift force and aerodynamic moment about the elastic axis, respectively. $L^{b}$ vector is representative of the forcing associated with each of the bending generalized coordinates, and likewise $M_{e a}^{t}$ vector is representative of the forcing associated with each of the rotational generalized coordinates.

\subsubsection{Quasisteady Aerodynamics}

Next, the aerodynamic loading terms will be restated as a combination of added aerodynamic inertia, damping and stiffness matrices, which would lead to the following relations for the quasisteady aerodynamics model:

$$
\left\{\begin{array}{c}
\left\{L^{b}\right\} \\
\left\{M_{e a}^{t}\right\}
\end{array}\right\}=\left[M_{\text {aero }}\right]\left\{\begin{array}{c}
\{\ddot{\xi}\} \\
\{\ddot{\gamma}\}
\end{array}\right\}+\left[B_{\text {aero }}(U)\right]\left\{\begin{array}{c}
\{\dot{\xi}\} \\
\{\dot{\gamma}\}
\end{array}\right\}+\left[K_{\text {aero }}(U)\right]\left\{\begin{array}{c}
\{\xi\} \\
\{\gamma\}
\end{array}\right\}
$$

Similar to the airfoil model, the added inertia terms are included with the quasisteady aerodynamics. Then, the aeroelastic system can be represented as:

$$
\left[M_{a e}\right]\left\{\begin{array}{c}
\{\ddot{\xi}\} \\
\{\ddot{\gamma}\}
\end{array}\right\}+\left[B_{a e}(U)\right]\left\{\begin{array}{c}
\{\dot{\xi}\} \\
\{\dot{\gamma}\}
\end{array}\right\}+\left[K_{a e}(U)\right]\left\{\begin{array}{c}
\{\xi\} \\
\{\gamma\}
\end{array}\right\}=\left\{\begin{array}{c}
\{0\} \\
\{0\}
\end{array}\right\}
$$


where

$$
\begin{aligned}
& {\left[M_{a e}\right]=\left[\begin{array}{cc}
\left(m(x)+\frac{1}{2} \rho b^{2} C_{L \alpha}\right)[I]_{n \times n} & \left(m(x) b x_{\theta}-\frac{1}{2} \rho b^{3} C_{L \alpha} a_{h}\right)\left[A_{i j}\right]_{n \times m} \\
\left(m(x) b x_{\theta}-\frac{1}{2} \rho b^{3} C_{L \alpha} a_{h}\right)\left[A_{i j}\right]_{m \times n}^{T} & \left(I_{e a}(x)+\frac{1}{2} \rho b^{4} C_{L \alpha}\left(\frac{1}{8}+a_{h}^{2}\right)\right)[I]_{m \times m}
\end{array}\right]} \\
& {\left[B_{a e}\right]=\left[\begin{array}{cc}
{\left[B_{1}\right]_{n \times n}+\rho U b C_{L \alpha}[I]_{n \times n}} & {\left[B_{2}\right]_{n \times m}+\rho U b^{2} C_{L \alpha}\left(1-a_{h}\right)\left[A_{i j}\right]_{n \times m}} \\
{\left[B_{3}\right]_{m \times n}-\rho U b^{2} C_{L \alpha}\left(\frac{1}{2}+a_{h}\right)\left[A_{i j}\right]_{m \times n}^{T}} & {\left[B_{4}\right]_{m \times m}-\rho U b^{3} C_{L \alpha} a_{h}\left(\frac{1}{2}-a_{h}\right)[I]_{m \times m}}
\end{array}\right]} \\
& {\left[K_{a e}\right]=\left[\begin{array}{cc}
E I(x)\left[\alpha^{4}\right]_{n \times n} & \rho U^{2} b C_{L \alpha}\left[A_{i j}\right]_{n \times m} \\
{[0]_{m \times n}} & G J(x)\left[\vartheta^{2}\right]_{m \times m}-\rho U^{2} b^{2} C_{L \alpha}\left(\frac{1}{2}+a_{h}\right)[I]_{m \times m}
\end{array}\right]}
\end{aligned}
$$

where $a_{h}$ represents the distance between the mid-chord point and the rotational axis, normalized by the half-chord length; $C_{L \alpha}$ is the lift curve slope; $\rho$ and $U$ are free flow air density and air speed, respectively (See Appendix E for further details).

\section{Mach Correction Factor}

Similar to the 2-D airfoil, the compressibility effects have to be considered in the solution. They are included in the aerodynamic forcing using a Prandtl-Glauert Mach Correction Factor in the lift curve slope, using Eq. (3.10), which was illustrated in the previous chapter.

\section{3-D Aerodynamic Lift Reduction Factor}

The dynamics of the system does not so far consider any effects of the 3-D aerodynamic flow due to tip vortex effects. These effects reduce the lift force towards the tip of the wing. In order to include the 3-D aerodynamic effects, a very simple lift reduction factor, which was illustrated in fig. (5.1), was applied to the lift coefficient as:

$$
\bar{C}_{L \alpha}=\bar{C}_{L \alpha}^{2 D} \times\left(1-x^{2}\right)
$$

where $\bar{C}_{L \alpha}^{2} D$ represents the 2-D lift curve slope for incompressible flow.

\section{Eigenvalue Analysis of Discrete System}

The cantilever wing modal analysis is based on a set of second order ordinary differential equations presented in eq. (5.7). To solve this equation, a general harmonic solution was used to 
arrive at a quadratic eigenvalue problem stated as:

$$
\left[\lambda^{2} M_{a e}+\lambda B_{a e}+K_{a e}\right]\{\mathbf{X}\}=\{0\}
$$

where $\lambda$ represents the characteristic roots of this aeroelastic system and $\{X\}$ represents the eigenvectors that are associated with each of these characteristic roots. Identical to the analysis of the airfoil stability, this Quadratic Eigenvalue Problem will be reduced to a Standard Eigenvalue Problem by using a state space representation. The general form of these equations in the state space is:

$$
[A]\{X\}=\lambda\{X\}
$$

Similar to the analysis of the rigid wing model, the $\lambda$ 's and $\{\mathbf{X}\}$ represent the characteristic roots of the system and their associated eigenvectors, respectively. The eigenvalues and the eigenvectors are:

$$
\begin{gathered}
\lambda_{i}=-\beta_{i} \pm j\left(\omega_{d}\right)_{i} \\
\{X\}_{i}=\left\{\xi_{i}, \gamma_{i}, \dot{\xi}_{i}, \dot{\gamma}_{i}\right\}^{T} \quad i=1,2,3
\end{gathered}
$$

Typically, the number of these characteristic roots depends on the size of the discretized aeroelastic model. In these case studies, since six basis functions were used to represent the continuous cantilever system, the solution yields six complex conjugate root pairs. As before, each pair represents the modal properties of each mode, describing its damped frequency and decay rate. Fig. (5.2) illustrates the modal transformation of the six modes of the representative aeroelastic system as a function of airspeed. This system is described by Case 1 parameters, given in Appendix C. For a better illustration of the coalescing modes, fig. (5.3) provides a zoomed view of fig. (5.2). The observations from these figures describe the same qualitative features as the equivalent rigid wing model. As airspeed increases up to a certain point, the modal frequencies of the two interacting modes coalesce and simultaneously the modal decays of these modes diverge. At the onset of flutter one of the modal decays becomes zero. 

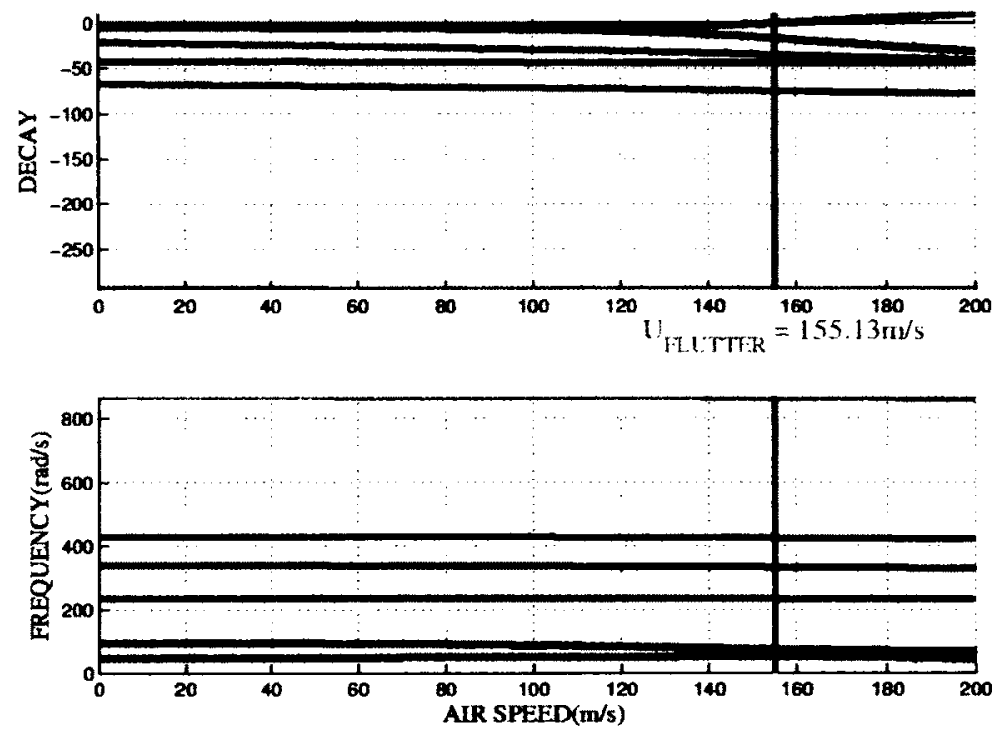

Figure 5.2: Evolution of the modal parameters with respect to increasing airspeed. Top plot describes modal decay rates; bottom plot describes damped modal frequencies
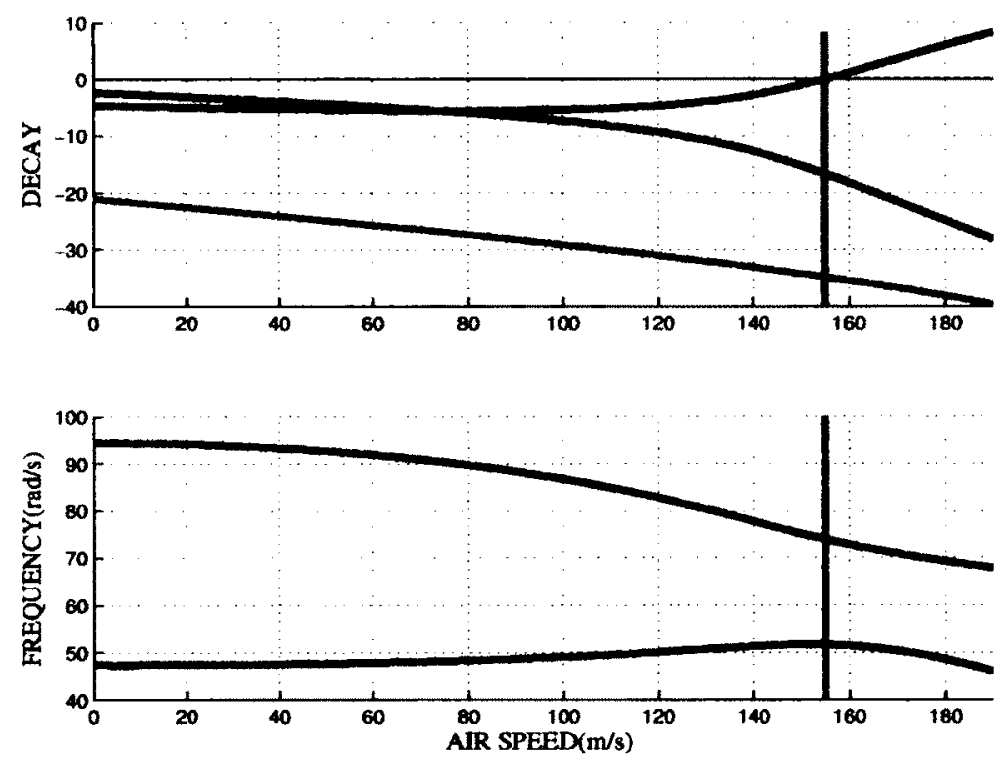

Figure 5.3: Evolution of the modal parameters with respect to increasing airspeed. Top plot describes modal decay rates; bottom plot describes damped modal frequencies 


\subsubsection{Unsteady Aerodynamics}

The unsteady aerodynamic effects are introduced into the model using the Wagner's Function via an additional degree of freedom in $z(x, t)=\sum_{i=1}^{m} \Omega_{i}(x) \varsigma_{i}(t)$. The basis functions $\Omega_{i}(x)$ that were used to represent this degree of freedom are the same basis functions used to represent the rotational degree of freedom (See Appendix E for more details).

In order to express the unsteady effects due to circulatory forcing, a two term Wagner Function approximation [88] was used to describe the diminishing influences of the "starting vortices" onto the wing as they travel downstream. With the additional degree of freedom in $z(x, t)$, an extra set of odes is derived to describes the lag effects of the circulatory forcing. The resulting general aeroelastic system is given by:

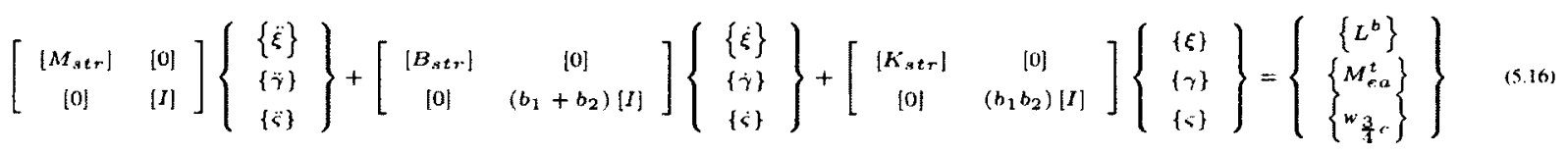

where $\left[M_{s t r}\right],\left[B_{s t r}\right]$ and $\left[K_{s t r}\right]$ are the structural matrices with a similar definition provided in the quasisteady aerodynamics analysis; $\left\{L^{b}\right\}$ and $\left\{M_{e a}^{t}\right\}$ represent vectors of aerodynamic lift and moment about elastic axis for each respective generalized coordinate in bending and twist. $\{\xi\}$ and $\{\gamma\}$ are previously defined vectors of generalized coordinates of the wing for each respective shape function in bending and twist. There is a new vector of generalized coordinates in $\{\varsigma\}$, representing the lag states, and an associated force vector $\left\{w_{\frac{3}{4} c}\right\}$, representing the effective downwash at the three-quarter chord point. For unsteady effects, the forcing terms become explicit functions of time, acceleration, velocity, displacement and airspeed:

$$
\begin{aligned}
& \left\{L^{b}\right\}=L^{b}(U,\{\gamma\},\{\dot{\xi}\},\{\dot{\gamma}\},\{\ddot{\xi}\},\{\ddot{\gamma}\}, t) \\
& \left\{M_{e a}^{t}\right\}=M_{e a}^{t}(U,\{\gamma\},\{\dot{\xi}\},\{\dot{\gamma}\},\{\ddot{\xi}\},\{\ddot{\gamma}\}, t) \\
& \left\{w_{\frac{3}{4} c}\right\}=w_{\frac{3}{4} c}(\{\gamma\},\{\dot{\xi}\},\{\dot{\gamma}\})
\end{aligned}
$$

Restating all of the aerodynamic loading terms in matrix form leads to the expression: 


$$
\left\{\begin{array}{c}
\left\{L^{b}\right\} \\
\left\{M_{e a}^{t}\right\} \\
\left\{w_{\frac{3}{4} c} c\right.
\end{array}\right\}=\left[M_{\text {aero }}\right]\left\{\begin{array}{c}
\{\ddot{\xi}\} \\
\{\ddot{\gamma}\} \\
\{\ddot{\zeta}\}
\end{array}\right\}+\left[B_{a e r o}(U)\right]\left\{\begin{array}{c}
\{\dot{\xi}\} \\
\{\dot{\gamma}\} \\
\{\dot{\zeta}\}
\end{array}\right\}+\left[K_{\text {aero }}(U)\right]\left\{\begin{array}{c}
\{\xi\} \\
\{\gamma\} \\
\{\varsigma\}
\end{array}\right\}
$$

where $\left[M_{a e r o}\right],\left[B_{a e r o}\right]$ and $\left[K_{\text {aero }}\right]$ are aerodynamic mass, damping and stiffness matrices respectively. The aerodynamic damping and stiffness matrices are non-symmetric. The full representation of these equations of motion can be found in Appendix E. A combination of the structural and aerodynamic matrices yields the final aeroelastic system of equations shown below:

$$
\left[M_{a e}\right]\left\{\begin{array}{c}
\{\ddot{\xi}\} \\
\{\ddot{\gamma}\} \\
\{\ddot{\zeta}\}
\end{array}\right\}+\left[B_{a e}\right]\left\{\begin{array}{c}
\{\dot{\xi}\} \\
\{\dot{\gamma}\} \\
\{\dot{\zeta}\}
\end{array}\right\}+\left[K_{a e}\right]\left\{\begin{array}{c}
\{\xi\} \\
\{\gamma\} \\
\{\varsigma\}
\end{array}\right\}=\left\{\begin{array}{c}
\{0\} \\
\{0\} \\
\{0\}
\end{array}\right\}
$$

Based on the convergence analysis, it was determined that only three basis functions were needed to obtain accurate results for unsteady effects. Since 3 extra basis functions were used to represent the unsteady effects, 3 additional odes were introduced to the original set of odes. The individual aeroelastic matrix terms of this aeroelastic system are illustrated below (See Appendix E):

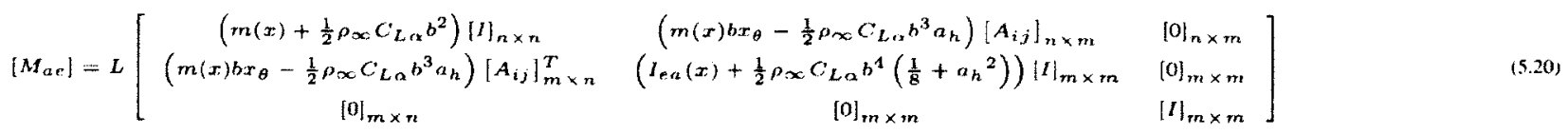

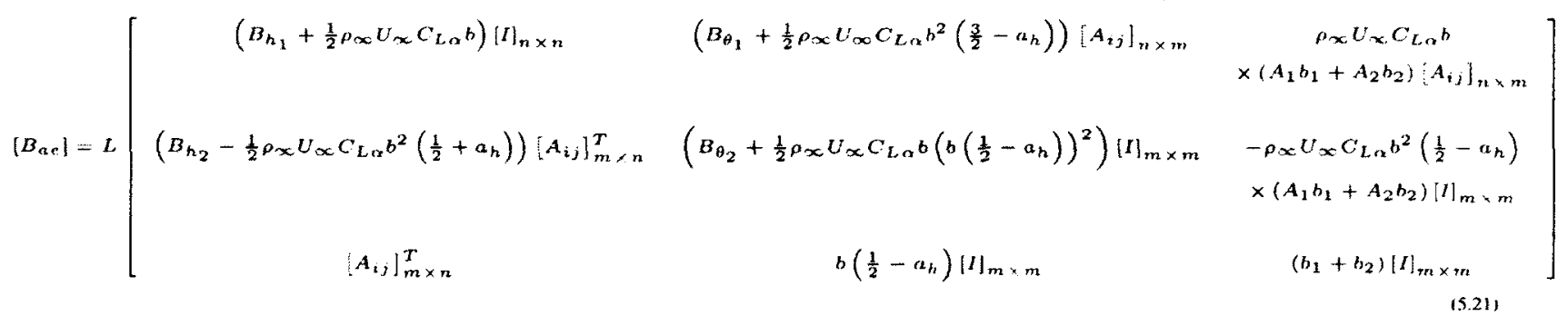

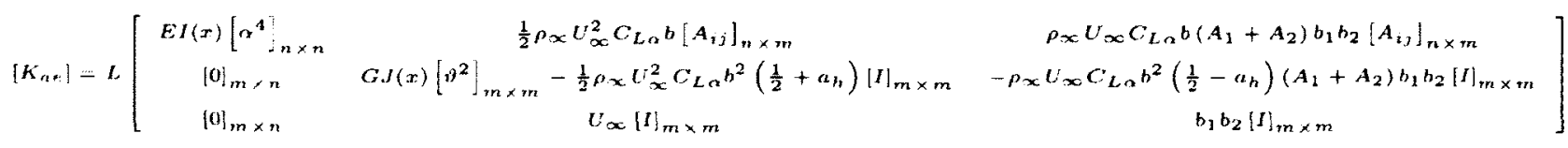




\section{Eigenvalue Analysis of Discrete System}

When the cantilever wing is forced with unsteady aerodynamics, the eigenvalue analysis of the representative system yields a number of additional characteristic roots to the original set of complex conjugate root pairs representing the oscillatory aeroelastic modes. The additional roots are real and distinct, which represent the overdamped modes from the addition of the extra degree of freedom $z(x, t)$, which accounts for the unsteady aerodynamics. The two figures below, figs. (5.4) and (5.5), illustrate the evolution of modal parameters with respect to varying airspeed of the six oscillatory modes of the aeroelastic system. The modal properties of overdamped modes are not shown in these figures. Fig. (5.5) is a zoomed image of fig. (5.4), which focuses on the modes that interact in the flutter mechanism. This representative system is based on Case 1 parameters, which are shown in Appendix $\mathbf{C}$.
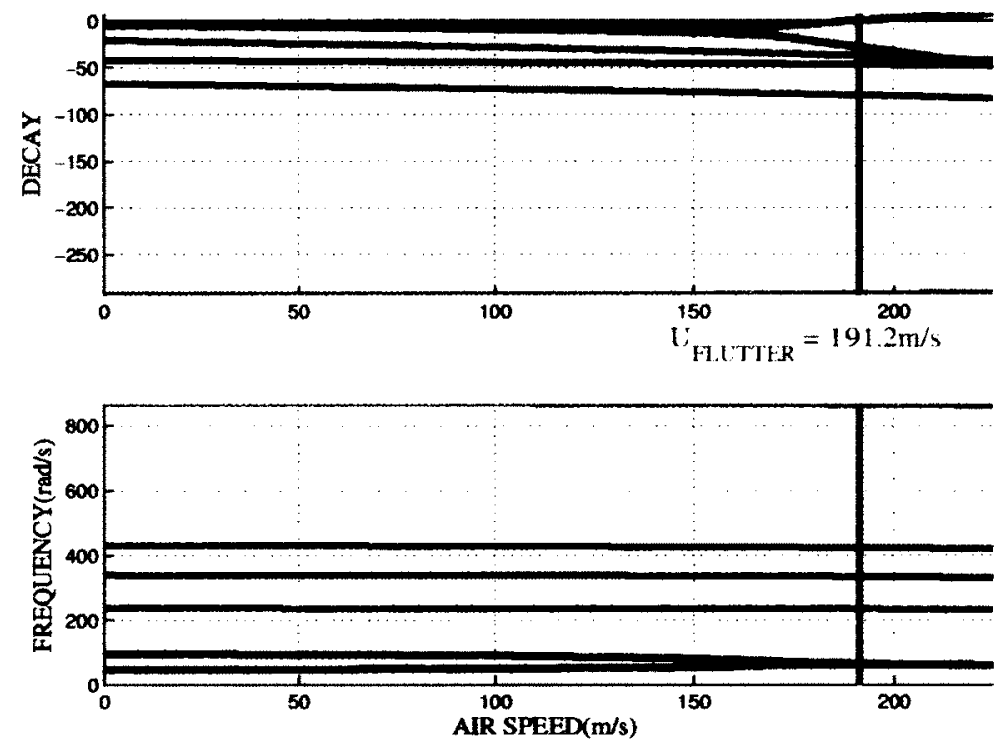

Figure 5.4: Evolution of the oscillatory modes' modal parameters with respect to increasing airspeed. Top plot describes modal decay rates; bottom plot describes damped modal frequencies 

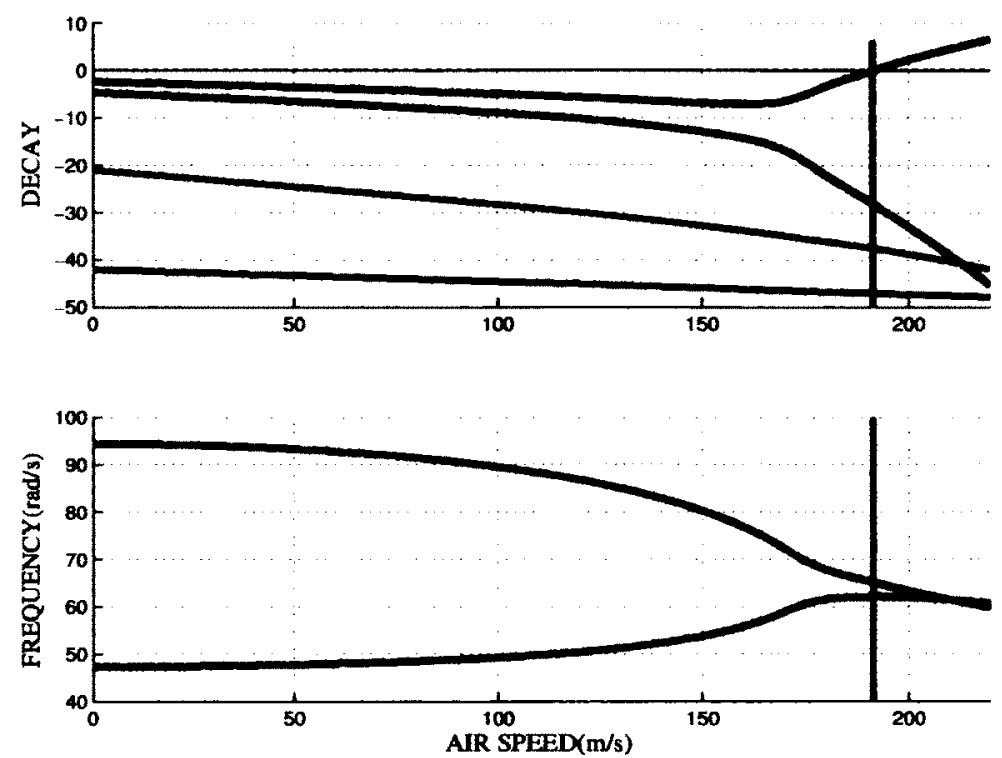

Figure 5.5: Evolution of the oscillatory modes' modal parameters with respect to increasing airspeed. Top plot describes modal decay rates; bottom plot describes damped modal frequencies

\subsection{Flutter Speed Estimation}

This section presents both the deterministic and probabilistic flutter speed results. The mapping plots between the flutter speed and the random system parameters will not be provided for the cantilever wing analysis due to the complexities involved in obtaining the analytical relationships from the Routh-Hurwitz stability criteria (i.e. involves characteristic roots of a higher order polynomial, therefore the size of sub-determinants becomes very large, see [1]).

\subsubsection{CASE 1}

For this case, the baseline parameters of a uniform cantilever wing have been taken from Borello et. al. [36]. Based on these parameters, they derived an equivalent 2-D simplified rigid model of a wing, which was presented in the previous chapter. This chapter will examine the results of the cantilever model and later compares these results with the 2-D equivalent model in order to examine the validity of the simplified model. The parameters of this system are shown in tbl. (5.1). Moreover, the results from Borello et. al. [36] were also used to validate the computer 
code developed in this investigation.

\begin{tabular}{||l|c|l|}
\hline PARAMETER & Symbol & 3D Wing \\
\hline \hline mass & $m$ & $35.7187 \mathrm{~kg} / \mathrm{m}$ \\
normalized radius of & & \\
gyration & $r_{g}$ & 0.5 \\
heave/bending stiffness & $k_{h} / E I$ & $9.77 \times 10^{6} \mathrm{Nm}^{2}$ \\
pitch/torsional stiffness & $k_{\theta} / G J$ & $9.89 \times 10^{5} \mathrm{Nm}^{2}$ \\
chord length & $c$ & $1.8288 \mathrm{~m}$ \\
span & $L$ & $6.096 \mathrm{~m}$ \\
normalized static imbalance & $x_{\theta}$ & 0.2 \\
damping ratio (mode 1) & $\xi_{1}$ & 0.05 \\
damping ratio (mode 2) & $\xi_{2}$ & 0.05 \\
free stream fluid density & $\rho_{\infty}$ & $1.225 \mathrm{~kg} / \mathrm{m}^{3}$ \\
normalized distance btw & & \\
EA and mid-chord & $a_{h}$ & -0.34 \\
lift coefficient gradient & $\bar{C}_{l \alpha}$ & $2 \pi\left(1-\eta^{2}\right)$ \\
\hline \hline Num. of Samples & - & 100000 \\
\hline
\end{tabular}

Table 5.1: Model parameters for Case 1

\section{Quasisteady Analysis: Baseline Case}

The aeroelastic frequencies and decays are plotted in fig. (5.6) as a function of airspeed. For an accurate computation of the modal properties of the coalescing modes, three bending and three torsional beam basis were used. A magnified view of the modal parameters of two coalescing
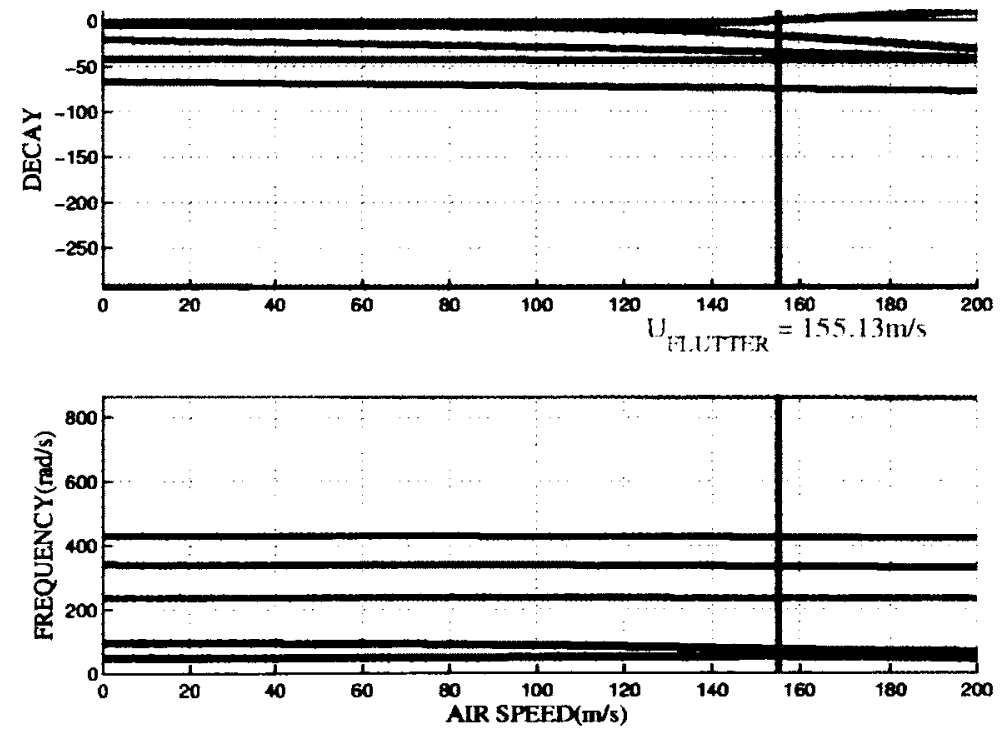

Figure 5.6: Evolution of the modal parameters with respect to airspeed 
modes is shown in fig. (5.7). As evident in this figure, the second mode becomes unstable through a mild-moderate flutter at a flutter speed of $155.13 \mathrm{~m} / \mathrm{s}$.
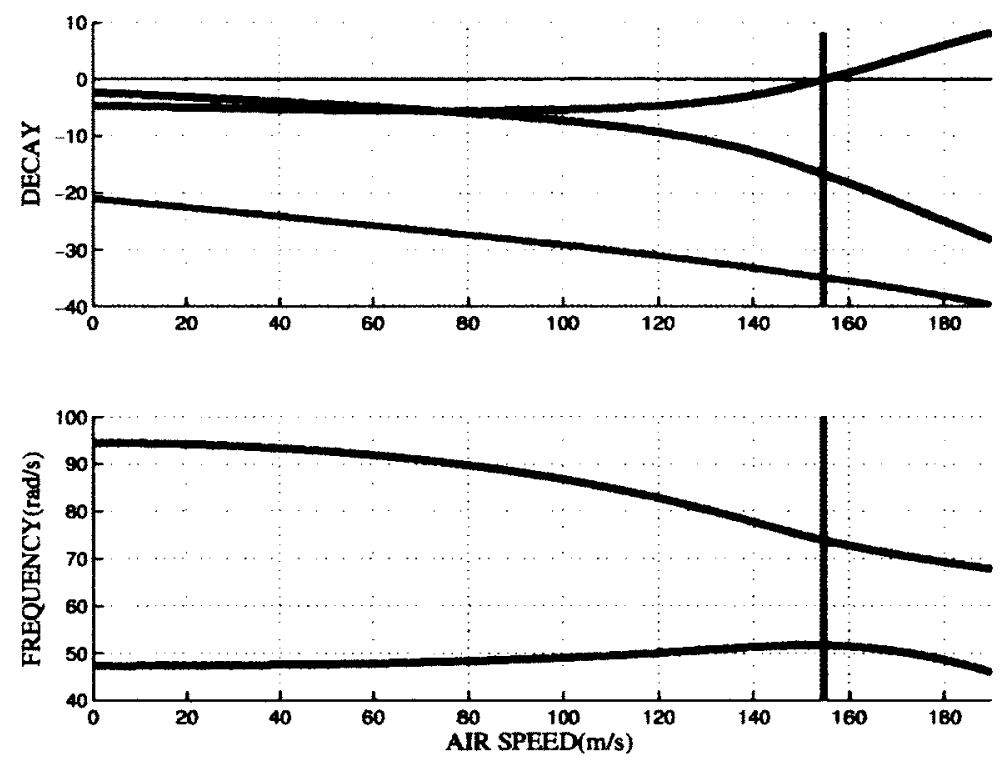

Figure 5.7: Magnified view of evolution of the modal parameters with respect to increasing airspeed

Fig. (5.8) shows the relative magnitude of the elements of the eigenvectors of each mode at no flow condition and at the proximity of the flutter speed. Although three bending and three torsional beam basis functions are used to approximate the dynamics of the cantilever, the two coalescing modes are mostly dominated by the first bending and first torsional basis functions. At flutter, the relative strength of each eigenvector is changed. Initially, the first mode contains dominant contribution from the first bending basis function. The second aeroelastic mode is dominated by the first torsional basis function. At the flutter point, both of the modes lose the relative strength of their dominant components.

The most evident differences in the comparison between these results and those of the rigid wing in Case 1 with quasisteady aerodynamics are a more explosive flutter type in this case and a much larger flutter speed. 

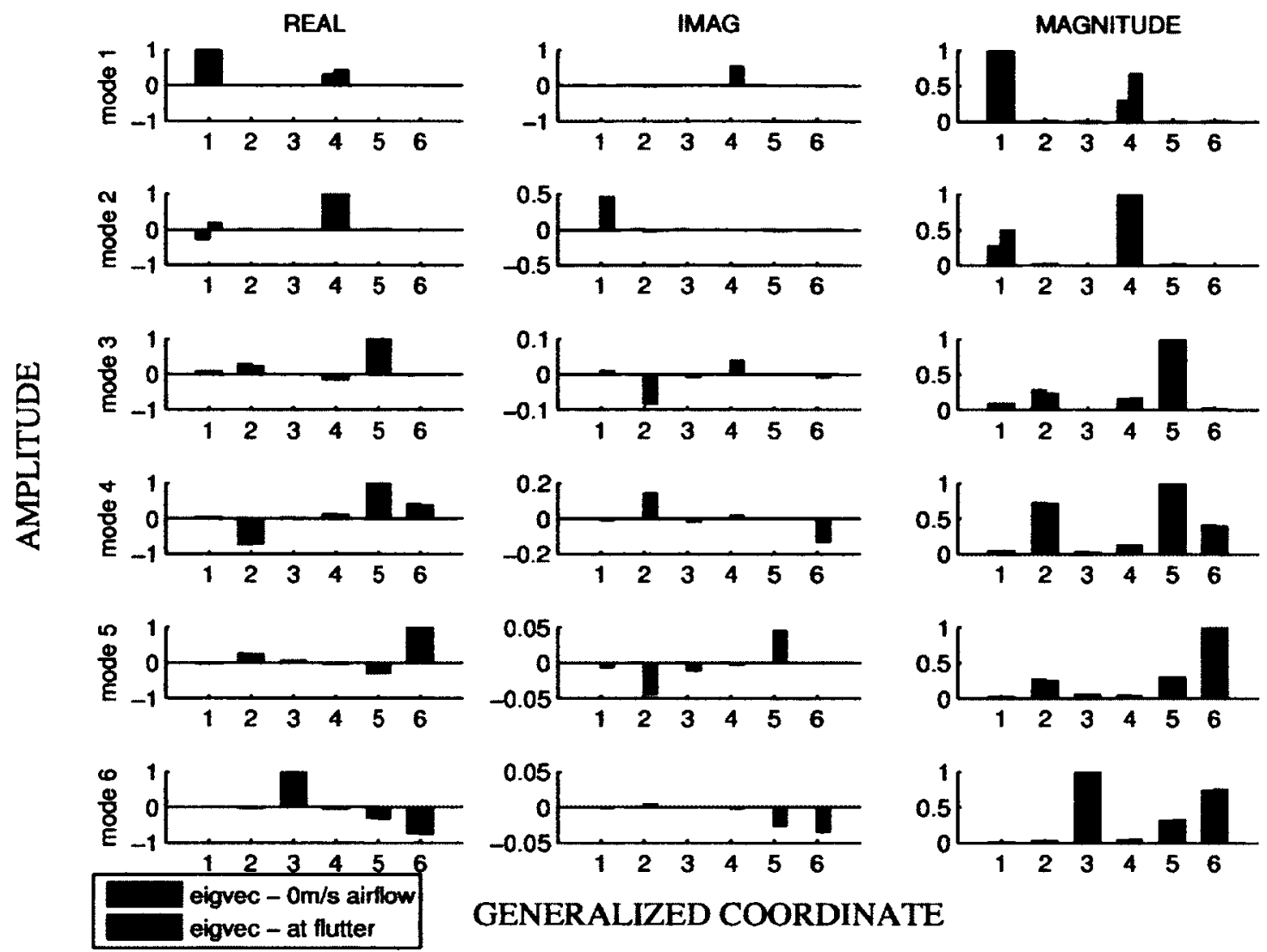

GENERALIZED COORDINATE

Figure 5.8: real part, imaginary part, and magnitude of eigenvectors of the vibration modes, illustrating relative strength of each component

\section{Quasisteady Analysis: Probabilistic Case}

Tables, 5.2 and 5.3, provide a summary of the results of probabilistic flutter speed estimation for Case 1 with quasisteady aerodynamics.

The resulting flutter speed pdfs for a truncated Gaussian distribution and a uniform distribution of the random structural parameters are illustrated in figs. (5.9) and (5.10), respectively. The boxed value represents the flutter speed COV. These results only include cases with large input uncertainty (10\% COV), since low structural parameter uncertainty ( $2.5 \% \mathrm{COV})$ produces approximately Gaussian pdfs. The following observations were noted from these figures. Although the uncertainties in the inertia (with exception of fully correlated inertia parameters) and stiffness contribute significantly to the uncertainty in the flutter speed, the uncertainty in the structural damping of the flutter mode also influences the randomness in the flutter speed 


\begin{tabular}{|c|c|c|c|}
\hline \multicolumn{2}{|c|}{ Input COV } & $2.5 \%$ & $10 \%$ \\
\hline Random Parameter & Distribution & \multicolumn{2}{c|}{ Flutter Speed COV \% } \\
\hline \hline \multirow{2}{*}{ mass } & Gaussian & 0.887 & 3.6 \\
\cline { 2 - 4 } & uniform & 0.886 & 3.58 \\
\hline radius of gyration & Gaussian & 0.967 & 3.88 \\
\cline { 2 - 4 } & uniform & 0.967 & 3.87 \\
\hline \multirow{2}{*}{ heave stiffness } & Gaussian & 0.574 & 2.3 \\
\cline { 2 - 4 } & uniform & 0.574 & 2.29 \\
\hline \multirow{2}{*}{ pitch stiffness } & Gaussian & 1.69 & 6.79 \\
\cline { 2 - 4 } & uniform & 1.69 & 6.78 \\
\hline \multirow{2}{*}{ damping ratio 1 } & Gaussian & 0.0461 & 0.184 \\
\cline { 2 - 4 } & uniform & 0.0461 & 0.184 \\
\hline damping ratio 2 & Gaussian & 0.271 & 1.09 \\
\cline { 2 - 4 } & uniform & 0.271 & 1.09 \\
\hline
\end{tabular}

Table 5.2: Results illustrating the uncertainty in the flutter speed due to independent uncertainty in the structural parameters

\begin{tabular}{|c|c|c|c|c|c|}
\hline \multicolumn{2}{|c|}{ Input COV } & $2.5 \%$ & $10 \%$ & $2.5 \%$ & $10 \%$ \\
\hline Random Parameter & Distribution & \multicolumn{3}{|c|}{ Flutter Speed COV (\%) } \\
\hline \hline \multicolumn{2}{|c|}{} & \multicolumn{3}{|c|}{ Uncorrelated Parameters } & Fully Correlated Parameters \\
\hline \hline $\begin{array}{c}\text { mass \& } \\
\text { radius of gyration }\end{array}$ & $\begin{array}{c}\text { Gaussian } \\
\text { Gaussian }\end{array}$ & 1.31 & 5.28 & 0.0813 & 0.348 \\
\hline $\begin{array}{c}\text { mass \& } \\
\text { radius of gyration }\end{array}$ & $\begin{array}{c}\text { uniform } \\
\text { uniform }\end{array}$ & 1.31 & 5.26 & 0.0812 & 0.336 \\
\hline $\begin{array}{c}\text { heave stiffness \& } \\
\text { pitch stiffness }\end{array}$ & $\begin{array}{c}\text { Gaussian } \\
\text { Gaussian }\end{array}$ & 1.79 & 7.16 & 1.11 & 4.49 \\
\hline $\begin{array}{c}\text { heave stiffness \& } \\
\text { pitch stiffness }\end{array}$ & $\begin{array}{c}\text { uniform } \\
\text { uniform }\end{array}$ & 1.78 & 7.14 & 1.11 & 4.48 \\
\hline $\begin{array}{c}\text { damping ratio 1 \& } \\
\text { damping ratio 2 }\end{array}$ & $\begin{array}{c}\text { Gaussian } \\
\text { Gaussian }\end{array}$ & 0.275 & 1.11 & 0.317 & 1.28 \\
\hline $\begin{array}{c}\text { damping ratio 1 \& } \\
\text { damping ratio 2 }\end{array}$ & $\begin{array}{c}\text { uniform } \\
\text { uniform }\end{array}$ & 0.275 & 1.10 & 0.317 & 1.27 \\
\hline \hline
\end{tabular}

Table 5.3: Results illustrating the uncertainty in the flutter speed due to correlated uncertainty in the structural parameters

due to a fairly mild nature of the flutter type that is experienced in this case. Uncertainty in the flutter speed increases for independent uncertainties in multiple system parameters. The correlation among the inertia and stiffness decreases uncertainty in the flutter speed (most notably in inertia), yet correlation in damping parameters leads to an increased uncertainty in the flutter speed. In contrast to the rigid wing case, the probabilistic characteristics of the flutter 
speed are less sensitive to the structural damping uncertainty due to a slightly more explosive nature of flutter. Some of the flutter speed pdfs indicate mild non-linear mapping effects. For such cases, the mean flutter speed no longer matches the baseline flutter speed. Due to only weak non-linear effects, the increase of the flutter speed uncertainty is almost proportional to an increase in structural parameter uncertainty.
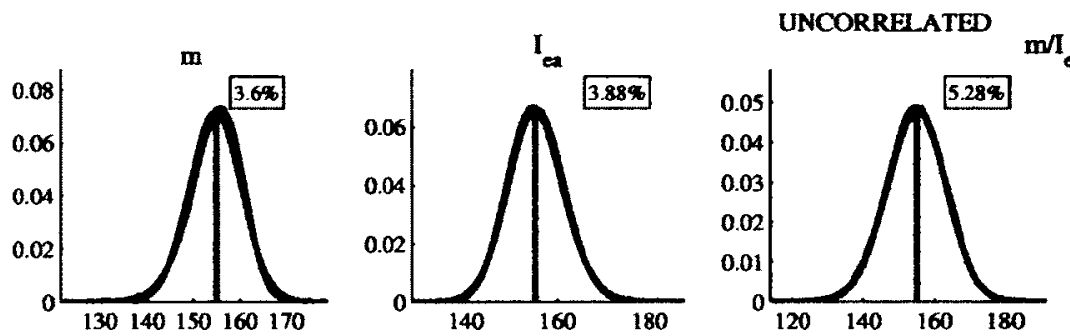

CORRELATED
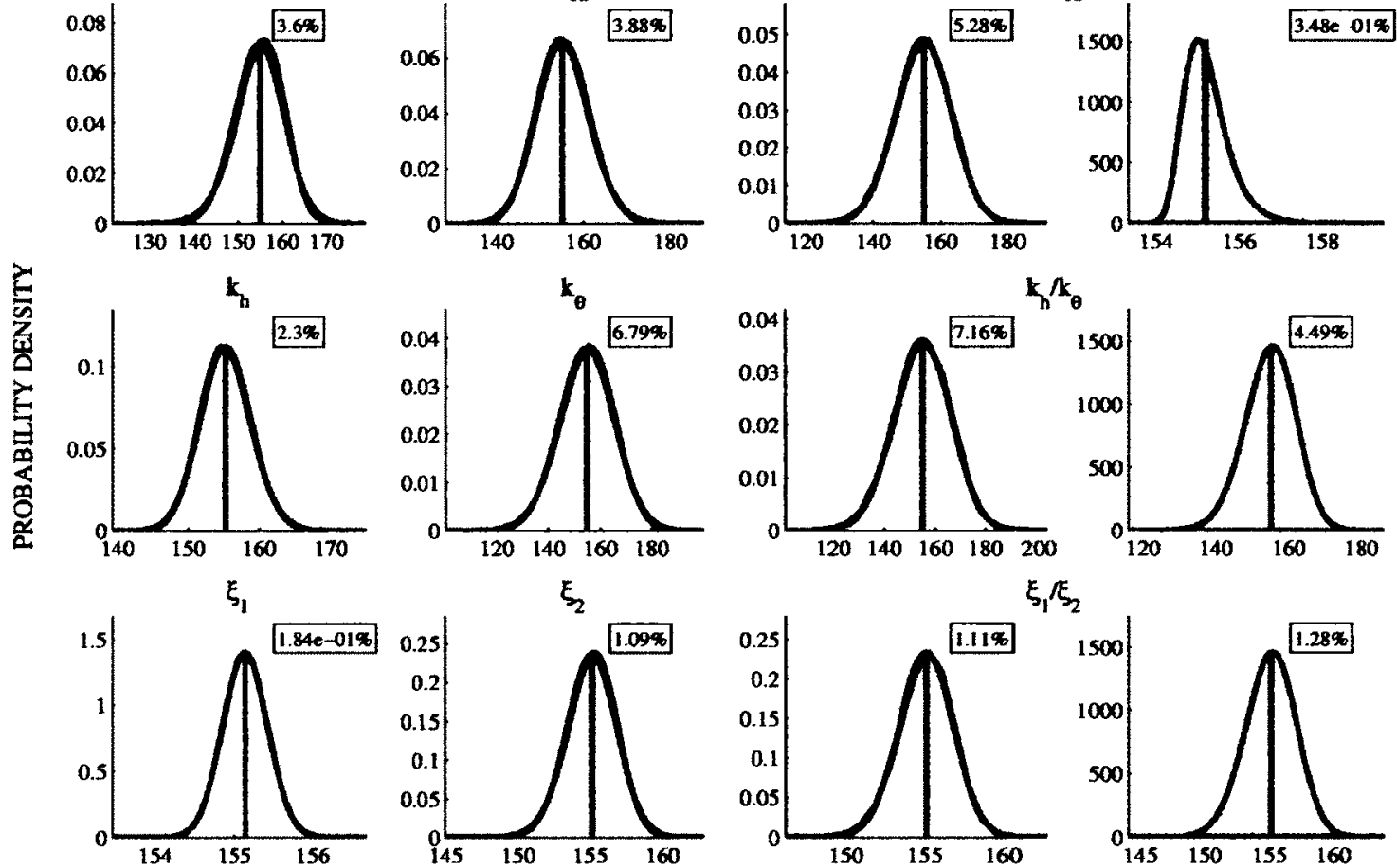

FLUTTER SPEED

Figure 5.9: Flutter speed pdfs (case 1 wing, quasisteady, 10\% COV) 

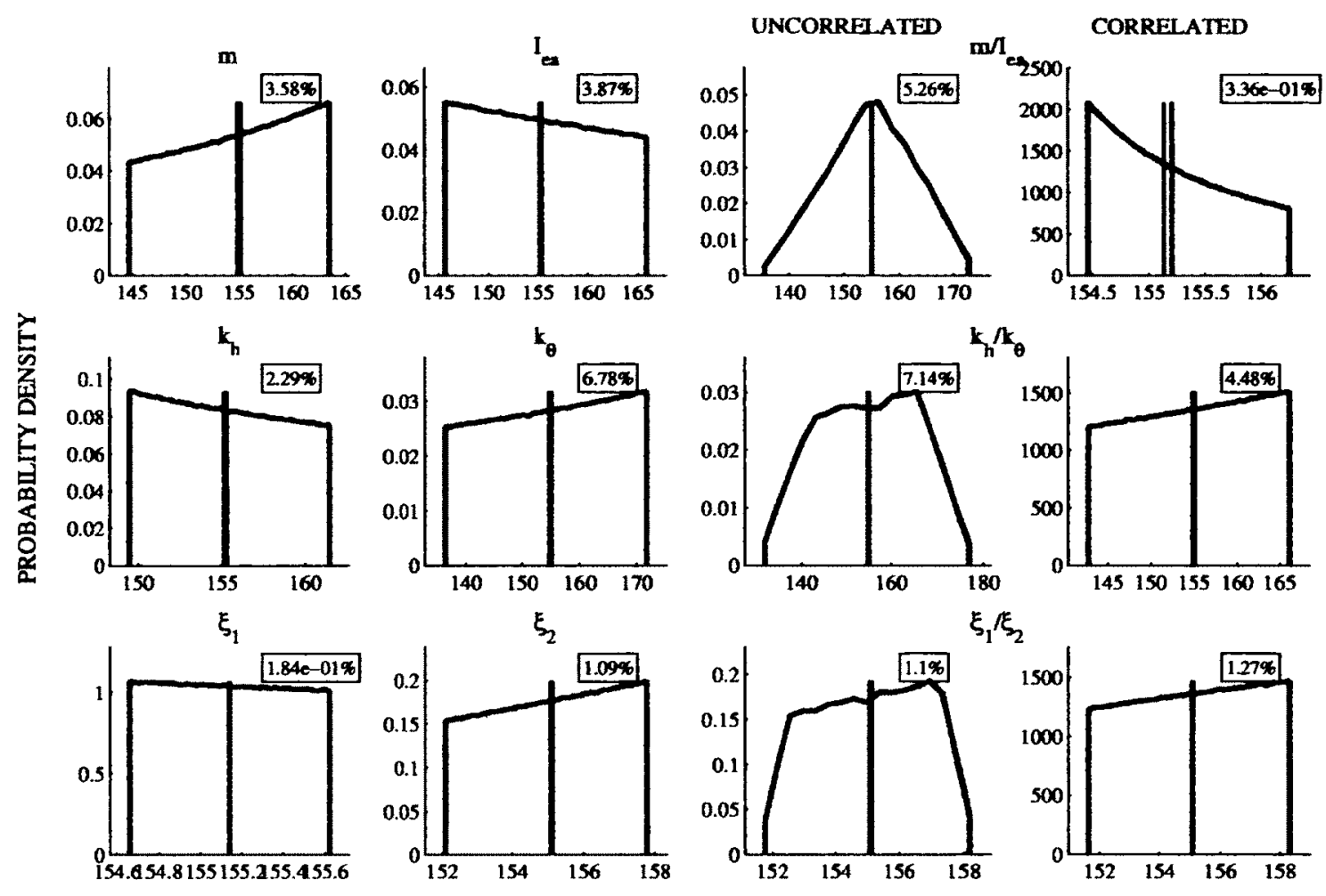

FLUTTER SPEED
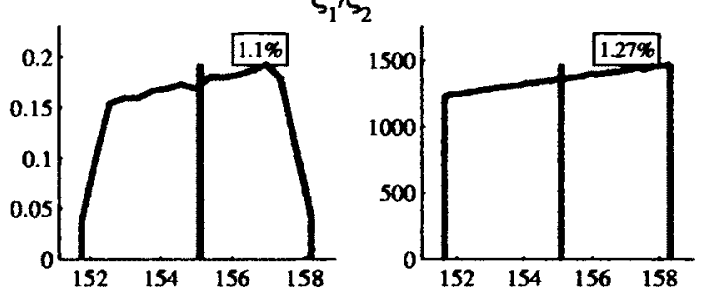

Figure 5.10: Flutter speed pdfs (case 1 wing, quasisteady, 10\% COV)

\section{Unsteady Analysis: Baseline Case}

Next, the baseline results for unsteady aerodynamic forcing are examined. Fig. (5.11) illustrates the modal parameters of the baseline system as a function of air speed. Fig. (5.12) shows a magnified view of the modal parameters of the coalescing modes. In contrast to the case with quasisteady aerodynamics, the unsteady aerodynamics lead to instability of the first mode through a more explosive flutter type (i.e. moderate explosive) with a larger flutter speed of $190.2 \frac{m}{s}$. In contrast to the rigid wing with unsteady aerodynamics, this case exhibits a milder flutter type and a higher flutter speed.

Fig. (5.13) shows the relative magnitude of elements of eigenvectors of each mode at no flow condition and at flutter speed. As the airspeed increases, the relative strength of individual components in each eigenvector changes. Similar to the case of quasisteady aerodynamics, initially the first mode exhibits a dominant first bending component. The second mode is first 
torsion component dominant. At the flutter point the two modes lose the relative strengths of their dominant components.
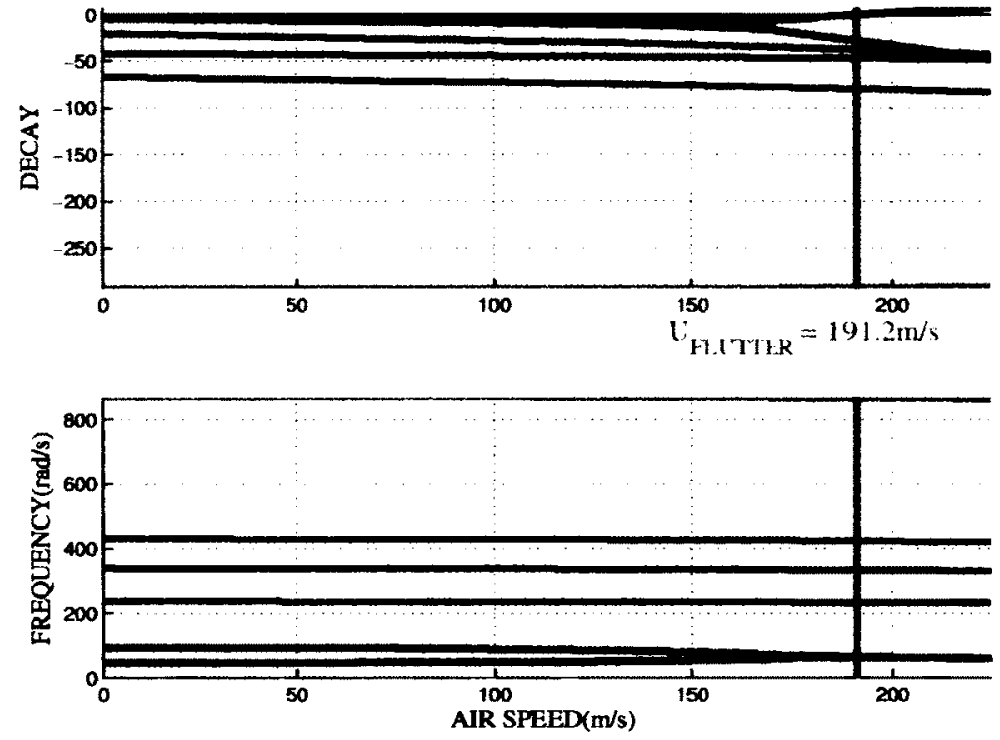

Figure 5.11: Evolution of modal parameters with respect to airspeed
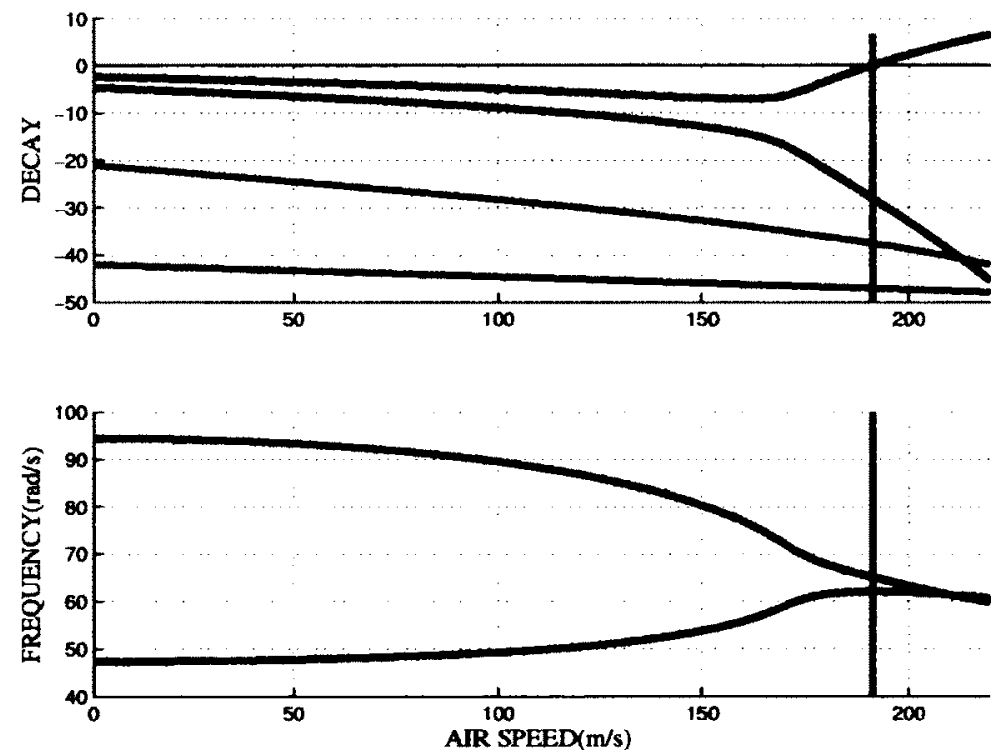

Figure 5.12: Magnified view of evolution of the modal parameters with respect to increasing airspeed 

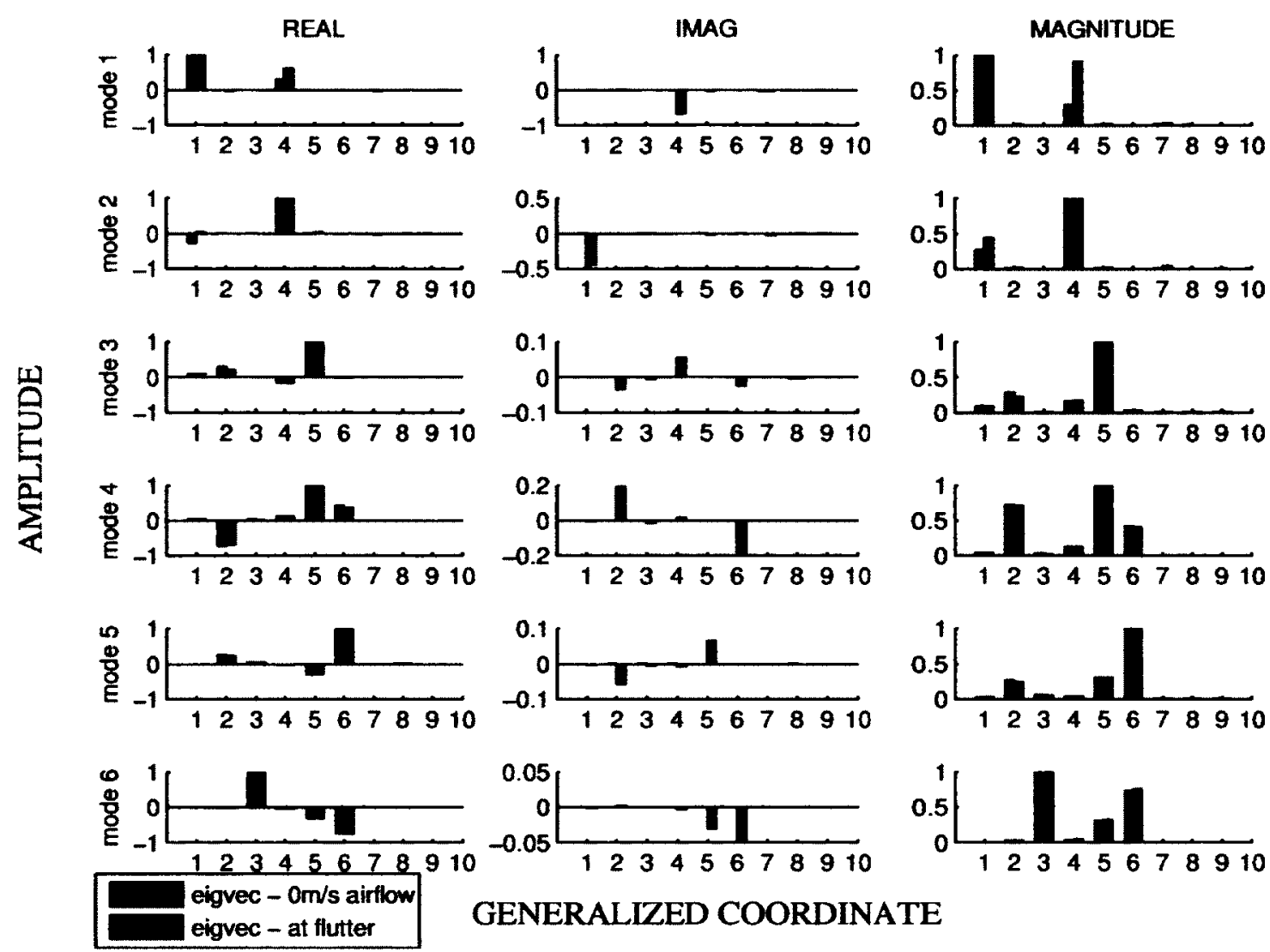

GENERALIZED COORDINATE

Figure 5.13: real part, imaginary part, and magnitude of eigenvectors of the vibration modes, illustrating relative strength of each component

\section{Unsteady Analysis: Probabilistic Case}

The probabilistic flutter speed estimation results with unsteady aerodynamics are presented in figs. (5.14) and (5.15) for a truncated Gaussian and uniform structural parameter distributions with a larger amount of uncertainty, respectively. A summary of these results is reported in tbls. (5.4) and (5.5). Similar to the quasisteady aerodynamics, only the results for large input uncertainty $(10 \% \mathrm{COV})$ will be examined in details.

Most of the conclusions are similar to the case with quasisteady aerodynamics. As expected from a more explosive flutter type, the randomness in inertia and stiffness parameters introduces significant uncertainty in the flutter speed and the effect of damping is not significant, as evident from figs. (5.14) and (5.15). In general, the most significant qualitative conclusions of the cantilever model and a rigid wing model retain similarities. 


\begin{tabular}{|c|c|c|c|}
\hline \multicolumn{2}{|c|}{ Input COV } & $2.5 \%$ & $10 \%$ \\
\hline Random Parameter & Distribution & \multicolumn{2}{c|}{ Flutter Speed COV \% } \\
\hline \hline \multirow{2}{*}{ mass } & Gaussian & 0.425 & 1.76 \\
\cline { 2 - 4 } & uniform & 0.424 & 1.73 \\
\hline radius of gyration & Gaussian & 0.690 & 2.76 \\
\cline { 2 - 4 } & uniform & 0.690 & 2.76 \\
\hline \multirow{2}{*}{ heave stiffness } & Gaussian & 0.360 & 1.45 \\
\cline { 2 - 4 } & uniform & 0.360 & 1.44 \\
\hline \multirow{2}{*}{ pitch stiffness } & Gaussian & 1.43 & 5.73 \\
\cline { 2 - 4 } & uniform & 1.43 & 5.72 \\
\hline \multirow{2}{*}{ damping ratio 1 } & Gaussian & 0.0898 & 0.359 \\
\cline { 2 - 4 } & uniform & 0.0898 & 0.359 \\
\hline \multirow{2}{*}{ damping ratio 2 } & Gaussian & 0.0509 & 0.204 \\
\cline { 2 - 4 } & uniform & 0.0509 & 0.204 \\
\hline
\end{tabular}

Table 5.4: Results illustrating the uncertainty in the flutter speed due to independent uncertainty in the structural parameters

\begin{tabular}{|c|c|c|c|c|c|}
\hline \multicolumn{2}{|c|}{ Input COV } & $2.5 \%$ & $10 \%$ & $2.5 \%$ & $10 \%$ \\
\hline Random Parameter & Distribution & \multicolumn{3}{c|}{ Flutter Speed COV (\%) } \\
\hline \hline \multicolumn{2}{|c|}{} & Uncorrelated Parameters & Fully Correlated Parameters \\
\hline \hline $\begin{array}{c}\text { mass \& } \\
\text { radius of gyration }\end{array}$ & $\begin{array}{c}\text { Gaussian } \\
\text { Gaussian }\end{array}$ & 0.812 & 3.28 & 0.267 & 1.1 \\
\hline $\begin{array}{c}\text { mass \& } \\
\text { radius of gyration }\end{array}$ & $\begin{array}{c}\text { uniform } \\
\text { uniform }\end{array}$ & 0.812 & 3.27 & 0.267 & 1.08 \\
\hline $\begin{array}{c}\text { heave stiffness \& } \\
\text { pitch stiffness }\end{array}$ & $\begin{array}{c}\text { Gaussian } \\
\text { Gaussian }\end{array}$ & 1.47 & 5.89 & 1.07 & 4.30 \\
\hline $\begin{array}{c}\text { heave stiffness \& } \\
\text { pitch stiffness }\end{array}$ & $\begin{array}{c}\text { uniform } \\
\text { uniform }\end{array}$ & 1.47 & 5.88 & 1.07 & 4.29 \\
\hline $\begin{array}{c}\text { damping ratio 1 \& } \\
\text { damping ratio 2 }\end{array}$ & $\begin{array}{c}\text { Gaussian } \\
\text { Gaussian }\end{array}$ & 0.103 & 0.414 & 0.141 & 0.563 \\
\hline $\begin{array}{c}\text { damping ratio 1 \& } \\
\text { damping ratio 2 }\end{array}$ & $\begin{array}{c}\text { uniform } \\
\text { uniform }\end{array}$ & 0.103 & 0.413 & 0.141 & 0.563 \\
\hline \hline
\end{tabular}

Table 5.5: Results illustrating the uncertainty in the flutter speed due to correlated uncertainty in the structural parameters 


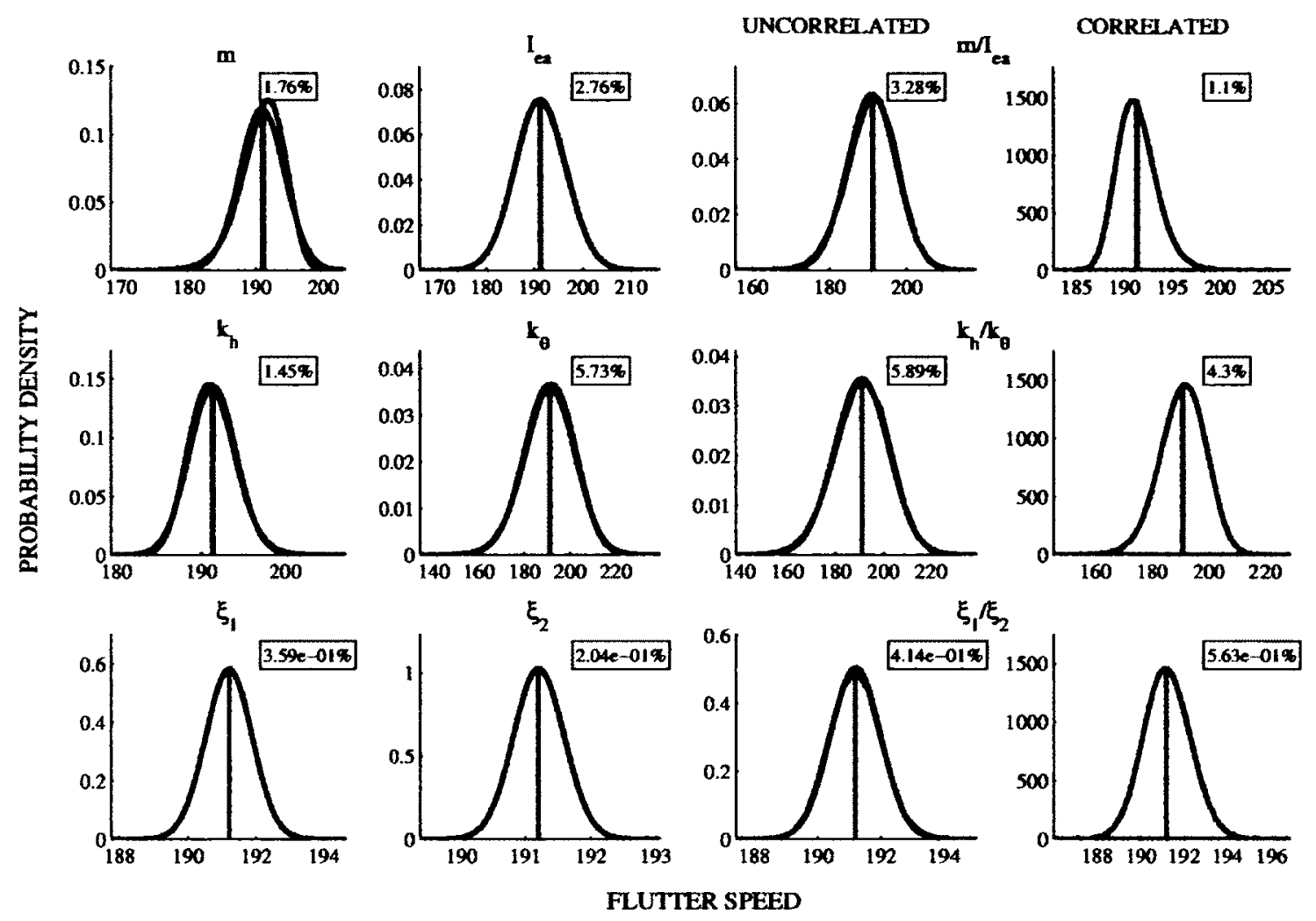

Figure 5.14: Flutter speed pdfs (case 1 wing, unsteady, 10\% COV) 

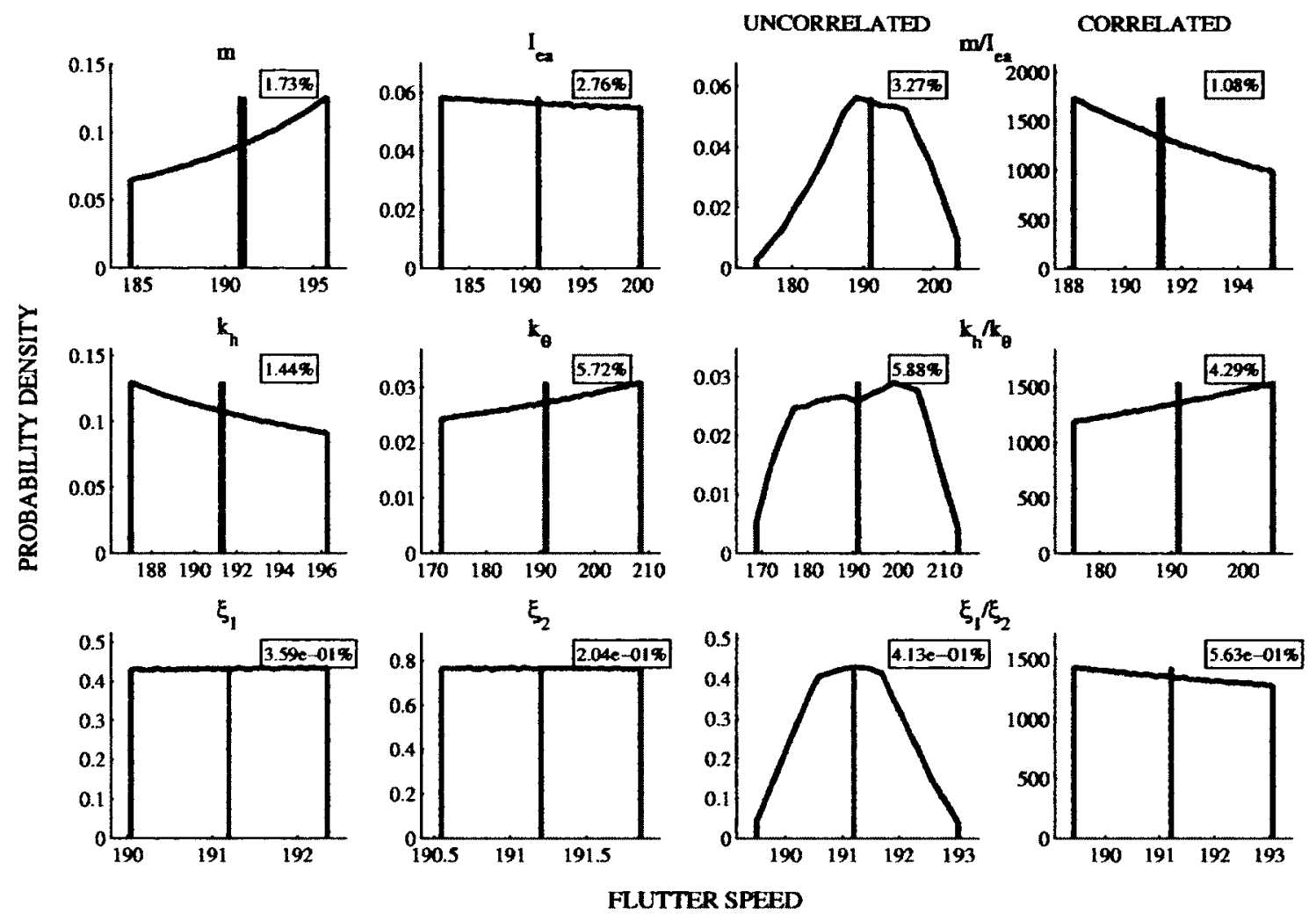

Figure 5.15: Flutter speed pdfs (case 1 wing, unsteady, 10\% COV)

Next, the probabilistic results of Case 2 study of a cantilever wing are examined.

\subsubsection{CASE 2}

The parameters of the baseline system representing Case 2 are reported in tbl. (5.6). As stated earlier, this case represents a canonical cantilever wing model that experiences contributions of the higher order basis functions in the aeroelastic modes, as discussed next. The arising complex motion exhibited by the cantilever cannot be adequately represented by the two dof of rigid wing. 


\begin{tabular}{||l|c|l|}
\hline PARAMETER & Symbol & 3D Wing \\
\hline \hline mass & $m$ & $35.7187 \mathrm{~kg} / \mathrm{m}$ \\
normalized radius of & & \\
gyration & $r_{g}$ & 1.05 \\
heave/bending stiffness & $k_{h} / E I$ & $9.77 \times 10^{6} \mathrm{Nm}^{2}$ \\
pitch/torsional stiffness & $k_{\theta} / G J$ & $3.956 \times 10^{5} \mathrm{Nm}^{2}$ \\
chord length & $c$ & $1.8288 \mathrm{~m}$ \\
span & $L$ & $6.096 \mathrm{~m}$ \\
normalized static imbalance & $x_{\theta}$ & 0.95 \\
damping ratio (mode 1) & $\xi_{1}$ & 0.04 \\
damping ratio (mode 2) & $\xi_{2}$ & 0.04 \\
free stream fluid density & $\rho_{\infty}$ & $1.225 \mathrm{~kg} / \mathrm{m}^{3}$ \\
normalized distance btw & & \\
EA and mid-chord & $a_{h}$ & -1.0 \\
lift coefficient gradient & $\bar{C}_{l \alpha}$ & $2 \pi\left(1-\eta^{2}\right)$ \\
\hline \hline Num. of Samples & - & 100000 \\
\hline
\end{tabular}

Table 5.6: Model parameters for Case 2

\section{Quasisteady Analysis: Baseline Case}

The aeroelastic frequencies and decays are plotted as a function of airspeed in fig. (5.16). Similar to Case 1, three bending and three torsional beam basis were used for accurate computation of the coalescing modes. A magnified view of the modal parameters of two coalescing modes is shown in fig. (5.17). As evident in this figure, the second mode becomes unstable through a moderate type of flutter occurring at a flutter speed of $160.21 \mathrm{~m} / \mathrm{s}$.

Fig. (5.18) shows the relative magnitude of the elements of eigenvectors for each mode at no flow condition and at flutter speed. Similar to Case 1, the stable coalescing mode (mode 1) consists mainly of the first bending and first torsional beam basis components. In contrast to Case 1, the unstable mode (mode 2) contains substantial contribution of the first bending, first and second torsional beam basis functions. Without any flow, the first aeroelastic mode exhibits is dominated by the first torsional basis component. The second mode indicates dominance of the first bending and the second torsional basis functions. At the flutter point, the relative strength of the first bending and first torsion basis components in the coalescing modes changes and the effect of the second torsional basis reduces for the unstable mode. 

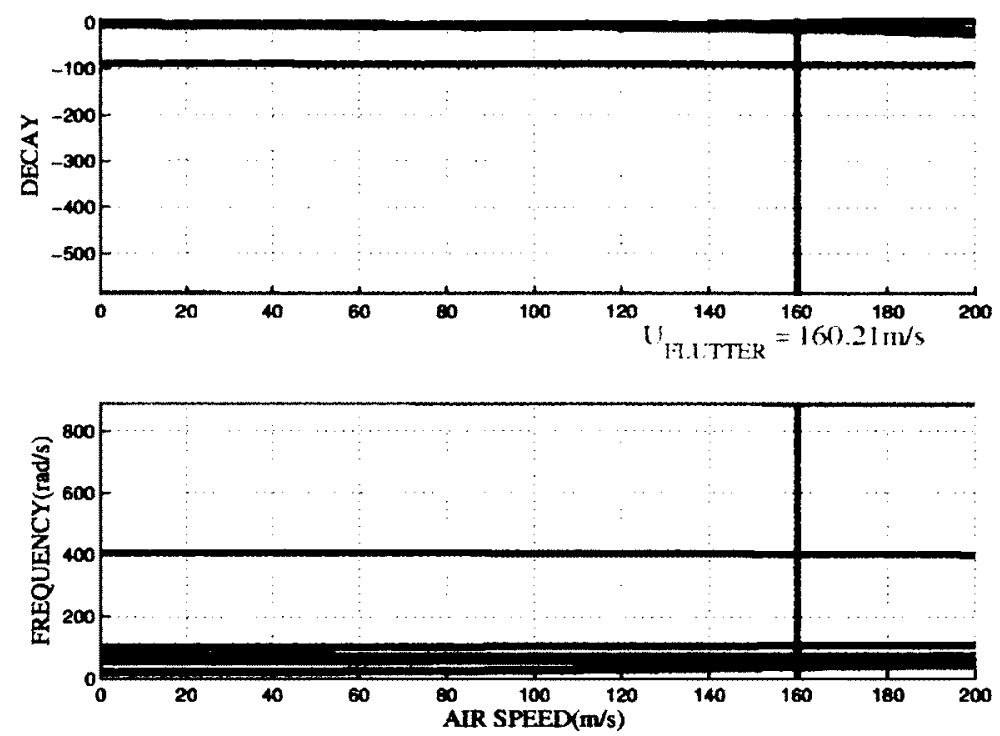

Figure 5.16: Evolution of modal parameters with respect to airspeed
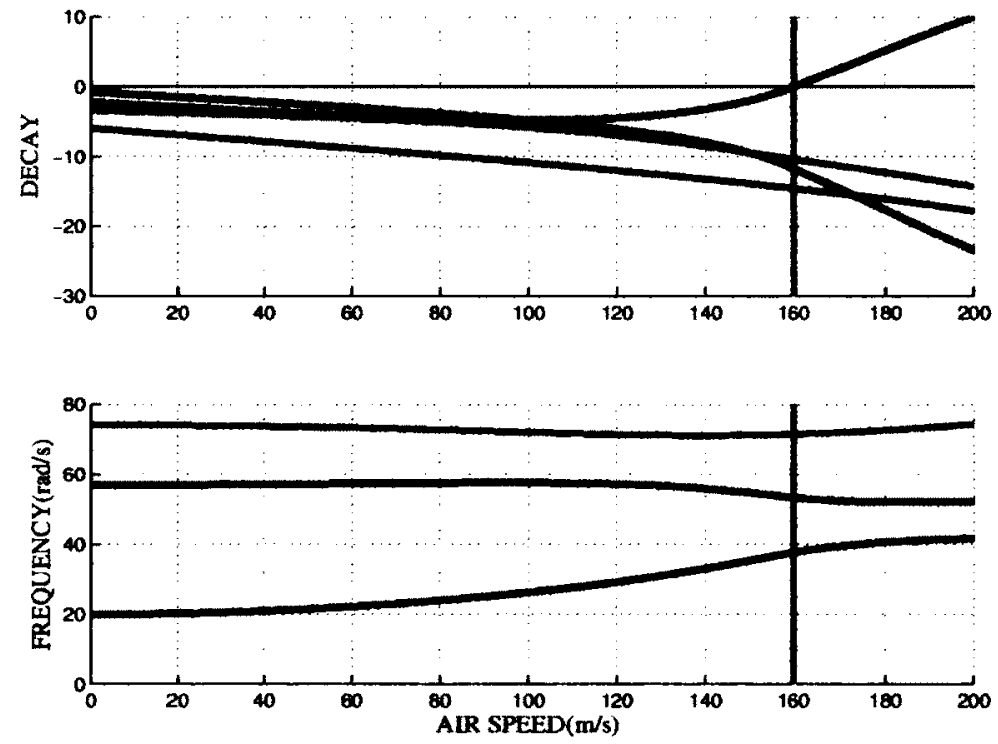

Figure 5.17: Magnified view of evolution of the modal parameters with respect to increasing airspeed 

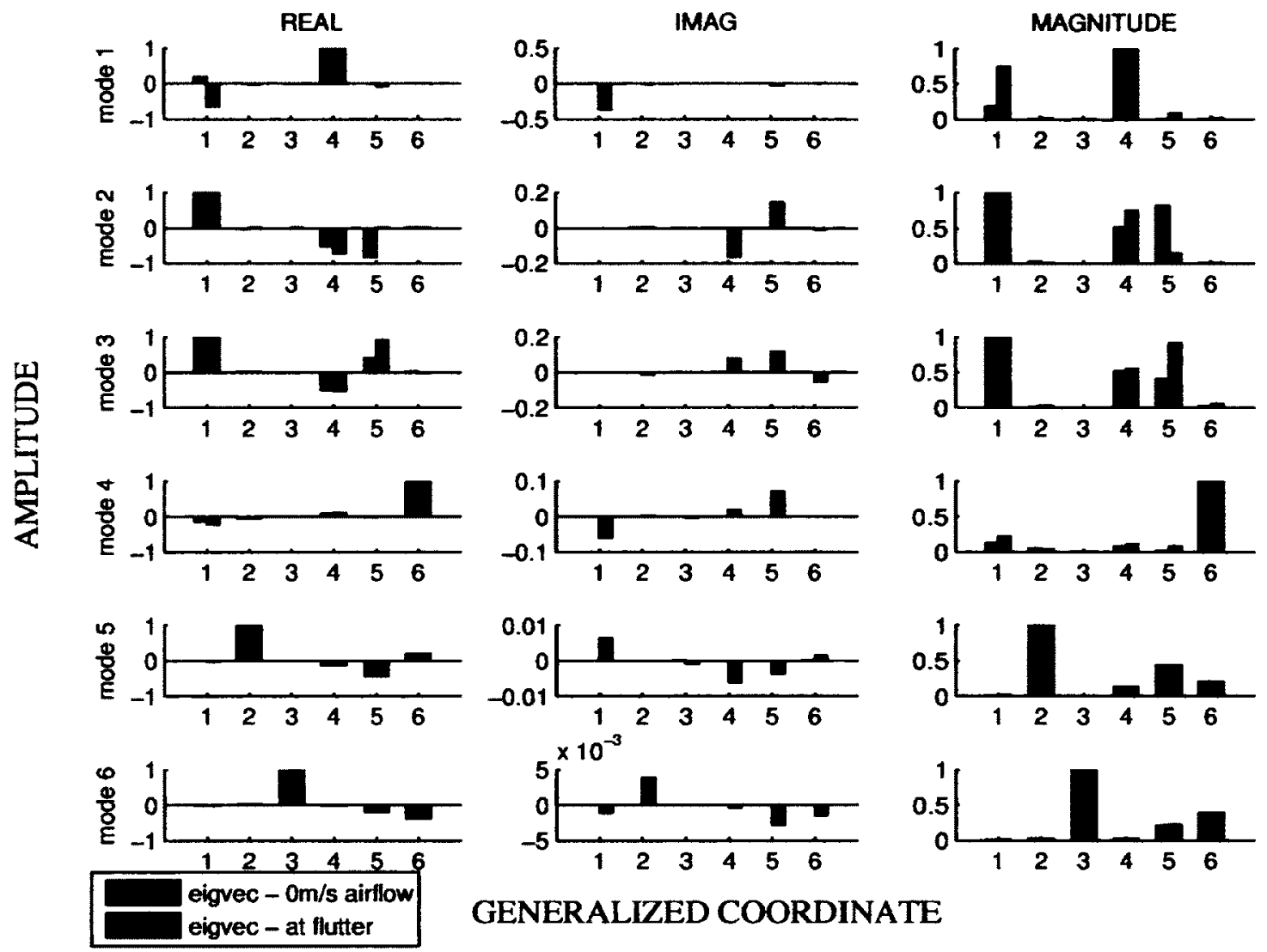

GENERALIZED COORDINATE

Figure 5.18: real part, imaginary part, and magnitude of eigenvectors of the vibration modes, illustrating relative strength of each component

In contrast to Case 2 of the rigid wing, the cantilever model exhibits a more explosive type of flutter and a significantly larger flutter speed.

\section{Quasisteady Analysis: Probabilistic Case}

Tbls. (5.7) and (5.8) report a brief summary of the probabilistic results for Case 2 study with quasisteady aerodynamics. Figs. (5.19) and (5.20) show the resulting flutter speed pdfs. These figures illustrate the results for parameter uncertainty with $10 \% \mathrm{COV}$, with a truncated Gaussian and a uniform distribution, respectively. From these results, the following observations were obtained. Uncertainty in most cases of the inertia and stiffness parameters produces larger effects on the flutter speed estimate than those due to damping. Uncertainty in the torsional stiffness parameter does not produce significant uncertainty in the flutter speed. From the study of the flutter speed mapping plots of the previous chapter, it is conjectured that the cause of 


\begin{tabular}{|c|c|c|c|}
\hline \multicolumn{2}{|c|}{ Input COV } & $2.5 \%$ & $10 \%$ \\
\hline Random Parameter & Distribution & \multicolumn{2}{c|}{ Flutter Speed COV \% } \\
\hline \hline \multirow{2}{*}{ mass } & Gaussian & 0.283 & 1.49 \\
\cline { 2 - 4 } & uniform & 0.279 & 1.28 \\
\hline radius of gyration & Gaussian & 0.244 & 1.17 \\
\cline { 2 - 4 } & uniform & 0.242 & 0.945 \\
\hline \multirow{2}{*}{ heave stiffness } & Gaussian & 1.33 & 5.32 \\
\cline { 2 - 4 } & uniform & 1.33 & 5.31 \\
\hline \multirow{2}{*}{ pitch stiffness } & Gaussian & 0.228 & 0.936 \\
\cline { 2 - 4 } & uniform & 0.228 & 0.923 \\
\hline \multirow{2}{*}{ damping ratio 1 } & Gaussian & 0.00472 & 0.0189 \\
\cline { 2 - 4 } & uniform & 0.00472 & 0.0189 \\
\hline damping ratio 2 & Gaussian & 0.177 & 0.712 \\
\cline { 2 - 4 } & uniform & 0.177 & 0.710 \\
\hline
\end{tabular}

Table 5.7: Results illustrating the uncertainty in the flutter speed due to independent uncertainty in the structural parameters

\begin{tabular}{|c|c|c|c|c|c|}
\hline \multicolumn{2}{|c|}{ Input COV } & $2.5 \%$ & $10 \%$ & $2.5 \%$ & $10 \%$ \\
\hline Random Parameter & Distribution & \multicolumn{3}{c|}{ Flutter Speed COV (\%) } \\
\hline \hline \multicolumn{2}{|c|}{} & \multicolumn{3}{|c|}{ Uncorrelated Parameters } & Fully Correlated Parameters \\
\hline \hline $\begin{array}{c}\text { mass \& } \\
\text { radius of gyration }\end{array}$ & $\begin{array}{c}\text { Gaussian } \\
\text { Gaussian }\end{array}$ & 0.378 & 2.19 & 0.0335 & 0.143 \\
\hline $\begin{array}{c}\text { mass \& } \\
\text { radius of gyration }\end{array}$ & $\begin{array}{c}\text { uniform } \\
\text { uniform }\end{array}$ & 0.374 & 1.88 & 0.0334 & 0.138 \\
\hline $\begin{array}{c}\text { heave stiffness \& } \\
\text { pitch stiffness }\end{array}$ & $\begin{array}{c}\text { Gaussian } \\
\text { Gaussian }\end{array}$ & 1.34 & 5.40 & 1.10 & 4.43 \\
\hline $\begin{array}{c}\text { heave stiffness \& } \\
\text { pitch stiffness }\end{array}$ & $\begin{array}{c}\text { uniform } \\
\text { uniform }\end{array}$ & 1.34 & 5.39 & 1.10 & 4.42 \\
\hline $\begin{array}{c}\text { damping ratio 1 \& } \\
\text { damping ratio 2 }\end{array}$ & $\begin{array}{c}\text { Gaussian } \\
\text { Gaussian }\end{array}$ & 0.177 & 0.712 & 0.173 & 0.692 \\
\hline $\begin{array}{c}\text { damping ratio 1 \& } \\
\text { damping ratio 2 }\end{array}$ & $\begin{array}{c}\text { uniform } \\
\text { uniform }\end{array}$ & 0.177 & 0.710 & 0.173 & 0.691 \\
\hline \hline
\end{tabular}

Table 5.8: Results illustrating the uncertainty in the flutter speed due to correlated uncertainty in the structural parameters

such low sensitivity is the low gradient of the flutter speed with respect to the torsional stiffness parameter within the uncertainty region. Uncertainty in the multiple uncorrelated structural parameters causes a larger variance in the flutter speed estimate. The correlation among all, inertia, stiffness and damping parameters reduces the uncertainty in the flutter speed. The uncertainty in the inertia parameters leads to significant non-linear effects in the flutter speed pdfs. 

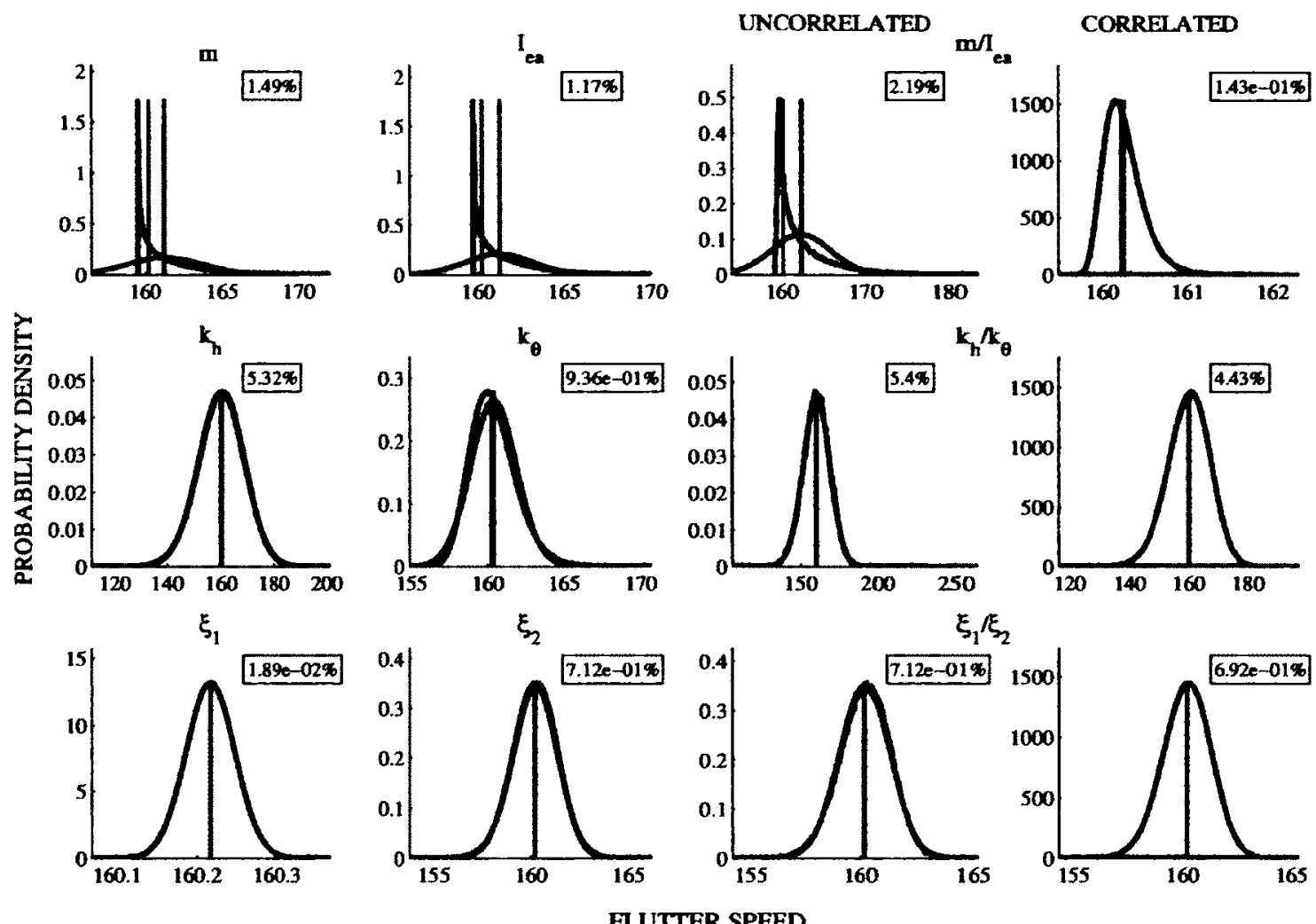

FLUTTER SPEED

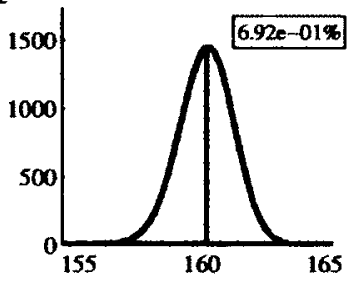

Figure 5.19: Flutter speed pdfs (case 2 wing, quasisteady, 10\% COV)

For such cases, the mean flutter speed does not match the baseline flutter speed, as a large positive skewness and a minimum cutoff are observed in the flutter speed pdfs. In cantilever wing analysis, the most notable differences between the cantilever and the rigid wing arises from a more profound non-linear effect due to uncertainty in inertia parameters and a less significant influence of damping due to a more explosive flutter type, as evident from figs. (5.19) and (5.20).

\section{Unsteady Analysis: Baseline Case}

Fig. (5.21) shows the modal properties as a function of increasing air speed of the baseline system for unsteady aerodynamics. Fig. (5.22) shows a magnified view of the modal parameters of the coalescing modes. In contrast to the quasisteady aerodynamics, the first mode becomes unstable, the flutter type becomes moderate-explosive and the flutter speed increases to $187.91 \mathrm{~m} / \mathrm{s}$. 

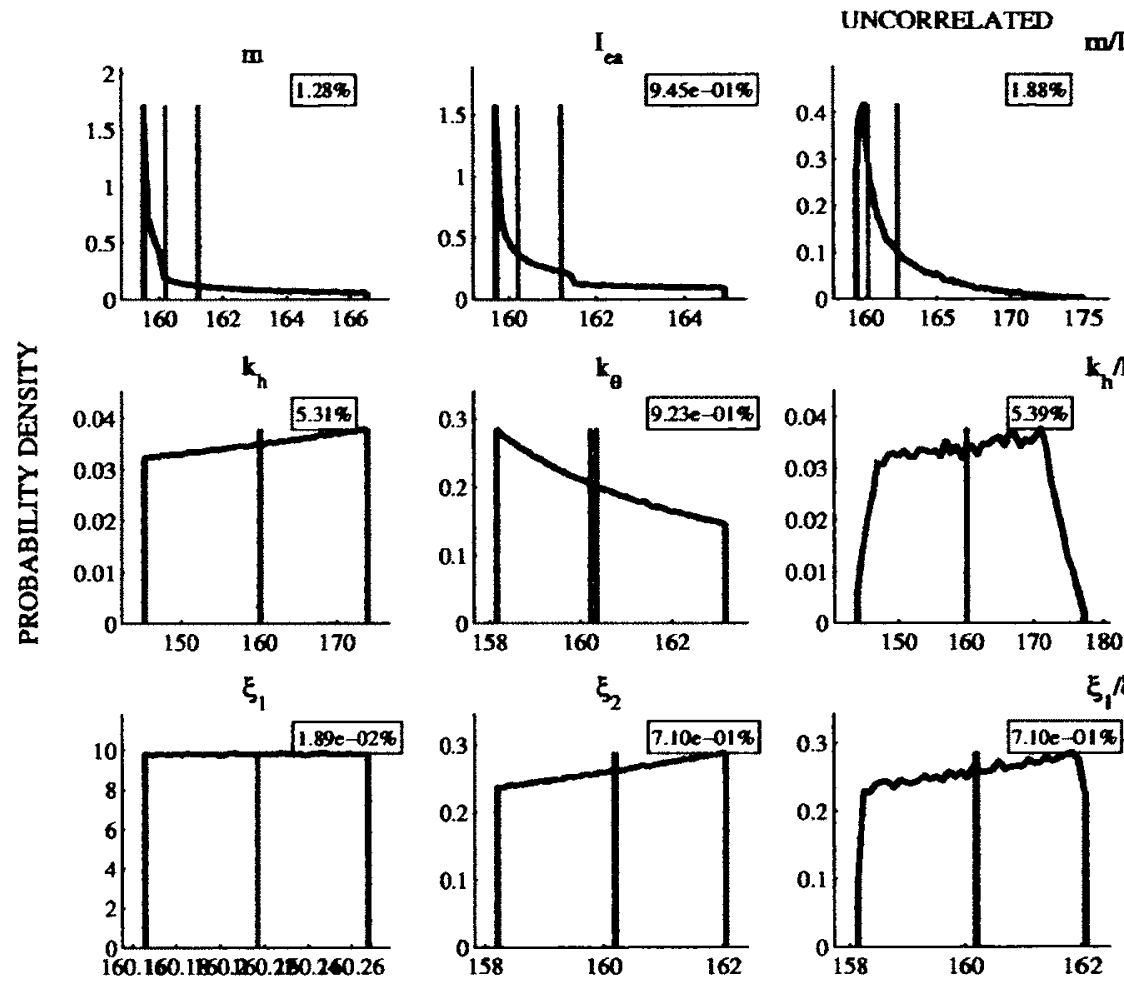

CORREIATED
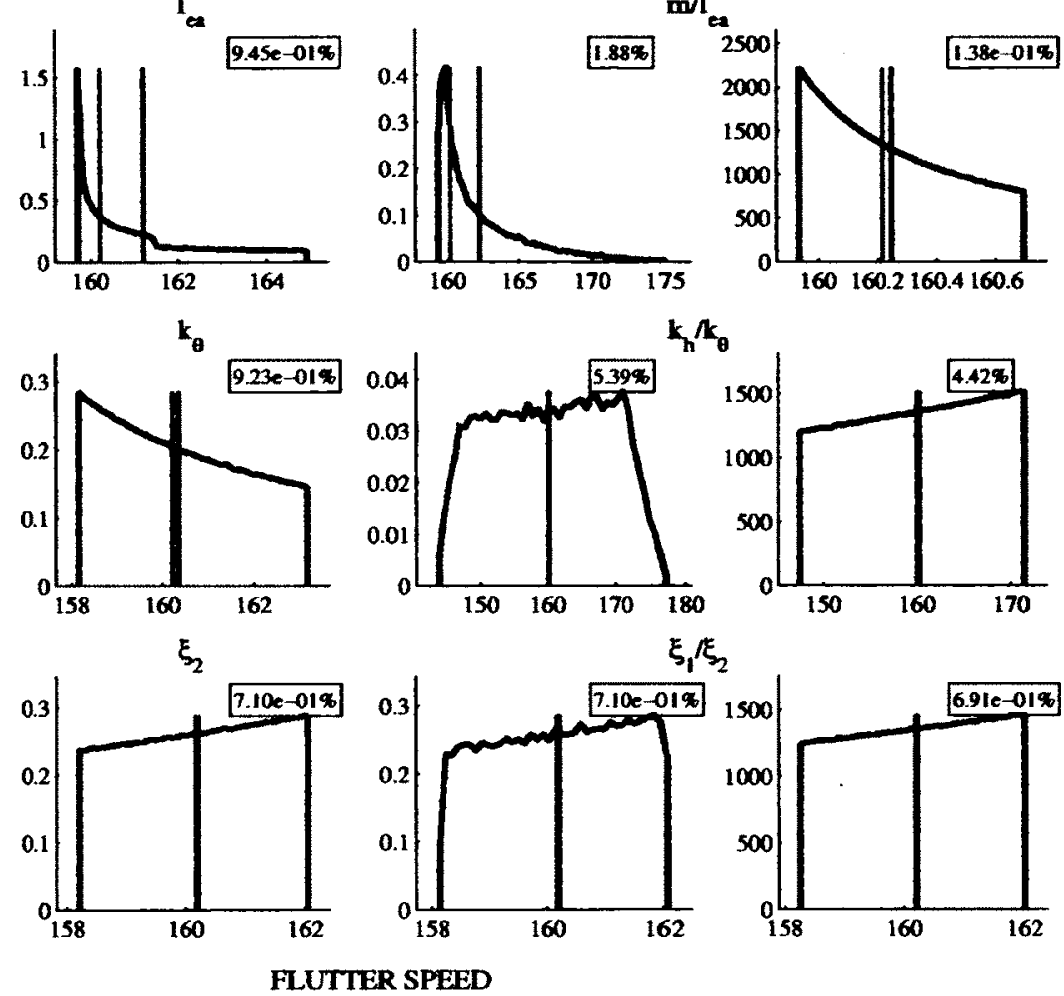

Figure 5.20: Flutter speed pdfs (case 2 wing, quasisteady, $10 \% \mathrm{COV}$ )
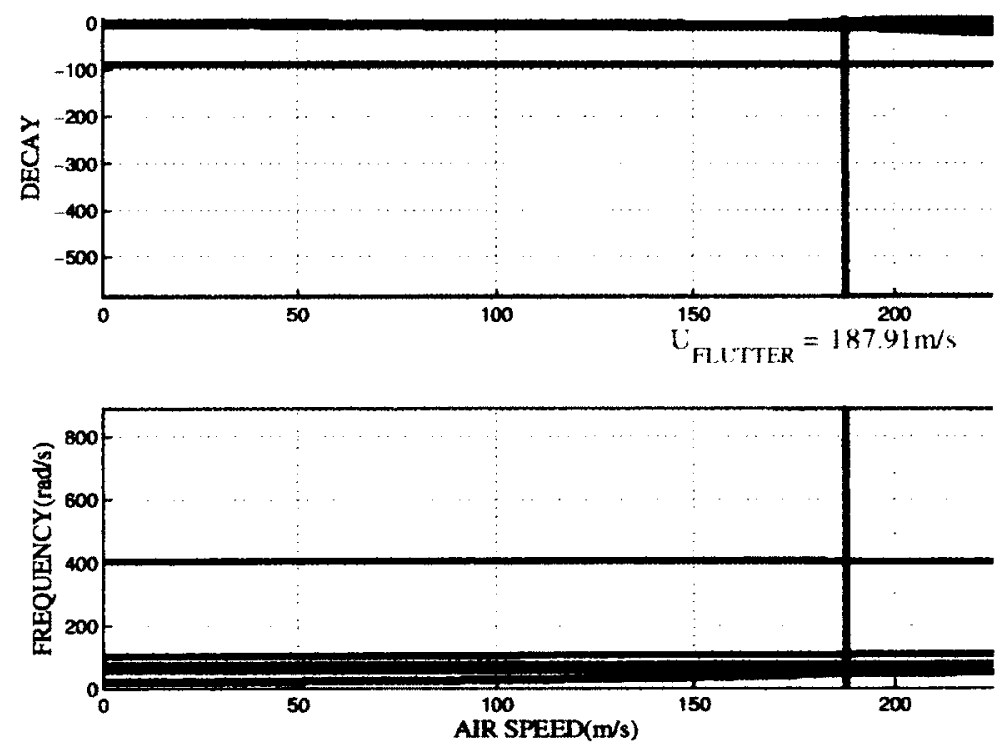

Figure 5.21: Evolution of modal parameters with respect to airspeed 

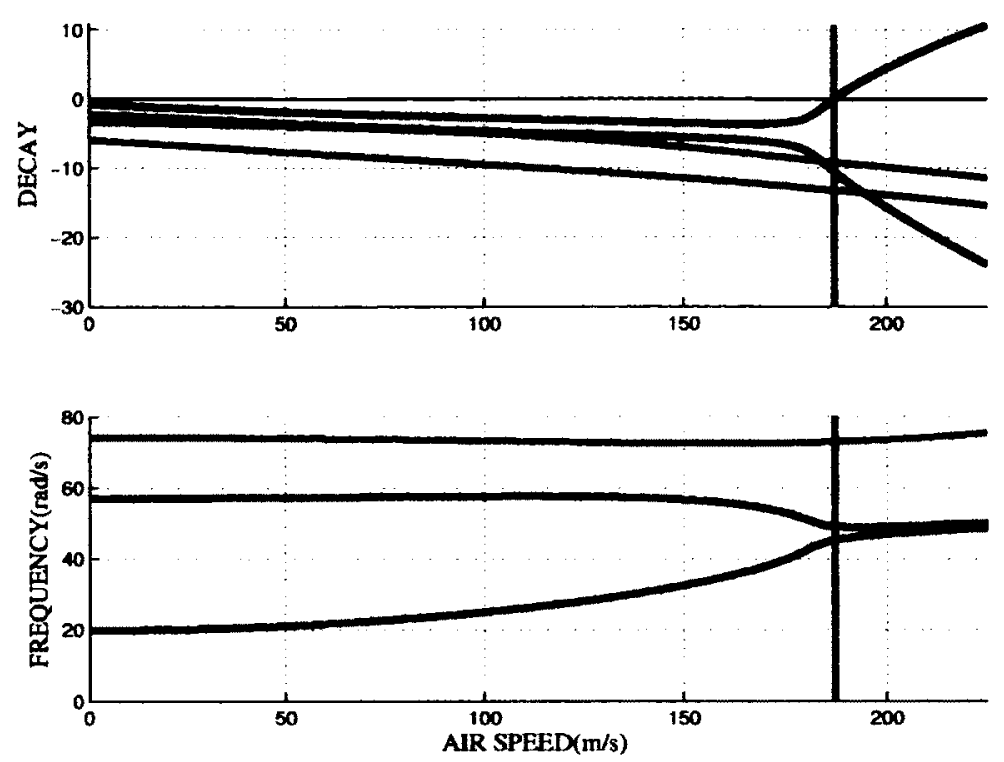

Figure 5.22: Magnified view of evolution of the modal parameters with respect to increasing airspeed

Fig. (5.23) illustrates the relative magnitude of the elements of eigenvectors for each mode at no flow condition and at flutter speed. As in quasisteady analysis, the first mode consists mainly of the first bending and first torsional beam basis and the second mode contains substantial contributions of the first bending, first and second torsional beam basis functions. For unsteady aerodynamics, the first mode becomes unstable. The relative strength of each eigenvector changes with an increase of airspeed. Without air flow, the first mode exhibits dominant first torsional component and the second mode shows dominant contributions of the first bending and second torsion basis, with a significant contribution of the first torsional basis component. At flutter point, both modes lose the relative strength of their dominant components and furthermore, the stable mode experiences a drastic reduction of the second torsional basis component.

In contrast to the rigid wing (as evident from fig.(3.24)), where the second mode becomes unstable, the cantilever exhibits instability through the first mode, as shown in fig.(5.22). Additionally, it is noted that a similar type of flutter is experienced for both cases, but the onset of flutter is delayed for the cantilever case. 

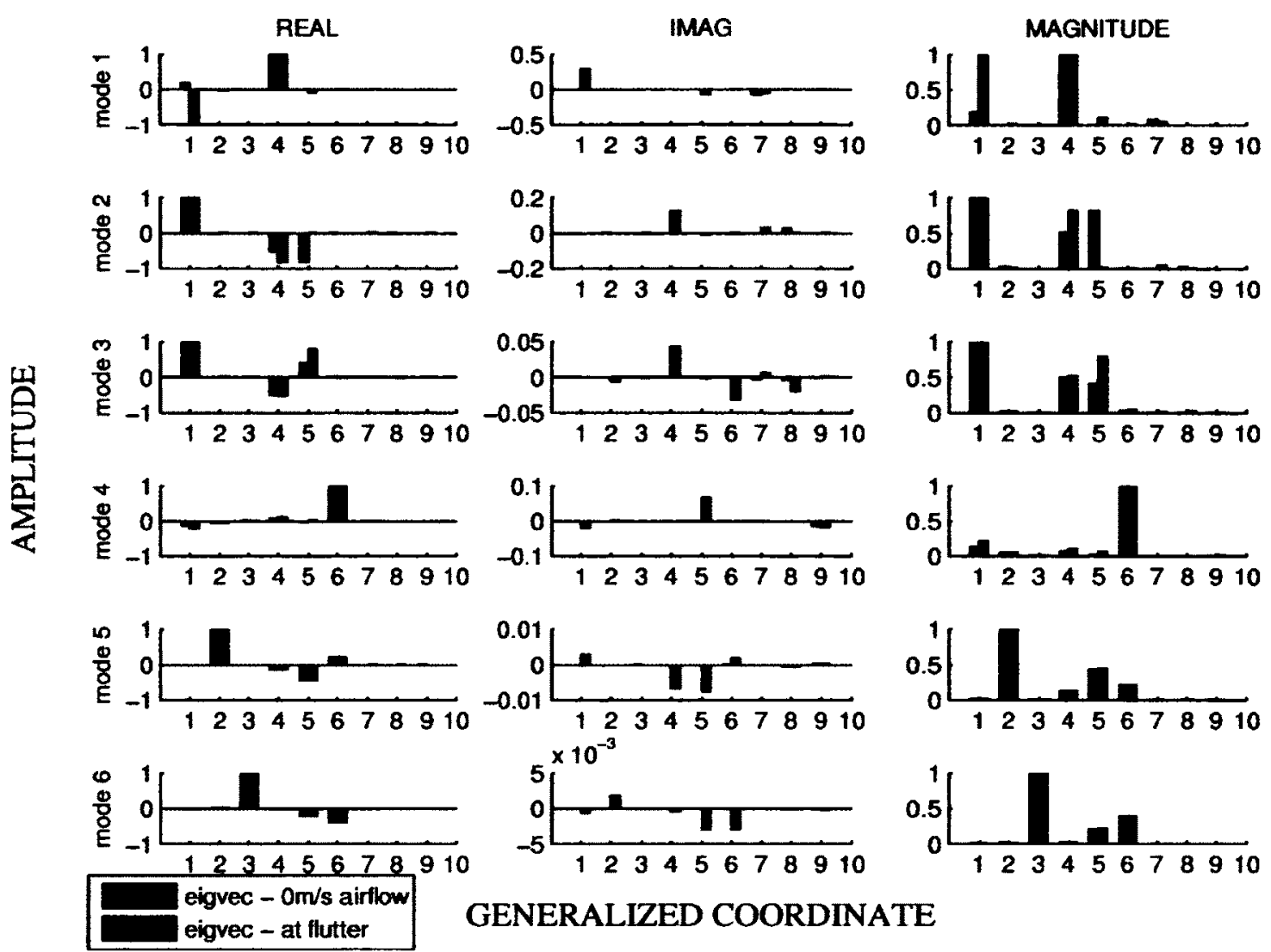

GENERALIZED COORDINATE

Figure 5.23: real part, imaginary part, and magnitude of eigenvectors of the vibration modes, illustrating relative strength of each component

\section{Unsteady Analysis: Probabilistic Case}

Tbls. (5.9) and (5.10) summarize the probabilistic flutter speed results for analysis with unsteady aerodynamics. Figs. (5.24) and (5.25) show the resulting flutter speed pdfs corresponding to a truncated Gaussian and a uniform distribution of structural parameters, respectively, having a $10 \% \mathrm{COV}$. From these figures the following observations are obtained. Uncertainty in most cases of the inertia and stiffness parameters produces larger effects on the flutter speed estimate than that of damping, as expected with a moderate-explosive type of flutter. Uncertainty in the torsional stiffness, mass and mass moment of inertia parameters does not produce significant uncertainty in the flutter speed. The effect of multiple independent structural parameter uncertainties produce a larger variance in the flutter speed estimate. In this case, the correlation effects in inertia and stiffness parameters reduce the uncertainty in the flutter speed, yet 


\begin{tabular}{|c|c|c|c|}
\hline \multicolumn{2}{|c|}{ Input COV } & $2.5 \%$ & $10 \%$ \\
\hline Random Parameter & Distribution & \multicolumn{2}{c|}{ Flutter Speed COV \% } \\
\hline \hline \multirow{2}{*}{ mass } & Gaussian & 0.251 & 1.13 \\
\cline { 2 - 4 } & uniform & 0.250 & 1.06 \\
\hline radius of gyration & Gaussian & 0.0416 & 0.468 \\
\cline { 2 - 4 } & uniform & 0.0384 & 0.259 \\
\hline \multirow{2}{*}{ heave stiffness } & Gaussian & 1.08 & 4.34 \\
\cline { 2 - 4 } & uniform & 1.08 & 4.32 \\
\hline \multirow{2}{*}{ pitch stiffness } & Gaussian & 0.0133 & 0.0593 \\
\cline { 2 - 4 } & uniform & 0.0132 & 0.0556 \\
\hline damping ratio 1 & Gaussian & 0.0230 & 0.0922 \\
\cline { 2 - 4 } & uniform & 0.0230 & 0.0921 \\
\hline damping ratio 2 & Gaussian & 0.00226 & 0.0106 \\
\cline { 2 - 4 } & uniform & 0.00224 & 0.00971 \\
\hline
\end{tabular}

Table 5.9: Results illustrating the uncertainty in the flutter speed due to independent uncertainty in the structural parameters

\begin{tabular}{|c|c|c|c|c|c|}
\hline \multicolumn{2}{|c|}{ Input COV } & $2.5 \%$ & $10 \%$ & $2.5 \%$ & $10 \%$ \\
\hline Random Parameter & Distribution & \multicolumn{3}{c|}{ Flutter Speed COV (\%) } \\
\hline \hline \multicolumn{1}{|c|}{$\begin{array}{c}\text { mass \& } \\
\text { radius of gyration }\end{array}$} & $\begin{array}{c}\text { Gaussian } \\
\text { Gaussian }\end{array}$ & 0.256 & 1.28 & 0.212 & 0.862 \\
\hline $\begin{array}{c}\text { mass \& } \\
\text { radius of gyration }\end{array}$ & $\begin{array}{c}\text { uniform } \\
\text { uniform }\end{array}$ & 0.254 & 1.13 & 0.212 & 0.856 \\
\hline $\begin{array}{c}\text { heave stiffness \& } \\
\text { pitch stiffness }\end{array}$ & $\begin{array}{c}\text { Gaussian } \\
\text { Gaussian }\end{array}$ & 1.08 & 4.34 & 1.06 & 4.29 \\
\hline $\begin{array}{c}\text { heave stiffness \& } \\
\text { pitch stiffness }\end{array}$ & $\begin{array}{c}\text { uniform } \\
\text { uniform }\end{array}$ & 1.08 & 4.32 & 1.06 & 4.28 \\
\hline $\begin{array}{c}\text { damping ratio 1 \& } \\
\text { damping ratio 2 }\end{array}$ & $\begin{array}{c}\text { Gaussian } \\
\text { Gaussian }\end{array}$ & 0.0231 & 0.0928 & 0.0253 & 0.101 \\
\hline $\begin{array}{c}\text { damping ratio 1 \& } \\
\text { damping ratio 2 }\end{array}$ & $\begin{array}{c}\text { uniform } \\
\text { uniform }\end{array}$ & 0.0231 & 0.0927 & 0.0252 & 0.101 \\
\hline \hline
\end{tabular}

Table 5.10: Results illustrating the uncertainty in the flutter speed due to correlated uncertainty in the structural parameters

correlation of damping increases the uncertainty. The uncertainty in the inertia parameters (except for correlated case), torsional stiffness and structural damping ratio of the second mode show significant non-linear effects in the flutter speed pdfs. For such cases, the mean flutter speed does not match the baseline flutter speed. While comparing the probabilistic results of the analysis with the quasisteady and unsteady aerodynamics, the effects of uncertainty are 

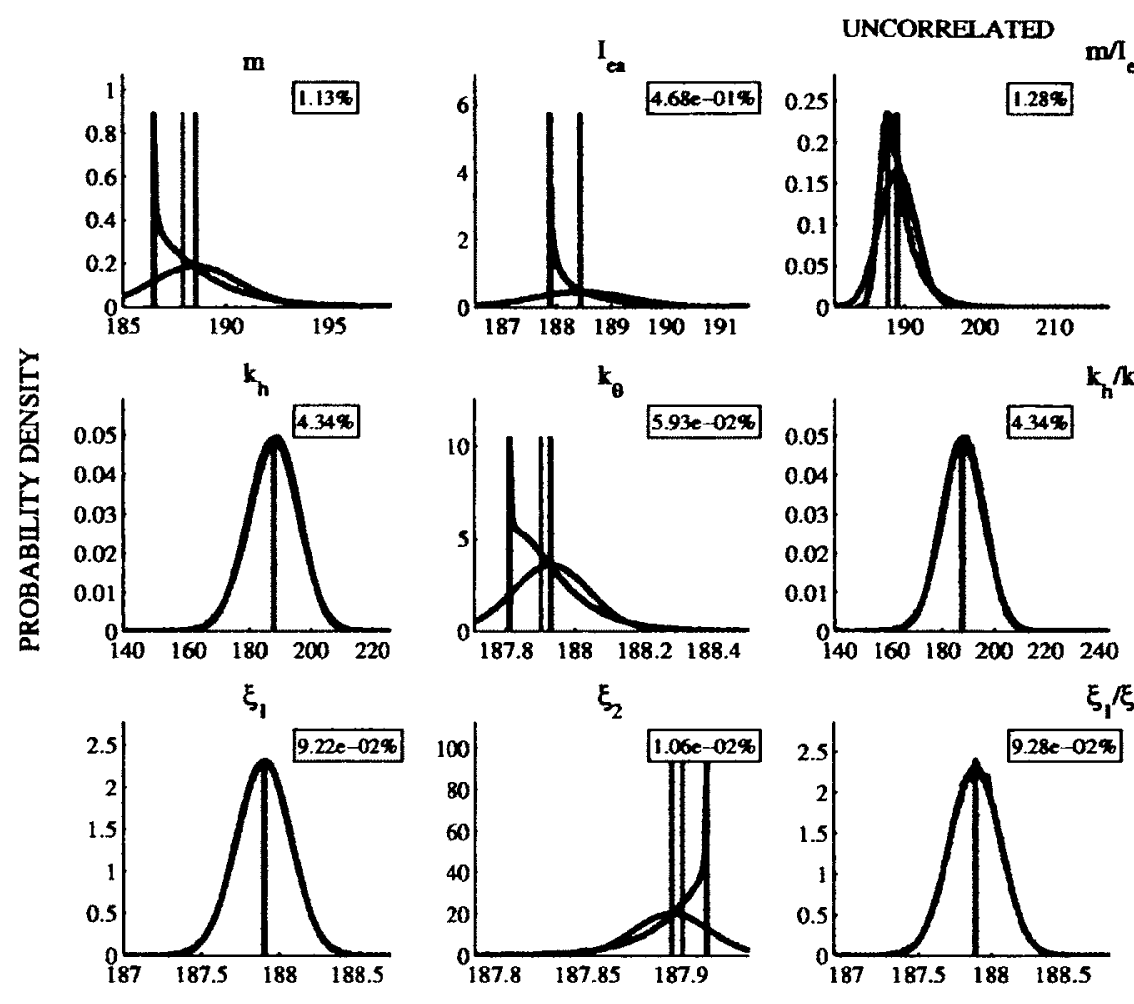

CORRFLATED
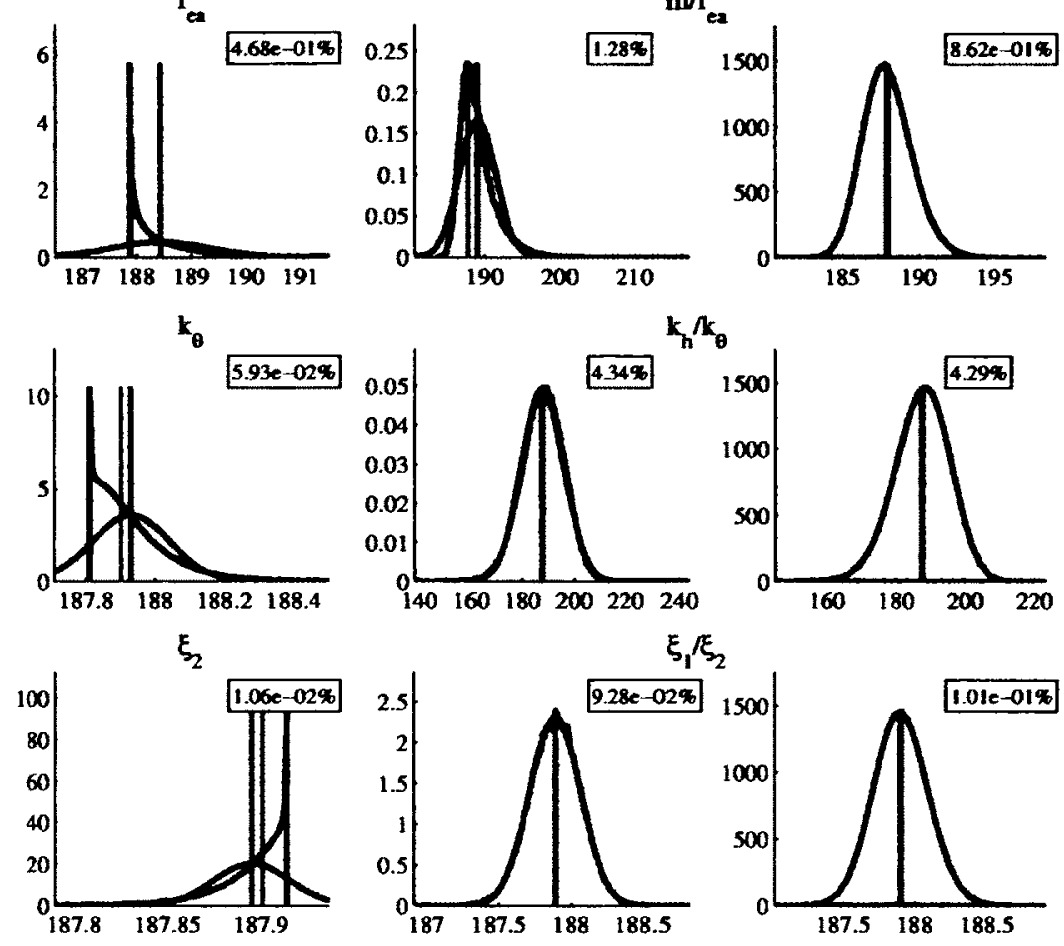

$\xi_{1} \xi_{2}$

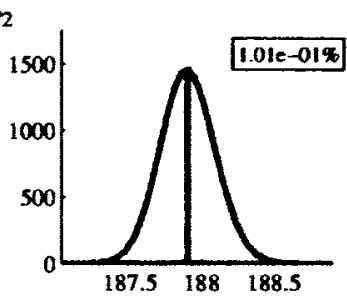

FLUTTER SPEED

Figure 5.24: Flutter speed pdfs (case 2 wing, unsteady, 10\% COV)

significantly smaller for the unsteady case in most cases. The exceptions to this observation are the cases of uncertainty in the structural damping of the first mode and the correlated inertia parameters. In contrast to the results from the rigid wing model, illustrated in figs. (3.26) and (3.27), the flutter speed pdfs for the cantilever show significant non-linear effects (i.e. skewness and cut-off speeds).

As a continuation of this investigation, the following chapter examines the characteristic roots for a cantilever wing from probabilistic prospective. Similar to the case of rigid wing, the probabilistic results of modal parameters provide significant insight into the mechanics of the coalescence flutter as delineated in the next chapter. 

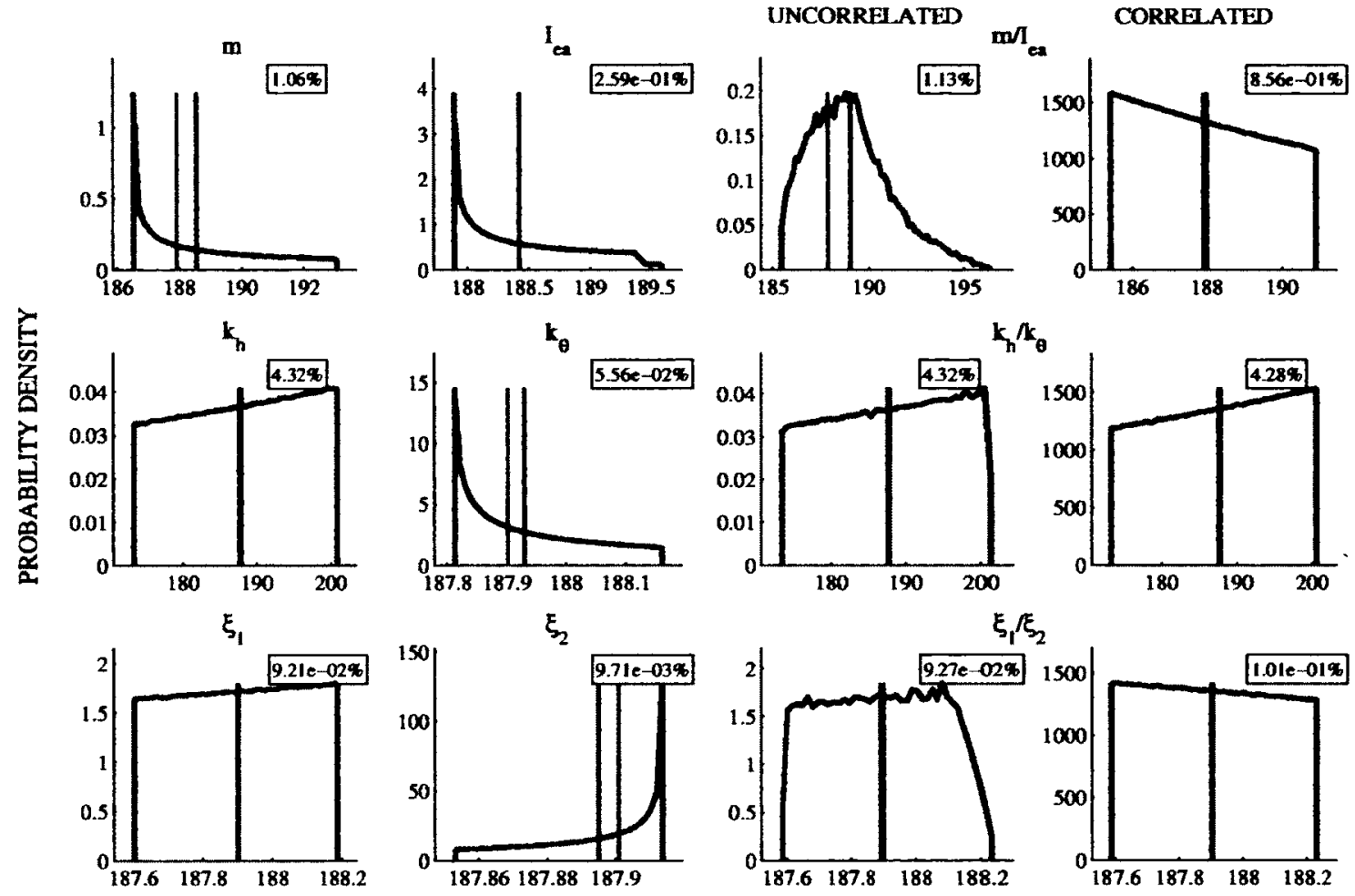

FLUTTER SPEED

Figure 5.25: Flutter speed pdfs (case 2 wing, unsteady, 10\% COV) 


\section{Chapter 6}

\section{A Cantilever Wing: Characteristic Roots}

In this chapter, a probabilistic analysis of the modal parameters of a cantilever wing is conducted in detail. Similar to the study of the rigid wing model, the density plots were used to study the statistics of the modal properties of complex aeroelastic modes in order to avoid the difficulties of tracking the individual aeroelastic modes. For the cantilever wing, these density plots become especially useful because the modal parameters experience much greater statistical overlap, due to mode veering attributed to the effect of randomness.

Several important points should be noted. The modal decay rates $\beta_{i}(U), i=1,2, \ldots, 6$ and the modal frequencies $\omega_{d, i}(U), i=1,2, \ldots, 6$ are complicated non-linear functions of the structural inertia, stiffness and damping parameters. For the unsteady aerodynamics, there are additional six (6) non-oscillatory modes that arise due to effects of circulatory forcing (but their probabilistic results are not shown for clarity of results). The physical mechanism of coalescence flutter dictates a strong relationship among the decay rate of the coalescing modes and the spacing between the coalescing frequencies. It is therefore natural to expect that the decay rates and frequencies will exhibit strong statistical correlation due to uncertainty in the system parameters. In general the frequencies and decay rates exhibit strong non-stationary trend with respect to the airspeed as the pdfs of the modal parameters substantially changes with the airspeed. This section is devoted to understanding the complex statistical interplay among the modal frequencies and decays. 


\subsection{CASE 1}

\subsubsection{Quasisteady Results}

The following probabilistic results illustrate the transformation of the modal properties as a function of airspeed. For each case, two figures will be provided, one illustrating modal properties of all modes and the second showing the magnified view of the same results focusing on the modes involved in the flutter mechanism. The effects of individual parameter uncertainty is considered first.

Fig. (6.1) shows the probabilistic results of the modal parameters for uncertain mass. Fig. (6.2) provides a magnified view focusing specifically on the modal parameters of modes involved in the flutter mechanism. The following observations are noted. The frequency pdfs of the coalescing modes show only weakly non-stationary trends, reflecting a milder flutter type. Furthermore, the pdf of frequency of the second (i.e. flutter) mode, show some non-Gaussian trend in the vicinity of flutter speed. The modal decay pdfs of the coalescing modes show significant non-stationarity, as their variances increase as function of airspeed. The uncertainty in mass has greater effect on the uncertainty in the frequency of the first coalescing (stable) mode, which exhibits predominantly bending motion, as evident fig. (5.8). However, the uncertainty of the modal decays in both coalescing modes is affected by the uncertainty in mass.

The effects of uncertainty in mass moment of inertia is considered next, with resulting modal parameter pdfs shown in fig. (6.3) and the magnified view of the coalescing modes provided by fig. (6.4). The observations for this case are very similar to the case of uncertain mass, but the most significant uncertainty effects appearing in the torsion dominated mode instead of the bending dominant mode. The frequency pdfs of the coalescing modes show only weak non-stationary trends. Furthermore, the frequency pdf of the first (i.e. stable) mode, show some non-Gaussian features in the vicinity of flutter speed (although not very clear in the scale of the graph). The modal decay pdfs of the coalescing modes show significant non-stationarity as their variances increase as function of airspeed. The uncertainty in the modal frequency of the torsion dominated (second) coalescing mode is most affected by the uncertainty in the mass 

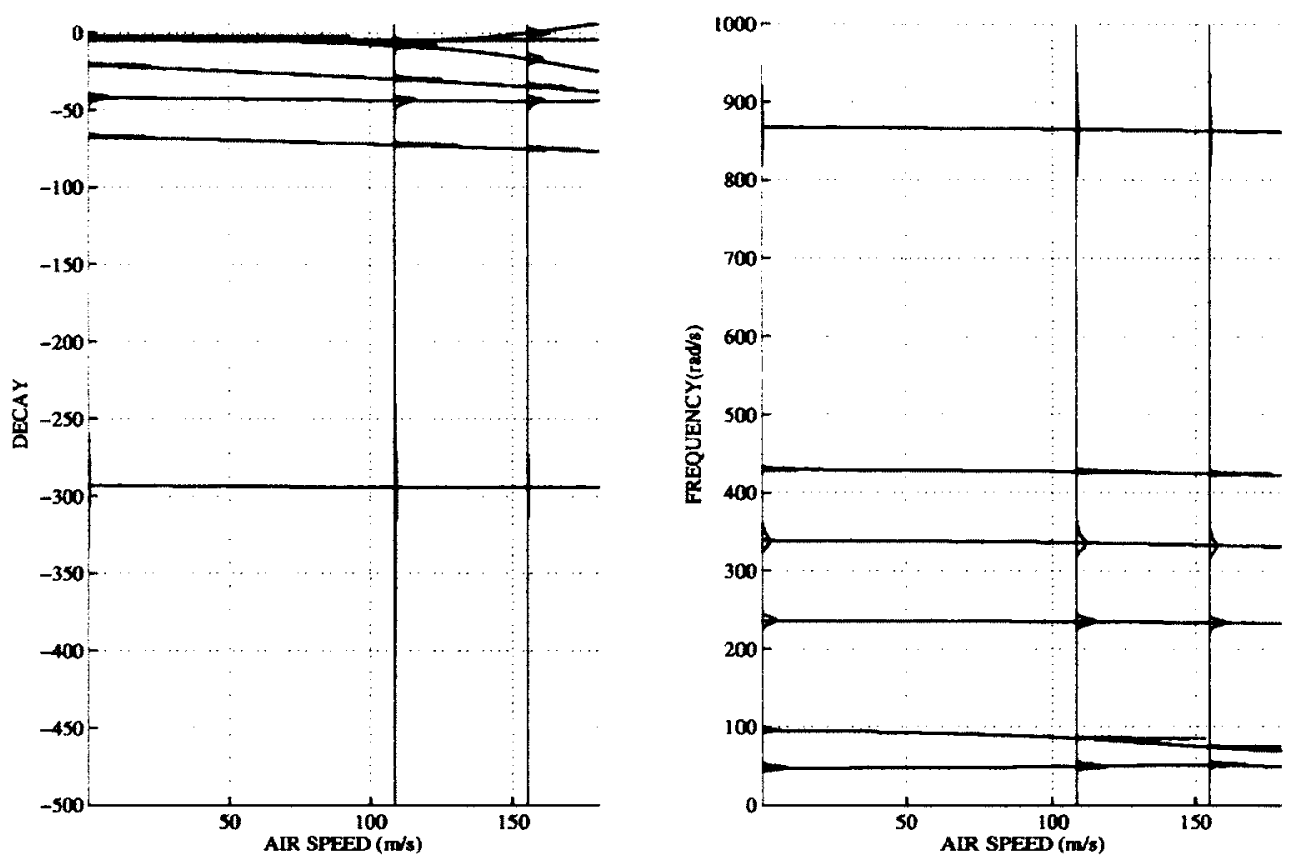

Figure 6.1: Modal parameter pdfs resulting from randomness in mass with $10 \% \mathrm{COV}$, obtained at 3 test airspeeds: $0 \mathrm{~m} / \mathrm{s}, 109 \mathrm{~m} / \mathrm{s}, 155.13 \mathrm{~m} / \mathrm{s}$
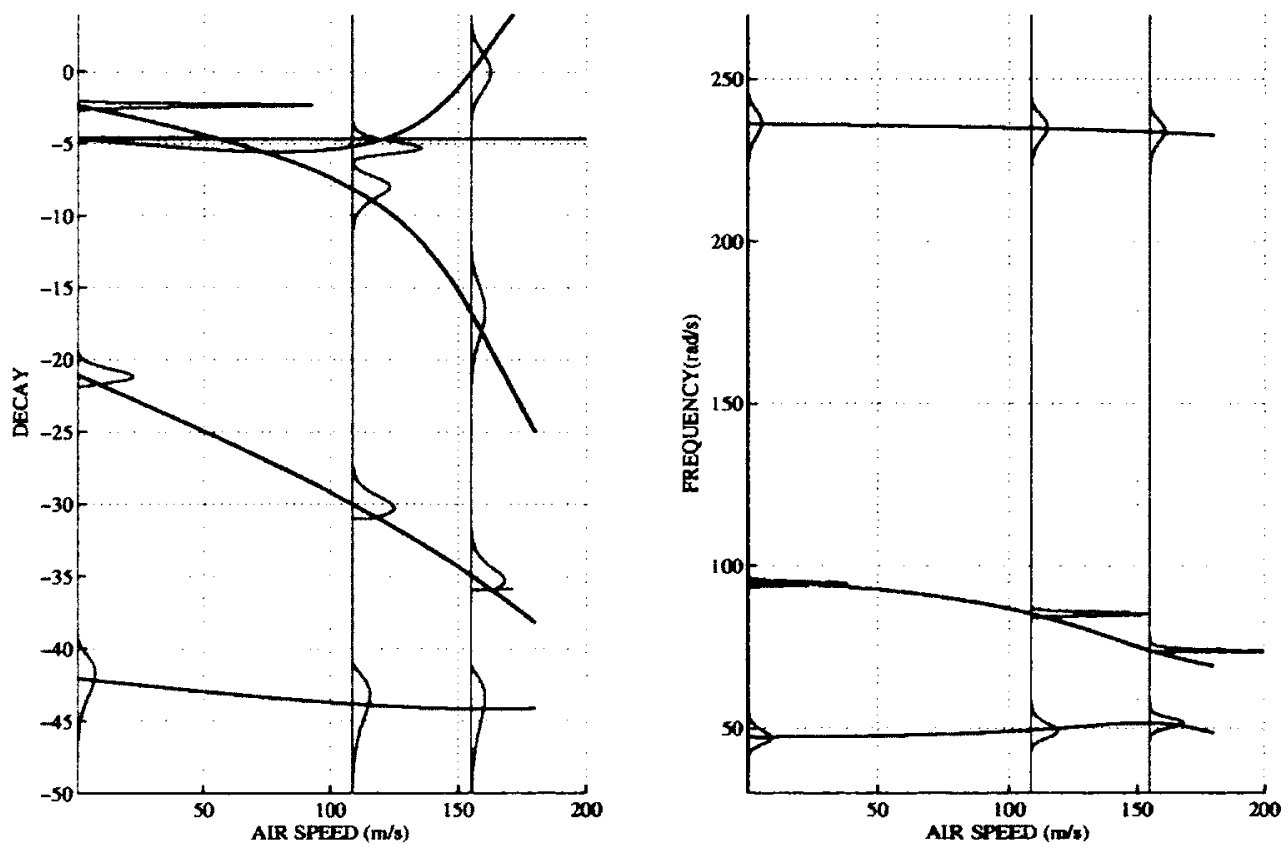

Figure 6.2: Magnified view of coalescing mode's modal parameter pdfs for uncertainty in mass 

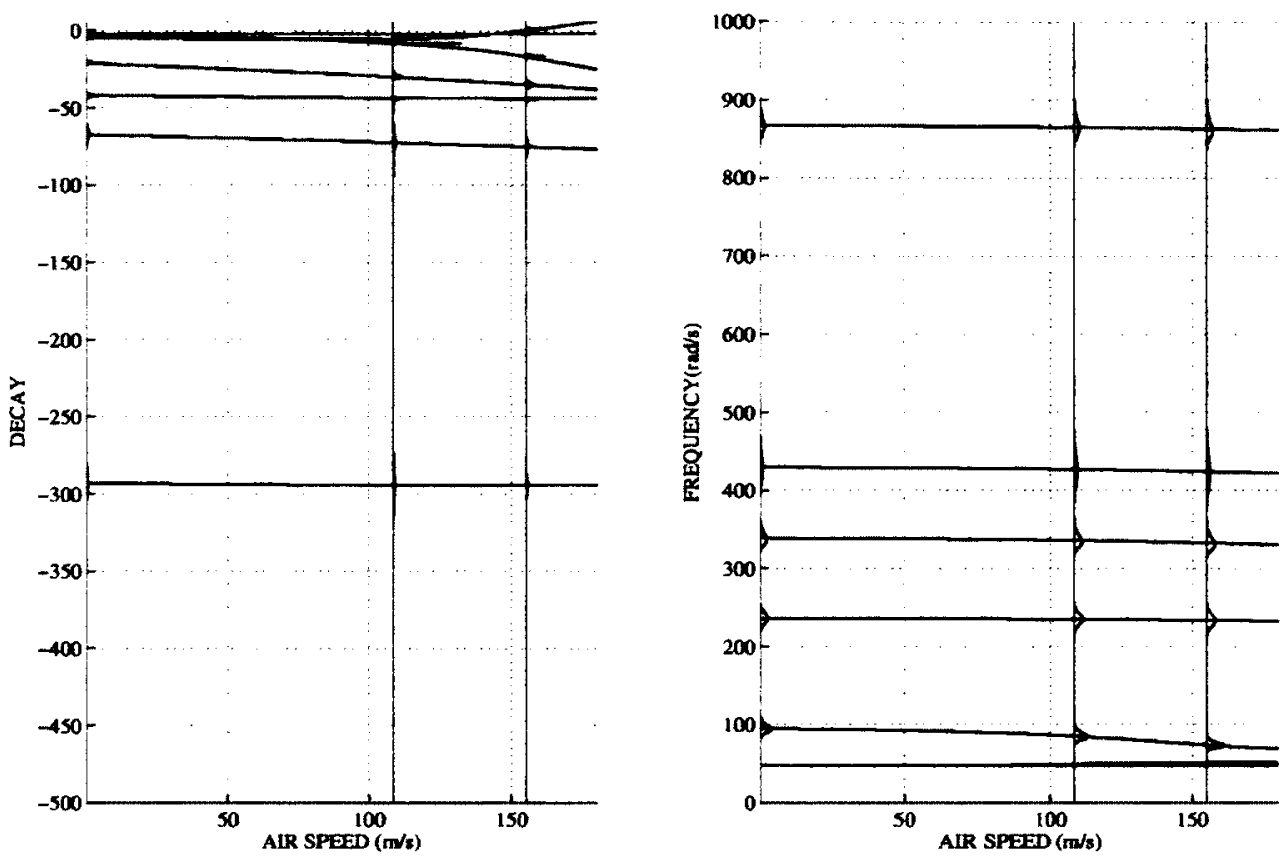

Figure 6.3: Modal parameter pdfs resulting from randomness in mass moment of inertia with $10 \%$ COV, obtained at 3 test airspeeds: $0 \mathrm{~m} / \mathrm{s}, 109 \mathrm{~m} / \mathrm{s}, 155.13 \mathrm{~m} / \mathrm{s}$
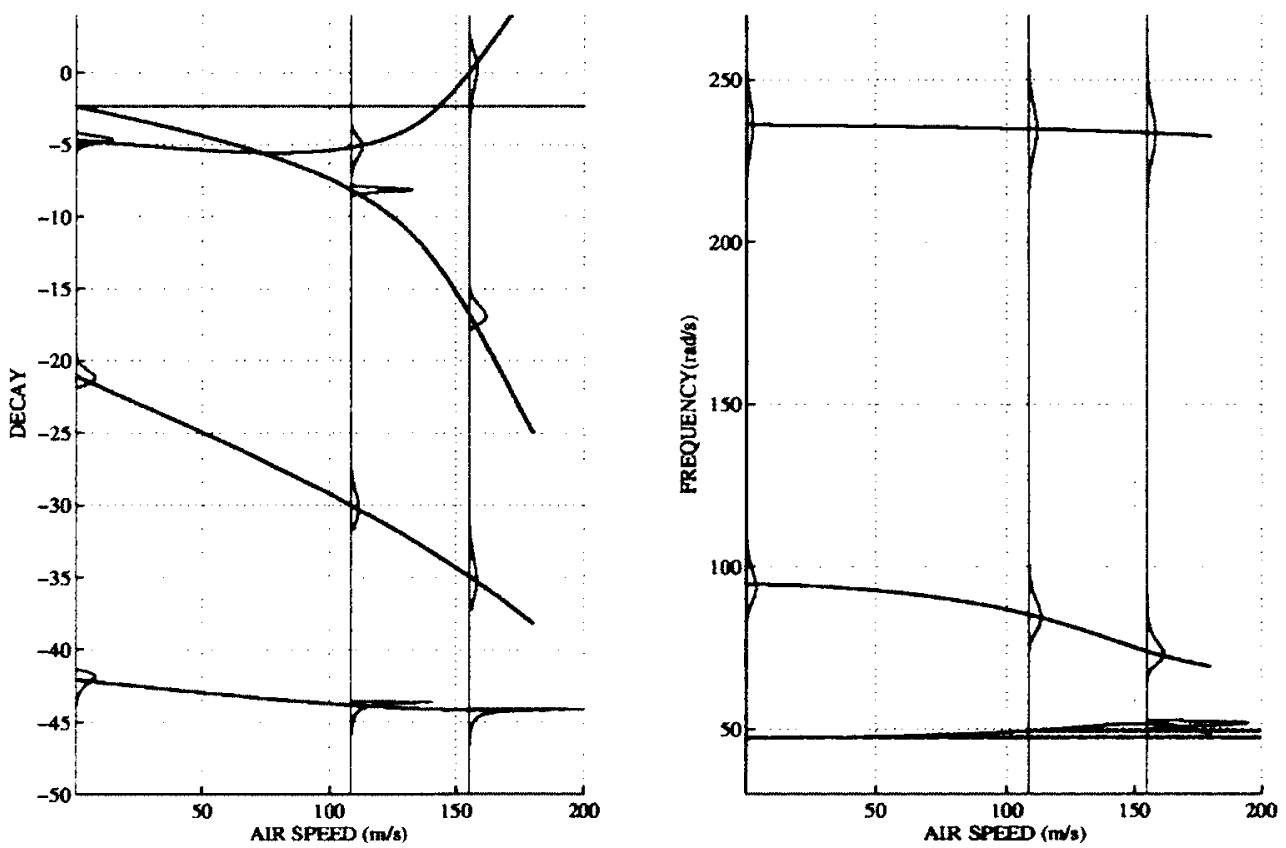

Figure 6.4: Magnified view of coalescing mode's modal parameter pdfs for uncertainty in mass moment of inertia 
moment of inertia. The uncertainty in mass moment of inertia influences the uncertainty in the modal decays of both coalescing modes.

Then, the effects of stiffness uncertainty are examined next. Figs. (6.5) and (6.6) show the effects of uncertainty in the bending stiffness on the modal parameters of the system. Figs. (6.7) and (6.8) illustrate the effects of torsional stiffness uncertainty on the modal properties of the aeroelastic system. From these figures, it is evident that the effects of bending stiffness uncertainty are almost similar to those of uncertain mass. The effects of torsional stiffness uncertainty also resemble the case of uncertain mass moment of inertia.

From the probabilistic results of uncertain inertia and stiffness and the resulting flutter speed pdfs, illustrated in fig. (5.9), note that the effect of growing variance in the modal decay of the flutter mode produces a large variance in the flutter speed pdfs.

The uncertainty of damping parameters has been shown to cause less significant effects in the flutter speed pdfs. Figs. (6.9) and (6.11) illustrate the effects of the first and second structural damping parameter uncertainties on the modal parameter pdfs, respectively. Figs. (6.10) and (6.12) provide a magnified view of the modal properties of the coalescing modes for the respective cases. There are no notable effects of uncertainty on the modal frequencies of the coalescing modes. The pdfs of the modal decays do not show large changes in variance as function of airspeed, which may be attributed to a milder type of flutter. Only in the vicinity of flutter, the modal decays of the coalescing modes show very slight changes in variance. For uncertainty in the first structural damping ratio, the most significant uncertainty effects are experienced in the decay of the first (stable) mode. The variance of the second mode is not significant. For uncertainty in the second structural damping ratio, the most significant effects of uncertainty are experienced in the decay of the second (flutter) mode. The variance of the first mode is not significant. 

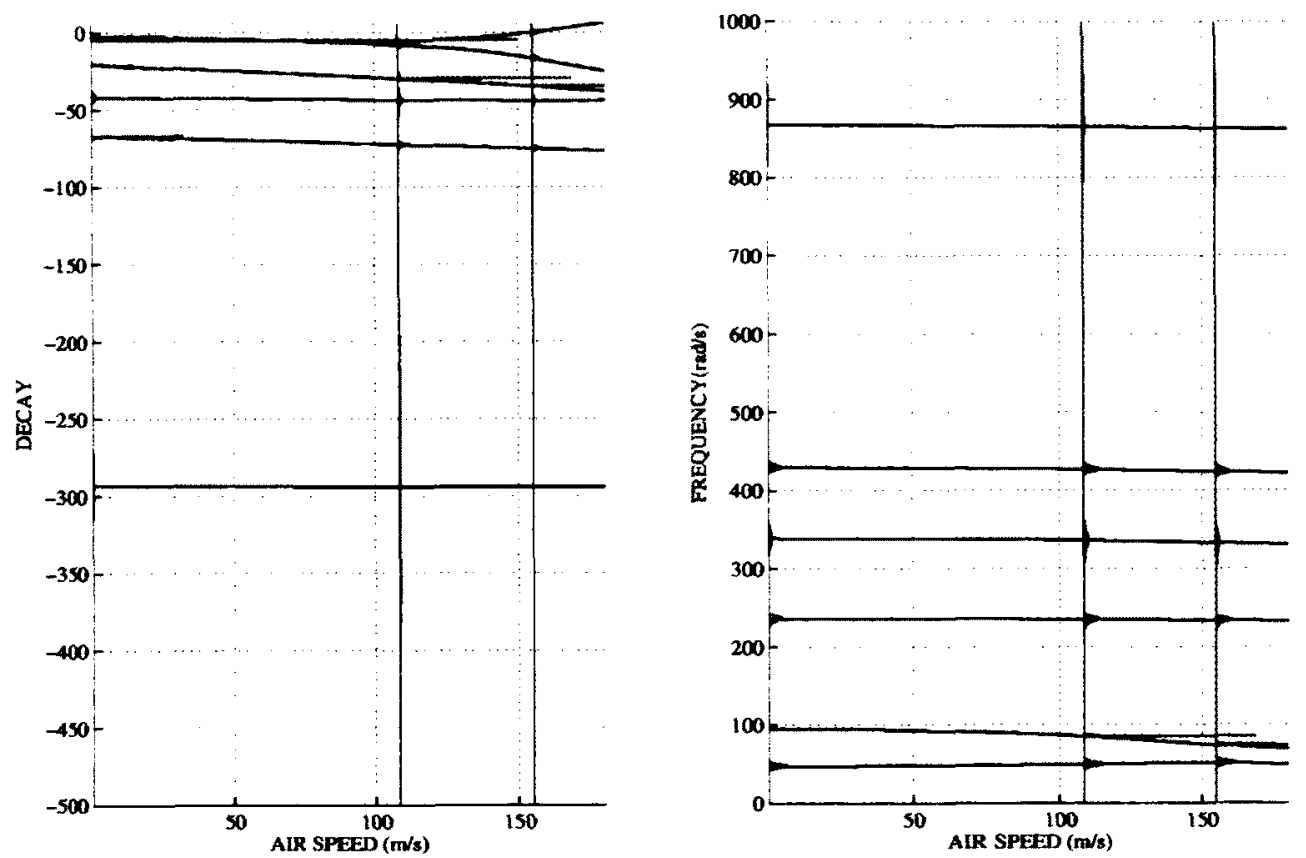

Figure 6.5: Modal parameter pdfs resulting from randomness in bending stiffness with $10 \%$ COV, obtained at 3 test airspeeds: $0 \mathrm{~m} / \mathrm{s}, 109 \mathrm{~m} / \mathrm{s}, 155.13 \mathrm{~m} / \mathrm{s}$
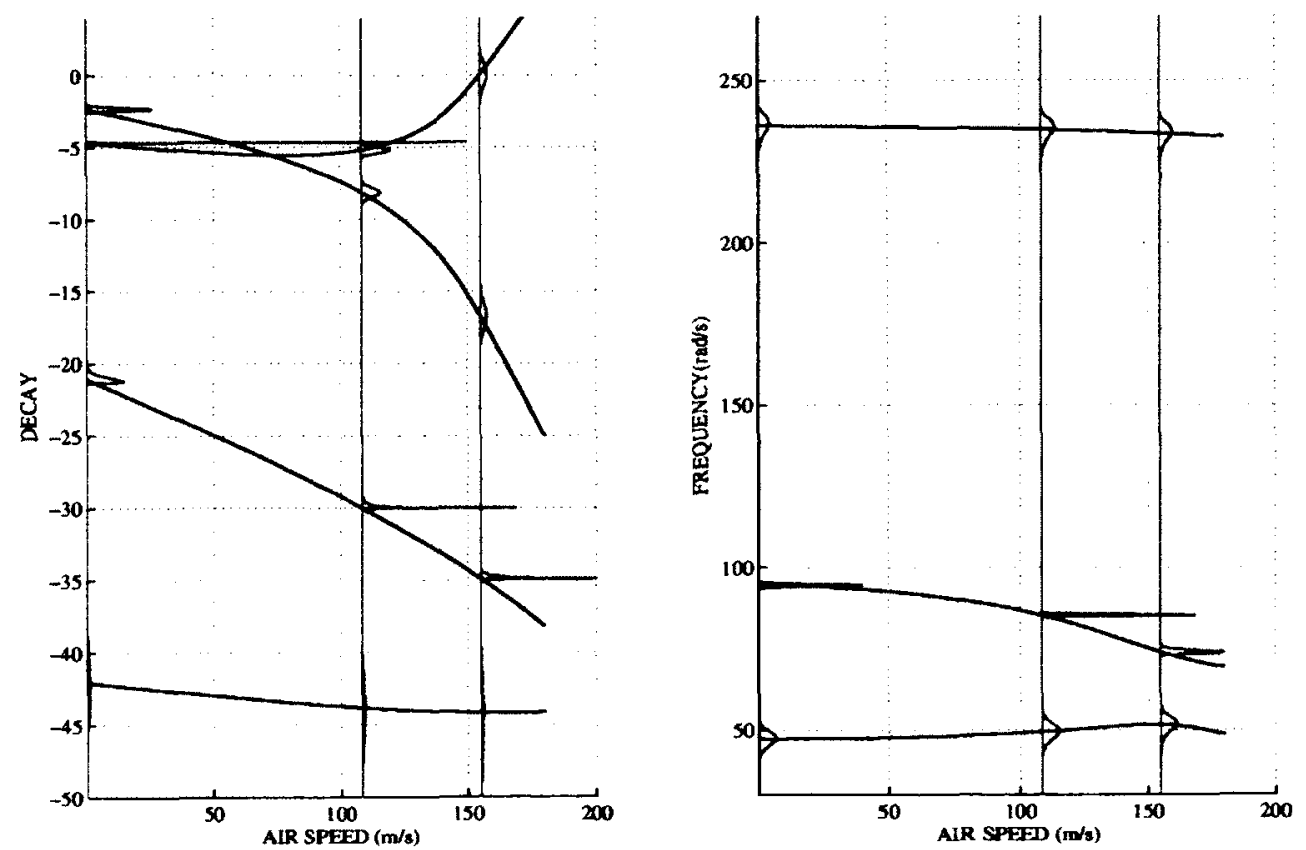

Figure 6.6: Magnified view of coalescing mode's modal parameter pdfs for uncertainty in bending stiffness 

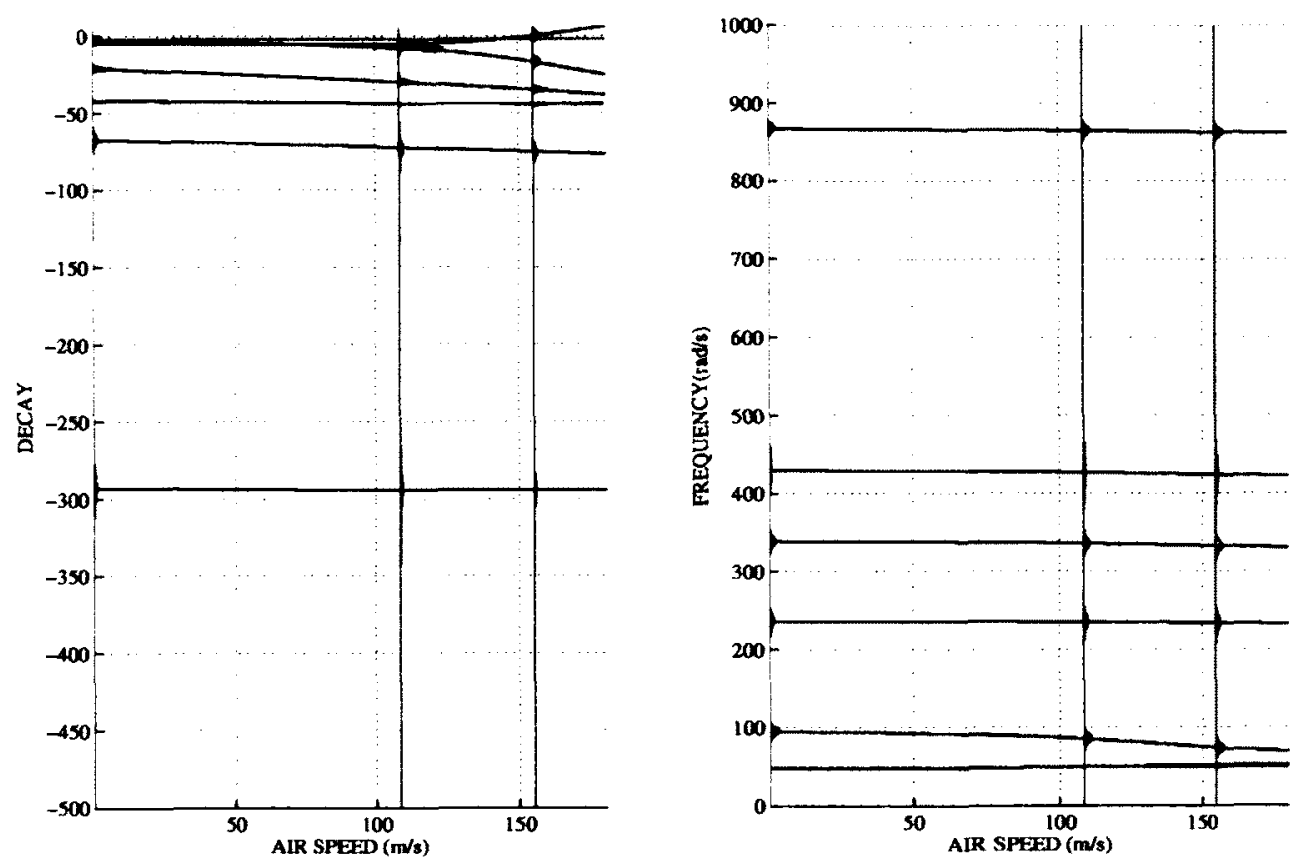

Figure 6.7: Modal parameter pdfs resulting from randomness in torsional stiffness with $10 \%$ COV, obtained at 3 test airspeeds: $0 \mathrm{~m} / \mathrm{s}, 109 \mathrm{~m} / \mathrm{s}, 155.13 \mathrm{~m} / \mathrm{s}$
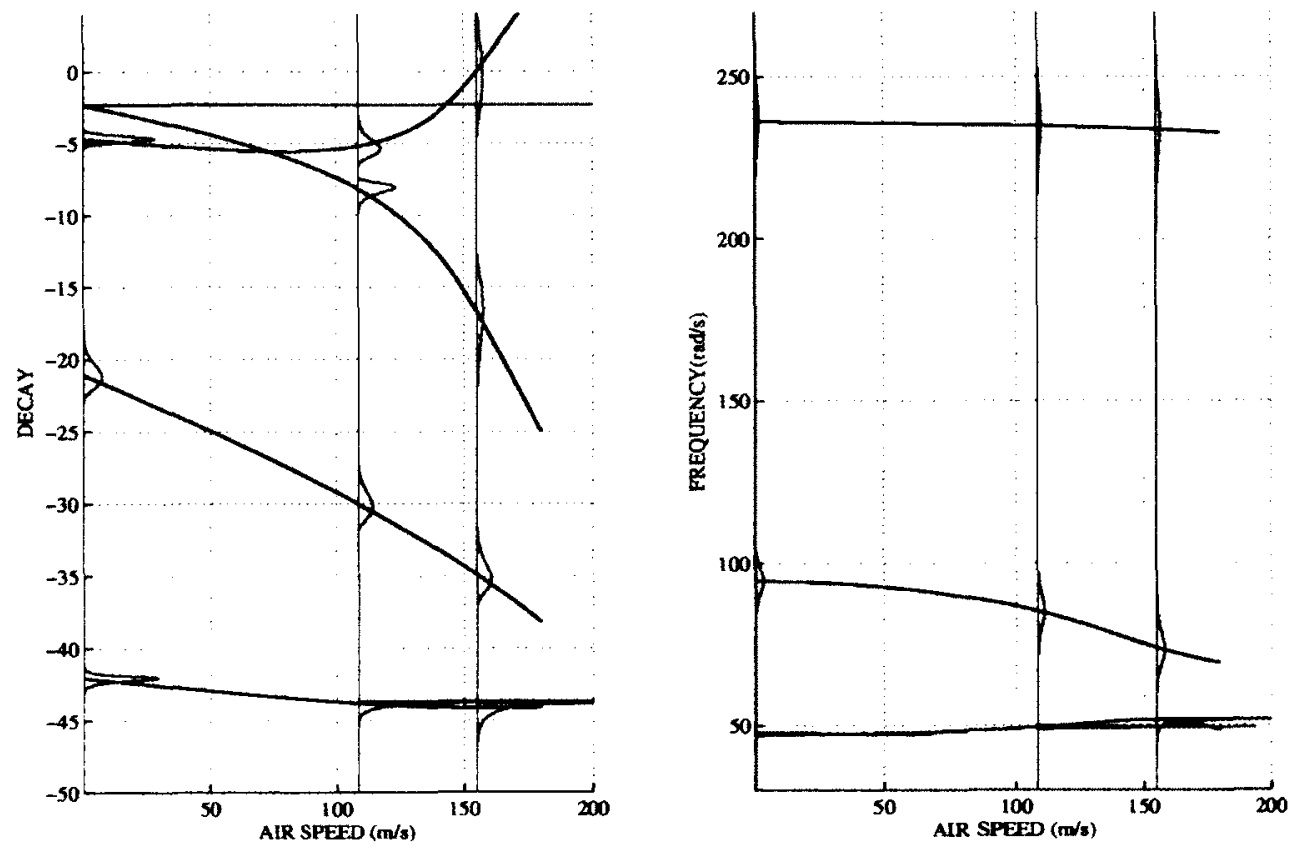

Figure 6.8: Magnified view of coalescing mode's modal parameter pdfs for uncertainty in torsional stiffness 

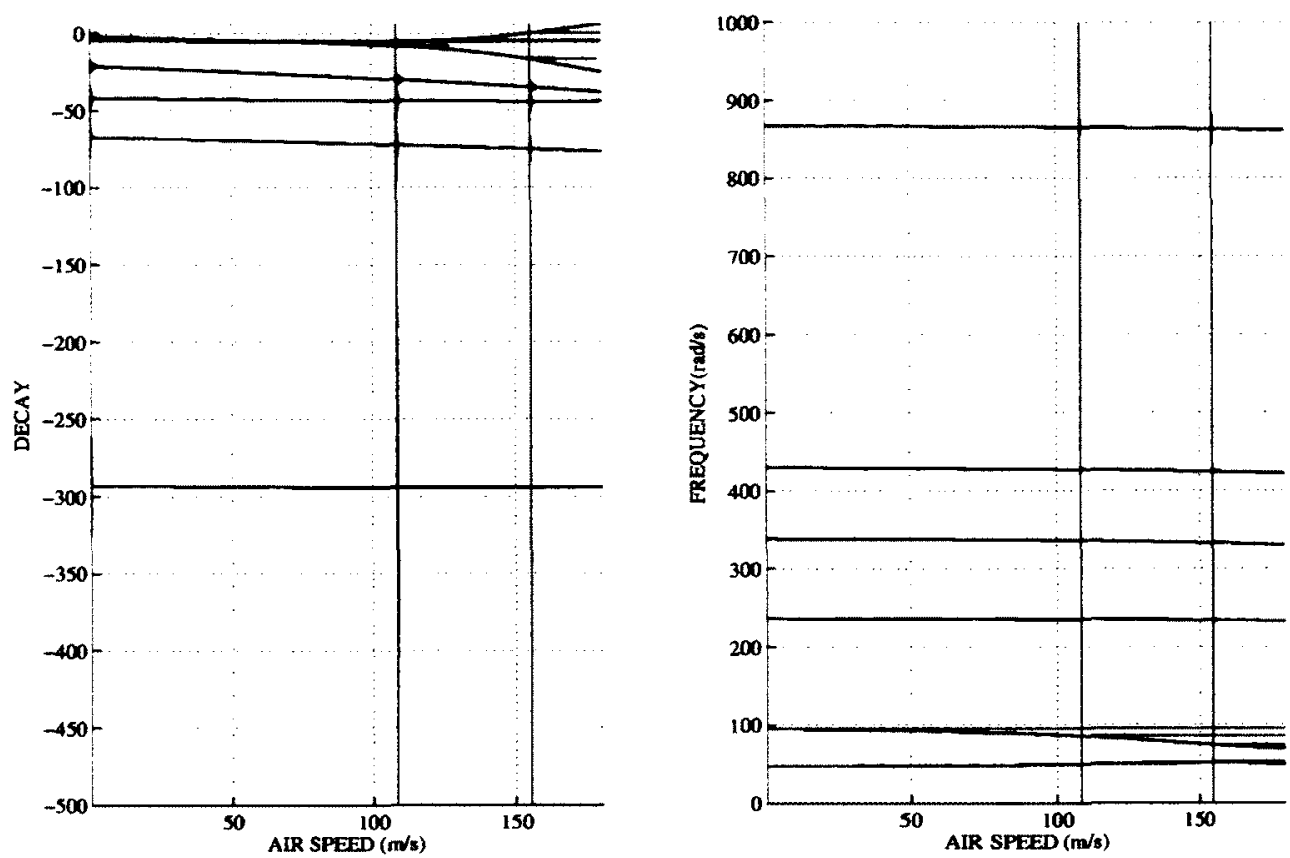

Figure 6.9: Modal parameter pdfs resulting from randomness in first structural damping ratio with $10 \%$ COV, obtained at 3 test airspeeds: $0 \mathrm{~m} / \mathrm{s}, 109 \mathrm{~m} / \mathrm{s}, 155.13 \mathrm{~m} / \mathrm{s}$
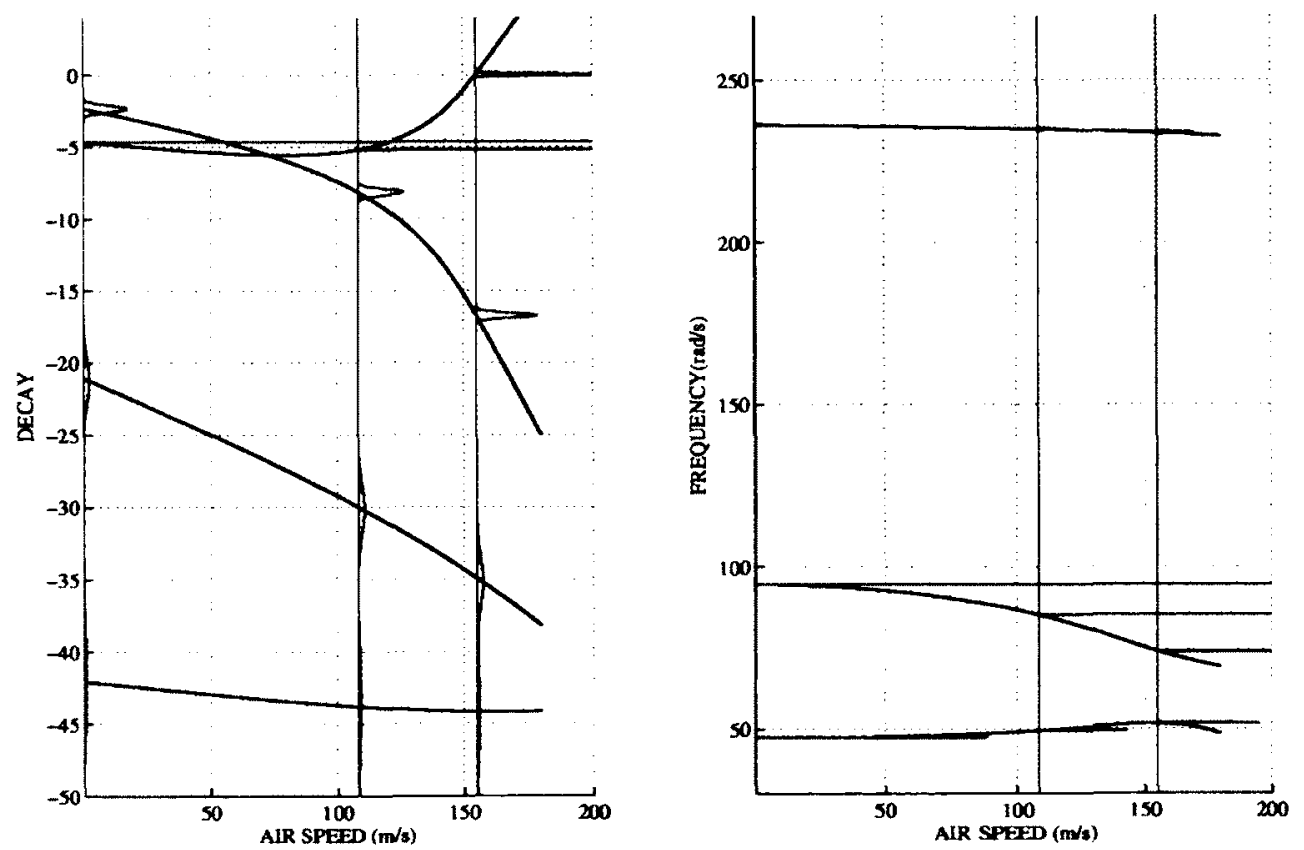

Figure 6.10: Magnified view of coalescing mode's modal parameter pdfs for uncertainty in first structural damping ratio 

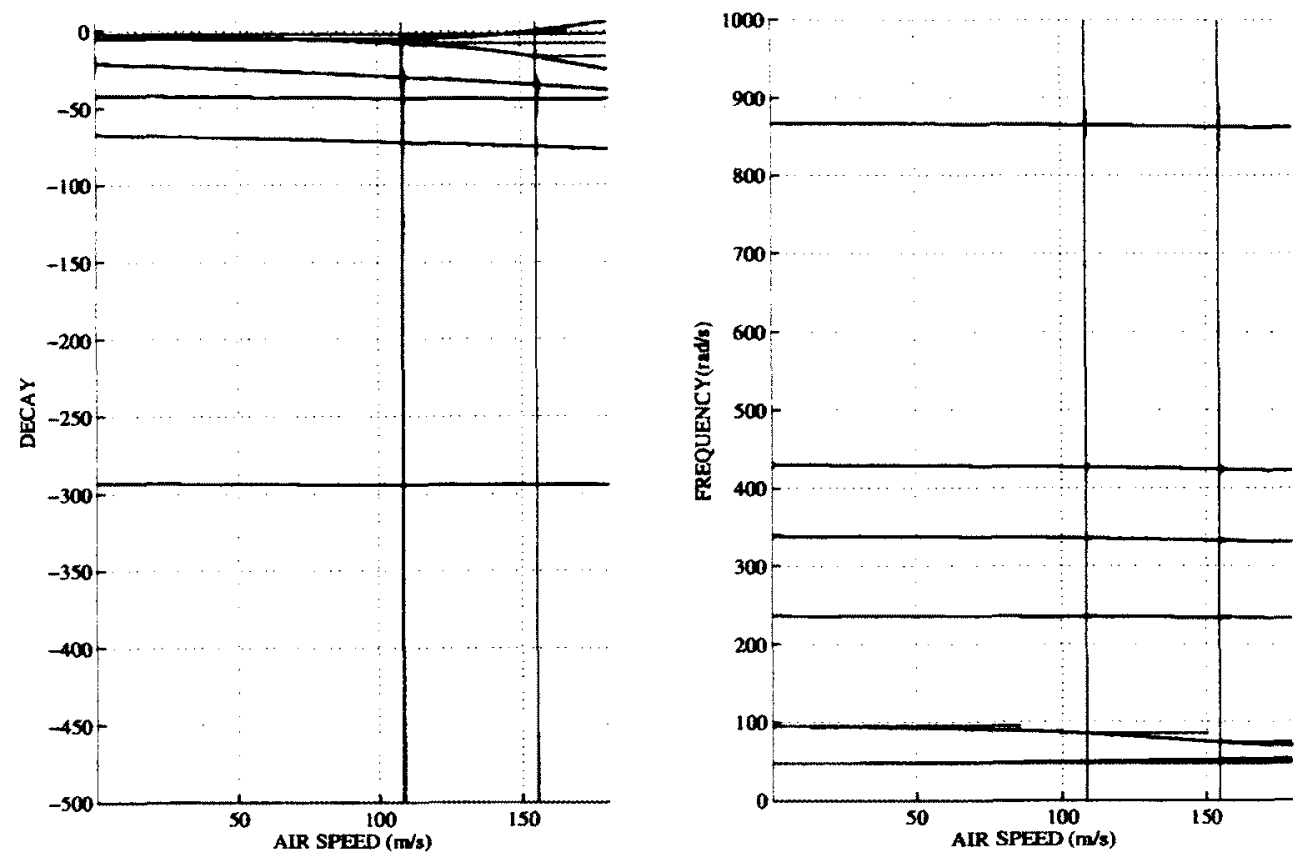

Figure 6.11: Modal parameter pdfs resulting from randomness in second structural damping ratio with $10 \% \operatorname{COV}$, obtained at 3 test airspeeds: $0 \mathrm{~m} / \mathrm{s}, 109 \mathrm{~m} / \mathrm{s}, 155.13 \mathrm{~m} / \mathrm{s}$
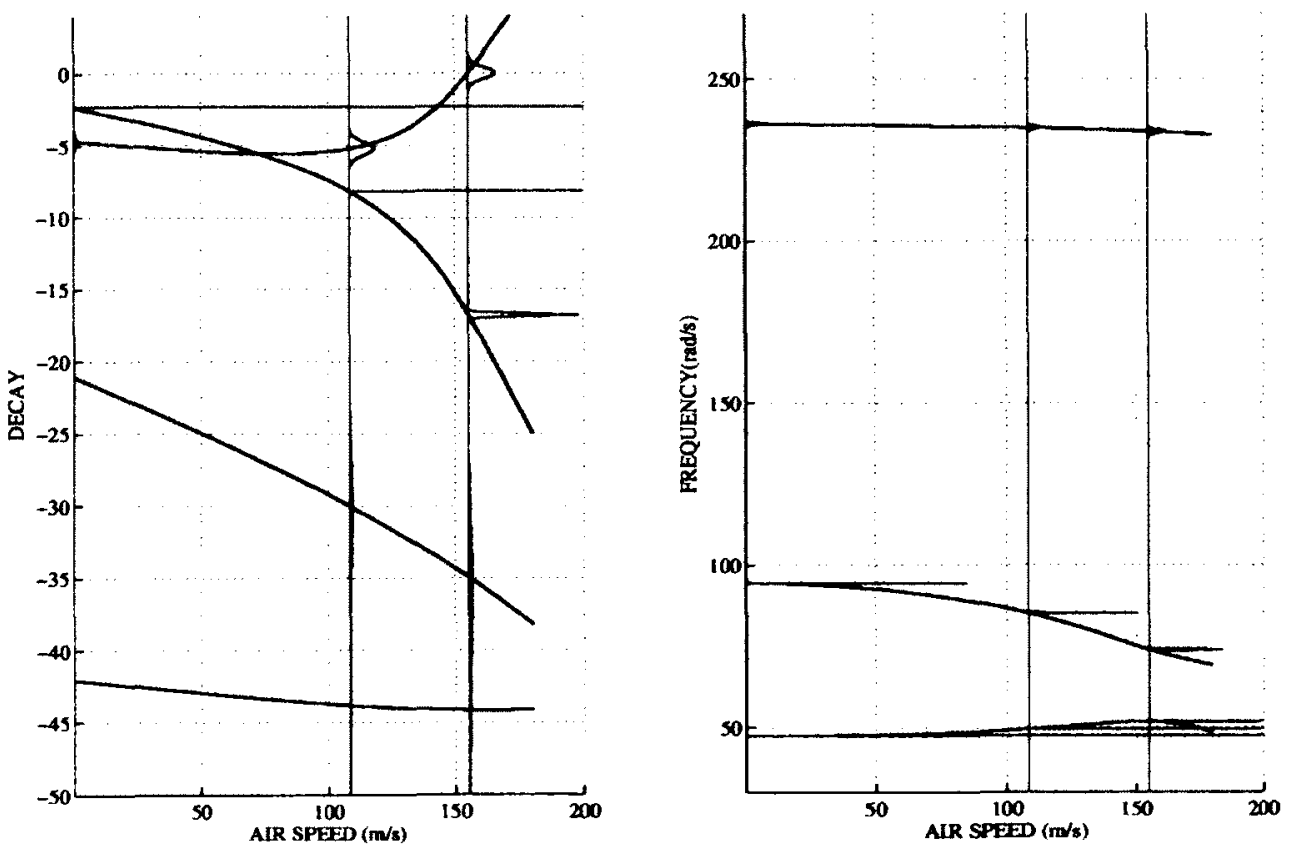

Figure 6.12: Magnified view of coalescing mode's modal parameter pdfs for uncertainty in second structural damping ratio 


\section{Multiple Parameter Uncertainty}

The analysis examines effects of multiple parameter variations where the random parameters are fully uncorrelated. Figs. (6.13)-(6.18) show the resulting pdfs of modal parameters as a function of airspeed for random inertia, stiffness and damping parameters. When the uncertain parameters are uncorrelated, the uncertainty in the modal parameter pdfs increases. The uncertainty in the uncorrelated inertia parameters introduces statistical overlapping effects in the modal decays.

Next, the effects of full correlation between the random parameters are examined. Figs. (6.19)-(6.24) illustrate the modal parameter pdfs for fully correlated inertia, stiffness and damping parameters. The following are the observations that were gathered from these results. The uncertainty in the modal frequencies of the coalescing modes is not significantly affected by the correlation between the random parameters. However, correlation between the random parameters may lead to changes in correlations between the modal frequencies and the modal decays. For uncertainty in inertia parameters, the correlation between the modal frequencies leads to a reduced growth of variance in the modal decays (as function of airspeed), especially evident in the flutter mode. Such reduction of variance in the flutter mode may imply a lower uncertainty in the flutter speed pdf due to structural parameter correlation. For uncertainty in the stiffness properties, similar trend in variance of the modal decays is experienced in both modes. The reduction of variance in the flutter mode implies a lower uncertainty in the flutter speed pdf due to effects of structural parameter correlation. The correlation between random structural damping parameters mostly affects the correlation between the modal decays. In this case, the correlation between modal decays of the coalescing modes leads to a consistent increase in the variance of the modal decays at every airspeed.

Lastly, a comparison of the probabilistic modal parameter results of the 3-D cantilever wing model and the rigid wing model (represented by Case 1 system parameters), for quasisteady aerodynamics, is obtained. Qualitatively, the results are very similar. Only a minor difference is noted for the cantilever model, where a more explosive flutter type leads to greater nonstationary effects in the modal pdfs in vicinity of flutter. 

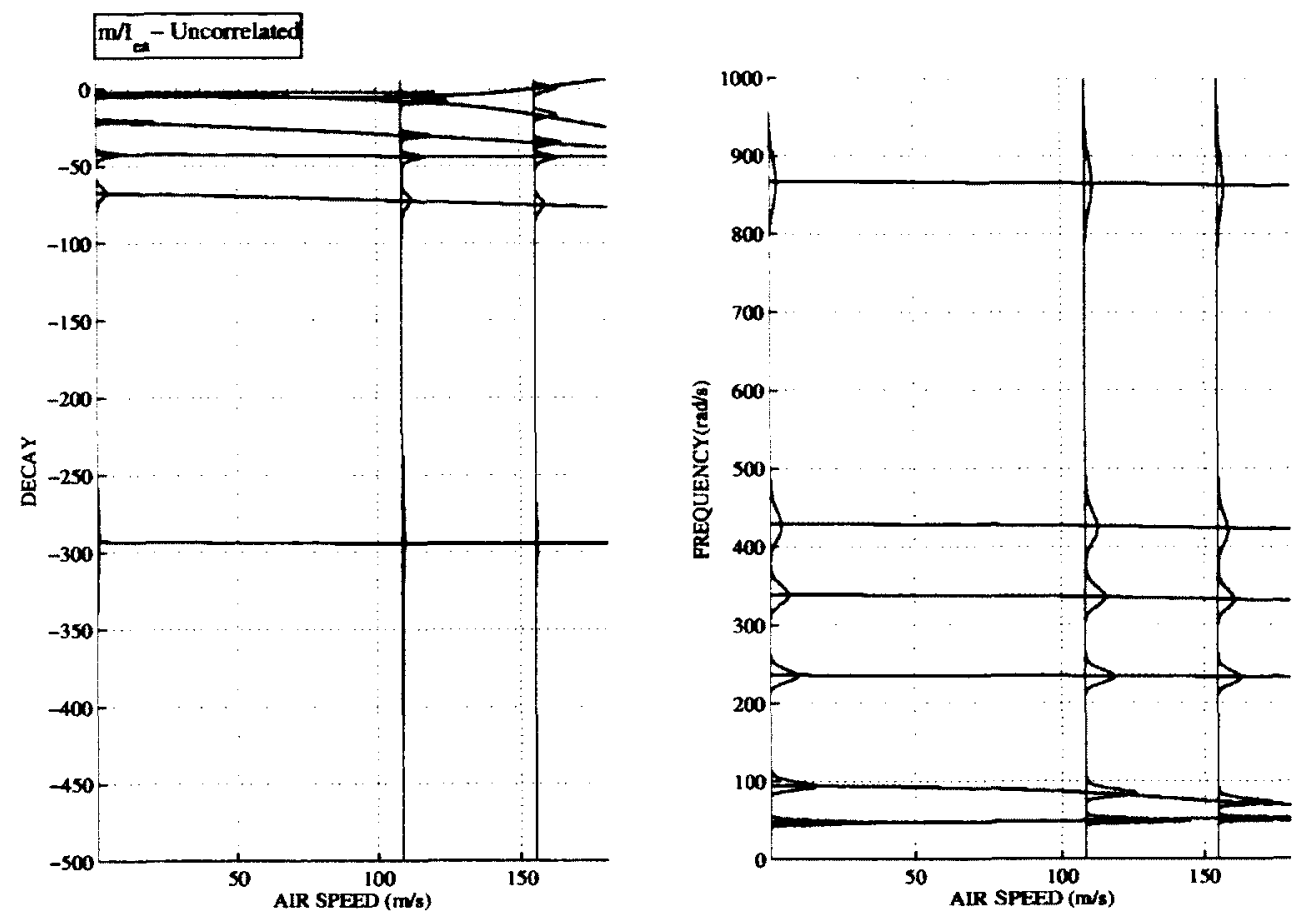

Figure 6.13: Modal parameter pdfs resulting from randomness in uncorrelated inertia terms with $10 \%$ COV, obtained at 3 test airspeeds: $0 \mathrm{~m} / \mathrm{s}, 109 \mathrm{~m} / \mathrm{s}, 155.13 \mathrm{~m} / \mathrm{s}$
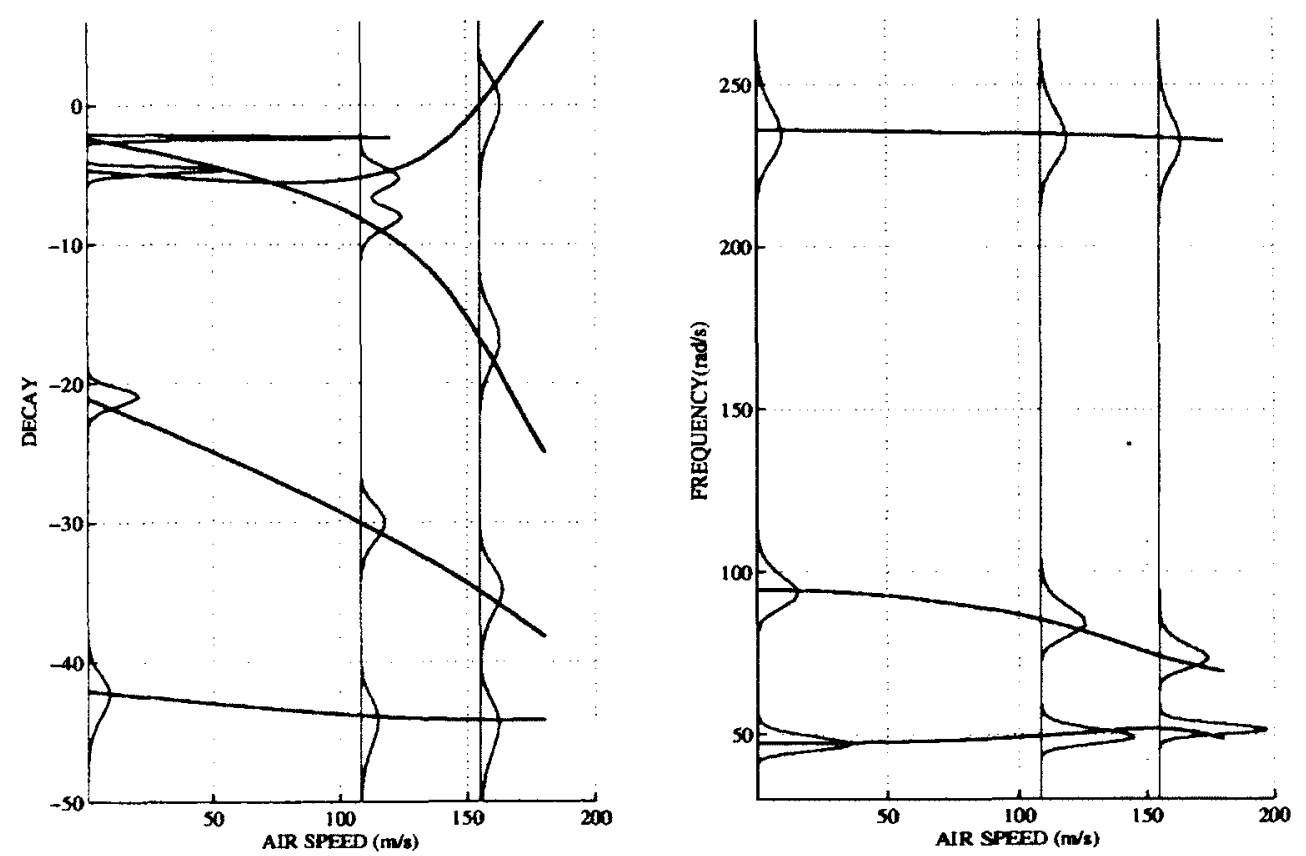

Figure 6.14: Magnified view of coalescing mode's modal parameter pdfs for uncertainty in uncorrelated inertia terms 

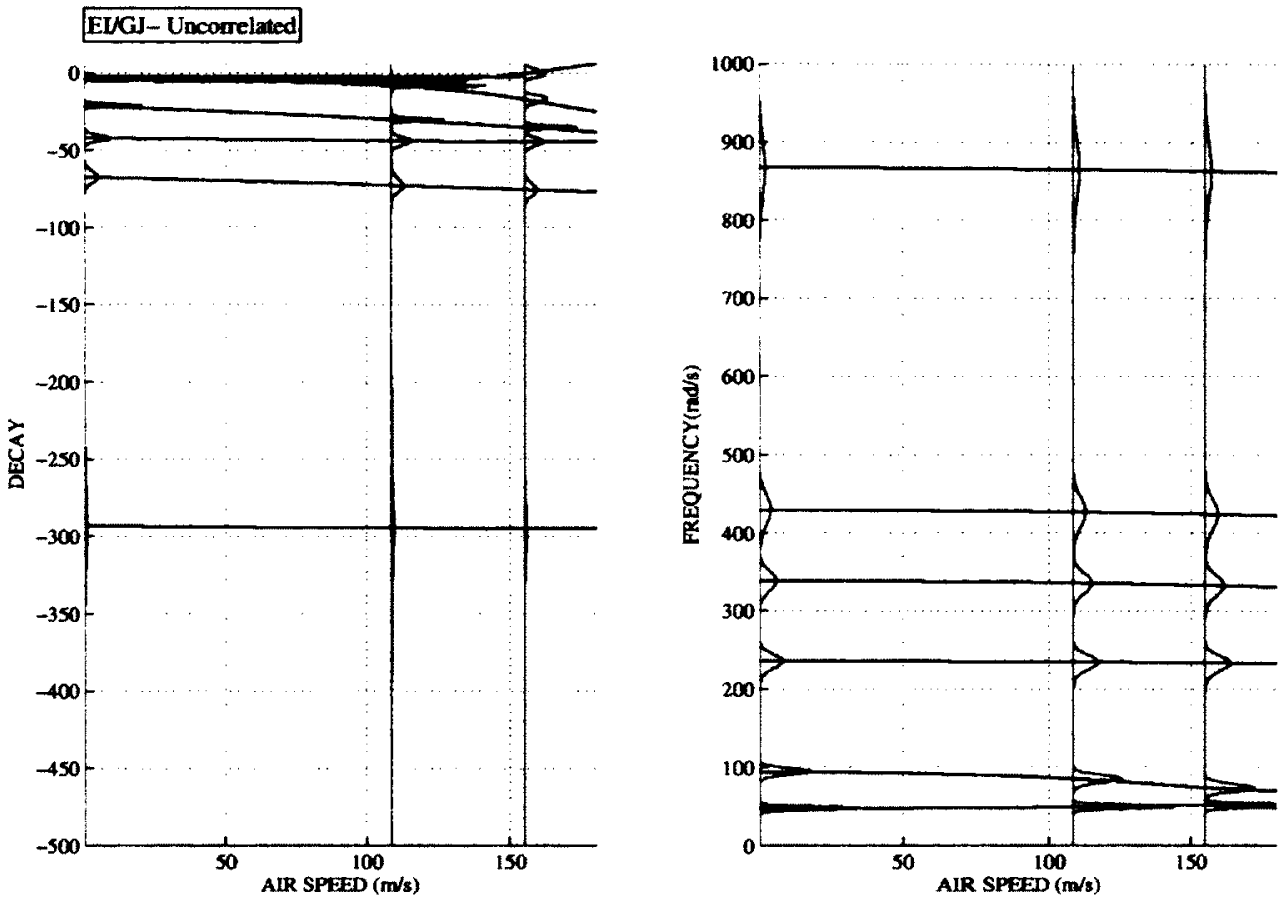

Figure 6.15: Modal parameter pdfs resulting from randomness in uncorrelated stiffness terms with $10 \% \mathrm{COV}$, obtained at 3 test airspeeds: $0 \mathrm{~m} / \mathrm{s}, 109 \mathrm{~m} / \mathrm{s}, 155.13 \mathrm{~m} / \mathrm{s}$
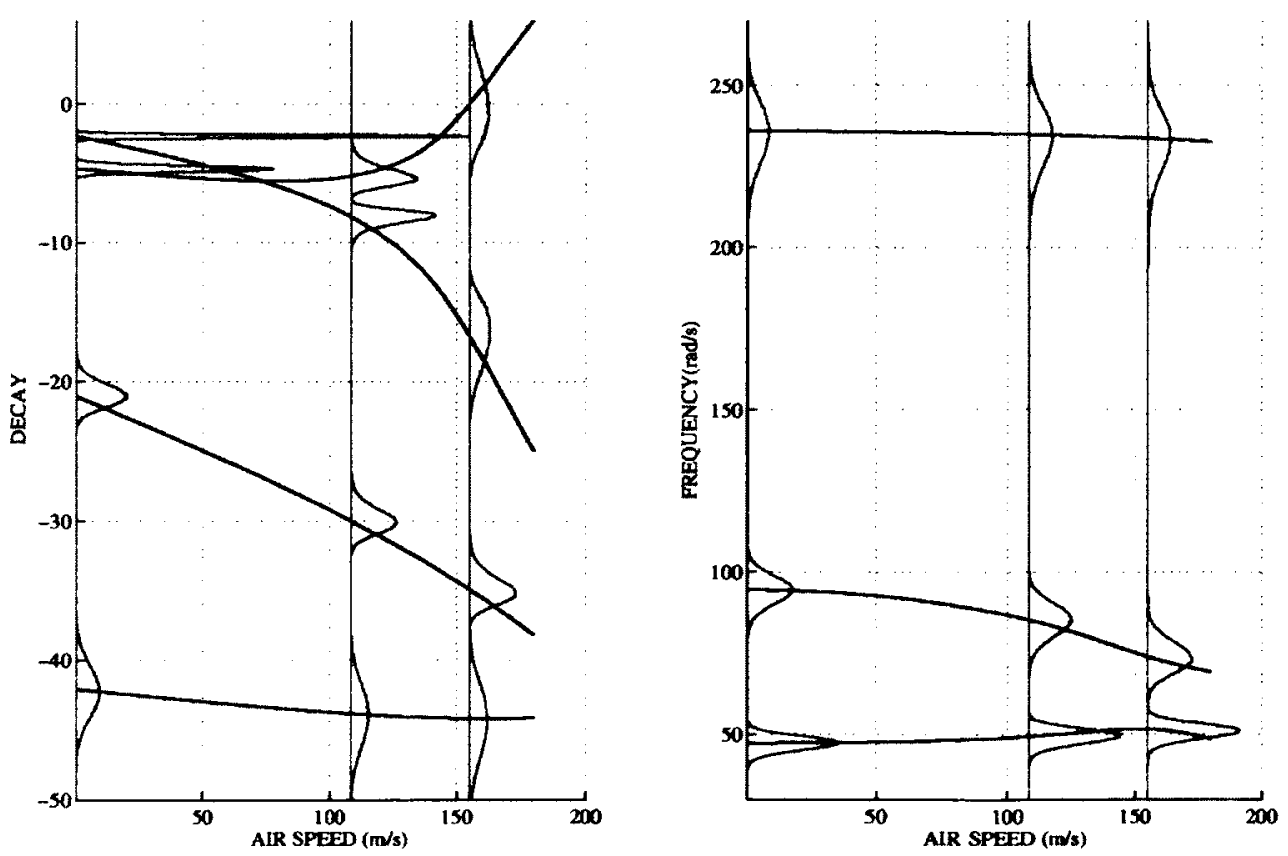

Figure 6.16: Magnified view of coalescing mode's modal parameter pdfs for uncertainty in uncorrelated stiffness terms 

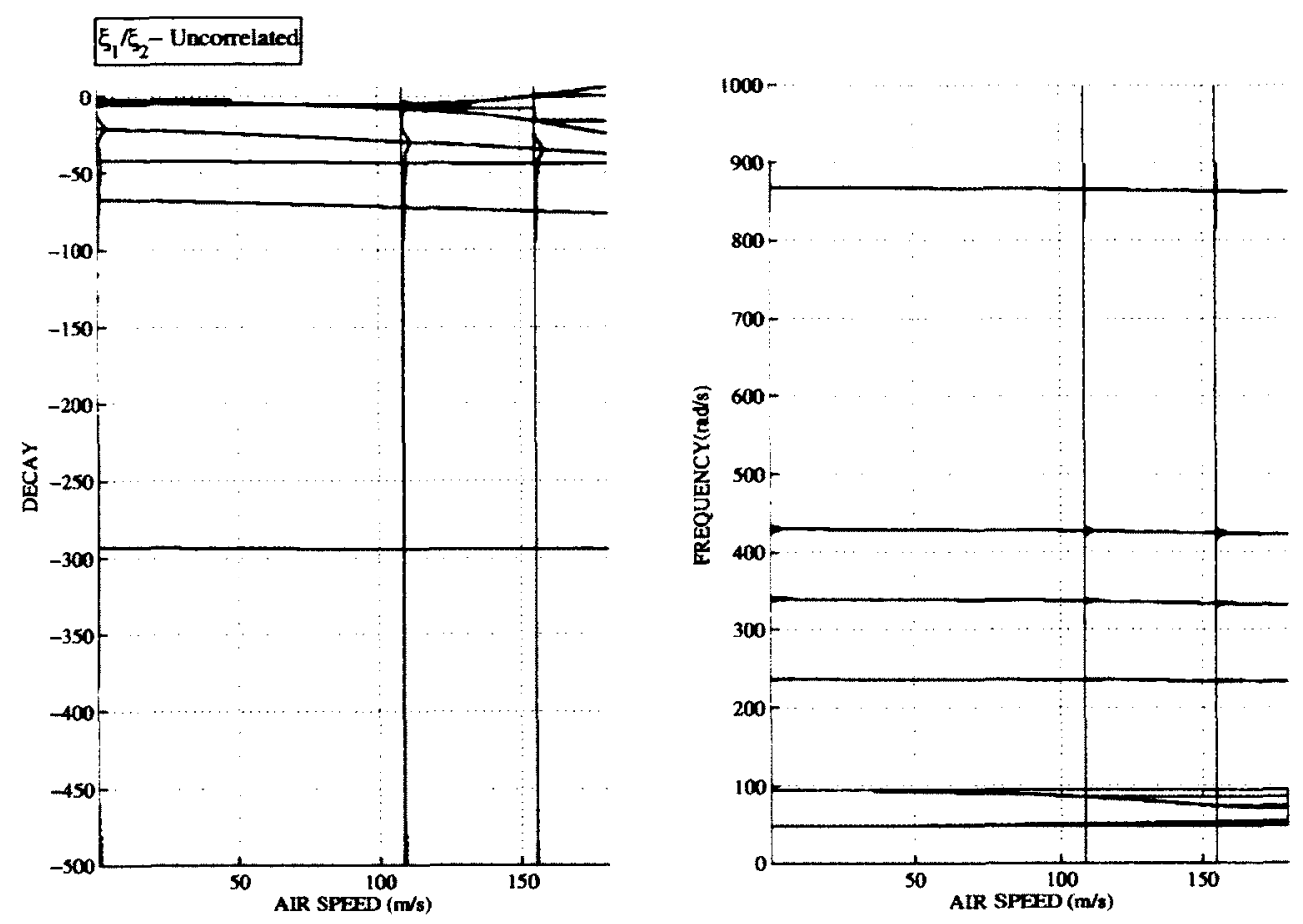

Figure 6.17: Modal parameter pdfs resulting from randomness in uncorrelated damping terms with $10 \% \mathrm{COV}$, obtained at 3 test airspeeds: $0 \mathrm{~m} / \mathrm{s}, 109 \mathrm{~m} / \mathrm{s}, 155.13 \mathrm{~m} / \mathrm{s}$
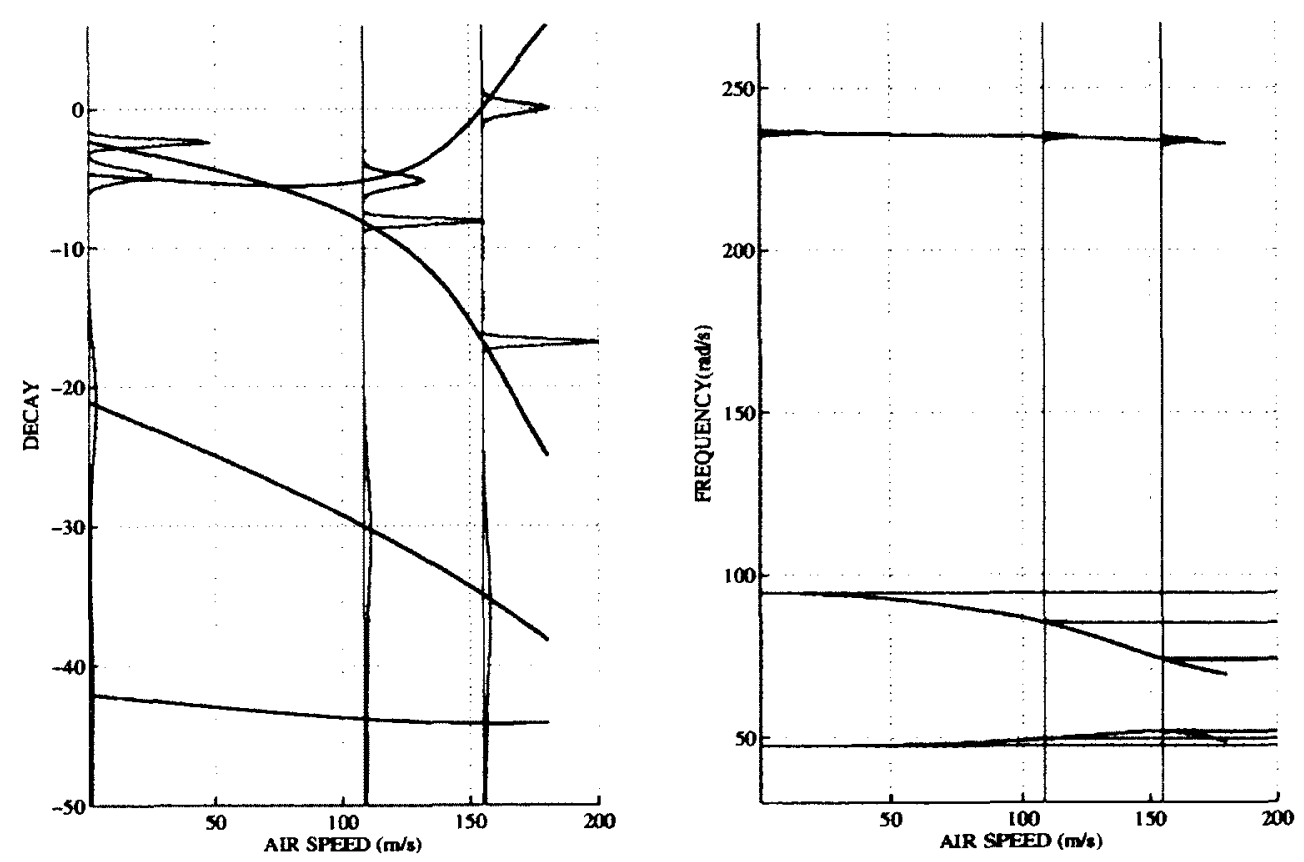

Figure 6.18: Magnified view of coalescing mode's modal parameter pdfs for uncertainty in uncorrelated damping terms 

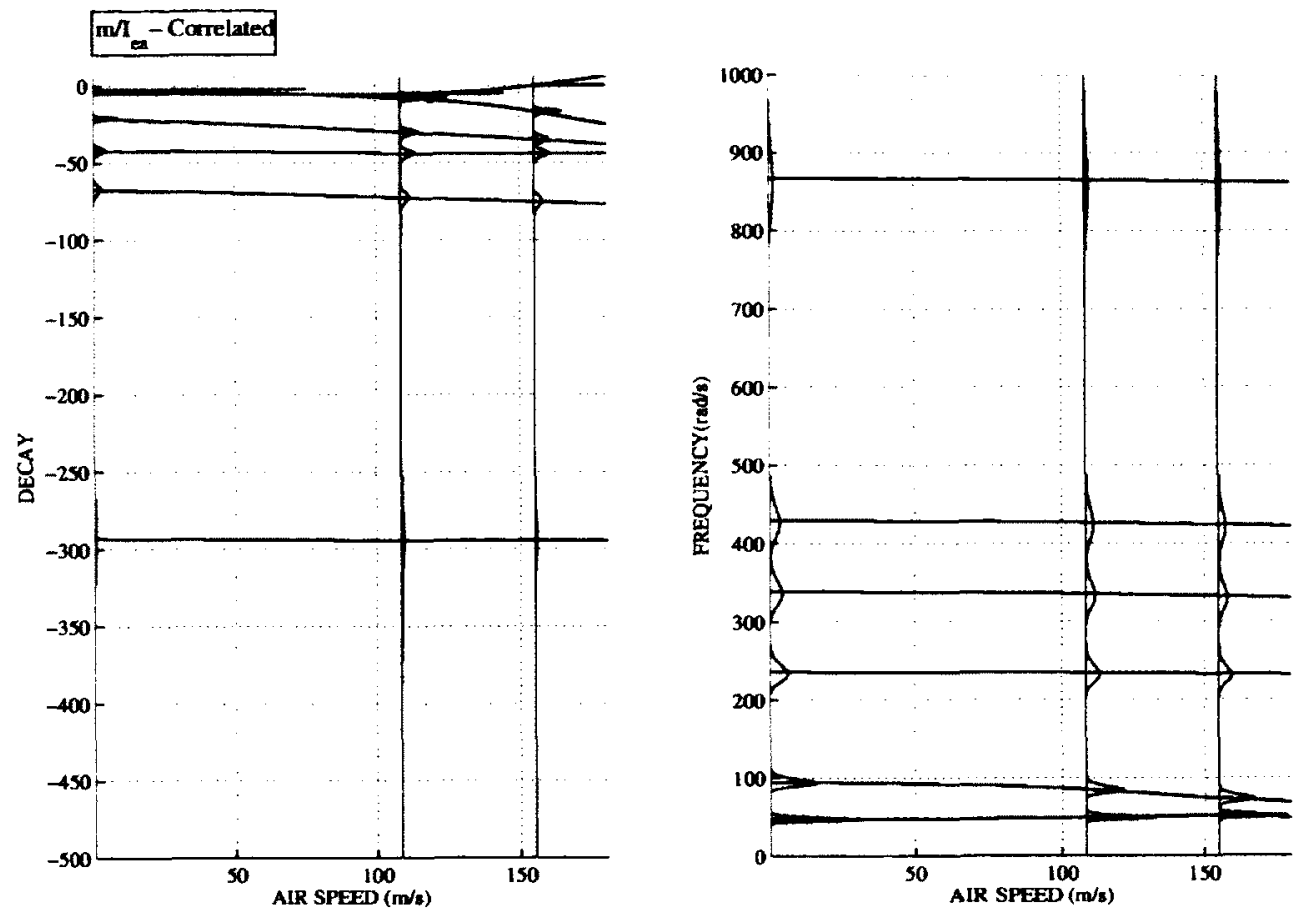

Figure 6.19: Modal parameter pdfs resulting from randomness in correlated inertia terms with $10 \%$ COV, obtained at 3 test airspeeds: $0 \frac{\mathrm{m}}{\mathrm{s}}, 109 \frac{\mathrm{m}}{\mathrm{s}}, 155.13 \frac{\mathrm{m}}{\mathrm{s}}$
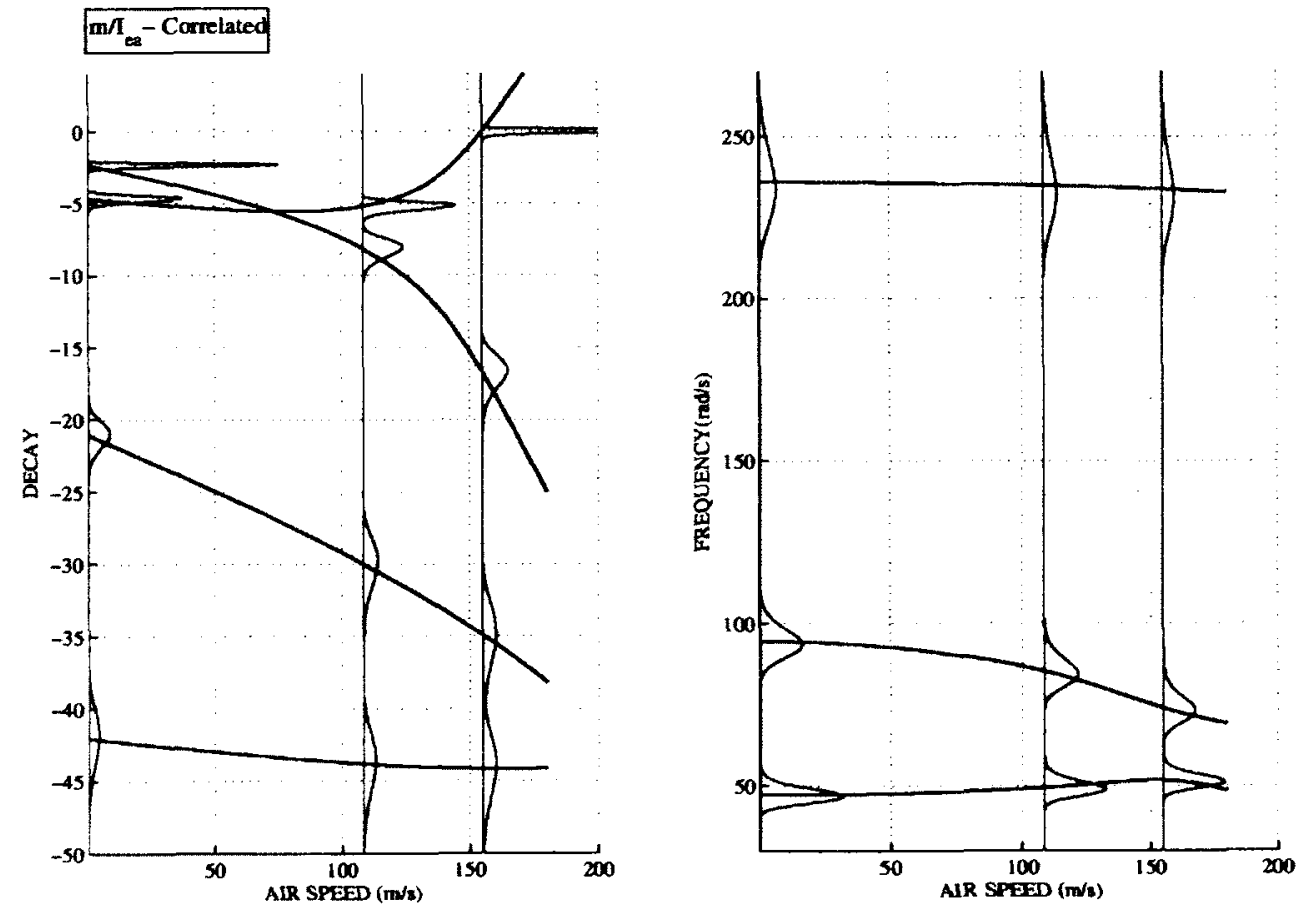

Figure 6.20: Magnified view of coalescing mode's modal parameter pdfs for uncertainty in correlated inertia terms 

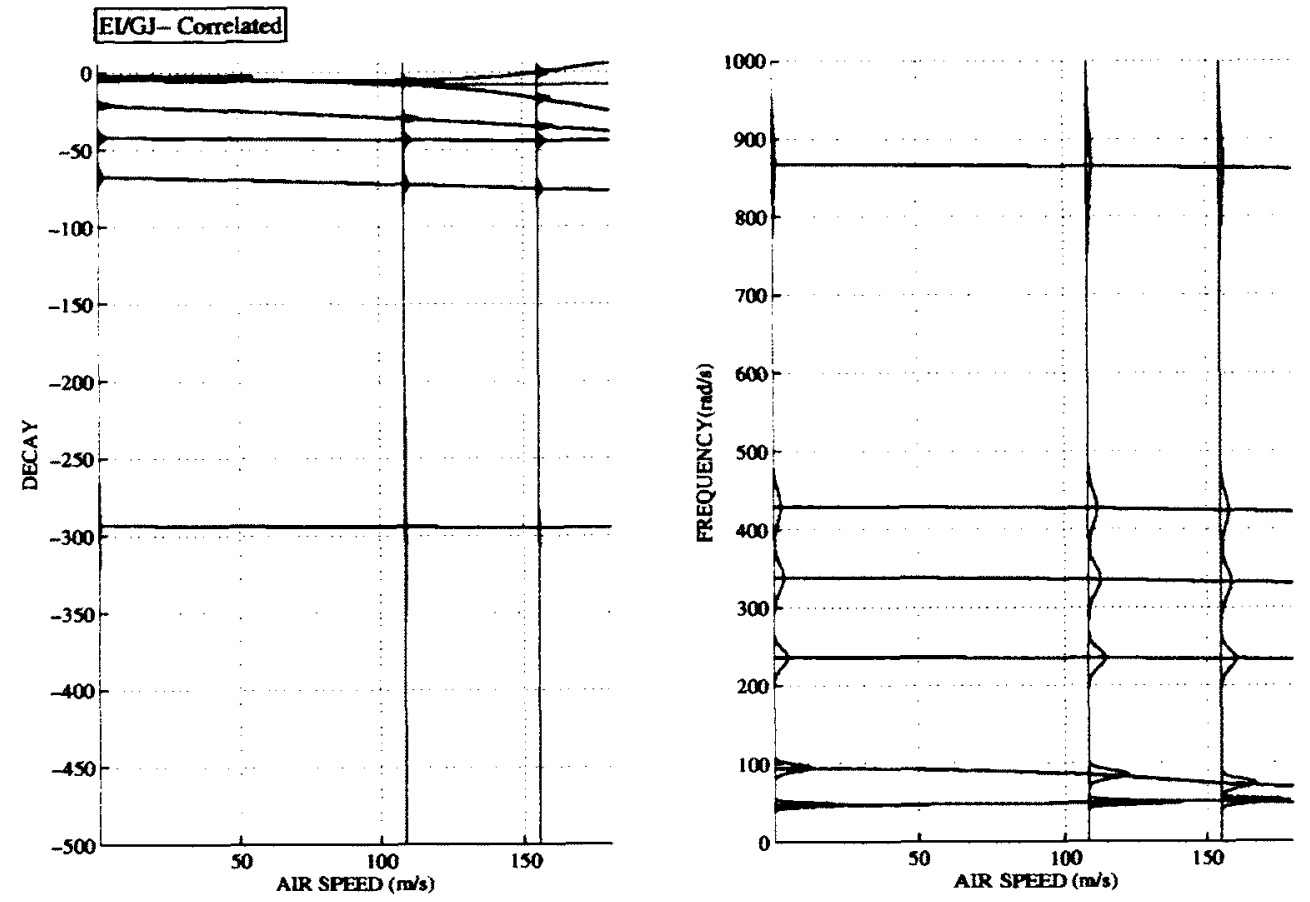

Figure 6.21: Modal parameter pdfs resulting from randomness in correlated stiffness terms with $10 \%$ COV, obtained at 3 test airspeeds: $0 \frac{\mathrm{m}}{\mathrm{s}}, 109 \frac{\mathrm{m}}{\mathrm{s}}, 155.13 \frac{\mathrm{m}}{\mathrm{s}}$
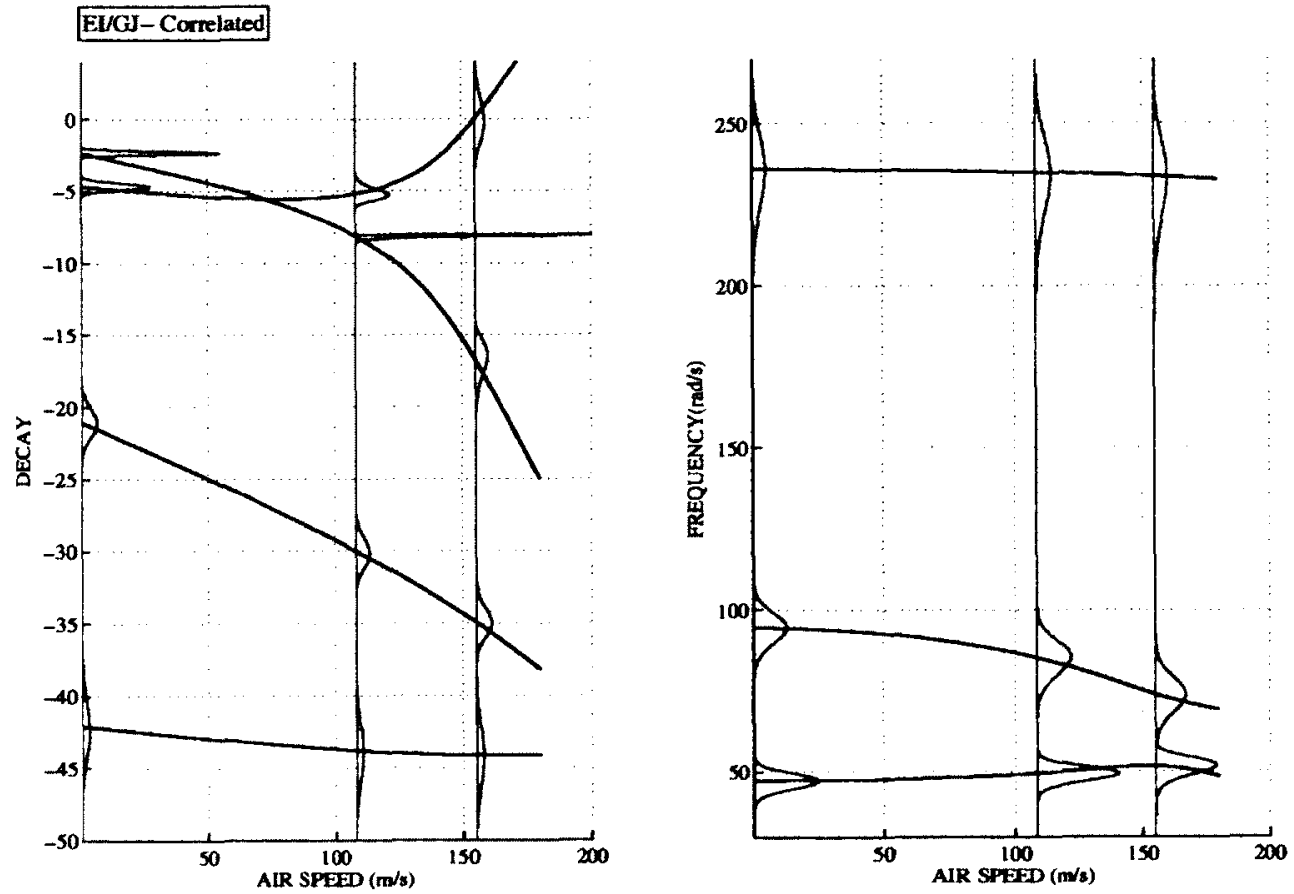

Figure 6.22: Magnified view of coalescing mode's modal parameter pdfs for uncertainty in correlated stiffness terms 

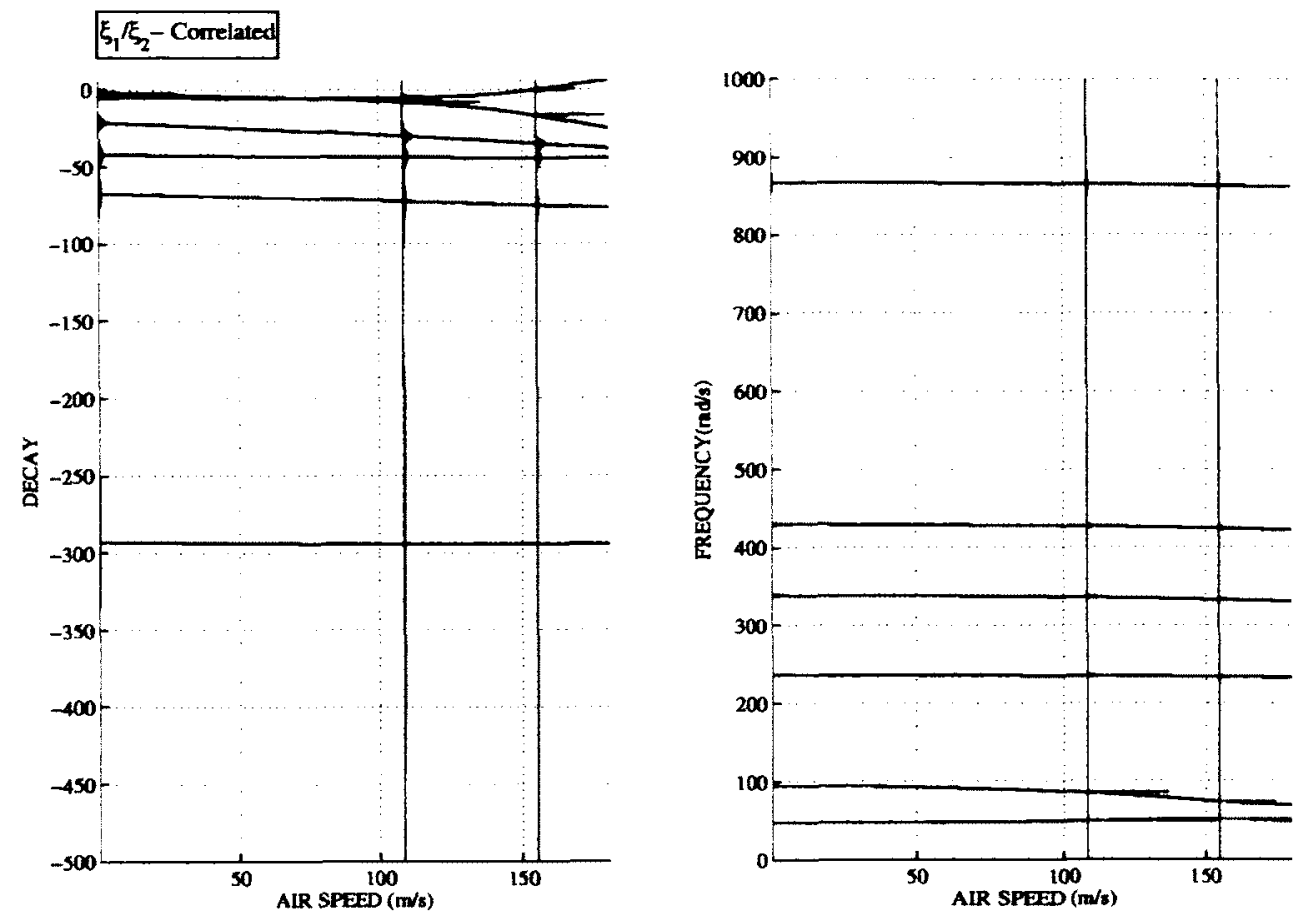

Figure 6.23: Modal parameter pdfs resulting from randomness in correlated damping terms with $10 \%$ COV, obtained at 3 test airspeeds: $0 \frac{\mathrm{m}}{\mathrm{s}}, 109 \frac{\mathrm{m}}{\mathrm{s}}, 155.13 \frac{\mathrm{m}}{\mathrm{s}}$
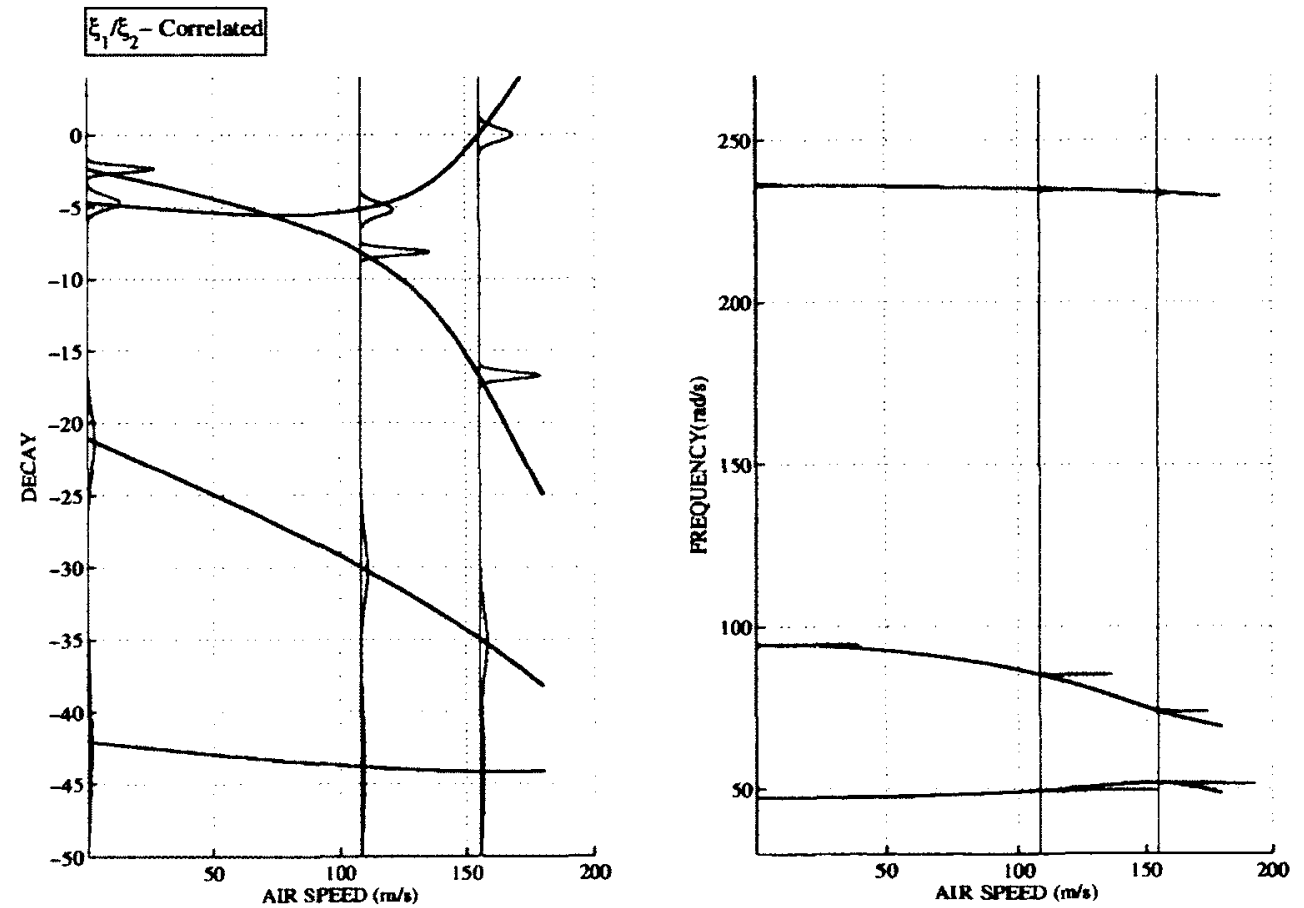

Figure 6.24: Magnified view of coalescing mode's modal parameter pdfs for uncertainty in correlated damping terms 


\subsubsection{Unsteady Results}

This section examines the effect of structural parameter uncertainty on the modal parameters for unsteady aerodynamics. The effect of individual parameter uncertainty will be investigated first. These results show only minor differences with respect to the results of quasisteady aerodynamics, mainly due to the effect of a more explosive flutter type.

Figs. (6.25) and (6.27) show the effects of uncertainty in the mass and mass moment of inertia parameters on the modal parameter pdfs, respectively. Figs. (6.26) and (6.28) provide a magnified view focusing specifically on the modal parameters of modes involved in the flutter mechanism. The frequency pdfs of the coalescing modes show strong non-stationary trends in the flutter region, reflecting a more explosive flutter type. The uncertainty in mass has greater effect on the uncertainty in frequency of the first (unstable) mode, which exhibits predominantly bending motion. Uncertainty in mass moment of inertia has greater influence on the frequency of the second (stable) mode, which exhibits predominantly torsional motion. For both cases, the decays of both coalescing modes are greatly affected. These modal decay pdfs show
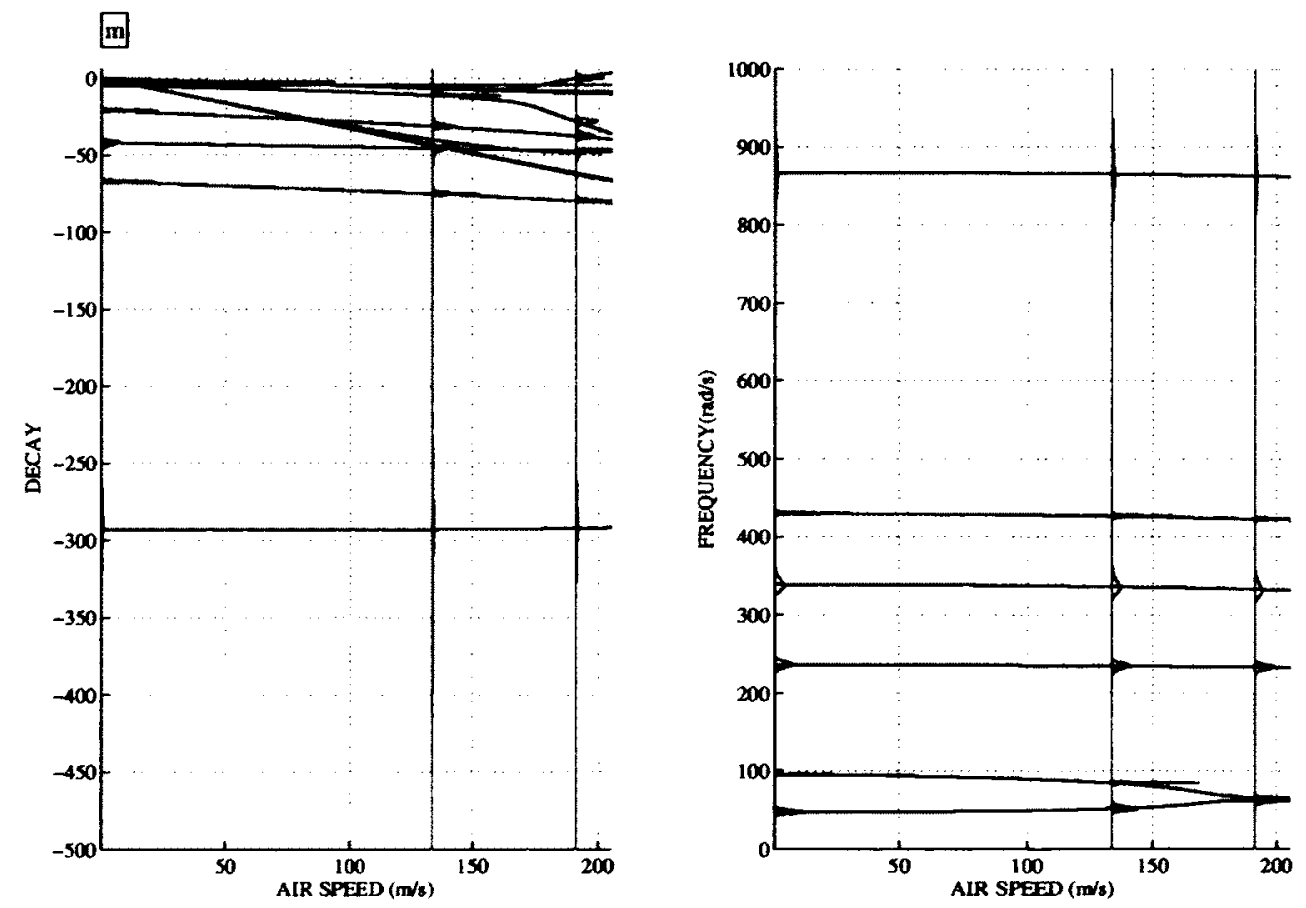

Figure 6.25: Modal parameter pdfs resulting from randomness in mass with $10 \% \mathrm{COV}$, obtained at 3 test airspeeds: $\approx 0 \mathrm{~m} / \mathrm{s}, 135.0 \mathrm{~m} / \mathrm{s}, 190.2 \mathrm{~m} / \mathrm{s}$ 

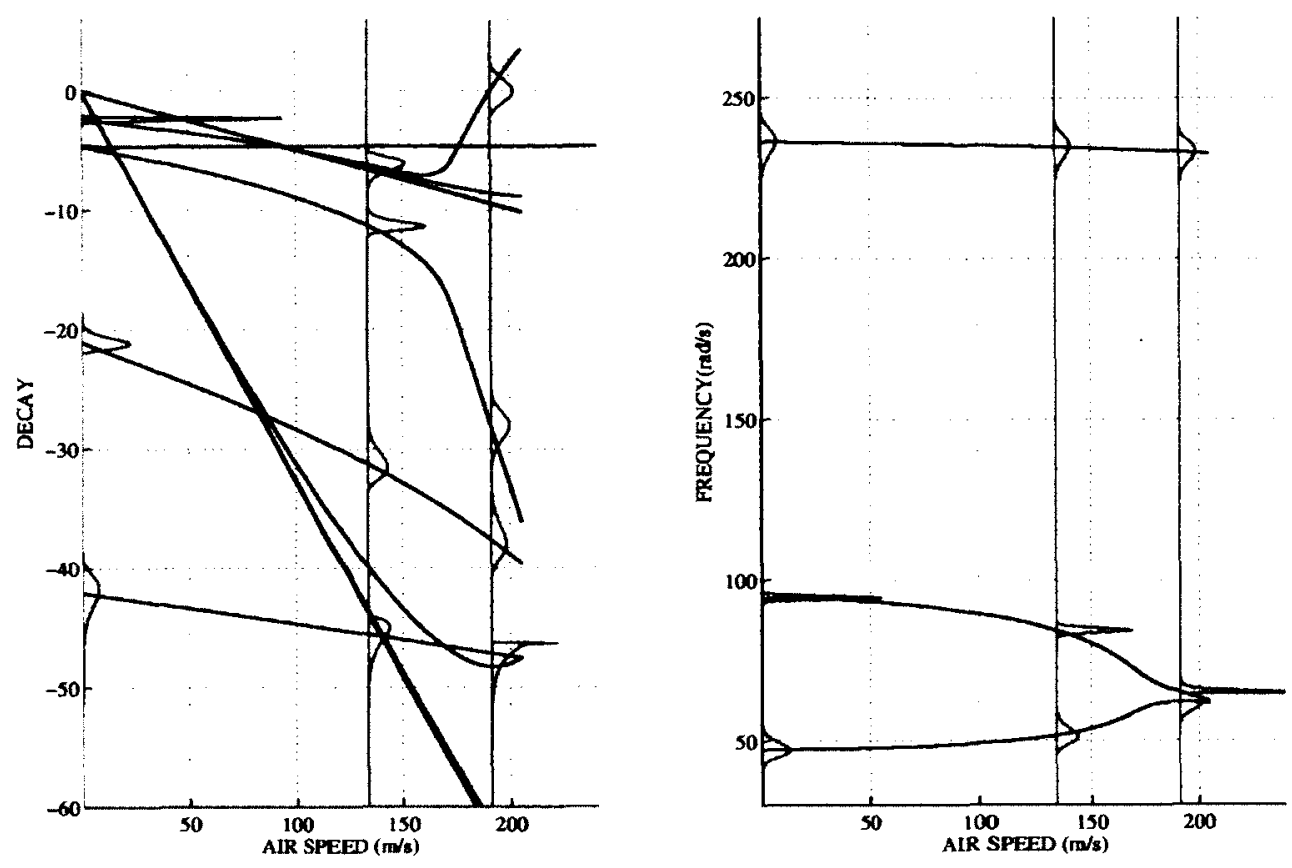

Figure 6.26: Magnified view of coalescing mode's modal parameter pdfs for uncertainty in mass
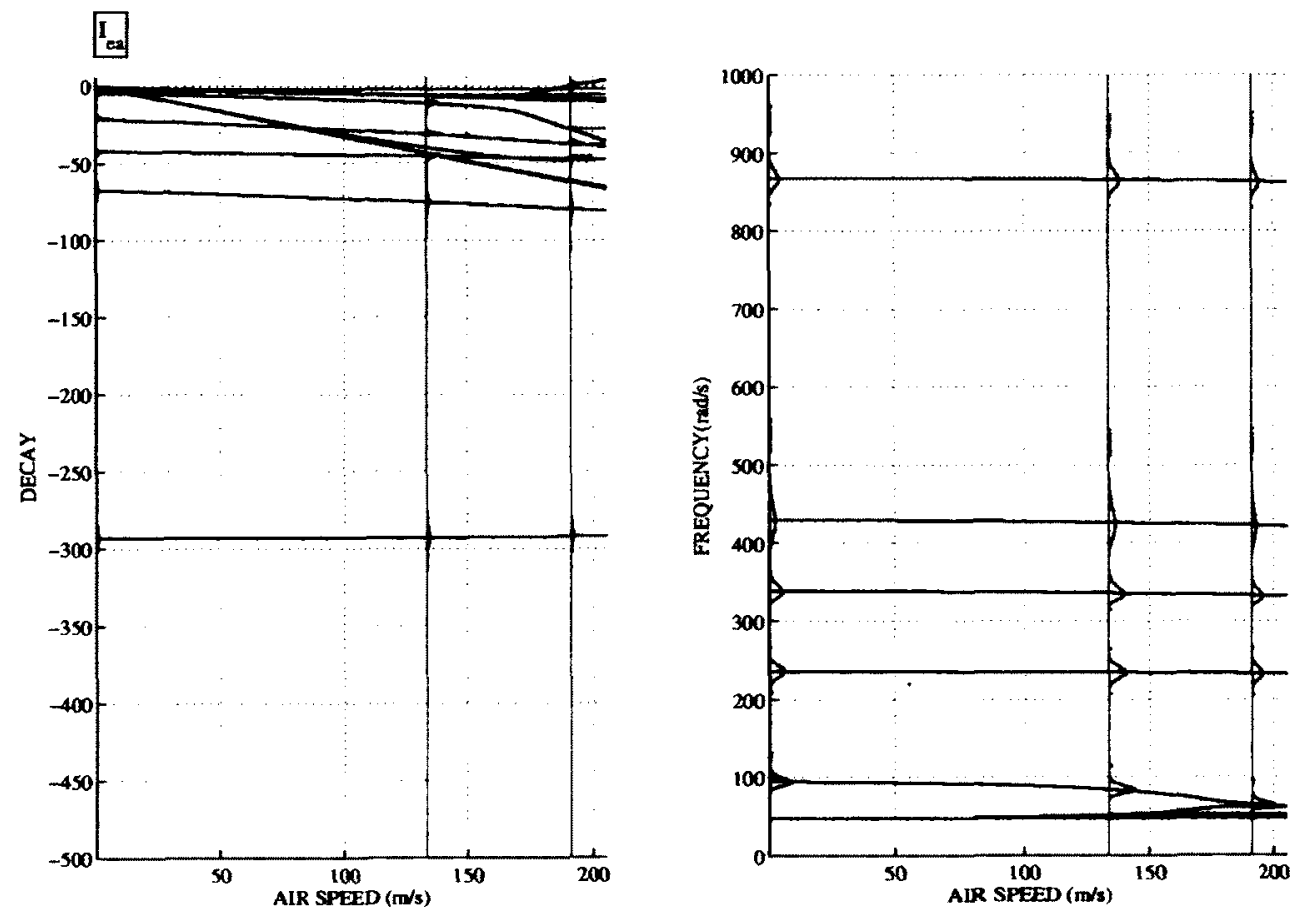

Figure 6.27: Modal parameter pdfs resulting from randomness in mass moment of inertia with $10 \% \mathrm{COV}$, obtained at 3 test airspeeds: $\approx 0 \mathrm{~m} / \mathrm{s}, 135.0 \mathrm{~m} / \mathrm{s}, 190.2 \mathrm{~m} / \mathrm{s}$ 

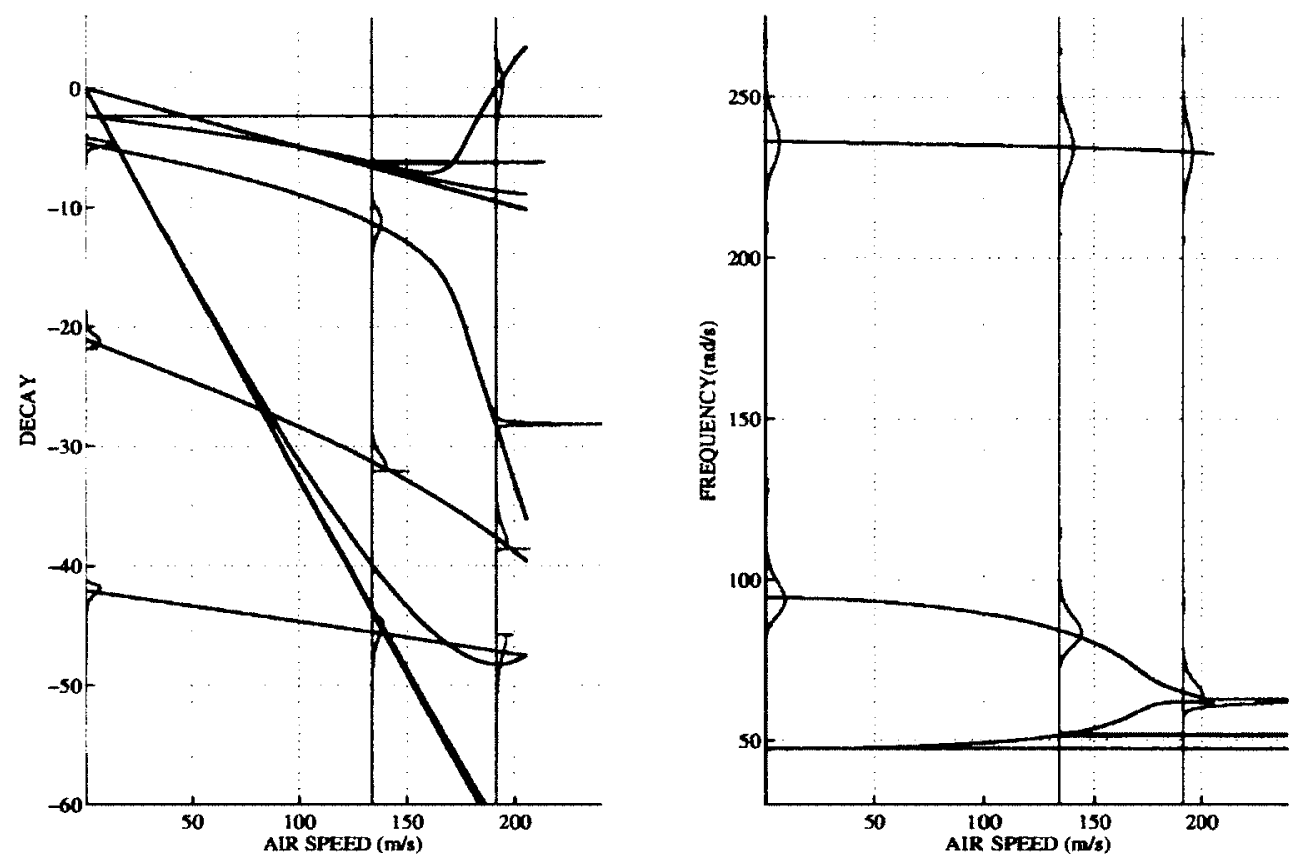

Figure 6.28: Magnified view of coalescing mode's modal parameter pdfs for uncertainty in mass moment of inertia

significant non-stationarity, where the variance increases as function of airspeed. For unsteady aerodynamics, a significant statistical overlap of the coalescing modal frequencies is observed near the flutter point.

The effects of stiffness uncertainty are illustrated in figs. (6.29) and (6.31) show the effects of bending stiffness and torsional stiffness uncertainty on the modal parameters of the system, respectively. Figs. (6.30) and (6.32) show the magnified view of the coalescing modes. As in quasisteady analysis, these figures indicate that effects of stiffness parameter uncertainty are almost identical to those of the uncertainty in the respective inertia parameter.

From the probabilistic results for uncertain inertia and stiffness parameters and the flutter speed pdfs from fig. (5.14), it is seen that the effect of growing variance in the modal decay of the flutter mode produces a substantial variance in the flutter speed pdfs. However, it should be noted that the growth in variance of the decay of the flutter mode is more substantial in cases of uncertainty of mass moment of inertia and the torsional stiffness, therefore the resulting flutter speed pdf for these cases experiences greater variance. 

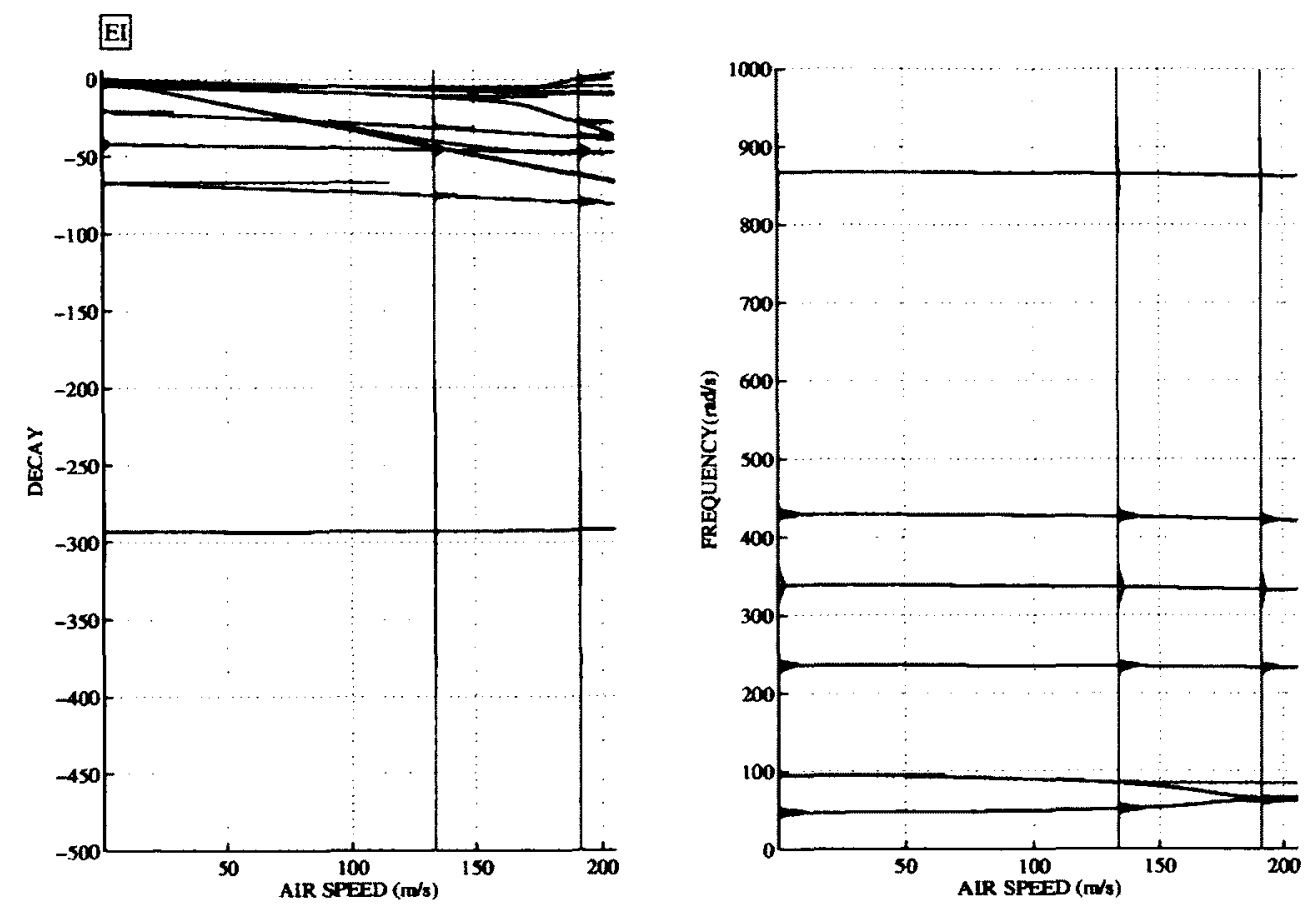

Figure 6.29: Modal parameter pdfs resulting from randomness in bending stiffness with $10 \%$ COV, obtained at 3 test airspeeds: $\approx 0 \mathrm{~m} / \mathrm{s}, 135.0 \mathrm{~m} / \mathrm{s}, 190.2 \mathrm{~m} / \mathrm{s}$
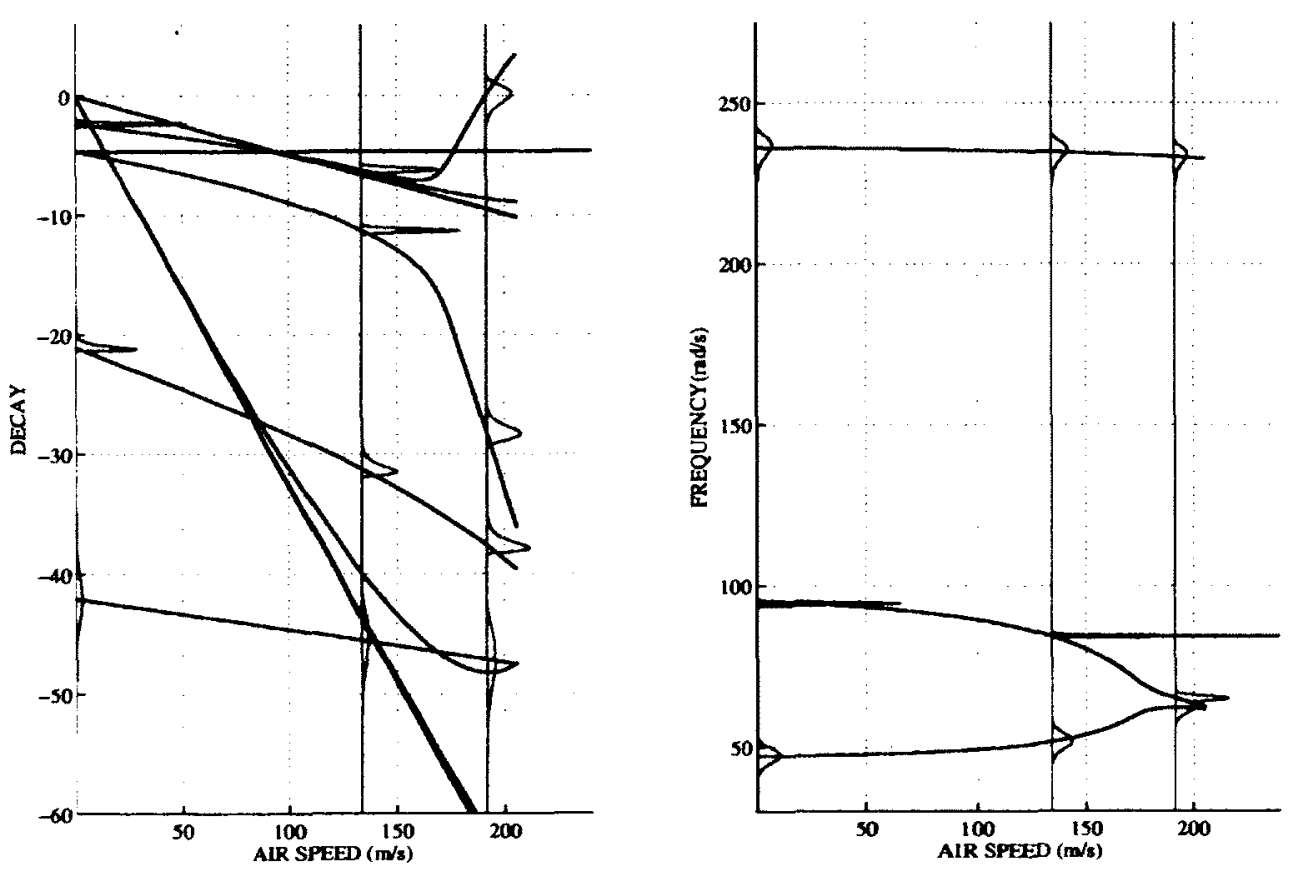

Figure 6.30: Magnified view of coalescing mode's modal parameter pdfs for uncertainty in bending stiffness 
四
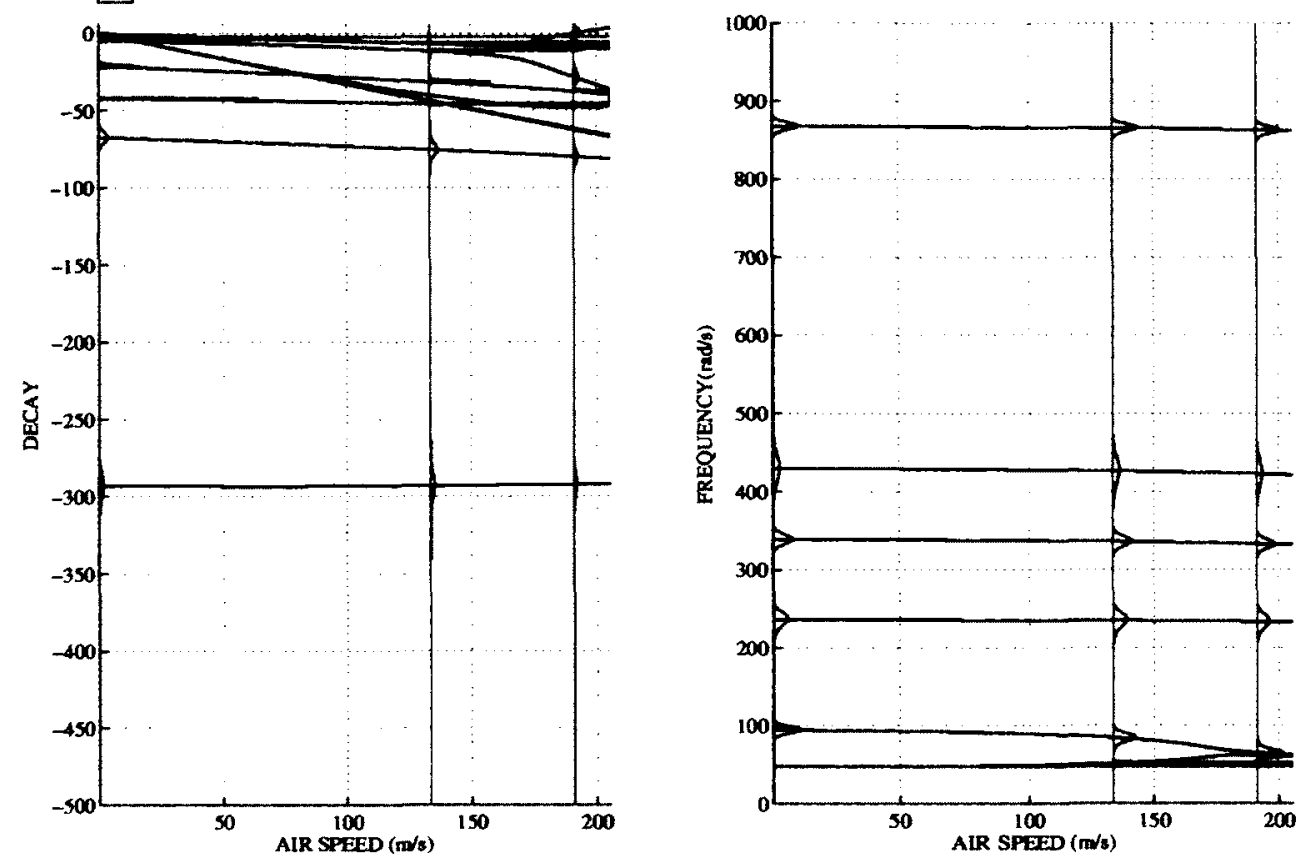

Figure 6.31: Modal parameter pdfs resulting from randomness in torsional stiffness with $10 \%$ COV, obtained at 3 test airspeeds: $\approx 0 \mathrm{~m} / \mathrm{s}, 135.0 \mathrm{~m} / \mathrm{s}, 190.2 \mathrm{~m} / \mathrm{s}$
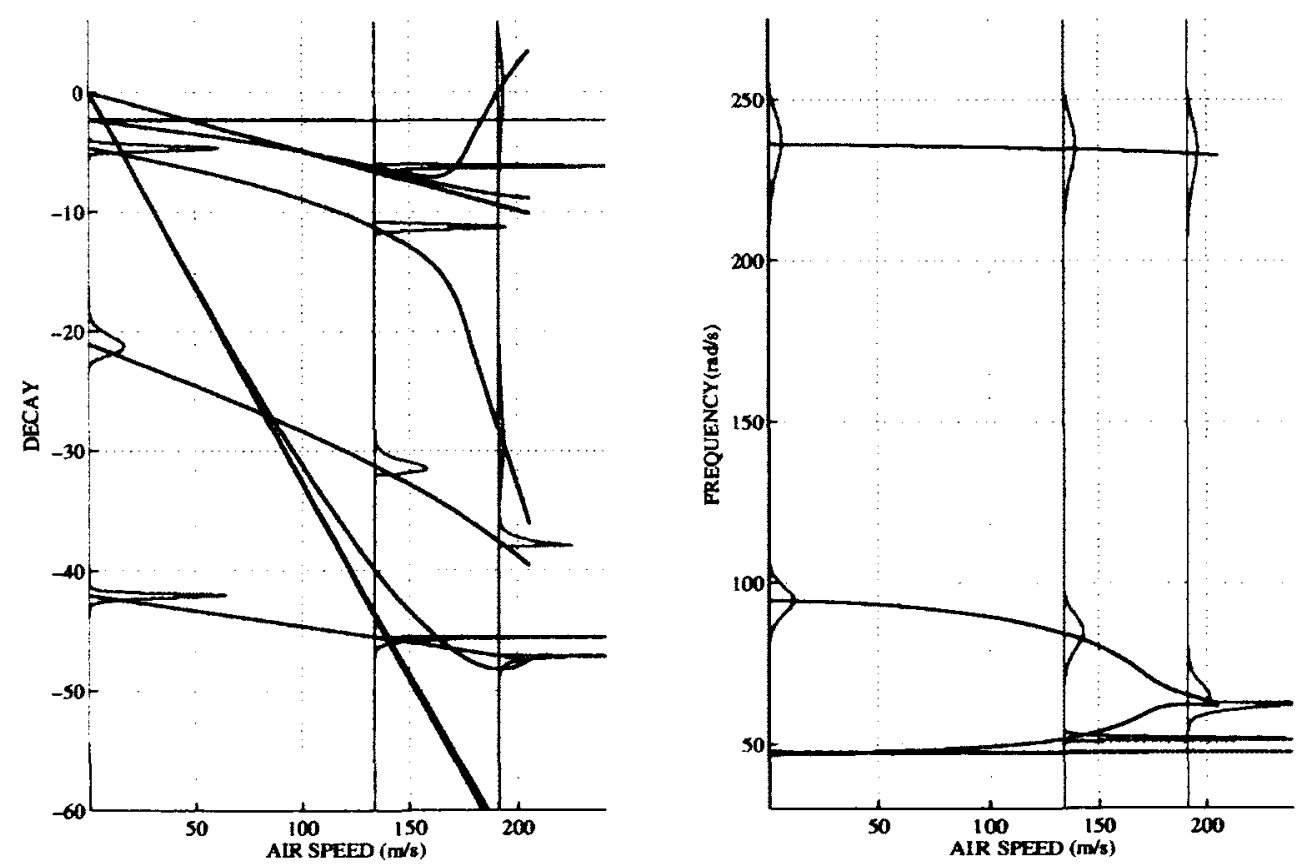

Figure 6.32: Magnified view of coalescing mode's modal parameter pdfs for uncertainty in torsional stiffness 
The effects of uncertainty of the damping parameters are illustrated in figs. (6.33) and (6.35) for uncertainty in the first and second structural damping parameters, respectively. Figs. (6.34) and (6.36) provide the magnified view of the modal properties of the coalescing modes for each respective case. The resulting observations are almost identical to the case of quasisteady aerodynamic forcing. There are no notable influence effects of uncertainty on the modal frequencies of the coalescing modes. The modal decay pdfs do not show large changes of variance as function of increasing airspeed. Only in vicinity of flutter the modal decays of the coalescing modes show very slight changes in variance. For uncertainty in the first structural damping ratio the most significant uncertainty is experienced by the decay of the first (flutter) mode. The variance of the second mode is not significant. For uncertainty in the second structural damping ratio, the decay of the second (non-flutter) mode is most affected. The variance of the first mode is insignificant.
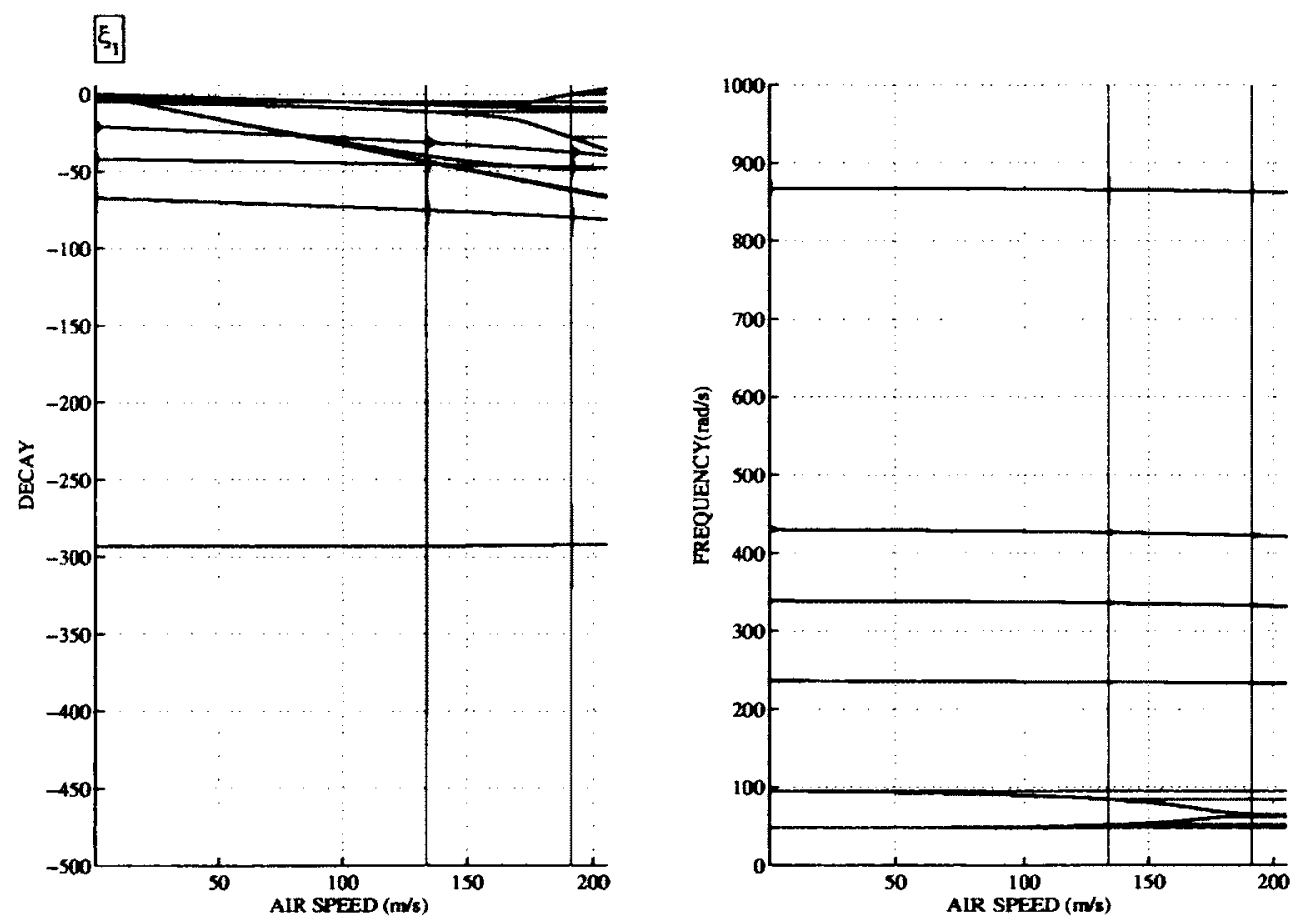

Figure 6.33: Modal parameter pdfs resulting from randomness in first structural damping ratio with $10 \% \mathrm{COV}$, obtained at 3 test airspeeds: $\approx 0 \mathrm{~m} / \mathrm{s}, 135.0 \mathrm{~m} / \mathrm{s}, 190.2 \mathrm{~m} / \mathrm{s}$ 

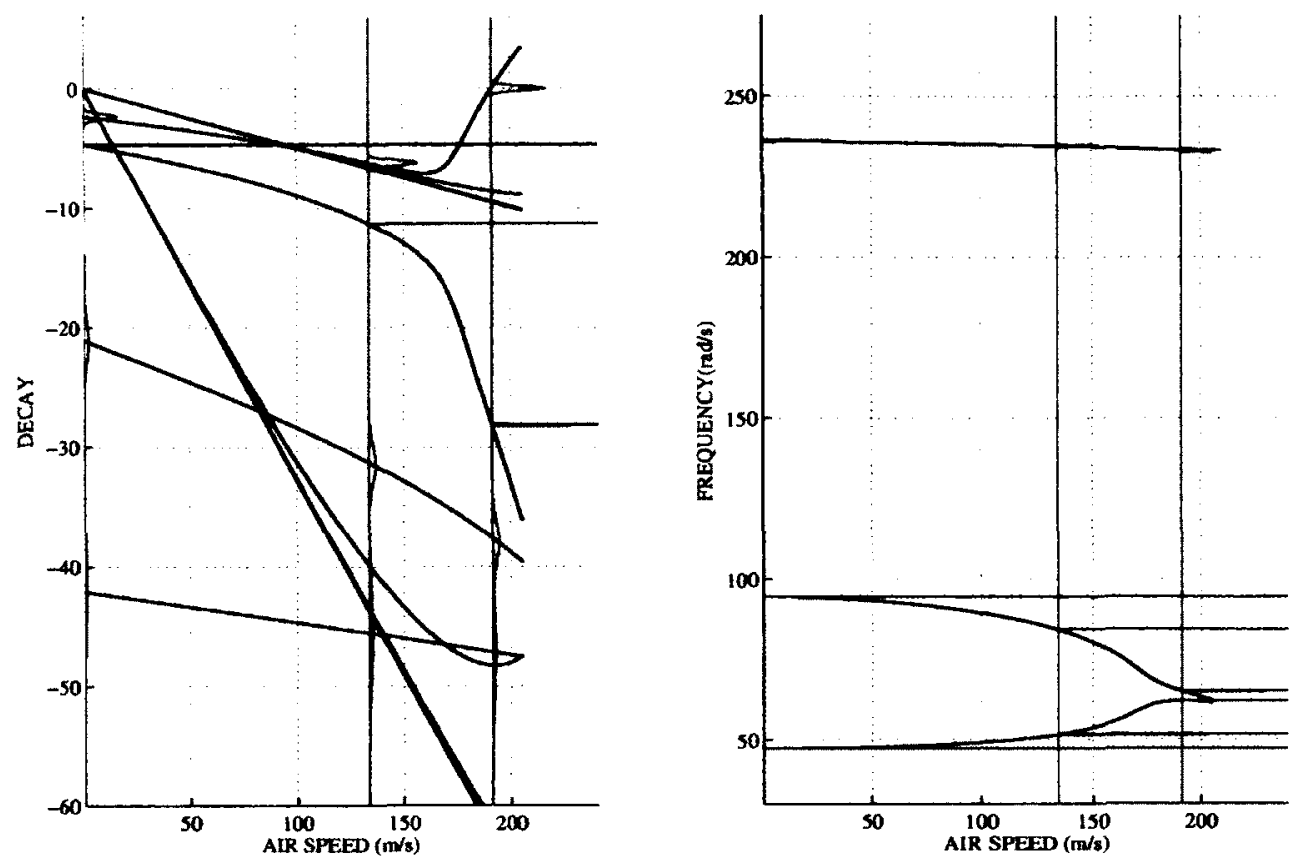

Figure 6.34: Magnified view of coalescing mode's modal parameter pdfs for uncertainty in first structural damping ratio
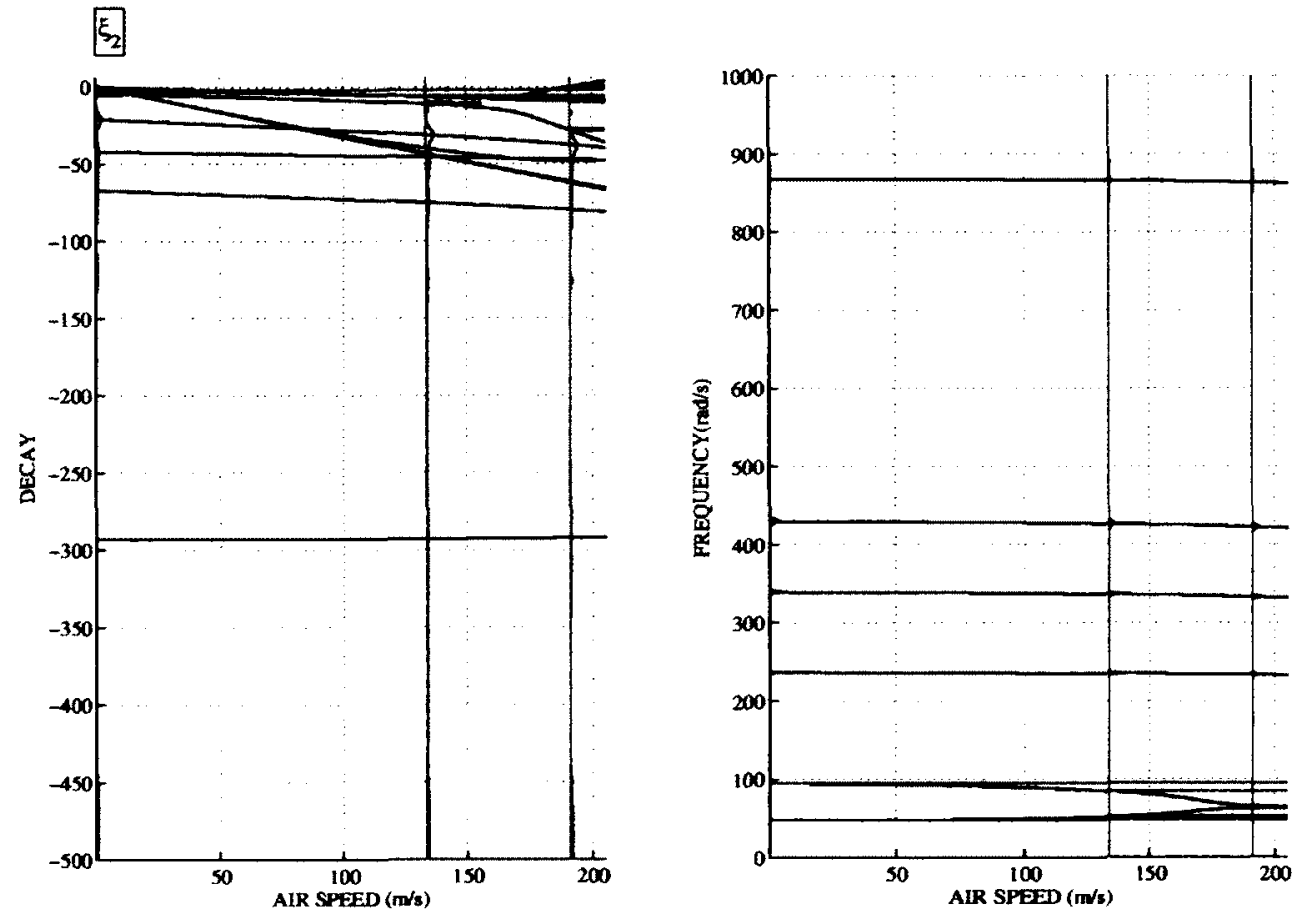

Figure 6.35: Modal parameter pdfs resulting from randomness in second structural damping ratio with $10 \%$ COV, obtained at 3 test airspeeds: $\approx 0 \mathrm{~m} / \mathrm{s}, 135.0 \mathrm{~m} / \mathrm{s}, 190.2 \mathrm{~m} / \mathrm{s}$ 

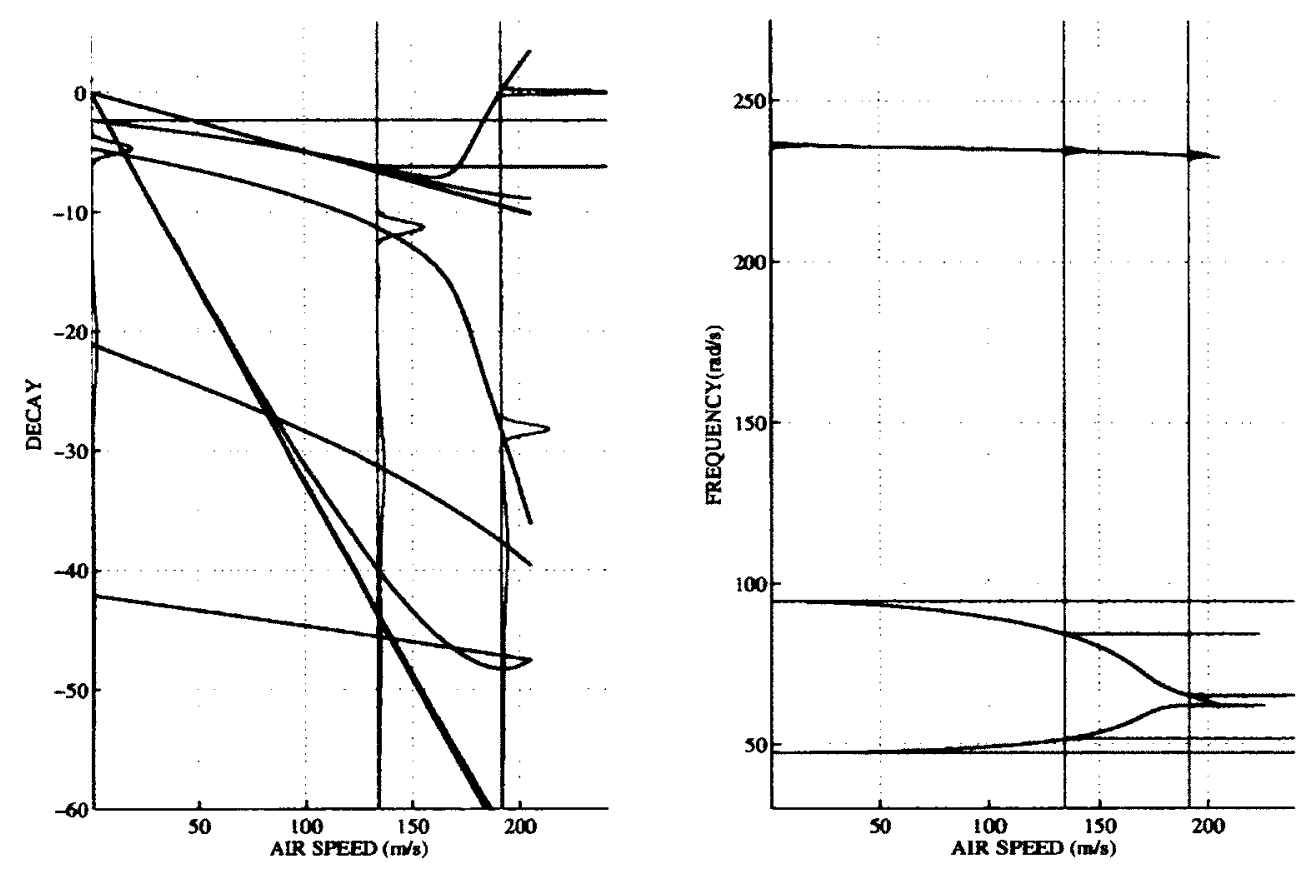

Figure 6.36: Magnified view of coalescing mode's modal parameter pdfs for uncertainty in second structural damping ratio

\section{Multiple Parameter Uncertainty}

The effects of uncertainty in multiple parameters are examined next. Figs. (6.37)-(6.42) show the resulting transformations of the modal parameter pdfs as a function of airspeed for independent inertia, stiffness and damping parameters. All of the results indicate that uncertainty effects of the independent multiple parameters lead to an increase of the uncertainty in the modal parameter pdfs. The resulting larger variance of modal decay of the flutter mode produces a larger variance in the flutter speed (evident in fig. (5.14). It should also be noted that modal frequencies of the coalescing modes experience full overlapping effects.

Next, the effects of full correlation between the random parameters are examined. Figs. (6.43)-(6.48) illustrate the probabilistic modal parameter transformation results for fully correlated inertia, stiffness and damping parameters. These results are identical with the results of analysis with quasisteady aerodynamics. Due to correlation between the random structural parameters, the following observations are deduced from these results. The uncertainty of the 

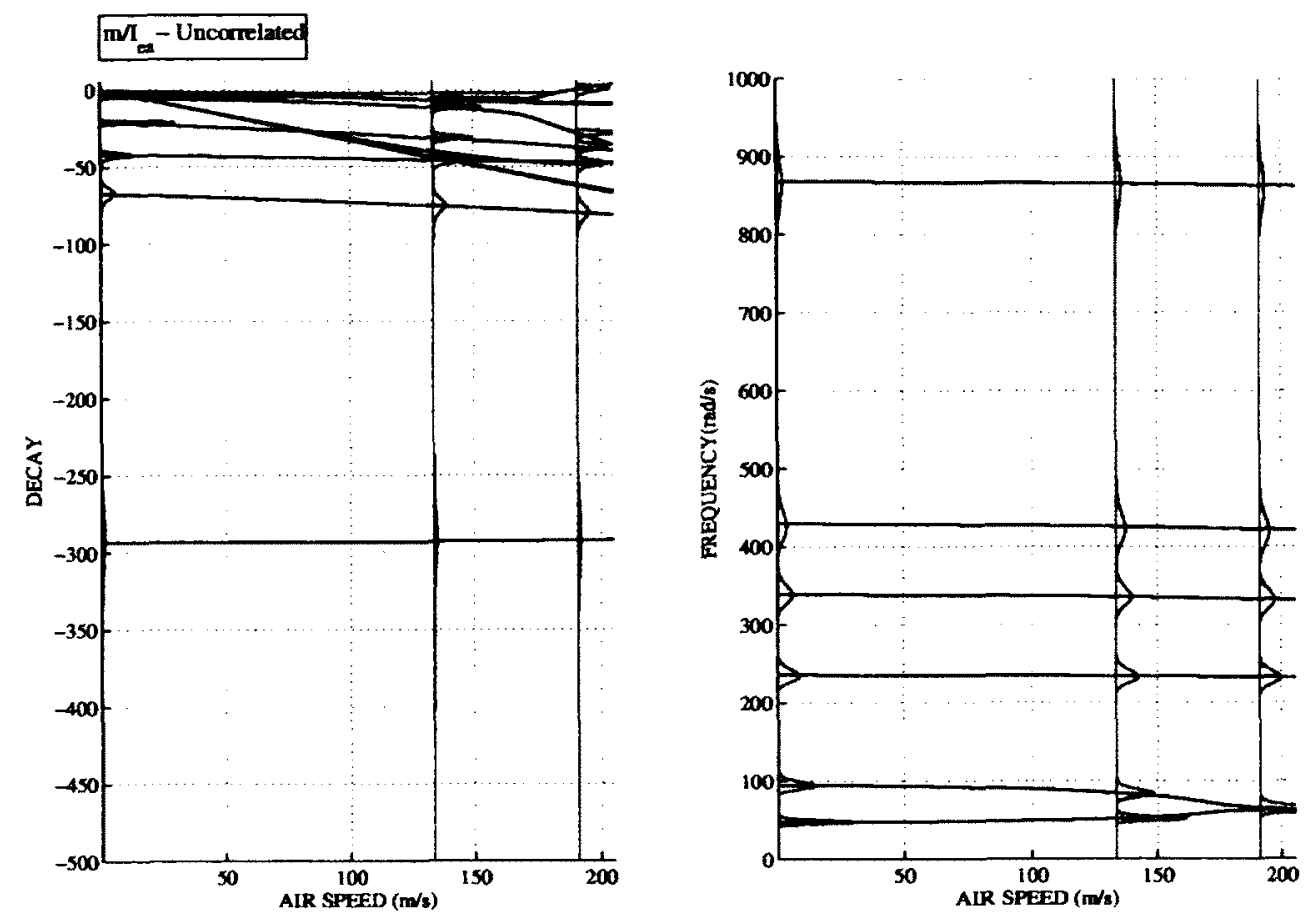

Figure 6.37: Modal parameter pdfs resulting from randomness in uncorrelated inertia terms with $10 \% \mathrm{COV}$, obtained at 3 test airspeeds: $\approx 0 \mathrm{~m} / \mathrm{s}, 135.0 \mathrm{~m} / \mathrm{s}, 190.2 \mathrm{~m} / \mathrm{s}$
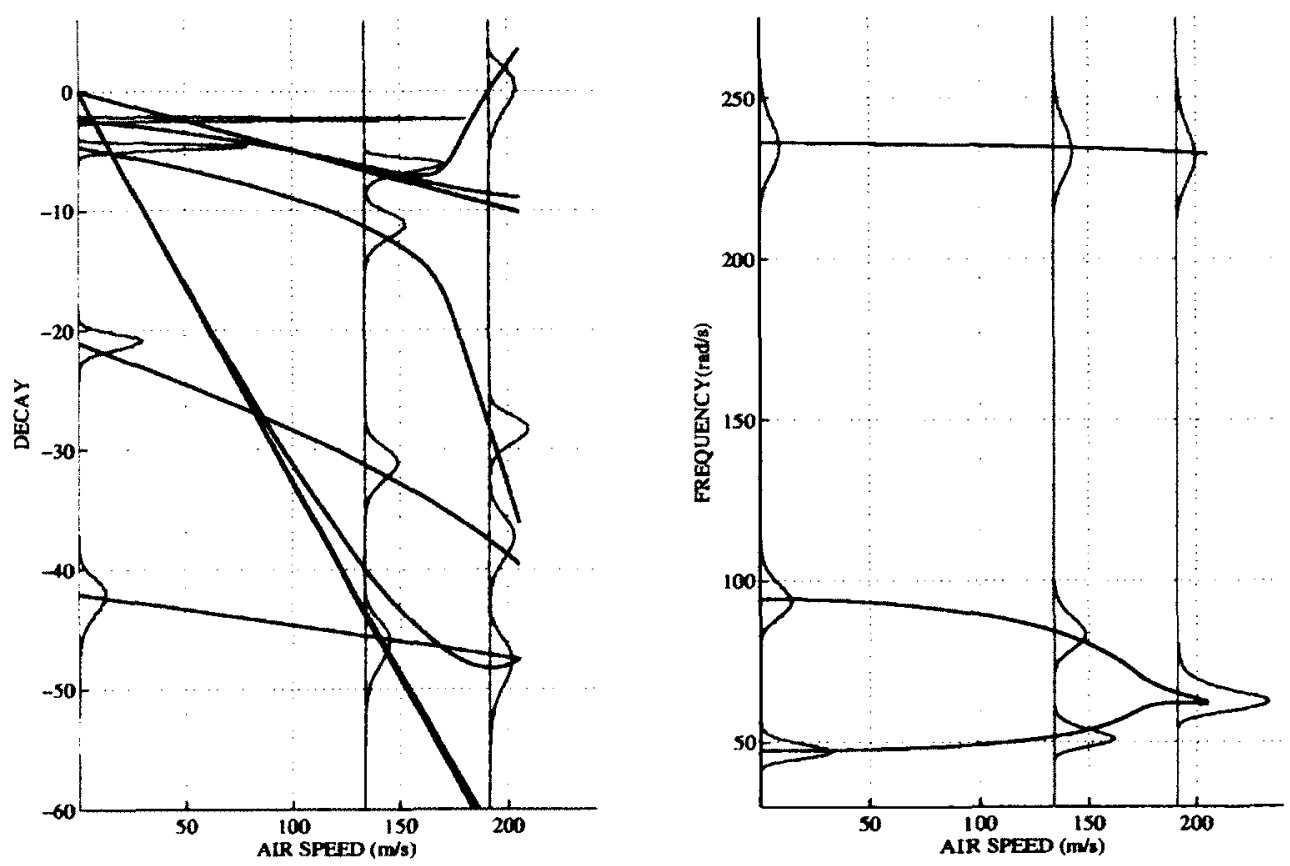

Figure 6.38: Magnified view of coalescing mode's modal parameter pdfs for uncertainty in uncorrelated inertia terms 

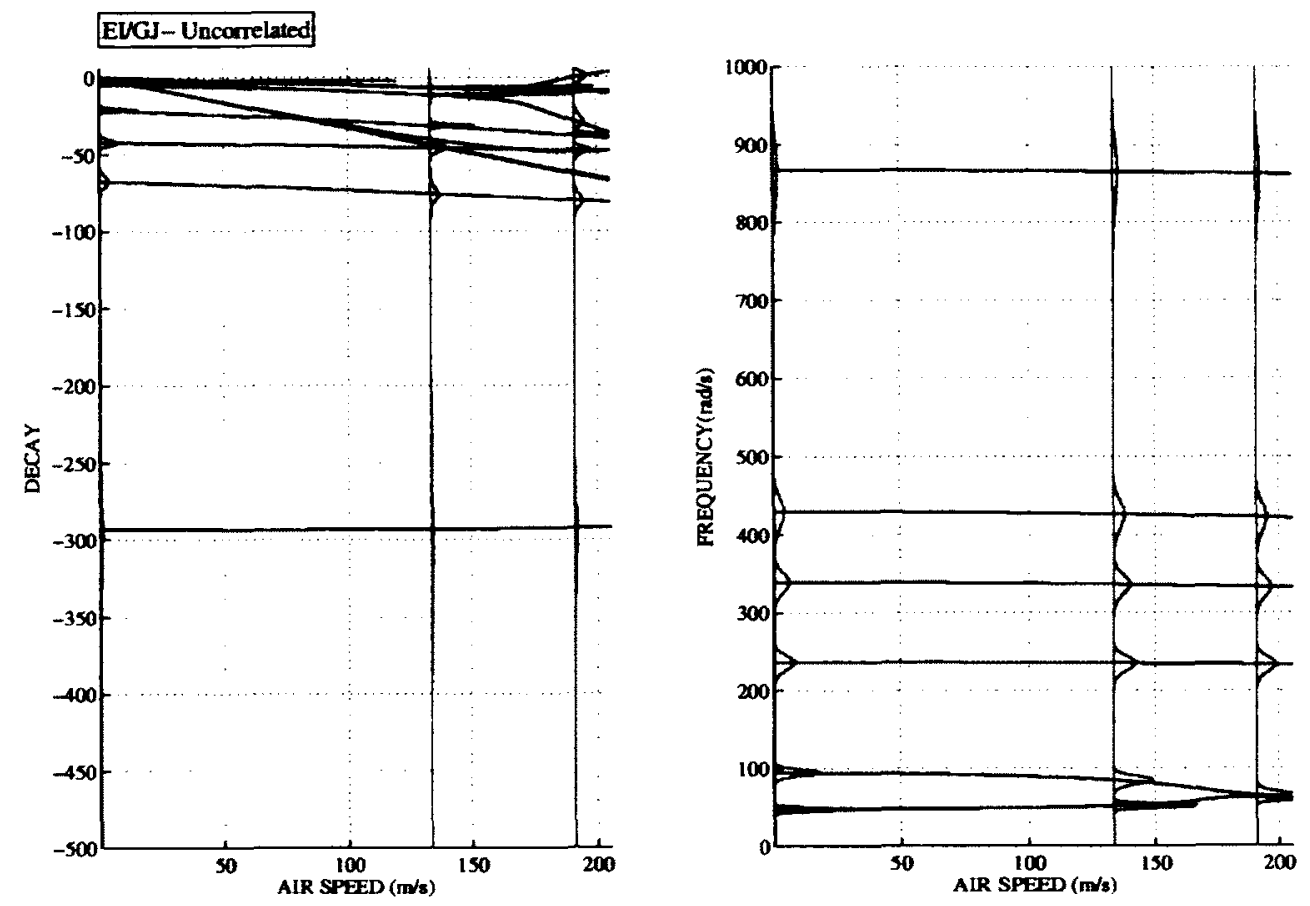

Figure 6.39: Modal parameter pdfs resulting from randomness in uncorrelated stiffness terms with $10 \%$ COV, obtained at 3 test airspeeds: $\approx 0 \mathrm{~m} / \mathrm{s}, 135.0 \mathrm{~m} / \mathrm{s}, 190.2 \mathrm{~m} / \mathrm{s}$
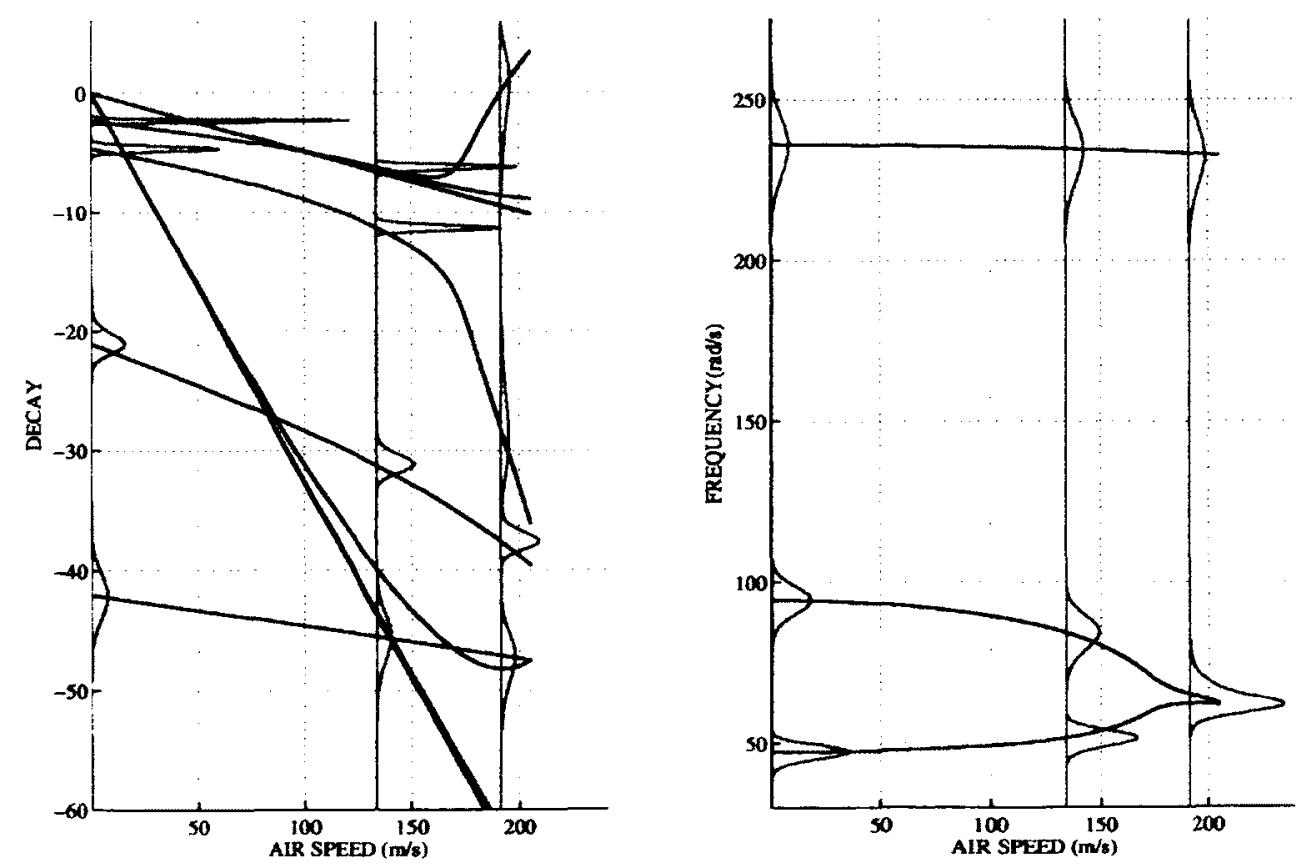

Figure 6.40: Magnified view of coalescing mode's modal parameter pdfs for uncertainty in uncorrelated stiffness terms 

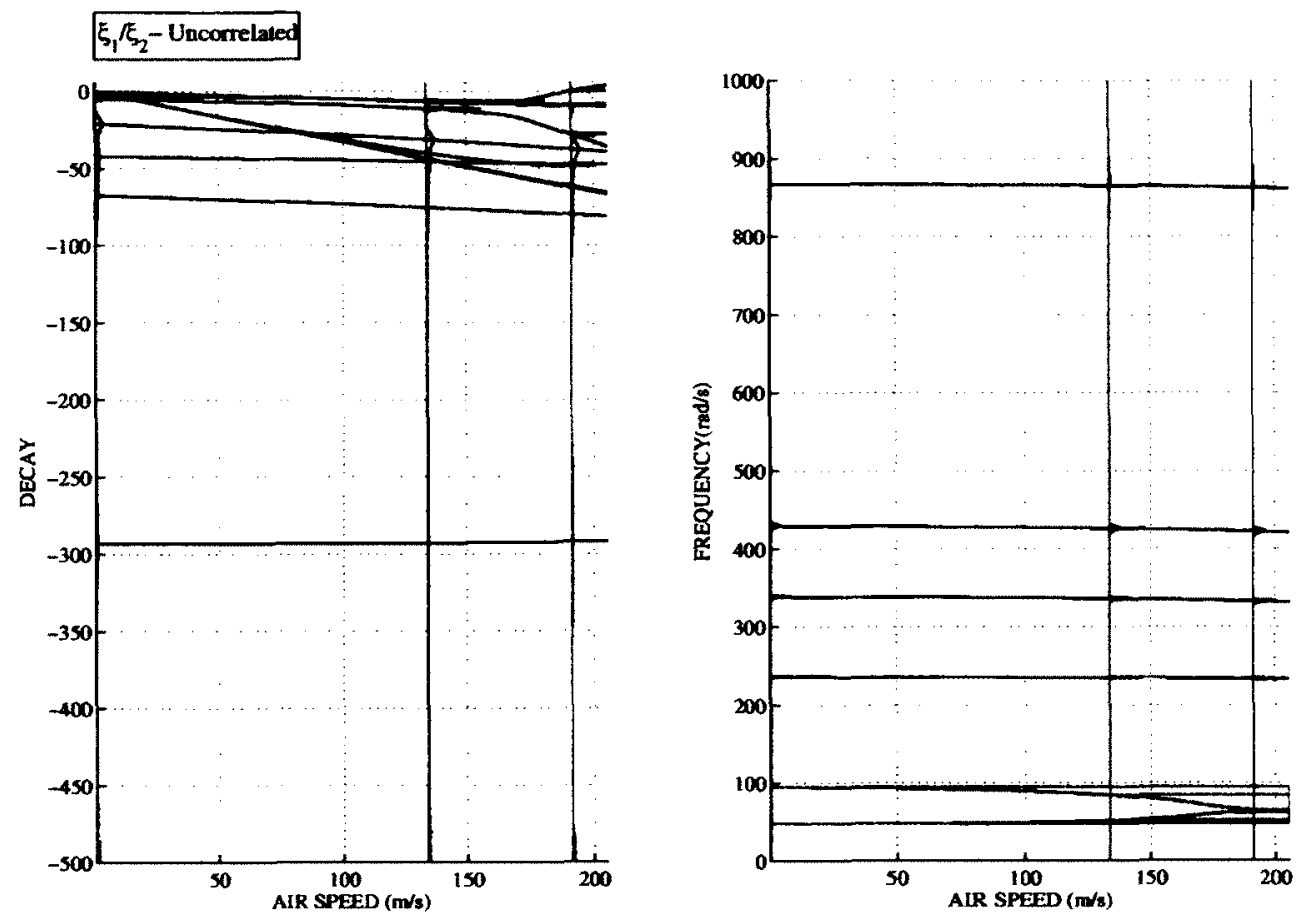

Figure 6.41: Modal parameter pdfs resulting from randomness in uncorrelated damping terms with $10 \% \mathrm{COV}$, obtained at 3 test airspeeds: $\approx 0 \mathrm{~m} / \mathrm{s}, 135.0 \mathrm{~m} / \mathrm{s}, 190.2 \mathrm{~m} / \mathrm{s}$
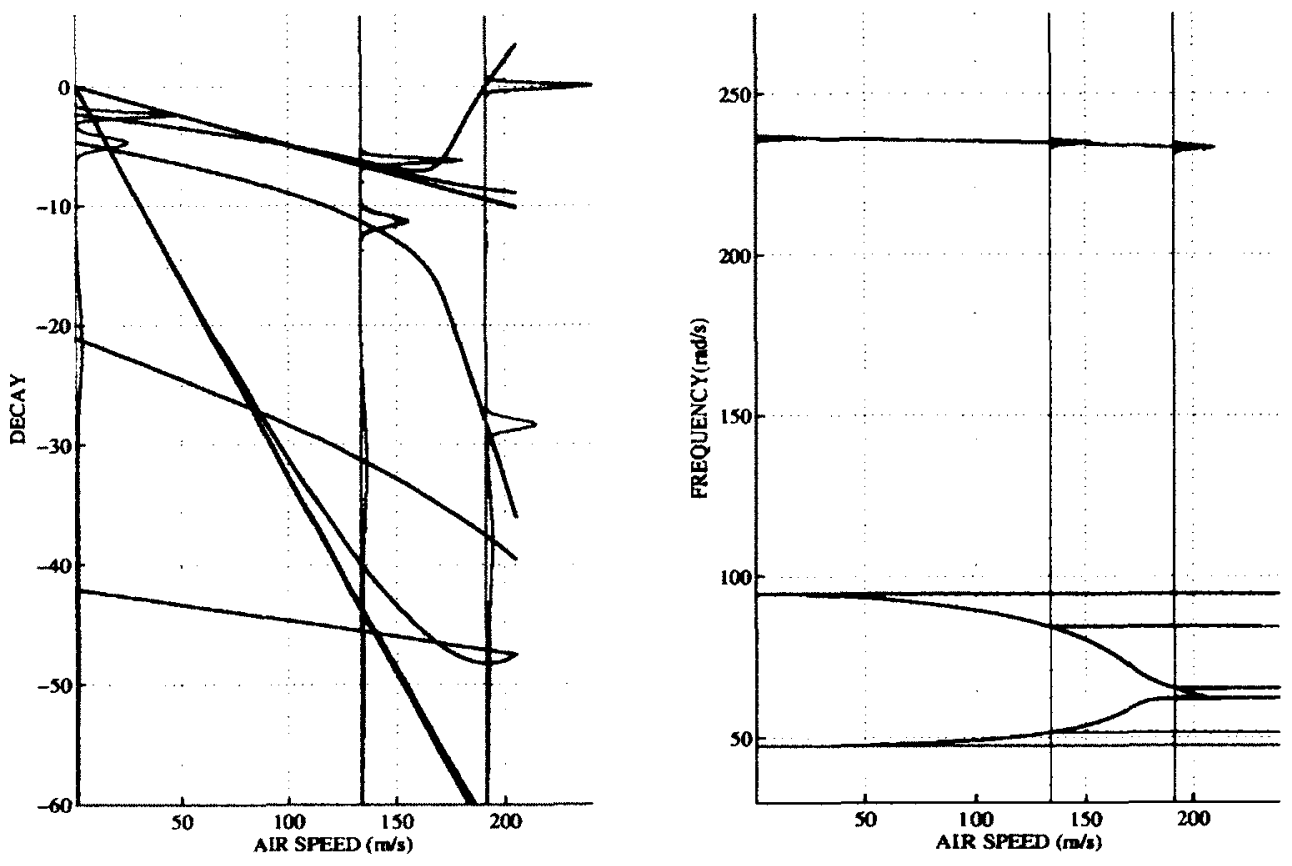

Figure 6.42: Magnified view of coalescing mode's modal parameter pdfs for uncertainty in uncorrelated damping terms 

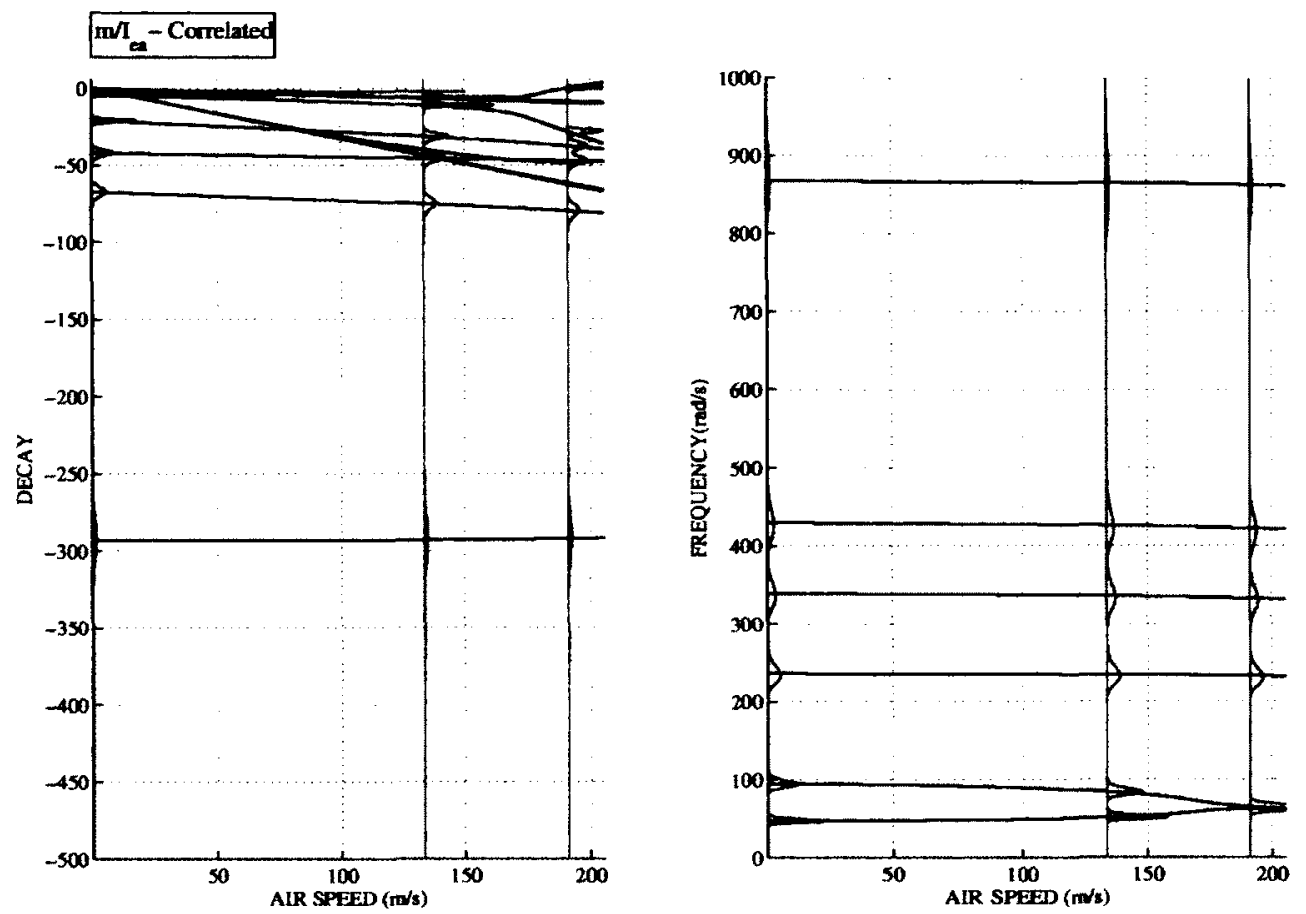

Figure 6.43: Modal parameter pdfs resulting from randomness in correlated inertia terms with $10 \%$ COV, obtained at 3 test airspeeds: $\approx 0 \mathrm{~m} / \mathrm{s}, 135.0 \mathrm{~m} / \mathrm{s}, 190.2 \mathrm{~m} / \mathrm{s}$
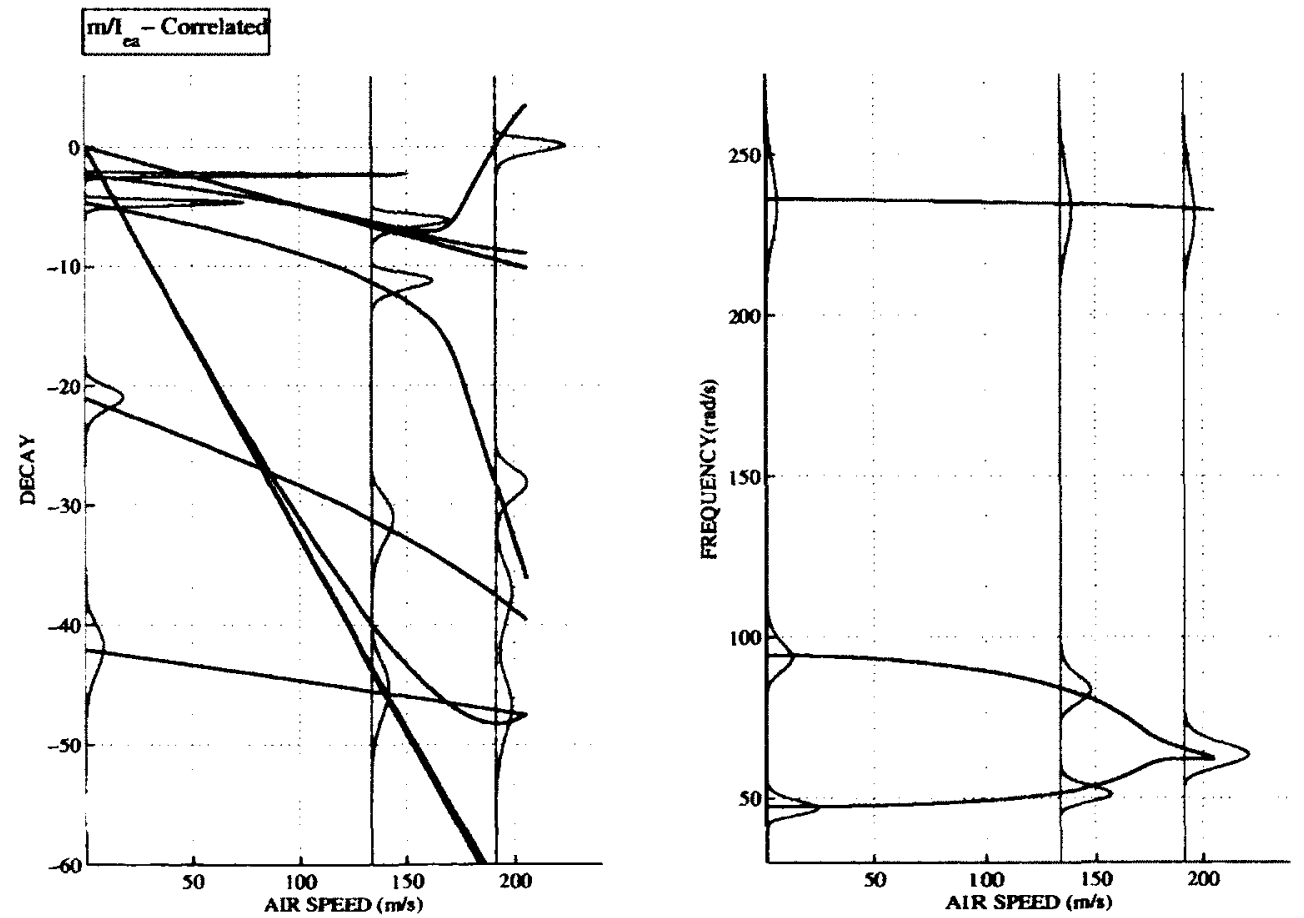

Figure 6.44: Magnified view of coalescing mode's modal parameter pdfs for uncertainty in correlated inertia terms 

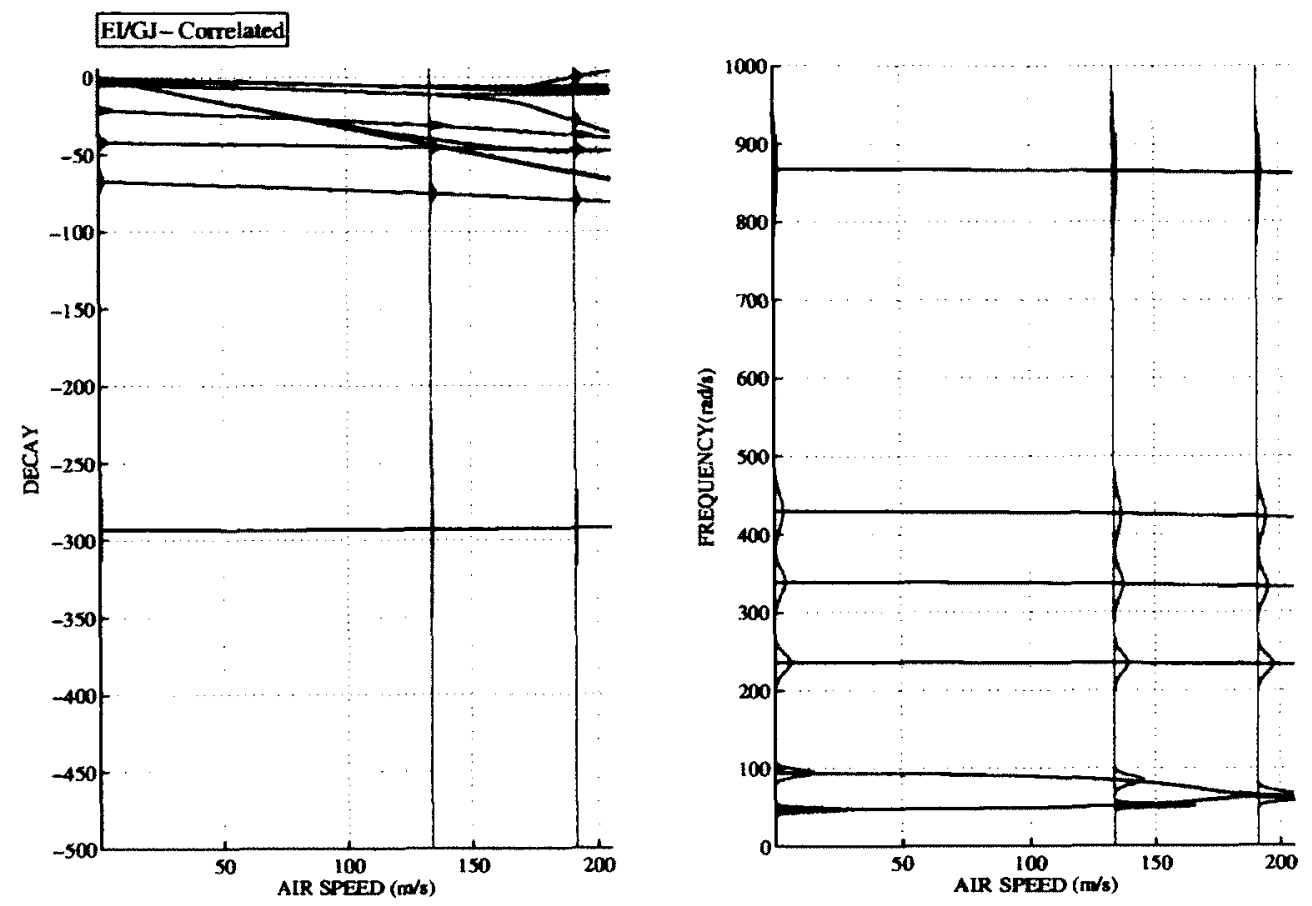

Figure 6.45: Modal parameter pdfs resulting from randomness in correlated stiffness terms with $10 \%$ COV, obtained at 3 test airspeeds: $\approx 0 \mathrm{~m} / \mathrm{s}, 135.0 \mathrm{~m} / \mathrm{s}, 190.2 \mathrm{~m} / \mathrm{s}$
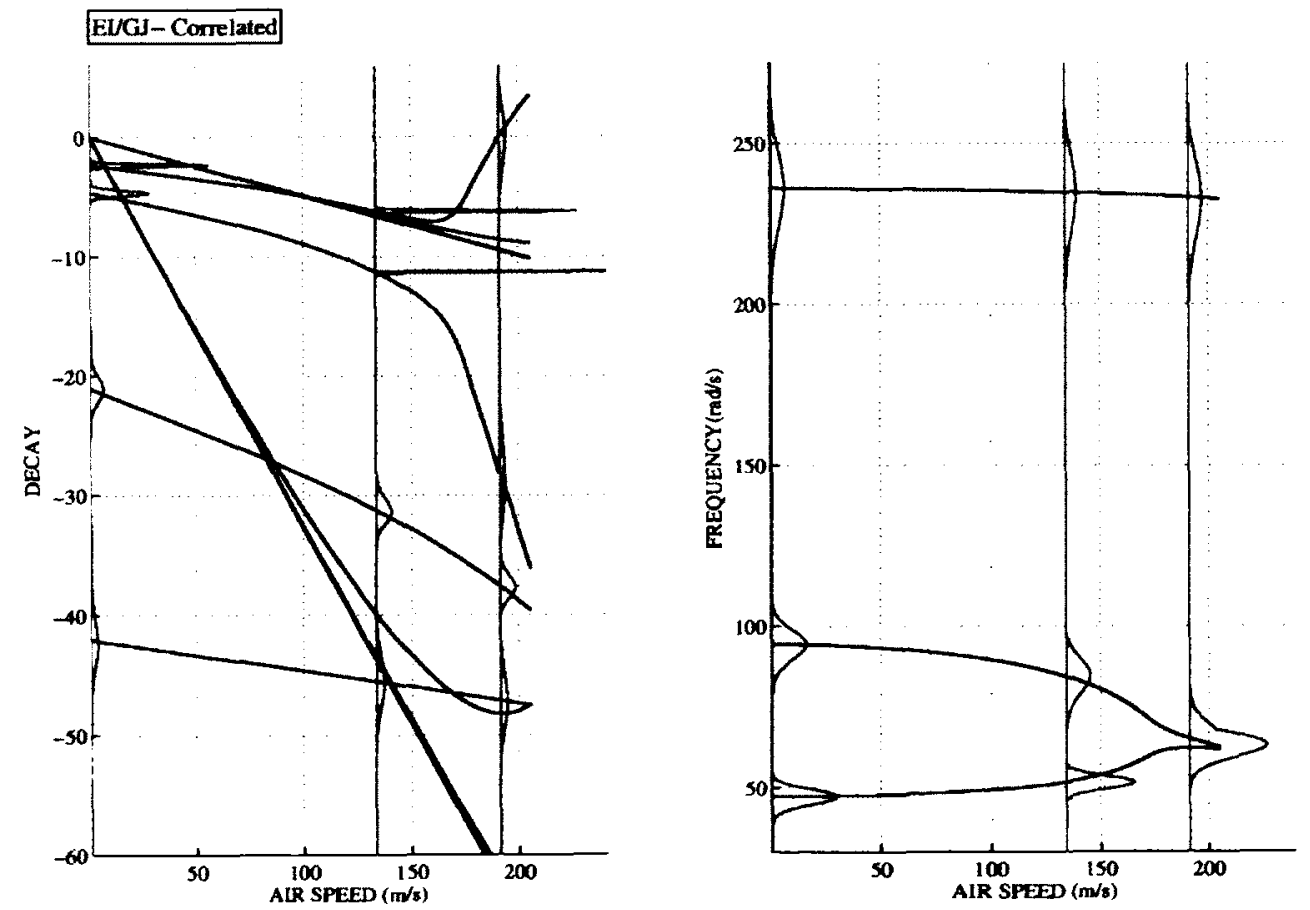

Figure 6.46: Magnified view of coalescing mode's modal parameter pdfs for uncertainty in correlated stiffness terms 

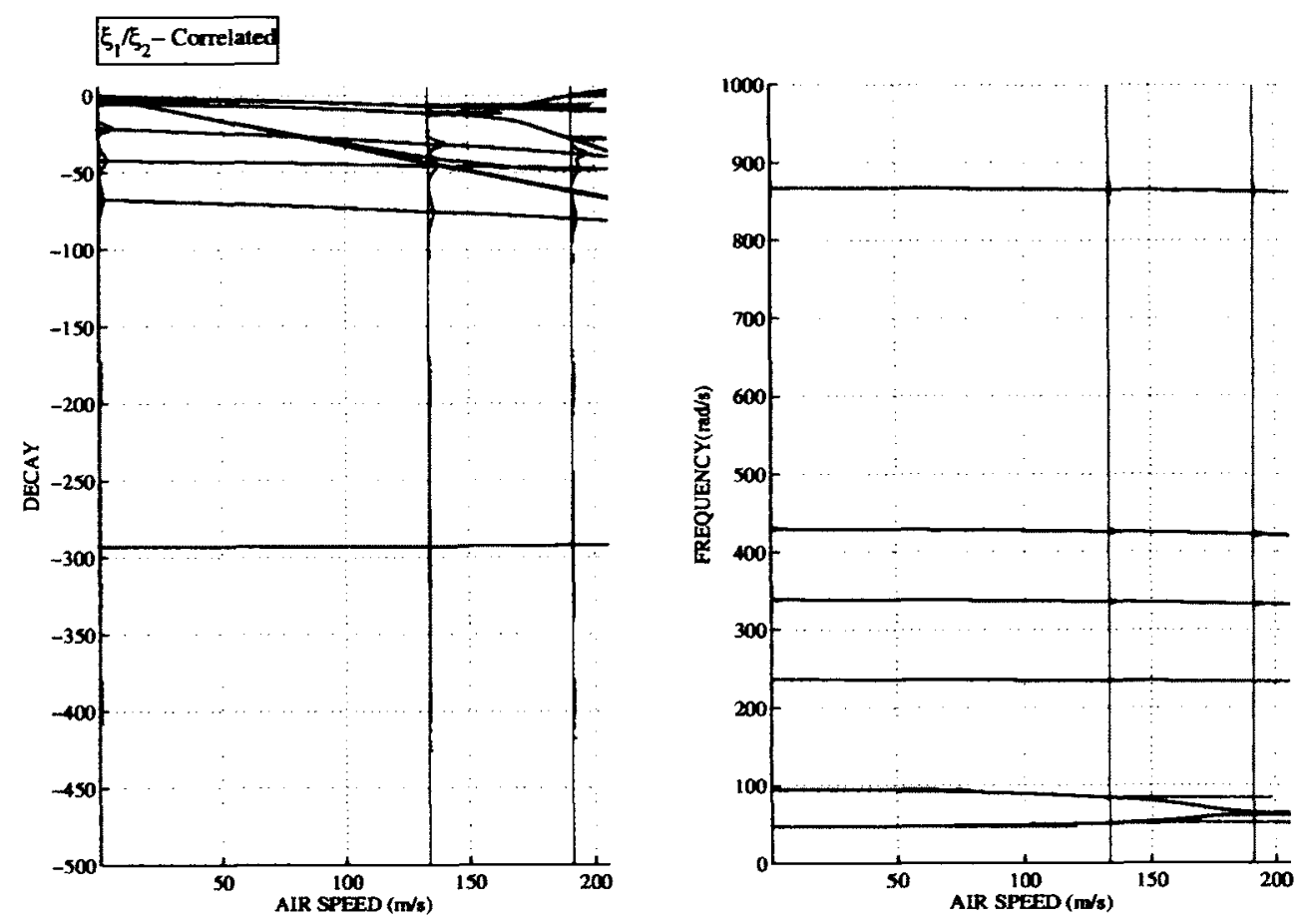

Figure 6.47: Modal parameter pdfs resulting from randomness in correlated damping terms with $10 \%$ COV, obtained at 3 test airspeeds: $\approx 0 \mathrm{~m} / \mathrm{s}, 135.0 \mathrm{~m} / \mathrm{s}, 190.2 \mathrm{~m} / \mathrm{s}$
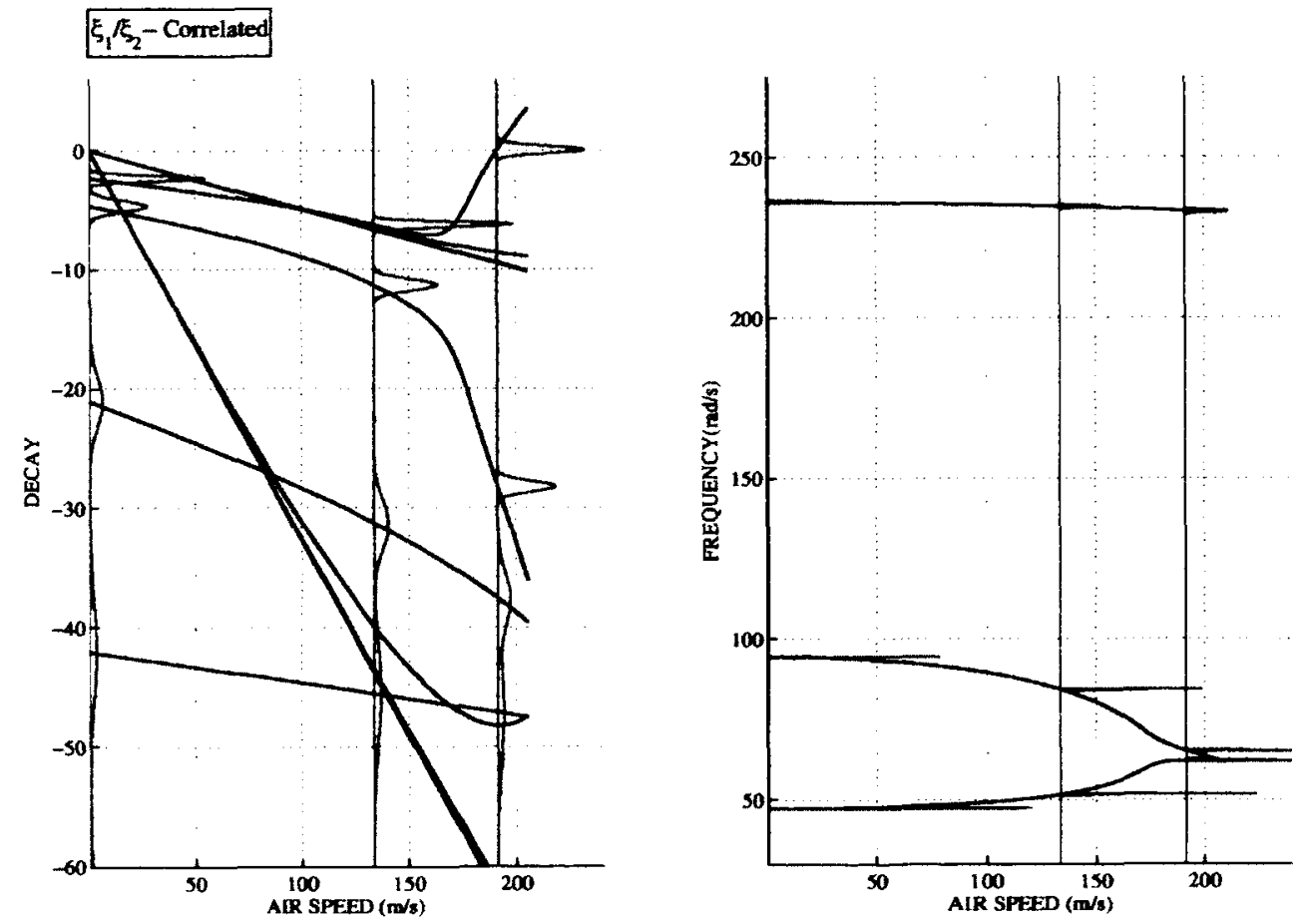

Figure 6.48: Magnified view of coalescing mode's modal parameter pdfs for uncertainty in correlated damping terms 
modal frequency parameters of the coalescing modes does not experience significant effects from correlation between the random parameters. However, correlation between the random parameters of course may lead to changes in correlations between the modal frequencies and the modal decays. For uncertainty in inertia and stiffness parameters, the correlation between modal frequencies lead to a reduced growth of variance in the modal decay of the flutter mode (as function of airspeed) in the vicinity of flutter. The correlation between random structural damping parameters effects the correlation between the modal decays. In this case, this particular correlation between modal decays of the coalescing modes leads to a consistent increase in the variance of the modal decays at every airspeed.

\subsubsection{Comparison of Quasisteady and Unsteady Results}

At this point, the probabilistic results of modal parameter transformations with quasisteady aerodynamics should be compared with those of the unsteady aerodynamics. Although, these effects were noted in the above results, the main observations should be stated. First, a more explosive flutter type in the unsteady loading case leads to a greater non-stationary effects of the modal parameters in region of strong frequency coalescence, as exhibited by the greater rate of change in the variance of the modal frequency pdfs. Furthermore, since the frequency coalescence is more substantial for cases with a more explosive flutter type, with unsteady aerodynamics, the modal frequencies of the coalescing modes experience significant statistical overlapping due to mode veering.

\subsection{Comparison of Cantilever and Rigid Wing Model Results}

Lastly, a comparison of the probabilistic modal parameter transformation results between the 3-D cantilever wing model and the rigid wing model, which are represented by Case 1 system parameters with unsteady aerodynamic forcing, is provided. Two main differences are noted. Mainly a more explosive flutter type is experienced by the rigid wing aeroelastic model and the cantilever model becomes unstable through the first mode, whereas the second mode becomes 
unstable for the rigid wing. Although the flutter type experienced by the rigid wing is more explosive, the modal frequency pdfs of the coalescing modes in the cantilever model experience significant overlaps due to a greater coalescence of frequencies. Due to a more explosive flutter type, greater non-stationary effects of the modal parameters are experienced for the rigid wing in the region of strong frequency coalescence, as exhibited by the greater rate of change in the variance of the modal frequency pdfs. The modal decay pdfs of the coalescing modes of the rigid wing show significant overlap. These effects do not exist in the cantilever model.

\subsection{CASE 2}

\subsubsection{Quasisteady Results}

The probabilistic results of modal parameter trends for the aeroelastic system described by Case 2 parameters are provided in this section. For this case it will become evident that there is significant overlap that occurs between the modal parameter pdfs, which presents some difficulties in interpreting the actual physics in the problem. The analysis begins with the presentation of results for individual parameter uncertainties in the aeroelastic system for quasisteady aerodynamics.

Figs. (6.49)-(6.52) show the effects of uncertainty in inertia parameters on the probabilistic results of modal parameters, along with a magnified view of the modes involved in the flutter mechanism. The frequency pdfs of the coalescing modes show non-stationary trends in the flutter region, reflecting a more explosive flutter type. The uncertainty of both inertia parameters produces a more significant effect on the uncertainty in the frequency of the second (unstable) mode, which exhibits predominantly bending motion. For mass parameter uncertainty the frequency pdf of the second mode indicates a non-Gaussian trend in the vicinity of flutter. The

modal decay and frequency pdfs of coalescing modes show small amounts of variance. The decays show non-Gaussian trends. The modal decay pdfs of the coalescing modes show significant non-stationarity, where the variance increases as function of airspeed, but only slightly. For uncertainty in the mass moment of inertia, the decays show strong non-Gaussian trends. The 

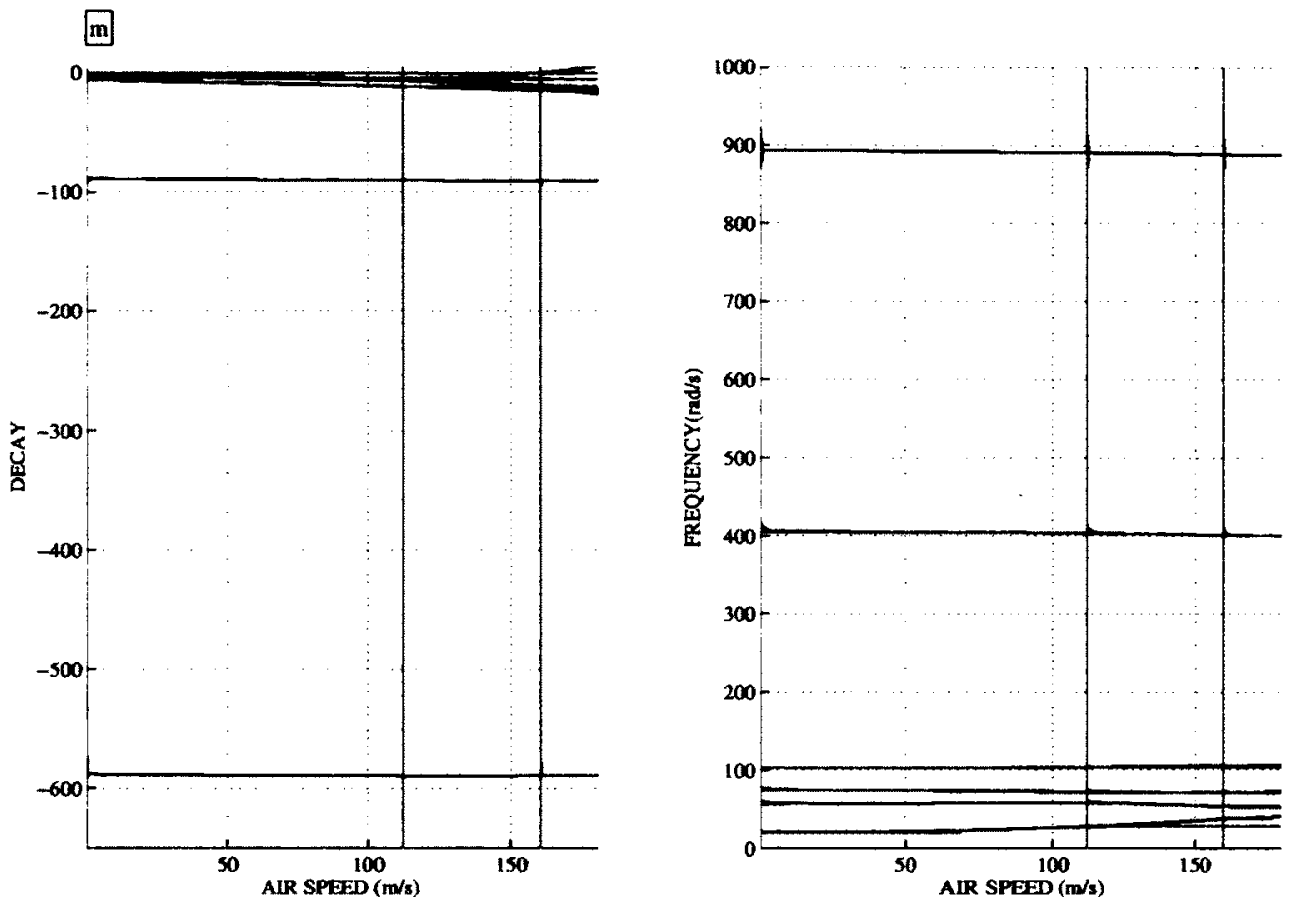

Figure 6.49: Modal parameter pdfs resulting from randomness in mass with $10 \% \mathrm{COV}$, obtained at 3 test airspeeds: $0 \mathrm{~m} / \mathrm{s}, 112 \mathrm{~m} / \mathrm{s}, 160.21 \mathrm{~m} / \mathrm{s}$
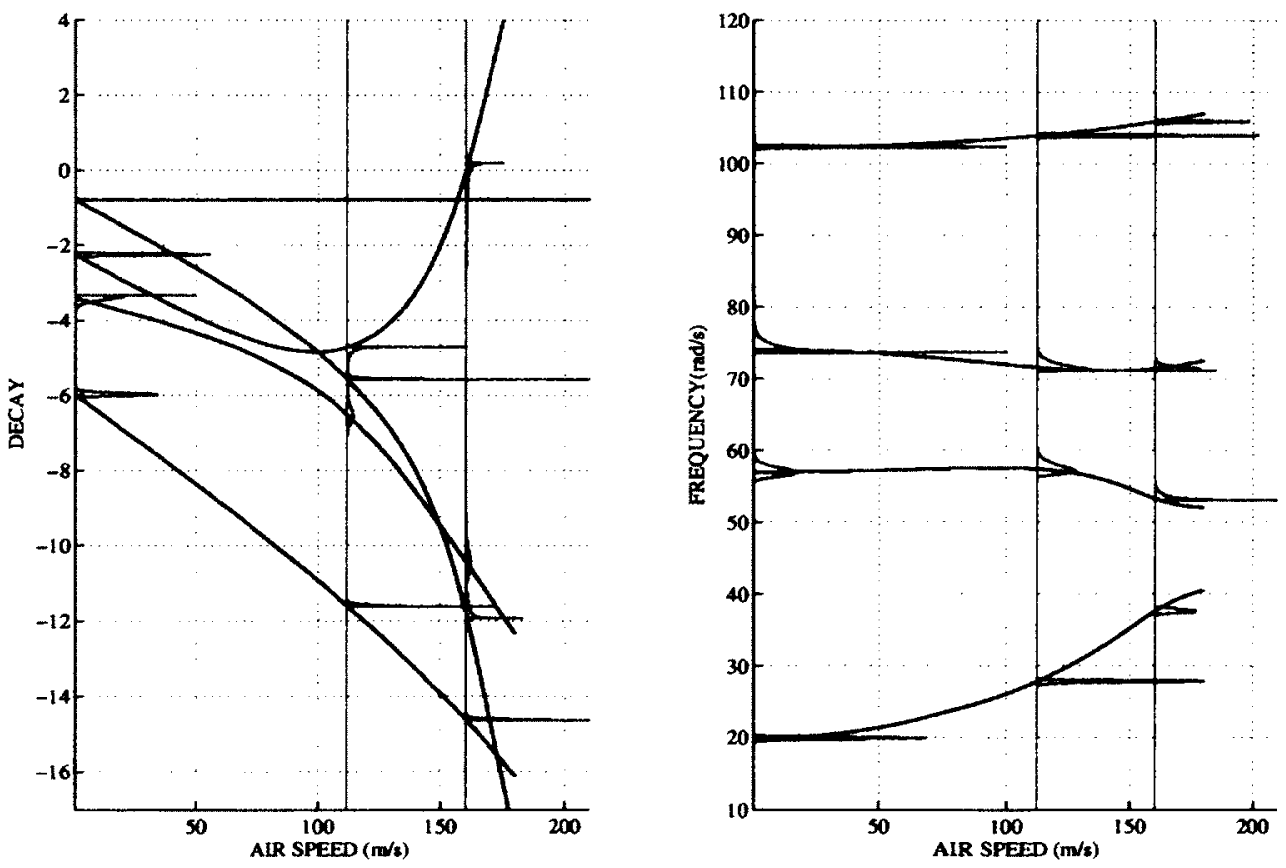

Figure 6.50: Magnified view of coalescing mode's modal parameter pdfs for uncertainty in mass 

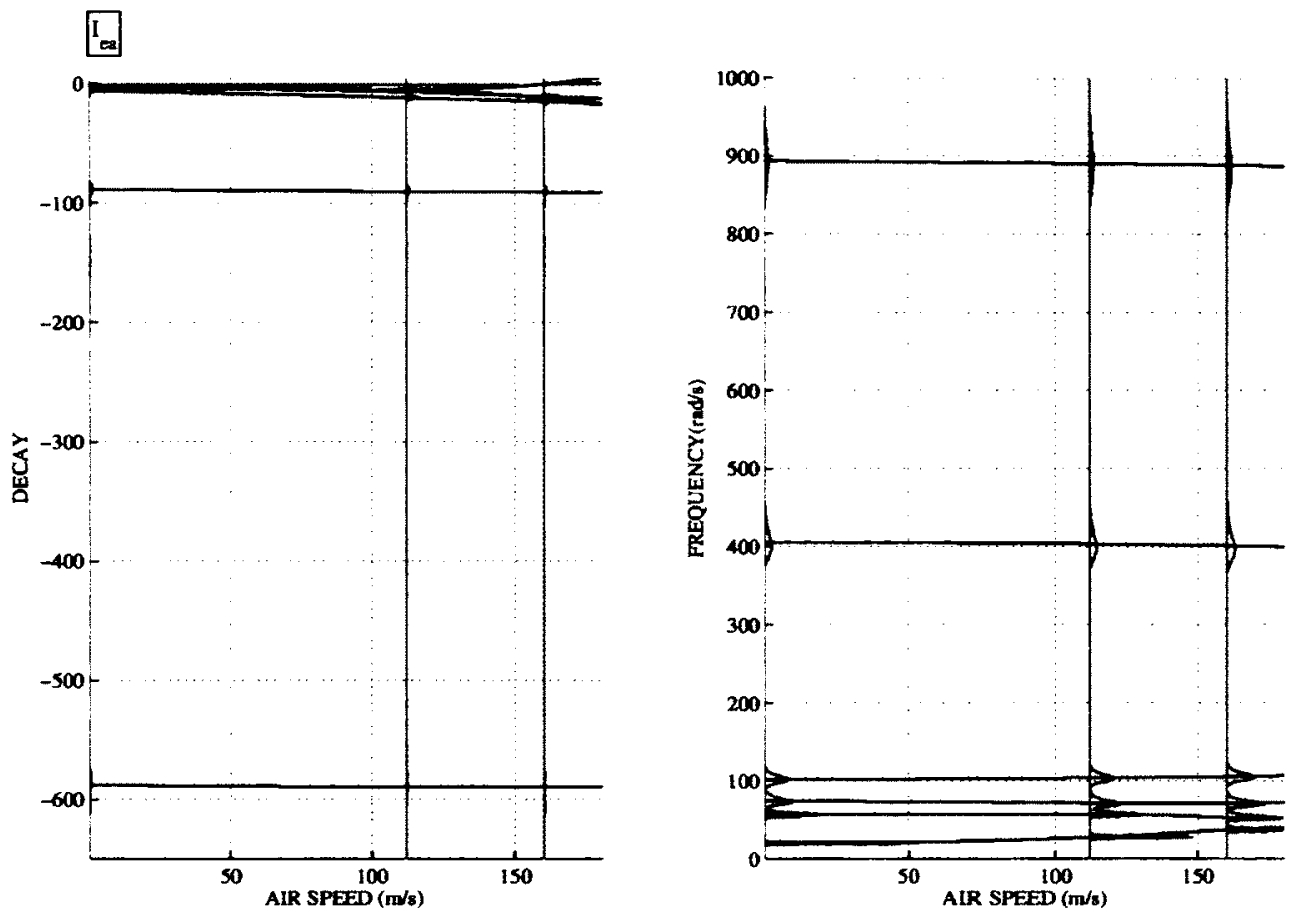

Figure 6.51: Modal parameter pdfs resulting from randomness in mass moment of inertia with $10 \% \mathrm{COV}$, obtained at 3 test airspeeds: $0 \mathrm{~m} / \mathrm{s}, 112 \mathrm{~m} / \mathrm{s}, 160.21 \mathrm{~m} / \mathrm{s}$
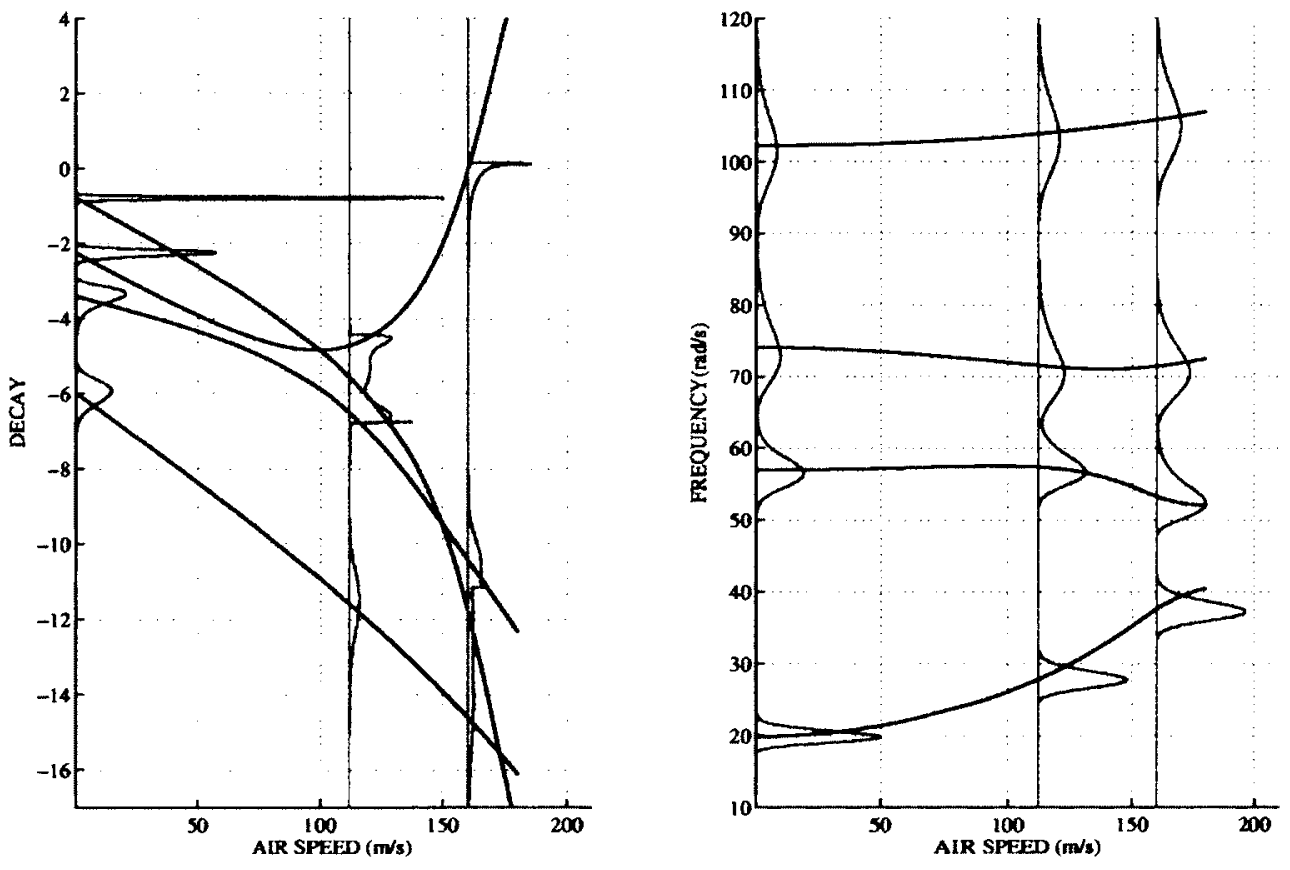

Figure 6.52: Magnified view of coalescing mode's modal parameter pdfs for uncertainty in mass moment of inertia 
modal decay pdfs of the coalescing modes show significant non-stationarity, where the variance experiences moderate increases as a function of airspeed. A significant statistical overlap of the modal decays is evident.

Similarly, the effects of stiffness uncertainty are illustrated in figs. (6.53)-(6.56), which illustrate the effects of bending stiffness and torsional stiffness uncertainty on the modal parameters of the system. Figs. (6.54) and (6.56) show a magnified view of the coalescing modes for each respective uncertainty case. The frequency pdfs of the coalescing modes show non-stationary trends in the flutter region, reflecting a more explosive flutter type. The uncertainty of both stiffness parameters produces a more significant effect on the uncertainty of frequency of the unstable mode, which exhibits predominantly bending motion. For bending stiffness uncertainty the frequency pdfs of both coalescing modes indicate an increase of variance in the flutter region. The modal decay pdfs of the coalescing modes show significant non-stationarity with large increase in variance as a function of airspeed. For uncertainty in the torsional stiffness the frequency pdfs of both coalescing modes indicate a decrease of variance in the flutter region. The modal decay pdfs of the coalescing modes show significant non-stationarity, where the variance experiences only a moderate increase as a function of airspeed, but shows less significant growth in the flutter region. For both cases, a significant statistical overlap of the modal decays is evident.

The probabilistic results of inertia and stiffness parameter variations are examined along with the flutter speed pdfs from fig. (5.19). Note that the effect of moderately growing variance in the modal decay of the flutter mode produced by inertia parameter uncertainty leads to only moderate variance in the flutter speed pdfs. A large growth of variance in modal decay of the flutter mode with bending stiffness uncertainty produces large variance in the flutter speed pdf. For case of torsional stiffness uncertainty, the low growth of variance in the modal decay of the flutter mode produces very low variance in the flutter speed pdf.

The uncertainty effects of the damping parameters are illustrated in figs. (6.57)-(6.60) for uncertainty in the first and second structural damping parameters, respectively. Figs. (6.58) and (6.60) provide the magnified view of the modal properties of the coalescing modes for each 

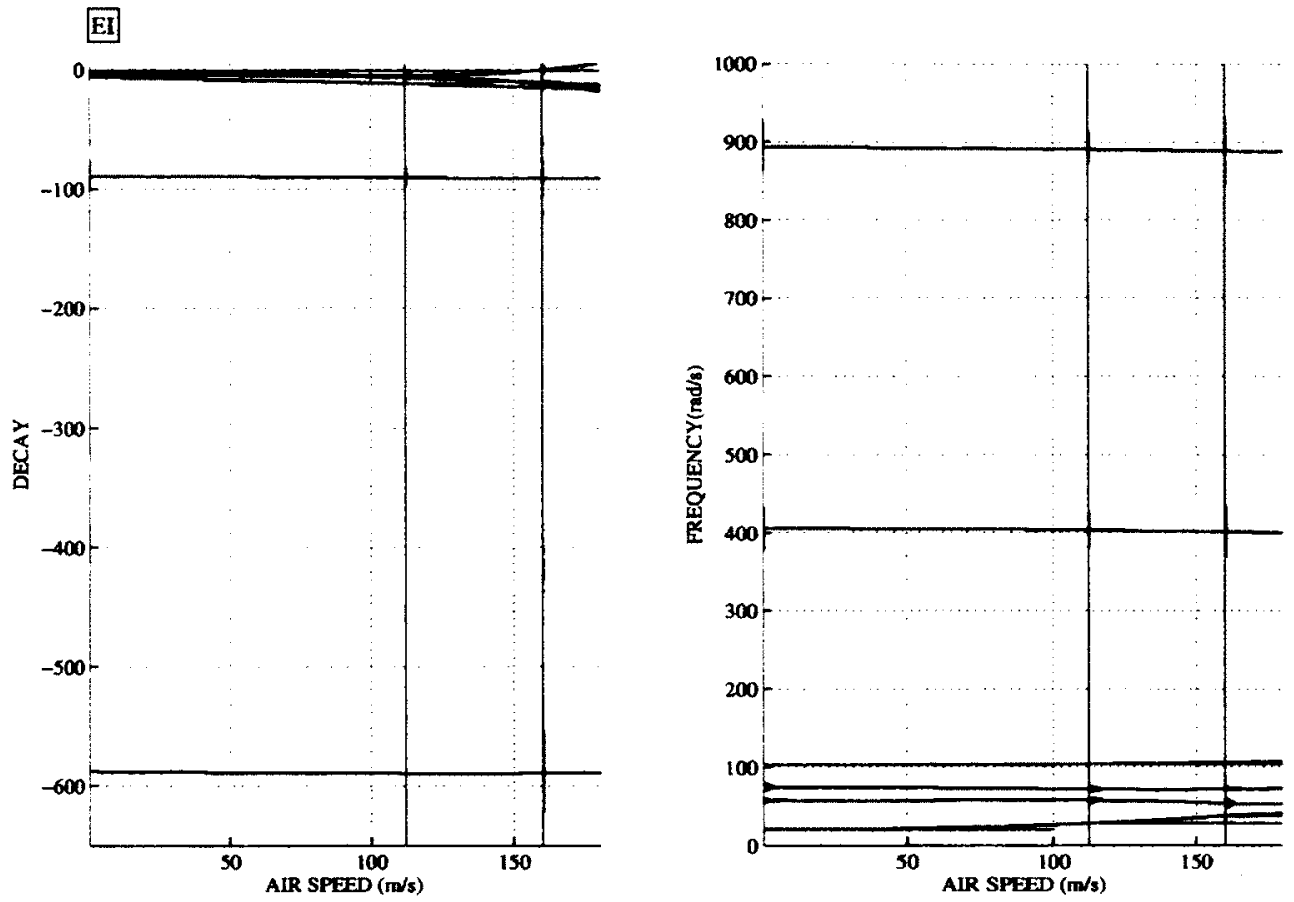

Figure 6.53: Modal parameter pdfs resulting from randomness in bending stiffness with $10 \%$ COV, obtained at 3 test airspeeds: $0 \mathrm{~m} / \mathrm{s}, 112 \mathrm{~m} / \mathrm{s}, 160.21 \mathrm{~m} / \mathrm{s}$
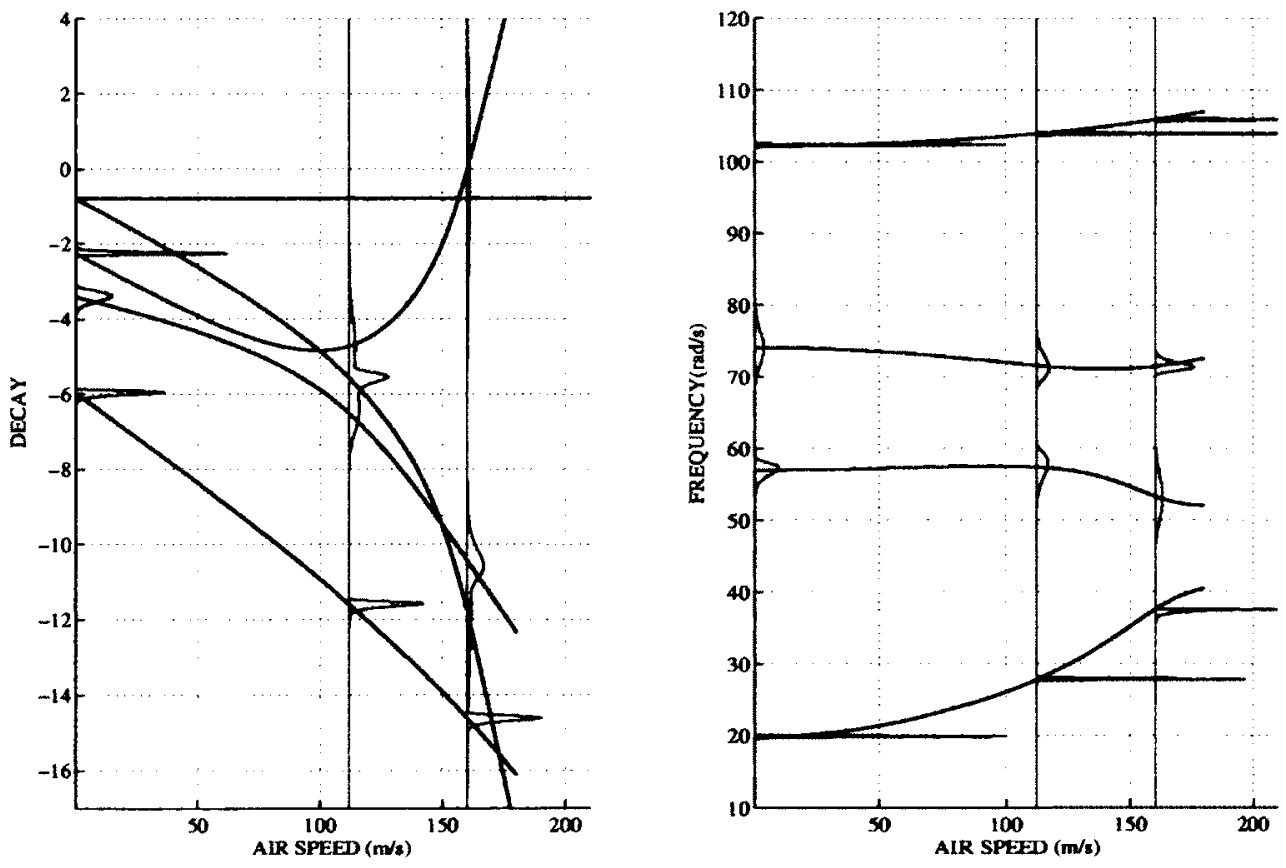

Figure 6.54: Magnified view of coalescing mode's modal parameter pdfs for uncertainty in bending stiffness 
(G)

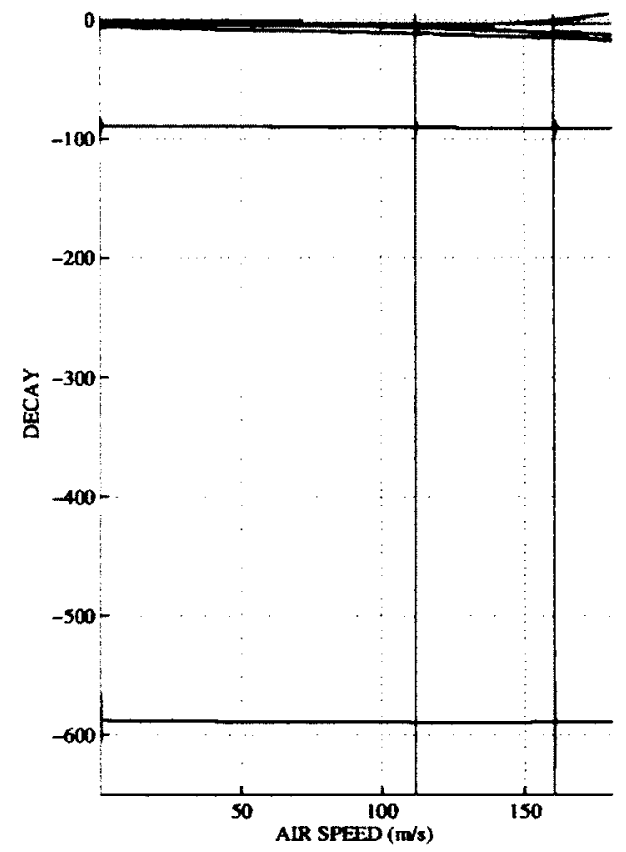

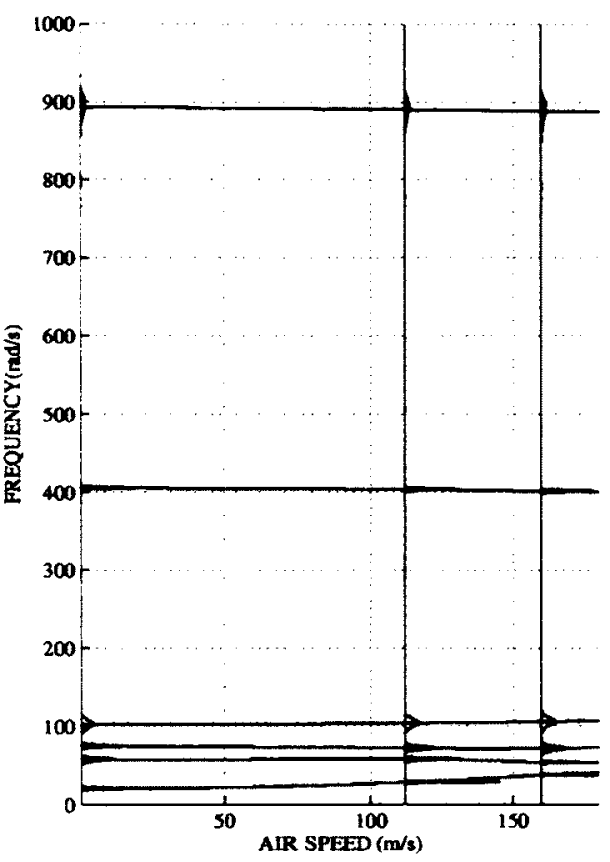

Figure 6.55: Modal parameter pdfs resulting from randomness in torsional stiffness with $10 \%$ COV, obtained at 3 test airspeeds: $0 \mathrm{~m} / \mathrm{s}, 112 \mathrm{~m} / \mathrm{s}, 160.21 \mathrm{~m} / \mathrm{s}$
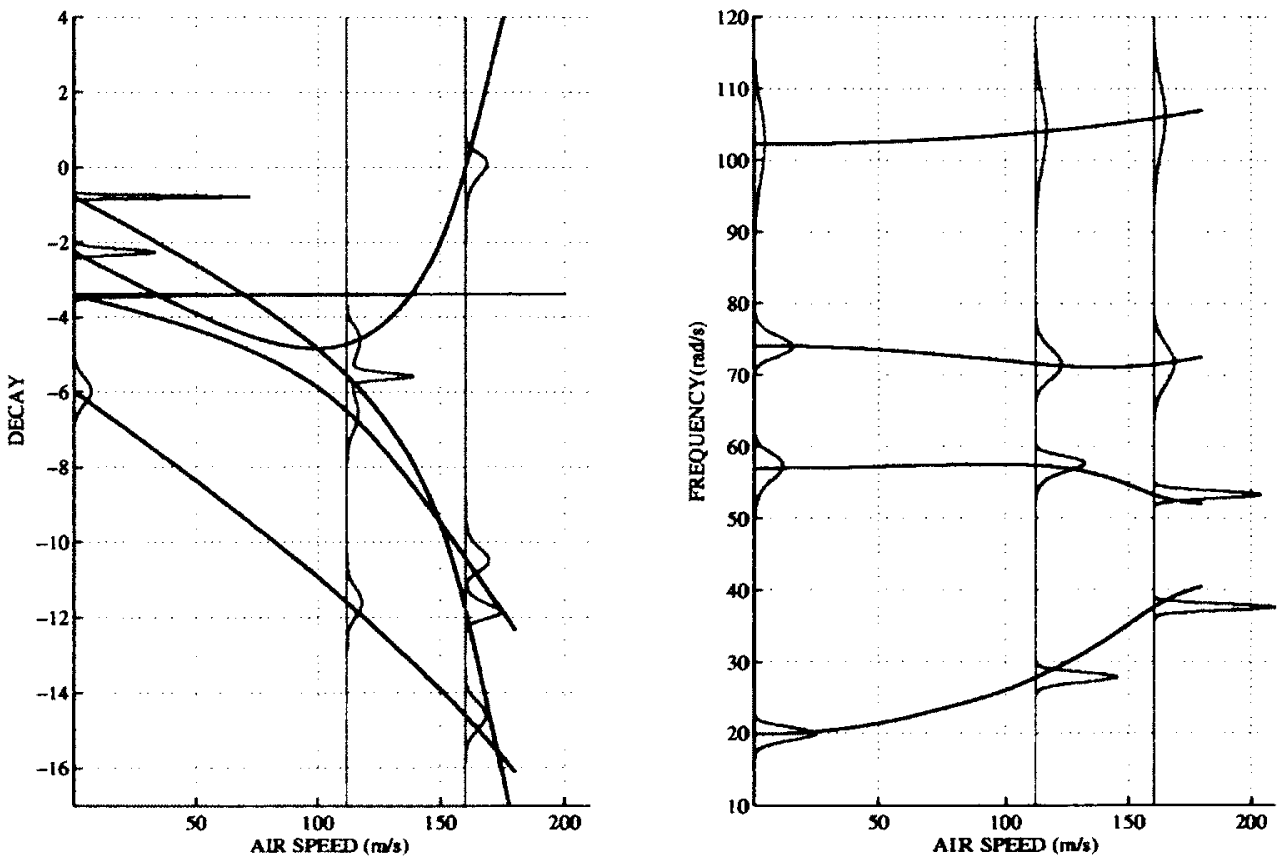

Figure 6.56: Magnified view of coalescing mode's modal parameter pdfs for uncertainty in torsional stiffness 

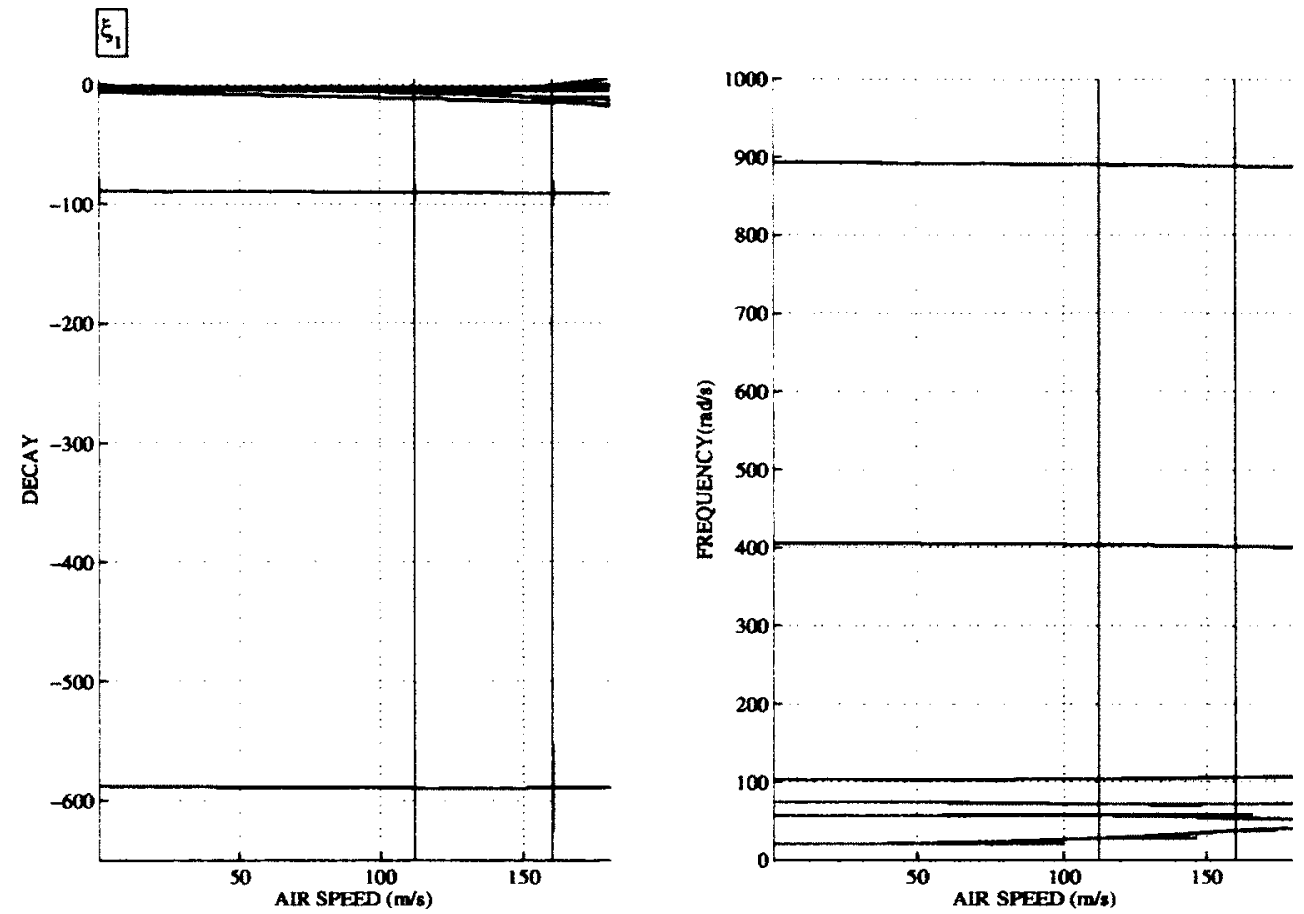

Figure 6.57: Modal parameter pdfs resulting from randomness in first structural damping ratio with $10 \% \mathrm{COV}$, obtained at 3 test airspeeds: $0 \mathrm{~m} / \mathrm{s}, 112 \mathrm{~m} / \mathrm{s}, 160.21 \mathrm{~m} / \mathrm{s}$
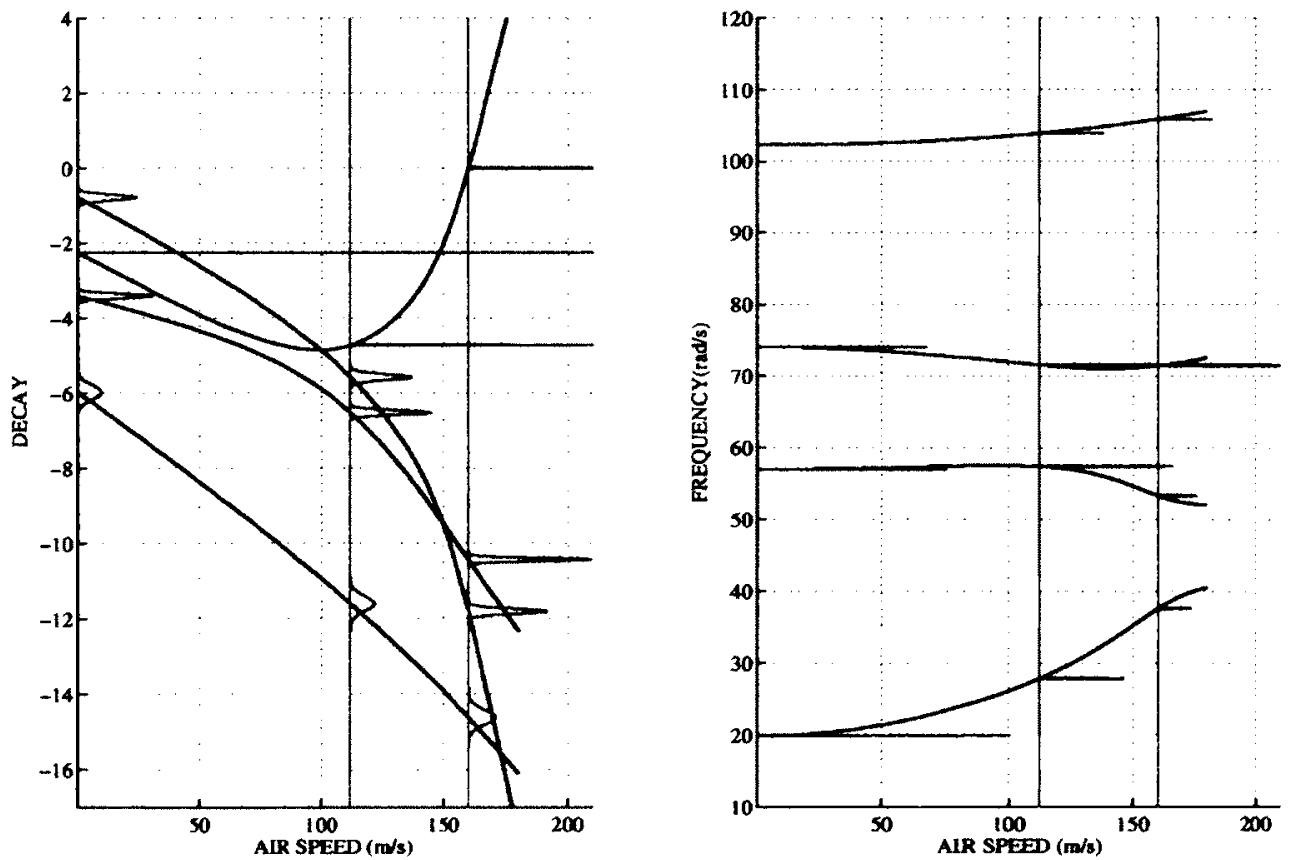

Figure 6.58: Magnified view of coalescing mode's modal parameter pdfs for uncertainty in first structural damping ratio 

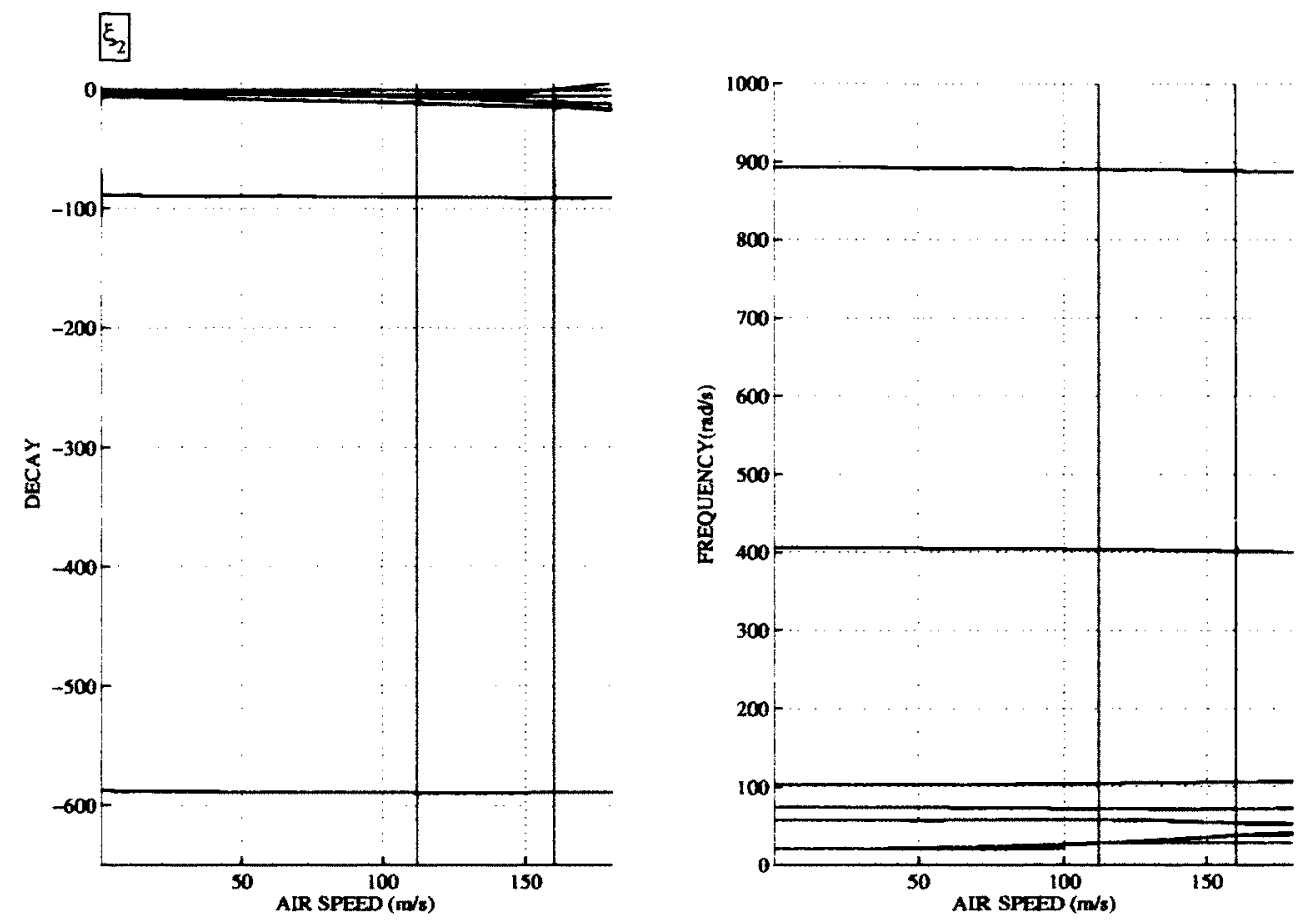

Figure 6.59: Modal parameter pdfs resulting from randomness in second structural damping ratio with $10 \%$ COV, obtained at 3 test airspeeds: $0 \mathrm{~m} / \mathrm{s}, 112 \mathrm{~m} / \mathrm{s}, 160.21 \mathrm{~m} / \mathrm{s}$
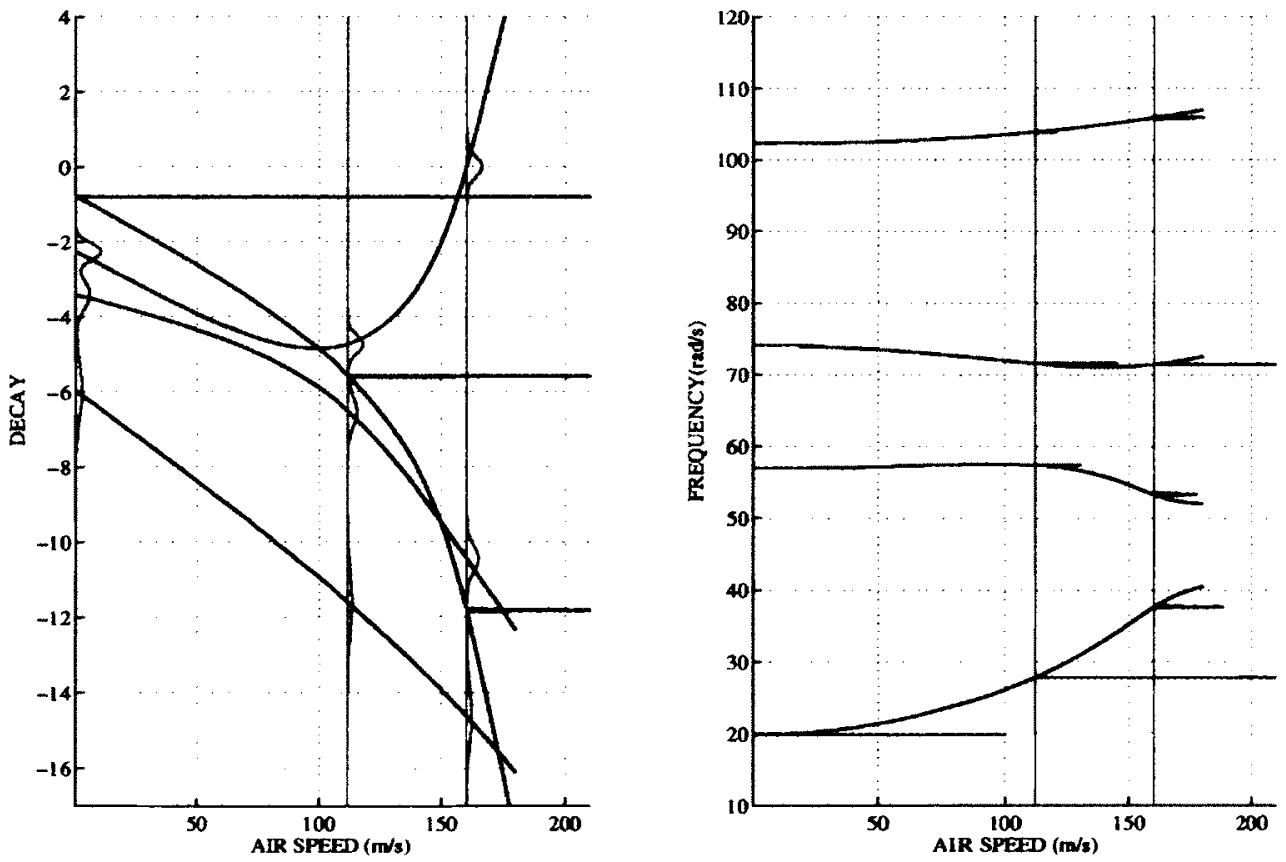

Figure 6.60: Magnified view of coalescing mode's modal parameter pdfs for uncertainty in second structural damping ratio 
respective case. The resulting observations are similar to Case 1 with quasisteady aerodynamics.

\section{Multiple Parameter Uncertainty}

The effects of uncertainty in multiple parameters are very similar to the previous case. Figs. (6.61)-(6.66) show the resulting transformations of the modal parameter pdfs as a function of airspeed for independent inertia, stiffness and damping parameters. The results indicate that uncertainty of multiple uncorrelated parameters increases the uncertainty of the modal parameter pdfs. The resulting high variance of modal decay of the flutter mode produces significant variance in the flutter speed (evident in fig. (5.19)). There are however some differences that are noted in comparison to the previous case study. Mainly, the uncertainty in inertia causes the flutter mode decay to experience non-Gaussian effects in vicinity of flutter, and additionally, both inertia and stiffness parameter uncertainties lead to significant statistical overlap of the modal decay pdfs.

The effects of full correlation between the random parameters are examined in Figs. (6.67)(6.72) illustrate the probabilistic modal parameter results for fully correlated inertia, stiffness and damping parameters. The uncertainty of the modal frequency parameters of the coalescing modes is not significantly influenced by the correlation between the random parameters. However, correlation between the random parameters may lead to changes in correlations between the modal frequencies and the modal decays. For uncertainty in inertia and stiffness parameters, the correlation between modal frequencies leads to a reduced growth of variance in the modal decay of the flutter mode (as function of airspeed) in the vicinity of flutter. The reduction of variance in the flutter mode decay implies a lower uncertainty in the flutter speed pdf due to structural parameter correlation. In case of random inertia effects, the flutter mode decay no longer shows non-Gaussian features in the flutter region. The correlation between random structural damping affects the correlation between the modal decays. In such a case, this particular correlation between modal decays of the coalescing modes leads to a consistent decrease in the variance of the modal decays at every airspeed, leading to a reduction of variance in the flutter speed pdf. 

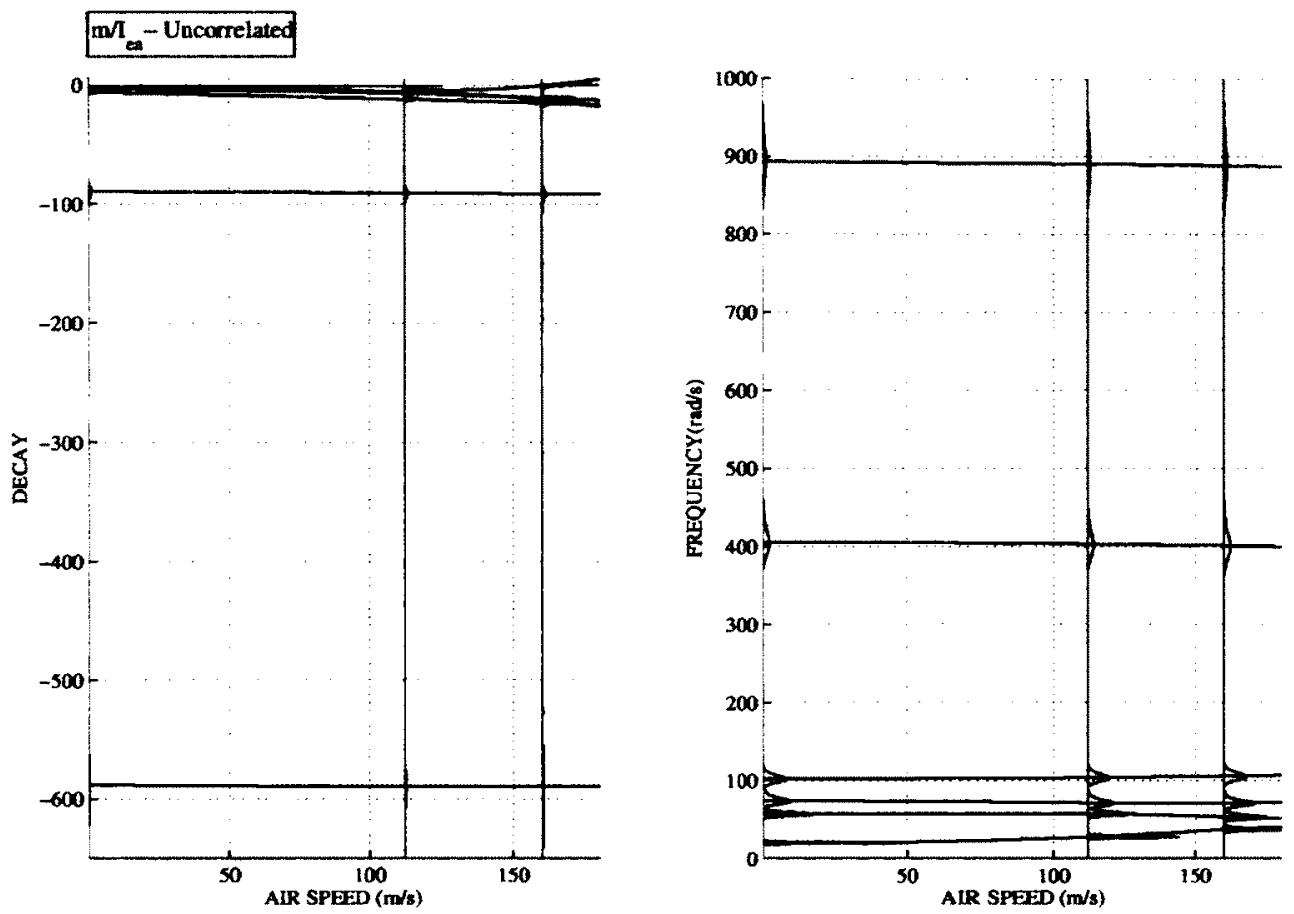

Figure 6.61: Modal parameter pdfs resulting from randomness in uncorrelated inertia terms with $10 \%$ COV, obtained at 3 test airspeeds: $0 \mathrm{~m} / \mathrm{s}, 112 \mathrm{~m} / \mathrm{s}, 160.21 \mathrm{~m} / \mathrm{s}$
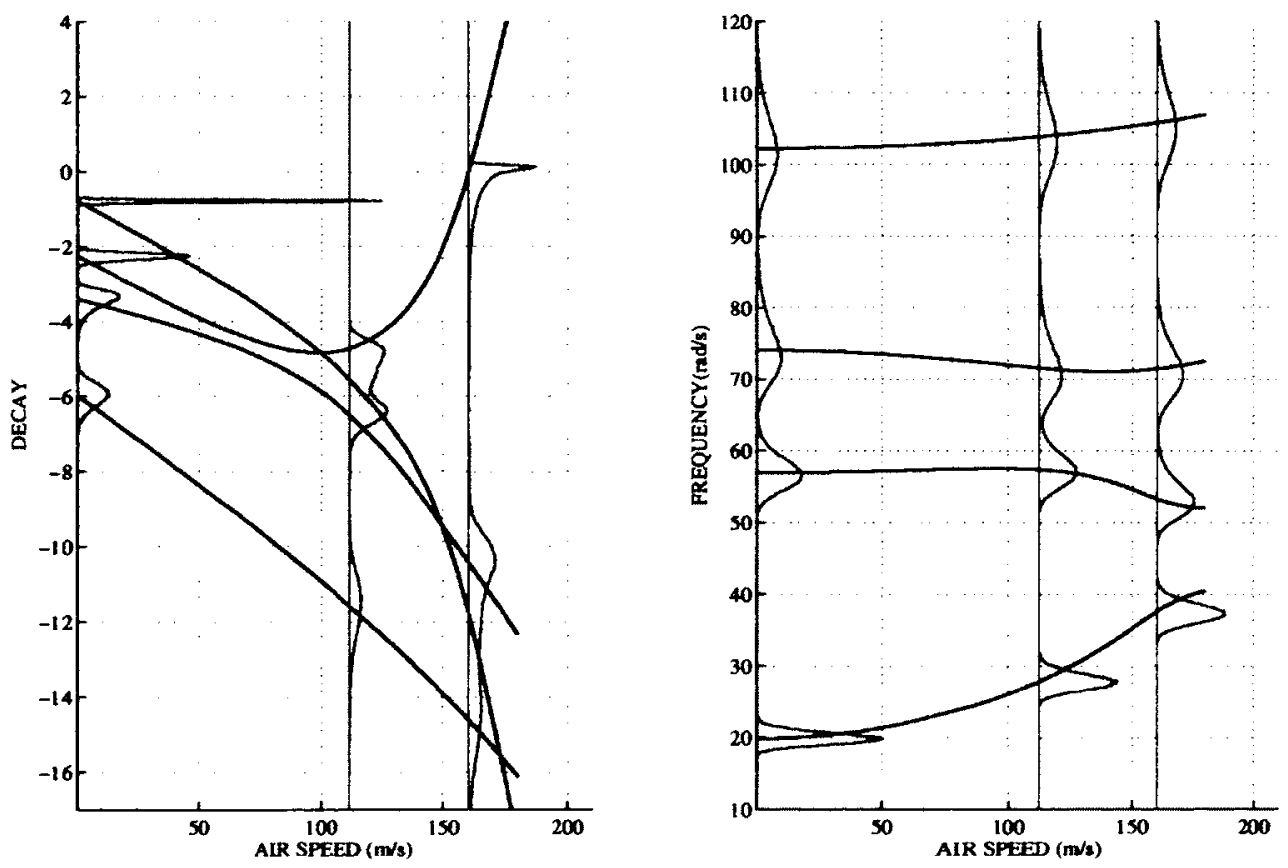

Figure 6.62: Magnified view of coalescing mode's modal parameter pdfs for uncertainty in uncorrelated inertia terms 

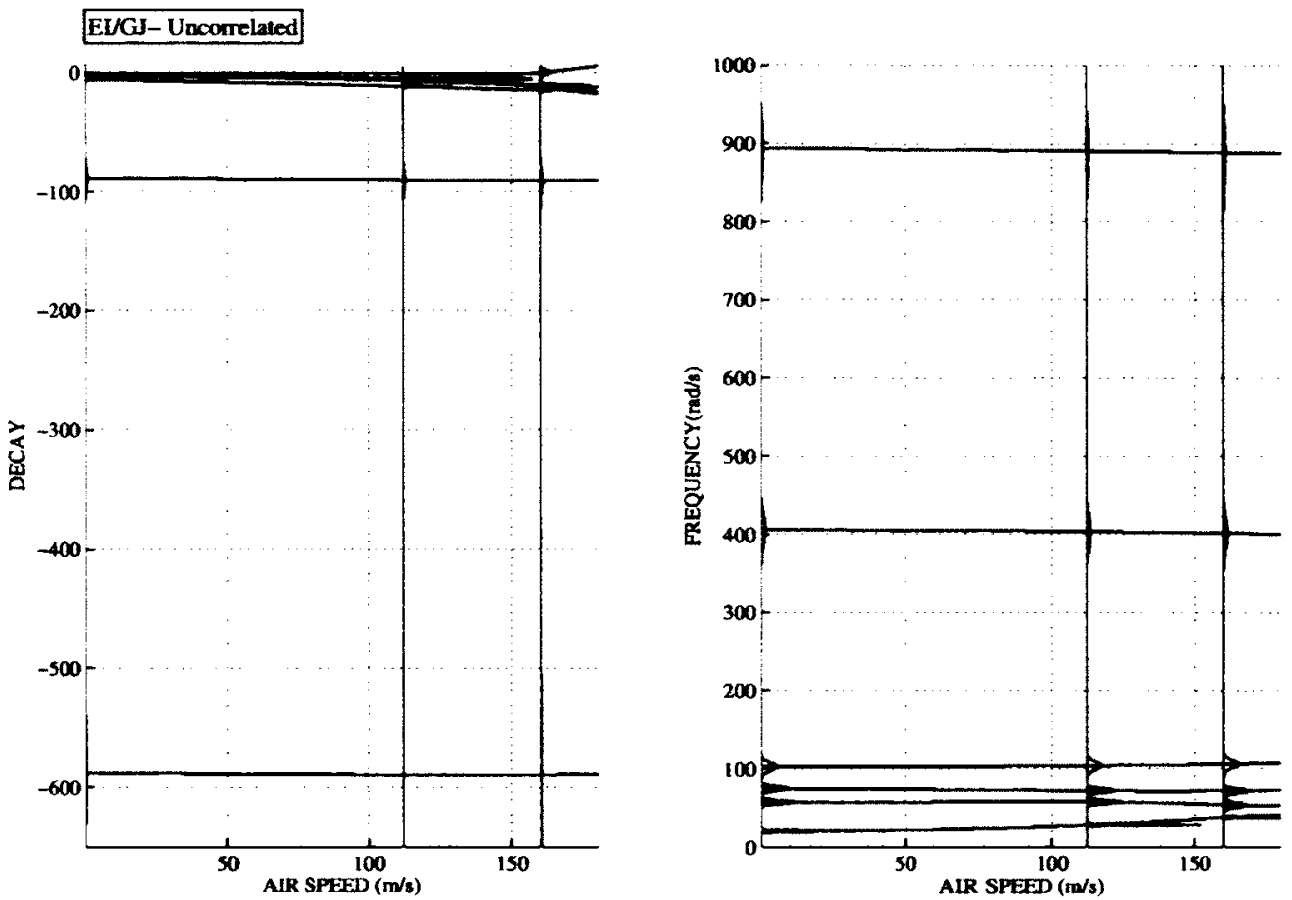

Figure 6.63: Modal parameter pdfs resulting from randomness in uncorrelated stiffness terms with $10 \%$ COV, obtained at 3 test airspeeds: $0 \mathrm{~m} / \mathrm{s}, 112 \mathrm{~m} / \mathrm{s}, 160.21 \mathrm{~m} / \mathrm{s}$
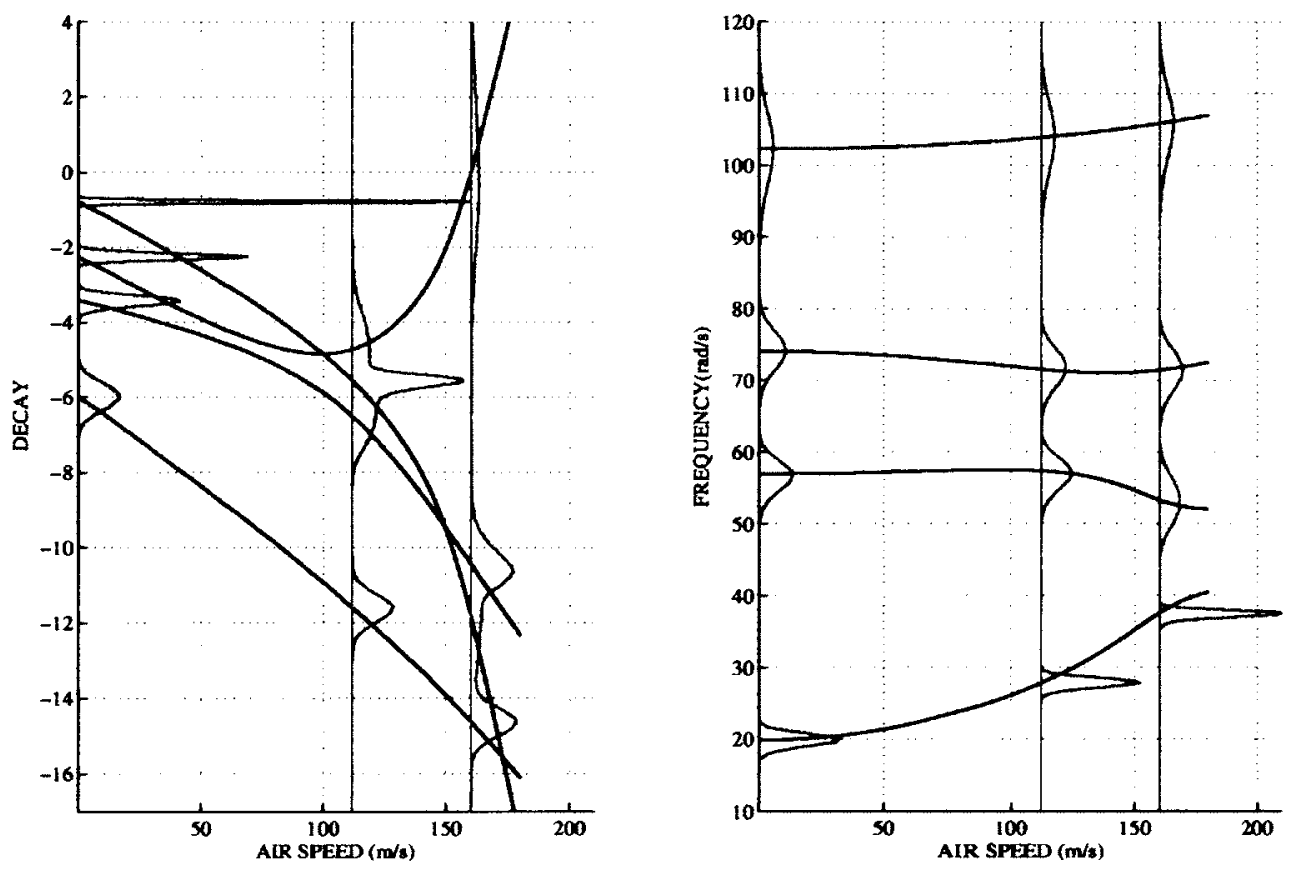

Figure 6.64: Magnified view of coalescing mode's modal parameter pdfs for uncertainty in uncorrelated stiffness terms 

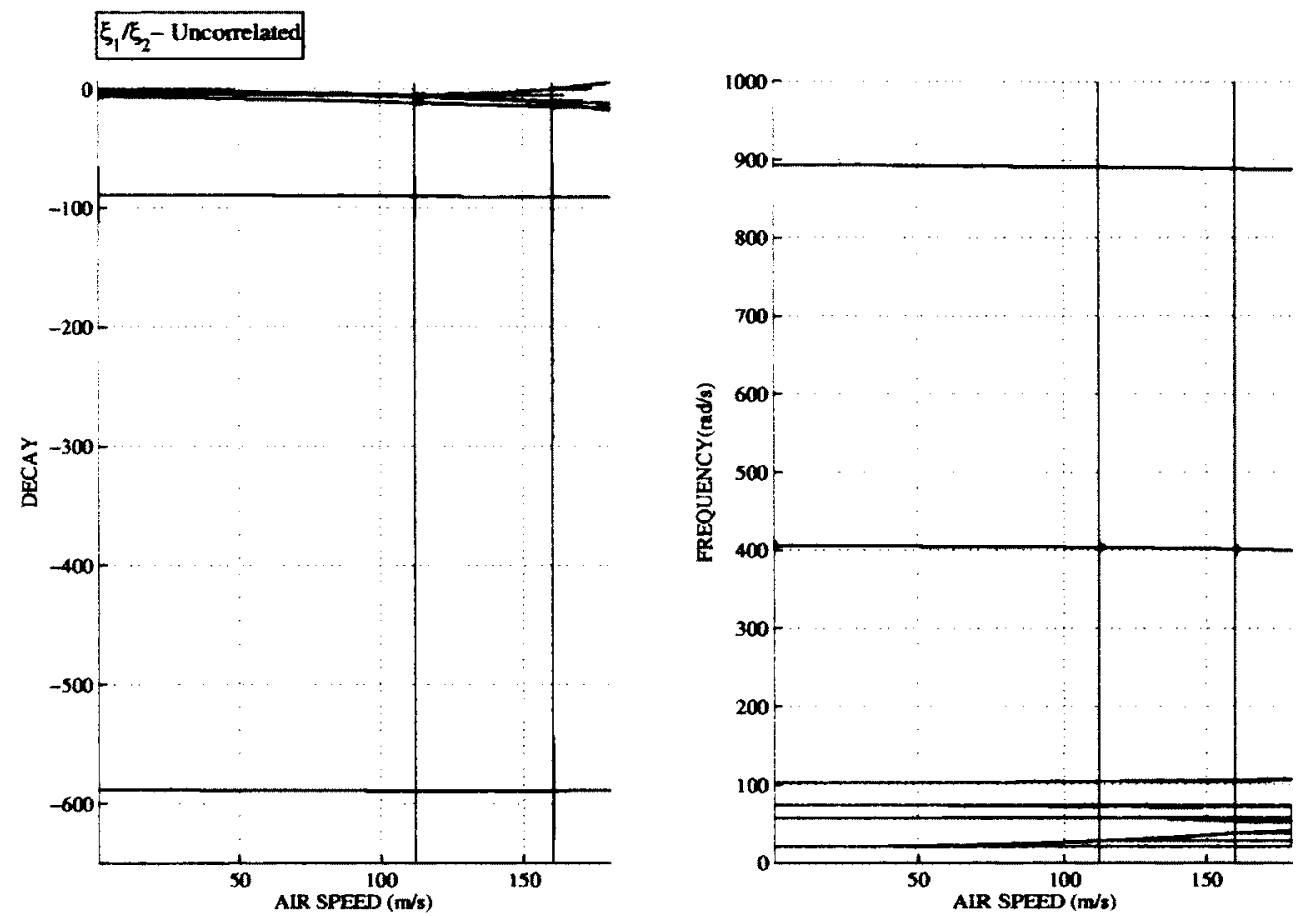

Figure 6.65: Modal parameter pdfs resulting from randomness in uncorrelated damping terms with $10 \%$ COV, obtained at 3 test airspeeds: $0 \mathrm{~m} / \mathrm{s}, 112 \mathrm{~m} / \mathrm{s}, 160.21 \mathrm{~m} / \mathrm{s}$
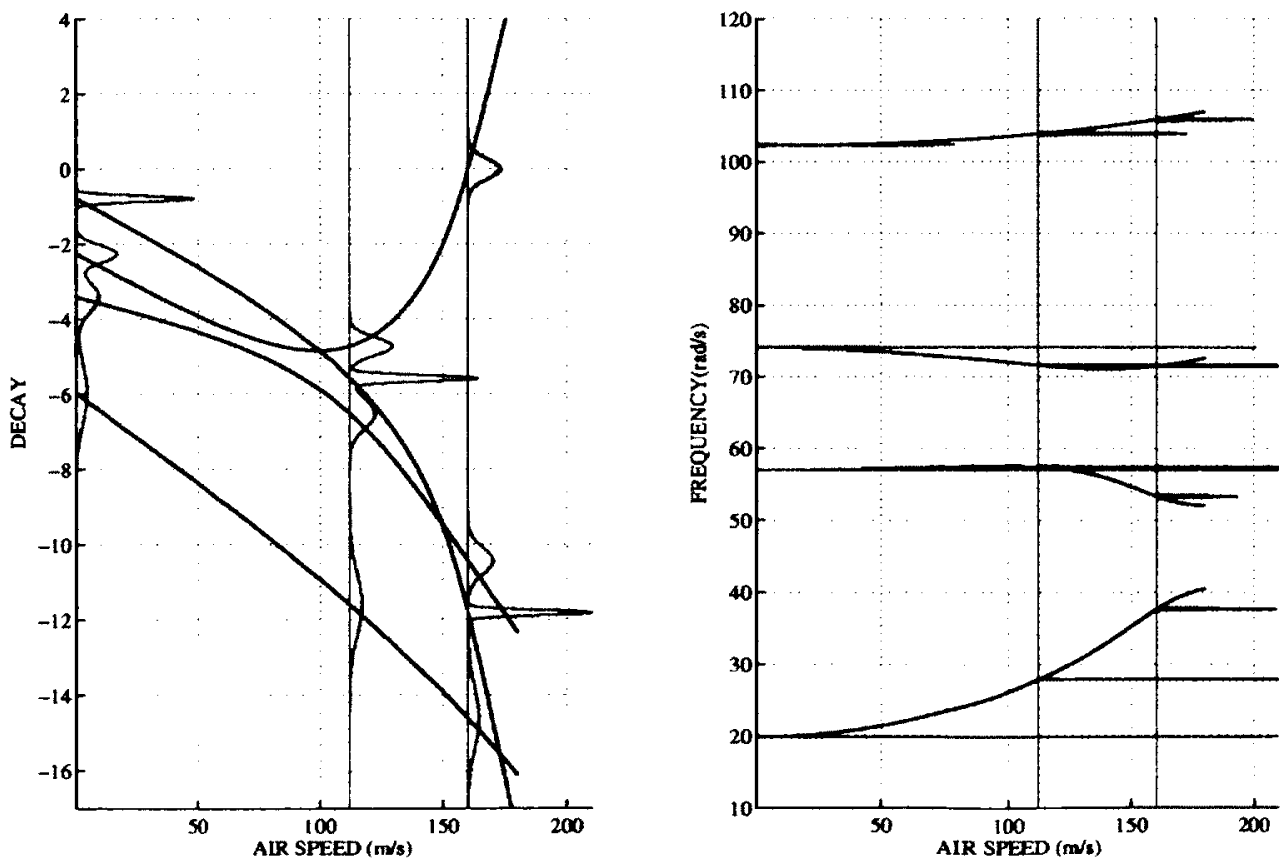

Figure 6.66: Magnified view of coalescing mode's modal parameter pdfs for uncertainty in uncorrelated damping terms 

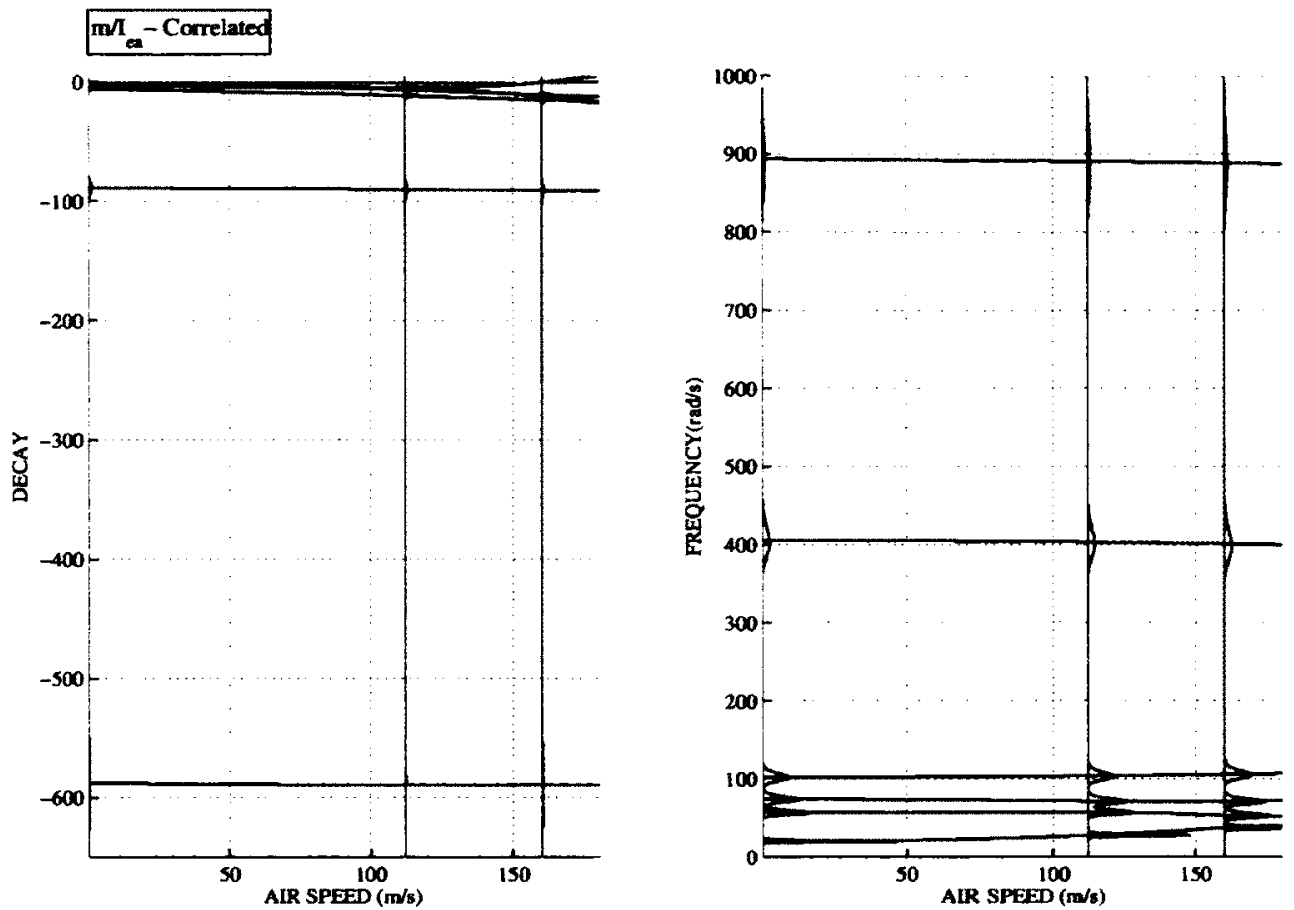

Figure 6.67: Modal parameter pdfs resulting from randomness in correlated inertia terms with $10 \%$ COV, obtained at 3 test airspeeds: $0 \mathrm{~m} / \mathrm{s}, 112 \mathrm{~m} / \mathrm{s}, 160.21 \mathrm{~m} / \mathrm{s}$
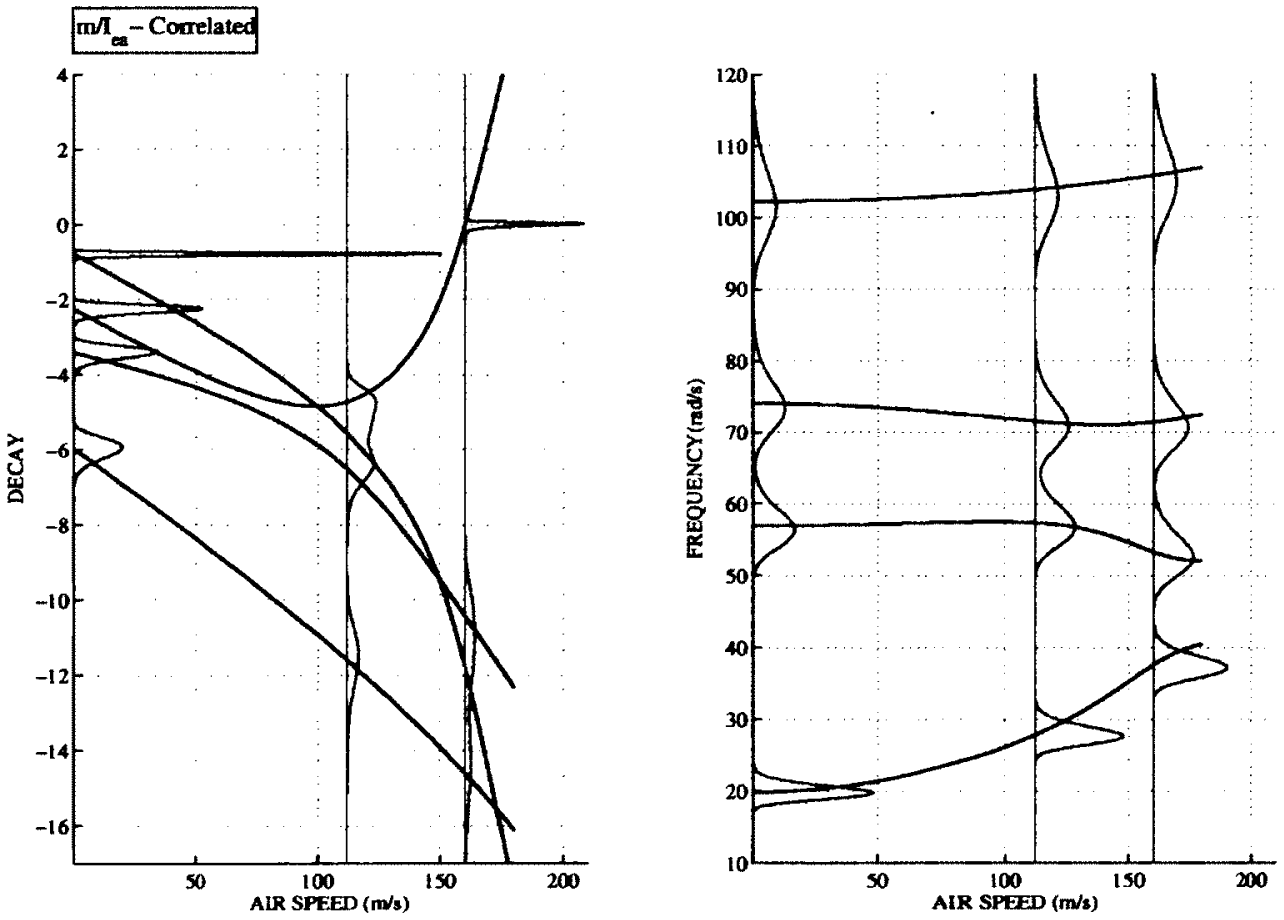

Figure 6.68: Magnified view of coalescing mode's modal parameter pdfs for uncertainty in correlated inertia terms 

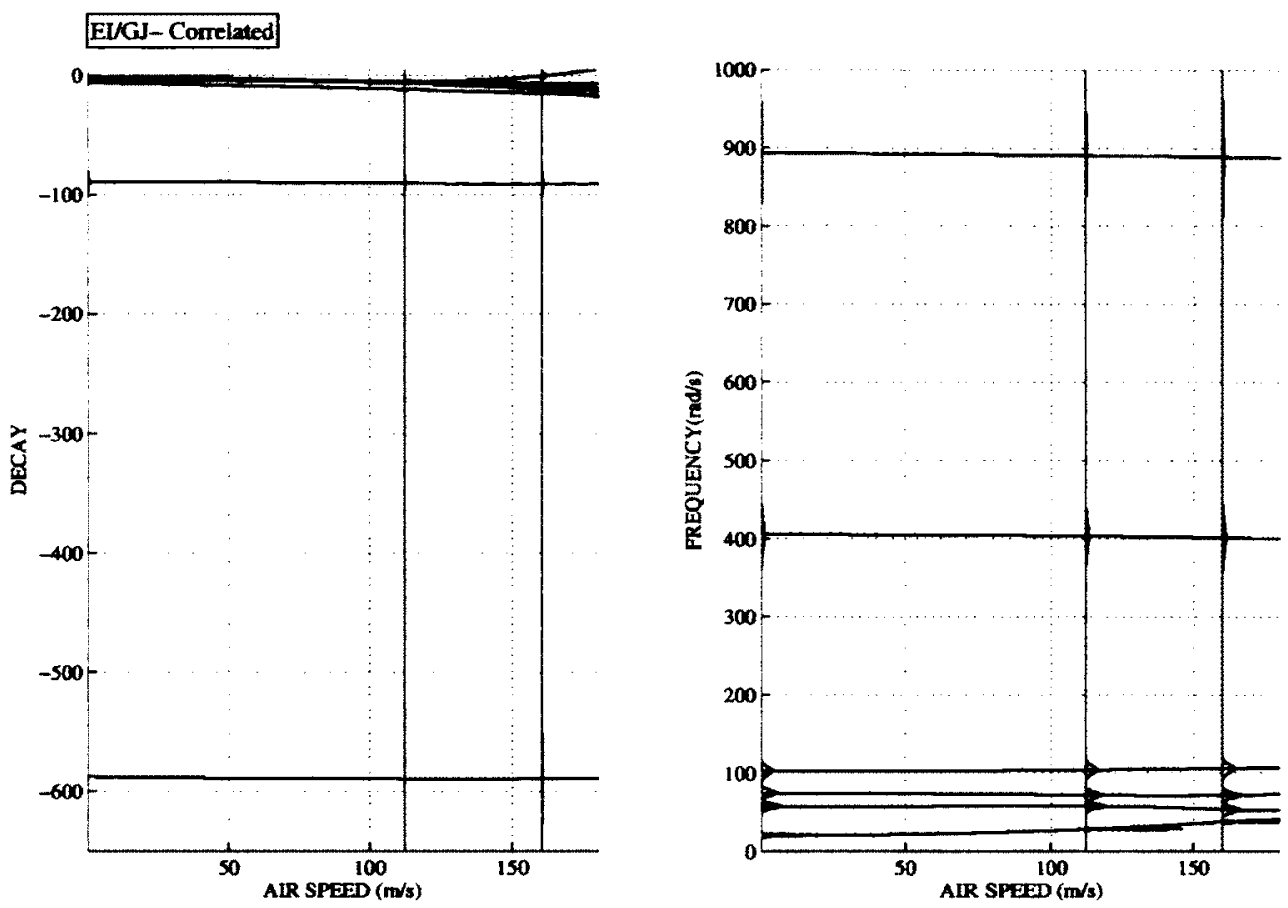

Figure 6.69: Modal parameter pdfs resulting from randomness in correlated stiffness terms with $10 \%$ COV, obtained at 3 test airspeeds: $0 \mathrm{~m} / \mathrm{s}, 112 \mathrm{~m} / \mathrm{s}, 160.21 \mathrm{~m} / \mathrm{s}$
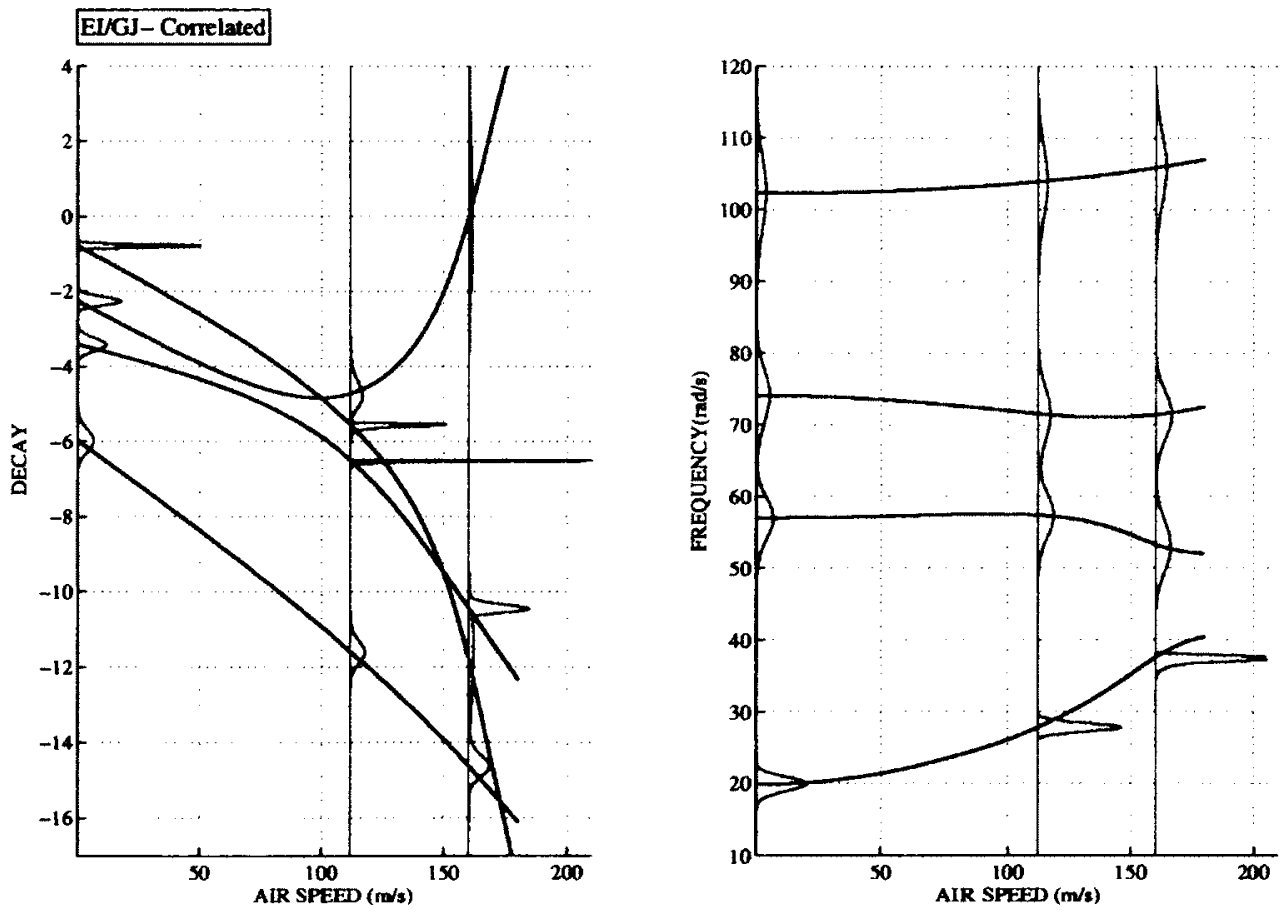

Figure 6.70: Magnified view of coalescing mode's modal parameter pdfs for uncertainty in correlated stiffness terms 

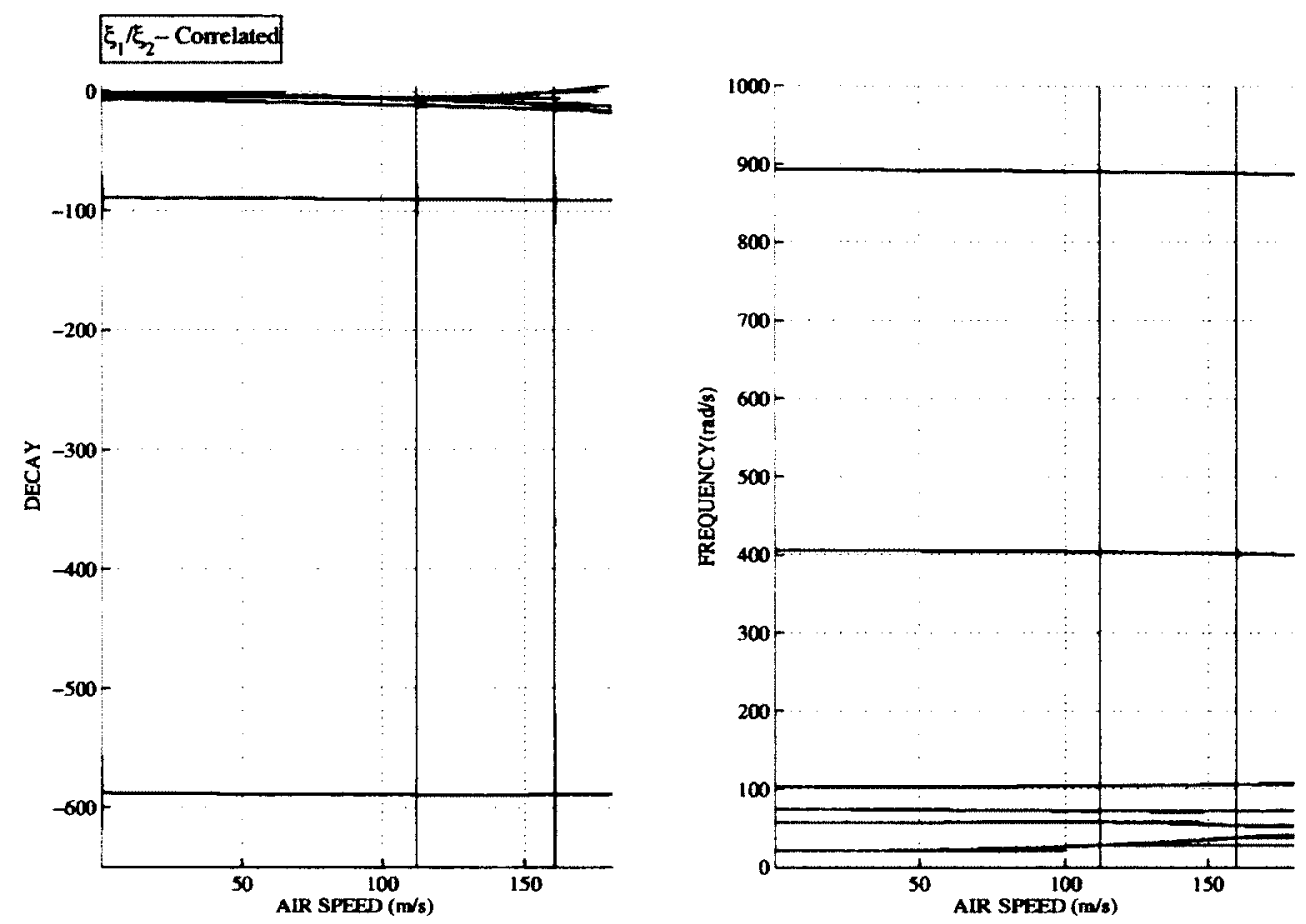

Figure 6.71: Modal parameter pdfs resulting from randomness in correlated damping terms with $10 \% \operatorname{COV}$, obtained at 3 test airspeeds: $0 \mathrm{~m} / \mathrm{s}, 112 \mathrm{~m} / \mathrm{s}, 160.21 \mathrm{~m} / \mathrm{s}$
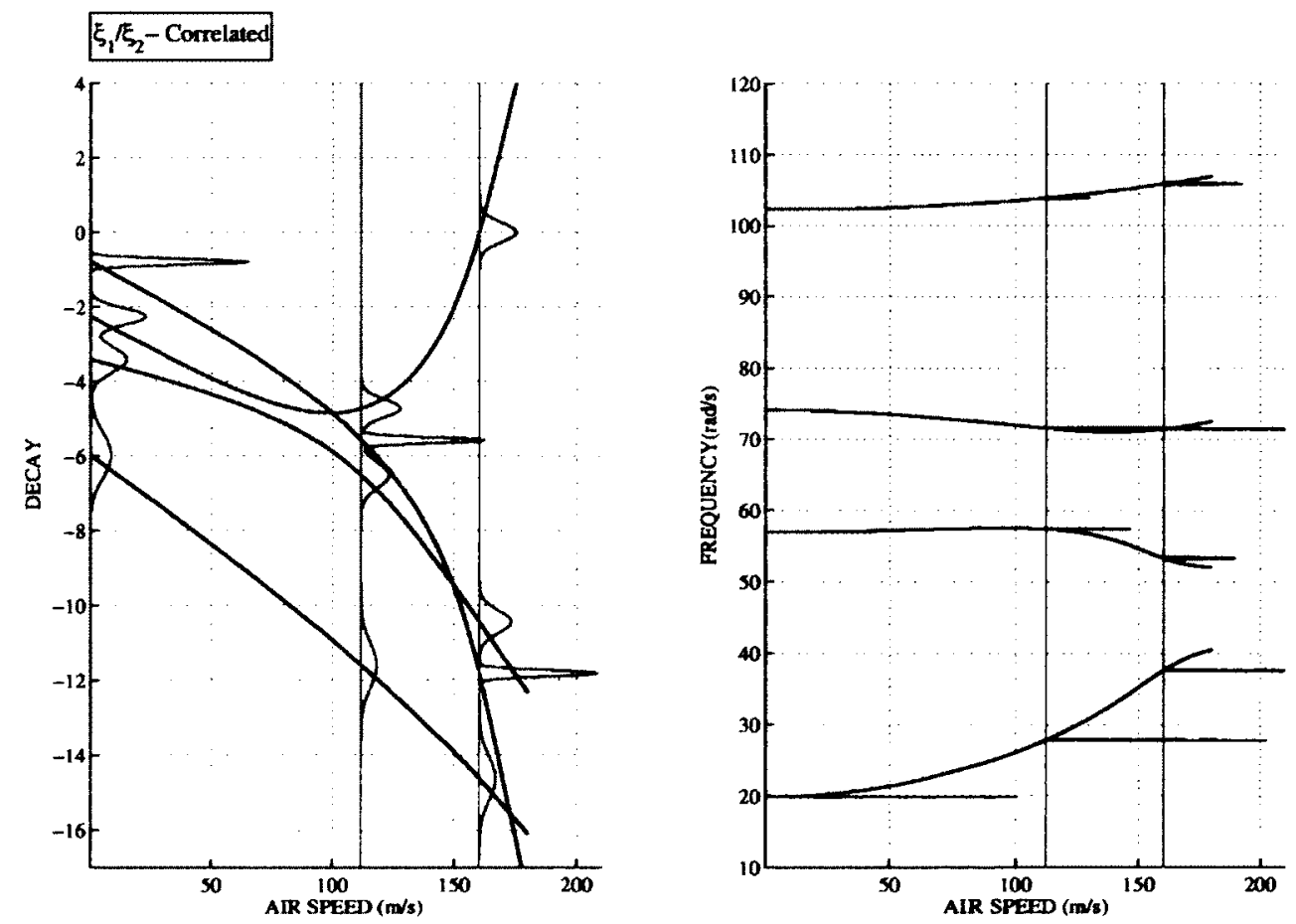

Figure 6.72: Magnified view of coalescing mode's modal parameter pdfs for uncertainty in correlated damping terms 


\subsubsection{Unsteady Results}

Next, the effects of unsteady aerodynamic forcing are examined. Figs. (6.73)-(6.76) show the effects of uncertainty in inertia parameters on the probabilistic modal parameter transformations, along with a magnified view of the modes involved in the flutter mechanism. The observations are very similar to the case of quasisteady aerodynamics. The frequency pdfs of the coalescing modes show significant non-stationary trends in the flutter region, indicating a more explosive flutter type. The uncertainty of both inertia parameters have significant effect on the uncertainty in the frequency of the second (stable) mode, which exhibits predominantly bending motion. For uncertain mass parameter, the frequency pdfs of both coalescing modes indicate a non-Gaussian trend in the flutter region. The modal decay and frequency pdfs of coalescing modes show only small amounts of variance. The decays show high non-Gaussian transformation. The modal decay pdfs of the coalescing modes show significant non-stationary trends with a moderately increasing variance with respect to airspeed. For uncertainty in the mass moment of inertia, the frequency pdfs of the coalescing modes show significant statistical overlap in the flutter region. The modal decay pdfs of the coalescing modes show significant non-stationarity with non-Gaussian features. The variance of the flutter mode decay experiences a reduction of variance in the flutter region. A significant statistical overlap of the modal decays is also evident.

The effects of stiffness uncertainty are illustrated in figs. (6.77)-(6.80), which illustrate the effects of bending stiffness and torsional stiffness uncertainty. Figs. (6.78) and (6.80) show the magnified plots of the coalescing modes for respective cases. The following observations were noted. The frequency pdfs of the coalescing modes show significant non-stationary trends in the flutter region, indicating a more explosive flutter type. The uncertainty of both stiffness parameters has significant effect on the frequency of the second mode, which exhibits predominantly bending motion. For uncertain bending stiffness, the frequency pdfs of both modes experience a high variance in the flutter region. The pdf of the first mode indicates a non-Gaussian trend. Additionally, there is statistical overlapping of the frequency pdfs. The modal decay pdfs of the coalescing modes show significant non-stationary trends, and significant variance increase and 

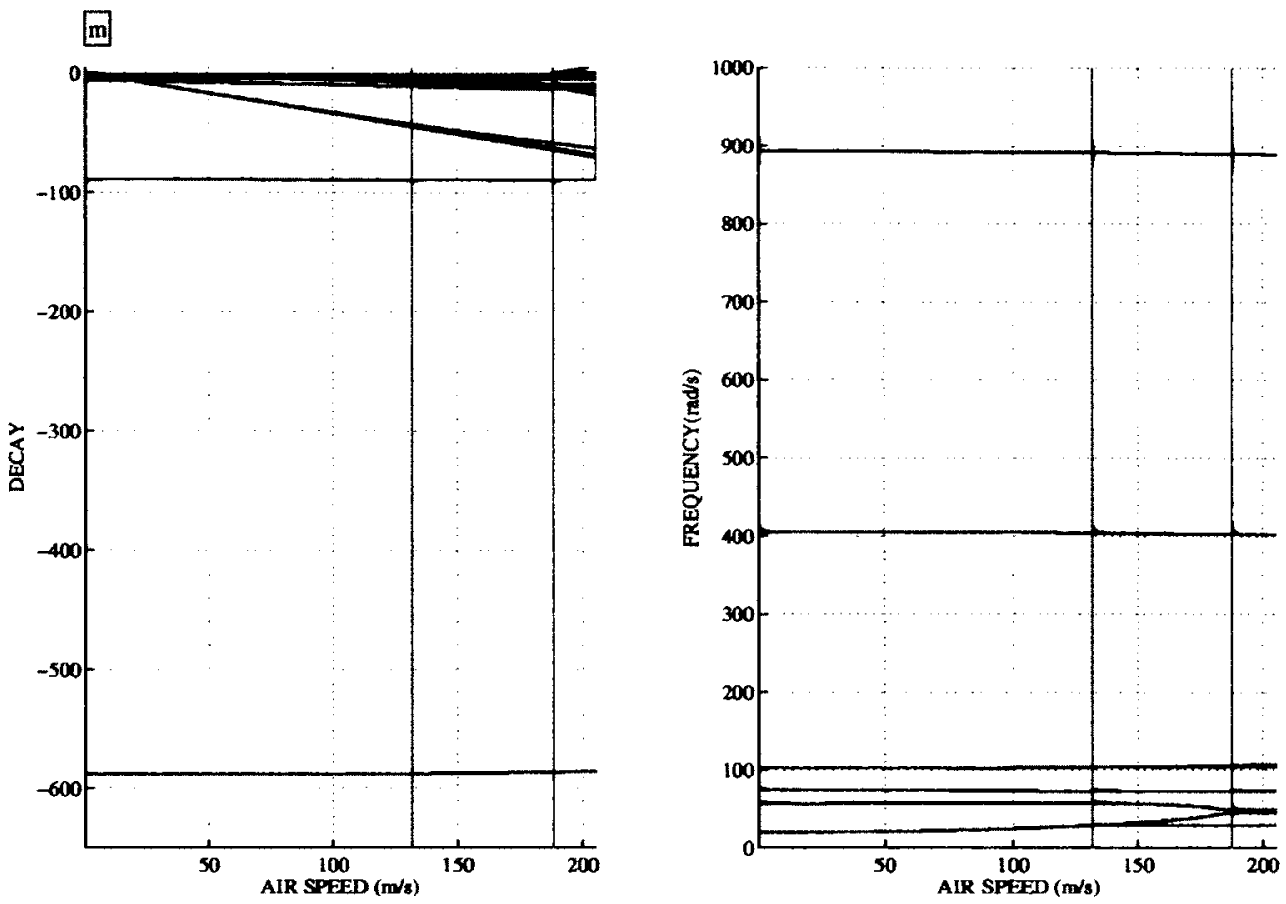

Figure 6.73: Modal parameter pdfs resulting from randomness in mass with $10 \% \mathrm{COV}$, obtained at 3 test airspeeds: $\approx 0 \mathrm{~m} / \mathrm{s}, 131.0 \mathrm{~m} / \mathrm{s}, 187.91 \mathrm{~m} / \mathrm{s}$
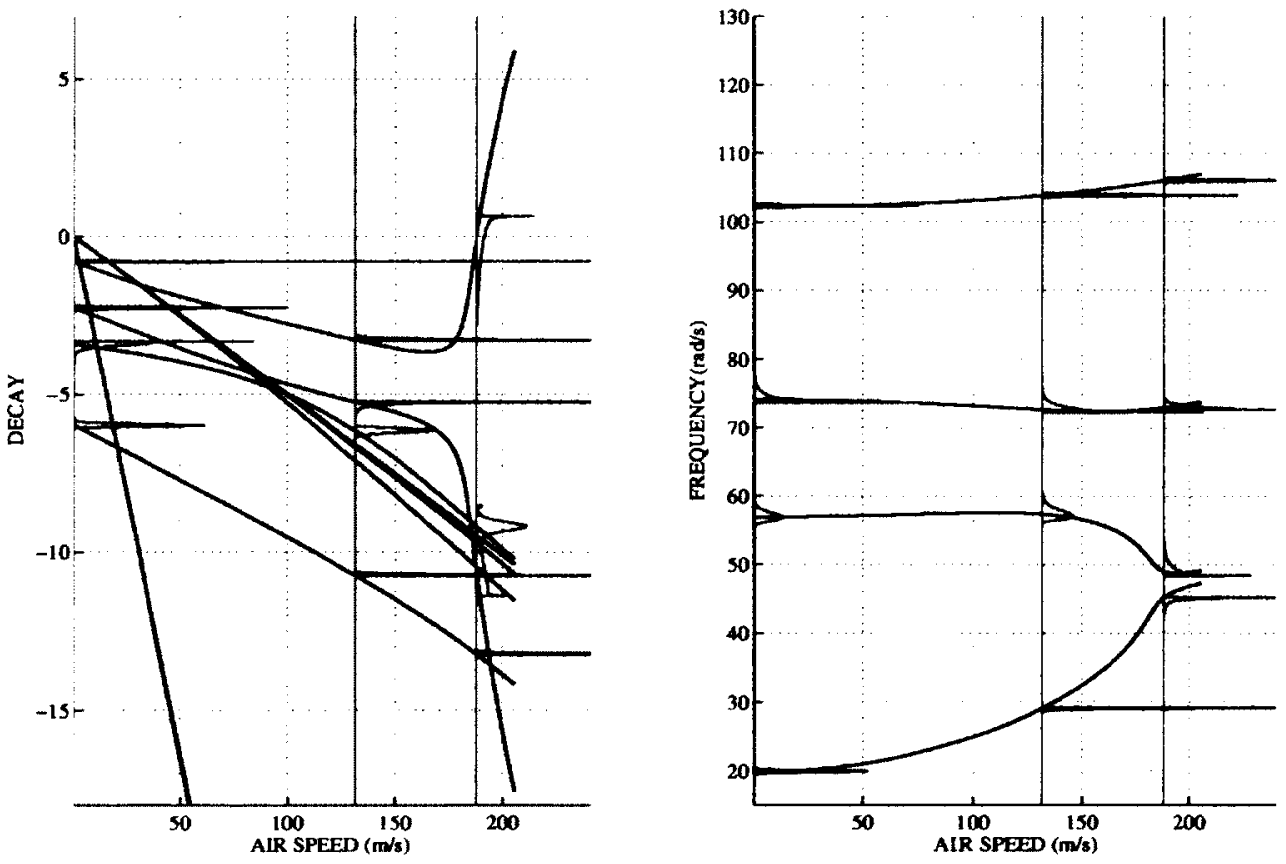

Figure 6.74: Magnified view of coalescing mode's modal parameter pdfs for uncertainty in mass 

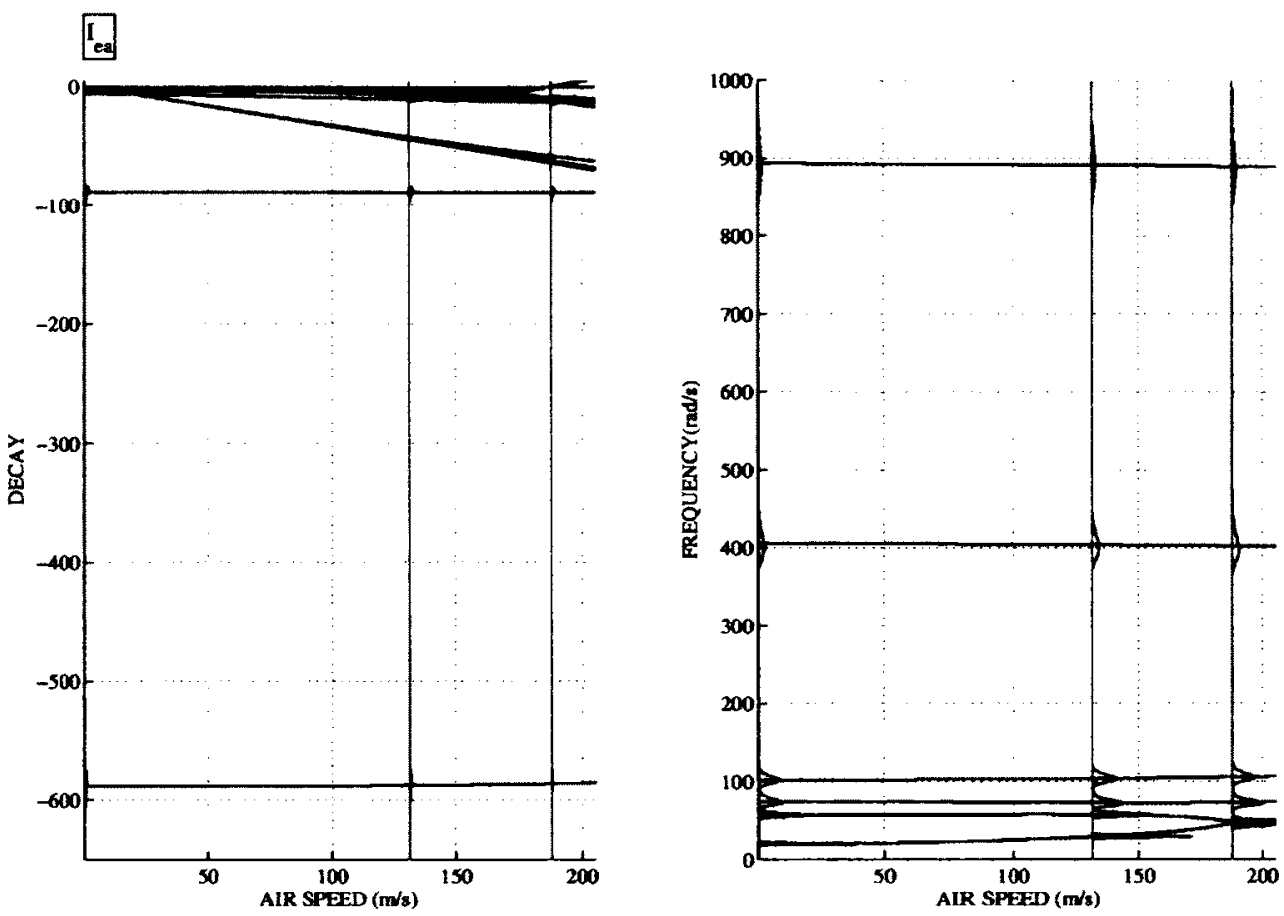

Figure 6.75: Modal parameter pdfs resulting from randomness in mass moment of inertia with $10 \%$ COV, obtained at 3 test airspeeds: $\approx 0 \mathrm{~m} / \mathrm{s}, 131.0 \mathrm{~m} / \mathrm{s}, 187.91 \mathrm{~m} / \mathrm{s}$
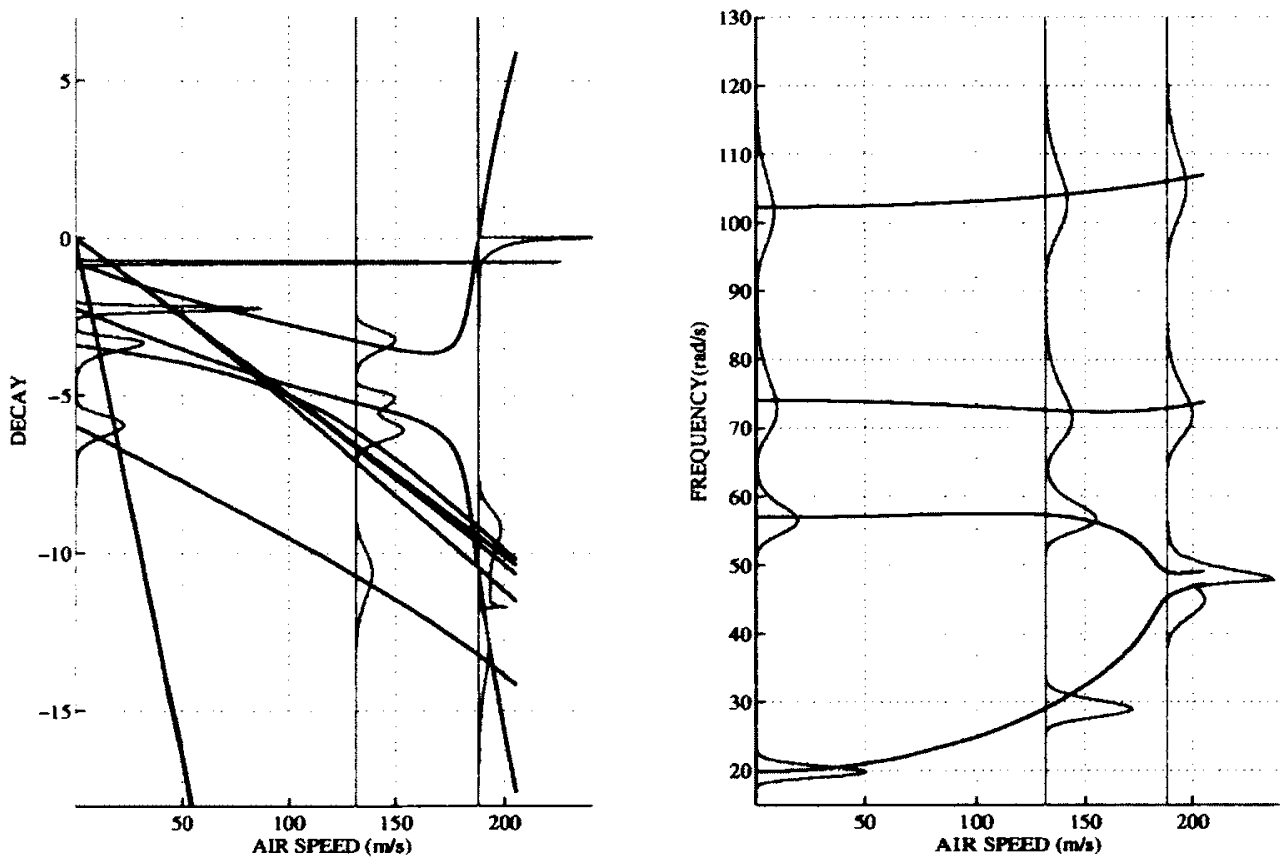

Figure 6.76: Magnified view of coalescing mode's modal parameter pdfs for uncertainty in mass moment of inertia 

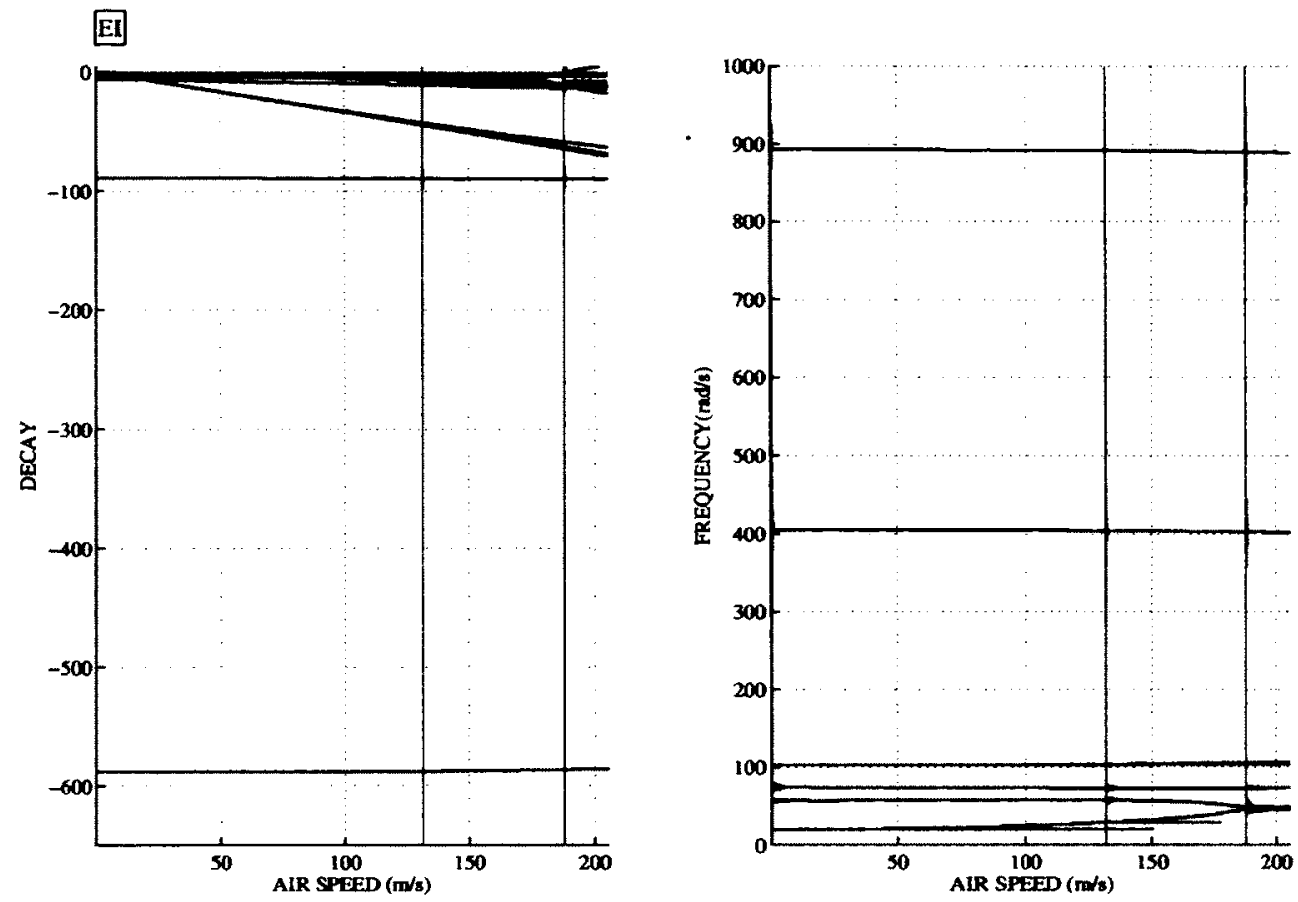

Figure 6.77: Modal parameter pdfs resulting from randomness in bending stiffness with $10 \%$ COV, obtained at 3 test airspeeds: $\approx 0 \mathrm{~m} / \mathrm{s}, 131.0 \mathrm{~m} / \mathrm{s}, 187.91 \mathrm{~m} / \mathrm{s}$
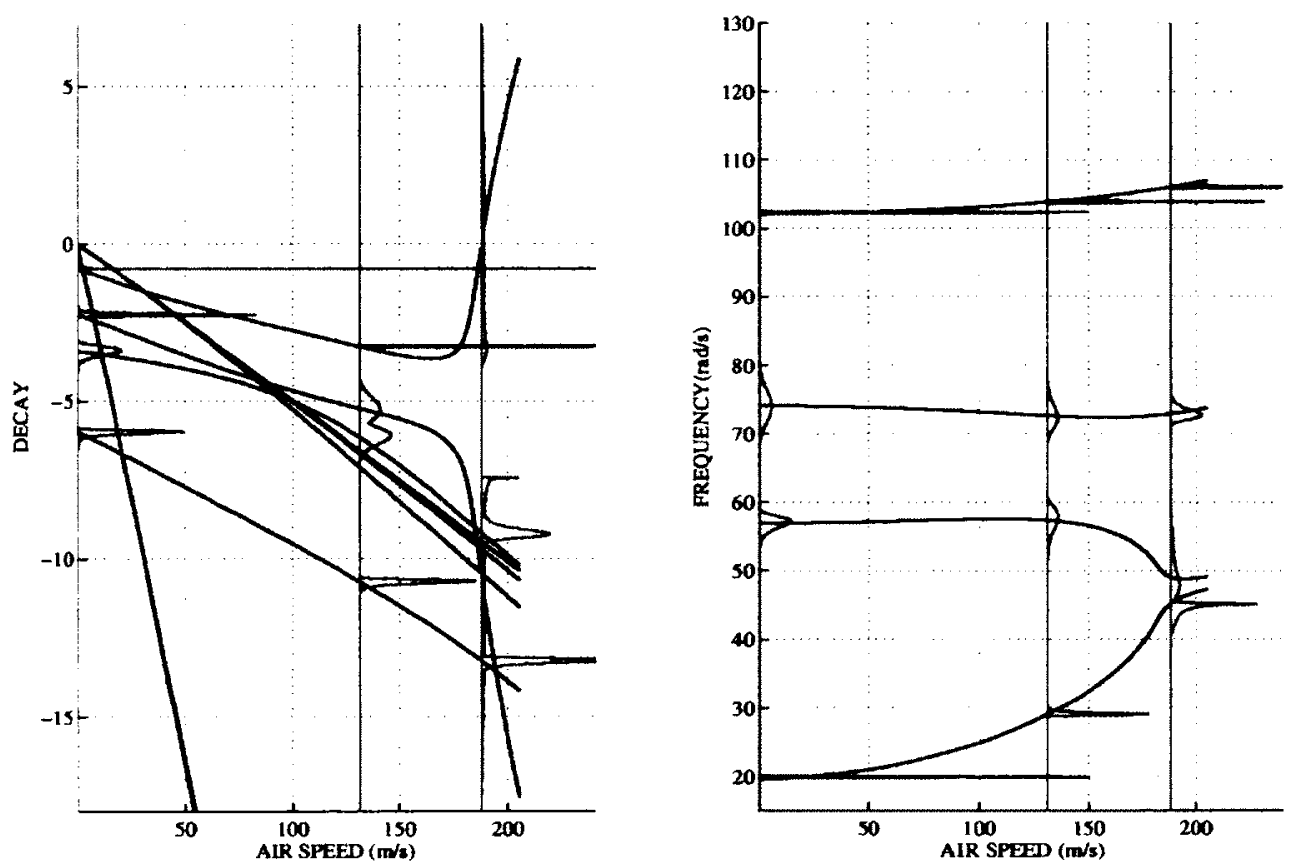

Figure 6.78: Magnified view of coalescing mode's modal parameter pdfs for uncertainty in bending stiffness 
G
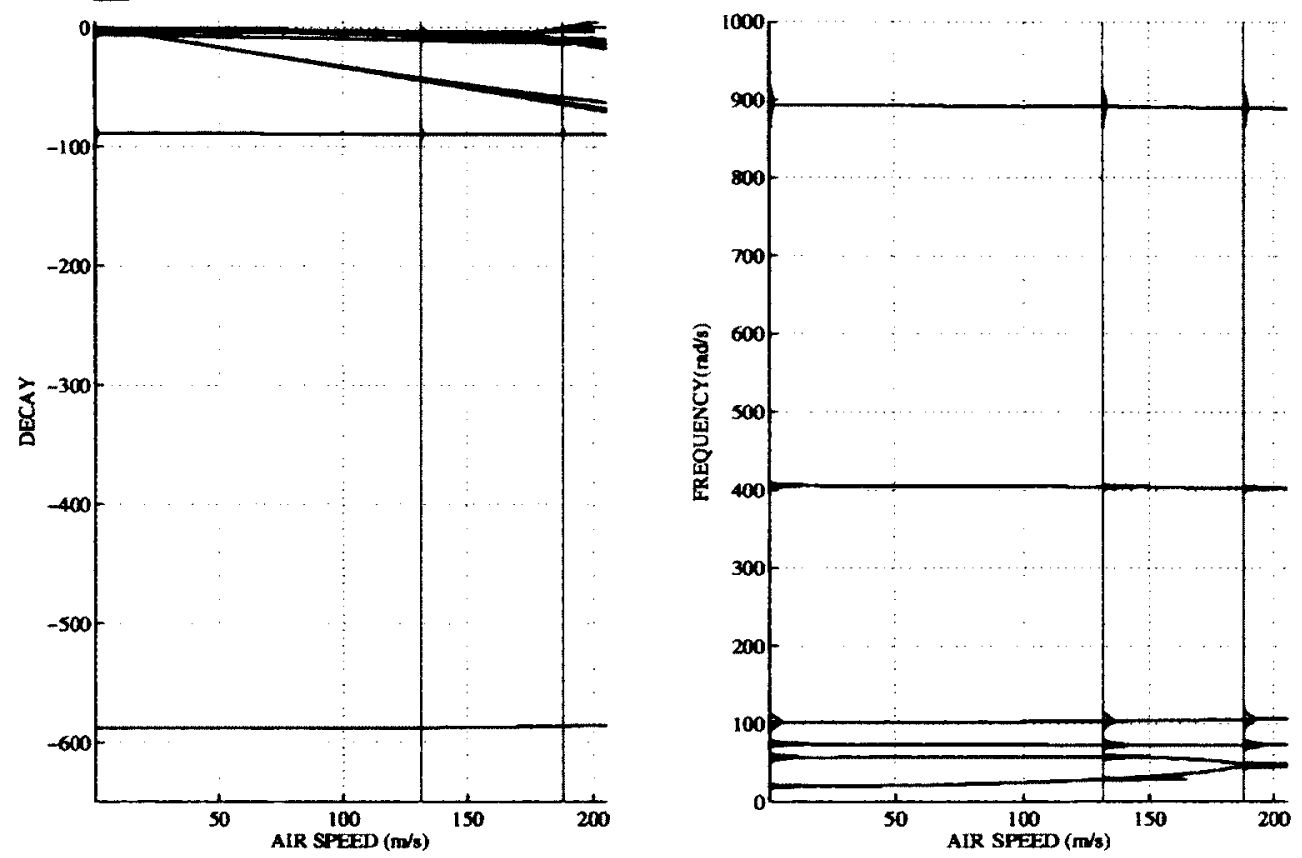

Figure 6.79: Modal parameter pdfs resulting from randomness in torsional stiffness with $10 \% \mathrm{COV}$, obtained at 3 test airspeeds: $\approx 0 \mathrm{~m} / \mathrm{s}, 131.0 \mathrm{~m} / \mathrm{s}, 187.91 \mathrm{~m} / \mathrm{s}$
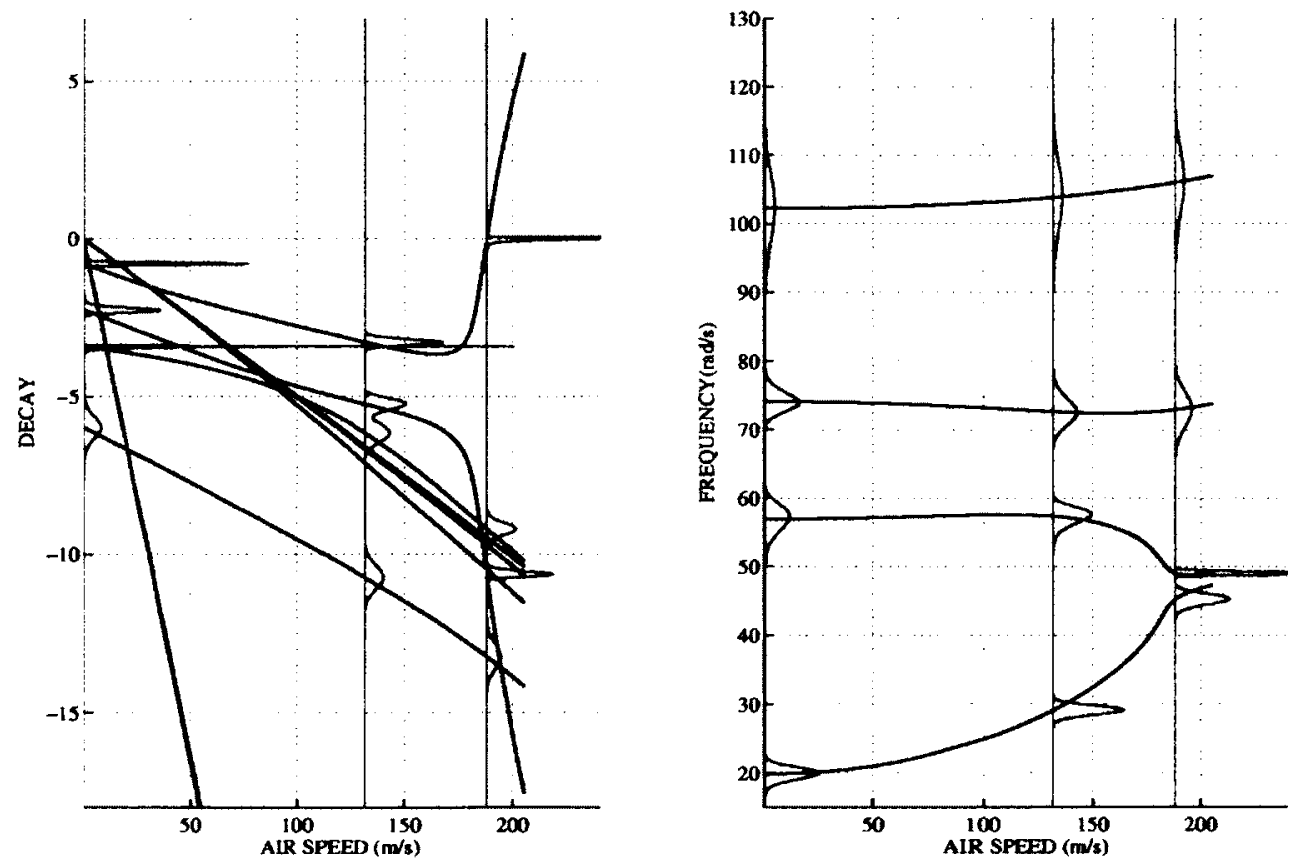

Figure 6.80: Magnified view of coalescing mode's modal parameter pdfs for uncertainty in torsional stiffness 
a non-Gaussian trend of the flutter mode in the flutter region. For uncertainty in the torsional stiffness, the frequency pdfs of the coalescing modes show significant statistical overlap in the flutter region. The modal decay pdfs of the coalescing modes show significant non-stationarity with strong non-Gaussian trends. The variance of the flutter mode decay experiences a reduction of variance in the flutter region. For uncertain torsional stiffness, the frequency pdfs of both coalescing modes show significant non-stationary trends in the flutter region, resulting in a change of variance. The modal decay pdfs of the coalescing modes show significant nonstationarity, but the variance of decay in the flutter mode experiences only slight changes with respect to the airspeed. In both cases a significant statistical overlap of the modal decays is evident.

From the probabilistic results of the random inertia and stiffness and the flutter speed pdfs in fig. (5.24), note that a moderate growth of variance in the modal decay of the flutter mode for uncertainty in the mass parameter leads to a moderate variance in the flutter speed pdf. Then, a reduction of variance in the flutter mode decay in the flutter region produces a low uncertainty in the flutter speed pdf. For uncertainty in the bending stiffness, a large growth of variance in the flutter mode decay results in a large variance of the flutter speed pdf. For uncertainty in the torsional stiffness, a low and almost constant variance in the decay of the flutter mode are experienced.

The effects of uncertainty in the damping parameters are illustrated in figs. (6.81)-(6.84). Figs. (6.82) and (6.84) provide the magnified view of the modal properties of the coalescing modes for the respective cases. The resulting observations are similar to the Case 1 study for unsteady aerodynamics. Most notably, for uncertainty in the first structural damping ratio, the decay of the first (flutter) mode is most affected. The variance of the second mode is not significant. The flutter speed pdf has a low level of uncertainty, as evident in fig. (5.24). For uncertainty in the second structural damping ratio, the decay of the second (non-flutter) mode is most affected. The variance of the first mode is insignificant. The uncertainty of the second structural damping ratio also introduces very low uncertainty in the flutter speed. 

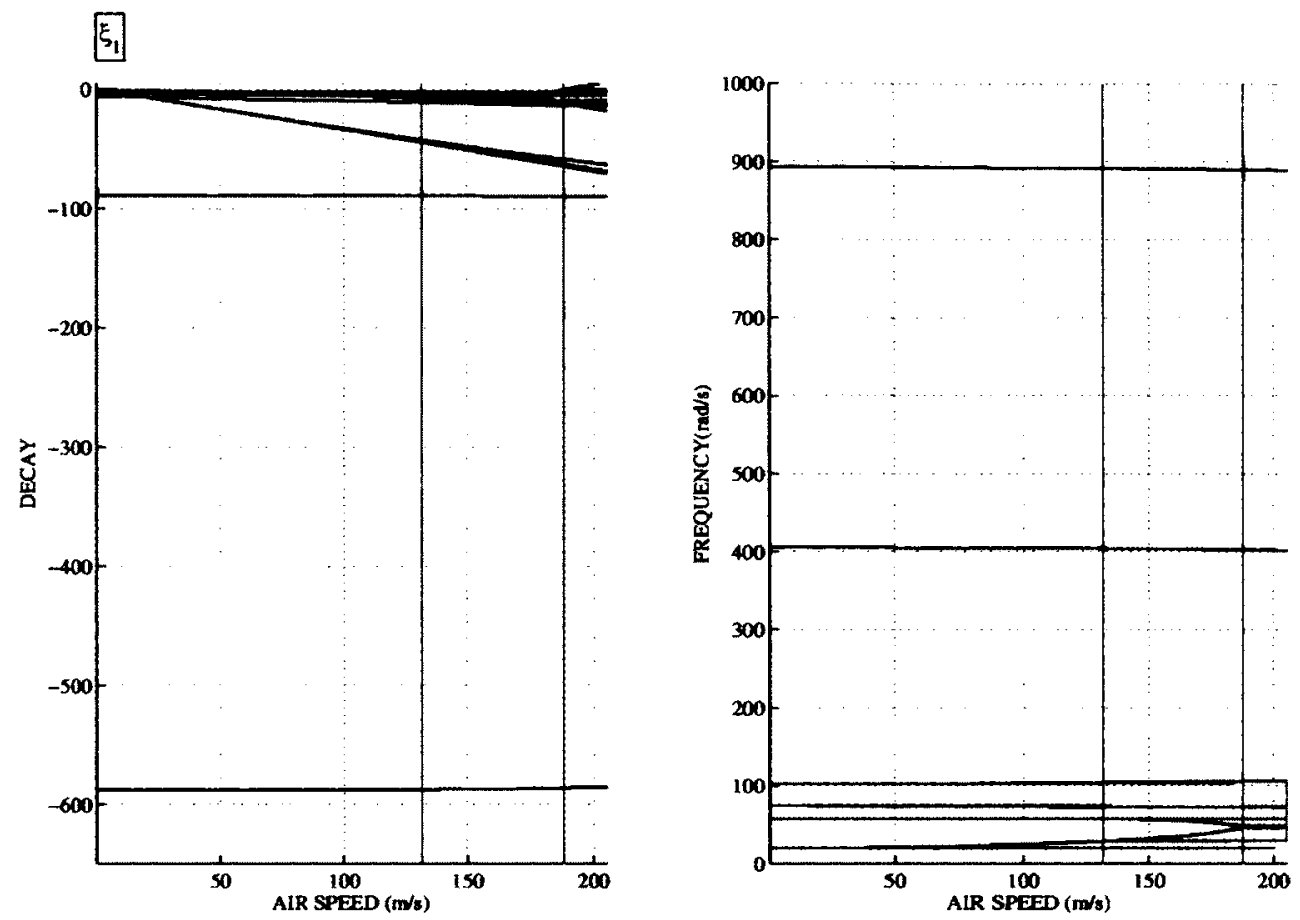

Figure 6.81: Modal parameter pdfs resulting from randomness in first structural damping ratio with $10 \% \mathrm{COV}$, obtained at 3 test airspeeds: $\approx 0 \mathrm{~m} / \mathrm{s}, 131.0 \mathrm{~m} / \mathrm{s}, 187.91 \mathrm{~m} / \mathrm{s}$
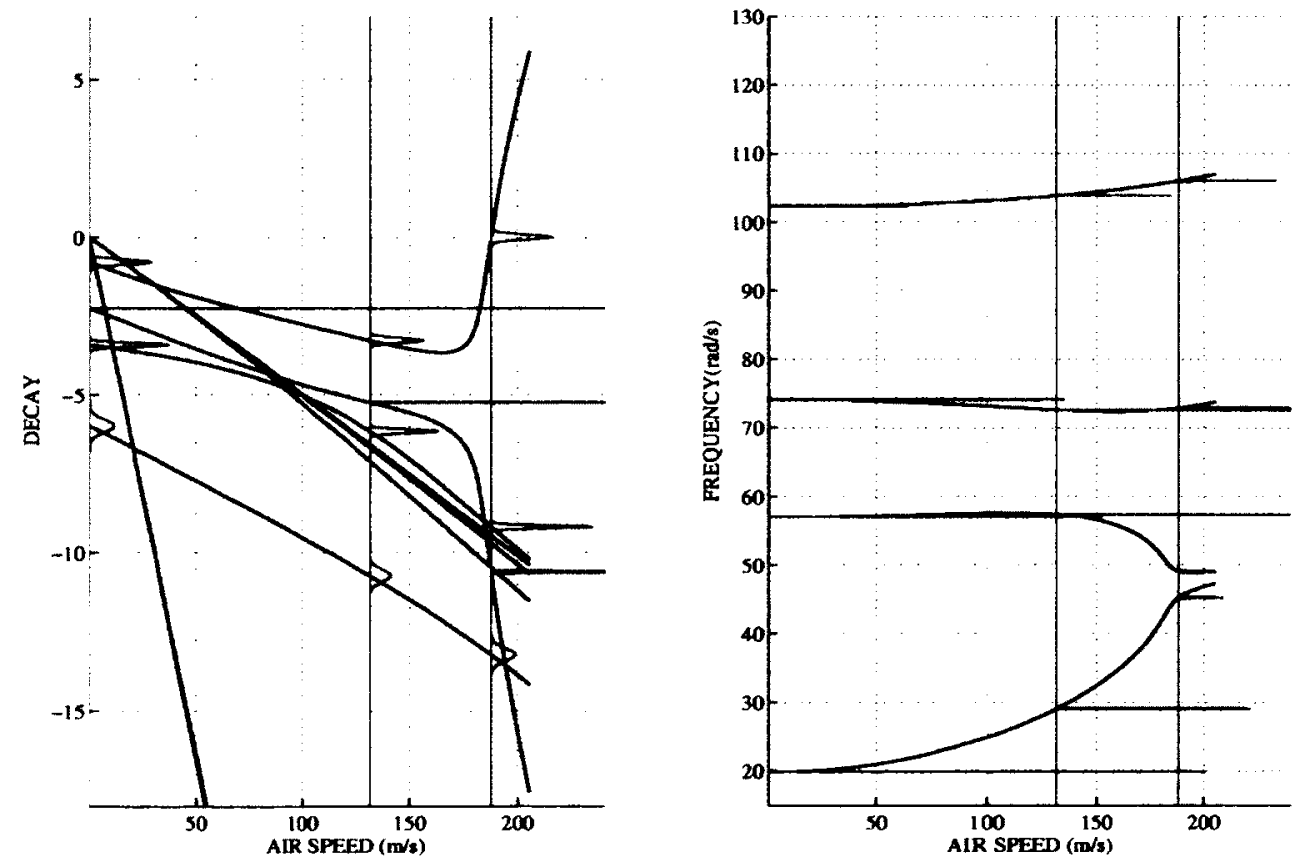

Figure 6.82: Magnified view of coalescing mode's modal parameter pdfs for uncertainty in first structural damping ratio 

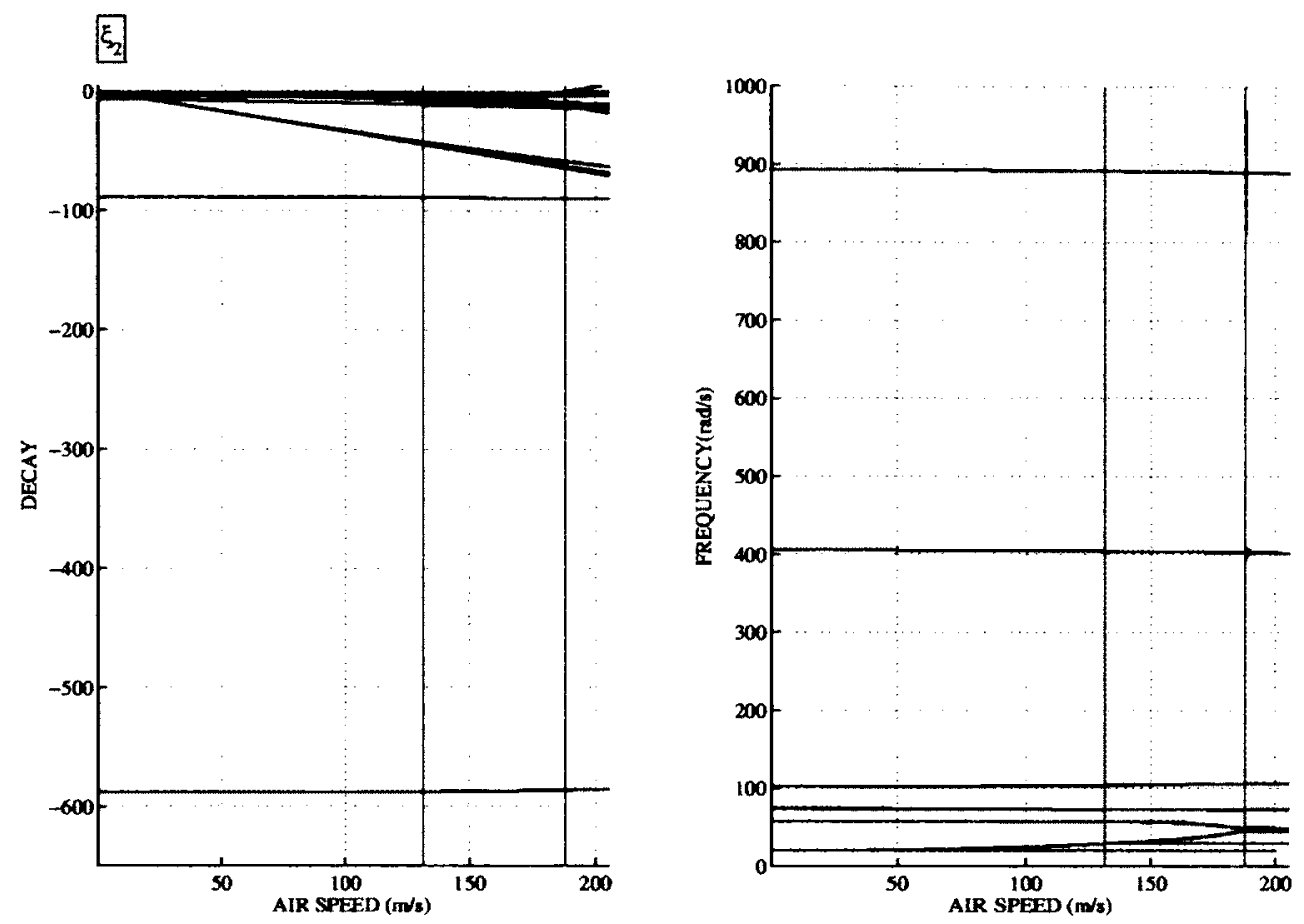

Figure 6.83: Modal parameter pdfs resulting from randomness in second structural damping ratio with $10 \% \mathrm{COV}$, obtained at 3 test airspeeds: $\approx 0 \mathrm{~m} / \mathrm{s}, 131.0 \mathrm{~m} / \mathrm{s}, 187.91 \mathrm{~m} / \mathrm{s}$
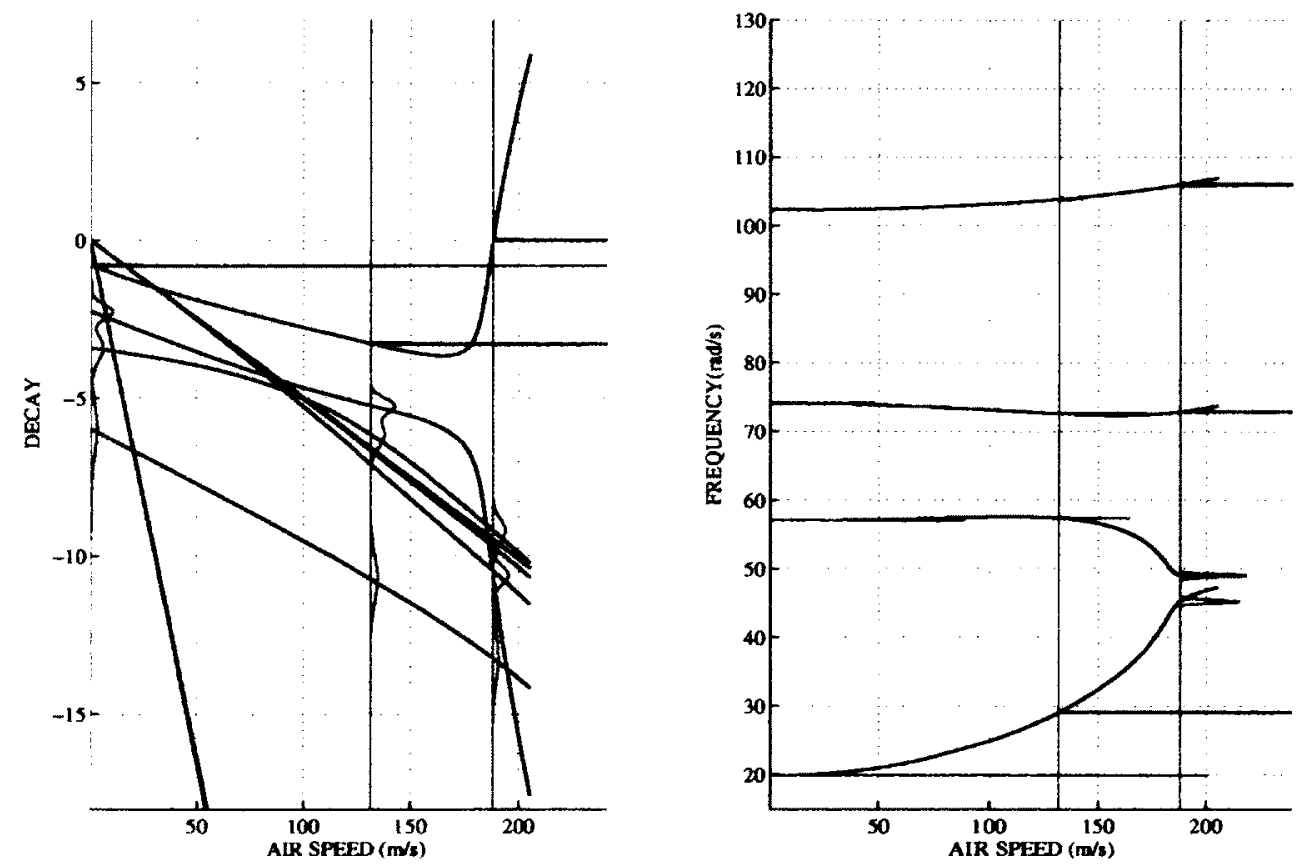

Figure 6.84: Magnified view of coalescing mode's modal parameter pdfs for uncertainty in second structural damping ratio 


\section{Multiple Parameter Uncertainty}

The effects of uncertainty in multiple parameters are very similar to the previous cases. Figs. (6.85)-(6.90) show the resulting the modal parameter pdfs as a function of airspeed for uncorrelated inertia, stiffness and damping parameters. The results indicate that uncertainty of multiple uncorrelated parameters increases the uncertainty of the modal parameter pdfs. The resulting larger variance of modal decay of the flutter mode produces a larger variance in the flutter speed (evident in fig. (5.24)). There are however some differences that are noted in comparison to the previous case study. Mainly, the uncertainty in inertia and stiffness parameters causes the modal decay and frequency pdfs to experience significant statistical overlap.

The effects of uncertainty with full correlation between the random parameters are examined in figs. (6.91)-(6.96). From the analysis of these figures, it is seen that the correlation between these random parameters does not cause significant changes in the probabilistic results of the modal parameters. Only from the probabilistic flutter speed results, shown in fig. (5.24), it can be assumed that: the uncertainty in the flutter mode decay decreases due to inertia and stiffness parameter correlation and the uncertainty in the flutter mode decay increases due to a damping parameter correlation. However, these effects are very minor.

\subsubsection{Comparison of Quasisteady and Unsteady Aerodynamics}

At this point, the probabilistic results of modal parameter transformations with quasisteady aerodynamic forcing are compared with those of unsteady aerodynamic forcing. The unsteady aerodynamics induce a more explosive flutter type, which leads to significant non-stationary effects on the modal parameters in the region of strong frequency coalescence. Additionally, a slightly stronger non-Gaussian trend is introduced in the flutter mode decay for the uncertain torsional stiffness and second mode damping ratio. Since the frequency coalescence is more profound in the case of unsteady aerodynamics, the modal frequencies of the coalescing modes experience significant statistical overlapping due to mode veering. 

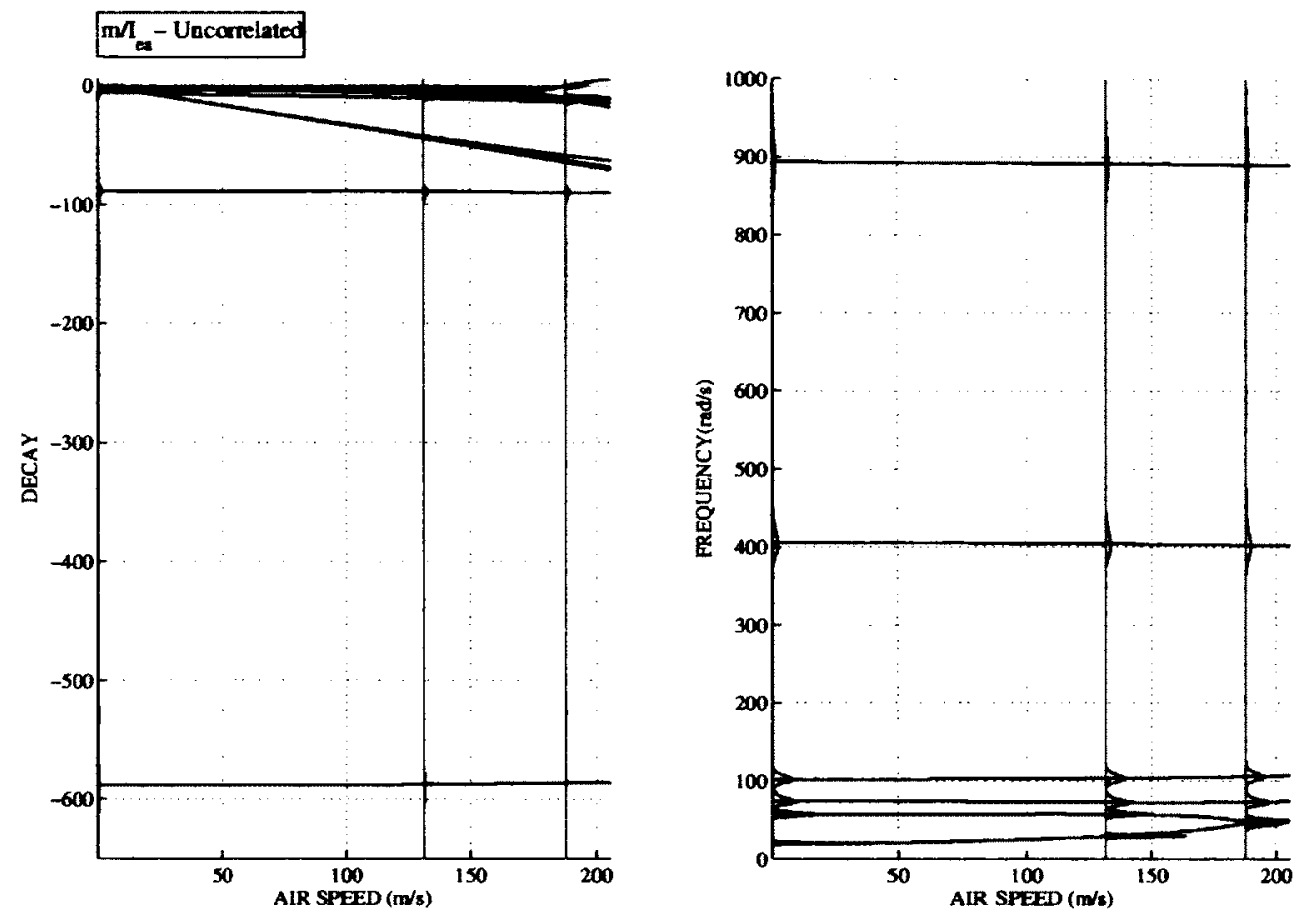

Figure 6.85: Modal parameter pdfs resulting from randomness in uncorrelated inertia terms with $10 \% \mathrm{COV}$, obtained at 3 test airspeeds: $\approx 0 \mathrm{~m} / \mathrm{s}, 131.0 \mathrm{~m} / \mathrm{s}, 187.91 \mathrm{~m} / \mathrm{s}$
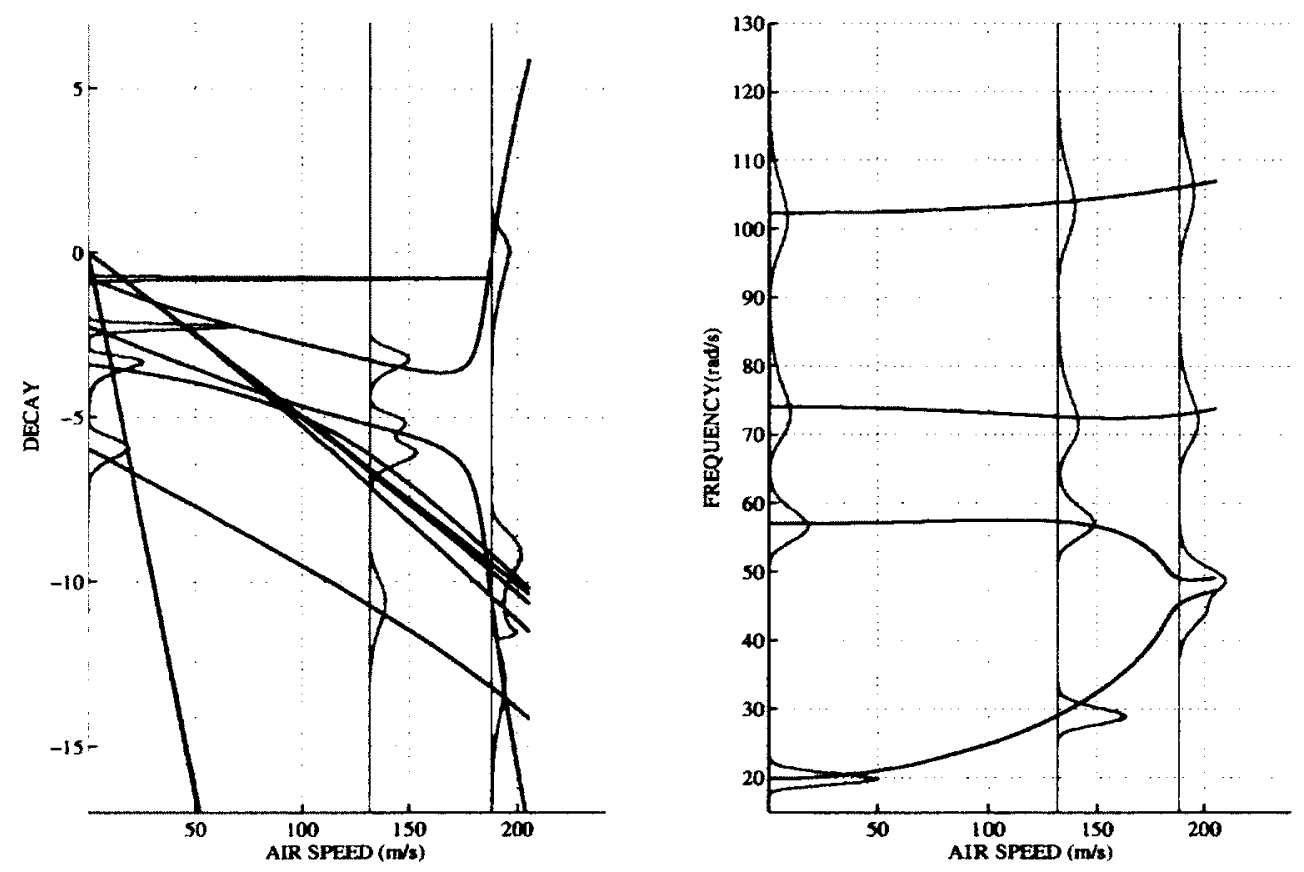

Figure 6.86: Magnified view of coalescing mode's modal parameter pdfs for uncertainty in uncorrelated inertia terms 

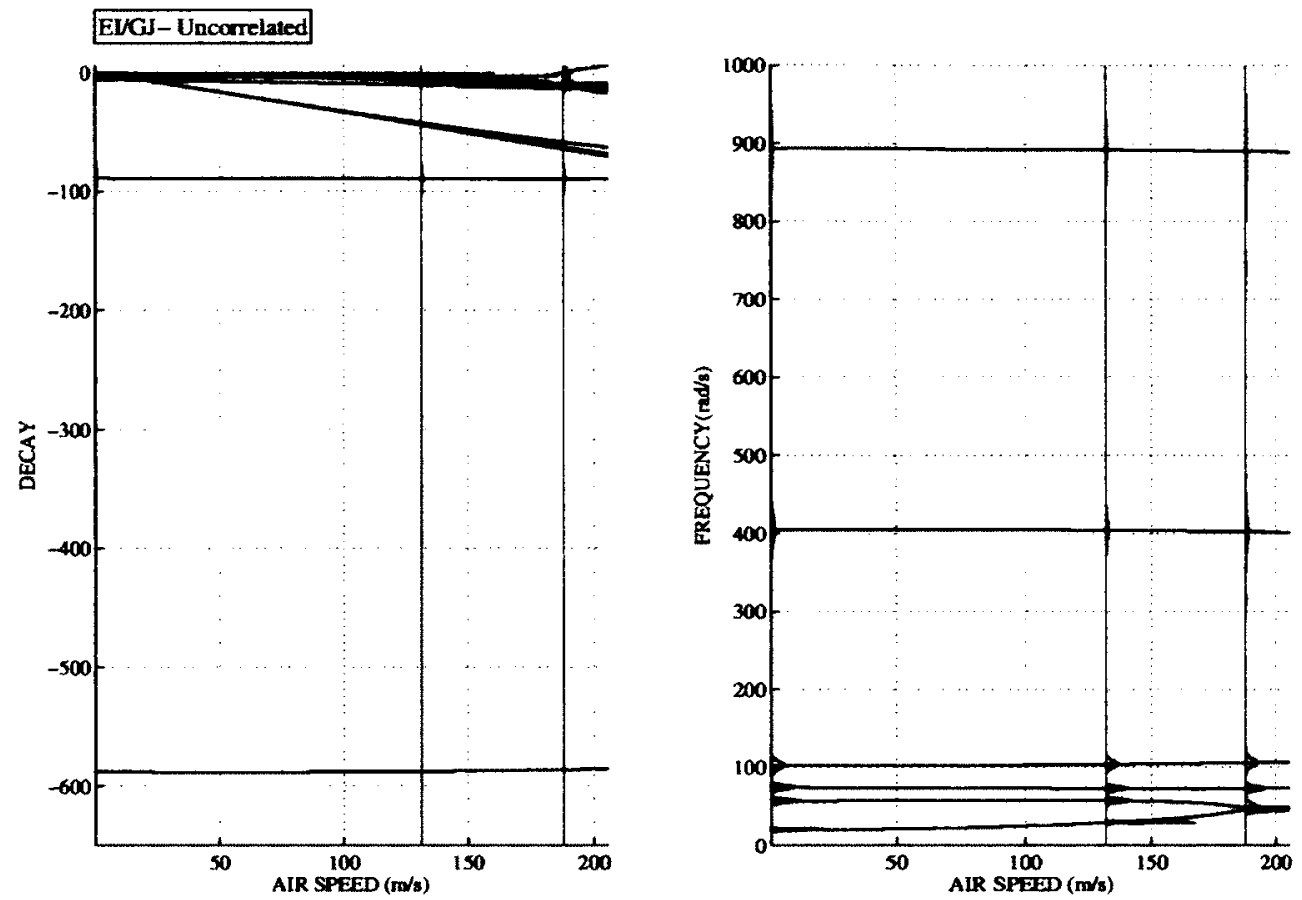

Figure 6.87: Modal parameter pdfs resulting from randomness in uncorrelated stiffness terms with $10 \% \mathrm{COV}$, obtained at 3 test airspeeds: $\approx 0 \mathrm{~m} / \mathrm{s}, 131.0 \mathrm{~m} / \mathrm{s}, 187.91 \mathrm{~m} / \mathrm{s}$
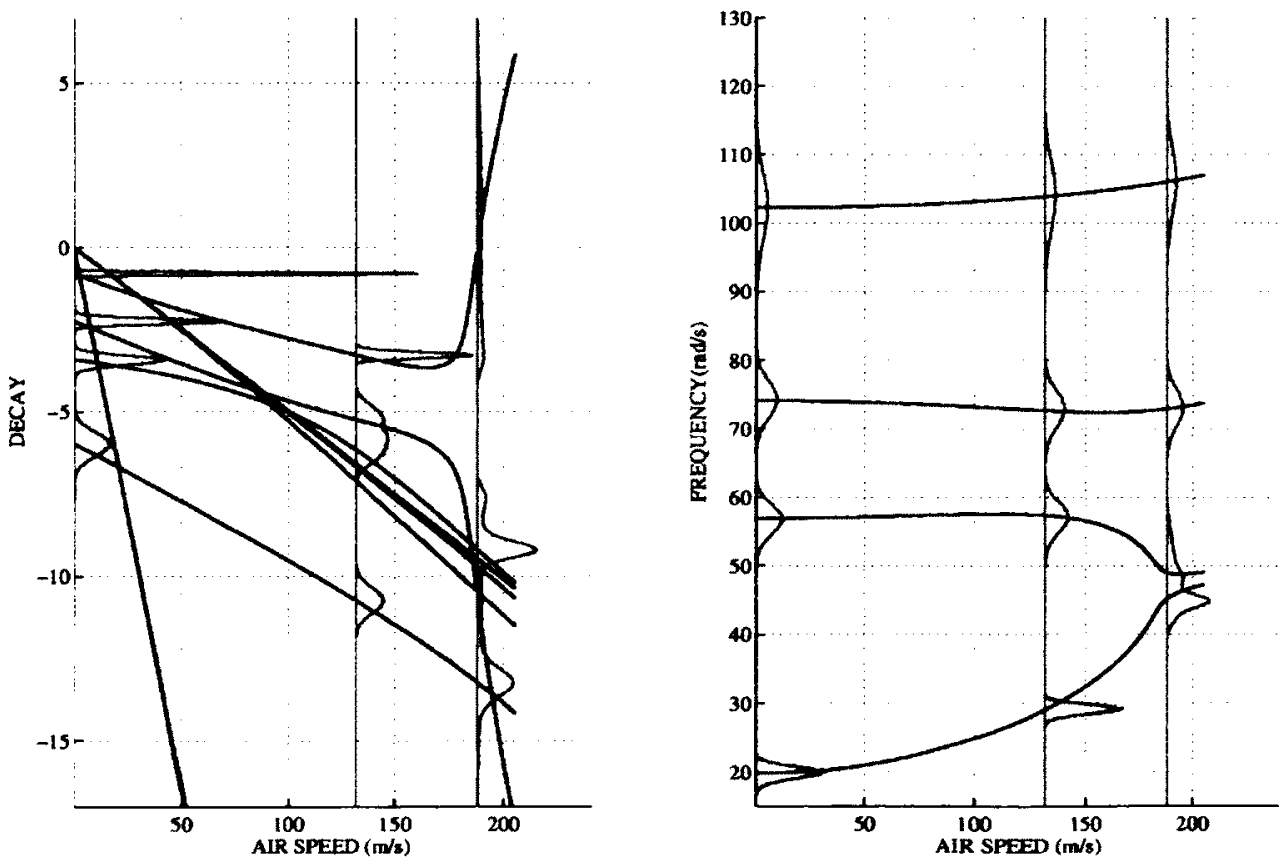

Figure 6.88: Magnified view of coalescing mode's modal parameter pdfs for uncertainty in uncorrelated stiffness terms 

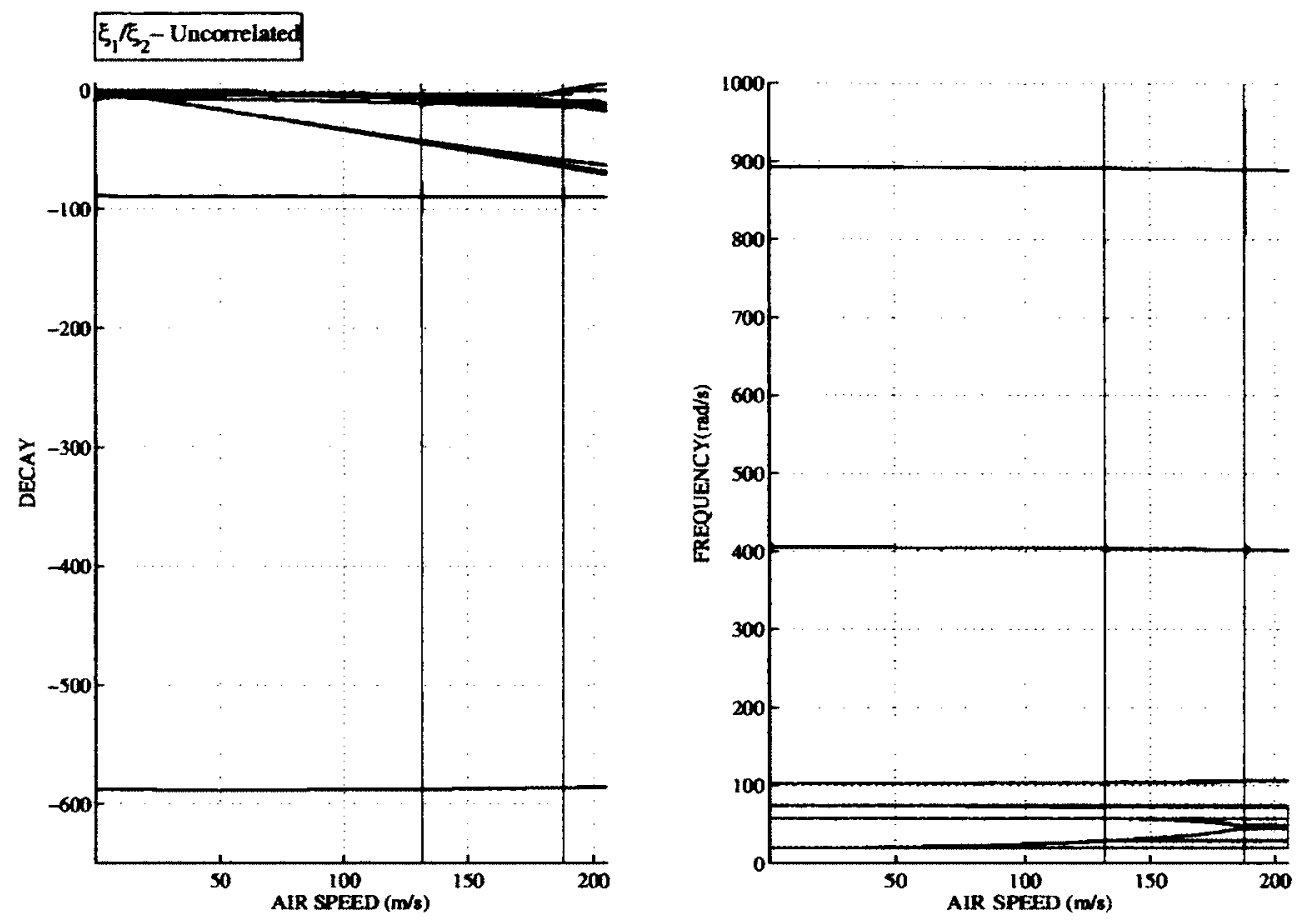

Figure 6.89: Modal parameter pdfs resulting from randomness in uncorrelated damping terms with $10 \% \mathrm{COV}$, obtained at 3 test airspeeds: $\approx 0 \mathrm{~m} / \mathrm{s}, 131.0 \mathrm{~m} / \mathrm{s}, 187.91 \mathrm{~m} / \mathrm{s}$
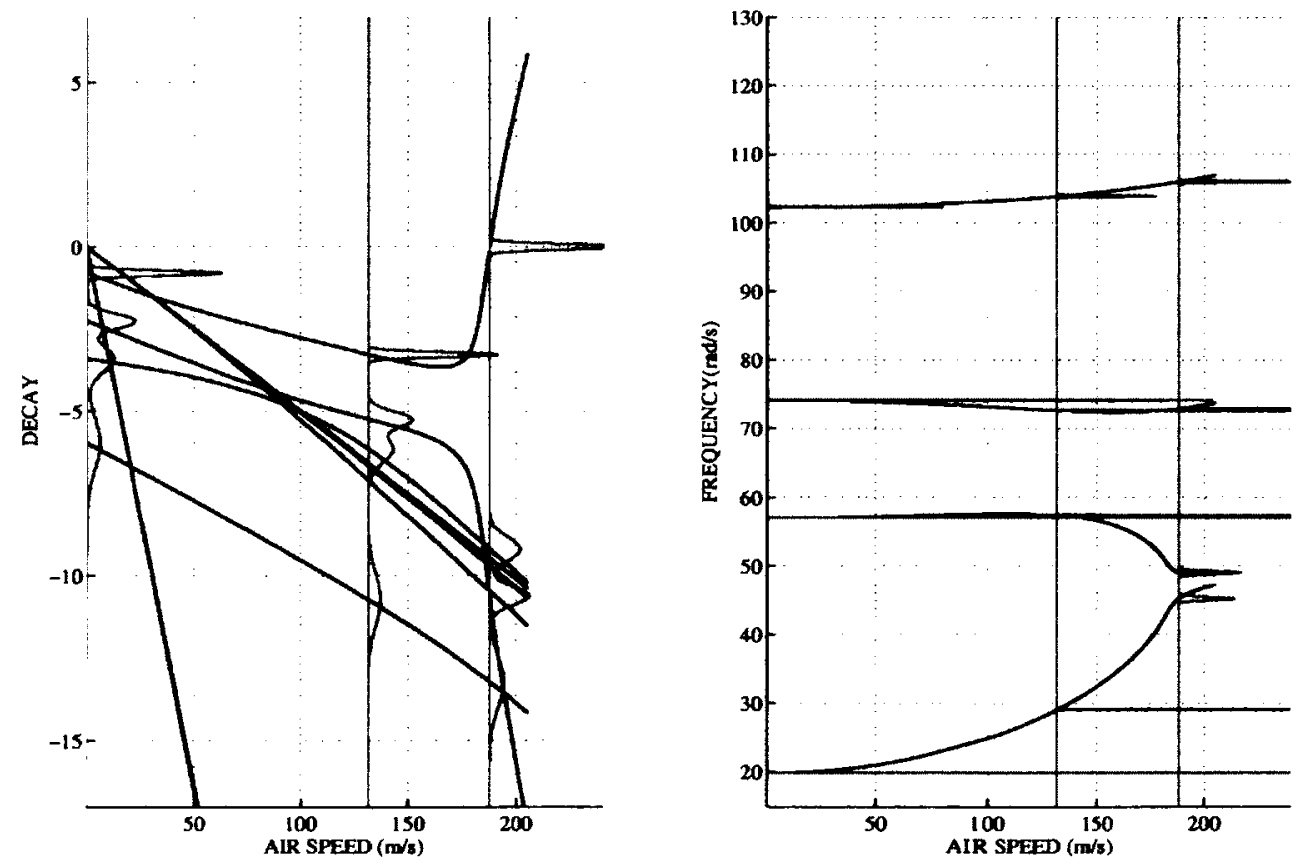

Figure 6.90: Magnified view of coalescing mode's modal parameter pdfs for uncentainty in uncorrelated damping terms 

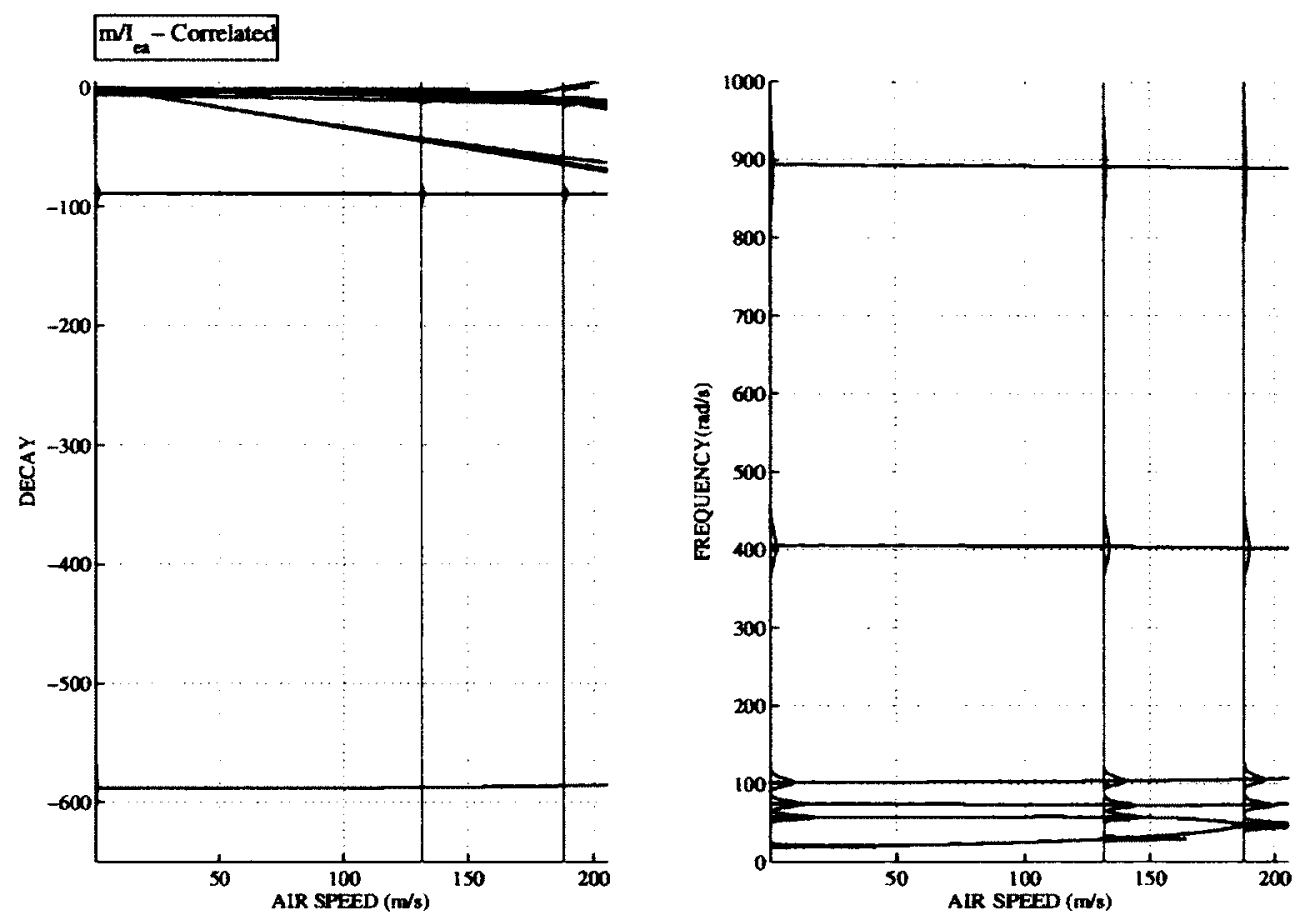

Figure 6.91: Modal parameter pdfs resulting from randomness in correlated inertia terms with $10 \%$ COV, obtained at 3 test airspeeds: $\approx 0 \mathrm{~m} / \mathrm{s}, 131.0 \mathrm{~m} / \mathrm{s}, 187.91 \mathrm{~m} / \mathrm{s}$
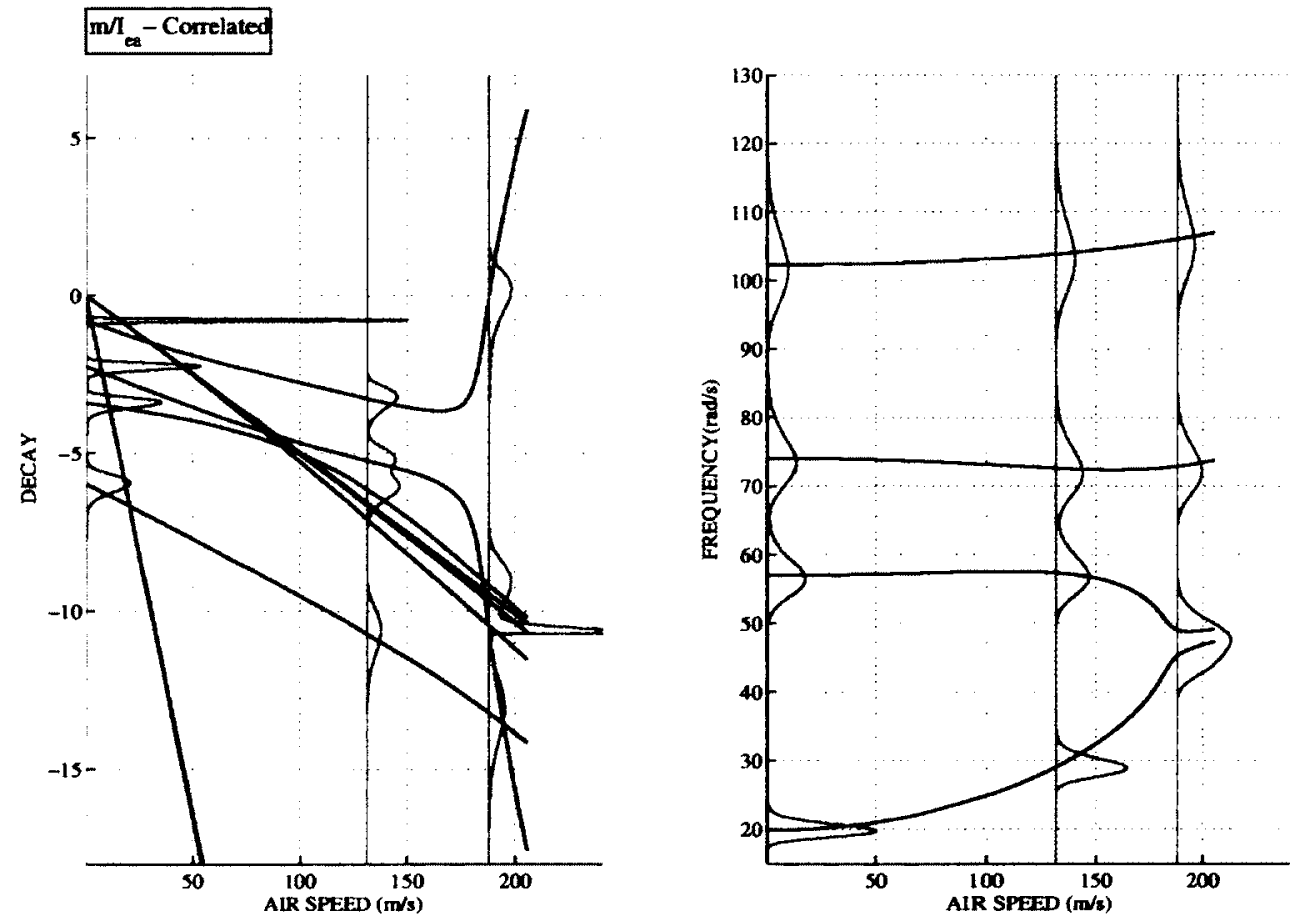

Figure 6.92: Magnified view of coalescing mode's modal parameter pdfs for uncertainty in correlated inertia terms 

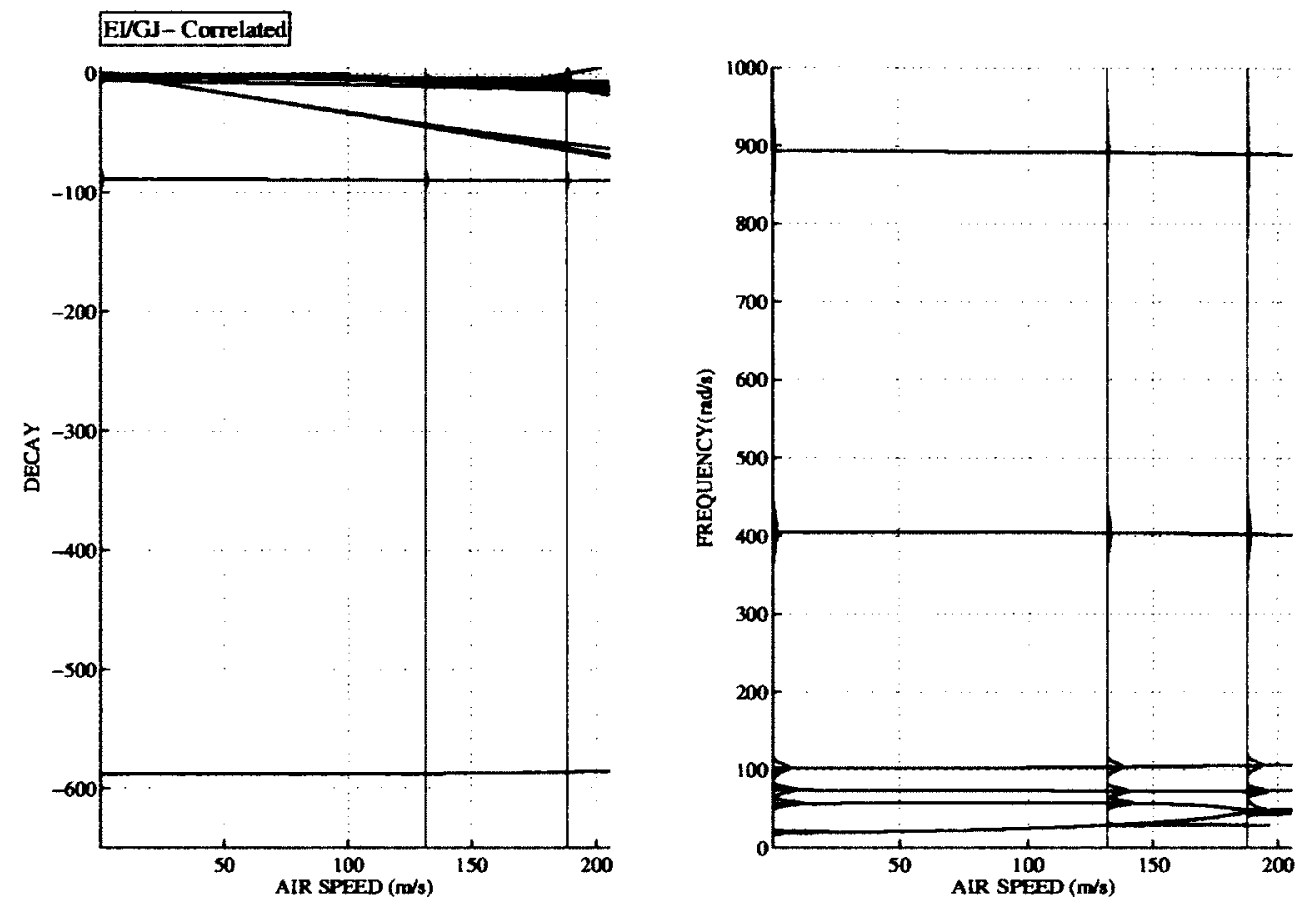

Figure 6.93: Modal parameter pdfs resulting from randomness in correlated stiffness terms with $10 \%$ COV, obtained at 3 test airspeeds: $\approx 0 \mathrm{~m} / \mathrm{s}, 131.0 \mathrm{~m} / \mathrm{s}, 187.91 \mathrm{~m} / \mathrm{s}$
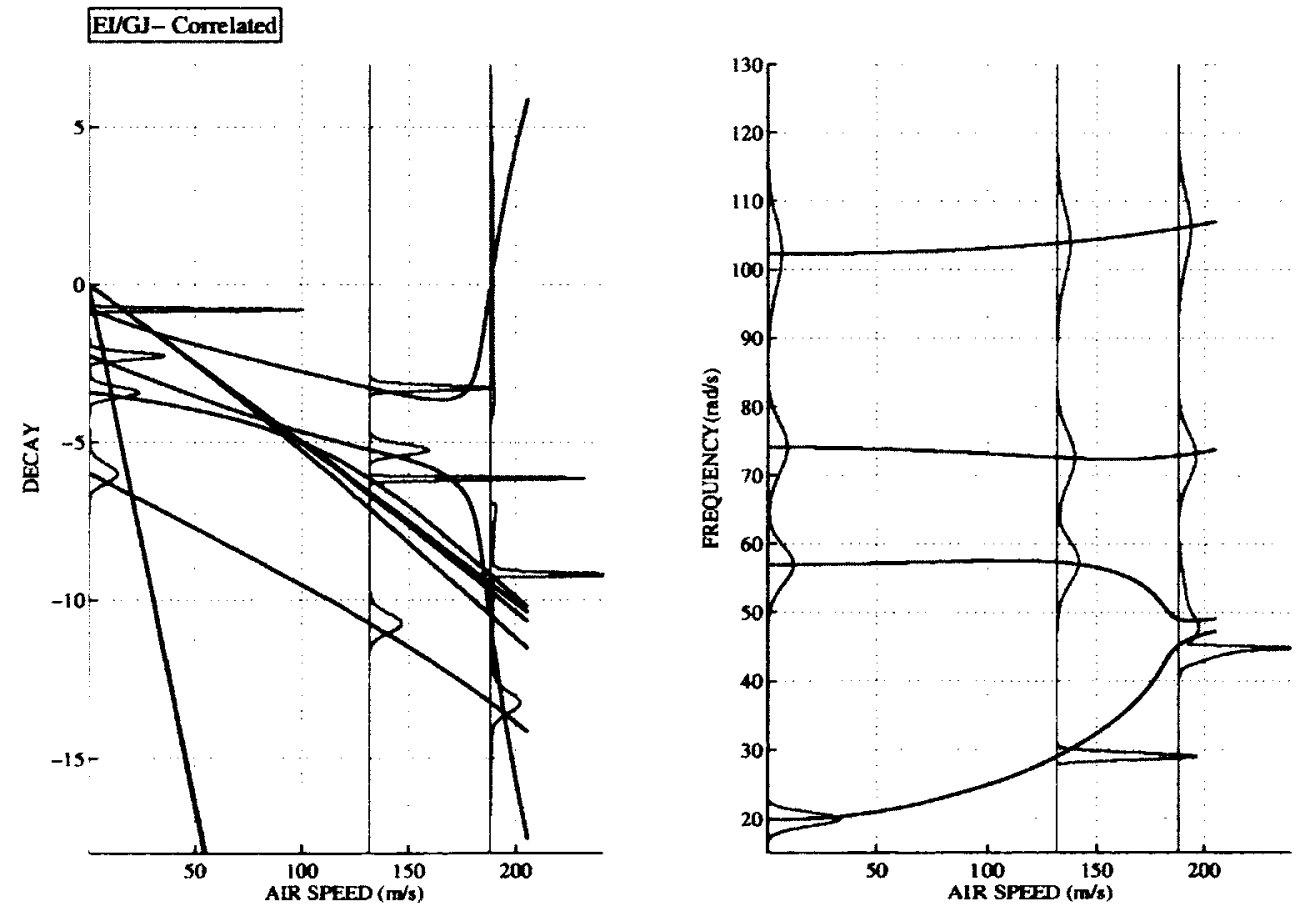

Figure 6.94: Magnified view of coalescing mode's modal parameter pdfs for uncertainty in correlated stiffness terms 

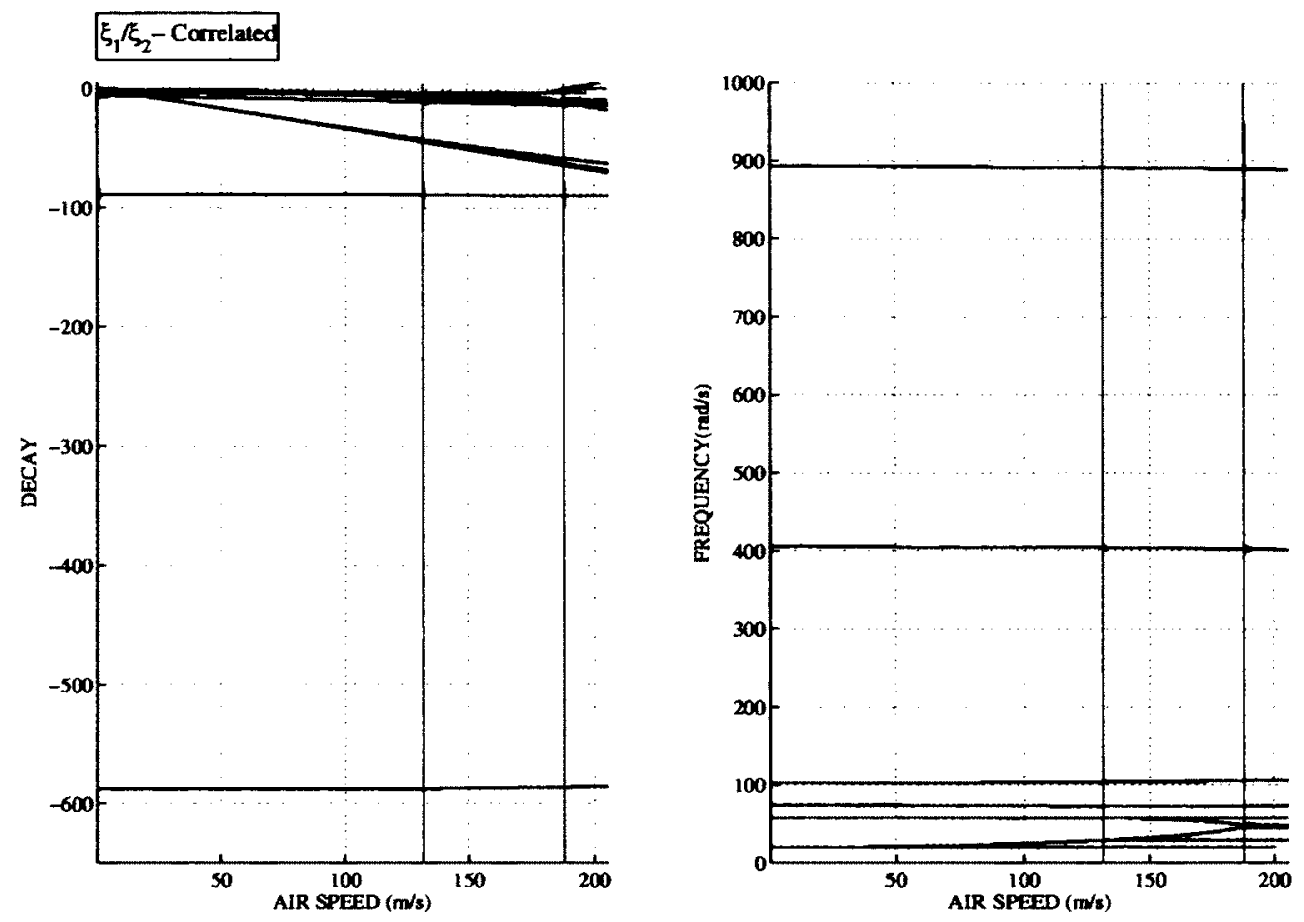

Figure 6.95: Modal parameter pdfs resulting from randomness in correlated damping terms with $10 \% \mathrm{COV}$, obtained at 3 test airspeeds: $\approx 0 \mathrm{~m} / \mathrm{s}, 131.0 \mathrm{~m} / \mathrm{s}, 187.91 \mathrm{~m} / \mathrm{s}$
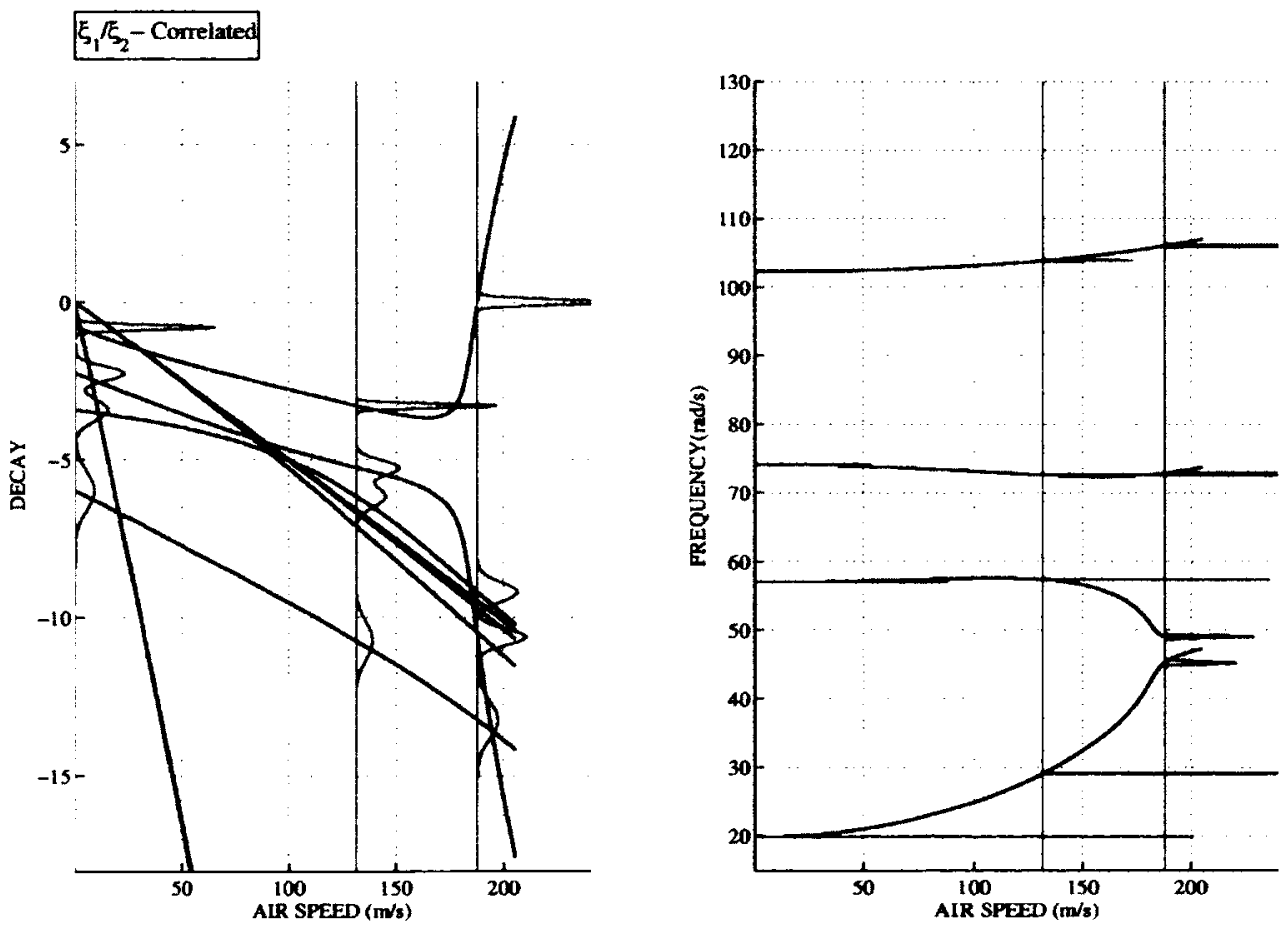

Figure 6.96: Magnified view of coalescing mode's modal parameter pdfs for uncertainty in correlated damping terms 


\subsection{Comparison of Cantilever and Rigid Wing Models for Quasisteady Aerodynamics}

The probabilistic results of the modal parameters between the 3-D cantilever wing and the rigid wing for quasisteady aerodynamics are compared. The results show significant differences. It has already been noted that the cantilever wing experiences a more explosive type of flutter. However, there are also additional probabilistic effects due to significant statistical overlap. These differences are summarized below. A more explosive type of flutter typically produces greater non-stationary effects of the modal parameters in the flutter region. The modal decay pdfs of the coalescing modes experience significant statistical overlap due to presence of additional modes. Uncertainty in the torsional parameters produces a greater influence on the frequency of the bending dominant (unstable) mode. A low uncertainty and greater non-Gaussian features are present in the decay of the flutter mode with uncertainty in inertia. For uncertainty in torsional stiffness, prior to flutter, a larger variance is observed in the flutter mode decay.

\subsection{Comparison of Cantilever and Rigid Wing Models for Unsteady Aerodynamics}

The probabilistic results for the modal parameters of the cantilever wing model and the rigid wing model are compared next for unsteady aerodynamics. There are some significant differences between the two models. The cantilever model exhibits the following additional features that are absent in the rigid wing model. For the cantilever model, the modal decay pdfs of the coalescing modes experience significant statistical overlap due to presence of multiple modes contributing to the response. The non-Gaussian features and lower variance in the decay of the flutter mode are evident with uncertainty in the torsional stiffness and the damping ratio of the second mode. Uncertainty in mass moment of inertia produces nearly Gaussian features in the pre-flutter decay and the flutter frequency of the first mode. Uncertainty in the torsional parameters has influence on the frequency of the bending dominant (first) mode. 


\section{Chapter 7}

\section{Conclusion}

The primary objective of this thesis is to examine the coalescence flutter problem from a probabilistic prospective. The investigation focuses on (1) a probabilistic flutter speed analysis and (2) a probabilistic characterization of the aeroelastic modal parameters. For numerical studies, an elastically mounted rigid wing and a uniform cantilever wing are considered for both quasisteady and unsteady aerodynamics. For the specific cases studied in this investigation, the conclusions are summarized as follows:

- In general, the effects of uncertainty in the flutter speed due to randomness of inertia and stiffness parameters proves to be significant. Uncertainty in damping, on the other hand, has some significance in cases of mild flutter type.

- The modal frequency pdfs of the coalescing modes show almost stationary trends for mild flutter types. However, for explosive flutter type, strong non-stationary trends in the pdfs of modal frequencies are observed in the flutter region. The modal decay pdfs show significant non-stationary features as a function of airspeed for inertia and stiffness parameter uncertainty.

- In most cases, the statistically correlated uncertainty in the inertia and stiffness parameter decreases the uncertainty of the flutter speeds.

- In contrast to independent parameter uncertainty, the correlation between the uncertain 
structural parameters significantly influence the probabilistic characteristics of the modal parameters.

- In numerous cases, the statistical overlap of the modal parameters is observed, which leads to significant non-Gaussian features in the probabilistic density plots. For explosive flutter the statistical overlap of the coalescing modal frequencies is more pronounced.

- In contrast to the 2-dof rigid wing, the statistical overlap is more prominent in the cantilever wing due to the participation of large number of structural modes in the structural response. 


\section{Appendix A}

\section{Equations of Motion for Rigid Wing Model}

The derivation of the aeroelastic system equations of motion for a 2-D rigid wing model are detailed in this section. This derivation is taken from texts by Hodges and Pierce [83] and Fung [1]. First some basic assumptions are stated:

- the section is thin and symmetric

- the amplitude of vibration is small

- the Reynolds Number is high, representative of inviscid flow

- the Mach Number is low, being representative of subsonic flight

These assumptions simplify the theoretical treatment of this problem and permit a linear representation of the aeroelastic system. The derivation of the aerodynamic forcing is based on the Thin Airfoil theory, which uses the Potential Flow theory to capture the flow around the airfoil.

Fig. (A.1) shows the schematic of the symmetric rigid airfoil undergoing heave and pitch motion. Here the chord length of the airfoil is denoted by $c$ and the half-chord by $b$. The mass center (CG), aerodynamic center (AC) and elastic axis (EA) locations are also illustrated in this figure. The mass center is the center of gravity of this airfoil. The aerodynamic center is a point on the airfoil, where the aerodynamic moment remains constant with respect to changing angle of attack (in low angle range and high Reynolds Number). For subsonic flow, this point is the quarter-chord point of the airfoil aft leading edge. The shear 


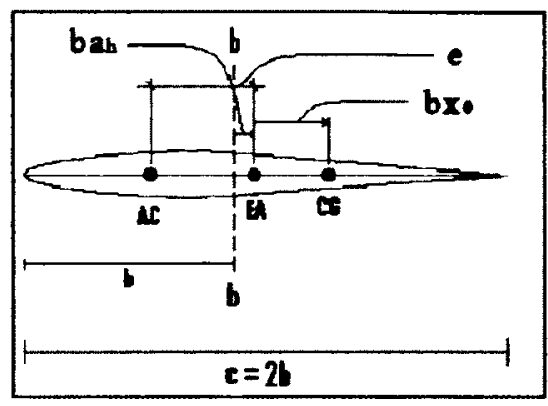

Figure A.1: Airfoil with definition of geometric parameters center is assumed to coincide with the elastic axis of the airfoil. Additionally, certain distance parameters associated to these three points need to be noted. As seen in the figure, $x_{\theta}$ is normalized mass static unbalance with respect to the rotational axis and $a_{h}$ is normalized distance

between the half-chord point and the elastic axis. The normalization constant is the half-chord $b$. The parameter $e$ represents the distance between the rotation axis and the aerodynamic center (AC).

The following figure A.2 shows the location of the springs associated with the structural model. There are two linear springs that provide stiffness against motion in heave

and pitch. The stiffness in heave and pitch is represented by $k_{h}$ and $k_{\theta}$, respectively. These springs introduce a reaction force proportional to the deflections of the airfoil, which resists the deflection. The rotational spring is assumed to be aligned with the elastic axis, and in effect the shear center, therefore the elastic axis serves as both, the rotational

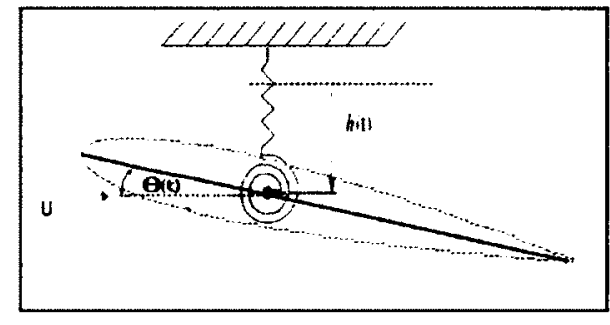

Figure A.2: Airfoil with definition of the translational and rotational springs and translational axis.

Prior to derivation of equations of motion, a set of degrees of freedom describing the motions will be defined and a brief description of the aerodynamic forces will be included. Figure A.3 shows the degrees of freedom in blue, which are (translational) heave $(h)$ and 


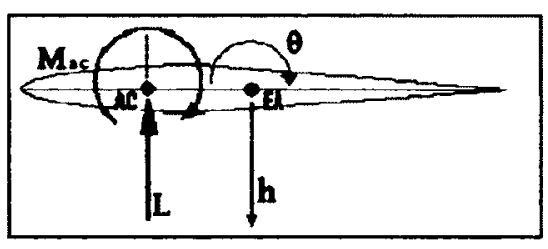

Figure A.3: Definition of the forcing acting on airfoil, lift and moment, as well as the degrees of freedom in heave and pitch about elastic axis

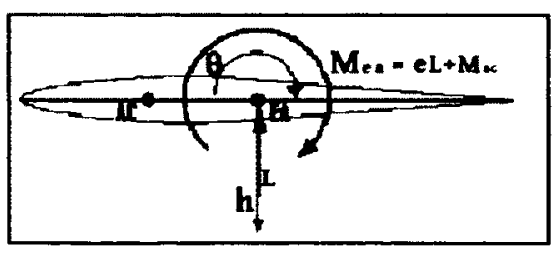

Figure A.4: Redefinition of lift and moment about aerodynamic center into equivalent forces about elastic axis

(rotational) pitch $(\theta)$ at the elastic axis. The two forces associated with these motions are lift $(L)$ and moment $\left(M_{a c}\right)$, which are taken about the aerodynamic center. To simplify the analysis, the aerodynamic forces are calculated with respect to the elastic axis (see in fig. (A.4)).

The dynamics of the aeroelastic system can be expressed as [1]:

$$
\left[M_{s t r}\right]\left\{\begin{array}{c}
\ddot{h} \\
\ddot{\theta}
\end{array}\right\}+\left[B_{s t r}\right]\left\{\begin{array}{c}
\dot{h} \\
\dot{\theta}
\end{array}\right\}+\left[K_{s t r}\right]\left\{\begin{array}{l}
h \\
\theta
\end{array}\right\}=\left\{\begin{array}{c}
-L \\
M_{e a}
\end{array}\right\}
$$

where $\left[M_{s t r}\right]\left[B_{s t r}\right]$ and $\left[K_{s t r}\right]$ are the system structural inertia, damping and stiffness matrices, which are given by:

$$
\left[M_{s t r}\right]=\left[\begin{array}{cc}
m & m b x_{\theta} \\
m b x_{\theta} & I_{e a}
\end{array}\right] \quad\left[K_{s t r}\right]=\left[\begin{array}{cc}
k_{h} & 0 \\
0 & k_{\theta}
\end{array}\right] \quad\left[B_{s t r}\right]=\alpha_{0}\left[M_{s t r}\right]+\alpha_{1}\left[K_{s t r}\right]
$$

The structural damping properties are represented by viscous and proportional Rayleigh damping, which gives a good approximation of the damping properties for a simple structural system vibrating at low amplitudes [92]. The proportionality is set with respect to the mass and stiffness matrices. The coefficients $\alpha_{0}$ and $\alpha_{1}$ are taken to attain the prescribed damping ratios, $\xi_{i}$ and $\xi_{j}$, of the two structural modes. Based on $\xi_{i}$ and $\xi_{j}$, the $\alpha_{0}$ and $\alpha_{1}$ are obtained as follows:

$$
\left[\begin{array}{cc}
\frac{1}{2 \omega_{i}} & \frac{\omega_{i}}{2} \\
\frac{1}{2 \omega_{j}} & \frac{\omega_{j}}{2}
\end{array}\right]\left\{\begin{array}{c}
\alpha_{0} \\
\alpha_{1}
\end{array}\right\}=\left\{\begin{array}{c}
\xi_{i} \\
\xi_{j}
\end{array}\right\}
$$

where $\omega_{i}$ and $\omega_{j}$ are the natural frequencies of the two modes used to formulate the proportional damping. In this study the two chosen modes, $i$ and $j$, are the two coalescing modes. For studies 
where a greater number of modes are involved in the solution, the development of the damping matrix may take other forms. However, since coalescence flutter problem primarily depends on the two coalescing modes, a two modal damping approximation is acceptable.

Returning to the definition of variables of eq. (A.2), the $m$ represents the distributed mass of the wing and $I_{e a}$ represents the mass moment of inertia of the wing about the elastic axis. The $I_{e a}$ can be restated as $I_{e a}=I_{c}+m r_{g}^{2}$, where $I_{c}$ represents the mass moment of inertia of the wing about the center of gravity and $r_{g}=b x_{\theta}$.

\section{Aerodynamic Loading}

\section{Quasisteady Aerodynamics}

To calculate the aerodynamic loads, the airfoil is assumed to undergo small amplitude vibrations, at a high Reynolds Number regime in the subsonic flow [1]. The steady aerodynamic lift force can be calculated as:

$$
\begin{gathered}
L_{\text {steady }}=\rho_{\infty} U_{\infty}^{2} b C_{L} \quad \text { where } \quad C_{L}=\left(\frac{\partial C_{L}}{\partial \theta}\right) \theta_{\text {eff }}=C_{L \alpha} \theta_{\text {eff }} \\
\text { and } \quad \theta_{\text {eff }}=\left(\alpha_{i}+\theta\right)
\end{gathered}
$$

The $\rho_{\infty}$ and $U_{\infty}$ are the free stream air density and air velocity respectively. $\alpha_{i}$ is an initial angle of incidence (assumed to be zero in this study) and $\theta$ is the static angle of attack representing the pitch motion. $\theta_{\text {eff }}$ is the effective angle of attack, which combines the effect both components of the angle between the air flow and the airfoil chord-line; and $C_{L}$ is the lift coefficient

also restated as $C_{L}(\theta)=C_{L \alpha} \theta_{\text {eff }}$. When linear, 2-D and incompressible aerodynamics are considered, $C_{L \alpha}$ becomes $2 \pi$.

In quasisteady aerodynamics, the heave and pitch motions lead to the following effective angle of attack [1]:

$$
\theta_{e f f}=\left(\theta(t)+\frac{\dot{h}(t)}{U_{\infty}}+\frac{b\left(\frac{1}{2}-a_{h}\right) \dot{\theta}(t)}{U_{\infty}}\right)
$$


leading to the following expression for the lift force:

$$
L(t)=\rho_{\infty} U_{\infty}^{2} b C_{L \alpha}\left(\theta(t)+\frac{\dot{h}(t)}{U_{\infty}}+\frac{b\left(\frac{1}{2}-a_{h}\right) \dot{\theta}(t)}{U_{\infty}}\right)
$$

Next, the expression of the quasisteady moment about the elastic axis will be provided. From prior derivations, it was shown that the moment expression can be introduced in the following form:

$$
M_{e a}(t)=e L(t)+M_{a c}
$$

Once again, it will be mentioned that $M_{a c}$, moment about aerodynamic center, remains constant if assumptions of small displacements and linear aerodynamics are applicable. Parameter $e$ in this expression represents the distance between the elastic axis and the aerodynamic center, which can be expressed as $b\left(\frac{1}{2}+a_{h}\right)$. Substituting eq. (A.7) into eq. (A.8), the aerodynamic moment can be expressed as:

$$
M_{e a}=b\left(\frac{1}{2}+a_{h}\right) \rho_{\infty} U_{\infty}^{2} b C_{L \alpha}\left(\theta(t)+\frac{\dot{h}(t)}{U_{\infty}}+\frac{b\left(\frac{1}{2}-a_{h}\right) \dot{\theta}(t)}{U_{\infty}}\right)+M_{a c}
$$

where $M_{a c}=-\frac{\rho_{\infty} U_{\infty} b^{3} C_{L \alpha}}{4} \dot{\theta}(t)$ is derived by the Thin Airfoil theory [1]. Finally, $M_{e a}$ can be expressed as:

$$
M_{\varepsilon a}=b^{2}\left(\frac{1}{2}+a_{h}\right) \rho_{\infty} U_{\infty}^{2} C_{L \alpha}\left(\theta(t)+\frac{\dot{h}(t)}{U_{\infty}}+\frac{b\left(\frac{1}{2}-a_{h}\right) \dot{\theta}(t)}{U_{\infty}}\right)-\frac{\rho_{\infty} U_{\infty} b^{3} C_{L \alpha}}{4} \dot{\theta}(t)
$$

The effect of added mass is next included in the calculation of the aerodynamic loading [1], yielding the following expressions for lift and moment:

$$
\begin{aligned}
-L(t)=-\frac{1}{2} \rho_{\infty} \mathcal{U}_{\infty}^{2} C_{L \alpha} & {\left[\frac{b^{2}}{U_{\infty}^{2}} \ddot{h}(t)-\frac{b^{3} a_{h}}{U_{\infty}^{2}} \ddot{\theta}(t)+\frac{2 b}{U_{\infty}} \dot{h}(t)+\frac{2 b^{2}\left(1-a_{h}\right)}{U_{\infty}} \dot{\theta}(t)+2 b \theta(t)\right] } \\
M_{e a}(t)=-\frac{1}{2} \rho_{\infty} \mathcal{U}_{\infty}^{2} C_{L \alpha}[ & {\left[-\frac{b^{3} a_{h}}{U_{\infty}^{2}} \ddot{h}(t)+\frac{b^{4}\left(\frac{1}{8}+a_{h}{ }^{2}\right)}{U_{\infty}^{2}} \ddot{\theta}(t)-\frac{2 b^{2}\left(\frac{1}{2}+a_{h}\right)}{U_{\infty}} \dot{h}(t)\right.} \\
& \left.-\frac{2 b^{3}\left(\frac{1}{2}-a_{h}\right) a_{h}}{U_{\infty}} \dot{\theta}(t)-2 b^{2}\left(\frac{1}{2}+a_{h}\right) \theta(t)\right]
\end{aligned}
$$

These additional effects arise due to the displacement of air particles by the oscillating wing. 
The added mass effects are included in the terms of eqs. (A.11) and (A.13) with the acceleration and velocity terms $\ddot{h}(t), \ddot{\theta}(t)$ and $\dot{\theta}(t)$. Typically, the effects of added mass (otherwise known as the non-circulatory effects) are included with the unsteady aerodynamic loading. In this study, these effects will be introduced into the quasisteady aerodynamics. This treatment does not affect the validity of the quasisteady model.

\section{Unsteady Aerodynamics}

For the unsteady aerodynamics, the circulatory components of the aeroelastic forces, stemming from the trailing edge vortex shedding are considered. As a trailing edge vortex is shed following a certain change in angle of attack, a steady-state in the aerodynamic load is achieved after the vortex has traveled approximately ten chord-lengths downstream of the airfoil. Within these ten chord-length bounds, the effects of trailing vortices are accounted by the Wagner's Function in time domain or the Theodorsen's Function in the frequency domain.

A two-term approximation of the Wagner's Function is given by [1]:

$$
\Phi(\tau)=1-0.165 \mathbf{e}^{-0.0455 \tau}-0.335 \mathbf{e}^{-0.3 \tau}
$$

where $\tau$ is a dimensionless time parameter defined as $\tau=\frac{U_{\infty} t}{b}$; In the dimensional form, the Wagner's function can be expressed as

$$
\Phi(t)=1-0.165 \mathbf{e}^{\frac{-0.0455 U_{\infty}}{b} t}-0.335 \mathbf{e}^{\frac{-0.3 U_{\infty}}{b} t}
$$

In the above expression, the circulatory component of lift can be approximated by:

$$
L_{c}(t)=\rho_{\infty} U_{\infty} b C_{L \alpha} \omega\left(\theta_{e f f}\right) \Phi(t)
$$

where $\omega\left(\theta_{\text {eff }}\right)$ is the downwash, which is the perpendicular component of velocity to the chord line. This component can be seen in fig. (A.5), where the downwash $\omega\left(\theta_{e f f}\right)$ is 


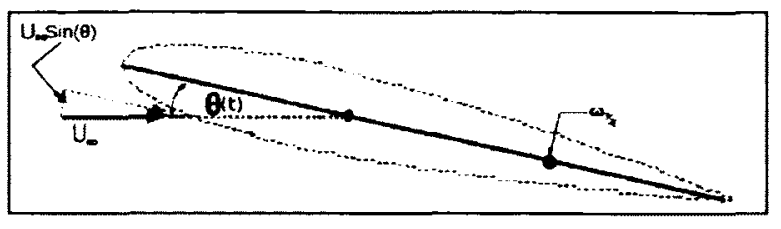

Figure A.5: Illustration of airfoil and component of velocity that represents downwash evaluated by $U_{\infty} \operatorname{Sin}\left(\theta_{\text {eff }}\right)$. Due to the assumption of small angular deflections, the downwash term can be restated as $\omega\left(\theta_{\text {eff }}\right)=U_{\infty} \theta_{\text {eff } f}$. In eq.

(A.16), the Wagner's function $\Phi$

introduces the transient effects caused by the trailing edge vortex shedding.

Using the Thin Airfoil theory, the effective downwash can be represented as [1]:

$$
\omega_{\frac{3 c}{4}}=U_{\infty} \theta_{e f f}=U_{\infty}\left(\theta(t)+\frac{\dot{h}}{U_{\infty}}+\frac{b\left(\frac{1}{2}-a_{h}\right) \dot{\theta}}{U_{\infty}}\right)
$$

Now, a differential lift term can be obtained due to an instantaneous differential change of downwash at time $t_{0}$. At time $t_{0}$ a vortex originates and travels downstream, inducing a reducing magnitude of the differential lift until a steady-state is reached. The differential lift introduced by this traveling vortex is represented as:

$$
d L_{c}(t)=\rho_{\infty} U_{\infty} b C_{L \alpha} d \omega_{\frac{3 c}{4}}\left(t_{0}\right) \Phi\left(t-t_{0}\right)
$$

Integrating the differential lift expression, we obtain the total circulatory lift force as:

$$
L_{c}(t)=\rho_{\infty} U_{\infty} b C_{L \alpha} \int_{0}^{t} \Phi(t-s) \frac{d \omega_{\frac{3 c}{4}}(s)}{d s} d s
$$

The circulatory lift force can be expressed as [1]:

$$
L_{c}(t)=\rho_{\infty} U_{\infty} b C_{L \alpha}\left[\omega_{\frac{3 c}{4}}(0) \Phi(t)+\int_{0}^{t} \Phi(t-s) \frac{d \omega_{\frac{3 c}{4}}(s)}{d s} d s\right]
$$

where the first term on the right hand side accounts for the initial conditions. From this equation, it becomes evident that the equations of motion are of integro-differential form. In order to solve them analytically, the integral needs to be transformed. This transformation was taken from a thesis [89].

Focusing on the bracketed term in the above equation, using the Wagner's function from eq. 
(A.15) and integrating by parts one obtains:

$$
\begin{aligned}
& \omega_{\frac{3 c}{4}}(0) \Phi(t)+\int_{0}^{t} \Phi(t-s) \frac{d \omega_{\frac{3 c}{4}}(s)}{d s} d s \\
= & \omega_{\frac{3 c}{4}}(t) \Phi(0)+\int_{0}^{t} \omega_{\frac{3 c}{4}}(s)\left[b_{1} A_{1} \mathrm{e}^{-b_{1}(t-s)}+b_{2} A_{2} \mathrm{e}^{-b_{2}(t-s)}\right] d s
\end{aligned}
$$

Using the Laplace transformation (assuming the initial conditions are negligible), eq. (A.21) becomes:

$$
\omega_{\frac{3 \mathrm{c}}{4}}(S) \Phi(0)+\left[\frac{S \omega_{\frac{3 c}{4}}(S)\left(b_{1} A_{1}+b_{2} A_{2}\right)+\omega_{\frac{3 c}{4}}(S)\left(A_{1}+A_{2}\right) b_{1} b_{2}}{\left(S+b_{1}\right)\left(S+b_{2}\right)}\right]
$$

Defining a new parameter

$$
z(S)=\frac{\omega_{\frac{3 c}{4}}(S)}{\left(S+b_{1}\right)\left(S+b_{2}\right)}
$$

eq. (A.22) is restated as:

$$
\omega_{\frac{3 c}{4}}(S) \Phi(0)+\left[S z(S)\left(b_{1} A_{1}+b_{2} A_{2}\right)+z(S)\left(A_{1}+A_{2}\right) b_{1} b_{2}\right]
$$

For zero initial condition $z(t=0)=0$, the inverse Laplace transform of eq. (A.24) yields:

$$
\omega_{\frac{3 c}{4}}(t) \Phi(0)+\dot{z}(t)\left(b_{1} A_{1}+b_{2} A_{2}\right)+z(t)\left(A_{1}+A_{2}\right) b_{1} b_{2}
$$

In terms of the variable $z(t)$, the expression for the circulatory lift force becomes:

$$
L_{c}(t)=\rho_{\infty} U_{\infty} b C_{L \alpha}\left[\omega_{\frac{3 c}{4}}(t) \Phi(0)+\dot{z}(t)\left(b_{1} A_{1}+b_{2} A_{2}\right)+z(t)\left(A_{1}+A_{2}\right) b_{1} b_{2}\right]
$$

In order to solve for three unknowns, $h(t), \theta(t)$ and $z(t)$ an additional equation of state has to be obtained in terms of $z(t)$. From eq. (A.23) note that:

$$
z(S)\left(S+b_{1}\right)\left(S+b_{2}\right)=z(S)\left(S^{2}+S\left(b_{1}+b_{2}\right)+b_{1} b_{2}\right)=\omega_{\frac{3 c}{4}}(S)
$$

For trivial initial conditions, $z(t=0)=0$ and $\dot{z}(t=0)=0$, eq. (A.27) leads to:

$$
\ddot{z}(t)+\dot{z}(t)\left(b_{1}+b_{2}\right)+z(t) b_{1} b_{2}=\omega_{\frac{3 c}{4}}(t)
$$


Thus, the equations of motion with unsteady aerodynamic forcing can be recast as:

$$
\left[\begin{array}{ccc}
m & m b x_{\theta} & 0 \\
m b x_{\theta} & I_{e a} & 0 \\
0 & 0 & 1
\end{array}\right]\left\{\begin{array}{c}
\ddot{h} \\
\ddot{\theta} \\
\ddot{z}
\end{array}\right\}+\left[\begin{array}{cc}
{\left[B_{s t r}\right.} & \{0\} \\
{[0]} & \left(b_{1}+b_{2}\right)
\end{array}\right]\left\{\begin{array}{c}
\dot{h} \\
\dot{\theta} \\
\dot{z}
\end{array}\right\}+\left[\begin{array}{ccc}
k_{h} & 0 & 0 \\
0 & k_{\theta} & 0 \\
0 & 0 & b_{1} b_{2}
\end{array}\right]\left\{\begin{array}{c}
h \\
\theta \\
z
\end{array}\right\}=\left\{\begin{array}{c}
-L(t) \\
M_{e a}(t) \\
\omega_{\frac{3 c}{t}}(t)
\end{array}\right\}
$$

where:

$$
\begin{aligned}
-L(t)= & -\frac{1}{2} \rho_{\infty} \mathcal{U}_{\infty}^{2} C_{L \alpha}\left[\frac{b^{2}}{U_{\infty}^{2}} \ddot{h}(t)-\frac{b^{3} a_{h}}{U_{\infty}^{2}} \ddot{\theta}(t)+\frac{b}{U_{\infty}} \dot{h}(t)\right. \\
& \left.+\frac{b^{2}\left(\frac{3}{2}-a_{h}\right)}{U_{\infty}} \dot{\theta}(t)+\frac{2 b\left(A_{1} b_{1}+A_{2} b_{2}\right)}{U_{\infty}} \dot{z}(t)+b \theta(t)+\frac{2 b\left(b_{1} b_{2}\left(A_{1}+A_{2}\right)\right)}{U_{\infty}} z(t)\right] \\
M_{e a}(t)= & -\frac{1}{2} \rho_{\infty} \mathcal{U}_{\infty}^{2} C_{L \alpha}\left[-\frac{b^{3} a_{h}}{U_{\infty}^{2}} \ddot{h}(t)+\frac{b^{4}\left(\frac{1}{8}+a_{h}{ }^{2}\right)}{U_{\infty}^{2}} \ddot{\theta}(t)-\frac{b^{2}\left(\frac{1}{2}+a_{h}\right)}{U_{\infty}} \dot{h}(t)+\frac{b\left(b\left(\frac{1}{2}-a_{h}\right)\right)^{2}}{U_{\infty}} \dot{\theta}(t)\right. \\
& \left.-\frac{2 b^{2}\left(\frac{1}{2}+a_{h}\right)\left(A_{1} b_{1}+A_{2} b_{2}\right)}{U_{\infty}} \dot{z}(t)-b^{2}\left(\frac{1}{2}+a_{h}\right) \theta(t)-\frac{2 b^{2}\left(\frac{1}{2}+a_{h}\right)\left(b_{1} b_{2}\left(A_{1}+A_{2}\right)\right)}{U_{\infty}} z(t)\right] \\
\omega_{\frac{3 c}{4}}(t)= & U_{\infty} \theta(t)+\dot{h}+b\left(\frac{1}{2}-a_{h}\right) \dot{\theta}
\end{aligned}
$$

Recall that $A_{1}=0.165, A_{2}=0.335, b_{1}=\frac{0.0455 U_{\infty}}{b}, b_{2}=\frac{0.3 U_{\infty}}{b}$. The non-circulatory effects remain completely unchanged from the quasisteady analysis. 


\section{Appendix B}

\section{State Space Transformation}

The dynamics of the aeroelastic system are given by:

$$
\left[M_{a e}\right]\left\{\begin{array}{c}
\ddot{h} \\
\ddot{\theta}
\end{array}\right\}+\left[B_{a e}\right]\left\{\begin{array}{c}
\dot{h} \\
\dot{\theta}
\end{array}\right\}+\left[K_{a e}\right]\left\{\begin{array}{l}
h \\
\theta
\end{array}\right\}=\{F\}
$$

Assuming $\left\{X_{1}\right\}=\left\{\begin{array}{c}h \\ \theta\end{array}\right\}$ and $\left\{X_{2}\right\}=\left\{\dot{X}_{1}\right\}=\left\{\begin{array}{c}\dot{h} \\ \dot{\theta}\end{array}\right\}$, the above second order system is converted into a first order model defined as

$$
\begin{aligned}
& \left\{\dot{X}_{1}\right\}=\left\{X_{2}\right\} \\
& \left\{\dot{X}_{2}\right\}=-\left[M_{a e}\right]^{-1}\left[B_{a e}\right]\left\{X_{2}\right\}-\left[M_{a e}\right]^{-1}\left[K_{a e}\right]\left\{X_{1}\right\}-\left[M_{a e}\right]^{-1}\{F\}
\end{aligned}
$$

In the matrix form, the above equation becomes

$$
\left\{\begin{array}{l}
\dot{h} \\
\dot{\theta} \\
\ddot{h} \\
\ddot{\theta}
\end{array}\right\}=\left[\begin{array}{cc}
{[0]} & {[I]} \\
-\left[M_{a e}\right]^{-1}\left[K_{a e}\right] & -\left[M_{a e}\right]^{-1}\left[B_{a e}\right]
\end{array}\right]\left\{\begin{array}{c}
h \\
\theta \\
\dot{h} \\
\dot{\theta}
\end{array}\right\}+\left\{\begin{array}{c}
0 \\
-\left[M_{a e}\right]^{-1}\{F\}
\end{array}\right\}
$$


Compactly, the above equation can be written as

$$
\{\dot{x}\}=[A]\{x\}+\{f\}
$$

where $[A]$ and $\{x\}$ represent the state matrix and state vector respectively. $\{f\}$ represents the external forcing vector, excluding the aerodynamic effects, which is not considered in this study (i.e. $\{f\}=\{0\}$ ). 


\section{Appendix C}

\section{System Parameters}

\begin{tabular}{||l|c|l|l||}
\hline PARAMETER & Symbol & 2D Airfoil & 3D Wing \\
\hline \hline mass & $m$ & $35.7187 \mathrm{~kg} / \mathrm{m}$ & $35.7187 \mathrm{~kg} / \mathrm{m}$ \\
normalized radius of & & & \\
gyration ,** $^{*}$ & $r_{g}$ & 0.5 & 0.5 \\
heave/bending stiffness & $k_{h} / E I$ & $8.75 \times 10^{4} \mathrm{~N} / \mathrm{m}^{2}$ & $9.77 \times 10^{6} \mathrm{Nm}^{2}$ \\
pitch/torsional stiffness & $k_{\theta} / G J$ & $6.57 \times 10^{4} \mathrm{~N} / \mathrm{rad}$ & $9.89 \times 10^{5} \mathrm{Nm}^{2}$ \\
chord length & $c$ & $1.8288 \mathrm{~m}$ & $1.8288 \mathrm{~m}$ \\
span & $L$ & $6.096 \mathrm{~m}$ & $6.096 \mathrm{~m}$ \\
normalized static imbalance & $x_{\theta}$ & 0.2 & 0.2 \\
damping ratio (mode 1) & $\xi_{1}$ & 0.05 & 0.05 \\
damping ratio (mode 2) & $\xi_{2}$ & 0.05 & 0.05 \\
free stream fluid density & $\rho_{\infty}$ & $1.225 \mathrm{~kg} / \mathrm{m}^{3}$ & $1.225 \mathrm{~kg} / \mathrm{m}^{3}$ \\
normalized distance btw & & & -0.34 \\
EA and mid-chord & $a_{h}$ & -0.34 & $2 \pi\left(1-\eta^{2}\right)$ \\
lift coefficient gradient & $\bar{C}_{l \alpha * *}$ & $2 \pi$ & 100000 \\
\hline \hline Num. of Samples & - & 50000 & \\
\hline
\end{tabular}

Table C.1: Model parameters for Case 1 


\begin{tabular}{||l|c|l|l||}
\hline PARAMETER & Symbol & 2D Airfoil & 3D Wing \\
\hline \hline mass & $m$ & $35.7187 \mathrm{~kg} / \mathrm{m}$ & $35.7187 \mathrm{~kg} / \mathrm{m}$ \\
normalized radius of & & & \\
gyration ${ }^{* * *}$ & $r_{g}$ & 1.05 & 1.05 \\
heave/bending stiffness & $k_{h} / E I$ & $8.74608 \times 10^{4} \mathrm{~N} / \mathrm{m}^{2}$ & $9.77 \times 10^{6} \mathrm{Nm}^{2}$ \\
pitch/torsional stiffness & $k_{\theta} / G J$ & $2.62688 \times 10^{4} \mathrm{~N} / \mathrm{rad}$ & $3.956 \times 10^{5} \mathrm{Nm}^{2}$ \\
chord length & $c$ & $1.8288 \mathrm{~m}$ & $1.8288 \mathrm{~m}$ \\
span & $L$ & $6.096 \mathrm{~m}$ & $6.096 \mathrm{~m}$ \\
normalized static imbalance* & $x_{\theta}$ & 0.95 & 0.95 \\
damping ratio (mode 1) & $\xi_{1}$ & 0.04 & 0.04 \\
damping ratio (mode 2) & $\xi_{2}$ & 0.04 & 0.04 \\
free stream fluid density & $\rho_{\infty}^{* * *}$ & $1.225 \mathrm{~kg} / \mathrm{m}^{3}$ & $1.225 \mathrm{~kg} / \mathrm{m}^{3}$ \\
normalized distance btw & & & \\
EA and mid-chord* & $\rho_{h}$ & -1.0 & -1.0 \\
lift coefficient gradient & & $2 \pi\left(1-\eta^{2}\right)$ \\
\hline \hline Num. of Samples & $\bar{C}_{l \alpha}$ & $2 \pi$ & 100000 \\
\hline
\end{tabular}

Table C.2: Model parameters for Case 2

\begin{tabular}{||l|c|l|}
\hline PARAMETER & Symbol & 2D Airfoil \\
\hline \hline mass & $m$ & $75 \mathrm{~kg} / \mathrm{m}$ \\
normalized radius of gyration ${ }^{* * * *}$ & $r_{g}$ & 0.47 \\
heave stiffness & $k_{h}$ & $7.5 \times 10^{4} \mathrm{~N} / \mathrm{m}^{2}$ \\
pitch stiffness & $k_{\theta}$ & $2.12 \times 10^{4} \mathrm{~N} / \mathrm{rad}$ \\
chord length & $c$ & $1.8288 \mathrm{~m}$ \\
span & $L$ & $6.096 \mathrm{~m}$ \\
normalized static imbalance* & $x_{\theta}$ & 0.2 \\
damping ratio (mode 1) & $\xi_{1}$ & 0.05 \\
damping ratio (mode 2) & $\xi_{2}$ & 0.05 \\
free stream fluid density & $\rho_{\infty}$ & $1.225 \mathrm{~kg} / \mathrm{m}^{3}$ \\
normalized distance btw EA and mid-chord & $a_{h}$ & -0.34 \\
lift coefficient gradient ${ }^{* * * *}$ & $\bar{C}_{l \alpha}$ & $2 \pi$ \\
\hline \hline Num. of Samples & - & 50000 \\
\hline
\end{tabular}

Table C.3: Model parameters for Case 3

\section{NOTES:}

* $\quad$ parameters have been normalized by half-chord length $b=\frac{c}{2}$

** radius of gyration is defined about Center of Gravity

*** density defined at standard conditions

**** $\eta$ is a non-dimensional span defined as: $\eta=\frac{x}{L}$; additionally, the coefficient for compressibility effects is not included 


\section{Appendix D}

\section{Analytical Expression for Flutter Speed}

Using the Routh-Hurwitz stability criterion, a semi-analytical relationship can be obtained for the flutter speed as function of the system parameters. Using the Laplace transformation of eq. (3.3) for a 2-D rigid wing model with quasisteady aerodynamic forcing the following system matrix is obtained:

$$
\left[\begin{array}{cc}
s^{2} M_{a e(1,1)}+s B_{a e(1,1)}+K_{a e(1,1)} & s^{2} M_{a e(1,2)}+s B_{a e(1,2)}+K_{a e(1,2)} \\
s^{2} M_{a e(2,1)}+s B_{a e(2,1)}+K_{a e(2,1)} & s^{2} M_{a e(2,2)}+s B_{a e(2,2)}+K_{a e(2,2)}
\end{array}\right],
$$

Setting the determinant of this dynamic matrix equal to zero leads to a root finding problem defined by the following polynomial:

$$
P_{4} s^{4}+P_{3} s^{3}+P_{2} s^{2}+P_{1} s+P_{0}=0
$$

$P_{4}, P_{3}, P_{2}, P_{1}, P_{0}$ are all functions of airspeed $\left(U_{\infty}\right)$. According to the Routh-Hurwitz stability criterion [1], which assesses the system stability based on evaluation of its sub-determinants, the system becomes unstable if:

$$
P_{1}\left(P_{3} \times P_{2}-P_{4} \times P_{1}\right)-P_{0} \times P_{3}^{2}=0
$$


The flow velocity that satisfies eq. (D.3) is identified as the flutter speed. This equivalence is reached when one of the modal decays reaches a value of zero [1]. 


\section{Appendix E}

\section{Continuous Aeroelastic System}

The equations illustrated in this section were taken from references $[1,83,92]$. The coupled bending and torsional oscillations of a uniform cantilever are described next for both quasisteady and unsteady aerodynamics. The linear dynamics of the cantilever is modeled by EulerBernoulli's beam together with a rod model.

\section{Quasisteady Aerodynamics}

$$
\begin{aligned}
& m \frac{\partial^{2} h(x, t)}{\partial t^{2}}+m b x_{\theta} \frac{\partial^{2} \theta(x, t)}{\partial t^{2}}+B_{1} \frac{\partial h(x, t)}{\partial t}+B_{2} \frac{\partial \theta(x, t)}{\partial t}+B_{3} \frac{\partial^{5} h(x, t)}{\partial x^{4} \partial t}+E l \frac{\partial^{4} h(x, t)}{\partial x^{4}}=-L(x, t) \\
& I_{e, a} \frac{\partial^{2} \theta(x, t)}{\partial t^{2}}+m b x_{\theta} \frac{\partial^{2} h(x, t)}{\partial t^{2}}+B_{4} \frac{\partial h(x, t)}{\partial t}+B_{5} \frac{\partial \theta(x, t)}{\partial t}+B_{6} \frac{\partial^{3} \theta(x, t)}{\partial x^{2} \partial t}-G J \frac{\partial^{2} \theta(x, t)}{\partial x^{2}}=M_{e, a}(x, t)
\end{aligned}
$$

where

$$
\begin{aligned}
-L(x, t)=-\frac{1}{2} \rho_{\infty} U_{\infty}^{2} C_{L \alpha} & {\left[\frac{b^{2}}{U_{\infty}^{2}} \frac{\partial^{2} h(x, t)}{\partial t^{2}}-\frac{b^{3} a_{h}}{U_{\infty}^{2}} \frac{\partial^{2} \theta(x, t)}{\partial t^{2}}+\frac{2 b}{U_{\infty}} \frac{\partial h(x, t)}{\partial t}\right.} \\
& \left.+\frac{2 b^{2}\left(1-a_{h}\right)}{U_{x}} \frac{\partial \theta(x, t)}{\partial t}+2 b \theta(x, t)\right] \\
M_{e, \alpha}(x, t)=-\frac{1}{2} \rho_{x} U_{\infty}^{2} C_{L a} & {\left[-\frac{b^{3} a_{h}}{U_{\infty}^{2}} \frac{\partial^{2} h(x, t)}{\partial t^{2}}+\frac{b^{4}\left(\frac{1}{8}+a_{h}{ }^{2}\right)}{U_{\infty}^{2}} \frac{\partial^{2} \theta(x, t)}{\partial t^{2}}-\frac{2 b^{2}\left(\frac{1}{2}+a_{h}\right)}{U_{x}} \frac{\partial h(x, t)}{\partial t}\right.} \\
& \left.-\frac{2 b^{3}\left(\frac{1}{2}-a_{h}\right) a_{h}}{U_{\infty}} \frac{\partial \theta(x, t)}{\partial t}-2 b^{2}\left(\frac{1}{2}+a_{h}\right) \theta(x, t)\right]
\end{aligned}
$$

Here $x$ is a span-wise coordinate that measures the distance from the root of the wing and $t$ is a time coordinate. The $m$ and $I_{e a}$ represent the distributed mass and mass moment of inertia about the axis of rotation, respectively. $B_{1}-B_{6}$ represent the proportional structural damping coefficients due to bending and torsional motions. $E$ and $G$ are the modulus of elasticity and the 
shear modulus, respectively. $I$ and $J$ are the second moment of area and the polar moment of inertia, respectively; $b$ is the half-chord length and $x_{\theta}$ is a geometric measure of offset between the center of gravity and the axis of rotation, normalized by half-chord; $a_{h}$ represents the distance between the mid-chord point and the rotational axis, normalized by the half-chord; $C_{L \alpha}$ is the lift curve slope. $h$ and $\theta$ are the vertical (bending) and rotational (torsional) displacements of the elastic axis of the uniform wing. $\rho_{\infty}$ and $U_{\infty}^{2}$ are the free flow air density and air speed, respectively. $L$ and $M_{e a}$ are the distributed lift and moment forces, respectively, acting on the wing.

\section{Unsteady Aerodynamics}

Then, the equations of motion are restated, where the aerodynamic loading takes into account the unsteady effects. In time domain these equations become [1]:

$$
\begin{aligned}
& \bar{m} \frac{\partial^{2} h(x, t)}{\partial t^{2}}+\bar{m}_{x_{\theta}} \frac{\partial^{2} \theta(x, t)}{\partial t^{2}}+\bar{B}_{1} \frac{\partial h(x, t)}{\partial t}+\bar{B}_{2} \frac{\partial \theta(x, t)}{\partial t}+\bar{B}_{3} \frac{\partial^{5} h(x, t)}{\partial x^{4} \partial t}+E I \frac{\partial^{4} h(x, t)}{\partial x^{4}}=-\bar{L}(x, t) \quad \text { (E.3) } \\
& \bar{T}_{e a} \frac{\partial^{2} \theta(x, t)}{\partial t^{2}}+\bar{m}_{b} x_{\theta} \frac{\partial^{2} h(x, t)}{\partial t^{2}}+\bar{B}_{4} \frac{\partial h(r, t)}{\partial t}+\bar{B}_{5} \frac{\partial \theta(x, t)}{\partial t}+\bar{B}_{b} \frac{\partial^{3} \theta(r, t)}{\partial x^{2} \partial t}-G J \frac{\partial^{2} \theta(r, t)}{\partial x^{2}}=\bar{M}_{e a}(x, t) \quad \text { (E.4) } \\
& \frac{\partial^{2} z(x, t)}{\partial t^{2}}+\left(b_{1}+b_{2}\right) \frac{\partial z(r, t)}{\partial t}+b_{1} b_{2} z(x, t)=x^{3} \frac{3}{4} c(x, t)
\end{aligned}
$$

where

$$
\begin{aligned}
& -\bar{L}(x, t)=-\frac{1}{2} \rho_{\infty} U_{\infty}^{2} C_{L \alpha}\left[\frac{b^{2}}{U_{\infty}^{2}} \frac{\partial^{2} h(x, t)}{\partial t^{2}}-\frac{b^{3} a_{h}}{U_{\infty}^{2}} \frac{\partial^{2} \theta(x, t)}{\partial t^{2}}+\frac{h}{U_{\infty}} \frac{\partial h(x, t)}{\partial t}\right. \\
& \left.+\frac{\hbar^{2}\left(\frac{3}{2}-a_{h}\right)}{U_{\infty}} \frac{\partial \theta(x, t)}{\partial t}+b \theta(x, t)+\frac{2 b}{U_{\infty}}\left(A_{1} b_{1}+A_{2} b_{2}\right) \frac{\partial z(x, t)}{\partial t}+\frac{2 b}{U_{\infty}} b_{1} b_{2}\left(A_{1}+A_{2}\right) z(x, t)\right] \\
& \bar{M}_{e a}(x, t)=-\frac{1}{2} \rho_{\infty} U_{\infty}^{2} C_{L \alpha}\left[-\frac{b^{3} a_{h}}{U_{\infty}^{2}} \frac{\partial^{2} h(x, t)}{\partial t^{2}}+\frac{b^{4}\left(\frac{1}{8}+a_{h}{ }^{2}\right)}{U_{\infty}^{2}} \frac{\partial^{2} \theta(x, t)}{\partial t^{2}}-\frac{b^{2}\left(\frac{1}{2}+a_{h}\right)}{U_{\infty}} \frac{\partial h(x, t)}{\partial t}+\frac{b^{3}\left(\frac{1}{2}-a_{h}\right)^{2}}{U_{\infty}} \frac{\partial \theta(x, t)}{\partial t}\right. \\
& \left.-b^{2}\left(\frac{1}{2}+a_{h}\right) \theta(x, t)-\frac{2 b^{2}}{U_{x}}\left(\frac{1}{2}+a_{h}\right)\left(A_{1} b_{1}+A_{2} b_{2}\right) \frac{\partial z(x, t)}{\partial t}-\frac{2 b^{2}}{U_{x}}\left(\frac{1}{2}+a_{h}\right) b_{1} b_{2}\left(A_{1}+A_{2}\right) z(x, t)\right] \\
& u_{3} \frac{3}{2}(x, t)=U x \theta(x, t)+\frac{\partial h(x, t)}{\partial t}+b\left(\frac{1}{2}-a_{h}\right) \frac{\partial \theta(x, t)}{\partial t}
\end{aligned}
$$

here, $z$ is a parameter that accounts for the unsteady effects of the trailing vortices and $A_{1}=$ $0.165, A_{2}=0.335, b_{1}=\frac{0.0455 U_{\infty}}{b}, b_{2}=\frac{0.3 U_{\infty}}{b}$.

In this investigation, the frequency domain approach, based on a Theodorsen's function, was used to validate the time domain approach by Wagner at the point of flutter onset. In 
Theodorsen's approach the aerodynamic forces have the following expressions [1]:

$$
\begin{aligned}
-\bar{L}(x, t)=-\frac{1}{2} \rho_{x} U_{\infty}^{2} C_{L \alpha} & {\left[\frac{b^{2}}{V_{\infty}^{2}} \frac{\partial^{2} h(x, t)}{\partial t^{2}}-\frac{b^{3} a_{h}}{U_{\infty}^{2}} \frac{\partial^{2} \theta(x, t)}{\partial t^{2}}+\frac{2 b C(k)}{V_{\infty}} \frac{\partial h(x, t)}{\partial t}\right.} \\
& \left.+\frac{b^{2}\left(1+2 C(k)\left(\frac{1}{2}-a_{h}\right)\right)}{U_{\infty}} \frac{\partial \theta(x, t)}{\partial t}+2 b C(k) \theta(x, t)\right] \\
\bar{M}_{e a}(x, t)=-\frac{1}{2} \rho_{x} U_{x}^{2} C_{L \alpha} & {\left[-\frac{b^{3} a_{h}}{U_{\infty}^{2}} \frac{\partial^{2} h(x, t)}{\partial t^{2}}+\frac{h^{4}\left(\frac{1}{g}+a_{h}^{2}\right)}{U_{\infty}^{2}} \frac{\partial^{2} \theta(x, t)}{\partial t^{2}}-\frac{2 b^{2} C(k)\left(\frac{1}{2}+a_{h}\right)}{U_{\infty}} \frac{\partial h(x, t)}{\partial t}\right.} \\
& \left.+\frac{b^{3}\left(\frac{1}{2}-a_{h}\right)\left(1-2 C(k)\left(\frac{1}{2}+a_{h}\right)\right)}{U_{\infty}} \frac{\partial \theta(x, t)}{\partial t}-2 b^{2} C(k)\left(\frac{1}{2}+a_{h}\right) \theta(x, t)\right]
\end{aligned}
$$

where the Theodorsen's function $C(k)$ accounts for the effect of the unsteady load that arises due to the trailing vortices and $k$ is the reduced frequency $k=\frac{\omega b}{U_{\infty}}$, where $\omega$ is the frequency of oscillation [1]. The complex representation of the Theodorsen's function is given by expression $C(k)=F(k)+i G(k)$ and its associated real and imaginary components are plotted in fig. (D.1) below.
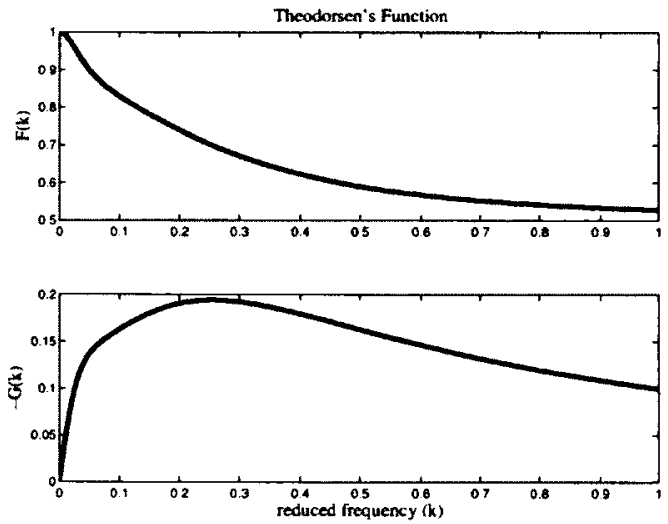

Figure D.1: Theodorsen's function, $C(k)$, at top $F(k)$ and bottom $G(k)$ [1]

\section{Finite Dimensional Representation}

Using the uniform cantilever modes for both bending and torsion as the basis functions, are illustrated in Appendix G, the solution is approximated as: 


$$
\begin{aligned}
& h(x, t) \approx \sum_{i=1}^{n} \Psi_{i}(x) \xi_{i}(t) \\
& \theta(x, t) \approx \sum_{i=1}^{m} \Omega_{i}(x) \gamma_{i}(t) \\
& z(x, t) \approx \sum_{i=1}^{n} \Psi_{i}(x) \iota_{i}(t)+\sum_{j=1}^{m} \Omega_{j}(x) \varsigma_{j}(t)
\end{aligned}
$$

However, for simplicity we only consider the effect of pitch motion in the approximation of downwash $w_{\frac{3}{4} c}(x, t)$ expressed as:

$$
w_{\frac{3}{4} c}(x, t)=U_{\infty} \theta(x, t)+b\left(\frac{1}{2}-a_{h}\right) \frac{\partial \theta(x, t)}{\partial t}
$$

Therefore, the approximation of $z(x, t)$ becomes:

$$
z(x, t) \approx \sum_{j=1}^{m} \Omega_{j}(x) \varsigma_{j}(t)
$$

Using Galerkin projection ( [91]), the discrete system is represented as follows. 


\section{Quasisteady Aerodynamics}

$$
\begin{aligned}
& \bar{m} L\left[\begin{array}{cc}
{[I]_{n \times n}} & b x_{\theta}\left[A_{i j}\right]_{n \times m} \\
b x_{\theta}\left[A_{i j}\right]_{m \times n}^{T} & \frac{\bar{I}_{e x}}{\bar{m}}[I]_{m \times m}
\end{array}\right]\left\{\begin{array}{c}
\ddot{\xi}(t)_{n} \\
\ddot{\gamma}(t)_{m}
\end{array}\right\}+\left[B_{s t r}\right]\left\{\begin{array}{c}
\dot{\xi}(t)_{n} \\
\dot{\gamma}(t)_{m}
\end{array}\right\} \\
& +L\left[\begin{array}{cc}
E I\left[\alpha^{4}\right]_{n \times n} & {[0]_{n \times m}} \\
{[0]_{m \times n}} & G J\left[\vartheta^{2}\right]_{m \times m}
\end{array}\right]\left\{\begin{array}{c}
\xi(t)_{n} \\
\gamma(t)_{m}
\end{array}\right\}= \\
& =-\frac{1}{2} \rho_{\infty} L U_{\infty}^{2} C_{L \alpha}\left[\left[\begin{array}{cc}
\frac{b^{2}}{U_{\infty}^{2}}[I]_{n \times n} & -\frac{b^{3} a_{h}}{U_{\infty}^{2}}\left[A_{i j}\right]_{n \times m} \\
-\frac{b^{3} a_{h}}{U_{\infty}^{2}}\left[A_{i j}\right]_{m \times n}^{T} & \frac{b^{4}\left(\frac{1}{8}+a_{h}{ }^{2}\right)}{U_{\infty}^{2}}[I]_{m \times m}
\end{array}\right]\left\{\begin{array}{c}
\ddot{\xi}(t)_{n} \\
\ddot{\gamma}(t)_{m}
\end{array}\right\}\right. \\
& +\left[\begin{array}{cc}
\frac{2 b}{U_{\infty}}[I]_{n \times n} & \frac{2 b^{2}\left(1-a_{h}\right)}{U_{\infty}}\left[A_{i j}\right]_{n \times m} \\
-\frac{2 b^{2}\left(\frac{1}{2}+a_{h}\right)}{U_{\infty}}\left[A_{i j}\right]_{m \times n}^{T} & -\frac{2 b^{3}\left(\frac{1}{2}-a_{h}\right) a_{h}}{U_{\infty}}[I]_{m \times m}
\end{array}\right]\left\{\begin{array}{c}
\dot{\xi}(t)_{n} \\
\dot{\gamma}(t)_{m}
\end{array}\right\} \\
& \left.+\left[\begin{array}{cc}
{[0]_{n \times n}} & 2 b\left[A_{i j}\right]_{n \times m} \\
{[0]_{m \times n}} & -2 b^{2}\left(\frac{1}{2}+a_{h}\right)[I]_{m \times m}
\end{array}\right]\left\{\begin{array}{c}
\xi(t)_{n} \\
\gamma(t)_{m}
\end{array}\right\}\right]
\end{aligned}
$$

where $L$ represents the wing span, $\alpha_{i, i} \approx 1.8751 / L, 4.69409 / L, 7.85476 / L$ for $i=1,2,3$ and $\vartheta_{i, i}=\frac{(2 i-1) \pi}{2 L}$ are individual elements of diagonal structural stiffness matrix. The matrix coupling terms are represented by [83]:

$$
\left[A_{i j}\right]_{n \times m}=\left(\int_{0}^{L} \Psi_{i}(x) \Omega_{j}(x) d x\right) / L
$$

\section{Unsteady Aerodynamics}

\section{Time Domain:}

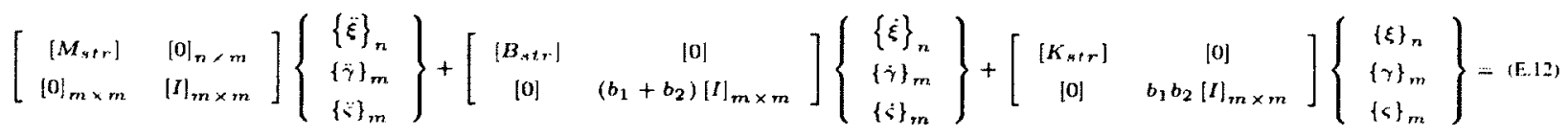




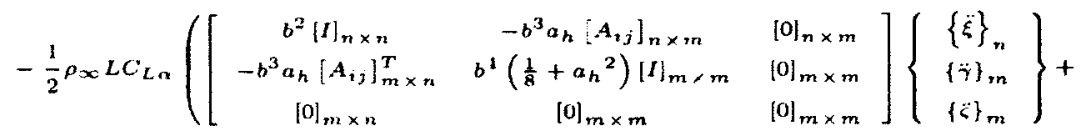

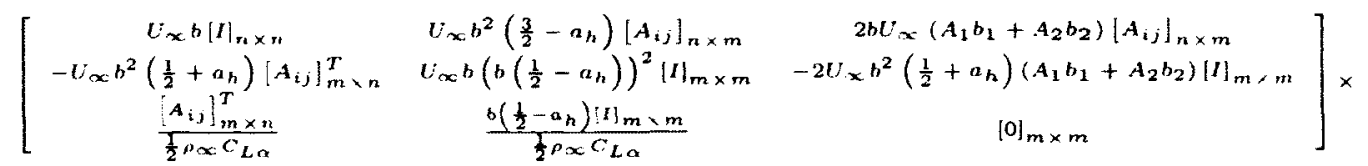

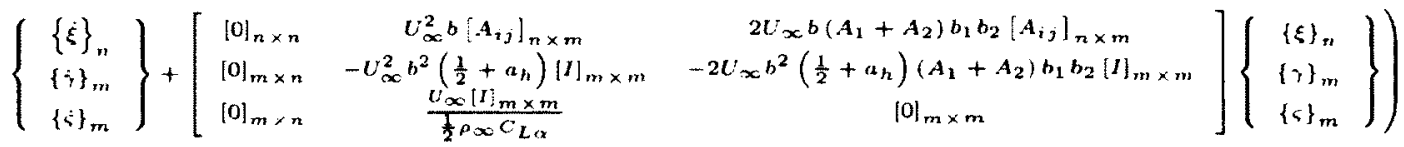

where $M_{s t r}, B_{s t r}$ and $K_{s t r}$ are the inertia, damping and stiffness structural matrices of the system with quasisteady aerodynamic loading.

Frequency Domain:

$$
\begin{aligned}
& {\left[M_{s t r}\right]\left\{\begin{array}{c}
\{\ddot{\xi}\}_{n} \\
\{\ddot{\gamma}\}_{m}
\end{array}\right\}+\left[B_{s t r}\right]\left\{\begin{array}{c}
\{\dot{\xi}\}_{n} \\
\{\dot{\gamma}\}_{m}
\end{array}\right\}+\left[K_{s t r}\right]\left\{\begin{array}{c}
\{\xi\}_{n} \\
\{\gamma\}_{m}
\end{array}\right\}=} \\
& =-\frac{1}{2} \rho_{\infty} L U_{\infty}^{2} C_{L \alpha}\left[\left[\begin{array}{cc}
\frac{b^{2}}{U_{\infty}^{2}}[I]_{n \times n} & -\frac{b^{3} a_{h}}{U_{\infty}^{2}}\left[A_{i j}\right]_{n \times m} \\
-\frac{b^{3} a_{h}}{U_{\infty}^{2}}\left[A_{i j}\right]_{m \times n}^{T} & \frac{b^{4}\left(\frac{1}{8}+a_{h}^{2}\right)}{U_{\infty}^{2}}[I]_{m \times m}
\end{array}\right]\left\{\begin{array}{c}
\ddot{\xi}(t)_{n} \\
\ddot{\gamma}(t)_{m}
\end{array}\right\}\right. \\
& +\left[\begin{array}{cc}
\frac{2 b C(k)}{U_{\infty}}[I]_{n \times n} & \frac{b^{2}\left(1+2 C(k)\left(\frac{1}{2}-a_{h}\right)\right)}{U_{\infty}}\left[A_{i j}\right]_{n \times m} \\
-\frac{2 b^{2} C(k)\left(\frac{1}{2}+a_{h}\right)}{U_{\infty}}\left[A_{i j}\right]_{m \times n}^{T} & \frac{b^{3}\left(\frac{1}{2}-a_{h}\right)\left(1-2 C(k)\left(\frac{1}{2}+a_{h}\right)\right)}{U_{\infty}}[I]_{m \times m}
\end{array}\right]\left\{\begin{array}{c}
\dot{\xi}(t)_{n} \\
\dot{\gamma}(t)_{m}
\end{array}\right\} \\
& \left.+\left[\begin{array}{cc}
{[0]_{n \times n}} & 2 b C(k)\left[A_{i j}\right]_{n \times m} \\
{[0]_{m \times n}} & -2 b^{2} C(k)\left(\frac{1}{2}+a_{h}\right)[I]_{m \times m}
\end{array}\right]\left\{\begin{array}{c}
\xi(t)_{n} \\
\gamma(t)_{m}
\end{array}\right\}\right]
\end{aligned}
$$




\section{Appendix F}

\section{Basis Functions}

The derivation of the fundamental bending and torsional basis functions is detailed in [83].

These basis functions are the vibration modes of the uncoupled, undamped uniform cantilever.

Flexural modes:

$$
\begin{aligned}
\Psi_{1}(x) & =\left[\cos \left(\frac{1.8751 x}{L}\right)-\cosh \left(\frac{1.8751 x}{L}\right)\right. \\
& \left.-\left[\frac{\cos (1.8751)+\cosh (1.8751)}{\sin (1.8751)+\sinh (1.8751)}\right]\left(\sin \left(\frac{1.8751 x}{L}\right)-\sinh \left(\frac{1.8751 x}{L}\right)\right)\right] \\
\Psi_{2}(x) & =\left[\cos \left(\frac{4.69409 x}{L}\right)-\cosh \left(\frac{4.69409 x}{L}\right)\right. \\
& \left.-\left[\frac{\cos (4.69409)+\cosh (4.69409)}{\sin (4.69409)+\sinh (4.69409)}\right]\left(\sin \left(\frac{4.69409 x}{L}\right)-\sinh \left(\frac{4.69409 x}{L}\right)\right)\right] \\
\Psi_{3}(x) & =\left[\cos \left(\frac{7.85476 x}{L}\right)-\cosh \left(\frac{7.85476 x}{L}\right)\right. \\
& \left.-\left[\frac{\cos (7.85476)+\cosh (7.85476)}{\sin (7.85476)+\sinh (7.85476)}\right]\left(\sin \left(\frac{7.85476 x}{L}\right)-\sinh \left(\frac{7.85476 x}{L}\right)\right)\right]
\end{aligned}
$$

Torsional modes:

$$
\Omega_{1}(x)=\sin \left(\frac{\pi}{2 L} x\right) \quad \Omega_{2}(x)=\sin \left(\frac{3 \pi}{2 L} x\right) \quad \Omega_{3}(x)=\sin \left(\frac{5 \pi}{2 L} x\right)
$$

These mode shapes are plotted in fig. (D.1). The wing span $L$ is s set to $6.096 \mathrm{~m}$. 

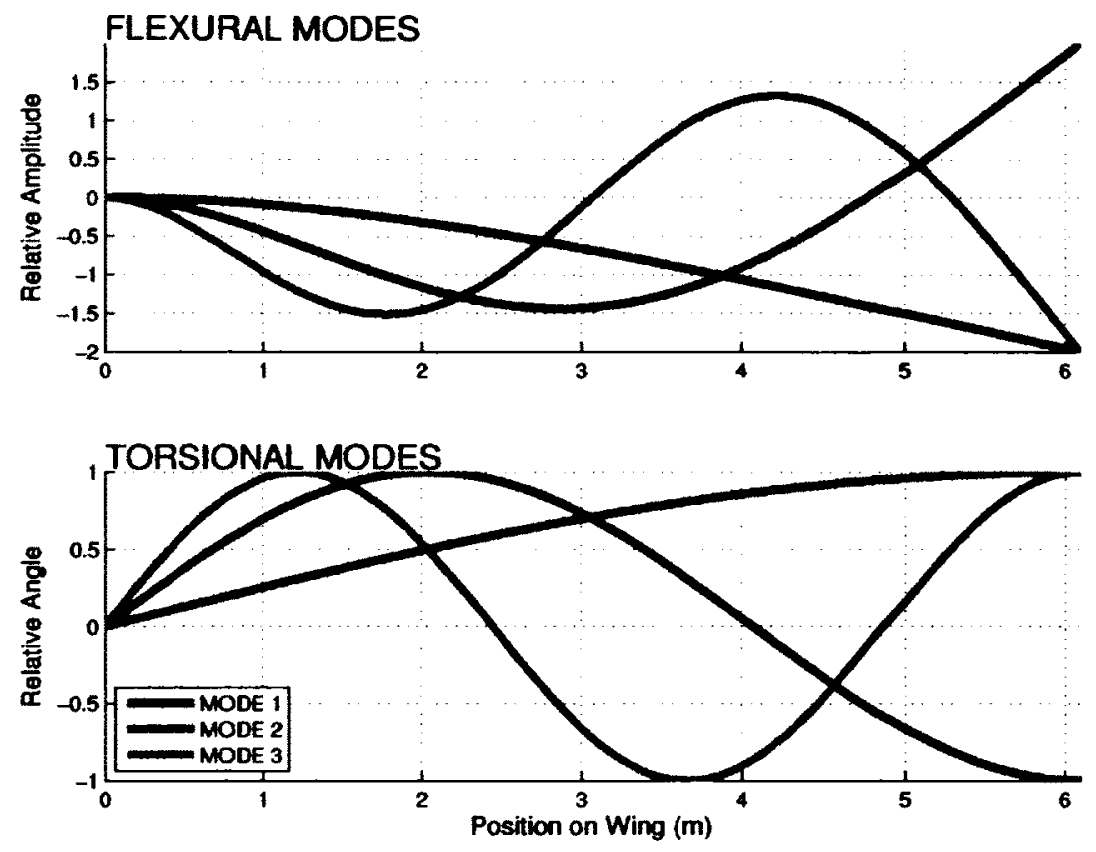

Figure D.1: Top figure depicts first 3 flexural mode shapes; bottom figure depicts first 3 torsional mode shapes 


\section{Bibliography}

[1] Y. Fung. An Introduction to the Theory of Aeroelasticity. John Wiley \& Sons Inc., New York, 1955.

[2] H. Flomenhoft. The Revolution in Structural Dynamics. Dynaflo Pr., 1997.

[3] I. Garrick and W. Reed III. Historical development of aircraft flutter. Journal of Aircraft, 18(11):897-912, 1981.

[4] E. Hirschel, H. Prem, and G. Madelung. Aeronautical Research in Germany from Lilienthal until Today. Springer, 2004.

[5] D. Pitt et al. Probabilistic aeroelastic analysis and its implications on flutter margin requirements. In 49th Structural Dynamics and Materials Conference, Schaumburg, IL, april 2008. AIAA/ASME/ASCE/AHS/ASC. 2008-2198.

[6] M. Shinozuka. Monte carlo solution of structural dynamics. Int. Journal of Computers \& Structures, 2:855-874, 1972.

[7] D. Moss and H. Benaroya. A discrete inverse vibration problem with parameter uncertainty. Applied Mathematics and Computation, 69(2-3):313-333, 1995.

[8] D. Ghiocel. Stochastic field models for advanced engineering applications. In Structural Dynamics and Materials Conference, Seattle, WA, april 2001. AIAA. 2001-1322.

[9] N. Lindsley. Uncertainty propagation through an aircraft system aeroelastic model. In 45th Aerospace Sciences Meeting and Exhibit, Reno, NV, january 2007. AIAA. 2007-1336.

[10] $M$. Chen et al. A stochastic analysis of transient two-phase flow in heterogeneous porous media. Water resources research, 42:1-14, 2006.

[11] G. Schueller and H. Pradlwarter. Uncertainty analysis of complex structural systems. Int. J. for Num. Met. in Eng., 80:881-913, 2009.

[12] H. Benaroya and E. Weinstein. Some remarks on random eigenvalues and structural dynamic models. In 29th Aerospace Sciences Meeting, Reno, NV, january 1995. AIAA.

[13] T. Theodorsen. General theory of aerodynamic instability and the mechanism of flutter. Technical report, 1935.

[14] T. Theodorsen and I. Garrick. Mechanism of flutter - a theoretical and experimental investigation of the flutter problem. Technical report, 1940. 
[15] M. Goland. The flutter of a uniform cantilever wing. Applied Mechanics, 12(4):A-197A-208, 1945.

[16] P. Marzocca, L. Librescu, and W. Silva. Aeroelastic response and flutter of swept aircraft wings. AIAA, 40(5):801-812, 2002.

[17] S. Fazelzadeh et al. Effects of rolling maneuver on divergence and flutter of aircraft wing store. Journal of Aircraft, 47(1):64-70, 2010.

[18] J. Schwars et al. Improved flutter boundary prediction for an isolated two-degree-offreedom airfoil. Journal of Aircraft, 46(6):2069-2076, 2009.

[19] E. Albano and W. Rodden. A doublet-lattice method for calculationg lift distributions on oscillating surfaces in subsonic flows. AIAA, 7(2):279-285, 1969.

[20] J. Moran. An Introduction to Theoretical and Computational Aerodyanmics. John Wiley \& Sons Inc., 1984.

[21] J. Anderson. Fundamentals of Aerodynamics. McGraw-Hill, 3rd edition, 2001.

[22] N. Minh et al. Aeroelastic complex mode analysis for coupled gust response of the akashi kaikyo bridge model. Journal of Wind Engineering and Industrial Aerodynamics, 88:307$324,2000$.

[23] O. Oiseth, A. Ronnquist, and R. Sibgjornsson. Finite element formulation of the selfexcited forces for time-domain assessment of wind-induced dynamic response and flutter stability limit of cable-supported bridges. Journal of Finite Elements in Analysis and Design, 50:173-183, 2012.

[24] J. Kim et al. Numerical investigation on the aeroelastic instability of a complete aircraft model. JSME Int. J., 48(2):212-217, 2005.

[25] J. Thomas, E. Dowell, and K. Hall. Modeling viscous transonic limit-cycle oscillation behavior using a harmonic balance approach. Journal of Aircraft, 41(6):1266-1274, 2004.

[26] C. Peng and J. Han. Numerical investigation of the effects of structural geometric and material nonlinearities on limit-cycle oscillation of a cropped delta wing. Journal of Fluids and Structures, 27:611-622, 2011.

[27] B. Castro, J. Ekaterinaris, and M. Platzer. Navier stokes analysis of wind-tunnel interference on transonic airfoil flutter. AIAA, 40(7):1269-1276, 2002.

[28] T. Miyata. Historical view of long-span bridge aerodynamics. J. of Wind Eng. and Indust. Aerodynamics, 91:1393-1410, 2003.

[29] M. Hansen. Aeroelastic instability problems for wind turbines. Wind Energy, 10:551-577, 2007.

[30] B. Yang. Integral formulas for non-self-adjoint distributed dynamic systems. AIAA, $34(10): 2132-2139,1996$. 
[31] M. Eldred, V. Venkayya, and W. Anderson. New mode tracking methods in aeroelastic analysis. AIAA, 33(7):1292-1299, 1995.

[32] C. Hoen. An engineering interpretation of the complex eigensolution of linear dynamic systems. In Conference and Exposition on Structural Dynamics, Orlando, FL, january 2005. IMAC.

[33] D. Ewins. Modal Testing theory, practice and application. Research Studies Press ltd., 2nd edition, 2000.

[34] N. Zimmerman. Elementary static aerodynamics adds significance and scope in flutter analysis. In Symposium on Structural Dynamics of High Speed Flight, Los Angeles, CA, april 1961. AIAA.

[35] C. Pettit. Uncertainty quantification in aeroelasticity: Recent results and research challenges. Journal of Aircraft, 41(5):1217-1229, 2004.

[36] F. Borello, E. Cestino, and G. Frulla. Structural uncertainty effect on classical wing flutter characteristics. Journal of Aerospace Engineering, 23(4):327-338, 2010.

[37] D. Ghosh and R. Ghanem. Random eigenvalue analysis of an airframe. In 45th Structural Dynamics and Materials Conference, Palm Springs, CA, april 2004. AIAA/ASME/ASCE/AHS/ASC. 2004-1533.

[38] D. Zhang and Z. Lu. An efficient, high-order perturbation approach for flow in random porous media via karhunen-loeve and polynomial expansions. Journal of Wind Engineering and Industrial Aerodynamics, 99:718-728, 2011.

[39] G. Abumeri and C. Chamis. Probabilistic structural analysis of a composite rotor for advanced engine applications. In 46th Structural Dynamics and Materials Conference, Austin, TX, april 2005. AIAA/ASME/ASCE/AHS/ASC. 2005-2216.

[40] D. Ghosh, R. Ghanem, and J. Red-Horse. Analysis of eigenvalues and modal interaction of stochastic systems. AIAA Journal, 43(10):2196-2201, 2005.

[41] R. Ghanem. Uncertainty quantification in computational and prediction science. Int. J. Numer. Meth. Engng, 80:671-672, 2009.

[42] M. Kurdi, N. Lindsley, and P. Beran. Uncertainty quantification of the goland wing's flutter boundary. In Atmospheric Flight Mechanics Conference and Exhibit, Hilton Head, SC, august 2007. AIAA. 2007-6309.

[43] D. Lobitz. Parameter sensitivities affecting the flutter speed of a mw-sized blade. In 43rd Aerospace Sciences Meeting and Exhibit, Reno, NV, january 2005. AIAA. 2005-0972.

[44] P. Bansal, D. Pitt, and N. Gurtowski. Effects of probabilistic aeroelastic uncertainties on flutter prediction. In International Forum on Aeroelasticity and Structural Dynamics, Paris, France, june 2011. IFASD.

[45] M. Shinozuka and E. Lenoe. A probabilistic model of for spacial distribution of material properties. Engineering Fracture Mechanics, 8:217-227, 1976. 
[46] M. Shinozuka and C. Astill. Random eigenvalue problems in structural analysis. AIAA, 10(4):456-462, april 1972.

[47] N. Lindsley, P. Beran, and C. Pettit. Effects of uncertainty on nonlinear plate aeroelastic response. In 43rd Structural Dynamics and Materials Conference, Denver, CO, april 2002. AIAA/ASME/ASCE/AHS/ASC. 2002-1271.

[48] N. Lindsley, P. Beran, and C. Pettit. Effects of uncertainty on nonlinear plate response in supersonic flow. In 9th Multidisciplinary Analysis and Optimization Conference, Atlanta, GA, september 2002. AIAA/ISSMO. 2002-5600.

[49] S. Murugan, R. Ganguli, and D. Harursampath. Effects of structural uncertainty on aeroelastic response of composite helicopter rotor. In 48th Structural Dynamics and Materials Conference, Honolulu, HI, april 2007. AIAA/ASME/ASCE/AHS/ASC. 2007-2298.

[50] C. Siva, M. Murugan, and R. Ganguli. Uncertainty quantification in helicopter performance using monte carlo simulations. Journal of Aircraft, 48(5):1503-1511, 2011.

[51] H. Millwater, K. Griffin, and D. Wieland. Probabilistic analysis of an advanced fighter/attack aircraft composite wing structure. In 41st Structural Dynamics and Materials Conference, Atlanta, GA, april 2000. AIAA/ASME/ASCE/AHS/ASC. 2000-1567.

[52] M. Sexstone. Aircraft structural mass property prediction using conceptual-level structural analysis. In 57th SAWE Annual Conference, Wichita, KS, May 1998. No2410.

[53] H. Benaroya. Random eigenvalues, algebraic methods and structural dynamic models. Applied Mathematics and Computation, 52(1):37-66, november 1992.

[54] B. Danowsky, F. Chavez, and M. Brenner. Formulation of an aircraft structural uncertainty model for robust flutter predictions. In 45th Structural Dynamics and Materials Conference, Palm Springs, CA, april 2004. AIAA/ASME/ASCE/AHS/ASC. 2004-1853.

[55] L. Becerra and I. Hernandez. Evaluation of the air density uncertainty: The effect to the correlation of input quantities and higher order terms in the taylor series expansion. Measurement Science and Technology, 17:2545-2550, 2006.

[56] D. Oh and L. Librescu. Free vibration and reliability of composite cantilevers featuring uncertain properties. Reliability Engineering and System Safety, 56:265-272, 1997.

[57] R. Ganesan. Probabilistic analysis of non-self adjoint mechanical systems with uncertain parameters. Int. J. Solid Structures, 33(5):675-688, 1994.

[58] R. Ghanem and D. Ghosh. Efficient characterization of the random eigenvalue problem in a polynomial chaos decomposition. International Journal for Numerical Methods in Engineering, 72:486-504, 2007.

[59] M. Ghommem et al. Stochastic modeling of incident gust effects on aerodynamic lift. Journal of Aircraft, 47(5):1720-1727, 2010.

[60] D. Millman, P. King, and P. Beran. Airfoil pitch-and-plunge bifurcation behavior with fourier chaos expansions. Journal of Aircraft, 42(2):376-384, 2005. 
[61] J. Witteveen, A. Loeven, and H. Bijl. Quantifying the effect of physical uncertainties in unsteady fluid-structure interaction problems. In 48th Structural Dynamics and Materials Conference, Honolulu, HI, april 2007. AIAA/ASME/ASCE/AHS/ASC. 2007-1942.

[62] J. Witteveen and $\mathrm{H}$. Bijl. Uncertainty quantification in fluid-structure interaction simulations using a simplex elements stochastic collocation approach. In 19th Computational Fluid Dynamics, San Antonio, TX, june 2009. AlAA. 2009-3671.

[63] D. Millman, P. King, R. Maple, and P. Beran. Predicting uncertainty propagation in a highly nonlinear system with a stochastic projection method. In 45th Structural Dynamics and Materials Conference, Palm Springs, CA, april 2004. AIAA/ASME/ASCE/AHS/ASC. 2004-1613.

[64] G. Loeven, J. Witteveen, and H. Bijl. Efficient uncertainty quantification in computational fluid-structure interactions. In 47th Structural Dynamics and Materials Conference, Newport, RI, May 2006. AIAA/ASME/ASCE/AHS/ASC. 2006-1634.

[65] G. Loeven, J. Witteveen, and H. Bijl. Probabilistic collocation: An efficient non-intrusive approach for arbitrarily distributed parametric uncertainties. In 45th Structural Dynamics and Materials Conference, Reno, NV, January 2007. AIAA. 2007-317.

[66] G. Loeven et al. Dynamic stall flutter analysis with uncertainties using multi-element probabilistic collocation. In 48th Structural Dynamics and Materials Conference, Honolulu, HI, april 2007. AIAA/ASME/ASCE/AHS/ASC. 2007-1964.

[67] A. Doostan and G. Iaccarino. Breaking the curse of dimensionality for a class of pdes with stochastic inputs. Center for Turbulence Research, pages 19-30, 2008.

[68] C. Chung, S. Shin, and T. Kim. Development and verification of an aircraft flutter analysis with uncertainty. In 48th Structural Dynamics and Materials Conference, Honolulu, HI, april 2007. AIAA/ASME/ASCE/AHS/ASC. 2007-2369.

[69] S. Castravete and R. Ibrahim. Effect of stiffness uncertainties on the flutter of a cantilever wing. AIAA Journal, 46(4):925-935, 2008.

[70] N. Zimmerman and J. Weissenburger. Prediction of flutter onset speed based on flight testing at subcritical speeds. Journal of Aircraft, 1(4):190-202, 1964.

[71] R. Lind. Flutter margins for multimode unstable couplings with associated flutter confidence. Journal of Aircraft, 46(5): 1563-1568, 2009.

[72] D. Poirel, S. Dunn, and J. Porter. Flutter-margin method accounting for modal parameter uncertainties. Journal of Aircraft, 42(5):1236-1243, 2005.

[73] J. Heeg. Stochastic characterization of flutter using historical wind tunnel data. In 48th Structural Dynamics and Materials Conference, Honolulu, HI, april 2007. AIAA/ASME/ASCE/AHS/ASC. 2007-1769.

[74] W. Silva et al. Advanced flutter and lco prediction tools for flight test risk and cost reduction - an international collaborative program for t\&e support. In U.S. Air Force T\&E Days, Nashville. TN, december 2005. AIAA. 
[75] S. Adhikari and M. Friswell. Random eigenvalue problems in structural dynamics. april 2004.

[76] S. Adhikari et al. Eigenvalue density of linear stochastic dynamical systems: A random matrix approach. Journal of Sound and Vibration, 331(5): 1042-1058, 2012.

[77] H. Meidani and R. Ghanem. A stochastic modal decomposition framework for the analysis of structural dynamics under uncertainties. In 53rd Structural Dynamics and Materials Conference, Honolulu, HI, april 2012. AIAA/ASME/ASCE/AHS/ASC.

[78] M. Mignolet and C. Soize. Nonparametric stochastic modeling of structures with uncertain boundary conditions and uncertain coupling between substructures. In 49th Structural Dynamics and Materials Conference, Schaumburg, IL, april 2008. AIAA/ASME/ASCE/AHS/ASC. 2008-2291.

[79] H. Khodaparast, J. Motterhead, and K. Badcock. Propagation of structural uncertainty to linear aeroelastic stability. Computers and Structures Journal, 88:223-236, 2010.

[80] T. Reddy, S. Mital, and G. Stefko. Probabilistic aeroelastic analysis of turbomachinery components. In 42nd Structural Dynamics and Materials Conference, Seattle, WA, april 2001. AIAA. 2001-1453.

[81] S. Adhikari. Rates of change of eigenvalues and eigenvectors in damped dynamic system. AIAA, 39(11):1452-1457, 1999.

[82] R. Plaut and K. Huseyin. Derivatives of eigenvalues and eigenvectors in non-self adjoint systems. AIAA, 11(2):250-251, 1973.

[83] D. Hodges and G. Pierce. Introduction to Structural Dynamics and Aeroelasticity. Press Syndicate of the University of Cambridge, 2002.

[84] R. Bisplinghoff, H. Ashley, and R. Halfman. Aeroelasticity. Addison-Wesley Publishing Company Inc., 2nd edition, 1957.

[85] O. Kirillov and A. Seyranian. Collapse of the keldysh chains and stability of continuous nonconservative systems. SIAM Review, 64(4):1383-1407, 2004.

[86] R. Plaut. Determining the nature of instability in nonconservative problems. AIAA, 10(7):967-968, 1972.

[87] F. Tisseur and K. Meerbergen. The quadratic eigenvalue problem. SIAM Review, 43(2):235-286, 2001.

[88] R. T. Jones. The unsteady lift of a wing of finite aspect ratio. Technical report, NACA Report 681, 1940.

[89] D. Poirel. Random Dynamics of a Structurally Nonlinear Airfoil in Turbulent Flow. PhD thesis, McGill University, Department of Mechanical Engineering, McGill University, Montreal, Canada, february 2001.

[90] J. Bendat and A. Piersol. Random Data. John Wiley \& Sons, 2nd edition, 1986. 
[91] M. Paidoussis. Fluid Structure Interactions Slender Bodies and Axial Flow, volume 1. Academic Press, 1st edition, 1998.

[92] R. Clough and J. Penzien. Dynamics of Structures. Computers and Structures Inc., 2nd edition, 2003. 